This page intentionally left blank. 


\title{
Sexually Transmitted Disease Surveillance 2017
}

\section{Division of STD Prevention September 2018}

\author{
U.S. Department of Health and Human Services \\ Centers for Disease Control and Prevention
}

National Center for HIV/AIDS, Viral Hepatitis, STD, and TB Prevention

Division of STD Prevention

Atlanta, Georgia, 30329-4027 


\section{Surveillance and Data Management Branch}

Division of STD Prevention

National Center for HIV/AIDS, Viral Hepatitis, STD, and TB Prevention

Centers for Disease Control and Prevention

Jim Braxton

Darlene Davis

Brian Emerson

Elaine Flagg

Jeremy Grey

LaZetta Grier

Alesia Harvey

Sarah Kidd

Jennifer Kim

Kristen Kreisel

Eloisa Llata

Kerry Mauk

Rodney Presley

Viani Ramirez

Steven Shapiro

Sancta St. Cyr

Mark Stenger

Elizabeth Torrone

Hillard Weinstock

Emily Weston

Niketta Womack

Others contributing to the production and dissemination of this publication

\section{Division of STD Prevention}

Diane Ballard

Gail Bolan

Harrell Chesson

Keith Davis

Lori Elmore

Jaeyoung Hong

Joseph Kang

Ellen Kersh

Mary McFarlane

Cau Pham

Raul Romaguera

Salina Smith

Guoyu Tao

Jo Valentine

National Center for HIV/AIDS, Viral Hepatitis, STD, and TB Prevention

Daniel Johnson

Brian Katzowitz

Rachel Powell

Publication of this report would not have been possible without the contributions of the state and territorial health departments, STD control programs, and public health laboratories that provided surveillance data to CDC. 


\section{Copyright Information}

All material contained in this report is in the public domain and may be used and reprinted without special permission; however, citation as to source is appreciated.

\section{Suggested Citation}

Centers for Disease Control and Prevention. Sexually Transmitted Disease Surveillance 2017. Atlanta: U.S. Department of Health and Human Services; 2018.

\section{Web Site}

The online version of this report is available at https://www.cdc.gov/std/stats

\section{Selected STD Surveillance and Prevention References and Web Sites}

STD Surveillance Reports 1993-2016

https://www.cdc.gov/std/stats/

\section{STD Data in the NCHHSTP AtlasPlus}

https://www.cdc.gov/nchhstp/atlas/

\section{STD Data on Wonder}

https://wonder.cdc.gov/std.html

\section{STD Data Management \& Information Technology}

https://www.cdc.gov/std/Program/data-mgmt.htm

\section{STD Fact Sheets}

https://www.cdc.gov/std/healthcomm/fact sheets.htm

\section{STD Treatment Guidelines}

https://www.cdc.gov/STD/treatment/

\section{STD Program Evaluation Guidelines}

https://www.cdc.gov/std/program/pupestd.htm

\section{STD Program Operation Guidelines}

https://www.cdc.gov/std/program/GL-2001.htm

Recommendations for Public Health Surveillance of Syphilis in the United States https://www.cdc.gov/std/SyphSurvReco.pdf

Gonococcal Isolate Surveillance Project (GISP)

https://www.cdc.gov/std/gisp/default.htm

STD Surveillance Network (SSuN)

https://www.cdc.gov/std/ssun/default.htm

\section{National Health and Nutrition Examination Survey (NHANES)}

https://www.cdc.gov/nchs/nhanes/index.htm 
This page intentionally left blank. 
STDs have long been an underestimated opponent in the public health battle. A 1997 Institute of Medicine (IOM) report described STDs as "hidden epidemics of tremendous health and economic consequence in the United States," and stated that the "scope, impact, and consequences of STDs are under recognized by the public and healthcare professionals." Since well before this report published, and two decades later, those facts remain unchanged.

Yet not that long ago, gonorrhea rates were at historic lows, syphilis was close to elimination, and we were able to point to advances in STD prevention, such as better chlamydia diagnostic tests and more screening, contributing to increases in detection and treatment of chlamydial infections. That progress has since unraveled. The number of reported syphilis cases is climbing after being largely on the decline since 1941, and gonorrhea rates are now increasing. This is especially concerning given that we are slowly running out of treatment options to cure Neisseria gonorrhoeae. Many young women continue to have undiagnosed chlamydial infections, putting them at risk for infertility.

Half of STDs are among young people ages 15 to 24 years. ${ }^{2}$ These infections can lead to long-term health consequences, such as infertility; they can facilitate HIV transmission; and they have stigmatized entire subgroups of Americans. Beyond the impact on an individual's health, STDs are also an economic drain on the US healthcare system, costing billions annually. ${ }^{3}$ To complicate the matter, STD public health programs are increasingly facing challenges and barriers in achieving their mission.

It is imperative that federal, state, and local programs employ strategies that maximize long-term population impact by reducing STD incidence and promoting sexual, reproductive, maternal, and infant health. The resurgence of syphilis, and particularly congenital syphilis, is not an arbitrary event, but rather a symptom of a deteriorating public health infrastructure and lack of access to health care. It is exposing hidden, fragile populations in need that are not getting the health care and preventive services they deserve. This points to our need for public health and health care action for each of the cases in this report, as they represent real people, not just numbers.

We also need to modernize surveillance to move beyond counting only those cases in persons who have access to diagnosis and treatment, to develop innovative strategies to understand the burden of disease in those who may not access care, and to improve our surveillance systems to collect the information needed to target prevention activities. Further, it will be important for us to measure and monitor the adverse health consequences of STDs, such as ocular and neurosyphilis, pelvic inflammatory disease, ectopic pregnancy, infertility, HIV, congenital syphilis, and neonatal herpes.

It is my hope that in future years, we will be reporting on progress, instead of more health inequity in our society. This is our challenge and our call to effectively respond to the information shared in this report.

Gail Bolan, M.D.

Director, Division of STD Prevention

National Center for HIV/AIDS, Viral Hepatitis, STD, and TB Prevention

US Centers for Disease Control and Prevention

\section{References}

1. Eng TR, Butler WT, editors; Institute of Medicine (US). Summary: The hidden epidemic: Confronting sexually transmitted diseases. Washington (DC): National Academy Press; 1997. p. 43.

2. Satterwhite CL, Torrone E, Meites E, et al. Sexually transmitted infections among US women and men: Prevalence and incidence estimates, 2008. Sex Transm Dis 2013; 40(3):187-193. DOI: 10.1097/ OLQ.0b013e318286bb53. Review.

3. Owusu-Edusei K Jr, Chesson HW, Gift TL, et al. The estimated direct medical cost of selected sexually transmitted infections in the United States, 2008. Sex Transm Dis 2013; 40(3):197-201. DOI: 10.1097/ OLQ.0b013e318285c6d2. 
This page intentionally left blank. 


\section{Preface}

Sexually Transmitted Disease Surveillance 2017 presents statistics and trends for STDs in the United States through 2017. This annual publication is intended as a reference document for policy makers, program managers, health planners, researchers, and others who are concerned with the public health implications of these diseases. The figures and tables in this edition supersede those in earlier publications of these data.

The surveillance information in this report is based on the following sources of data: (1) notifiable disease reporting from state and local STD programs; (2) projects that monitor STD positivity and prevalence in various settings, including the National Job Training Program, the STD Surveillance Network, and the Gonococcal Isolate Surveillance Project; and (3) national surveys and other data collection systems implemented by federal and private organizations.

Four STDs are nationally notifiable, chlamydia, gonorrhea, syphilis, and chancroid, and state and local STD control programs provide CDC with case reports for these conditions. These case reports are the data source for many of the figures and most of the statistical tables in this publication; however, it is important to note that these case reports reflect only a portion of STDs occurring in the US population. First, other common STDs, such as human papillomavirus (HPV) and herpes simplex virus (HSV) are not nationally notifiable diseases. Additionally, STDs are often asymptomatic and may not be diagnosed; therefore, case report data underestimate the number of infections that occurred.

Sexually Transmitted Disease Surveillance 2017 consists of four sections: the National Profile, the Special Focus Profiles, the Tables, and the Appendix. The National Profile section contains figures that provide an overview of STD morbidity in the United States. The accompanying text identifies major findings and trends for selected STDs. The Special Focus Profiles section contains figures and text that describe STDs in selected populations that are a focus of national and state prevention efforts. The Tables section provides statistical information about STDs at county, metropolitan statistical area, regional, state, and national levels. The Appendix includes information on how to interpret the STD surveillance data used to produce this report, as well as information about Healthy People 2020 STD objectives and progress toward meeting these objectives, Government Performance and Results Act goals and progress toward meeting these goals, and STD surveillance case definitions.
Any comments and suggestions that would improve future publications are appreciated and should be sent to:

Director, Division of STD Prevention

National Center for HIV/AIDS, Viral Hepatitis, STD, and TB Prevention

Centers for Disease Control and Prevention

1600 Clifton Road NE, Mailstop US12-2

Atlanta, Georgia 30329-4027 
This page intentionally left blank. 


\section{Guide to Acronyms}

\begin{tabular}{|c|c|}
\hline $\mathrm{AI} / \mathrm{AN}$ & American Indians/Alaska Natives \\
\hline ARLN & Antibiotic Resistance Laboratory Network \\
\hline $\mathrm{CDC}$ & Centers for Disease Control and Prevention \\
\hline CI & confidence interval \\
\hline CIA & chemiluminescence immunoassay \\
\hline $\mathrm{CIN} 2+$ & cervical intraepithelial neoplasia grades 2 and 3 \\
\hline $\mathrm{CS}$ & congenital syphilis \\
\hline CSF & cerebrospinal fluid \\
\hline CSTE & Council of State and Territorial Epidemiologists \\
\hline $\mathrm{CT}$ & Chlamydia \\
\hline EIA & enzyme immunoassay \\
\hline EP & ectopic pregnancy \\
\hline FTA-ABS & fluorescent treponemal antibody absorbed \\
\hline $\mathrm{GC}$ & Gonorrhea \\
\hline GISP & Gonococcal Isolate Surveillance Project \\
\hline GU & Guam \\
\hline HCUP & Healthcare Cost and Utilization Project \\
\hline $\mathrm{HD}$ & health department \\
\hline HEDIS & Healthcare Effectiveness Data and Information Set \\
\hline HMOs & health maintenance organizations \\
\hline HIV & human immunodeficiency virus \\
\hline HP2020 & Healthy People 2020 \\
\hline $\mathrm{HPV}$ & human papillomavirus \\
\hline $\mathrm{HSV}$ & herpes simplex virus \\
\hline IHC & immunohistochemistry \\
\hline MHA-TP & microhemagglutination assay for antibody to Treponema pallidum \\
\hline $\mathrm{MICs}$ & minimum inhibitory concentrations \\
\hline MPC & mucopurulent cervicitis \\
\hline MSAs & metropolitan statistical areas \\
\hline MSM & gay, bisexual, and other men who have sex with men \\
\hline MSMW & men who have sex with both men and women \\
\hline MSW & men who have sex with women only \\
\hline NAATs & nucleic acid amplification tests \\
\hline NCHHSTP & National Center for HIV/AIDS, Viral Hepatitis, STD, and TB Prevention \\
\hline $\mathrm{NCHS}$ & National Center for Health Statistics \\
\hline NHOPI & Native Hawaiians/Other Pacific Islanders \\
\hline NDTI & National Disease and Therapeutic Index \\
\hline NETSS & National Electronic Telecommunications System for Surveillance \\
\hline NGU & nongonococcal urethritis \\
\hline NHANES & National Health and Nutrition Examination Survey \\
\hline NJTP & National Job Training Program \\
\hline NNDSS & National Notifiable Diseases Surveillance System \\
\hline OMB & Office of Management and Budget \\
\hline $\mathrm{P} \& \mathrm{~S}$ & primary and secondary \\
\hline PCR & polymerase chain reaction \\
\hline PID & pelvic inflammatory disease \\
\hline PR & Puerto Rico \\
\hline RPR & rapid plasma reagin \\
\hline $\mathrm{SSuN}$ & STD Surveillance Network \\
\hline STD & sexually transmitted disease \\
\hline STI & sexually transmitted infection \\
\hline TP-PA & T. pallidum particle agglutination \\
\hline VDRL & Venereal Disease Research Laboratory \\
\hline VI & Virgin Islands \\
\hline WBC & white blood cell \\
\hline
\end{tabular}


This page intentionally left blank. 


\section{Table of Contents}

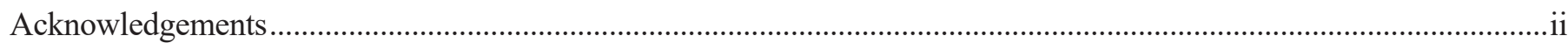

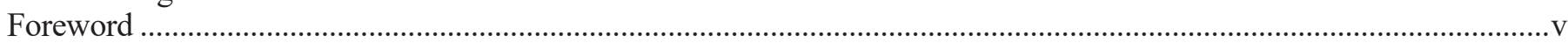

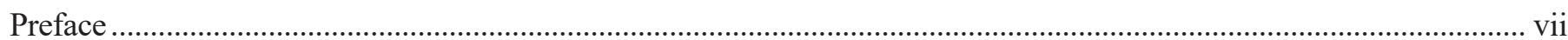

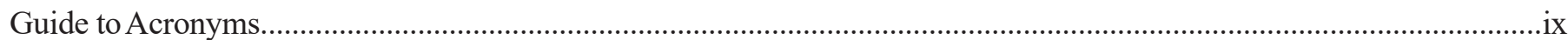

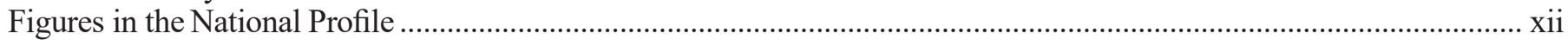

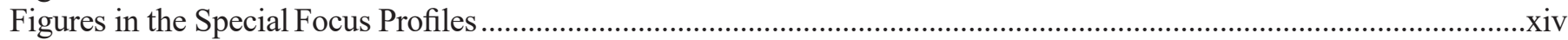

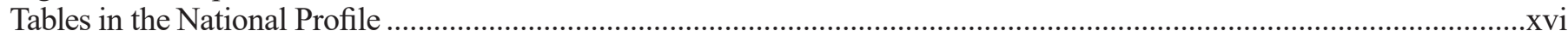

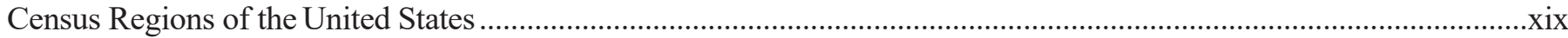

\section{National Profile}

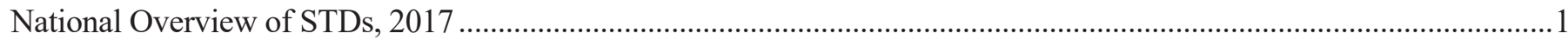

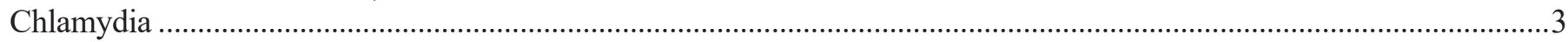

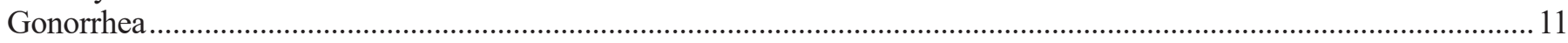

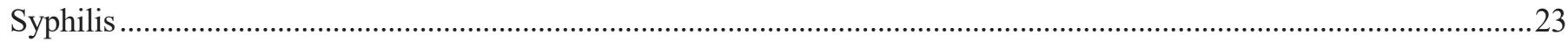

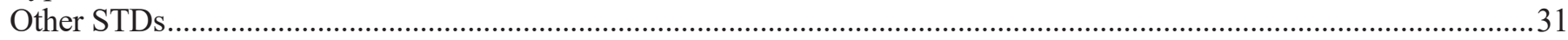

\section{Special Focus Profiles}

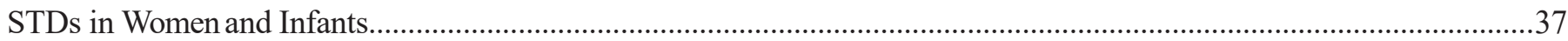

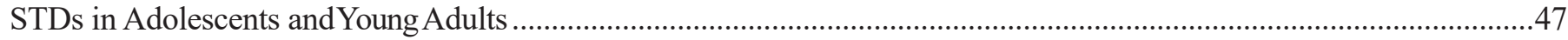

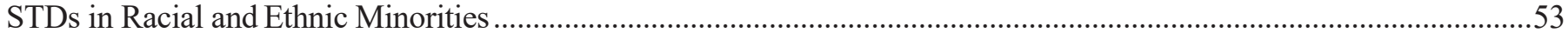

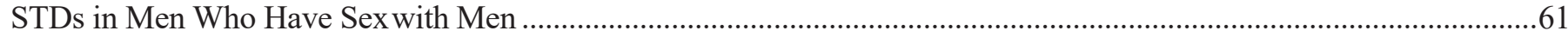

\section{Tables}

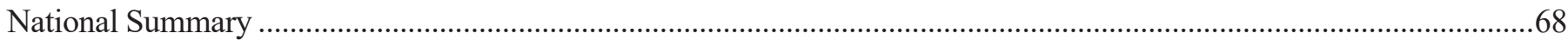

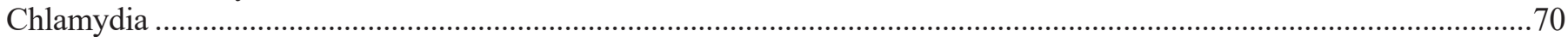

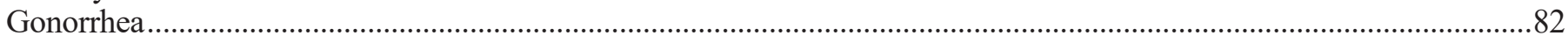

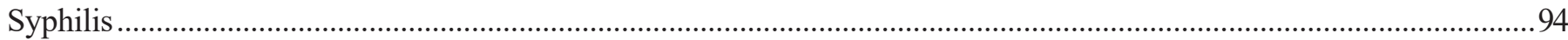

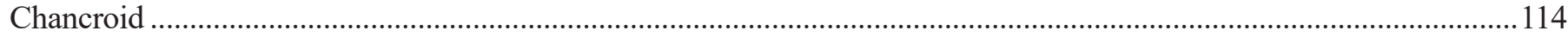

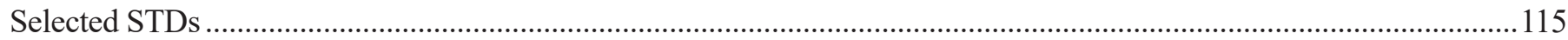

\section{Appendix}

A. Interpreting STD Surveillance Data

TableA1. Selected STDs - Percentage of Unknown, Missing, or Invalid Values for Selected Variables

by State and by Nationally NotifiableSTD, 2017......

TableA2. Reported Cases of STDs by Reporting Source and Sex, UnitedStates, 2017 ….....................................127

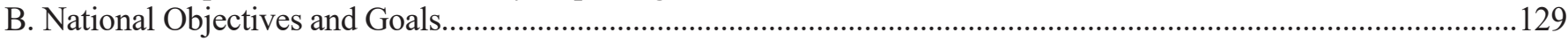

Table B1. Healthy People 2020 (HP2020) Sexually Transmitted Diseases Objectives ............................................130

Table B2. Government Performance and Results Act (GPRA) Sexually Transmitted Diseases Goals,

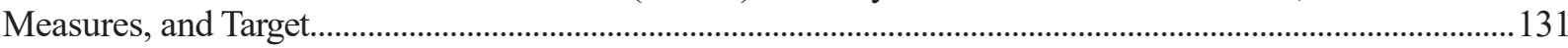

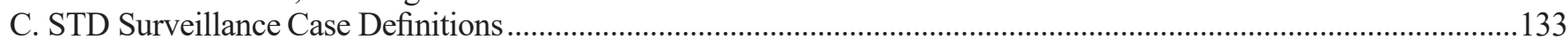

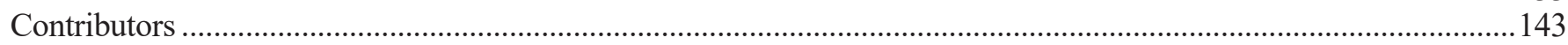




\section{Chlamydia}

Figure 1.

Figure 2.

Figure 3.

Figure 4.

Figure 5.

Figure 6.

Chlamydia — Rates of Reported Cases by Sex, United States, 2000-2017 .............................................. 4

Chlamydia — Rates of Reported Cases by Region, United States, 2008-2017.......................................... 4

Chlamydia - Rates of Reported Cases by State, United States and Outlying Areas, 2017 ........................5

Chlamydia — Rates of Reported Cases by County, United States, 2017 .................................................... 5

Chlamydia — Rates of Reported Cases by Age Group and Sex, United States, 2017.................................. 6

Chlamydia - Rates of Reported Cases Among Women Aged 15-44 Years by Age Group,

United States, 2008-2017...

Figure 7. Chlamydia - Rates of Reported Cases Among Men Aged 15-44 Years by Age Group,

United States, 2008-2017 .......................................................................................................................

Figure 8. Chlamydia - Rates of Reported Cases by Race and Hispanic Ethnicity, United States,

Figure 9. $\quad$ Chlamydia - Percentage of Reported Cases Among Men by Reporting Source,

Figure 10. Chlamydia - Percentage of Reported Cases Among Women by Reporting Source,

United States, 2008-2017 ................................................................................................

Figure 11. Chlamydia — National Estimates of Prevalence Among Persons Aged 14-39 Years by Sex,
Race, Hispanic Ethnicity, or Age Group, National Health and Nutrition Examination Survey

(NHANES), 2013-2016....

Figure 12. Chlamydia - National Estimates of Prevalence Among Sexually-Active Women Aged

14-39 Years by Race, Hispanic Ethnicity, and Age Group, National Health and Nutrition

Examination Survey (NHANES), 2013-2016..

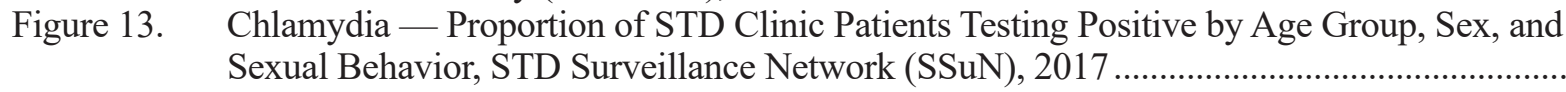

Sexual Behavior, STD Surveillance Network (SSuN), 2017.

\section{Gonorrhea}

Figure 14.

Figure 15.

Gonorrhea - Rates of Reported Cases by Year, United States, 1941-2017.....

Figure 16.

Figure 17.

Gonorrhea - Rates of Reported Cases by Region, United States, 2008-2017

Gonorrhea - Rates of Reported Cases by State, United States and Outlying Areas, 2017 ....................... 12

Figure 18.

Gonorrhea - Rates of Reported Cases by County, United States, 2017

Gonorrhea — Rates of Reported Cases by Sex, United States, 2008-2017 ............................................. 13

Figure 19.

Gonorrhea - Rates of Reported Cases by Age Group and Sex, United States, 2017 ...

Figure 20.

Gonorrhea - Rates of Reported Cases Among Women Aged 15-44 Years by Age Group,

United States, 2008-2017.

Figure 21. Gonorrhea - Rates of Reported Cases Among Men Aged 15-44 Years by Age Group,

United States, 2008-2017...

Figure 22. Gonorrhea - Rates of Reported Cases by Race and Hispanic Ethnicity,

United States, 2013-2017...

Figure 23. Gonorrhea - Percentage of Reported Cases Among Men by Reporting Source,

United States, 2008-2017...

Figure 24. Gonorrhea - Percentage of Reported Cases Among Women by Reporting Source,

United States, 2008-2017...

Figure 25. Estimated Proportion of MSM, MSW, and Women Among Gonorrhea Cases by Jurisdiction,

STD Surveillance Network (SSuN), 2017.

Figure 26. Gonorrhea - Estimated Rates of Reported Gonorrhea Cases by MSM, MSW, and Women,

STD Surveillance Network (SSuN), 2010-2017.

Figure 27. Gonorrhea - Proportion of STD Clinic Patients Testing Positive by Age Group, Sex, and

Sexual Behavior, STD Surveillance Network (SSuN), 2017 ...

Figure 28. Location of Participating Sentinel Sites and Regional Laboratories, Gonococcal Isolate

Surveillance Project (GISP), United States, 2017 
Figure 29. Neisseria gonorrhoeae - Percentage of Isolates with Elevated Azithromycin Minimum Inhibitory Concentrations (MICs) $(\geq 2.0 \mu \mathrm{g} / \mathrm{ml})$, Elevated Ceftriaxone MICs $(\geq 0.125 \mu \mathrm{g} / \mathrm{ml})$, and Elevated Cefixime MICs ( $\geq 0.25 \mu \mathrm{g} / \mathrm{ml})$, Gonococcal Isolate Surveillance Project (GISP), 2008-2017 .....18

Figure 30. Neisseria gonorrhoeae - Distribution of Gentamicin Minimum Inhibitory Concentrations (MICs) by Year, Gonococcal Isolate Surveillance Project (GISP), 2015-2017 ....

Figure 31. Neisseria gonorrhoeae - Prevalence of Tetracycline, Penicillin, or Fluoroquinolone Resistance or Elevated Cefixime, Ceftriaxone, or Azithromycin Minimum Inhibitory Concentrations (MICs), by Year - Gonococcal Isolate Surveillance Project (GISP), 2000-2017

Figure 32. Susceptibility Patterns of Neisseria gonorrhoeae Isolates to Antimicrobials, Gonococcal Isolate Surveillance Project (GISP), 2017.

Figure 33. Distribution of Primary Antimicrobial Drugs Used to Treat Gonorrhea Among Participants, Gonococcal Isolate Surveillance Project (GISP), 1988-2017......

Figure 34. Gonorrhea - Estimated Proportion of Cases by Treatment Regimen Received and Jurisdiction, STD Surveillance Network (SSuN), 2017

\section{Syphilis}

Figure 35.

Figure 36.

Figure 37.

Syphilis — Rates of Reported Cases by Stage of Infection, United States, 1941-2017... 23

Primary and Secondary Syphilis — Rates of Reported Cases by Region, United States, 2008-2017 .........24 Primary and Secondary Syphilis — Rates of Reported Cases by State, United States and Outlying

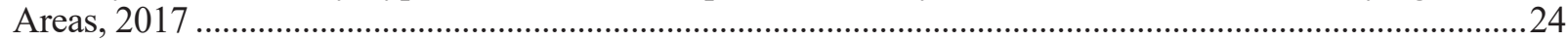

Figure 38. Primary and Secondary Syphilis — Rates of Reported Cases by County, United States, 2017 .................25

Figure 39. Primary and Secondary Syphilis — Distribution of Cases by Sex and Sexual Behavior, United States, 2017 …......................................................................................................................25

Figure 40. Primary and Secondary Syphilis - Rates of Reported Cases by Sex and Male-to-Female Rate

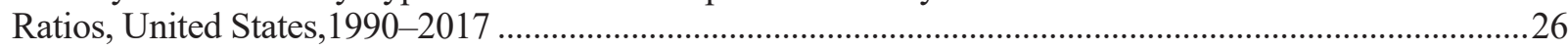

Figure 41. Primary and Secondary Syphilis - Reported Cases by Sex and Sexual Behavior, 37 States,

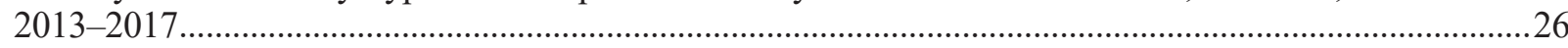

Figure 42. Primary and Secondary Syphilis - Rates of Reported Cases by Age Group and Sex,

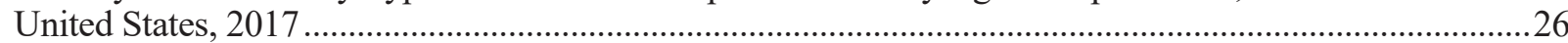

Figure 43. Primary and Secondary Syphilis - Rates of Reported Cases Among Women Aged 15-44 Years by Age Group, United States, 2008-2017.

Figure 44. Primary and Secondary Syphilis - Rates of Reported Cases Among Men Aged 15-44 Years by Age Group, United States, 2008-2017 ..... .27

Figure 45. Primary and Secondary Syphilis - Rates of Reported Cases by Race and Hispanic Ethnicity,

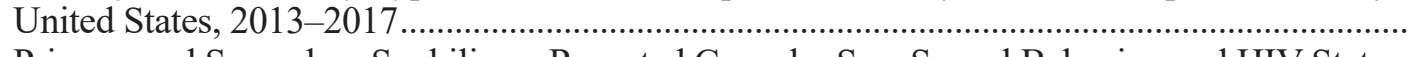

Figure 46. Primary and Secondary Syphilis — Reported Cases by Sex, Sexual Behavior, and HIV Status,

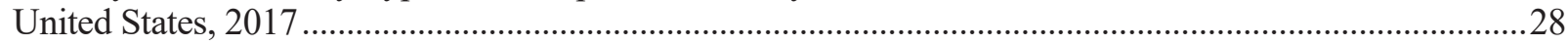

Figure 47. Primary and Secondary Syphilis — Reported Cases by Reporting Source and Sex, United States, 2008-2017......

Figure 48. Primary and Secondary Syphilis - Percentage of Reported Cases by Sex, Sexual Behavior, and Selected Reporting Sources, United States, 2017.

Figure 49. Congenital Syphilis - Reported Cases by Year of Birth and Rates of Reported Cases of Primary and Secondary Syphilis Among Women Aged 15-44 Years, United States, 2008-2017......

\section{Other STDs}

Figure 50.

Figure 51.

Figure 52.

Figure 53.

Figure 54.
Chancroid - Reported Cases by Year, United States, 1941-2017.

Female Enrollees in Private Health Plans Aged 15-39 Years, by Age Group and Year, 2007-2014 32

Anogenital Warts - Prevalence per 1000 Person-Years Among Enrollees in Private Health

Plans Aged 15-39 Years by Sex, Age Group, and Year, 2006-2014

Herpes Simplex Virus Type 2 - National Estimates of Trends in Age-Adjusted Seroprevalence

Among Persons Aged 14-49 Years by Race and Hispanic Ethnicity, National Health and Nutrition

Examination Survey (NHANES), 1999-2000 through 2015-2016

Trichomonas vaginalis and Other Vaginal Infections Among Females — Initial Visits to

Physicians' Offices, United States, 1966-2016. 


\section{STDs in Women and Infants}

Figure A. Pelvic Inflammatory Disease - Initial Visits to Physicians' Offices Among Women Aged

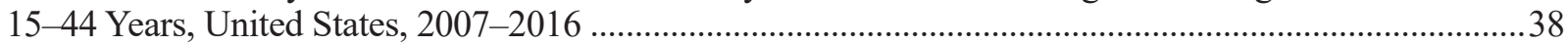

Figure B. Trends in the Percentage of Acute Pelvic Inflammatory Disease (PID) Emergency Department (ED) Visits Among Women Aged 15-44 Years by Age Group, United States, 2006-2013...................................39

Figure C. Pelvic Inflammatory Disease - National Estimates of Lifetime Prevalence Among Sexually Experienced Women Aged 18-44 Years by Race, Hispanic Ethnicity, and Previous STI Diagnosis, National Health and Nutrition Examination Survey (NHANES), 2013-2014....

Figure D. Ectopic Pregnancy — Ratio Among Commercially Insured Women with Live Births Aged 15-44 Years by Age Group, 2005-2016....

Figure E. Chlamydia - Rates of Reported Cases Among Women by State, United States and Outlying Areas, $2017 .$.

Figure F. Gonorrhea - Rates of Reported Cases Among Women by State, United States and Outlying Areas, 2017

Figure G. Chlamydia - Positivity Among Women Aged 14-39 Years by Race, Hispanic Ethnicity, and Age Group in Clinics Providing Family Planning and Reproductive Health Services, STD Surveillance Network (SSuN), 2017 ....

Figure H. Chlamydia and Gonorrhea - Rates of Reported Cases Among Infants $<1$ Year of Age by Year and Specimen Source, United States, 2013-2017

Figure I. Primary and Secondary Syphilis — Rates of Reported Cases Among Women by State, United States and Outlying Areas, 2017

Figure J. Congenital Syphilis - Rates of Reported Cases Among Infants by Year of Birth and State, United States and Outlying Areas, 2017.

\section{STDs in Adolescents and Young Adults}

Figure K. Chlamydia - Rates of Reported Cases Among Women Aged 15-24 Years by State, United States and Outlying Areas, 2017

Figure L. Chlamydia - Rates of Reported Cases Among Men Aged 15-24 Years by State, United States and Outlying Areas, 2017

Figure M. Gonorrhea - Rates of Reported Cases Among Women Aged 15-24 Years by State, United States and Outlying Areas, 2017

Figure N.

Gonorrhea - Rates of Reported Cases Among Men Aged 15-24 Years by State,

United States and Outlying Areas, 2017

Figure O. Chlamydia - Prevalence Among Women Aged 16-24 Years Entering the National Job

Training Program (NJTP) by State of Residence, United States and Outlying Areas, 2017........................51

Figure P. Chlamydia - Prevalence Among Men Aged 16-24 Years Entering the National Job Training Program (NJTP) by State of Residence, United States and Outlying Areas, 2017.......................51

Figure Q. Gonorrhea - Prevalence Among Women Aged 16-24 Years Entering the National Job Training Program (NJTP) by State of Residence, United States and Outlying Areas, 2017 ........................52

Figure R. Gonorrhea - Prevalence Among Men Aged 16-24 Years Entering the National Job Training Program (NJTP) by State of Residence, United States and Outlying Areas, 2017. 


\section{STDs in Racial and Ethnic Minorities}

Figure S. Chlamydia - Rates of Reported Cases by Race, Hispanic Ethnicity, and Sex, United States, 2017 ..........54

Figure T. Chlamydia - Rate Ratios Among Women Aged 15-24 Years by Race, Hispanic Ethnicity, and Region, United States, 2017 ...............................................................................................................5

Figure U. Gonorrhea - Rates of Reported Cases by Race, Hispanic Ethnicity and Sex, United States, 2017 ...........56

Figure V. Gonorrhea - Rate Ratios by Race, Hispanic Ethnicity, and Region, United States, 2017 ........................56

Figure W. Primary and Secondary Syphilis - Reported Cases by Sex, Sexual Behavior, Race, and Hispanic

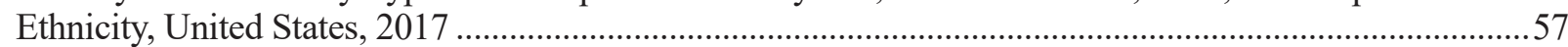

Figure X. Primary and Secondary Syphilis - Rates of Reported Cases by Race, Hispanic Ethnicity,

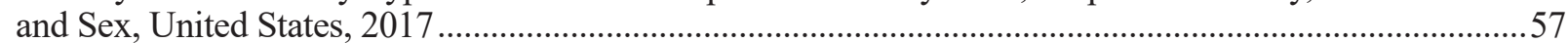

Figure Y. Primary and Secondary Syphilis - Rate Ratios by Sex, Race, Hispanic Ethnicity, and Region,

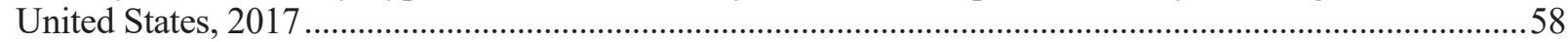

Figure Z. Congenital Syphilis — Rates of Reported Cases by Year of Birth, Race, and Hispanic Ethnicity of Mother, United States, 2008-2017

\section{STDs in Men Who Have Sex with Men}

Figure AA. Primary and Secondary Syphilis - Estimated Rates of Reported Cases Among MSM by State, United States, 2017. 62

Figure BB. Neisseria gonorrhoeae - Percentage of Urethral Isolates Obtained from MSM Attending STD Clinics, Gonococcal Isolate Surveillance Project (GISP), 1989-2017

Figure CC. Neisseria gonorrhoeae - Percentage of Urethral Isolates with Elevated Azithromycin Minimum Inhibitory Concentrations (MICs) $(\geq 2.0 \mu \mathrm{g} / \mathrm{ml})$ and Elevated Ceftriaxone MICs $(\geq 0.125 \mu \mathrm{g} / \mathrm{ml})$ by Reported Sex of Sex Partner, Gonococcal Isolate Surveillance Project (GISP), 2008-2017

Figure DD. Gonorrhea and Chlamydia - Proportion of MSM Attending STD Clinics Testing Positive for Urogenital Gonorrhea and Chlamydia by Jurisdiction, STD Surveillance Network (SSuN), 2017

Figure EE. Gonorrhea and Chlamydia - Proportion of MSM Attending STD Clinics Testing Positive for Rectal Gonorrhea and Chlamydia by Jurisdiction, STD Surveillance Network (SSuN), 2017

Figure FF. Gonorrhea - Proportion of MSM Attending STD Clinics Testing Positive for Oropharyngeal Gonorrhea by Jurisdiction, STD Surveillance Network (SSuN), 2017

Proportion of MSM Attending STD Clinics with Primary and Secondary Syphilis, Urogenital Gonorrhea, or Urogenital Chlamydia by HIV Status, STD Surveillance Network (SSuN), 2017.

Figure GG.

Gonontea, or Urogenital Chlamydia by HIV Status, STD Survellance Network (SSuN), 2017 .... .65 


\section{Tables in the National Profile}

\section{National Summary}

Table 1. Sexually Transmitted Diseases - Reported Cases and Rates of Reported Cases per 100,000 Population,

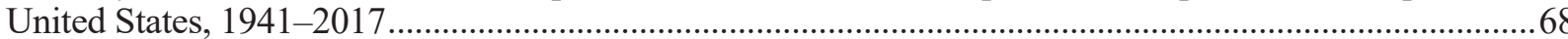

\section{Chlamydia}

Table 2.

Chlamydia - Reported Cases and Rates of Reported Cases by State, Ranked by Rates,

United States, 2017.

Table 3.

Chlamydia - Reported Cases and Rates of Reported Cases by State/Area and Region in

Alphabetical Order, United States and Outlying Areas, 2013-2017.

Table 4.

Chlamydia Among Women - Reported Cases and Rates of Reported Cases by State/Area and

Region in Alphabetical Order, United States and Outlying Areas, 2013-2017.

Table 5 .

Chlamydia Among Men - Reported Cases and Rates of Reported Cases by State/Area and

Region in Alphabetical Order, United States and Outlying Areas, 2013-2017....

Table 6.

Table 7.

Areas (MSAs) in Alphabetical Order, United States, 2013-2017...

Chlamydia Among Women - Reported Cases and Rates of Reported Cases in Selected

Metropolitan Statistical Areas (MSAs) in Alphabetical Order, United States, 2013-2017.

Table 8 .

Chlamydia Among Men - Reported Cases and Rates of Reported Cases in Selected Metropolitan

Statistical Areas (MSAs) in Alphabetical Order, United States, 2013-2017 ..............................................76

Table 9. Chlamydia - Reported Cases and Rates of Reported Cases in Counties and Independent Cities

Ranked by Number of Reported Cases, United States, 2017 ....................................................................77

Table 10 Chlamydia - Reported Cases and Rates of Reported Cases by Age Group and Sex,

United States, 2013-2017.

Table 11A. Chlamydia - Reported Cases by Race/Hispanic Ethnicity, Age Group, and Sex,

United States, 2017

Table 11B. Chlamydia - Rates of Reported Cases per 100,000 Population by Race/Hispanic Ethnicity, Age Group, and Sex, United States, 2017.

Table 12. Chlamydia Among Women Aged 15-24 Years — Reported Cases and Rates of Reported Cases by Age, United States, 2013-2017.

\section{Gonorrhea}

Table 13. Gonorrhea - Reported Cases and Rates of Reported Cases by State, Ranked by Rates, United States, 2017

Table 14. Gonorrhea - Reported Cases and Rates of Reported Cases by State/Area and Region in

Alphabetical Order, United States and Outlying Areas, 2013-2017.

Table 15. Gonorrhea Among Women - Reported Cases and Rates of Reported Cases by State/Area and

Region in Alphabetical Order, United States and Outlying Areas, 2013-2017.

Table 16.

Gonorrhea Among Men - Reported Cases and Rates of Reported Cases by State/Area and

Region in Alphabetical Order, United States and Outlying Areas, 2013-2017.....

Table 17.

Gonorrhea - Reported Cases and Rates of Reported Cases in Selected Metropolitan Statistical

Areas (MSAs) in Alphabetical Order, United States, 2013-2017...

Table 18.

Gonorrhea Among Women - Reported Cases and Rates of Reported Cases in Selected

Metropolitan Statistical Areas (MSAs) in Alphabetical Order, United States, 2013-2017.

Table 19. Gonorrhea Among Men - Reported Cases and Rates of Reported Cases in Selected

Metropolitan Statistical Areas (MSAs) in Alphabetical Order, United States, 2013-2017.

Table 20. Gonorrhea - Reported Cases and Rates of Reported Cases in Counties and Independent Cities

Ranked by Number of Reported Cases, United States, 2017.

Table 21. Gonorrhea - Reported Cases and Rates of Reported Cases by Age Group and Sex,

United States, 2013-2017...

Table 22A. Gonorrhea - Reported Cases by Race/Hispanic Ethnicity, Age Group, and Sex,

United States, 2017. 
Table 22B. Gonorrhea - Rates of Reported Cases per 100,000 Population by Race/Hispanic Ethnicity, Age Group, and Sex, United States, 2017...

Table 23. Gonorrhea Among Women Aged 15-24 Years - Reported Cases and Rates of Reported

Cases by Age, United States, 2013-2017

\section{Syphilis}

Table 24.

All Stages of Syphilis - Reported Cases and Rates of Reported Cases by State/Area and Region in Alphabetical Order, United States and Outlying Areas, 2013-2017.

Table 25 .

All Stages of Syphilis - Reported Cases and Rates of Reported Cases in Selected Metropolitan

Statistical Areas (MSAs) in Alphabetical Order, United States, 2013-2017.

Table 26.

Primary and Secondary Syphilis - Reported Cases and Rates of Reported Cases by State, Ranked

by Rates, United States, 2017.

Table 27.

and Region in Alphabetical Order, United States and Outlying Areas, 2013-2017

Table 28 .

Table 29.

by State/Area and Region in Alphabetical Order, United States and Outlying Areas, 2013-2017.

Primary and Secondary Syphilis Among Men - Reported Cases and Rates of Reported Cases by

State/Area and Region in Alphabetical Order, United States and Outlying Areas, 2013-2017.

Table 30. Primary and Secondary Syphilis - Reported Cases and Rates of Reported Cases in Selected

Metropolitan Statistical Areas (MSAs) in Alphabetical Order, United States, 2013-2017.

Table 31 .

Selected Metropolitan Statistical Areas (MSAs) in Alphabetical Order, United States, 2013-2017

Table 32 .

Primary and Secondary Syphilis Among Men - Reported Cases and Rates of Reported Cases in Selected Metropolitan Statistical Areas (MSAs) in Alphabetical Order, United States, 2013-2017 .......... 102

Table 33. Primary and Secondary Syphilis - Reported Cases and Rates of Reported Cases in Counties and Independent Cities Ranked by Number of Reported Cases, United States, 2017

Table 34 .

Table 35A. and Sex, United States, 2013-2017.

Primary and Secondary Syphilis — Reported Cases by Race/Hispanic Ethnicity, Age Group, and Sex, United States, 2017

Table 35B.

Primary and Secondary Syphilis — Rates of Reported Cases per 100,000 Population by

Race/Hispanic Ethnicity, Age Group, and Sex, United States, 2017

Table 36.

Early Latent Syphilis - Reported Cases and Rates of Reported Cases by State/Area and Region

in Alphabetical Order, United States and Outlying Areas, 2013-2017....

Table 37.

Early Latent Syphilis - Reported Cases and Rates of Reported Cases in Selected Metropolitan

Statistical Areas (MSAs) in Alphabetical Order, United States, 2013-2017....

Table 38 .

Region in Alphabetical Order, United States and Outlying Areas, 2013-2017.

Table 39.

Table 40.

Late and Late Latent Syphilis - Reported Cases and Rates of Reported Cases in Selected Metropolitan Statistical Areas (MSAs) in Alphabetical Order, United States, 2013-2017.

Congenital Syphilis - Reported Cases and Rates of Reported Cases by State, Ranked by Rates, United States, 2017

Table 41.

Congenital Syphilis - Reported Cases and Rates of Reported Cases by Year of Birth, State/Area

and Region in Alphabetical Order, United States and Outlying Areas, 2013-2017

Table 42 .

Congenital Syphilis - Reported Cases and Rates of Reported Cases per 100,000 Live Births by

Year of Birth and Race/Hispanic Ethnicity of Mother, United States, 2013-2017

\section{Chancroid}

Table 43.

Chancroid - Reported Cases and Rates of Reported Cases by State/Area in Alphabetical Order, United States and Outlying Areas, 2013-2017.

\section{Selected STDs}

Table 44. Selected STDs and Complications - Initial Visits to Physicians' Offices, National Disease and Therapeutic Index (NDTI), United States, 1966-2016 


\section{Appendix}

Table A1. Selected STDs - Percentage of Unknown, Missing, or Invalid Values for Selected Variables by State and by Nationally Notifiable STD, 2017 .......

Table A2.

Table B1.

Table B2.

Reported Cases of STDs by Reporting Source and Sex, United States, 2017

Healthy People 2020 (HP 2020) Sexually Transmitted Diseases Objectives...................................................... 130

Government Performance and Results Act (GPRA) Sexually Transmitted Diseases Goals,

Measures, and Target 


\section{Census Regions of the United States}
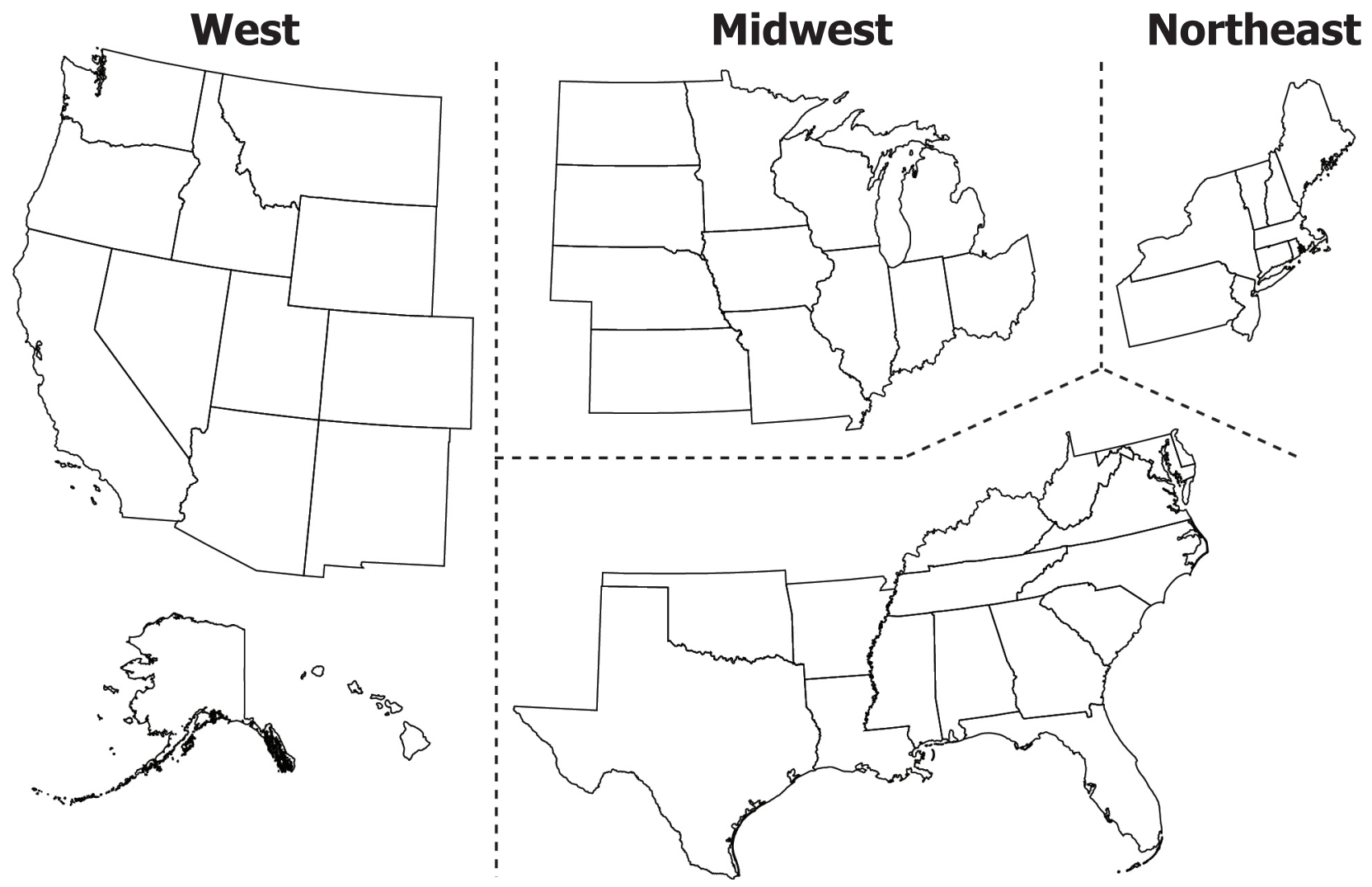

West

Alaska

Arizona

California

Colorado

Hawaii

Idaho

Montana

Nevada

New Mexico

Oregon

Utah

Washington

Wyoming
Midwest

Illinois

Indiana

Iowa

Kansas

Michigan

Minnesota

Missouri

Nebraska

North Dakota

Ohio

South Dakota

Wisconsin
South

Alabama

Arkansas

Delaware

District of Columbia

Florida

Georgia

Kentucky

Louisiana

Maryland

Mississippi

North Carolina

Oklahoma

South Carolina

Tennessee

Texas

Virginia

West Virginia
Northeast

Connecticut

Maine

Massachusetts

New Hampshire

New Jersey

New York

Pennsylvania

Rhode Island

Vermont 
This page intentionally left blank. 
other men who have sex with men (collectively referred to as MSM) being tested and diagnosed with a chlamydial infection due to increased availability of urine testing and extragenital screening.

\section{Gonorrhea}

In 2017, a total of 555,608 cases of gonorrhea were reported to CDC, making it the second most common notifiable condition in the United States. Rates of reported gonorrhea increased $75.2 \%$ since the historic low in 2009 and increased $18.6 \%$ since 2016. During 2016-2017, rates of reported gonorrhea increased among both males and females, in all regions of the United States, and among all racial and Hispanic ethnicity groups.

During 2016-2017, the rate of reported gonorrhea increased 19.3\% among men and $17.8 \%$ among women. The magnitude of the increase among men suggests either increased transmission, increased case ascertainment (e.g., through increased extra-genital screening among MSM), or both. The concurrent increase in cases reported among women, suggests parallel increases in heterosexual transmission, increased screening among women, or both.

Antimicrobial resistance remains an important consideration in the treatment of gonorrhea. Therapy with ceftriaxone and azithromycin is now the only CDC recommended treatment for gonorrhea. ${ }^{2}$ Since 2008 , the percentage of isolates with elevated ceftriaxone minimum inhibitory concentrations (MICs) has remained low and was only $0.2 \%$ in 2017. During 2014-2017, the percentage of isolates with elevated azithromycin MICs increased from $2.5 \%$ to $4.4 \%$. Continued monitoring of susceptibility patterns to these antibiotics is critical.

\section{Syphilis}

In 2017, a total of 30,644 cases of primary and secondary (P\&S) syphilis, the most infectious stages of the disease, were reported in the United States, yielding a rate of 9.5 cases per 100,000 population. Since reaching a historic low in 2000 and 2001, the rate of P\&S syphilis has increased almost every year, increasing 10.5\% during 2016-2017. Rates increased among both males and females, among all racial and Hispanic ethnicity groups, and in $72.0 \%$ of states and the District of Columbia.

During 2000-2017, the rise in the P\&S syphilis rate was primarily attributable to increased cases among men and, specifically, among MSM. In 2017, men accounted for almost $90 \%$ of all cases of P\&S syphilis and MSM accounted for $68.2 \%$ of reported $\mathrm{P} \& \mathrm{~S}$ syphilis cases among women or men with information about sex of sex partners. In states with consistent information on sex of sex partner, the number of P\&S syphilis cases increased $8.6 \%$ among MSM, 17.8\% among MSW, and $24.9 \%$ among women during 2016 2017. Among P\&S syphilis cases with known HIV-status, $45.5 \%$ of MSM, $8.8 \%$ of MSW, and $4.5 \%$ of women were HIV-positive in 2017.

The 2013 rate of congenital syphilis ( 9.2 cases per 100,000 live births) marked the first increase in congenital syphilis since 2008. Since 2013, the rate of congenital syphilis has increased each year. In 2017, there were a total of 918 reported cases of congenital syphilis, including 64 syphilitic stillbirths and 13 infant deaths. The national rate of 23.3 cases per 100,000 live births represents a $43.8 \%$ increase relative to 2016 and a $153.3 \%$ increase relative to 2013. This increase in the congenital syphilis rate has paralleled increases in $\mathrm{P} \& \mathrm{~S}$ syphilis among all women and reproductive-aged women during 2013-2017 (155.6\% and 142.8\% increase, respectively).

\section{References}

1. Institute of Medicine. The Hidden Epidemic: Confronting Sexually Transmitted Diseases. Washington DC: The National Academy Press; 1997.

2. Centers for Disease Control and Prevention. Sexually transmitted diseases treatment guidelines, 2015. MMWR Morb Mortal Wkly Rep 2015; 64(No. RR-3): 1-137. 


\section{Chlamydia}

\section{Background}

Chlamydia, caused by infection with Chlamydia trachomatis, is the most common notifiable disease in the United States. It is among the most prevalent of all STDs, and since 1994, has comprised the largest proportion of all STDs reported to CDC (Table 1). Studies also demonstrate the high prevalence of chlamydial infections in the general US population, particularly among young women. ${ }^{1}$

Chlamydial infections in women are usually asymptomatic. ${ }^{2}$ Untreated infection can result in pelvic inflammatory disease (PID), which is a major cause of infertility, ectopic pregnancy, and chronic pelvic pain. Data from randomized controlled trials of chlamydia screening suggested that screening programs can lead to a reduction in the incidence of PID. ${ }^{3,4}$ As with other inflammatory STDs, chlamydial infection could facilitate the transmission of HIV infection..$^{5}$ In addition, pregnant women infected with chlamydia can pass the infection to their infants during delivery, potentially resulting in ophthalmia neonatorum, which can lead to blindness, and pneumonia. ${ }^{6}$ Because of the large burden of disease and risks associated with infection, CDC recommends annual chlamydia screening for all sexually active women younger than age 25 years and women $\geq 25$ years at increased risk for infection (e.g., women with new or multiple sex partners). ${ }^{7}$

The Healthcare Effectiveness Data and Information Set (HEDIS) contains a measure which assesses chlamydia screening coverage of sexually active young women who receive medical care through commercial or Medicaid managed care organizations. Among sexuallyactive women aged 16-24 years in commercial health maintenance organization (HMO) plans, chlamydia screening increased from $23.1 \%$ in 2001 to $48.3 \%$ in 2016. Among sexually-active women aged 16-24 years covered by Medicaid, screening rates increased from $40.4 \%$ in 2001 to $57.3 \%$ in $2016 .{ }^{8}$ Although chlamydia screening has expanded over the past two decades, many women who are at risk are still not being tested - reflecting, in part, the lack of awareness among some health care providers and the limited resources available to support these screenings.

\section{Interpreting Rates of Reported Cases of Chlamydia}

Trends in rates of reported cases of chlamydia are influenced by changes in incidence of infection, as well as changes in diagnostic, screening, and reporting practices. As chlamydial infections are usually asymptomatic, the number of infections identified and reported can increase as more people are screened even when incidence is flat or decreasing. During 2000-2011, the expanded use of more sensitive diagnostic tests (e.g., nucleic acid amplification tests [NAATs]) likely increased the number of infections identified and reported independently of increases in incidence. Also, although chlamydia has been a nationally notifiable condition since 1995, it was not until 2000 that all 50 states and the District of Columbia required reporting of chlamydia cases. National case rates prior to 2000 reflect incomplete reporting. The increased use of electronic laboratory reporting over the last decade or so also likely increased the proportion of diagnosed cases reported. Consequently, an increasing chlamydia case rate over time may reflect increases in incidence of infection, screening coverage, and use of more sensitive tests, as well as more complete reporting. Likewise, decreases in chlamydia case rates may suggest decreases in incidence of infection or screening coverage.

\section{Chlamydia - United States}

In 2017, a total of 1,708,569 chlamydial infections were reported to $\mathrm{CDC}$ in 50 states and the District of Columbia (Table 1). This case count corresponds to a rate of 528.8 cases per 100,000 population. During 2000-2011, the rate of reported chlamydial infection increased from 251.4 to 453.4 cases per 100,000 population (Figure 1, Table 1). During 2011-2013, the rate of reported cases decreased to 443.5 cases per 100,000 population, followed by an increase in the rate of reported cases over each of the next four years. During 2016-2017, the rate increased 6.9\%, from 494.7 to 528.8 cases per 100,000 population (Figure 1, Table 1).

\section{Chlamydia by Region}

In 2017, rates of reported cases of chlamydia were highest in the South (560.4 cases per 100,000 population, $6.8 \%$ increase from 2016), followed by the West (524.3, 8.8\% increase from 2016), Midwest (514.4, 5.6\% increase from 2016), and Northeast (483.3, 6.2\% increase from 2016) (Table 3). During 2008-2012, rates of reported cases of chlamydia increased in all regions (Figure 2). During 2012-2013, rates decreased in the Northeast, Midwest, and South and remained stable in the West. During 2013-2017, rates increased in all regions, with the largest increase occurring in the West (421.1 to 524.3 cases per 100,000 population, $24.5 \%$ increase) (Figure 2, Table 3).

\section{Chlamydia by State}

In 2017, rates of reported cases of chlamydia by state ranged from 226.1 cases per 100,000 population in West 


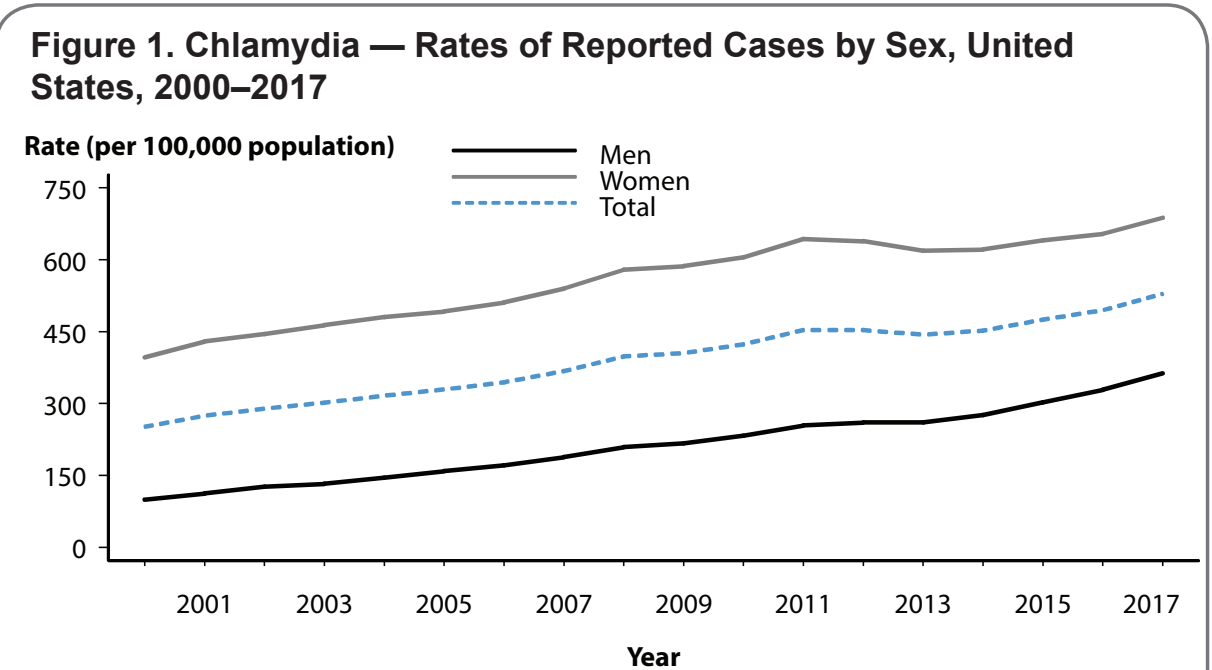

NOTE: Data collection for chlamydia began in 1984 and chlamydia was made nationally notifiable in 1995; however, chlamydia was not reportable in all 50 states and the District of Columbia until 2000. Refer to the National Notifiable Disease Surveillance System (NNDSS) website for more information: https://wwwn.cdc.gov/nndss/conditions/chlamydia-trachomatis-infection/

\section{Figure 2. Chlamydia - Rates of Reported Cases by Region, United States, 2008-2017}

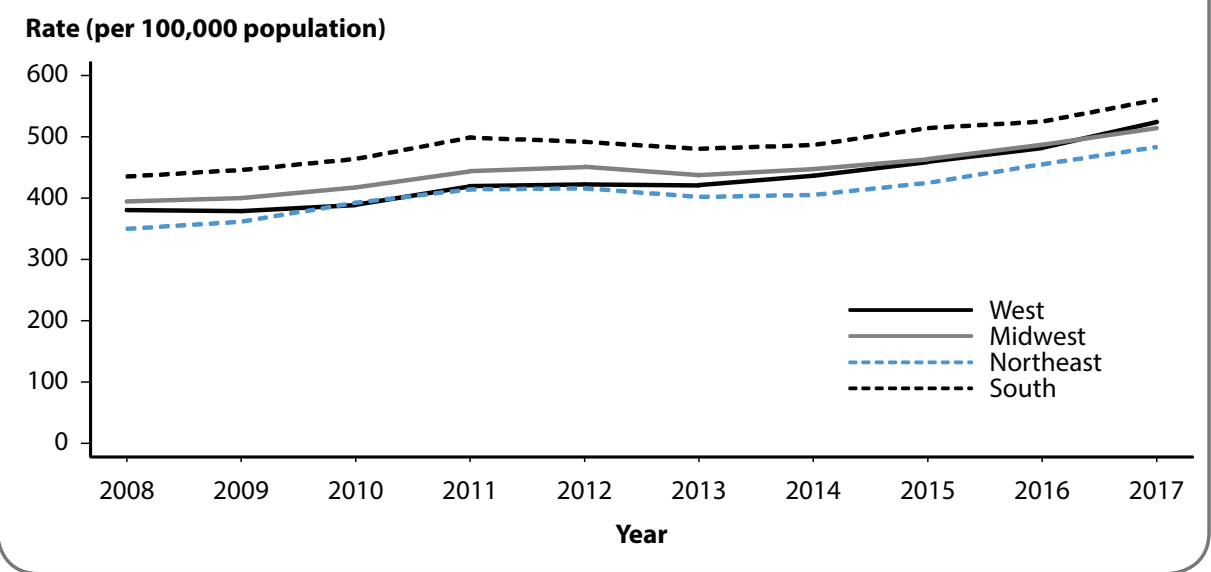

Virginia to 799.8 cases per 100,000 population in Alaska (Figure 3, Table $2)$; the rate in the District of Columbia was $1,337.0$ cases per 100,000 population (Table 3). During 20162017, rates of reported chlamydia cases increased in 46 states and the District of Columbia. The rate of reported chlamydia cases in 2017 was above the US total in 19 states (Table 2).

\section{Chlamydia by Metropolitan Statistical Area}

The rate of reported cases of chlamydia in the 50 most populous

\section{Chlamydia by County}

In $2017,630(20.1 \%)$ of 3,140 counties had rates of reported chlamydia higher than 542 cases per 100,000 population (Figure 4). Seventy counties and independent cities reported $43 \%$ of all chlamydia cases in 2017 (Table 9). Of the 70 counties and independent cities reporting the highest number of chlamydia cases, $46(65.7 \%)$ were located in the South and West (Table 9).

\section{Chlamydia by Sex}

In $2017,1,127,651$ cases of chlamydia were reported among females for a rate of 687.4 cases per 100,000 females (Table 4). After increasing each year during 2000 2011, the rate of reported chlamydia cases among females decreased during 2011-2013, followed by an increase in the rate of reported cases over each of the next four years (Figure 1). The total rate increase during 2013-2017 among females was $11.1 \%$.

Among males, 577,644 cases of chlamydia were reported in 2017 for a rate of 363.1 cases per 100,000 males (Table 5). The rate of reported cases among males increased each year during 2000-2017, with the exception of 2012-2013, when rates remained stable (Figure 1). During 2016-2017 alone, the rate among men increased $10.5 \%$; however, during 2013-2017, rates of reported cases among men increased $39.3 \%$ (compared with an $11.1 \%$ increase among women) (Tables 4 and 5). This pronounced increase among men could be attributed to either increased transmission or improved case identification (e.g., through intensified extra-genital screening efforts) among gay, bisexual, and other men who have sex with men (collectively referred to as MSM). This cannot be assessed, however, as most jurisdictions do not routinely report sex of sex partner or anatomic site of infection. 
Figure 3. Chlamydia - Rates of Reported Cases by State, United States and Outlying Areas, 2017

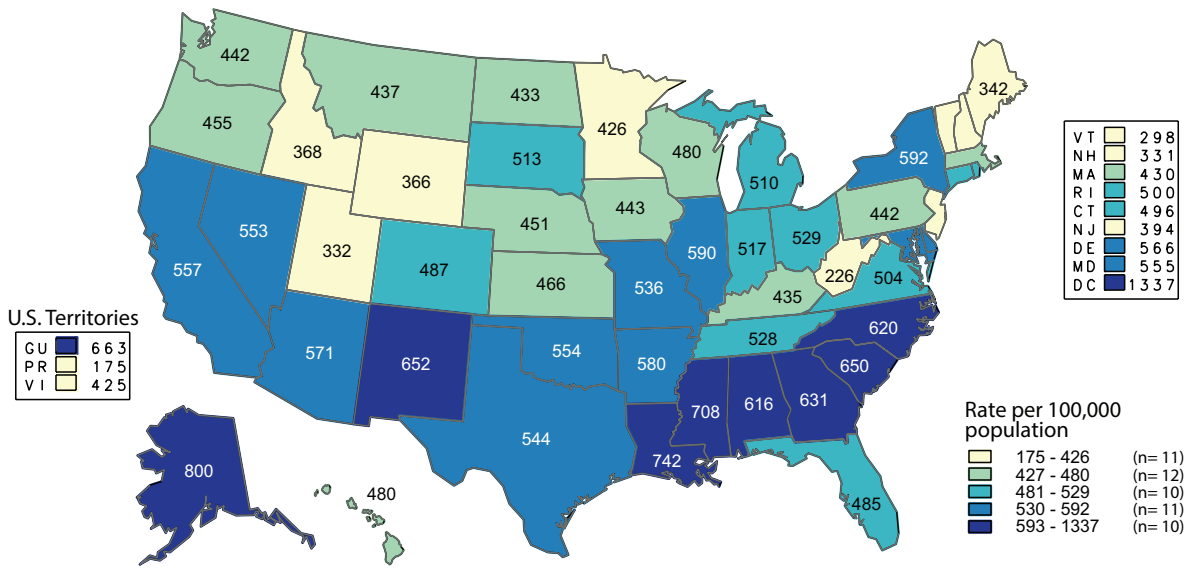

NOTE: The total rate of reported cases of chlamydia for the United States and outlying areas (including Guam, Puerto Rico, and the Virgin Islands) was 525.1 per 100,000 population. See Section A1.11 in the Appendix for more information on interpreting reported rates in the outlying areas.

ACRONYMS: $\mathrm{GU}=$ Guam; PR = Puerto Rico; VI = Virgin Islands.

Figure 4. Chlamydia - Rates of Reported Cases by County, United States, 2017

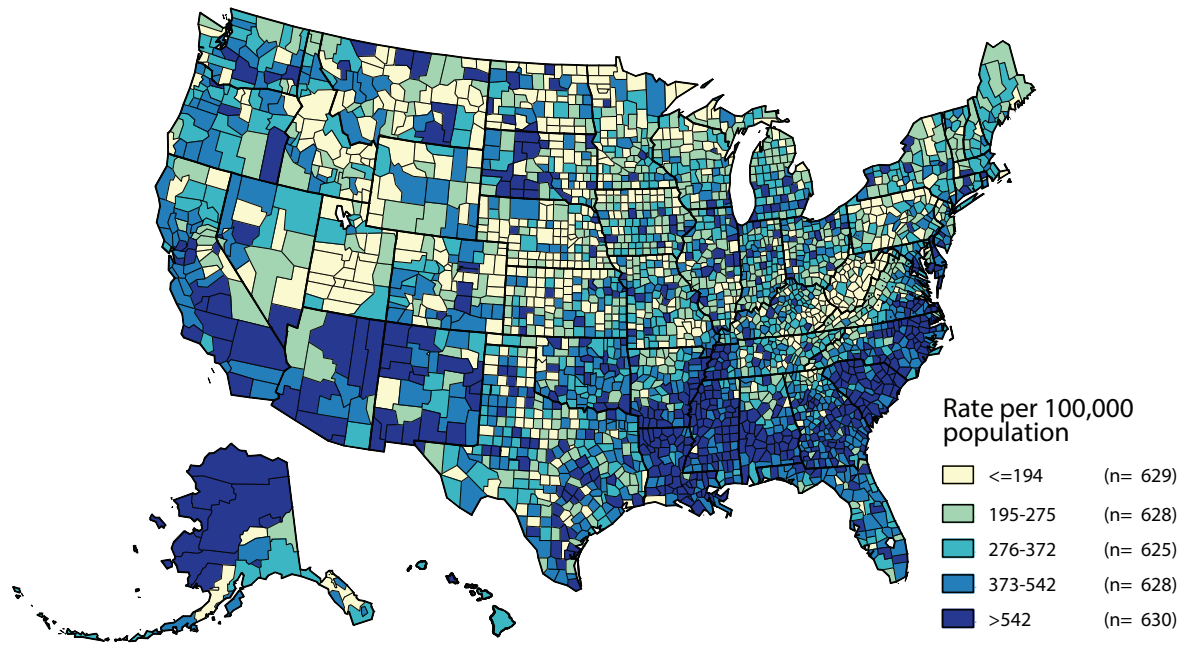

NOTE: Refer to the NCHHSTP AtlasPlus for further county-level rate information: https://www.cdc. gov/nchhstp/atlas

Despite this considerable increase in men, the rate of reported chlamydia cases among females was still about two times the rate among males in 2017, likely reflecting a larger number of women screened for this infection (Figure 1, Tables 4 and 5). The lower rate among men also suggests that many of the sex partners of women with chlamydia are not receiving a diagnosis of chlamydia or being reported as having chlamydia.

\section{Chlamydia by Age}

The rates of reported cases of chlamydia were highest among adolescents and young adults aged 15-24 years during 2013-2017 (Table 10). In 2017, the age-specific rate of reported cases of chlamydia among 15-19 year olds was 2072.4 cases per 100,000 population and the rate among 20-24 year olds was 2,820.3 cases per 100,000 population (Table $10)$.

In $2017,97.4 \%$ of all reported chlamydia cases in women were among those aged 15-44 years. The highest age-specific rates of reported cases of chlamydia in 2017 were among those aged 15-19 years (3,265.7 cases per 100,000 females) and $20-24$ years $(3,985.8$ cases per 100,000 females) (Figure 5, Table 10). Within these age groups, rates were highest among women aged 19 years $(5,398.6$ cases per 100,000 females) and 20 years $(5,141.4$ cases per 100,000 females) (Table 12).

Increases have been observed in recent years in rates of reported cases of chlamydia among all age groups in females aged 15-44 years (Figure 6). Specifically, the rate of reported cases among women aged 15-19 and 20-24 years increased over the last three and four years, respectively. The rate among 15-19 year olds increased 6.5\% during 2016-2017, with a total increase of $10.7 \%$ during 2014-2017 (2,949.3 to 3,265.7 cases per 100,000 females) (Table 10). The rate among 20-24 year olds increased $3.7 \%$ during 2016-2017, with a total increase of $10.9 \%$ during 2013-2017 $(3,594.2$ to $3,985.8$ cases per 100,000 females) (Table 10).

In $2017,94.2 \%$ of all reported chlamydia cases in men were among those aged 15-44 years. The agespecific rates of reported cases of chlamydia among men, although substantially lower than rates among women, were highest in those aged 20-24 years $(1,705.4$ cases per 100,000 males) (Figure 5, Table 10). Similar to trends in women, increases have been observed in rates of reported cases of chlamydia among all age groups in males aged 15-44 years (Figure 7). Specifically, the rate of reported cases among men aged 15-19 and 20-24 years increased 
Figure 5. Chlamydia - Rates of Reported Cases by Age Group and Sex, United States, 2017

Men

Rate (per 100,000 population)

Women

$50004000300020001000 \quad 0$ Age Group $0 \quad 10002000300040005000$

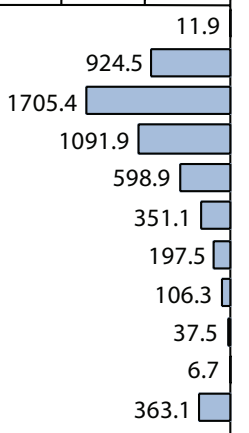

$10-14$
$15-19$
$20-24$
$25-29$
$30-34$
$35-39$
$40-44$
$45-54$
$55-64$
$65+$
Total
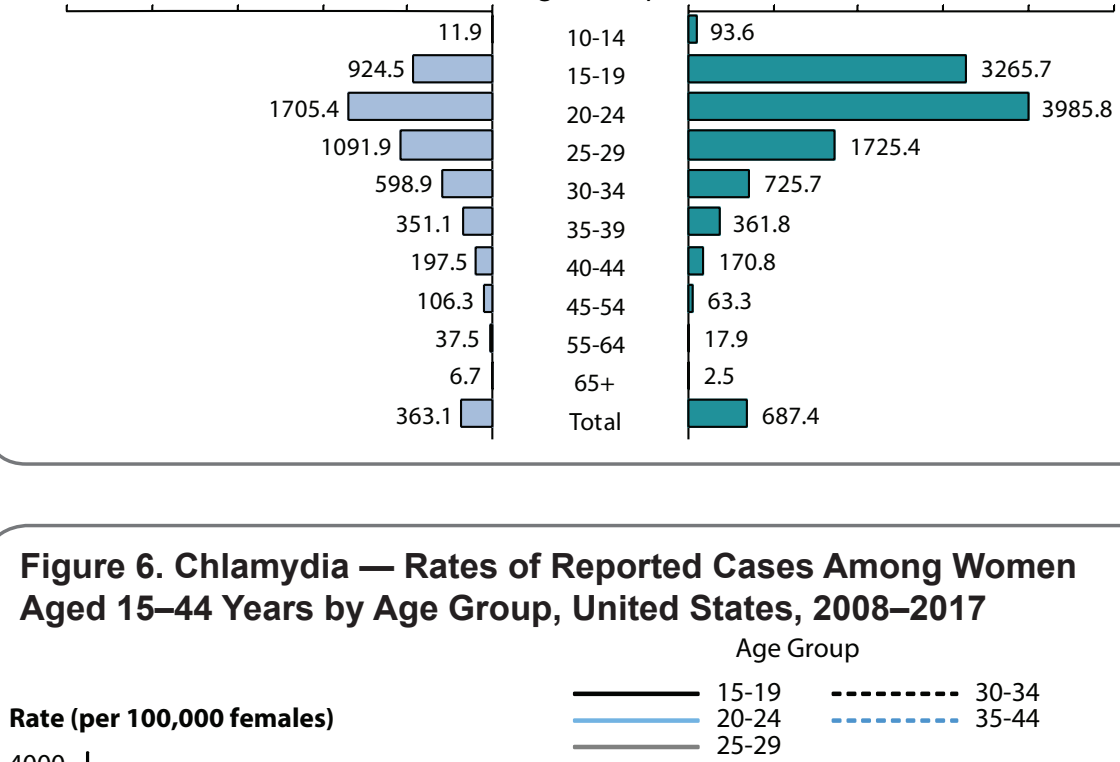

4000

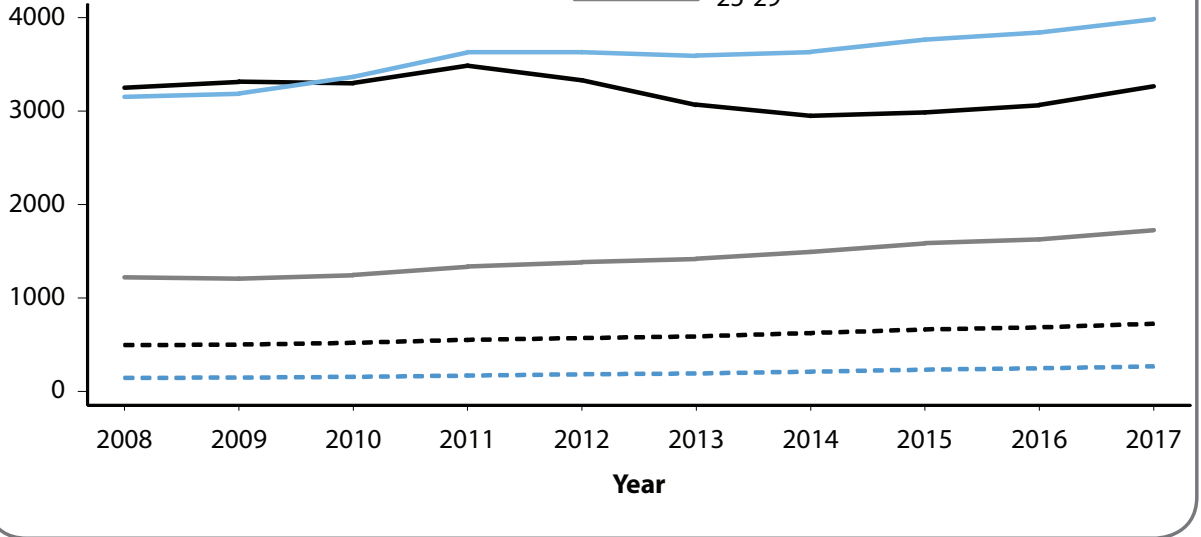

over the last three and four years, respectively. The rate among 15-19 year olds increased $11.1 \%$ during 2016-2017, with a total increase of $28.0 \%$ during $2014-2017$ (722.9 to 924.5 cases per 100,000 males). The rate among 20-24 year olds increased $7.8 \%$ during 2016-2017, with a total increase of $30.1 \%$ during $2013-2017$ $(1,310.9$ to $1,705.4$ cases per 100,000 males) (Table 10).

\section{Chlamydia by Race/Hispanic Ethnicity}

Rates of reported cases of chlamydia were highest among Black, American Indian/Alaska Native (AI/AN), and Native Hawaiian/Other Pacific
Table 11B). Overall, the rate of reported cases of chlamydia among Blacks was 5.6 times the rate among Whites $(1,175.8$ and 211.3 cases per 100,000 population, respectively). The rate among AI/ANs (781.2 cases per 100,000 population) was 3.7 times the rate among Whites. The rate among NHOPIs (715.4 cases per 100,000 population) was 3.4 times the rate among Whites. The rate among Hispanics (404.1 cases per 100,000 population) was 1.9 times the rate among Whites. The rate among Asians (129.6 cases per 100,000 population) was 0.6 times the rate among Whites.

During 2013-2017, rates of reported chlamydia cases increased among all racial and Hispanic ethnicity groups, with $\mathrm{AI} / \mathrm{ANs}$ increasing 3.7\%, Asians $29.6 \%$, Blacks $6.1 \%$, NHOPIs $19.4 \%$, Whites 20.2\%, Multirace 59.9\%, and Hispanics 10.5\% (Figure 8). During 2016-2017, rates increased among all racial and Hispanic ethnicity groups (AI/ANs: $5.0 \%$, Asians: $10.7 \%$, Blacks: 5.2\%, NHOPIs: $11.0 \%$, Whites: $5.8 \%$, Multirace: $11.4 \%$, and Hispanics 9.5\%) (Figure 8).

More information on chlamydia rates among race/Hispanic ethnicity groups can be found in the Special Focus Profiles.

\section{Chlamydia by Reporting Source}

In $2017,5.8 \%$ of chlamydia cases were reported from STD clinics, $78.6 \%$ were reported from venues outside of STD clinics, and $15.6 \%$ had an unknown reporting source (Table A2). Over time, the proportion of male cases reported from STD clinic sites has decreased substantially, from $30.5 \%$ in 2008 to $9.7 \%$ in 2017 (Figure 9). In 2017 , among women, only $3.8 \%$ of chlamydia cases were reported through an STD clinic (Table A2). A large proportion of cases among women $(31.6 \%)$ were reported from private physicians/HMOs in 2017 (Figure 10). Among men, 24.8\% of cases were reported from private physicians/HMOs (Figure 9).

\section{Chlamydia Prevalence in the Population}

The National Health and Nutrition Examination Survey (NHANES; see Section A2.4 in the Appendix) is a nationally representative survey of the US civilian, non-institutionalized population that provides an important measure of chlamydia disease burden in respondents aged 14-39 years. During 2013-2016, the overall prevalence of chlamydia 


\section{Figure 7. Chlamydia - Rates of Reported Cases Among Men Aged 15-44 Years by Age Group, United States, 2008-2017}

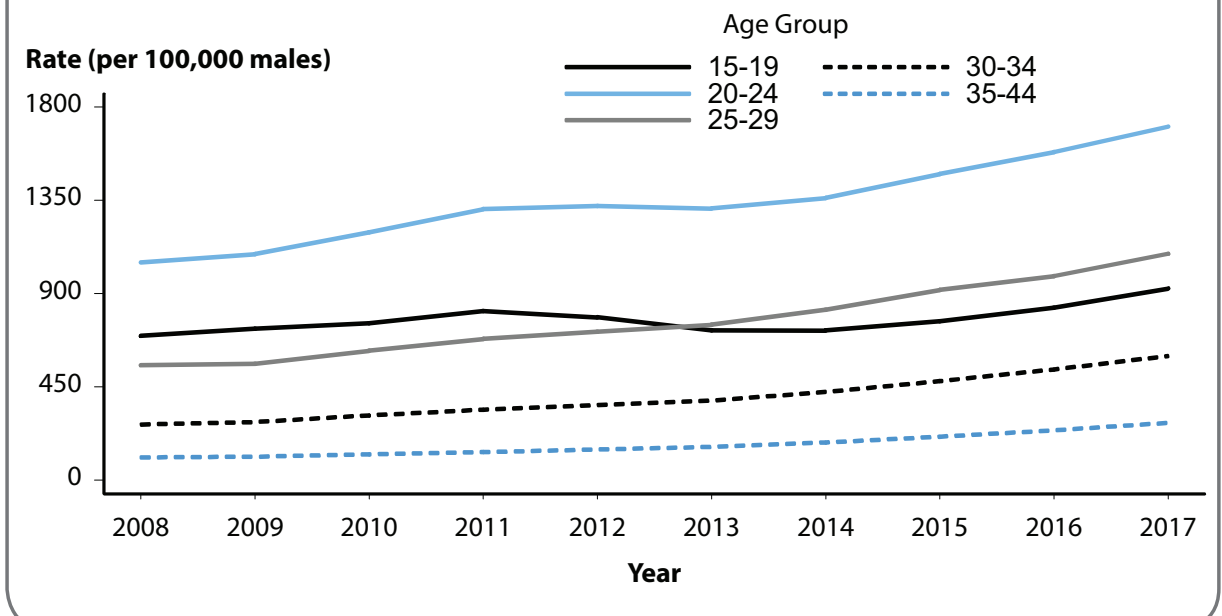

Figure 8. Chlamydia - Rates of Reported Cases by Race and
Hispanic Ethnicity, United States, 2013-2017

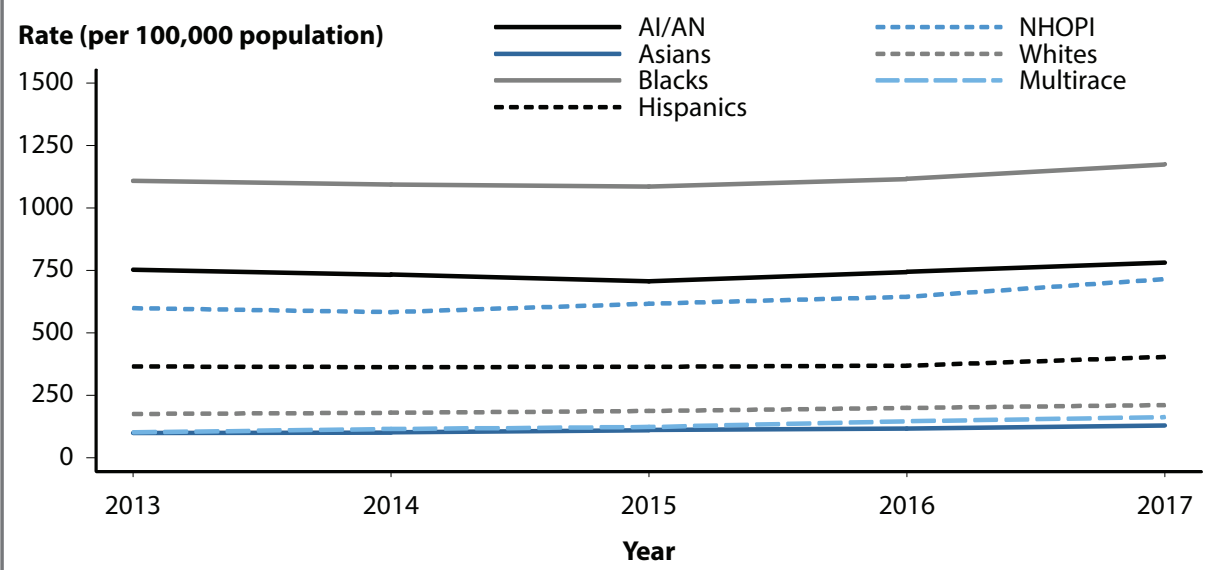

NOTE: Not all US jurisdictions reported cases in OMB-compliant Race categories in 2017. This may minimally under- or overestimate rates for Asians, NHOPI, or Multirace individuals. For completeness, data in this figure include cases reported from all jurisdictions. See Section A1.5 in the Appendix for information on reporting STD case data for race and Hispanic ethnicity.

ACRONYMS: AI/AN = American Indians/Alaska Natives; $\mathrm{NHOPI}=$ Native Hawaiians/Other Pacific Islanders; $\mathrm{OMB}=$ Office of Management and Budget.

among persons aged $14-39$ years was $1.7 \%$ (95\% Confidence Interval [CI]: 1.3-2.1) (Figure 11). Among sexually active females aged 14-24 years, the population targeted for screening, prevalence was $4.3 \%$ (95\% CI: 2.7-5.8), with the highest prevalence among Mexican American females $(10.0 \%, 95 \%$ CI: 4.0-15.9) (Figure 12).

\section{Chlamydia Positivity in Selected Populations}

The STD Surveillance Network $(\mathrm{SSuN})$ is an ongoing collaboration of 10 state, county, and city health departments collecting enhanced clinical and behavioral information among patients attending $30 \mathrm{STD}$ clinics in the SSuN jurisdictions (See Section A2.2 of the Appendix).
In 2017, the proportion of STD clinic patients testing positive for chlamydia varied by age, sex, and sexual behavior. Adolescent men who have sex with women only (MSW) had the highest positivity (39.2\%), either reflecting disproportionate testing of men with urethritis or targeted testing of partners of women diagnosed with chlamydia. Positivity among all those tested decreased with age, though the variation in positivity by age was not as pronounced for MSM (Figure 13). The overall positivity, represented by the average of the mean value by the $10 \mathrm{SSuN}$ jurisdictions, was $17.5 \%$ for MSM, $15.0 \%$ for MSW, and $11.5 \%$ for women.

\section{Chlamydia Among Special Populations}

More information on chlamydia among women of reproductive age, adolescents and young adults, MSM, and minority populations is presented in the Special Focus Profiles.

\section{Chlamydia Summary}

Chlamydia continues to be the most commonly reported nationally notifiable disease, with 1,708,569 cases reported in 2017 and increasing rates of reported cases over each of the last four years. Rates of reported chlamydia cases increased $6.9 \%$ during 2016-2017. The Southern region of the US reported the highest rate of chlamydial infection in 2017; the West reported the largest rate increase during 2016-2017. In 2017, the rate of reported cases of chlamydia in women was nearly two times the rate in men. However, during 2013-2017, the rate in men increased $39.3 \%$, whereas the rate in women increased only $11.1 \%$. Potential reasons for this considerable increase in male cases could be due to a true increase in infections or to improved screening coverage in men, especially increased extra-genital screening in MSM. 
Figure 9. Chlamydia - Percentage of Reported Cases Among Men by Reporting Source*, United States, 2008-2017

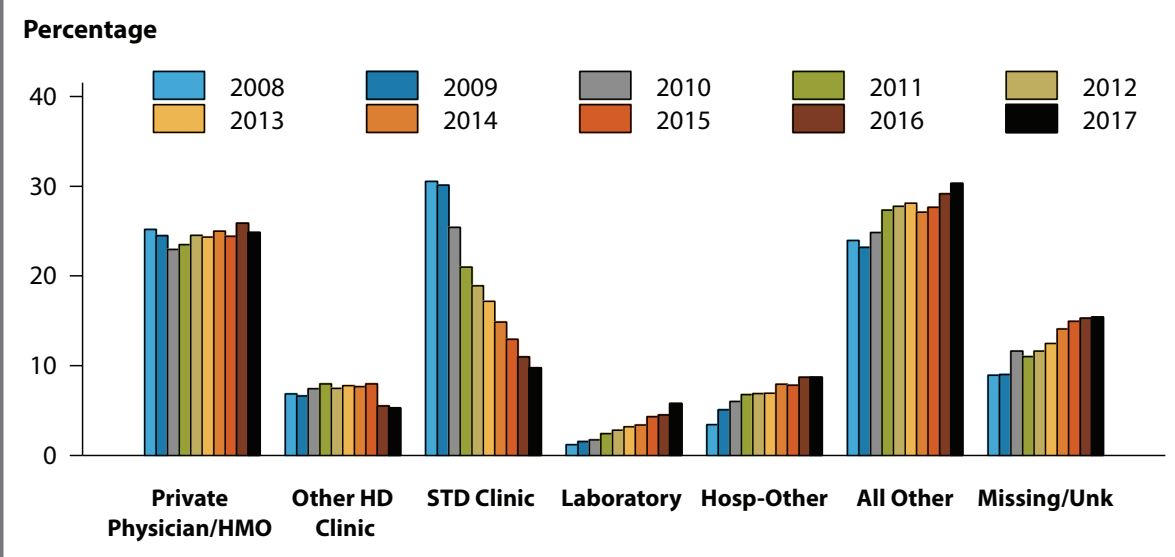

* Includes the top five reporting sources for chlamydia cases reported among men, plus those with reporting sources categorized as "All Other" and "Missing/Unknown".

NOTE: All Other includes: Family Planning, Drug Treatment, Tuberculosis Clinic, Correctional Facility, Blood Bank, Labor and Delivery, Prenatal Care, National Job Training Program, Schoolbased Clinic, Mental Health Provider, Indian Health Service, Military, Emergency Room, HIV Counseling and Testing Site, and Other.

ACRONYMS: $\mathrm{HMO}=$ health maintenance organization; $\mathrm{HD}=$ health department.

\section{Figure 10. Chlamydia - Percentage of Reported Cases Among Women by Reporting Source*, United States, 2008-2017}

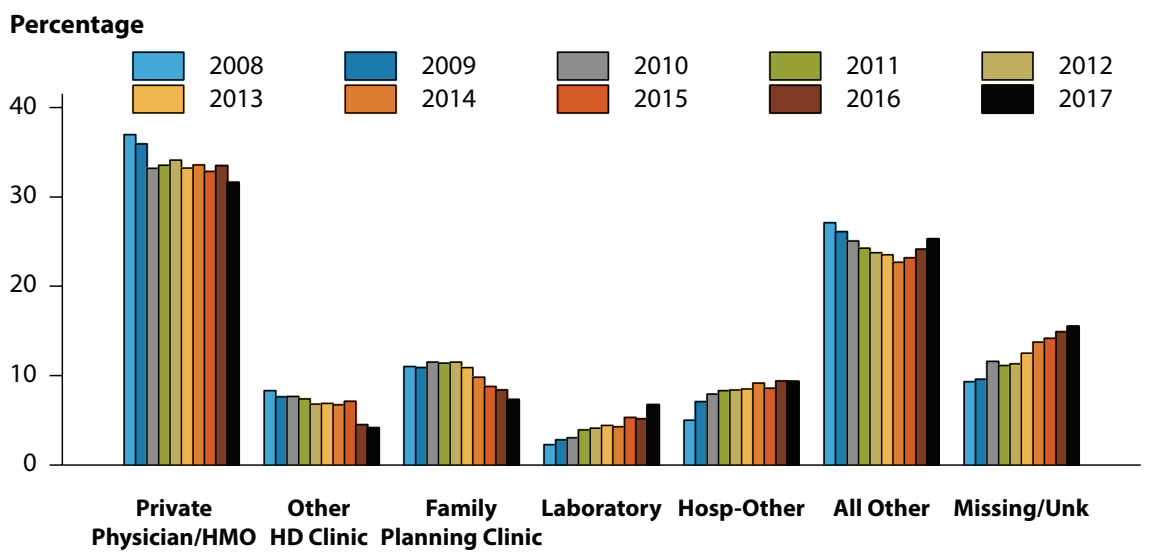

* Includes the top five reporting sources for chlamydia cases reported among women, plus those with reporting sources categorized as "All Other" and "Missing/Unknown".

NOTE: All Other includes: Drug Treatment, Tuberculosis Clinic, Correctional Facility, Blood Bank, Labor and Delivery, Prenatal Care, National Job Training Program, School-based Clinic, Mental Health Provider, Indian Health Service, Military, Emergency Room, STD Clinic, HIV Counseling and Testing Site, and Other.

ACRONYMS: $\mathrm{HMO}=$ health maintenance organization; $\mathrm{HD}=$ health department.
HMOs. Racial differences also persist; reported case rates among Blacks continue to be substantially higher than among all other racial/Hispanic ethnicity groups. Rates of reported cases of chlamydia were next highest among AI/AN and NHOPI women. Ultimately, both test positivity and the number of reported cases of $C$. trachomatis infections remain high among most age groups, racial/ Hispanic ethnicity groups, geographic areas, and both sexes.
The facilities reporting chlamydial infections have changed over the last 10 years or so, with most $(78.6 \%)$ chlamydia cases in 2017 reported from venues outside of STD clinics. The proportion of men being diagnosed with chlamydia in STD clinics decreased $68.2 \%$ from $30.5 \%$ in 2008 to $9.7 \%$ in 2017 ; in 2017 alone, approximately one-third of chlamydia cases among women were reported from private physicians/ 


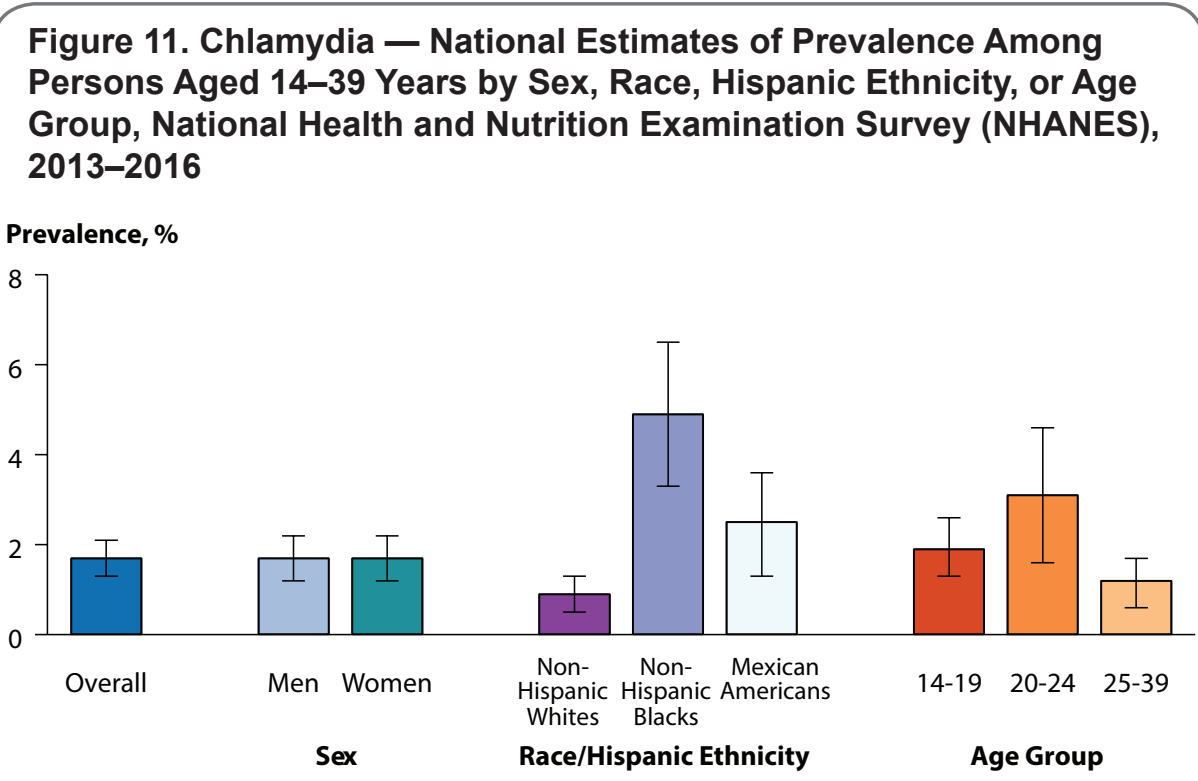

NOTE: Error bars indicate $95 \%$ confidence intervals. Overall prevalence estimates include all race and Hispanic ethnicity groups, including those not shown separately.

ADAPTED FROM: Torrone E, Papp J, Weinstock H. Prevalence of Chlamydia trachomatis genital infection among persons aged 14-39 years - United States, 2007-2012. MMWR Morb Mortal Wkly Rep 2014; 63(38):834-838.

Figure 12. Chlamydia - National Estimates of Prevalence Among Sexually-Active Women Aged 14-39 Years by Race, Hispanic Ethnicity, and Age Group, National Health and Nutrition Examination Survey (NHANES), 2013-2016

Prevalence, \%

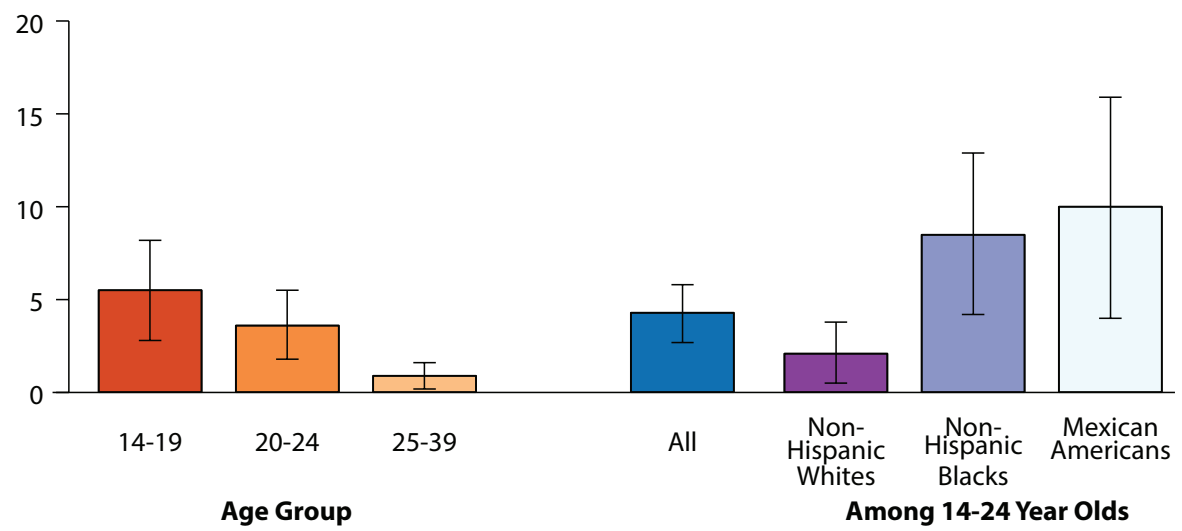

NOTE: Error bars indicate $95 \%$ confidence intervals. Overall prevalence estimates include all race and Hispanic ethnicity groups, including those not shown separately.

ADAPTED FROM: Torrone E, Papp J, Weinstock H. Prevalence of Chlamydia trachomatis genital infection among persons aged 14-39 years - United States, 2007-2012. MMWR Morb Mortal Wkly Rep 2014; 63(38):834-838. 


\section{Figure 13. Chlamydia - Proportion* of STD Clinic Patients Testing Positive $^{\dagger}$ by Age Group, Sex, and Sexual Behavior, STD Surveillance Network (SSuN), 2017}

\section{Percentage}

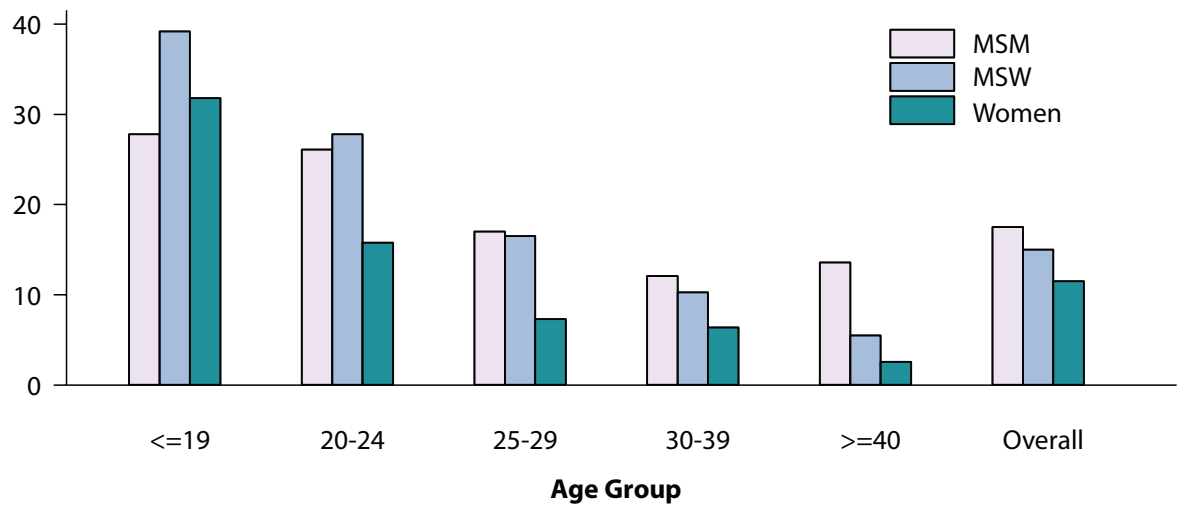

* Proportions represent the overall average of the mean value by jurisdiction.

${ }^{+}$Results are based on unique patients with known sexual behavior $(n=95,167)$ attending SSUN STD clinics who were tested $\geq 1$ times for chlamydia in 2017.

NOTE: See Section A2.2 in the Appendix for SSuN methods.

ACRONYMS: MSM = Gay, bisexual, and other men who have sex with men (collectively referred to as MSM); MSW = Men who have sex with women only.

\section{References}

1. Torrone E, Papp J, Weinstock H. Prevalence of Chlamydia trachomatis genital infection among persons aged $14-39$ years - United States, 2007-2012. MMWR 2014; 63(38): 834-838.

2. Stamm WE. Chlamydia trachomatis infections in the adult. In: Holmes KK, Sparling PF, Stamm WE, Piot P, Wasserheit JN, Corey L, Cohen MS, Watts DH. Sexually Transmitted Diseases. 4th ed. New York, NY: McGraw-Hill; 2008: 575-606.

3. Scholes D, Stergachis A, Heidrich FE, et al. Prevention of pelvic inflammatory disease by screening for cervical chlamydial infection. $\mathrm{N}$ Engl J Med 1996; 334(21): 1362-1366.

4. Oakeshott P, Kerry S, Aghaizu A, et al. Randomised controlled trial of screening for Chlamydia trachomatis to prevent pelvic inflammatory disease: The POPI (prevention of pelvic infection) trial. BMJ 2010; 340: c1642.

5. Fleming DT, Wasserheit JN. From epidemiological synergy to public health policy and practice: The contribution of other sexually transmitted diseases to sexual transmission of HIV infection. Sex Transm Infect 1999; 75(1): 3-17.

6. Hammerschlag MR, Chandler JW, Alexander $\mathrm{ER}$, et al. Longitudinal studies of chlamydial infection in the first year of life. Pediatr Infect Dis 1982; 1(6): 395-401.

7. Centers for Disease Control and Prevention. Sexually transmitted diseases treatment guidelines, 2015. MMWR Recomm Rep 2015; 64(RR-3): 1-137. Erratum in: MMWR 2015; 64(33): 924.

8. National Committee for Quality Assurance. The State of Healthcare Quality 2017. Available at: http://www.ncqa.org/report-cards/healthplans/state-of-health-care-quality/2017-tableof-contents/chlamydia-screening. Accessed June 29, 2018 


\section{Background}

Gonorrhea is the second most commonly reported notifiable disease in the United States. Infections due to Neisseria gonorrhoeae, like those resulting from Chlamydia trachomatis, are a major cause of pelvic inflammatory disease (PID) in the United States. PID can lead to serious outcomes in women, such as tubal infertility, ectopic pregnancy, and chronic pelvic pain. In addition, epidemiologic and biologic studies provide evidence that gonococcal infections facilitate the transmission of HIV infection. ${ }^{1}$ Together, sexual behavior and community prevalence can increase the risk of acquiring gonorrhea. Social determinants of health, such as socioeconomic status, discrimination, and access to quality health care, may contribute to the burden of gonorrhea in a community. ${ }^{2}$

$N$. gonorrhoeae has progressively developed resistance to each of the antimicrobials used for treatment of gonorrhea. Declining susceptibility to cefixime (an oral cephalosporin antibiotic) resulted in a change to the CDC treatment guidelines in 2015, so that dual therapy with ceftriaxone (an injectable cephalosporin) and azithromycin is now the only CDCrecommended treatment regimen for gonorrhea. ${ }^{3}$ The emerging threat of cephalosporin resistance highlights the need for continued surveillance of $N$. gonorrhoeae antimicrobial susceptibility.

The combination of persistently high gonorrhea morbidity in some populations and the threat of cephalosporin-resistant gonorrhea reinforces the need to better understand the epidemiology of gonorrhea.

\section{Interpreting Rates of Reported Cases of Gonorrhea}

Although gonorrhea case reporting is useful for monitoring disease trends, the number of gonorrhea cases reported to CDC is affected by many factors in addition to the actual occurrence of the infection within the population. Changes in the burden of gonorrhea may be masked by changes in screening practices (e.g., screening for chlamydia with tests that also detect $N$. gonorrhoeae infections or increased screening at extra-genital anatomic sites), the use of diagnostic tests with different test performance (e.g., the broader use of nucleic acid amplification tests [NAATs]), and changes in reporting practices. As with other STDs, the reporting of gonorrhea cases to CDC is incomplete. ${ }^{4}$ For these reasons, supplemental data on gonorrhea prevalence in persons screened in a variety of settings are useful in assessing the burden of disease in selected populations.

\section{Gonorrhea - United States}

In 2017, a total of 555,608 cases of gonorrhea were reported in the United States, yielding a rate of 171.9 cases per 100,000 population (Figure 14, Table 1). During 2016-2017, the rate of reported gonorrhea cases increased $18.6 \%$, and increased $75.2 \%$ since the historic low in 2009.

\section{Gonorrhea by Region}

The South had the highest rate of reported gonorrhea cases (194.0 cases per 100,000 population) among the four regions of the United States in 2017, followed by the Midwest (170.6 cases per 100,000 population), the West (169.0 cases per 100,000 population), and the Northeast (129.6 cases per 100,000 population) (Figure 15, Table 14). During 20162017, the gonorrhea rate increased in all four regions: $19.9 \%$ in the West, $19.5 \%$ in the Midwest, $19.0 \%$ in the Northeast, and $17.4 \%$ in the South (Figure 15, Table 14). During 20132017, the rate of gonorrhea in the

\section{Figure 14. Gonorrhea - Rates of Reported Cases by Year, United States, 1941-2017}

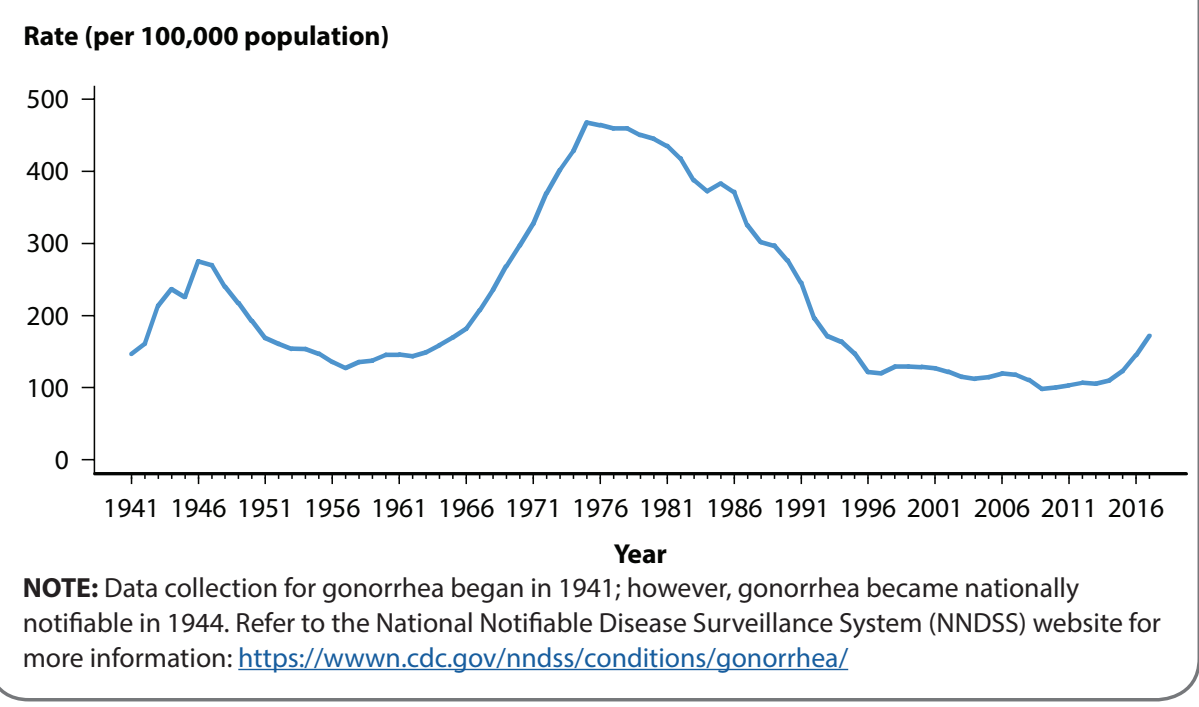


Figure 15. Gonorrhea - Rates of Reported Cases by Region, United States, 2008-2017

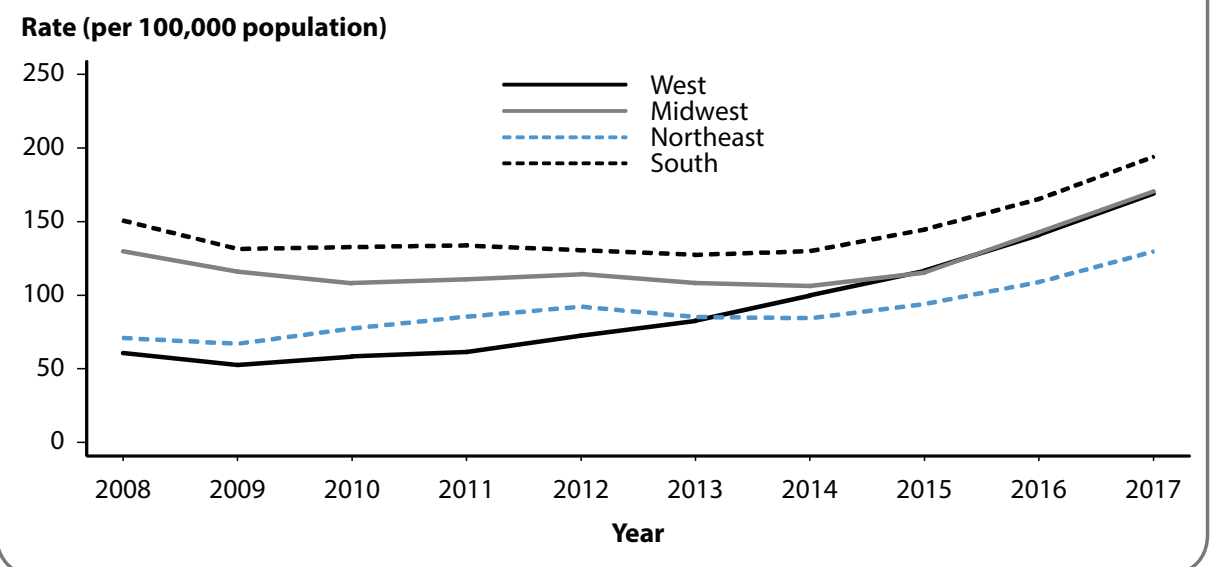

Figure 16. Gonorrhea - Rates of Reported Cases by State, United States and Outlying Areas, 2017

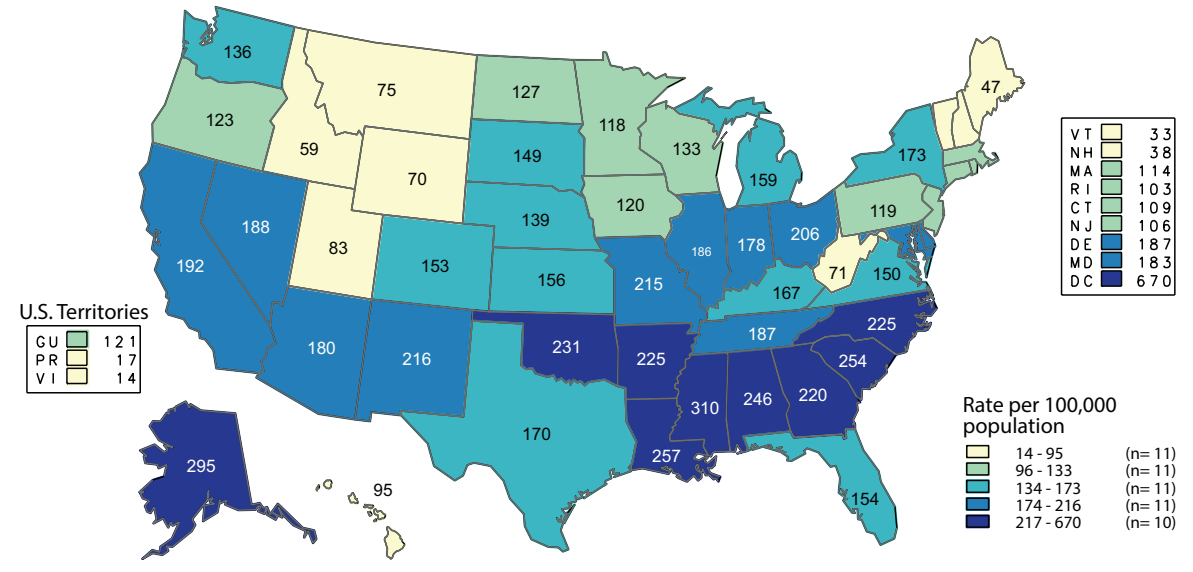

NOTE: The total rate of reported cases of gonorrhea for the United States and outlying areas (including Guam, Puerto Rico, and the Virgin Islands) was 170.3 per 100,000 population. See Section A1.11 in the Appendix for more information on interpreting reported rates in the outlying areas.

ACRONYMS: GU = Guam; PR = Puerto Rico; VI = Virgin Islands.

West increased by $104.4 \%$ ( 82.7 to 169.0 cases per 100,000 population), while other regions had lower overall increases during this time period (i.e., $57.7 \%$ in the Midwest, $52.3 \%$ in the South, and $52.1 \%$ in the Northeast).

\section{Gonorrhea by State}

In 2017, rates of reported gonorrhea cases per 100,000 population ranged by state from 32.5 in Vermont to

\section{Gonorrhea by Metropolitan Statistical Area}

The overall rate of reported gonorrhea cases in the 50 most populous metropolitan statistical areas (MSAs) was 191.5 cases per 100,000 population in 2017 , representing a $18.1 \%$ increase compared with the rate in 2016 (162.1 cases per 100,000 population) (Table 17). In 2017 , $61.3 \%$ of reported gonorrhea cases were reported by these MSAs. Since 2013, the gonorrhea rate among females in the 50 most populous MSAs has been lower than the rate among males (Tables 18 and 19). In 2017, the rate among females in these MSAs was 141.0 cases per 100,000 females, while the rate among males was 243.4 cases per 100,000 males.

\section{Gonorrhea by County}

In $2017,50.0 \%$ of reported gonorrhea cases occurred in just 70 counties or independent cities and 625 counties $(19.9 \%)$ in the United States had a rate of reported gonorrhea less than or equal to 25 cases per 100,000 population (Figure 17, Table 20). The rate ranged from 26 to 53 cases per 100,000 population in 628 counties $(20.0 \%)$, ranged from 54 to 92 cases per 100,000 population in 631 counties $(20.0 \%)$, ranged from 93 to 172 cases per 100,000 population in 632 counties $(20.1 \%)$, and was more than 172 cases per 100,000 population in 624 counties $(19.9 \%)$. As in previous years, counties with the highest gonorrhea rates were concentrated in the South.

\section{Gonorrhea by Sex} rate in the District of Columbia was 669.9 cases per 100,000 population (Figure 16, Tables 13 and 14).

During 2016-2017, gonorrhea rates increased in 47 states and the District of Columbia (94.1\%) and decreased in 3 states $(5.9 \%)$ (Table 14).
As was observed during 2013-2016, the rate of reported gonorrhea cases among males was higher than the rate among females in 2017 (Figure 18, Tables 15 and 16). During 20162017 , the gonorrhea rate among males increased $19.3 \%$ (169.7 to 202.5 


\section{Figure 17. Gonorrhea — Rates of Reported Cases by County, United States, 2017}

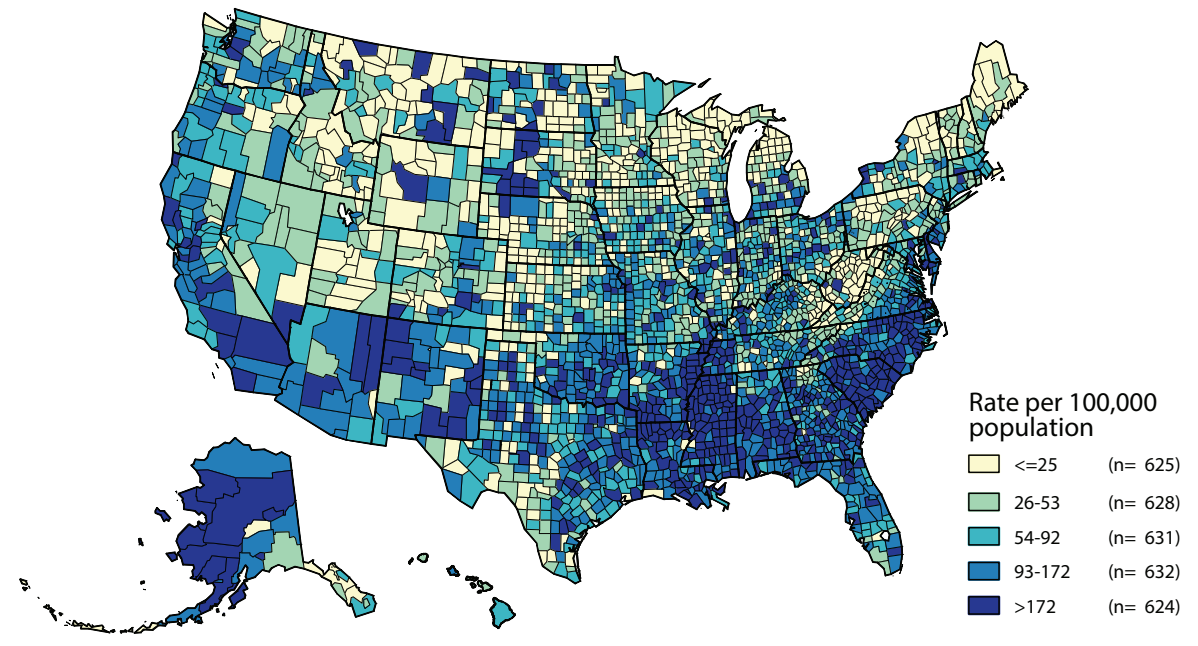

NOTE: Refer to the NCHHSTP AtlasPlus for further county-level rate information: https://www.cdc. gov/nchhstp/atlas/

\section{Figure 18. Gonorrhea - Rates of Reported Cases by Sex, United States, 2008-2017}

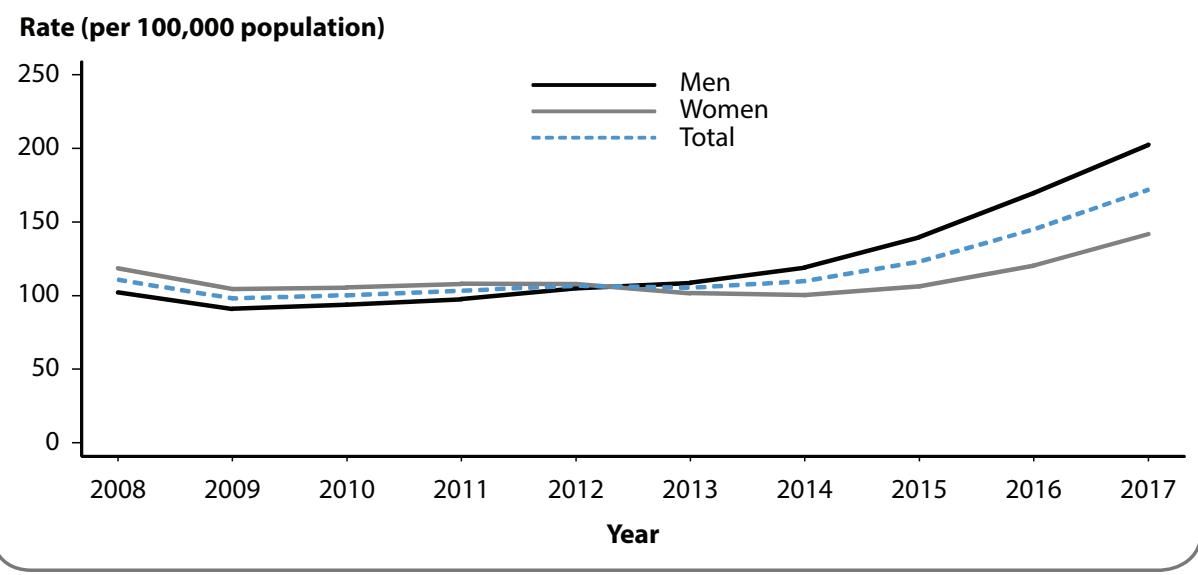

cases per 100,000 males) and the rate among females increased $17.8 \%$ (120.4 to 141.8 cases per 100,000 females). During 2013-2017, the rate among males increased $86.3 \%$ (108.7 to 202.5 cases per 100,000 males) and the rate among females increased $39.4 \%$ (101.7 to 141.8 cases per 100,000 females). The magnitude of the increase among males suggest either increased transmission or increased case ascertainment (e.g., through increased extra-genital screening) among gay, bisexual, and other men who have sex with men (collectively referred to as MSM). However, most jurisdictions do not routinely report sex of sex partner or site of infection for gonorrhea cases, so trends in gonorrhea rates among MSM over time cannot be assessed.

\section{Gonorrhea by Region and Sex}

In all regions, the rate of gonorrhea increased among both males and females during 2016-2017 and during
2013-2017 (Tables 15 and 16). The rate of reported gonorrhea cases among females increased the most in the West (21.4\% during 2016-2017 and $83.4 \%$ during 2013-2017) and Midwest (18.2\% during 2016-2017 and $37.5 \%$ during 2013-2017) (Table 15). However, the rate of reported gonorrhea cases among males increased the most in the Northeast $(21.5 \%)$ and the Midwest $(20.4 \%)$ during 2016-2017 and in the West (117.8\%) and Northeast (85.7\%) during 2013-2017 (Table 16).

\section{Gonorrhea by Age}

In 2017, rates of reported gonorrhea cases continued to be highest among adolescents and young adults (Figure 19, Table 21). In 2017, the highest rates among females were observed among those aged 20-24 years (684.8 cases per 100,000 females) and 15-19 years (557.4 cases per 100,000 females). Among males, the rate was highest among those aged 20-24 years ( 705.2 cases per 100,000 males) and 25-29 years (645.9 cases per 100,000 males).

In 2017, persons aged 15-44 years accounted for $91.8 \%$ of reported gonorrhea cases with known age. Among 15-19 year olds, rates increased $15.5 \%$ during 2016-2017. The gonorrhea rate also increased among other age groups during 2016-2017: $12.8 \%$ among those aged 20-24 years, $20.3 \%$ among those aged $25-29$ years, $24.2 \%$ among those aged $30-34$ years, $28.6 \%$ among those aged 35-39 years, and $26.2 \%$ among those aged $40-44$ years (Table 21). Among persons aged 15-44 years, increases were observed in all age groups for both men and women during 2016-2017 (Figures 20 and 21).

\section{Gonorrhea by Race/Hispanic Ethnicity}

In 2017, the rate of reported gonorrhea cases remained highest among Blacks (548.1 cases per 
Figure 19. Gonorrhea - Rates of Reported Cases by Age Group and Sex, United States, 2017

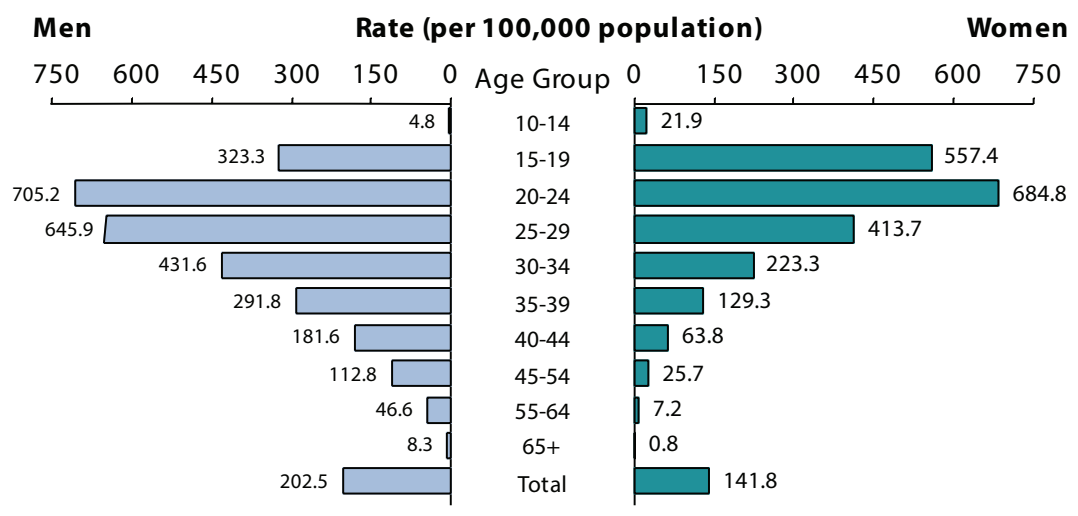

Figure 20. Gonorrhea - Rates of Reported Cases Among Women Aged 15-44 Years by Age Group, United States, 2008-2017

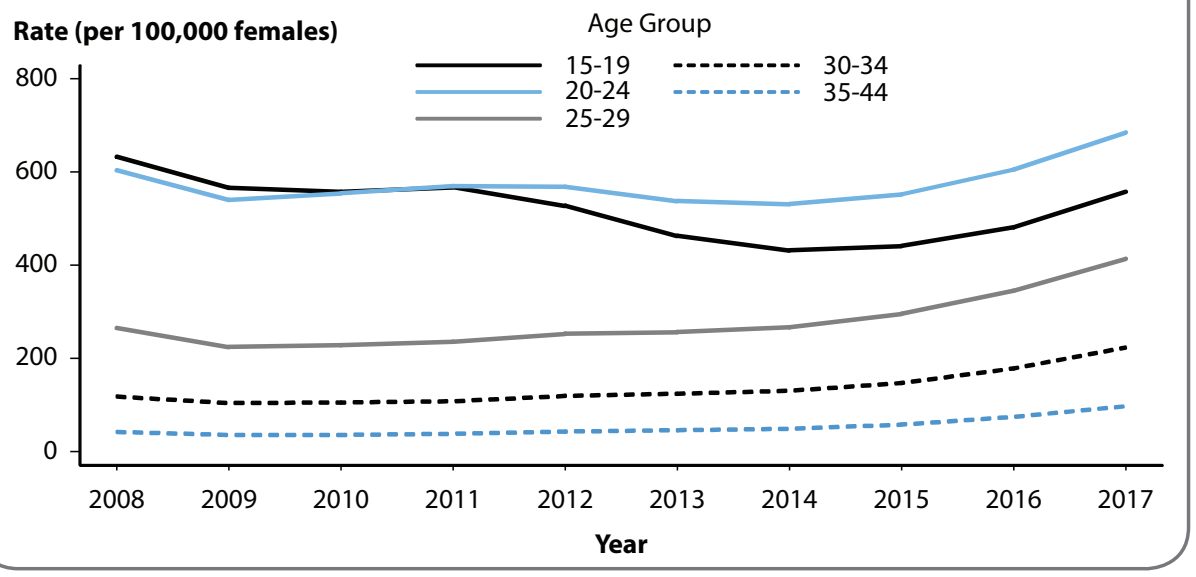

Figure 21. Gonorrhea - Rates of Reported Cases Among Men Aged 15-44 Years by Age Group, United States, 2008-2017

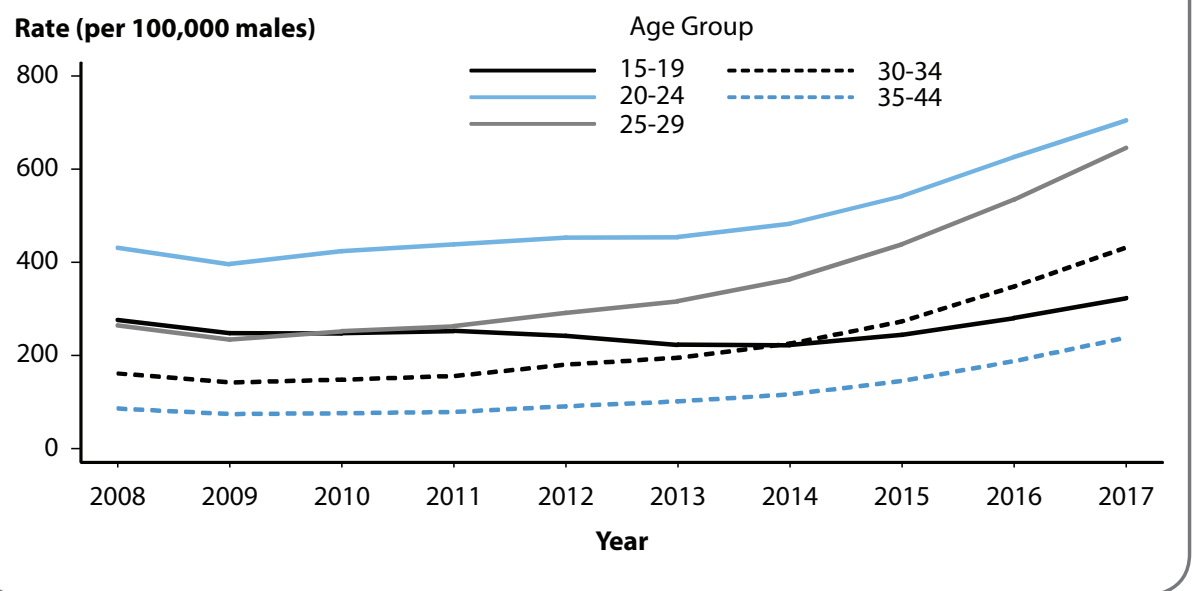

100,000 population) (Table 22B). The rate among Blacks was 8.3 times the rate among Whites (66.4 cases per 100,000 population). The gonorrhea rate among American Indians/Alaska Natives (AI/AN) (301.9 cases per 100,000 population) was 4.5 times that of Whites, the rate among Native Hawaiians/Other Pacific Islanders (NHOPI) (187.8 cases per 100,000 population) was 2.8 times that of Whites, the rate among Hispanics (113.7 cases per 100,000 population) was 1.7 times that of Whites, the rate among Multirace persons (76.9 cases per 100,000 population) was 1.2 times that of Whites, and the rate among Asians (34.7 cases per 100,000 population) was half the rate of Whites (Table 22B).

During 2013-2017, for all five years during that period, the gonorrhea rate increased among all race and Hispanic ethnicity groups: $176.6 \%$ among Multirace persons, $122.4 \%$ among Asians, 109.1\% among NHOPI, 100.6\% among Whites, 95.3\% among AI/AN, 77.4\% among Hispanics, and 36.2\% among Blacks (Figure 22).

More information on gonorrhea rates among race/Hispanic ethnicity groups can be found in the Special Focus Profiles.

\section{Gonorrhea by Reporting Source}

In $2017,9.3 \%$ of gonorrhea cases were reported from STD clinics, $76.7 \%$ were reported from venues outside of STD clinics, and $14.0 \%$ had an unknown reporting source (Table A2).

During 2008-2017, the percent of gonorrhea cases reported by STD clinics declined $64.4 \%$ among males and $57.9 \%$ among females; however, the percent of gonorrhea cases with missing/unknown reporting source increased $50.5 \%$ among males and 46.9\% among females (Figures 23 and 24). During 2016-2017, the percent 
Figure 22. Gonorrhea - Rates of Reported Cases by Race and Hispanic Ethnicity, United States, 2013-2017

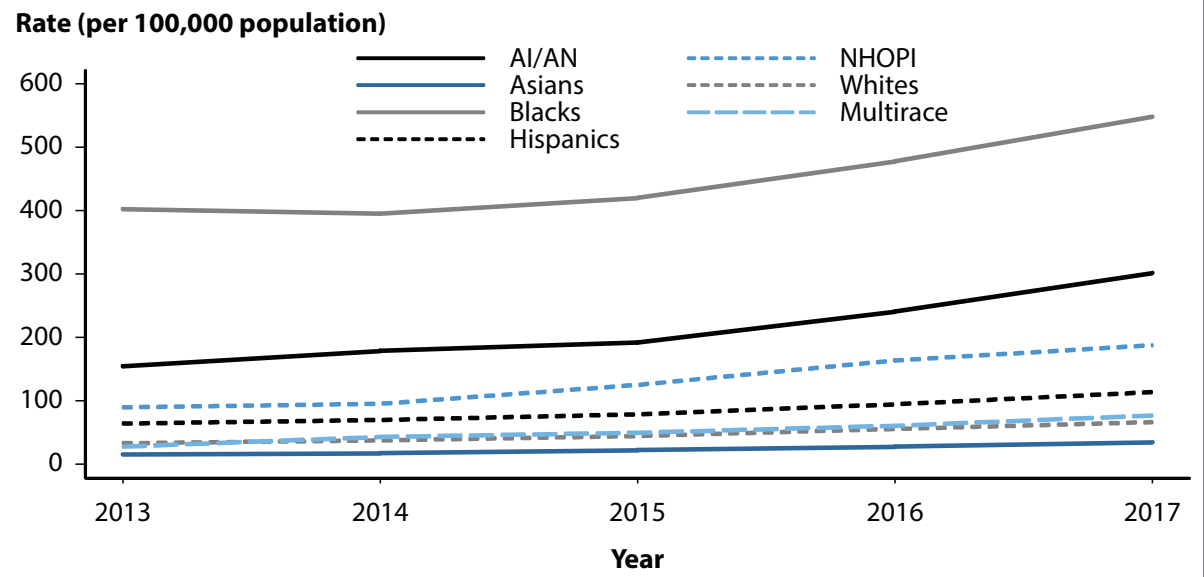

NOTE: Not all US jurisdictions reported cases in OMB-compliant Race categories in 2017. This may minimally under- or overestimate rates for Asians, NHOPI, or Multirace individuals. For completeness, data in this figure include cases reported from all jurisdictions. See Section A1.5 in the Appendix for information on reporting STD case data for race and Hispanic ethnicity. ACRONYMS: AI/AN = American Indians/Alaska Natives; NHOPI = Native Hawaiians/Other Pacific Islanders; $\mathrm{OMB}=$ Office of Management and Budget.

\section{Figure 23. Gonorrhea - Percentage of Reported Cases Among Men by Reporting Source*, United States, 2008-2017}

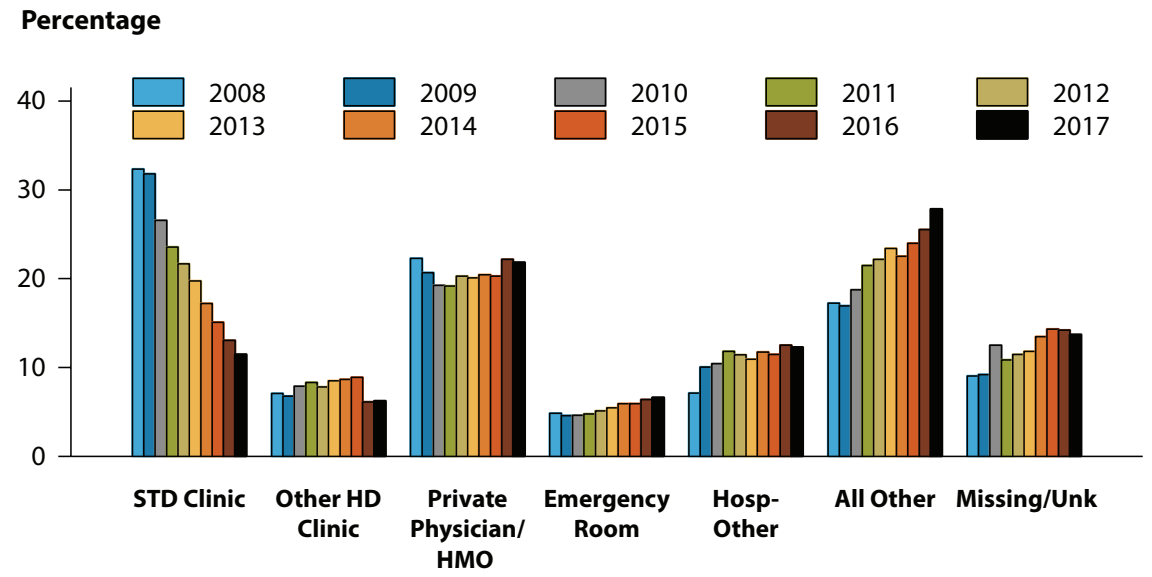

* Includes the top five reporting sources for gonorrhea cases reported among men, plus those with reporting sources categorized as "All Other" and "Missing/Unknown".

NOTE: All Other includes: Drug Treatment, Tuberculosis Clinic, Correctional Facility, Laboratory, Blood Bank, Labor and Delivery, Prenatal Care, National Job Training Program, School-based Clinic, Mental Health Provider, Indian Health Service, Military, Family Planning, HIV Counseling and Testing Site, and Other.

ACRONYMS: $\mathrm{HMO}=$ health maintenance organization; $\mathrm{HD}$ = health department.

of gonorrhea cases reported by STD clinics decreased $12.2 \%$ among males and $8.4 \%$ among females.

In 2017, the largest proportion of cases among men were reported by private physicians/health maintenance organizations (HMOs) (21.8\%), followed by other hospital clinics/ facilities (12.3\%), STD clinics $(11.5 \%)$, emergency rooms $(6.6 \%)$, and other health department clinics (6.2\%) (Figure 23). Among females, private physicians/HMOs (24.8\%) were the most common reporting source, followed by other hospital clinics/facilities (13.5\%), laboratories (7.8\%), STD clinics $(6.4 \%)$, and family planning clinics $(6.3 \%)$ (Figure 24).

\section{STD Surveillance Network}

The STD Surveillance Network $(\mathrm{SSuN})$ is an ongoing collaboration of states and independently funded cities collecting enhanced information on a representative sample of gonorrhea case reports received from all reporting sources in their jurisdiction. Enhanced gonorrhea case report data for 2017 were obtained from Cycle 3 of SSuN, which includes 10 jurisdictions randomly sampling cases reported in their jurisdictions. In 2017, SSuN collaborators interviewed 6,409 gonorrhea cases, representing $4.1 \%$ of all cases reported from participating jurisdictions. The estimated burden of disease represented by men who have sex with men (MSM; including men who have sex with both men and women), men who have sex with women only (MSW), and women varied substantially across collaborating sites based on weighted analysis (Figure 25). San Francisco had the highest proportion of cases estimated to be MSM (86.0\%), while Florida had the lowest proportion of MSM cases (20.6\%). In total, across all SSuN sites, $41.7 \%$ of gonorrhea cases were estimated to be among MSM, 25.5\% among MSW, and $32.9 \%$ among women.

Among six jurisdictions participating in SSuN continuously for the period 2010-2017, estimated rates of gonorrhea among MSM, MSW, and women were calculated by extending published estimates of the MSM population and are presented in Figure 26. ${ }^{5,6}$ The estimated gonorrhea case rate among MSM increased $283 \%$ during 2010-2017 from 1,368.6 cases per 100,000 MSM in 2010 to $5,241.8$ cases per 100,000 MSM in 2017. Over the same time period, 
Figure 24. Gonorrhea - Percentage of Reported Cases Among Women by Reporting Source*, United States, 2008-2017

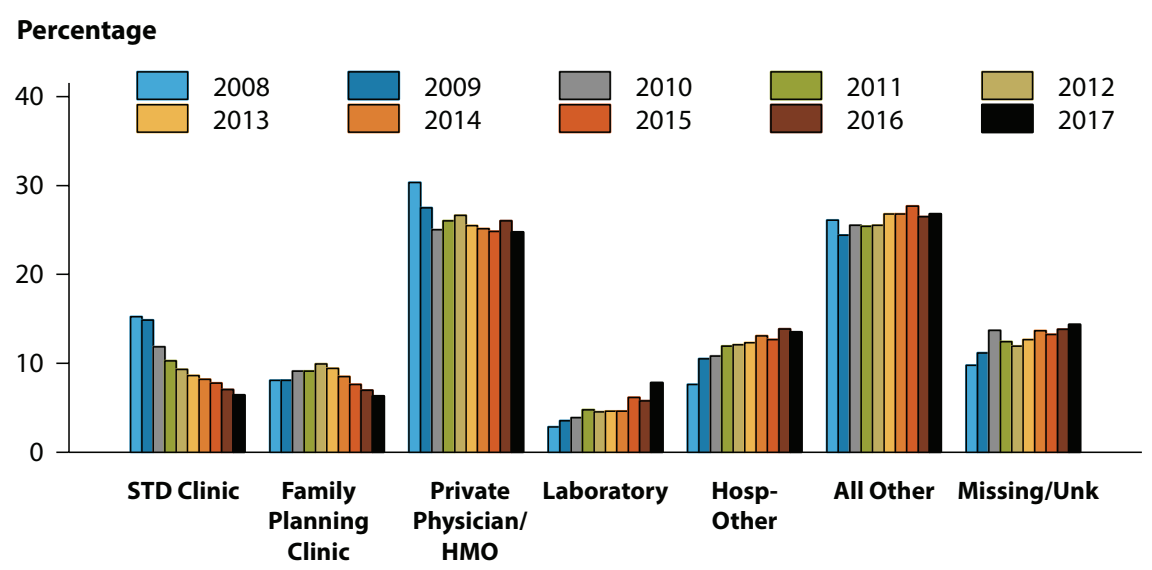

* Includes the top five reporting sources for gonorrhea cases reported among women, plus those with reporting sources categorized as "All Other" and "Missing/Unknown".

NOTE: All Other includes: Drug Treatment, Tuberculosis Clinic, Correctional Facility, Blood Bank, Labor and Delivery, Prenatal Care, National Job Training Program, School-based Clinic, Mental Health Provider, Indian Health Service, Military, Emergency Room, Other Health Department Clinic, HIV Counseling and Testing Site, and Other.

ACRONYMS: $\mathrm{HMO}=$ health maintenance organization.

Figure 25. Estimated Proportion* of MSM, MSW, and Women Among Gonorrhea Cases by Jurisdiction, STD Surveillance Network (SSuN), 2017

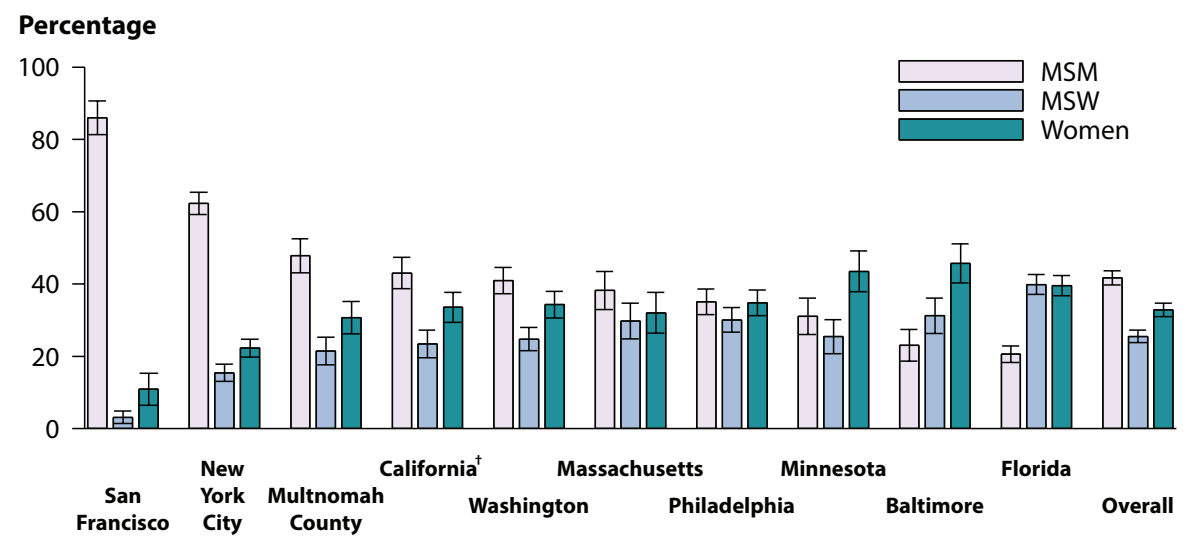

* Estimate based on weighted analysis of data obtained from interviews $(n=6,409)$ conducted among a random sample of reported gonorrhea cases during January to December 2017.

${ }^{\dagger}$ California data exclude San Francisco (shown separately).

NOTE: See section A2.2 in the Appendix for SSuN methods.

ACRONYMS: MSM = Gay, bisexual, and other men who have sex with men (collectively referred to as MSM); MSW = Men who have sex with women only.

case rates among MSW and women also increased by $53.9 \%$ and $88.3 \%$, respectively.

Collaborating SSuN jurisdictions also conduct sentinel surveillance on all patients seeking care in selected
STD clinics. Sentinel facility data for this report include information from patients attending STD clinics during 2017 in 10 funded jurisdictions. In 2017, the proportion of STD clinic patients who tested positive for gonorrhea varied by age group, sex, and sexual behavior (Figure 27). The overall prevalence, represented by the average of the mean value by the 10 SSuN jurisdictions, was $26.5 \%$ for MSM, $14.6 \%$ for MSW, and 7.4\% for women. Among those attending these clinics, MSM disproportionately had higher positivity rates when compared to MSW and women in all age groups. While positivity rates declined with increasing age in women, rates in MSW and MSM showed less consistent declines across age groups.

Additional information about SSuN methodology can be found in Section A2.2 of the Appendix.

\section{Gonococcal Isolate Surveillance Project}

Antimicrobial resistance remains an important consideration in the treatment of gonorrhea. ${ }^{3,-9}$ In 1986, the Gonococcal Isolate Surveillance Project (GISP), a national sentinel surveillance system, was established to monitor trends in antimicrobial susceptibilities of urethral $N$. gonorrhoeae strains in the United States. ${ }^{7}$ Data are collected from selected STD clinic sentinel sites and from regional laboratories (Figure 28).

Antimicrobial susceptibility is measured by the minimum inhibitory concentration (MIC), the lowest antimicrobial concentration that inhibits bacterial growth in the laboratory. Increases in MICs demonstrate that the bacteria can survive at higher antimicrobial concentrations in the laboratory. Monitoring of MIC trends is useful because increasing MICs can oftentimes be an early indicator of the emergence of antimicrobial resistance.

Information on the antimicrobial susceptibility criteria used in GISP can be found in Section A2.3 in the Appendix. More information about GISP and additional data can be found at https://www.cdc.gov/std/GISP. 


\section{Figure 26. Gonorrhea - Estimated ${ }^{*}$ Rates of Reported Gonorrhea Cases by MSM, MSW, and Women, STD Surveillance Network (SSuN) ${ }^{\dagger}, 2010-2017$}

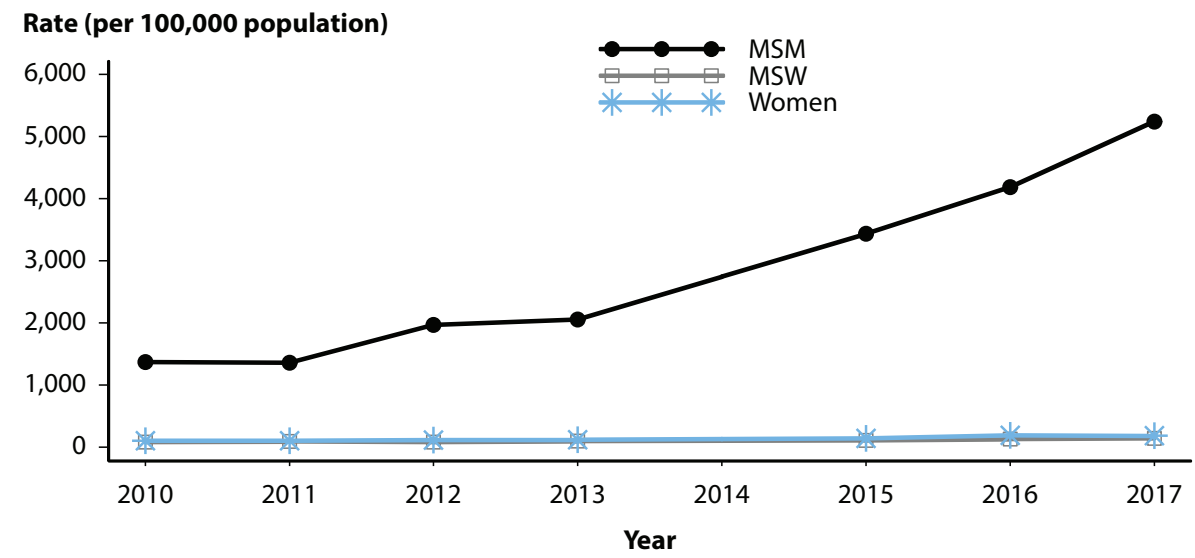

* Estimates based on interviews among a random sample of reported cases of gonorrhea $(\mathrm{N}=17,765)$; cases weighted for analysis.

+ Sites include Baltimore, Philadelphia, New York City, Washington State, San Francisco, and California (excluding San Francisco).

NOTE: Data not available for 2014; 2013-2015 trend interpolated; trend lines overlap for MSW and women in this figure. See section A2.2 in the Appendix for SSuN methods.

ADAPTED FROM: Stenger M, Pathela P, Anschuetz G, et al. Increases in the rate of Neisseria gonorrhoeae among gay, bisexual and other men who have sex with men (MSM) — findings from the STD Surveillance Network 2010-2015. Sex Transm Dis 2017; 44(7): 393-397.

ACRONYMS: MSM = Gay, bisexual, and other men who have sex with men (collectively referred to as MSM); MSW = Men who have sex with women only.

\section{Figure 27. Gonorrhea - Proportion* of STD Clinic Patients ${ }^{\dagger}$ Testing Positive by Age Group, Sex, and Sexual Behavior, STD Surveillance Network (SSuN), 2017}

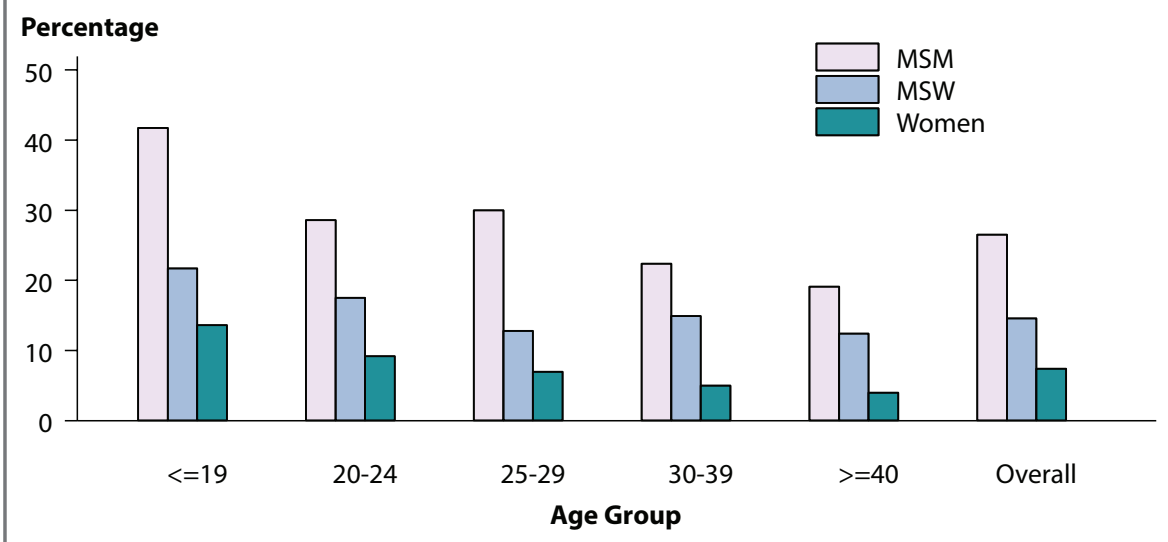

* Proportions represent the overall average of the mean value by jurisdiction.

+ Results are based on data obtained from unique patients with known sexual behavior $(n=94,893)$ attending SSuN STD clinics who were tested $\geq 1$ times for gonorrhea in 2017.

NOTE: See section A2.2 in the Appendix for SSUN methods.

ACRONYMS: MSM = Gay, bisexual, and other men who have sex with men (collectively referred to as MSM); MSW = Men who have sex with women only.

\section{Ceftriaxone Susceptibility}

Susceptibility testing for ceftriaxone began in 1987. During 2008-2017, the percentage of GISP isolates that exhibited elevated ceftriaxone MICs, defined as $\geq 0.125 \mu \mathrm{g} / \mathrm{ml}$, fluctuated between $0.05 \%$ and $0.4 \%$ (Figure 29). In $2017,0.2 \%$ of isolates had elevated ceftriaxone MICs. Five isolates with decreased ceftriaxone susceptibility $(\mathrm{MIC}=0.5 \mu \mathrm{g} / \mathrm{ml}$ ) have been previously identified in GISP: one from San Diego, California (1987), two from Cincinnati, Ohio (1992 and 1993), one from Philadelphia, Pennsylvania (1997), and one from Oklahoma City, Oklahoma (2012).

\section{Cefixime Susceptibility}

Susceptibility testing for cefixime began in 1992, was discontinued in 2007 , and was restarted in 2009. The percentage of isolates with elevated cefixime MICs $(\geq 0.25 \mu \mathrm{g} / \mathrm{ml})$ declined from $1.4 \%$ in 2011 to $0.4 \%$ in 2017 (Figure 29).

\section{Azithromycin Susceptibility}

Susceptibility testing for azithromycin began in 1992. Figure 29 displays the distribution of azithromycin MICs among GISP isolates collected during 2008-2017. Most isolates had MICs of $0.125-0.5 \mu \mathrm{g} / \mathrm{ml}$. During 2012 2014, the percentage of isolates with elevated azithromycin MICs ( $\geq 2 \mu \mathrm{g} / \mathrm{ml}$ ) ranged from $0.3 \%$ to $2.5 \%$ with a sharp increase during $2013-2014$ (0.6\% to $2.5 \%)$; during 2014-2017, the percentage increased from $2.5 \%$ to $4.4 \%$. No isolates with elevated azithromycin MICs had elevated ceftriaxone MICs in 2017.

\section{Susceptibility to Other Antimicrobials}

Susceptibility testing for gentamicin began in 2015. Between 2015 and $2017,66.7-75.3 \%$ of all tested isolates have had an MIC value of $8 \mu \mathrm{g} / \mathrm{mL}$ (Figure 30 ). None of the isolates tested in GISP have had an MIC above $16 \mu \mathrm{g} / \mathrm{mL}$.

In $2017,30.1 \%$ of isolates collected from GISP sites were resistant to ciprofloxacin, $23.1 \%$ to tetracycline, and $15.8 \%$ to penicillin (Figure 31 ). Although these antimicrobials are no 


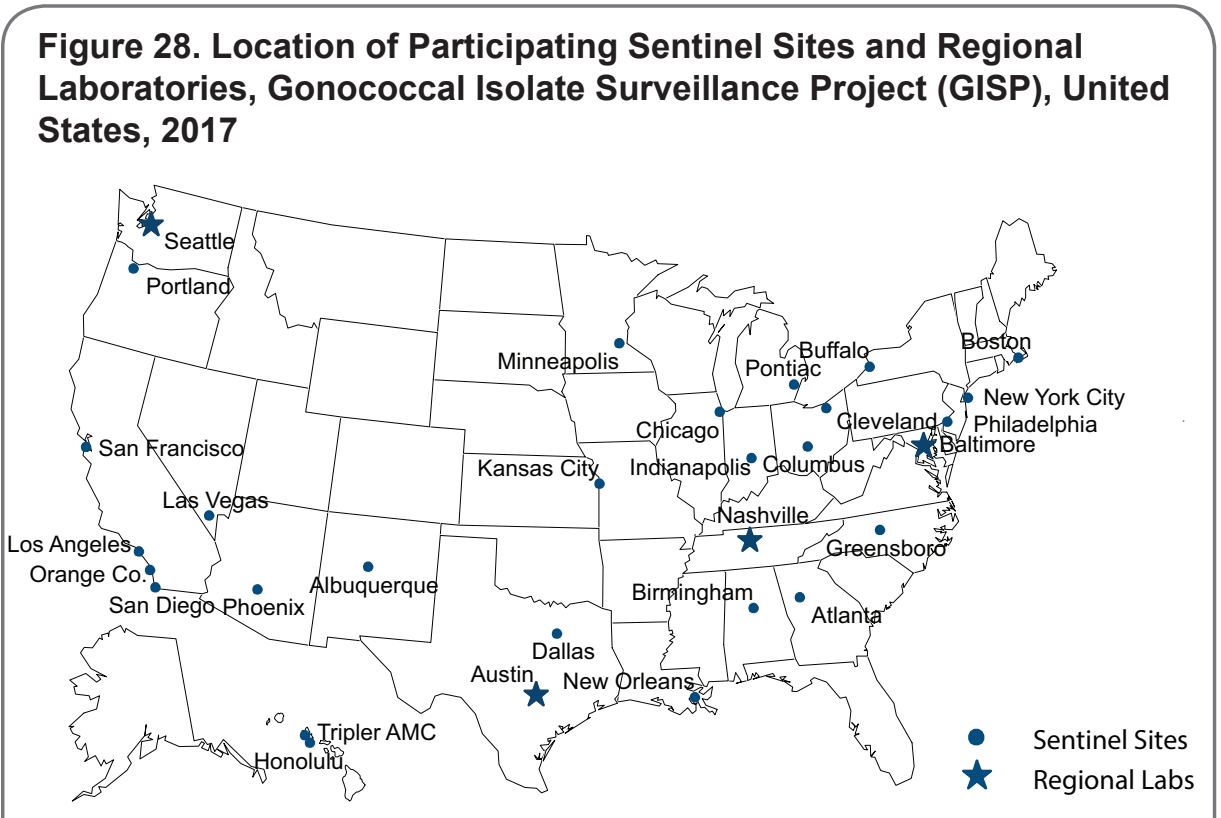

NOTE: Seattle is both a sentinel site and a regional laboratory.

\section{Figure 29. Neisseria gonorrhoeae - Percentage of Isolates with Elevated Azithromycin Minimum Inhibitory Concentrations (MICs) $(\geq 2.0 \mu \mathrm{g} / \mathrm{ml})$, Elevated Ceftriaxone MICs $(\geq 0.125 \mu \mathrm{g} / \mathrm{ml})$, and Elevated Cefixime MICs ( $\geq 0.25 \mu \mathrm{g} / \mathrm{ml})$, Gonococcal Isolate Surveillance Project (GISP), 2008-2017}

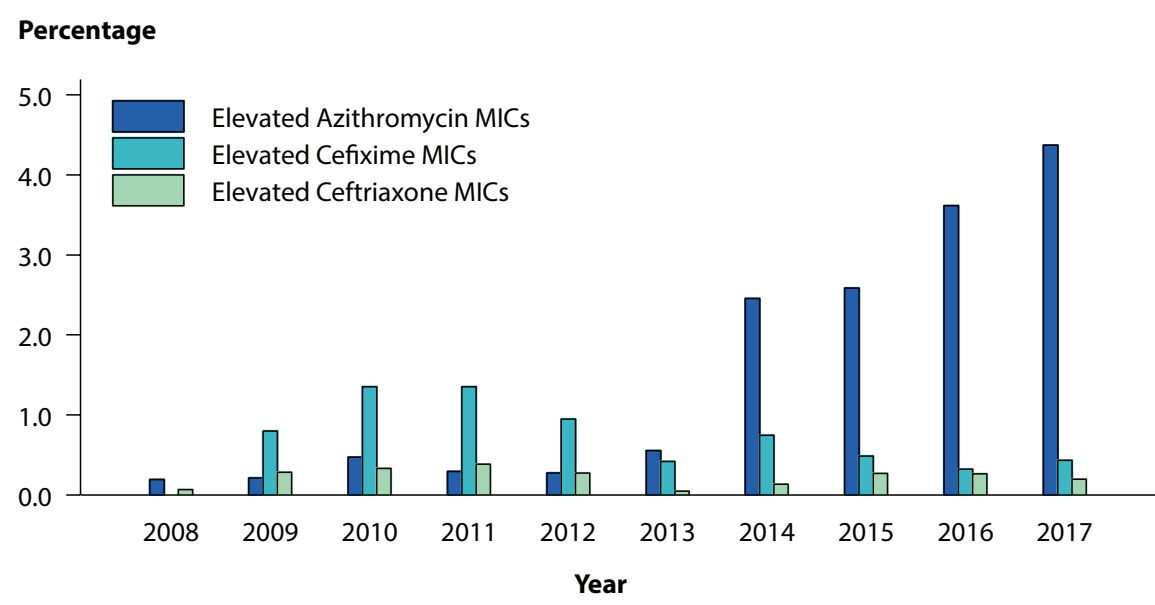

NOTE: Isolates not tested for cefixime susceptibility in 2008.

longer recommended for treatment of gonorrhea, the resistance phenotypes remain common. Of all the isolates collected in GISP in 2017, 4.6\% demonstrated resistance or elevated MICs to at least 3 antibiotics tested and $51.5 \%$ were susceptible to all antibiotics tested (Figure 32).
$84.0 \%$ in 2011 to $98.1 \%$ in 2017 . In $2017,1.1 \%$ of patients were treated with gentamicin $240 \mathrm{mg}$ and $0.1 \%$ were treated with cefixime $400 \mathrm{mg}$.

In 2017, based on weighted analysis of $\mathrm{SSuN}$ jurisdictions with documented treatment information (i.e., antimicrobials and dosages) for $\geq 90 \%$ of cases, $82.4 \%$ (95\% CI $=80.3-84.4)$ of reported patients with gonorrhea in SSuN jurisdictions received the recommended treatment for uncomplicated gonorrhea (Figure 34). The proportion of reported patients that received the recommended dual treatment ranged from $77.3 \%(95 \% \mathrm{CI}=72.5-82.1)$ in Massachusetts to $92.0 \%$ (95\% CI $=88.9-95.2)$ in Multnomah County, Oregon.

\section{Gonorrhea Among Special Populations}

More information about gonorrhea in race/Hispanic ethnicity groups, females of reproductive age, adolescents, and MSM can be found in the Special Focus Profiles.

\section{Gonorrhea Summary}

The national rate of reported gonorrhea cases reached a historic low in 2009, but increased each year during 2009-2012. After a temporary decrease in 2013, the gonorrhea rate increased again during 2014-2017. This increase was largely attributable to increases among men. Enhanced surveillance data suggest the largest increases are among MSM. However, high gonorrhea rates persist in certain geographic areas, among adolescents and young adults, and in some racial/Hispanic ethnicity groups. Additionally, continued surveillance for antimicrobial resistant gonorrhea is critical to monitor for the emergence of reduced susceptibility and resistance to cephalosporins and azithromycin. 


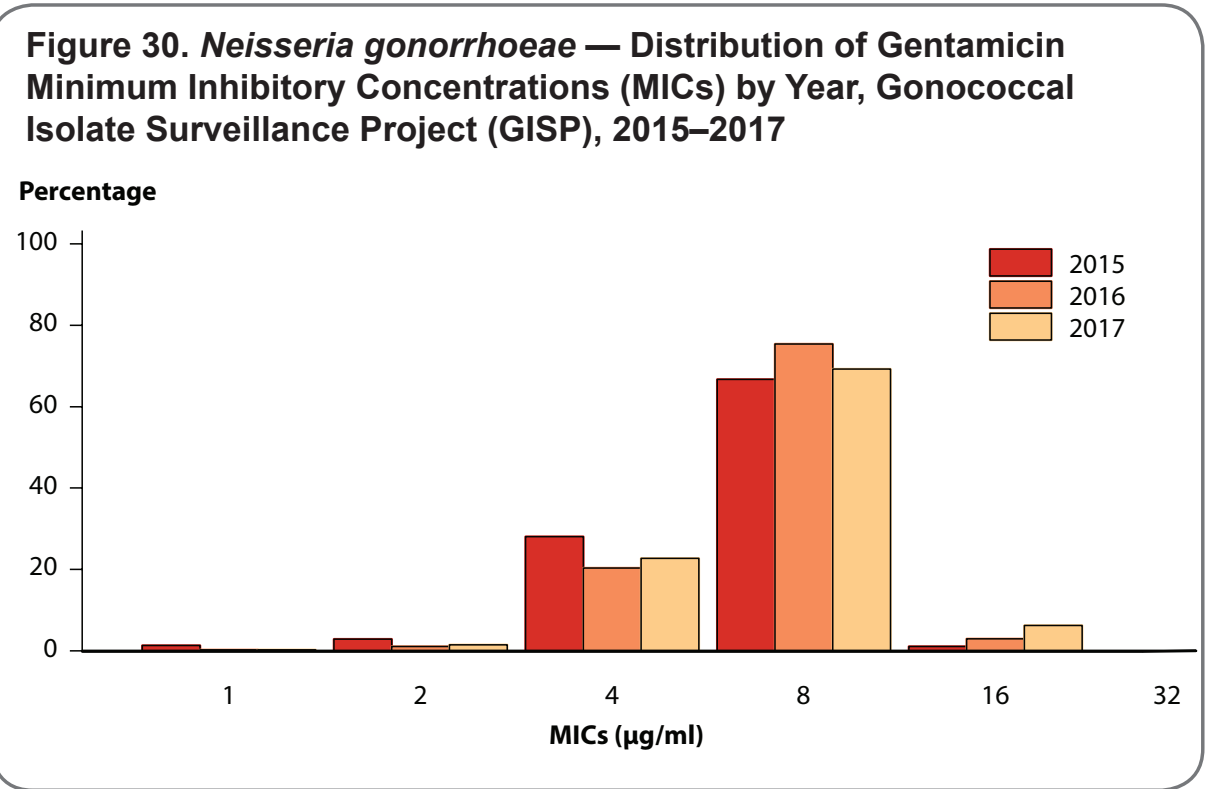

Figure 31. Neisseria gonorrhoeae - Prevalence of Tetracycline, Penicillin, or Fluoroquinolone Resistance* or Elevated Cefixime, Ceftriaxone, or Azithromycin Minimum Inhibitory Concentrations (MICs) $^{\dagger}$, by Year - Gonococcal Isolate Surveillance Project (GISP), 2000-2017

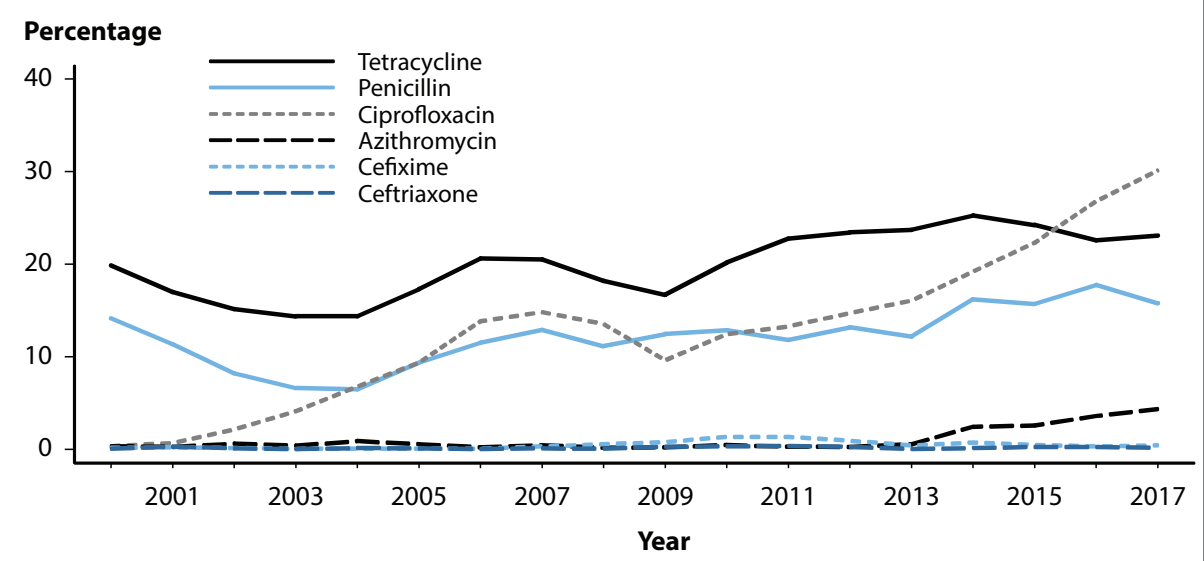

* Resistance: Fluoroquinolone (ciprofloxacin) $=\mathrm{MIC} \geq 1.0 \mu \mathrm{g} / \mathrm{mL}$; Penicillin $=\mathrm{MIC} \geq 2.0 \mu \mathrm{g} / \mathrm{mL}$ or B-lactamase positive; Tetracycline $=\mathrm{MIC} \geq 2.0 \mu \mathrm{g} / \mathrm{mL}$.

${ }^{\dagger}$ Elevated MICs: Azithromycin = MIC $\geq 1.0 \mu \mathrm{g} / \mathrm{mL}$ (2000-2004); $\geq 2.0 \mu \mathrm{g} / \mathrm{mL}$ (2005-2017); Ceftriaxone $=\mathrm{MIC} \geq 0.125 \mu \mathrm{g} / \mathrm{mL}$; Cefixime $=\mathrm{MIC} \geq 0.25 \mu \mathrm{g} / \mathrm{mL}$.

NOTE: Cefixime susceptibility was not tested in 2007 and 2008.

ADAPTED FROM: Kirkcaldy RD, Harvey A, Papp JR, et al. Neisseria gonorrhoeae antimicrobial susceptibility surveillance - The Gonococcal Isolate Surveillance Project, 27 Sites, United States, 2014. MMWR Surveill Summ 2016; 65(7):1-24. 
Figure 32. Susceptibility Patterns of Neisseria gonorrhoeae Isolates to Antimicrobials, Gonococcal Isolate Surveillance Project (GISP), 2017

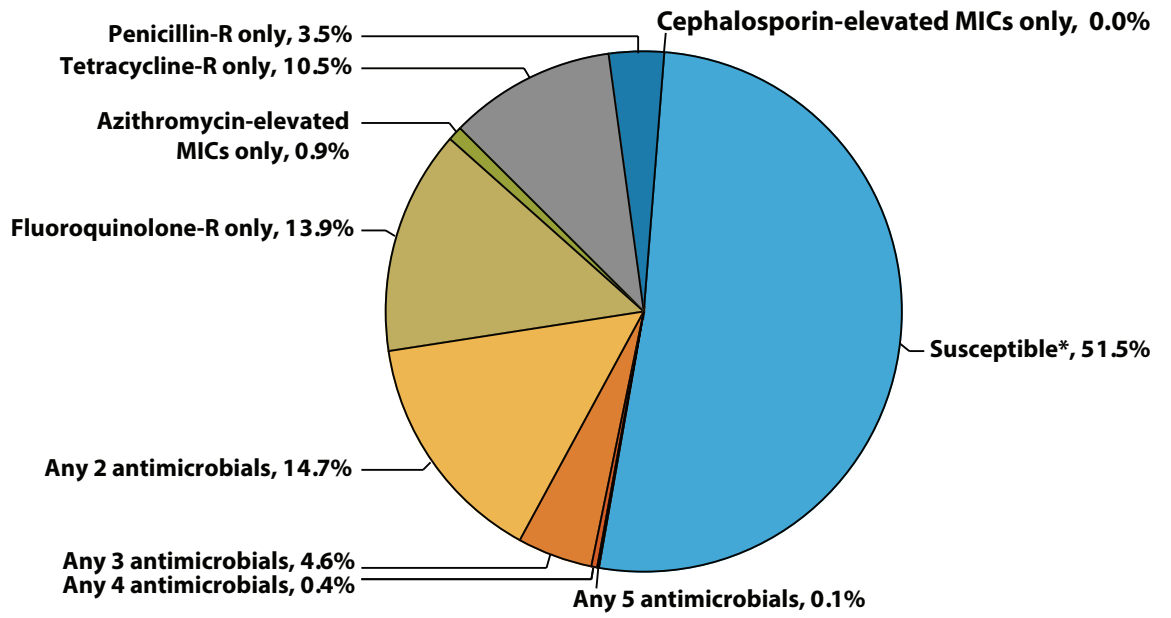

* Susceptible category only includes isolates with penicillin, tetracycline, and fluoroquinolone MIC values that are considered susceptible and isolates with ceftriaxone, cefixime, and azithromycin MIC values that are not considered elevated.

NOTE: Elevated MIC = Ceftriaxone: $\geq 0.125 \mu \mathrm{g} / \mathrm{ml}$; Cefixime: $\geq 0.25 \mu \mathrm{g} / \mathrm{ml}$; Azithromycin: $\geq 2.0 \mu \mathrm{g} / \mathrm{ml}$. Resistant (R) MIC = Tetracycline: $\geq 2.0 \mu \mathrm{g} / \mathrm{ml}$; Fluoroquinolone: $\geq 1.0 \mu \mathrm{g} / \mathrm{ml}$; Penicillin: $\geq 2.0 \mu \mathrm{g} / \mathrm{ml}$ or PPNG.

ACRONYMS: $\mathrm{R}=$ Resistant; $P$ PNG = Penicillinase-producing Neisseria gonorrhoeae and chromosomally-mediated penicillin-resistant N. gonorrhoeae; MIC = Minimum Inhibitory Concentration.

Figure 33. Distribution of Primary Antimicrobial Drugs Used to Treat Gonorrhea Among Participants, Gonococcal Isolate Surveillance Project (GISP), 1988-2017

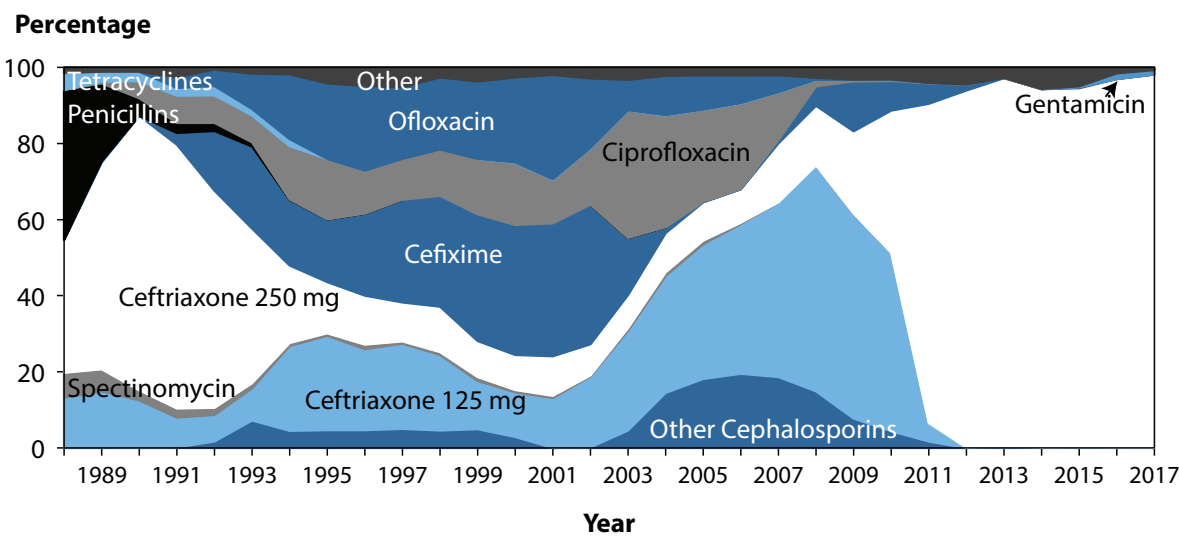

NOTE: For 2017, "Other" includes azithromycin $2 \mathrm{~g}(0.3 \%)$, no therapy $(0.3 \%)$, and other less frequently used drugs (0.1\%). 


\section{Figure 34. Gonorrhea - Estimated Proportion* of Cases by Treatment Regimen Received and Jurisdiction ${ }^{\dagger}$, STD Surveillance Network (SSuN), 2017}

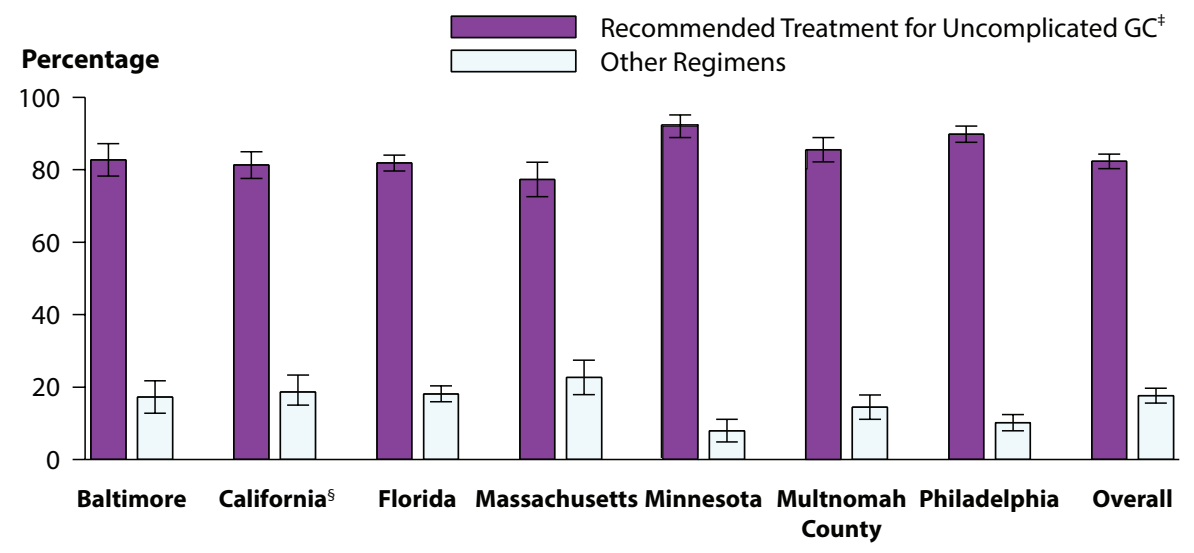

* Percentage and $95 \% \mathrm{Cl}$ reflect weighted estimates for all reported gonorrhea cases; minor variances cause category estimates to total to slightly more or less than overall case estimate. ${ }^{+}$Includes SSuN jurisdictions with documented treatment information (antimicrobials and dosages) for $\geq 90 \%$ of cases with complete investigations.

${ }^{\ddagger}$ The recommended treatment for uncomplicated gonorrhea is dual treatment with $250 \mathrm{mg}$ dose of Ceftriaxone [IM] plus $1 \mathrm{~g}$ dose of Azithromycin [PO].

${ }^{5}$ California data exclude San Francisco.

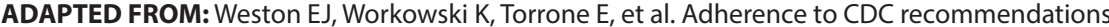
for the treatment of uncompllicated gonorrhea - STD Surveillance Network (SSuN), United States, 2016. MMWR Morb Mort Wkly Rep. 2018; 67:473-76.

ACRONYMS: $\mathrm{Cl}$ = Confidence interval; $\mathrm{GC}=$ Gonorrhea; IM = Intramuscular injection $\mathrm{PO}=$ By mouth

\section{References}

1. Fleming DT, Wasserheit JN. From epidemiological synergy to public health policy and practice: The contribution of other sexually transmitted diseases to sexual transmission of HIV infection. Sex Transm Infect 1999; 75(1): 3-17.

2. Hogben M, Leichliter JS. Social determinants and sexually transmitted disease disparities. Sex Transm Dis 2008; 35(12 Suppl): S13-18.

3. Centers for Disease Control and Prevention. Sexually transmitted diseases treatment guidelines, 2015. MMWR Recomm Rep 2015; 64(No. RR-3): 1-137.

4. Satterwhite CL, Torrone E, Meites E, et al. Sexually transmitted infections among US women and men: Prevalence and incidence estimates, 2008. Sex Transm Dis 2013; 40(3): 187-193. DOI: 10.1097/ OLQ.0b013e318286bb53. Review.

5. Grey JA, Bernstein KT, Sullivan PS, et al. Estimating the population sizes of men who have sex with men in US states and counties using data from the American Community Survey. JMIR Public Health Surveill 2016; 2(1):e14.

6. Stenger M, Pathela P, Anschuetz G, et al Increases in the rate of Neisseria gonorrhoeae among gay, bisexual and other men who have sex with men (MSM) - findings from the STD Surveillance Network 2010-2015. Sex Transm Dis 2017; 44(7): 393-397.

7. Centers for Disease Control and Prevention Update to CDC's sexually transmitted diseases treatment guidelines, 2006: Fluoroquinolones no longer recommended for treatment of gonococcal infections. MMWR Morb Mortal Wkly Rep 2007; 56(14): 332-336.

8. Centers for Disease Control and Prevention. Sexually transmitted diseases treatment guidelines, 2010. MMWR Recomm Rep 2010; 59(No.RR-12): 1-110.

9. Schwarcz S, Zenilman J, Schnell D, et al. National surveillance of antimicrobial resistance in Neisseria gonorrhoeae. JAMA 1990; 264(11): 1413-1417. 
This page intentionally left blank. 


\section{Background}

Syphilis, a genital ulcerative disease caused by the bacterium Treponema pallidum, is associated with significant complications if left untreated and can facilitate the transmission and acquisition of HIV infection..$^{1-3}$ Additionally, historical data demonstrate that untreated syphilis in pregnant women, if acquired during the four years before delivery, can lead to infection of the fetus in up to $80 \%$ of cases and may result in stillbirth or death of the infant in up to $40 \%$ of cases. ${ }^{4}$

In 2000 and 2001, the national rate of reported primary and secondary (P\&S) syphilis cases was 2.1 cases per 100,000 population, the lowest rate since reporting began in 1941 (Figure 35, Table 1). However, the P\&S syphilis rate has increased almost every year since 2000-2001. Since 2000, the rise in the rate of reported P\&S syphilis has been primarily attributable to increased cases among men and, specifically, among gay, bisexual, and other men who have sex with men (collectively referred to as MSM). MSM account for the majority of P\&S syphilis cases, and estimated rates are substantially higher among MSM compared with men who have sex with women only (MSW) or women. ${ }^{5}$ The number of cases among MSM has continued to increase, but within the last five years, cases among MSW and women have increased substantially as well. The increase in syphilis among women is of particular concern because it has been associated with a striking increase in congenital syphilis. These recent trends highlight the importance of national syphilis surveillance to better understand the current epidemiology of syphilis in the United States and to focus prevention efforts.
Interpreting Rates of Reported Cases of Syphilis

Left untreated, infection with syphilis can span decades. P\&S syphilis are the earliest stages of infection, reflect symptomatic disease, and are indicators of incident infection. ${ }^{6}$ For these reasons, trend analyses of syphilis focus on reported cases and rates of reported cases of $\mathrm{P} \& \mathrm{~S}$ syphilis. When referring to " $P \& S$ both primary and secondary cases, and "rate of P\&S syphilis" refers to this sum per unit population.

Changes in reporting and screening practices can complicate interpretation of trends over time. To minimize the effect of changes in reporting over time, trend data in this report are restricted to jurisdictions that consistently report data of interest (e.g., sex of sex partners) for each year of a given time period. Details of these restrictions are provided in the text and footnotes of the pertinent text and figures. syphilis", case counts are the sum of

\section{P\&S Syphilis - United States}

In 2017, a total of 30,644 cases of P\&S syphilis were reported in the United States, yielding a rate of 9.5 cases per 100,000 population (Figure 35, Table 1). This rate represents a $10.5 \%$ increase compared with 2016 (8.6 cases per 100,000 population), and a $72.7 \%$ increase compared with 2013 (5.5 cases per 100,000 population).

\section{P\&S Syphilis by Region}

In 2017, the West had the highest rate of reported $\mathrm{P} \& \mathrm{~S}$ syphilis cases (13.2 cases per 100,000 population), followed by the South ( 9.7 cases per 100,000 population), the Northeast (8.0 cases per 100,000 population), and the Midwest ( 6.2 cases per 100,000 population) (Table 27). During 2016-2017, the P\&S syphilis rate increased $16.8 \%$ in the West, $10.2 \%$ in the South, and $8.8 \%$ in the Midwest; the rate did not change in the Northeast (Figure 36, Table 27).

\section{Figure 35. Syphilis - Rates of Reported Cases by Stage of Infection, United States, 1941-2017}

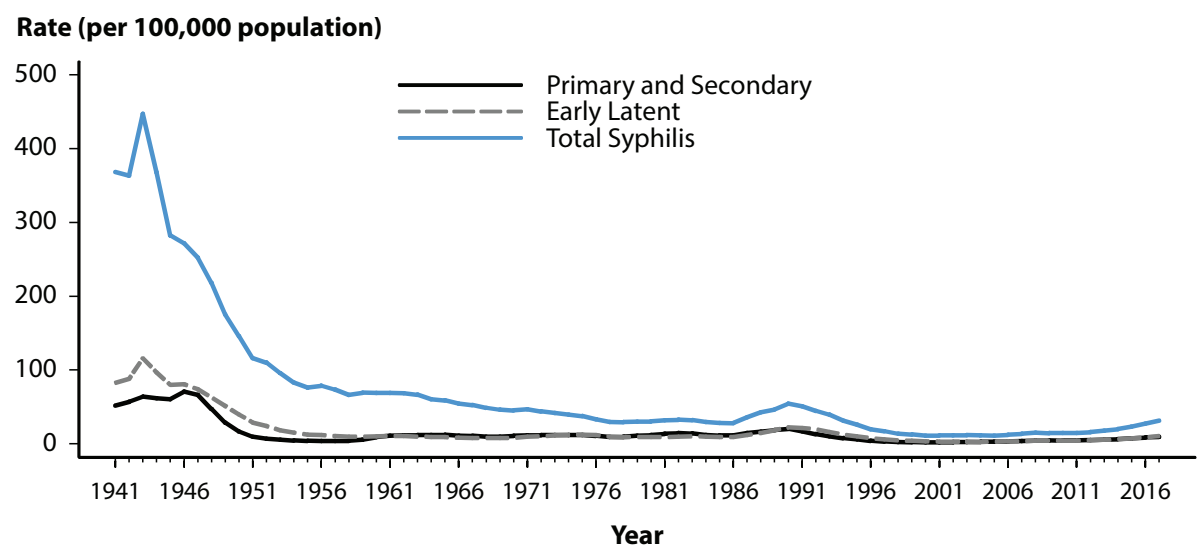

NOTE: Data collection for syphilis began in 1941; however, syphilis became nationally notifiable in 1944. Refer to the National Notifiable Disease Surveillance System (NNDSS) website for more information: https://wwwn.cdc.gov/nndss/conditions/syphilis/ 


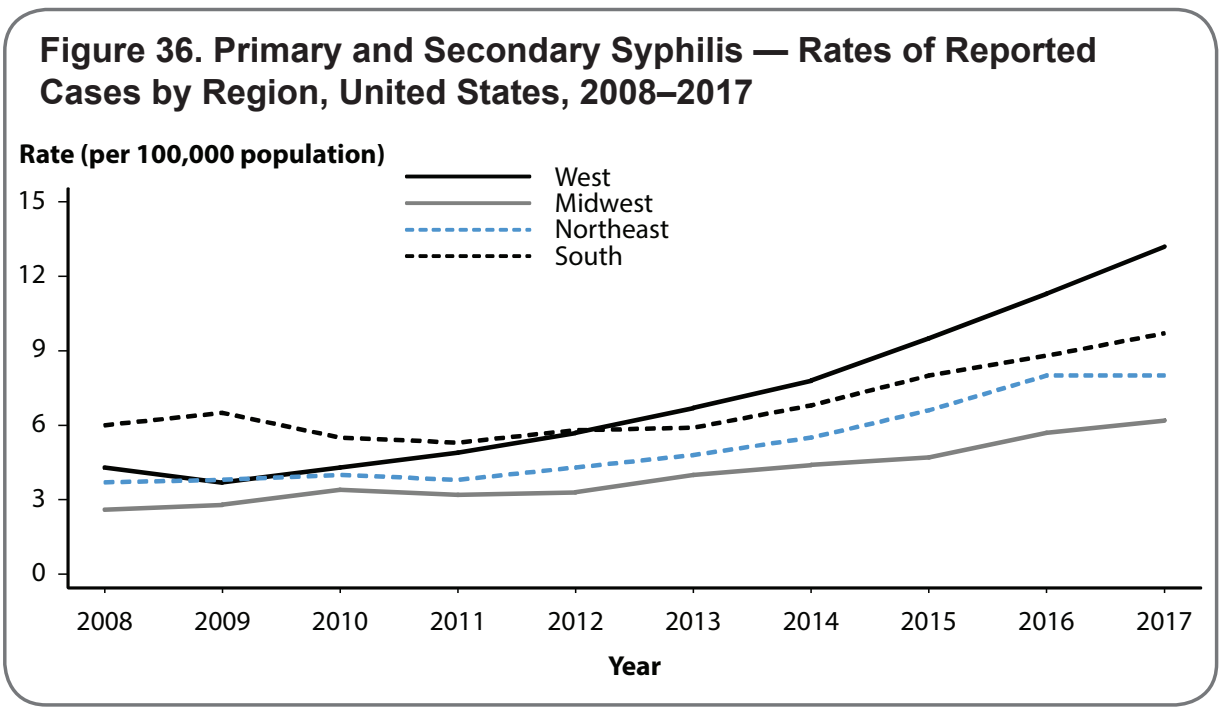

\section{P\&S Syphilis by State}

In 2017, rates of reported $P \& S$ syphilis cases per 100,000 population ranged by state from 0.7 in Wyoming to 20.0 in Nevada (Figure 37, Table 26). The rate of reported P\&S syphilis cases in the District of Columbia was 40.2 cases per 100,000 population. During 2016-2017, P\&S syphilis rates increased in $72 \%$ (36/50) of states and the District of Columbia, and remained stable or decreased in $28 \%(14 / 50)$ of states (Table 27).

\section{P\&S Syphilis by Metropolitan Statistical Area}

The overall rate of reported $\mathrm{P} \& \mathrm{~S}$ syphilis cases in the 50 most populous metropolitan statistical areas (MSAs) was 12.1 cases per 100,000 population in 2017 , which represents a $9.0 \%$ increase since 2016 (11.1 cases per 100,000 population) (Table 30). Overall, in 2017, 70.4\% of reported P\&S syphilis cases (72.9\% of male cases and $52.0 \%$ of female cases) were reported by these 50 MSAs. In 2017, the rate among women in these MSAs was 2.1 cases per 100,000 females, while the rate among men was 22.5 cases per 100,000 males (Tables 31 and 32).

\section{P\&S Syphilis by County}

In $2017,63 \%$ of reported $P \& S$ syphilis cases occurred in 70 counties or independent cities (Table 33). Of 3,140 counties in the United States, $531(16.9 \%)$ had a P\&S syphilis rate greater than 7.5 cases per 100,000 population, $522(16.6 \%)$ reported a rate from 3.9 to 7.5 cases per 100,000 population, $525(16.7 \%)$ reported a rate from $>0$ to 3.8 cases per 100,000 population, and 1,562 (49.7\%) counties reported no cases of $\mathrm{P} \& \mathrm{~S}$ syphilis in 2017 (Figure 38).

\section{P\&S Syphilis by Sex and Sexual Behavior}

As has been observed in previous years, in 2017 the rate of reported P\&S syphilis cases among men (16.9 cases per 100,000 males) was much higher than the rate among women ( 2.3 cases per 100,000 females), and men accounted for a large majority (87.7\%) of P\&S syphilis cases (Figure 39, Tables 28 and 29). Among men, the rate of P\&S syphilis has increased every year since 2000, and during 2016-2017, the rate among men increased $9.0 \%$ (Figure 40, Table 29). In contrast, the P\&S syphilis rate among women fluctuated between 0.8 and 1.7 cases per 100,000 females during 20002013, but has increased substantially since 2013 (Figure 40, Table 28). During 2013-2017, the P\&S syphilis rate among women more than doubled (increased 155.6\%). During 2016-2017, the P\&S syphilis rate among women increased $21.1 \%$.

These increases in male and female P\&S syphilis rates were observed in almost every region of the country during 2016-2017. Among men, the rate increased $14.5 \%$ in the

\section{Figure 37. Primary and Secondary Syphilis - Rates of Reported Cases by State, United States and Outlying Areas, 2017}

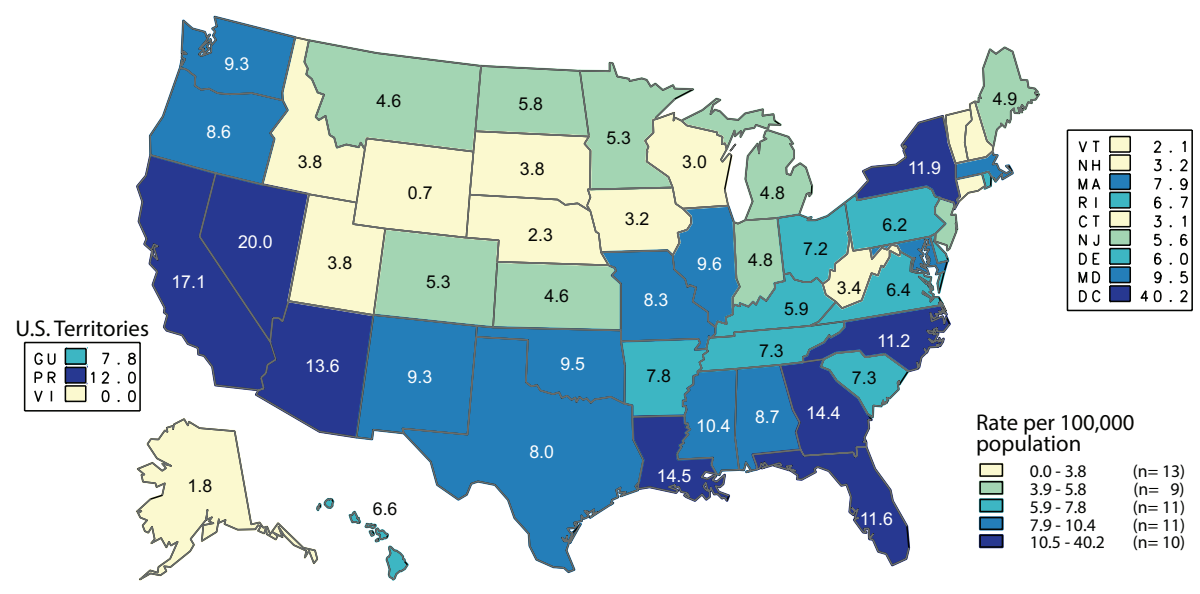

NOTE: The total rate of reported cases of primary and secondary syphilis for the United States and outlying areas (including Guam, Puerto Rico, and the Virgin Islands) was 9.5 per 100,000 population. See Section A1.11 in the Appendix for more information on interpreting reported rates in the outlying areas.

ACRONYMS: $\mathrm{GU}=$ Guam; PR = Puerto Rico; $\mathrm{Vl}=$ Virgin Islands. 


\section{Figure 38. Primary and Secondary Syphilis - Rates of Reported Cases by County, United States, 2017}

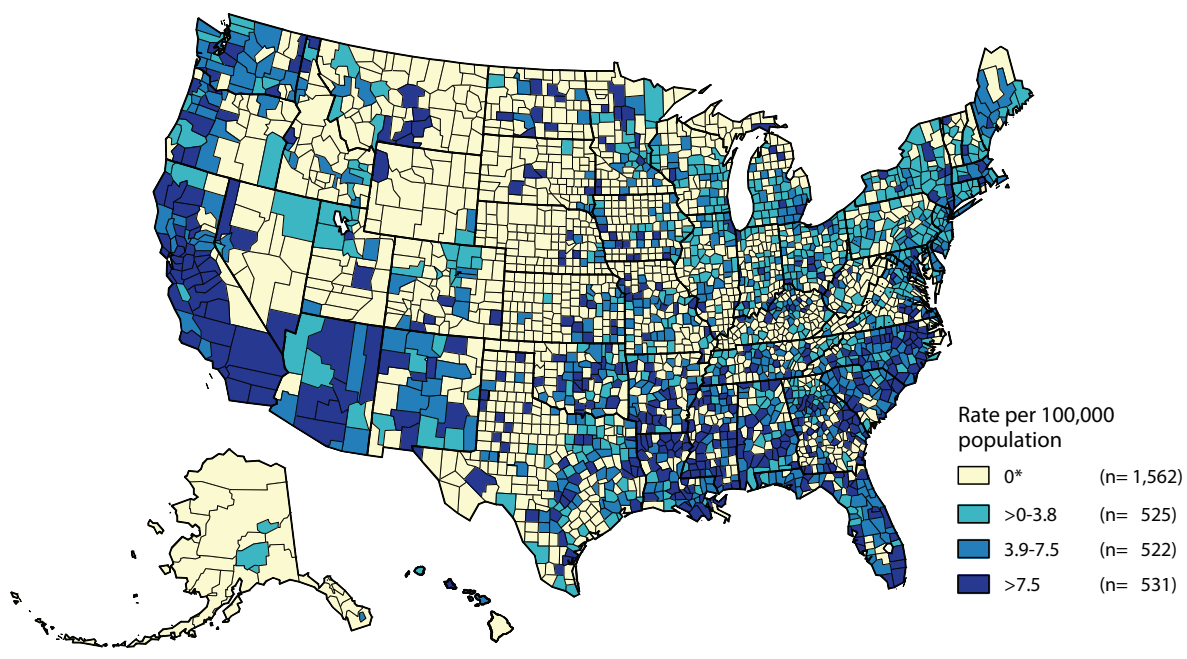

* In 2017, 1,562 (49.7\%) of 3,140 counties in the United States reported no cases of primary and secondary syphilis. Refer to the NCHHSTP AtlasPlus for further county-level rate information: https://www.cdc.gov/nchhstp/atlas/

\section{Figure 39. Primary and Secondary Syphilis - Distribution of Cases by Sex and Sexual Behavior, United States, 2017}

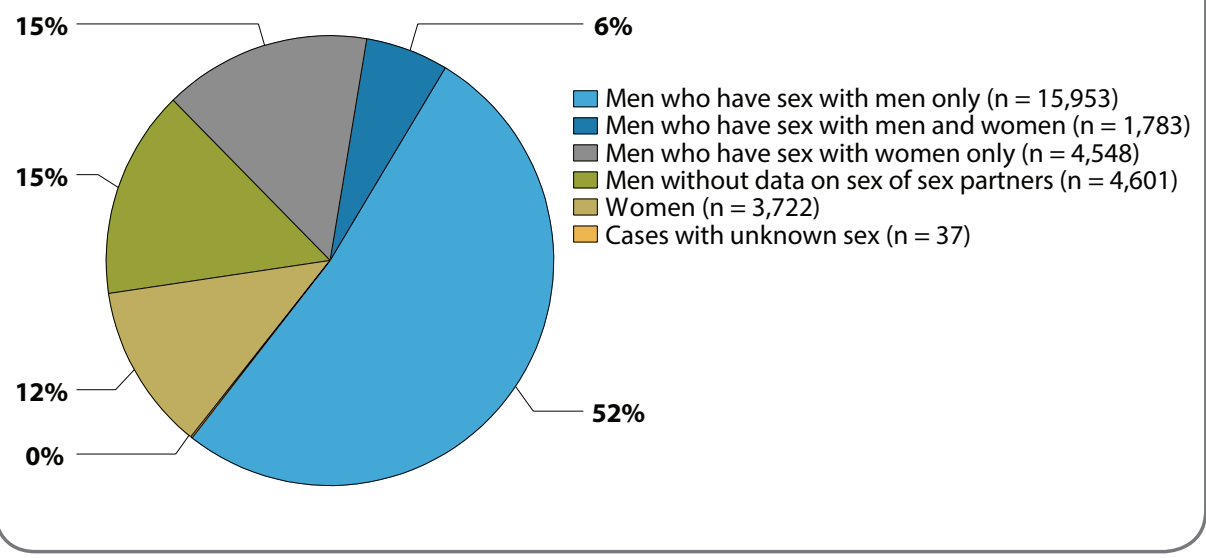

West, $8.3 \%$ in the South, and $7.8 \%$ in the Midwest; the rate decreased $0.7 \%$ in the Northeast (Table 29). Among women, the largest increases were observed in the West $(29.6 \%)$, followed by the South $(22.7 \%)$, the Northeast (11.1\%) and the Midwest $(8.3 \%)$ (Table 28$)$.

MSM continued to account for the majority of P\&S syphilis cases in 2017 (Figures 39 and 41). Of 30,644 reported P\&S syphilis cases in 2017, 17,736 (57.9\%) were among MSM, including 15,953 (52.1\%) cases among men who had sex with men only and $1,783(5.8 \%)$ cases among men who had sex with both men and women (Figure 39). Overall, $4,548(14.8 \%)$ cases were among MSW, 3,722 (12.1\%) were among women, 4,601 (15.0\%) were among men without information about sex of sex partner, and $37(0.1 \%)$ were cases reported with unknown sex. Among the 22,284 male cases with information on sex of sex partner, $79.6 \%$ occurred among MSM.
A total of 37 states were able to classify at least $70.0 \%$ of reported $\mathrm{P} \& \mathrm{~S}$ syphilis cases as MSM, MSW, or women each year during 20132017 (Figure 41). In these states, during 2016-2017, the number of cases increased $8.6 \%$ among MSM, $17.8 \%$ among MSW, and $24.9 \%$ among women.

\section{P\&S Syphilis by Age}

As in previous years, in 2017, rates of reported P\&S syphilis cases were highest among persons aged 25-29 years (Figure 42, Table 34). In 2017, the highest rates were observed among men aged 25-29 years (51.9 cases per 100,000 males), 20-24 years (41.1 cases per 100,000 males), and 30-34 years (39.3 cases per 100,000 males). The highest rates among women were among those aged 20-24 years ( 7.8 cases per 100,000 females) and those aged 25-29 years ( 7.1 cases per 100,000 females).

During 2016-2017, the overall rate of reported P\&S syphilis cases increased in all age groups among those aged 15 years or older (Table 34 ). Rates increased $9.8 \%$ among those aged 15-19 years, $7.8 \%$ among those aged 20-24 years, $10.7 \%$ among those aged $25-29$ years, $14.3 \%$ among those aged 30-34 years, $17.8 \%$ among those aged 35-39 years, $6.4 \%$ among those aged $40-44$ years, $4.3 \%$ among those aged $45-54$ years, $11.8 \%$ among those aged 55-64 years, and $16.7 \%$ among those aged 65 or older.

In 2017, persons aged 15-44 years accounted for $80.2 \%$ of reported $\mathrm{P} \& \mathrm{~S}$ syphilis cases with known age. Among men, during 2016-2017, the $\mathrm{P} \& \mathrm{~S}$ syphilis rate increased in all age groups among those aged 15-44 years. Among women, the rate decreased slightly among those aged 15-19 years, but increased in all older age groups (Figures 43 and 44, Table $34)$. 


\section{Figure 40. Primary and Secondary Syphilis - Rates of Reported Cases by Sex and Male-to-Female Rate Ratios, United States, 1990-2017}

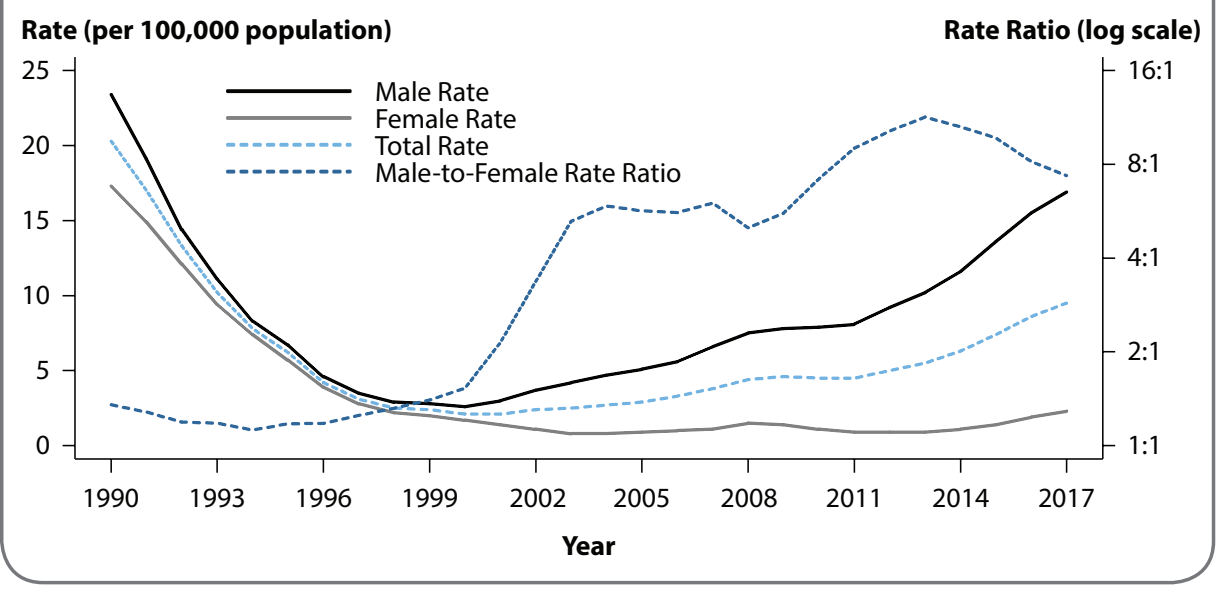

Figure 41. Primary and Secondary Syphilis - Reported Cases by Sex and Sexual Behavior, 37 States*, 2013-2017

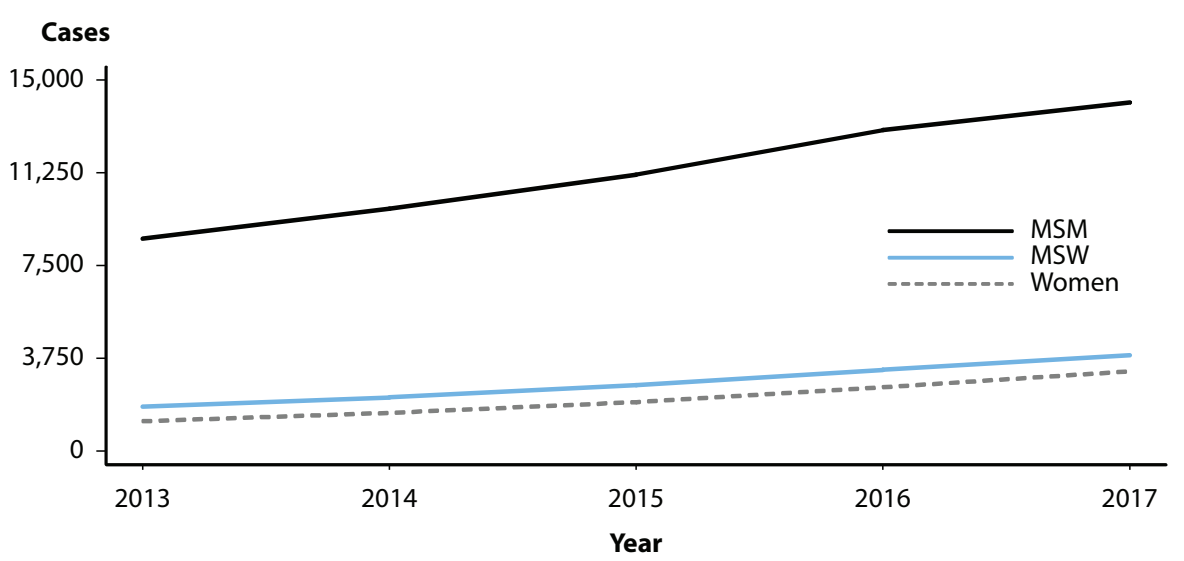

* 37 states were able to classify $\geq 70 \%$ of reported cases of primary and secondary syphilis as either MSM, MSW, or women for each year during 2013-2017.

ACRONYMS: MSM = Gay, bisexual, and other men who have sex with men (collectively referred to as MSM); MSW = Men who have sex with women only.

Figure 42. Primary and Secondary Syphilis - Rates of Reported Cases by Age Group and Sex, United States, 2017

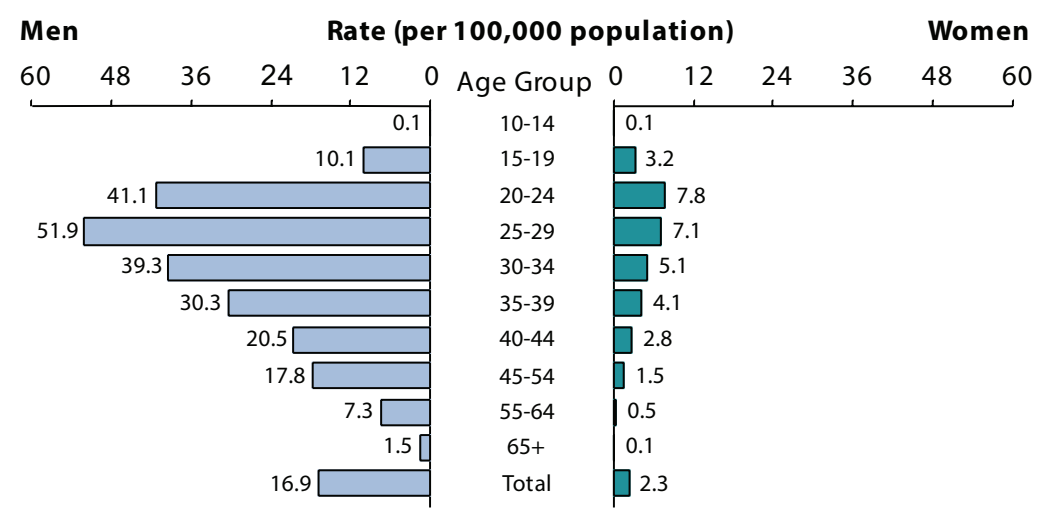

P\&S Syphilis by

Race/Hispanic Ethnicity

In 2017, the rate of reported $P \& S$ syphilis cases was highest among Blacks (24.2 cases per 100,000 population) (Table $35 \mathrm{~B})$. The $\mathrm{P} \& \mathrm{~S}$ syphilis rate among Blacks was 4.5 times the rate among Whites (5.4 cases per 100,000 population), the rate among Native Hawaiians/Other Pacific Islanders (NHOPI) (13.9 cases per 100,000 population) was 2.6 times the rate among Whites, the rate among Hispanics (11.8 cases per 100,000 population) was 2.2 times the rate among Whites, the rate among American Indians/Alaska Natives (AI/AN) (11.1 cases per 100,000 population) was 2.1 times the rate among Whites, and the rate among Asians (4.4 cases per 100,000 population) was 0.8 times the rate among Whites.

During 2013-2017, the P\&S syphilis rate increased among all race/ Hispanic ethnicity groups (Figure 45). The greatest increases during 20162017 were observed among AI/AN $(38.8 \%)$ and those who identified as Multiracial (31.7\%), followed by Asians (15.7\%), Whites (10.2\%), NHOPI (9.4\%), Hispanics (9.3\%), and Blacks (5.7\%).

More information on $\mathrm{P} \& \mathrm{~S}$ syphilis rates among racial/Hispanic ethnicity groups can be found in the Special Focus Profiles.

\section{P\&S Syphilis and HIV Co-infection}

Reported cases of P\&S syphilis continue to be characterized by a high rate of HIV co-infection, particularly among MSM (Figure 46). Among 2017 P\&S syphilis cases with known HIV status, $45.5 \%$ of cases among MSM were HIV-positive, compared with $8.8 \%$ of cases among MSW, and $4.5 \%$ of cases among women. 


\section{Figure 43. Primary and Secondary Syphilis - Rates of Reported Cases Among Women Aged 15-44 Years by Age Group, United States, 2008-2017}

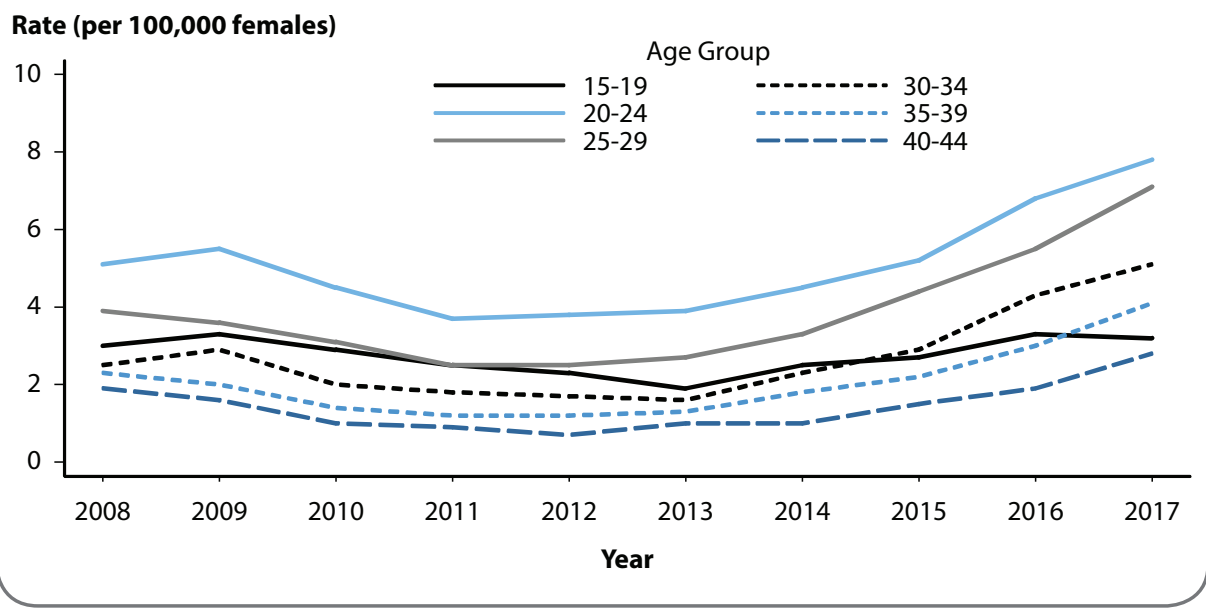

\section{Figure 44. Primary and Secondary Syphilis - Rates of Reported Cases Among Men Aged 15-44 Years by Age Group, United States, 2008-2017}

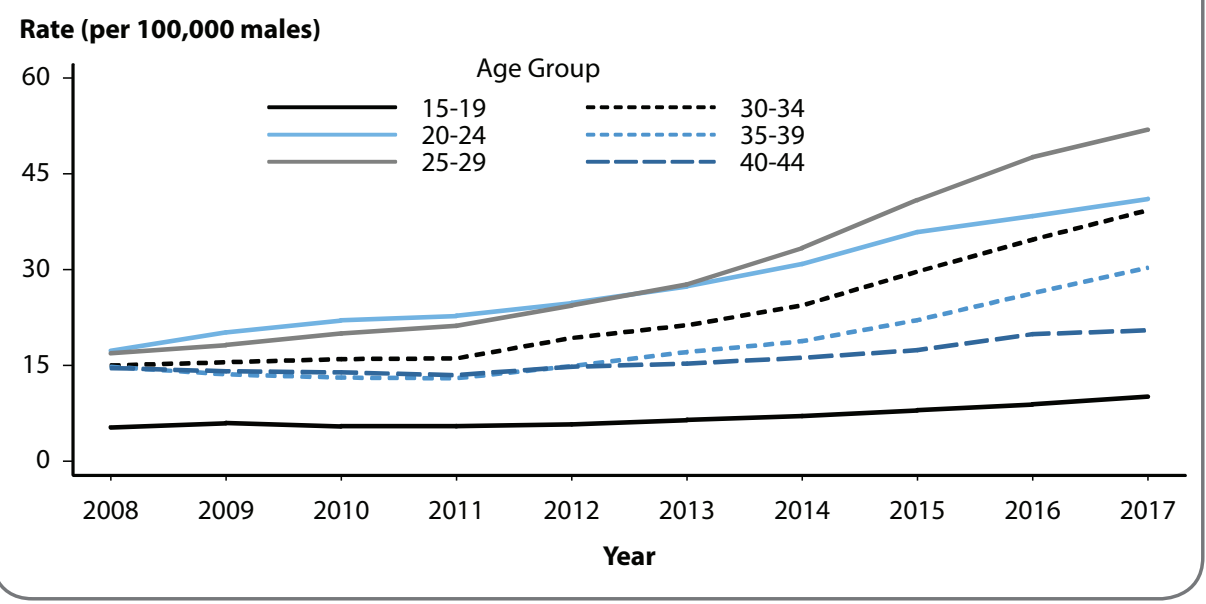

\section{P\&S Syphilis by Reporting Source}

In $2017,17.0 \%$ of $P \& S$ syphilis cases were reported from STD clinics, $71.7 \%$ were reported from venues outside of STD clinics, and $11.4 \%$ of cases had an unknown reporting source (Table A2). During 20162017, the number of P\&S syphilis cases reported by STD clinics and by non-STD clinic settings increased (Figure 47). However, the proportion of P\&S syphilis cases that were reported by STD clinics has declined over the last decade from $31.1 \%$ of cases in 2008 to $17.0 \%$ of cases in 2017. In 2017, private physicians/ health maintenance organizations (HMOs) and STD clinics were the most common reporting sources among MSM (29.8\% and 22.4\%, respectively), MSW (23.0\% and $19.8 \%$, respectively), and women (Figure 48).

\section{Congenital Syphilis}

After decreasing from 10.5 to 8.4 reported congenital syphilis cases per 100,000 live births during 2008(26.1\% and $14.4 \%$, respectively)
2012, the rate of reported congenital syphilis has subsequently increased each year since 2012 (Table 1). In 2017, there were a total of 918 reported cases of congenital syphilis, including 64 syphilitic stillbirths and 13 infant deaths, and the national rate was 23.3 cases per 100,000 live births. This rate represents a $43.8 \%$ increase relative to 2016 (16.2 cases per 100,000 live births) and a $153.3 \%$ increase relative to 2013 ( 9.2 cases per 100,000 live births). As has been observed historically, this increase in the congenital syphilis rate has paralleled increases in $\mathrm{P} \& \mathrm{~S}$ syphilis among all women and reproductiveaged women during 2013-2017 (155.6\% and $142.8 \%$ increases, respectively) (Figure 49, Table 28).

During 2013-2017, the increase in reported congenital syphilis cases was primarily attributable to an increase in the West. During this time period, the congenital syphilis rate increased $362.5 \%$ in the West, $107.7 \%$ in the South, $93.1 \%$ in the Northeast, and $43.8 \%$ in the Midwest (Table 41). During 2016-2017, the congenital syphilis rate increased $60.3 \%$ in the South, $40.7 \%$ in the West, $5.7 \%$ in the Northeast, and 5.7\% in the Midwest. In 2017, the highest congenital syphilis rates were reported from the West (37.0 cases per 100,000 live births), followed by the South (29.5 cases per 100,000 live births), Midwest (9.2 cases per 100,000 live births), and the Northeast (5.6 cases per 100,000 live births). In addition, rates were highest among Blacks (58.9 cases per 100,000 live births), followed by AI/AN (35.5 cases per 100,000 live births), Hispanics (33.5 cases per 100,000 live births), Whites (9.7 cases per 100,000 live births), and Asians/Pacific Islanders (4.3 cases per 100,000 live births) (Table 42). 
Figure 45. Primary and Secondary Syphilis - Rates of Reported Cases by Race and Hispanic Ethnicity, United States, 2013-2017

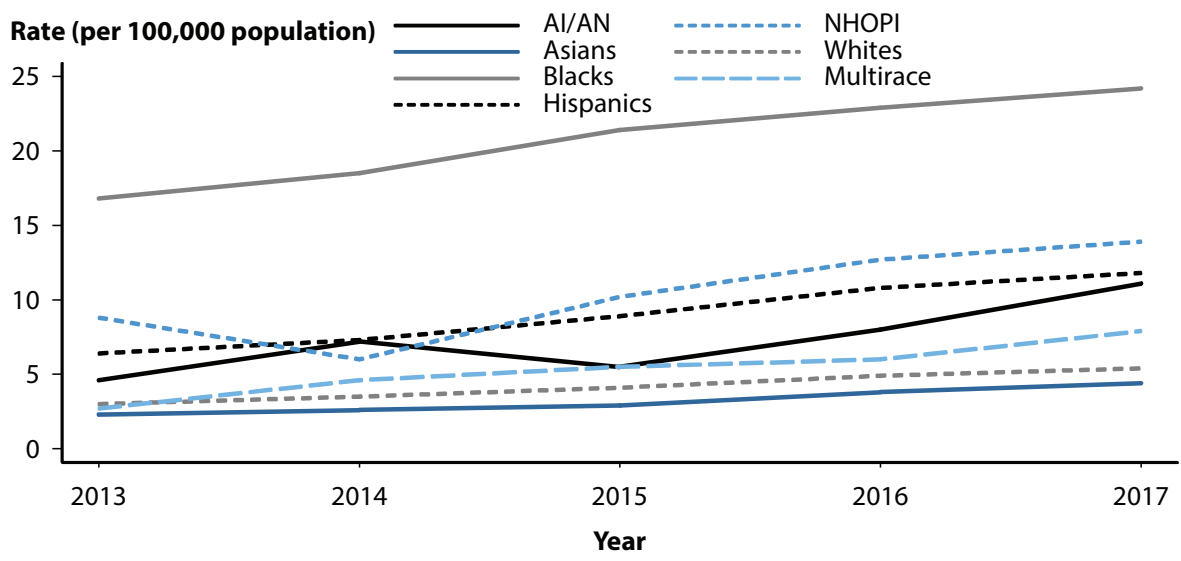

NOTE: Not all US jurisdictions reported cases in OMB-compliant Race categories in 2017. This may minimally under- or overestimate rates for Asians, NHOPI, or Multirace individuals. For completeness, data in this figure include cases reported from all jurisdictions. See Section A1.5 in the Appendix for information on reporting STD case data for race and Hispanic ethnicity. ACRONYMS: AI/AN = American Indians/Alaska Natives; NHOPI = Native Hawaiians/Other Pacific Islanders; $\mathrm{OMB}=$ Office of Management and Budget.

Figure 46. Primary and Secondary Syphilis - Reported Cases by Sex, Sexual Behavior, and HIV Status, United States, 2017

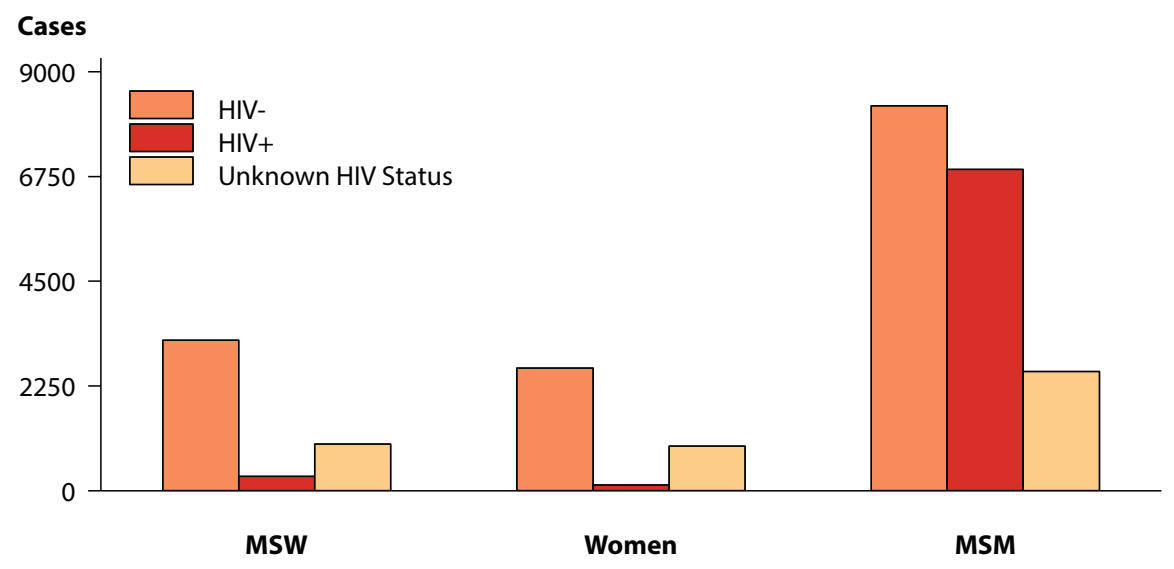

ACRONYMS: MSM = Gay, bisexual, and other men who have sex with men (collectively referred to as MSM); MSW = Men who have sex with women only.
Syphilis - All Stages (P\&S, Early Latent, Late, Late Latent, and Congenital)

In 2017, the total case count of reported syphilis (all stages combined: P\&S, early latent, late and late latent, and congenital) was the highest recorded since 1993. The total number of cases of syphilis (all stages) reported to CDC increased $15.3 \%$ during 2016-2017 (from 88,053 cases to 101,567 cases) (Table 1). The number of cases of early latent syphilis reported to CDC increased $17.6 \%$ (from 28,924 cases to 34,013 cases), and the number of cases of late and late latent syphilis increased $17.3 \%$ (from 30,676 cases to 35,992 cases) (Tables 1,36 , and 38 ).

\section{Syphilis among Special Populations}

More information about syphilis and congenital syphilis in racial/ Hispanic ethnicity groups, women of reproductive age, adolescents, and MSM can be found in the Special Focus Profiles.

\section{Syphilis Summary}

The national rate of reported $\mathrm{P} \& \mathrm{~S}$ syphilis cases reached an historic low in 2000 and 2001, but has increased almost every year since then. This increase was largely attributable to an increase among men, and in particular among MSM. However, in the last five years, rates have increased among both men and women, and the P\&S syphilis rate among women has more than doubled. Rates of reported congenital syphilis cases also increased substantially during 2013-2017 and during 2016-2017. MSM continued to account for the majority of reported $\mathrm{P} \& \mathrm{~S}$ syphilis cases in 2017. Nationally, the highest rates of P\&S syphilis in 2017 were observed among men aged 20-34 years, among men in the West, and among Black men. 
Figure 47. Primary and Secondary Syphilis - Reported Cases by Reporting Source and Sex, United States, 2008-2017

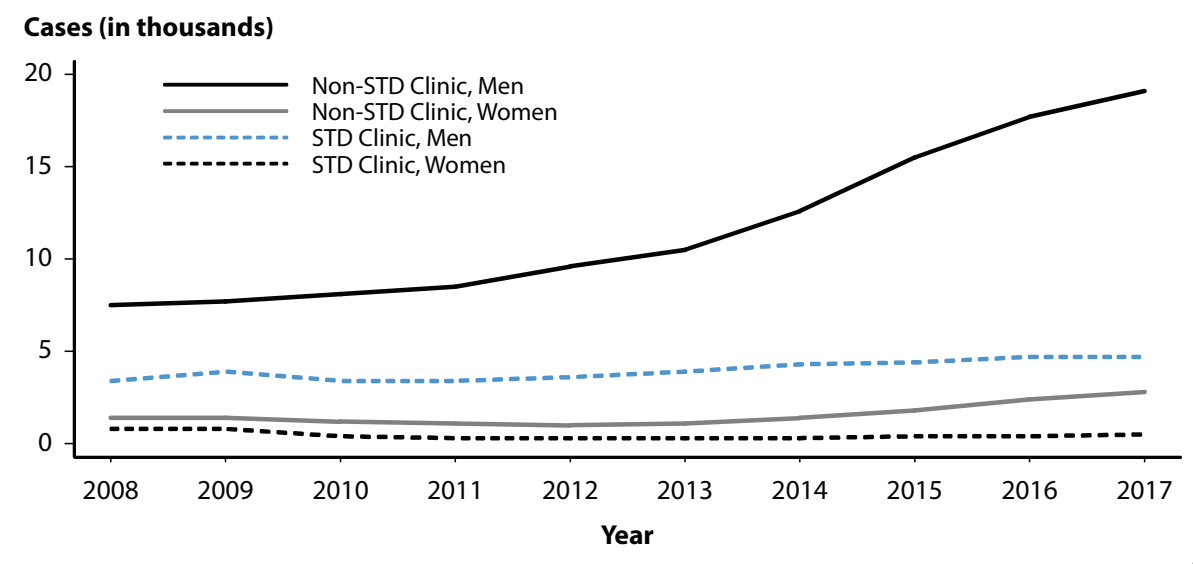

\section{Figure 48. Primary and Secondary Syphilis - Percentage of Reported Cases* by Sex, Sexual Behavior, and Selected Reporting Sources, United States, 2017}

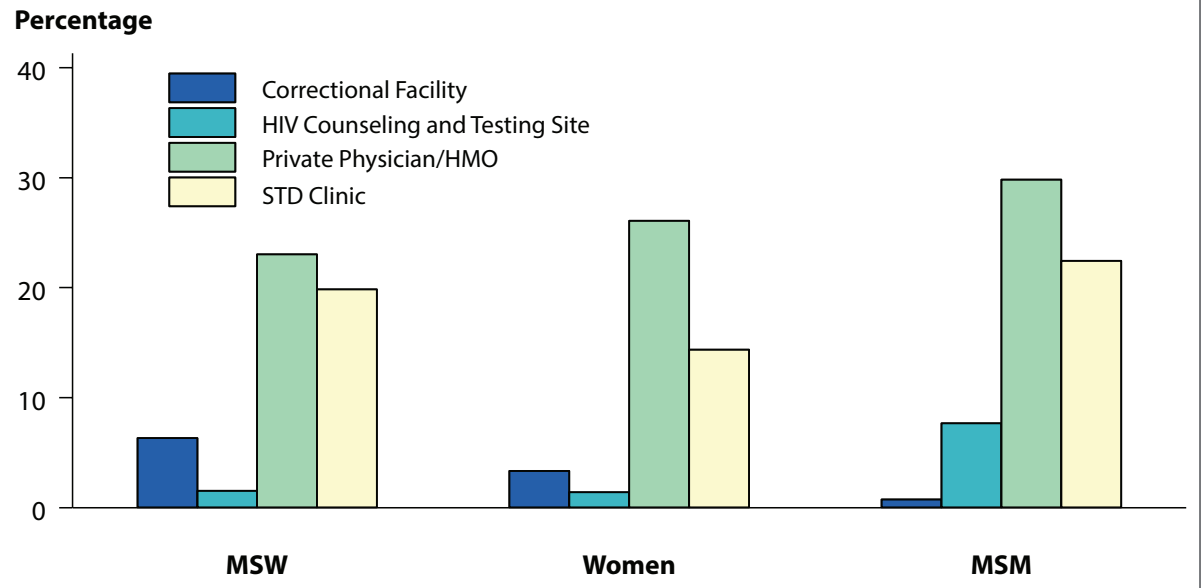

* Of all primary and secondary syphilis cases, $11.4 \%$ had a missing or unknown reporting source. Among all cases with a known reporting source, the reporting source categories presented represent $56.4 \%$ of cases; $43.6 \%$ were reported from sources other than those shown. ACRONYMS: $\mathrm{HMO}=$ health maintenance organization; $\mathrm{MSM}=\mathrm{Gay}$, bisexual, and other men who have sex with men (collectively referred to as MSM); MSW = Men who have sex with women only. 


\section{Figure 49. Congenital Syphilis - Reported Cases by Year of Birth and Rates of Reported Cases of Primary and Secondary Syphilis Among Women Aged 15-44 Years, United States, 2008-2017}

\section{CS Cases}

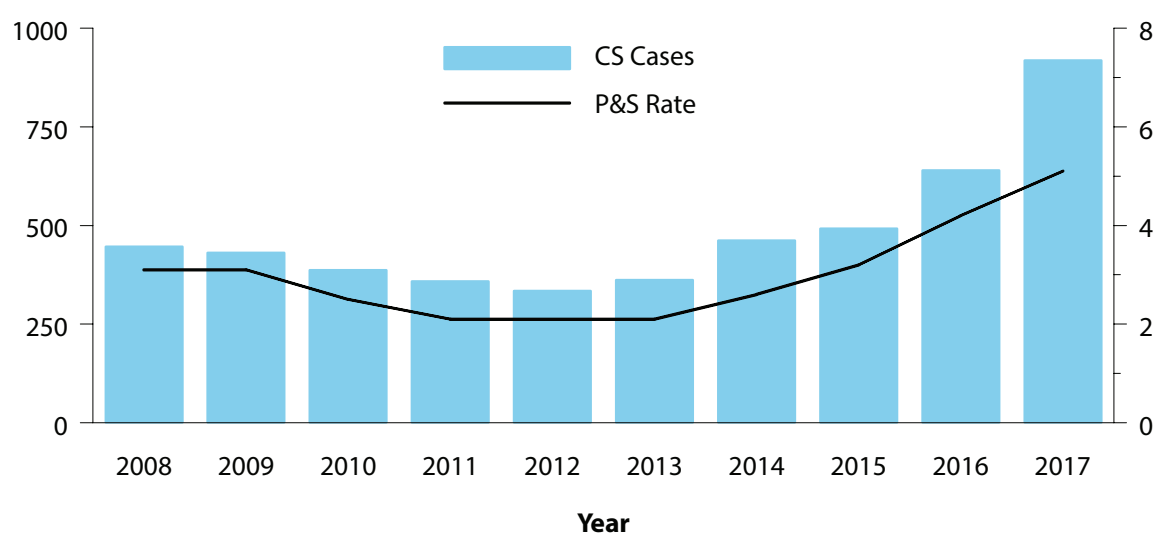

ACRONYMS: $\mathrm{CS}=$ Congenital syphilis; $\mathrm{P} \& S$ = Primary and secondary syphilis.

\section{References}

1. Jarzebowski W, Caumes E, Dupin N, et al. Effect of early syphilis infection on plasma viral load and CD4 cell count in human immunodeficiency virus- infected men: Results from the FHDH- ANRS CO4 cohort. Arch Intern Med 2012; 172(16): 1237-1243.

2. Buchacz K, Patel P, Taylor M, et al. Syphilis increases HIV viral load and decreases CD4 cell counts in HIV-infected patients with new syphilis infections. AIDS 2004; 18(15): 2075-2079.

3. Fleming DT, Wasserheit JN. From epidemiological synergy to public health policy and practice: The contribution of other sexually transmitted diseases to sexual transmission of HIV infection. Sex Trans Infect 1999; 75(1): 3-17.

4. Ingraham NR. The value of penicillin alone in the prevention and treatment of congenital syphilis. Acta Derm Venereol 1951; 31(Suppl 24): 60-88.

5. de Voux A, Kidd S, Grey JA, et al. State-specific rates of primary and secondary syphilis among men who have sex with men - United States, 2015. MMWR Morb Mortal Wkly Rep 2017; 66(13):349-354.

6. Peterman TA, Kahn RH, Ciesielski CA, et al. Misclassification of the stages of syphilis: implications for surveillance. Sex Transm Dis 2005; 32(3):144-149. 


\section{Chancroid}

Chancroid is caused by anogenital infection with the bacterium Haemophilus ducreyi. Clinical manifestations of chancroid include anogenital ulcers, and inguinal lymphadenopathy or buboes in up to $50 \%$ of cases. ${ }^{1}$ Reported cases of chancroid peaked in 1947 and then declined rapidly through 1957 , presumably due to the increasing use of antibiotics such as sulfonamides and penicillin, which were introduced in the late 1930s and early 1940s (Figure 50, Table 1). ${ }^{2,3}$ Numerous localized outbreaks, some of which were linked to commercial sex work, were identified during 1981-1990.4,5 Chancroid has declined since 1987, and since 2000 , the annual number of reported cases has been less than 100. During 2008-2017, the number of reported cases has fluctuated, ranging from 28 in 2009 to six in 2014. In 2017, a total of seven cases of chancroid were reported in the United States. Five states reported one or more cases of chancroid in 2017 (Table 43).

Although the overall decline in reported chancroid cases most likely reflects a decline in the incidence of this disease, these data should be interpreted with caution because $H$. ducreyi is difficult to culture and no molecular assays have been cleared by the Food and Drug Administration (FDA) for use in the United States. ${ }^{6}$

\section{Human Papillomavirus}

Human papillomavirus (HPV) is the most common sexually transmitted infection in the United States. ${ }^{7}$ Over 40 distinct HPV types can infect the genital tract; ${ }^{8}$ although most infections are asymptomatic and appear to resolve spontaneously within a few years. ${ }^{9}$ Prevalence of genital infection with any HPV type was $42.5 \%$ among United States adults aged $18-59$ years during

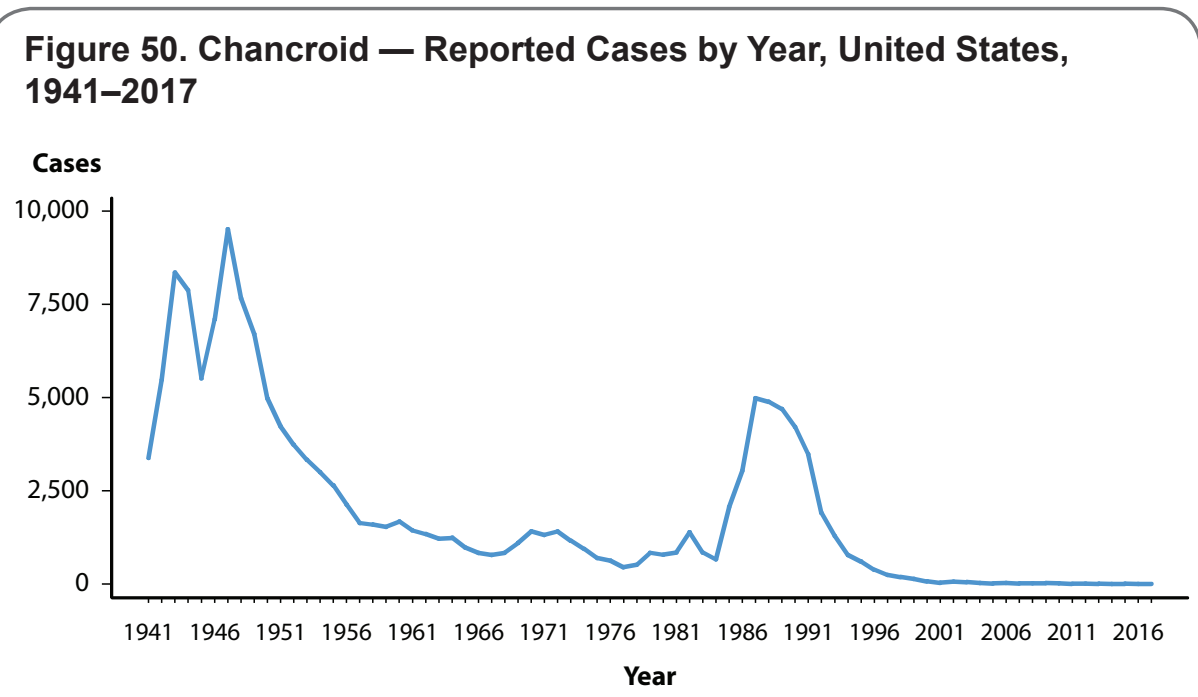

NOTE: Data collection for chancroid began in 1941; however, chancroid became nationally notifiable in 1944. Refer to the National Notifiable Disease Surveillance System (NNDSS) website for more information: https://wwwn.cdc.gov/nndss/conditions/chancroid/

2013-2014. ${ }^{10}$ Persistent infection with some HPV types can cause cancer and genital warts. ${ }^{11}$ HPV types 16 and 18 account for approximately $66 \%$ of cervical cancers in the United States, ${ }^{12}$ and approximately $25 \%$ of low-grade and $50 \%$ of high-grade cervical intraepithelial lesions, or dysplasia. ${ }^{13,14}$ HPV types 6 and 11 are responsible for approximately $90 \%$ of genital warts. ${ }^{15,16}$

Quadrivalent HPV vaccine, which targets HPV types 6, 11, 16, and 18, ${ }^{11}$ was licensed in the United States in mid-2006 for females ${ }^{17}$ and in late 2009 for males. ${ }^{18}$ Although a bivalent vaccine was also licensed for females, ${ }^{19}$ almost all HPV vaccine administered in the United States through late 2014 was quadrivalent. ${ }^{20}$ A 9-valent vaccine, which protects against the quadrivalent and 5 additional oncogenic HPV types (types 31, 33, 45, 52, and 58), was licensed in late 2014 for males and females. ${ }^{21}$ All HPV vaccines have been recommended for routine use in United States females aged 11-12 years, with catch-up vaccination through age $26 .{ }^{17,21}$ Since late 2011, routine use of the quadrivalent or 9-valent vaccine has been recommended for males aged 11-12, with catch-up vaccination through age $21 .{ }^{21-23}$ Vaccination through age 26 is recommended for gay, bisexual, and other men who have sex with men (collectively referred to as MSM) and persons who are immunocompromised (including those infected with HIV). ${ }^{21-23}$

HPV vaccine uptake in the United States remains lower than the Healthy People 2020 goal of $80 \%$ coverage. ${ }^{24}$ In 2016, a national survey found that $65 \%$ of girls aged $13-17$ years had received at least one dose of the HPV vaccine, and 50\% had received all doses in the series ${ }^{25}$ based on recommendations published in late $2016 .{ }^{23} \mathrm{HPV}$ vaccine uptake is lower among boys; $56 \%$ of those aged 13-17 years received at least one dose, but only $38 \%$ received all recommended doses. ${ }^{25}$

HPV infection is not a nationally notifiable condition. Cervicovaginal prevalence of any quadrivalent HPV vaccine type was estimated using data for females aged 14-34 years from 
the National Health and Nutrition Examination Survey (NHANES; see Section A2.4 in the Appendix). ${ }^{26}$ Prevalence decreased significantly from 2003-2006 (the pre-vaccine era) to 2011-2014 in specimens from females aged 14-19 years (from $11.5 \%$ to $3.3 \%$ ) and $20-24$ years (from $18.5 \%$ to $7.2 \%$ ); these are the age groups most likely to benefit from HPV vaccination. Among women aged 25-34 years, vaccine-type HPV prevalence did not differ significantly between the two time periods. An NHANES analysis of 2013-2014 HPV prevalence from penile swab specimens found low prevalence of quadrivalent HPV vaccine types in young males, which the authors attributed to male vaccination and/ or herd protection from female vaccination. $^{27}$

Health-care claims data from adolescents and adults with employer-provided private health insurance in the United States were used to examine the population effectiveness of HPV vaccination on clinical sequelae of HPV infection. Annual prevalence of high-grade histologically-detected cervical intraepithelial neoplasia grades 2 and 3 (CIN2+) during 2007-2014 was estimated using claims from 9 million females aged 15-39 years who received cervical cancer screening in a given calendar year. ${ }^{28}$ Prevalence of CIN2+ decreased significantly in females aged 15-19 and 20-24 years (Figure 51). Among those aged 15-19 years, annual percent change (APC) in CIN2+ prevalence was $-19.8 \%$ during 2007-2009 and -12.1\% during 2009-2014. For women aged 20-24 years, APC was $-6.7 \%$ during $2007-$ 2012, and $-12.5 \%$ during 2012-2014. No decreases in CIN2+ prevalence were observed among women aged 25-39 years. The observed decreases in high-grade cervical lesions only among young women provide ecologic evidence of population effectiveness of HPV vaccination on clinical sequelae of infection among privately-insured women in the United States.

Prevalence of anogenital warts was examined using health-care claims of privately-insured females and males aged 15-39 years during 2006-2014 (Figures 52A and 52B). ${ }^{29}$ Prevalence among adolescent females aged 1519 years declined non-significantly during 2006-2008, and then significantly decreased (APC $=-14.1)$ through 2014. Among women aged 20-24 years, genital wart prevalence was stable during 2006-2009, but declined significantly during 2009-2014 (APC=-12.9). Prevalence among women aged 25-29 years also decreased significantly from 2009-2014 (APC=-6.0). Prevalence increased or was stable during the entire period for women aged 30-39 years. These declines in genital wart prevalence among females aged 15-29 years extend the observations of a previous study using claims from 2003 through 2010, in which decreased prevalence was found only among adolescent females aged 1519 years. ${ }^{30}$ The observed declines in prevalence among increasingly older age groups would be expected from including more years of observation after the initiation of routine HPV vaccination for females in 2006. Among males, anogenital wart prevalence increased significantly during 2006-2009 for all age groups except those aged 15-19 years. ${ }^{29}$ From 2009 to 2014, rates decreased somewhat among male adolescents aged $15-19$ years (APC $=-5.4)$, but decreased significantly among men aged 20-24 years (APC $=-6.5)$.

Among those aged 25-29 years, prevalence declined non-significantly during 2010-2014 (APC=-1.7); prevalence increased or was stable throughout the entire period for men aged 30-39 years. The decreased prevalence observed among men aged 20-24 years is unlikely to be due to male vaccination for several reasons. Almost all men in this age group were aged 19 years or older since 2011, when HPV vaccine was first recommended for routine use in United States males ${ }^{22}$ and vaccination coverage in adult males through 2014 was extremely low. ${ }^{31}$ Also, the most likely sexual partners for men in this age group were females of a

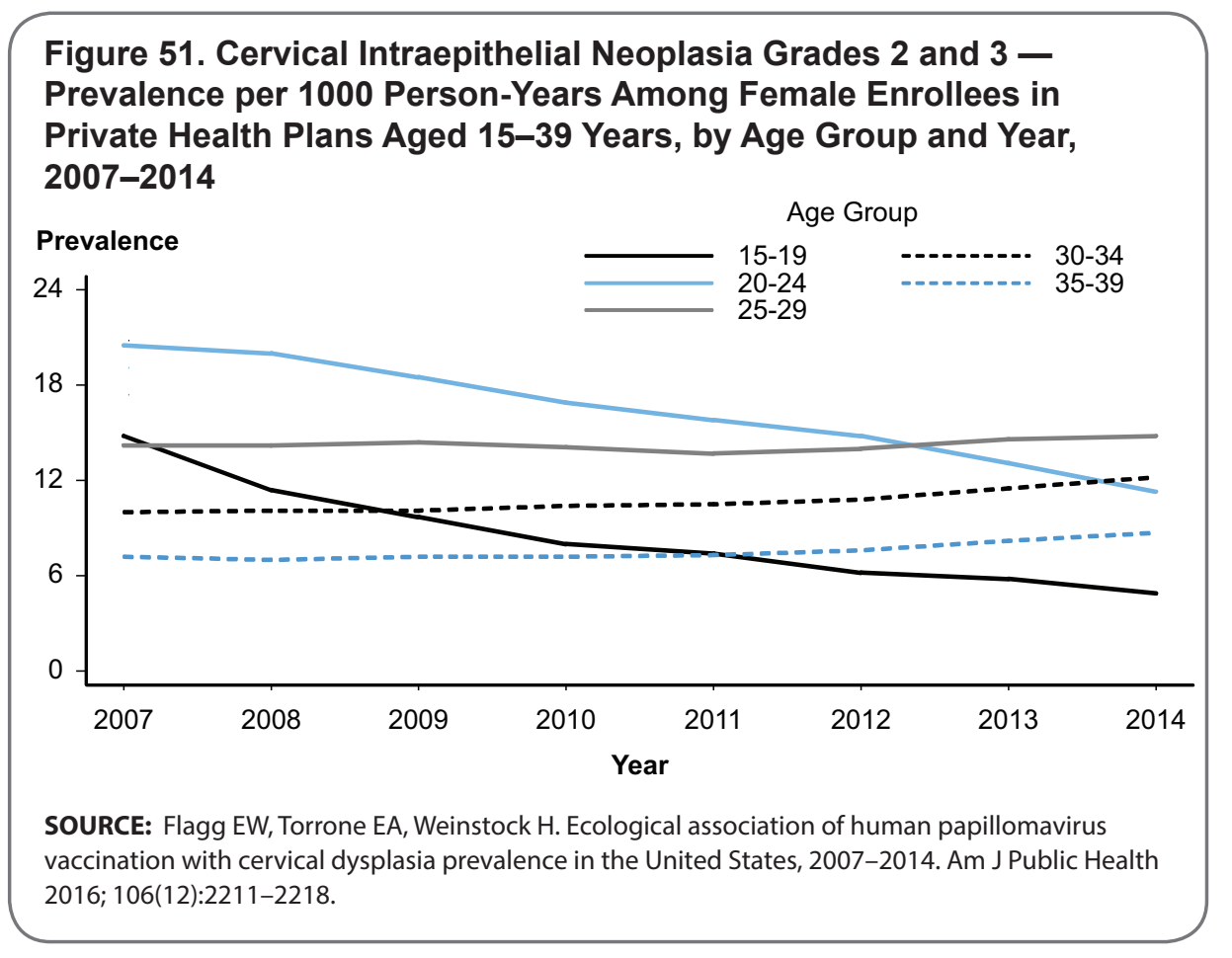




\section{Figure 52. Anogenital Warts - Prevalence per 1000 Person-Years Among Enrollees in Private Health Plans Aged 15-39 Years by Sex, Age Group, and Year, 2006-2014}

\section{A. Females}

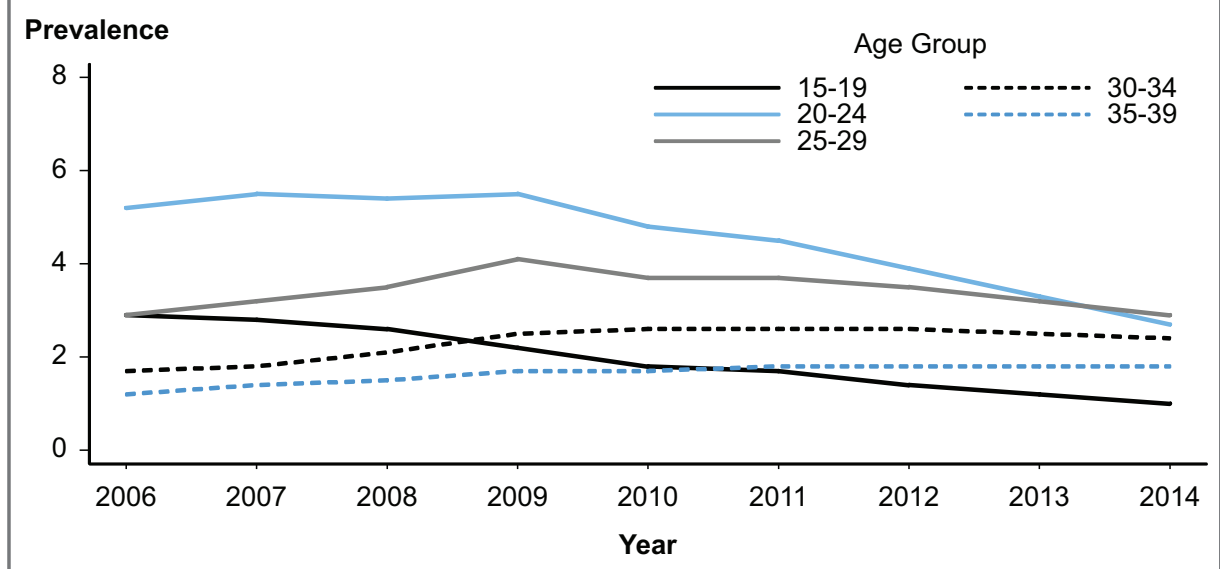

B. Males

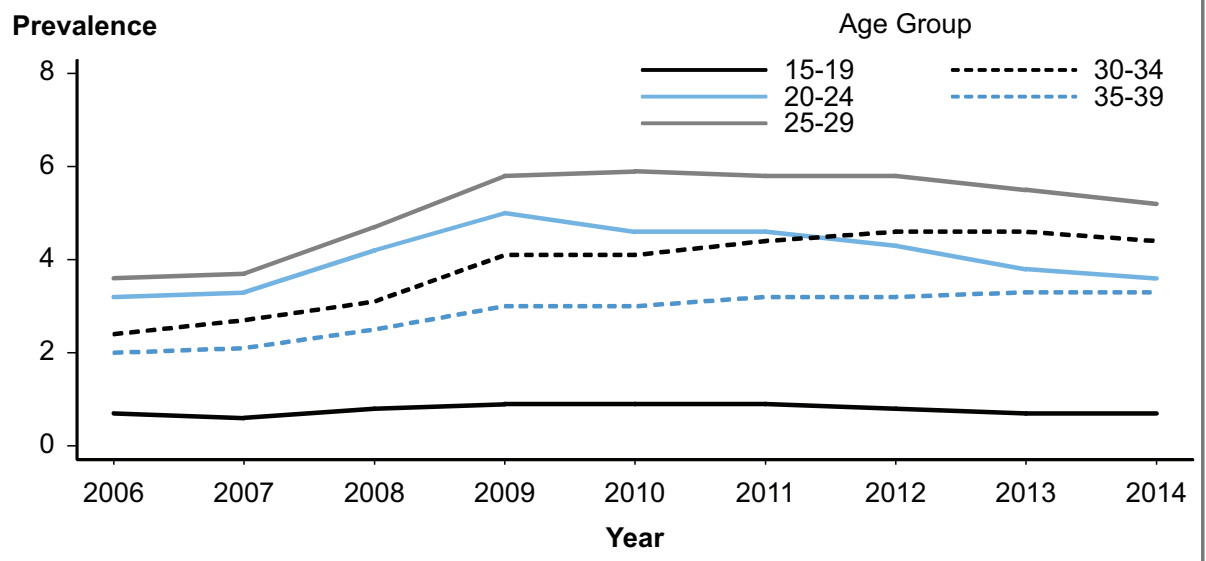

SOURCE: Flagg EW, Torrone EA. Declines in anogenital warts among age groups most likely to be impacted by human papillomavirus vaccination, United States, 2006-2014. Am J Public Health 2018; 108(1):112-119.

similar age or younger; ${ }^{32,33}$ therefore, the observed declines in genital wart prevalence among young men are consistent with herd protection from vaccination among females.

\section{Pelvic Inflammatory Disease}

For information on pelvic inflammatory disease, see Special Focus Profiles, STDs in Women and Infants.

\section{Herpes Simplex Virus}

Herpes simplex virus (HSV) is among the most prevalent of sexually transmitted infections. ${ }^{7,34}$ Although most infections are subclinical, ${ }^{35}$ clinical manifestations are characterized by recurrent, painful genital and/or anal lesions. ${ }^{36}$ Most genital HSV infections in the United States are caused by HSV type 2 (HSV-2), while HSV type 1
(HSV-1) infections are typically orolabial and acquired during childhood $; 5,37$ however, the prevalence of genital HSV-1 infections appears to be increasing among young adults. ${ }^{38,39}$

Genital HSV infection is not a nationally notifiable condition. Most persons with genital HSV infection have not received a diagnosis. ${ }^{40}$ The overall percentage of HSV-2 seropositive NHANES participants aged $14-49$ years who reported never being told by a doctor or healthcare professional that they had genital herpes did not change significantly between 1988-1994 and 2007-2010, and remained high $(90.7 \%$ and $87.4 \%$, respectively). ${ }^{40}$

NHANES data indicate the seroprevalence of HSV-2 in the United States has decreased from 1999-2000 to 2015-2016; agestandardized seroprevalence declined from $18.0 \%$ in $1999-2000$ to $12.1 \%$ in 2015-2016 (Figure 53). ${ }^{41}$ Although these declines were observed among all race and Hispanic ethnicity groups, HSV-2 seroprevalence was highest among non-Hispanic Blacks throughout the entire time period.

NHANES data also show that among adolescents aged $14-19$ years, HSV-1 seroprevalence has significantly decreased by almost $23 \%$, from $39.0 \%$ during $1999-2004$ to $30.1 \%$ during 2005-2010, indicating declining orolabial infection in this age group. $.^{37} \mathrm{HSV}-2$ seroprevalence in this age group was much lower, less than $2 \%$ in both time periods. ${ }^{37}$ Other studies have found that genital HSV-1 infections are increasing among young adults. ${ }^{38,39}$ This has been attributed, in part, to the decline in orolabial HSV-1 infections, because those who lack HSV-1 antibodies at sexual debut are more susceptible to genital HSV-1 infection. ${ }^{37,42}$ Increasingly common oral sex behavior among 
Figure 53. Herpes Simplex Virus Type 2 - National Estimates of Trends in Age-Adjusted Seroprevalence Among Persons Aged 14-49 Years by Race and Hispanic Ethnicity, National Health and Nutrition Examination Survey (NHANES), 1999-2000 through 2015-2016

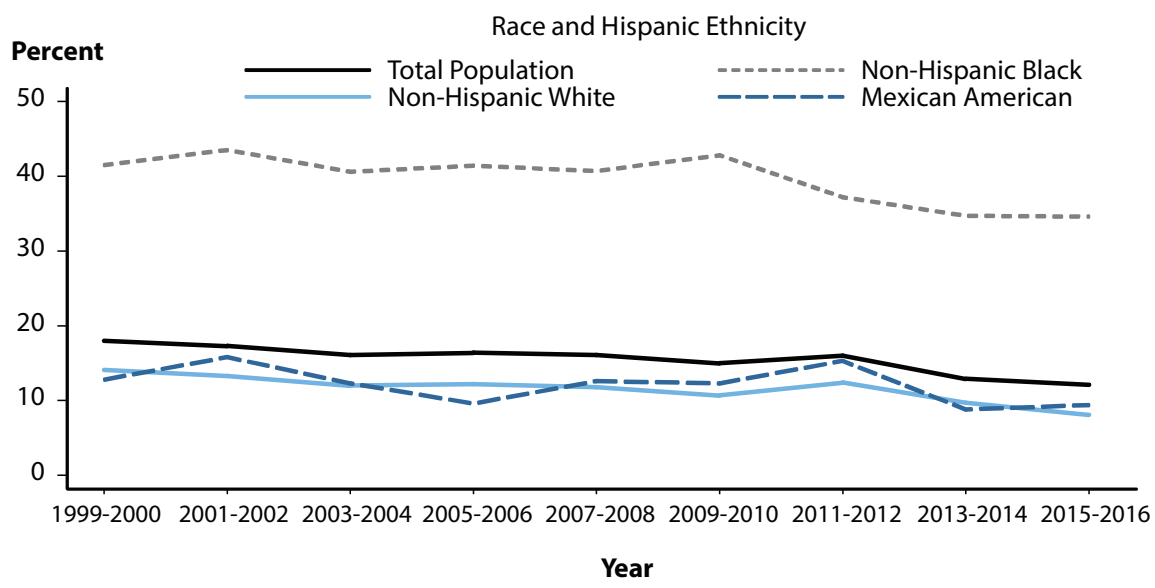

NOTE: Age-adjusted by the direct method to the 2000 US Census population, using age groups 14-19, 20-29, 30-39, and 40-49 years. Total population includes all race and Hispanic ethnicity groups, including those not shown separately.

SOURCE: McQuillan G, Kruszon-Moran D, Flagg EW, et al. Prevalence of herpes simplex virus type 1 and type 2 in persons aged 14-49: United States, 2015-2016. NCHS Data Brief, No 304. Hyattsville, MD: National Center for Health Statistics. 2018.

adolescents and young adults also has been suggested as a contributing factor. ${ }^{37,43}$ The absence of HSV-1 antibodies also increases the likelihood of developing symptomatic disease from newly-acquired (i.e., primary) genital HSV-2 infection. ${ }^{44}$ Young women may therefore be increasingly likely to first acquire HSV-1 infection genitally, or acquire a primary genital HSV-2 infection, during their childbearing years, ${ }^{42,45}$ and first-episode primary genital HSV infection during pregnancy increases the risk of neonatal HSV transmission. ${ }^{42,46}$ A recent analysis of NHANES data found that among pregnant women with three or fewer lifetime sex partners, seronegativity for both HSV-1 and HSV-2 increased from 1999-2006 to 2007-2014. ${ }^{47}$ This raises the possibility that pregnant women with fewer sex partners may have increased risk of acquiring genital HSV during pregnancy and vertically transmitting HSV to their neonates.
For information on neonatal HSV infections, see Special Focus Profiles, STDs in Women and Infants.

\section{Trichomonas vaginalis}

Trichomonas vaginalis is a common sexually transmitted protozoal infection associated with adverse health outcomes such as preterm birth and symptomatic vaginitis. ${ }^{7,48-50}$ It is not a nationally notifiable condition, and trend data are limited to estimates of initial physician office visits for this condition from the National Disease and Therapeutic Index (NDTI; see Section A2.5 in the Appendix) (Figure 54, Table 44). Visits appear to be fairly stable since the 1990's; the number of initial visits for $T$. vaginalis infection in 2016 was 222,000 . The 2017 NDTI data were not obtained in time to include them in this report. NHANES data during 2013-2014 indicated prevalence of $T$. vaginalis in urine specimens obtained from adult participants aged $18-59$ years was $0.5 \%$ among males and $1.8 \%$ among females; highest prevalences were observed among non-Hispanic Black males (4.2\%) and females $(8.9 \%) .{ }^{49}$ A separate analysis of NHANES data during 2013-2016 among men aged 18-59 years found higher prevalence among non-Hispanic Blacks. ${ }^{50} \mathrm{An}$ analysis of NHANES data during 2001-2004 from cervicovaginal swab specimens also found higher T. vaginalis prevalence among non-Hispanic Black females. ${ }^{51}$ 


\section{Figure 54. Trichomonas vaginalis and Other Vaginal Infections Among Females - Initial Visits to Physicians' Offices, United States, 1966-2016}

\section{Visits (in thousands)}

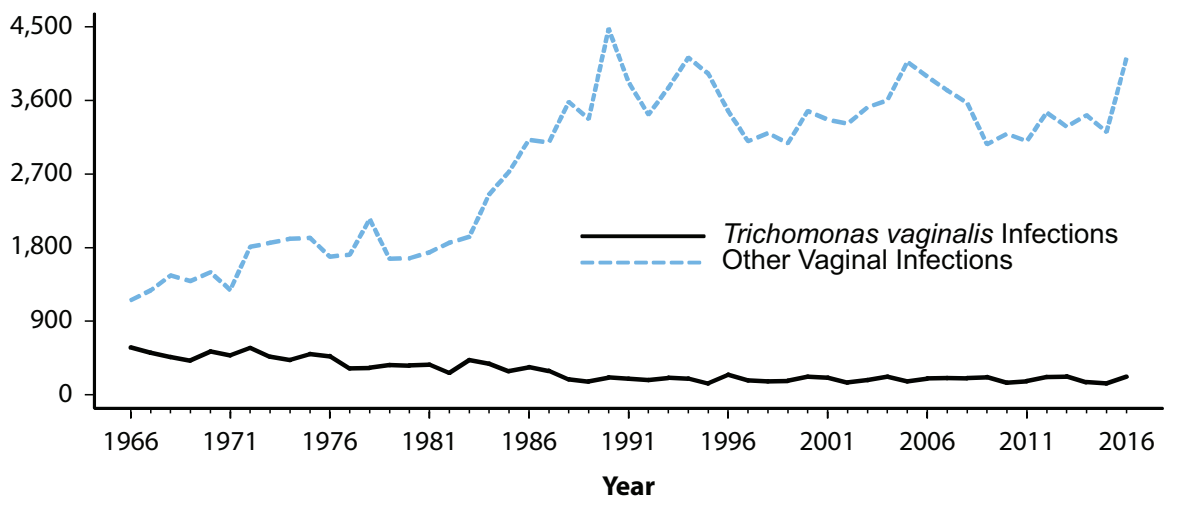

NOTE: The relative standard errors for Trichomonas vaginalis infection estimates range from $23 \%$ to $17 \%$ and for other vaginal infection estimates range from $13 \%$ to $8 \%$. See Section A2.5 in the Appendix and Table 44.

SOURCE: National Disease and Therapeutic Index, IMS Health, Integrated Promotional Services ${ }^{\mathrm{TM}}$, IMS Health Report, 1966-2016. The 2017 data were not obtained in time to include them in this report.

\section{References}

1. Lewis DA. Epidemiology, clinical features, diagnosis and treatment of Haemophilus ducreyi - a disappearing pathogen? Expert Rev Anti Infect Ther 2014; 12(6):687-696.

2. Steen R. Eradicating chancroid. Bull World Health Organ 2001; 79:818-826.

3. Hall L. "The Cinderella of medicine": Sexuallytransmitted diseases in Britain in the nineteenth and twentieth centuries. Genitourin Med 1993; 69:314-319.

4. Schmid GP, Sanders LL, Blount JH, et al. Chancroid in the United States.

Reestablishment of an old disease. JAMA 1987; 258(22):3265-3268.

5. Schulte JM, Martich FA, Schmid GP. Chancroid in the United States, 1981-1990: Evidence for underreporting of cases. MMWR Morb Mortal Wkly Rep Surveill Summ 1992; 41(SS-3):5761 .

6. Workowski KA, Bolan GA. Sexually transmitted diseases treatment guidelines, 2015. MMWR Morb Mortal Wkly Rep Recomm Rep 2015; 64(3):25-27.

7. Satterwhite CL, Torrone E, Meites E, et al. Sexually transmitted infections among US women and men: Prevalence and incidence estimates, 2008. Sex Transm Dis 2013; 40(3):187-193.

8. de Villiers E-M, Fauquet C, Broker TR, et al. Classification of papillomaviruses. Virology 2004; 324:17-27.
9. Insinga RP, Perez G, Wheeler CM, et al. Incident cervical HPV infections in young women: Transition probabilities for CIN and infection clearance. Cancer Epidemiol Biomarkers Prev 2011; 20(2):287-296.

10. McQuillan G, Kruszon-Moran D, Markowitz LE, et al. Prevalence of HPV in adults aged 18-69: United States, 2011-2014. NCHS Data Brief, No. 280. Hyattsville, MD: National Center for Health Statistics; 2017.

11. Doorbar J, Quint W, Banks L, et al. The biology and life-cycle of human papillomaviruses. Vaccine 2012; 30(Suppl 5):F55-F70.

12. Saraiya M, Unger ER, Thompson TD, et al. US assessment of HPV types in cancers: Implications for current and 9-valent HPV vaccines. J Natl Cancer Inst 2015; 107(6):djv086.

13. Clifford GM, Rana RK, Franceschi S, et al. Human papillomavirus genotype distribution in low-grade cervical lesions: Comparison by geographic region and with cervical cancer. Cancer Epidemiol Biomarkers Prev 2005; 14(5):1157-1164.

14. Porras C, Rodriguez AC, Hildesheim A, et al. Human papillomavirus types by age in cervical cancer precursors: Predominance of human papillomavirus 16 in young women. Cancer Epidemiol Biomarkers Prev 2009; 18(3):863865.

15. Garland SM, Steben M, Sings HL, et al. Natural history of genital warts: Analysis of the placebo arm of 2 randomized phase III trials of a quadrivalent human papillomavirus (types $6,11,16$, and 18) vaccine. J Infect Dis 2009; 199(6):805-814.

16. Gissmann L, Wolnik L, Ikenberg H, et al. Human papillomavirus types 6 and 11 DNA sequences in genital and laryngeal papillomas and in some cervical cancers. Proc Natl Acad Sci USA 1983; 80(2): 560-563.

17. Markowitz LE, Dunne EF, Saraiya M, et al. Quadrivalent human papillomavirus vaccine. Recommendations of the Advisory Committee on Immunization Practices (ACIP). MMWR Morb Mortal Wkly Rep Recomm Rep 2007; 56(RR-2):1-24.

18. Centers for Disease Control and Prevention. FDA licensure of quadrivalent human papillomavirus vaccine (HPV4, Gardasil) for use in males and guidance from the Advisory Committee on Immunization Practices (ACIP). MMWR Morb Mortal Wkly Rep 2010; 59(20):630-632.

19. Centers for Disease Control and Prevention. FDA licensure of bivalent human papillomavirus vaccine (HPV2, Cervarix) for use in females and updated HPV vaccination recommendations from the Advisory Committee on Immunization Practices (ACIP). MMWR Morb Mortal Wkly Rep 2010; 59(20):626-629.

20. Stokley S, Jeyarajah J, Yankey D, et al. Human papillomavirus vaccination coverage among adolescents, 2007-2013, and postlicensure vaccine safety monitoring, 2006-2014 United States. MMWR Morb Mortal Wkly Rep 2014; 63(29):620-624.

21. Petrosky E, Bocchini Jr. JA, Hariri S, et al. Use of 9-valent human papillomavirus (HPV) vaccine: Updated HPV vaccination recommendations of the Advisory Committee on Immunization Practices. MMWR Morb Mortal Wkly Rep 2015; 64(11):300-304.

22. Centers for Disease Control and Prevention. Recommendations on the use of quadrivalent human papillomavirus vaccine in males Advisory Committee on Immunization Practices (ACIP), 2011. MMWR Morb Mortal Wkly Rep 2011; 60(50):1705-1708.

23. Meites E, Kempe A, Markowitz LE. Use of a 2-dose schedule for human papillomavirus vaccination - updated recommendations of the Advisory Committee on Immunization Practices. MMWR Morb Mortal Wkly Rep 2016; 65(49):1405-1408.

24. HealthyPeople.gov. Healthy People 2020 Topics \& Objectives. Immunization and Infectious Diseases. Objectives IID-11.4 and IID-11.5. Available at: https://www.healthypeople. gov/2020/topics-objectives/topic/immunizationand-infectious-diseases/objectives. Accessed March 29, 2018.

25. Walker TY, Elam-Evans LD, Singleton JA, et al National, regional, state, and selected local area vaccination coverage among adolescents aged 13-17 years - United States, 2016. MMWR Morb Mortal Wkly Rep 2017; 66(33):874-882. 
26. Oliver SE, Unger ER, Lewis R, et al. Prevalence of human papillomavirus among females after vaccine introduction - National Health and Nutrition Examination Survey, United States, 2003-2014. J Infect Dis 2017; 216(5):594-603.

27. Gargano JW, Unger ER, Liu G, et al. Prevalence of genital human papillomavirus in males, United States, 2013-2014. J Infect Dis 2017; 215(7):1070-1079.

28. Flagg EW, Torrone EA, Weinstock H. Ecological association of human papillomavirus vaccination with cervical dysplasia prevalence in the United States, 2007-2014. Am J Public Health 2016; 106(12):2211-2218.

29. Flagg EW, Torrone EA. Declines in anogenital warts among age groups most likely to be impacted by human papillomavirus vaccination, United States, 2006-2014. Am J Public Health 2018; 108(1):112-119.

30. Flagg EW, Schwartz R, Weinstock H. Prevalence of anogenital warts among participants in private health plans in the United States, 2003-2010: Potential impact of human papillomavirus vaccination. Am J Public Health 2013; 103(8):1428-1435.

31. Williams WW, Lu P-J, O'Halloran A, et al. Surveillance of vaccination coverage among adult populations - United States, 2014. MMWR Morb Mortal Wkly Rep Surveill Summ 2016; 65(1):1-36.

32. Ford K, Sohn W, Lepkowsk J. American adolescents: Sexual mixing patterns, bridge partners, and concurrency. Sex Transm Dis 2002; 29(1):13-19.

33. Aral SO, Hughes JP, Stoner B, et al. Sexual mixing patterns in the spread of gonococcal and chlamydial infections. Am J Public Health 1999;89(6):825-833.

34. Smith JS, Robinson NJ. Age-specific prevalence of infection with herpes simplex virus types 2 and 1: A global review. J Infect Dis 2002; 186(Suppl 1):S3-S28.
35. Corey L, Wald A. Genital Herpes. In: Holmes KK, Sparling FP, Stamm WE, et al., eds. Sexually Transmitted Diseases. 4th ed. New York, NY: McGraw-Hill; 2008:399-437.

36. Kimberlin DW, Rouse DJ. Genital herpes. N Engl J Med 2004; 350(19):1970-1977.

37. Bradley H, Markowitz LE, Gibson T, et al. Seroprevalence of herpes simplex virus types 1 and 2 - United States, 1999-2010. J Infect Dis 2014; 209(3):325-333.

38. Bernstein DI, Bellamy AR, Hook EW III, et. al. Epidemiology, clinical presentation, and antibody response to primary infection with herpes simplex virus type 1 and type 2 in young women. Clin Infect Dis 2013; 56:344-351.

39. Roberts CM, Pfister JR, Spear SJ. Increasing proportion of herpes simplex virus type 1 as a cause of genital herpes infection in college students. Sex Transm Dis 2003; 30(10):797800.

40. Fanfair RN, Zaidi A, Taylor LD, et al. Trends in seroprevalence of herpes simplex virus type 2 among non-Hispanic Blacks and nonHispanic Whites aged 14 to 49 years - United States, 1988 to 2010. Sex Transm Dis 2013; 40(11):860-864.

41. McQuillan G, Kruszon-Moran D, Flagg EW, et al. Prevalence of herpes simplex virus type 1 and type 2 in persons aged 14-49: United States, 2015-2016. NCHS Data Brief, No. 304. Hyattsville, MD: National Center for Health Statistics. 2018

42. Kimberlin DW. The scarlet H. J Infect Dis 2014; 209(3):315-317.

43. Copen CE, Chandra A, Martinez G. Prevalence and timing of oral sex with opposite-sex partners among females and males aged 15-24 years: United States, 2007-2010. National Health Statistics Reports, No. 56. Hyattsville, MD: National Center for Health Statistics; 2012.
44. Langenberg AGM, Corey L, Ashley RL, et al. A prospective study of new infections with herpes simplex virus type 1 and type 2 . N Engl J Med 1999; 341:1432-1438.

45. Sampath A, Maduro G, Schillinger JA. Infant deaths due to herpes simplex virus, congenital syphilis, and HIV in New York City. Pediatrics 2016; 137(4):e20152387.

46. Brown ZA, Wald A, Morrow RA, et al. Effect of serologic status and cesarean delivery on transmission rates of herpes simplex virus from mother to infant. JAMA 2003; 289(2):203-209.

47. Patton ME, Bernstein K, Liu G, et al. Seroprevalence of herpes simplex virus types 1 and 2 among pregnant women and sexually active, non-pregnant women in the United States. Clin Infect Dis 2018; https://doi. org/10.1093/cid/ciy318.

48. French J, McGregor JA, Parker R. Readily treatable reproductive tract infections and preterm birth among Black women. Am J Obstet Gynecol 2006; 194:1717-1727.

49. Patel EU, Gaydos CA, Packman ZR, et al. Prevalence and correlates of Trichomonas vaginalis infection among men and women in the United States. Clin Infect Dis 2018;67(2):211-217.

50. Daugherty M, Glynn K, Byler T. The prevalence of Trichomonas vaginalis infection among US males, 2013-2016. Clin Infect Dis 2018; https://doi.org/10.1093/cid/ciy499.

51. Sutton M, Sternberg M, Koumans EH, et al. The prevalence of Trichomonas vaginalis infection among reproductive-age women in the United States, 2001-2004. Clin Infect Dis 2007; 45(10):1319-1326.

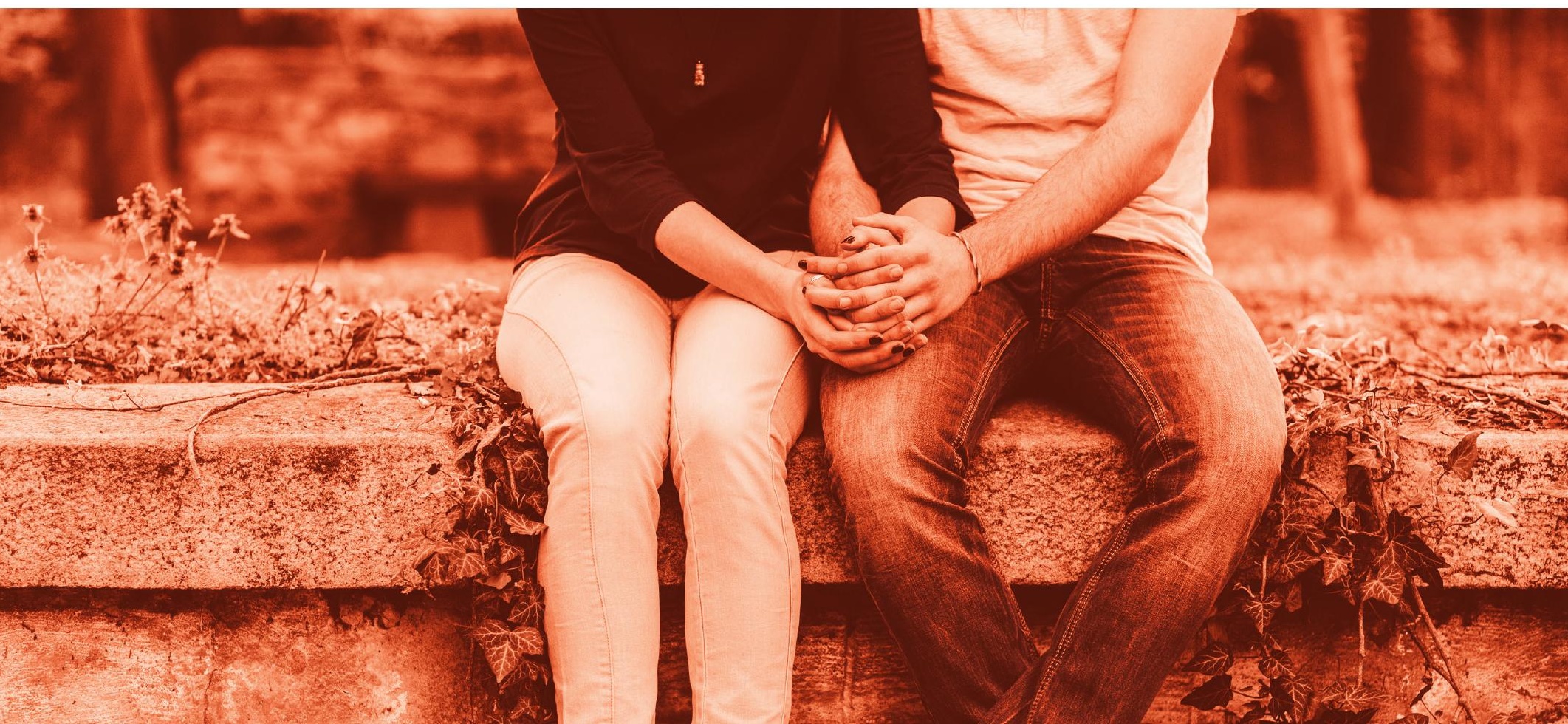




\section{Special Focus Profiles}

The Special Focus Profiles highlight trends and distribution of STDs in populations of particular interest to STD and HIV prevention programs in state and local health departments: women and infants, adolescents and young adults, racial and ethnic minority groups, and gay, bisexual, and other men who have sex with men (collectively referred to as MSM).

\section{STDs in Women and Infants}

\section{Background}

Women and their infants are uniquely vulnerable to the consequences of sexually transmitted infections (STI). While individual-level determinants, including high-risk behaviors, contribute to disease transmission and acquisition risk, it is widely accepted that social barriers to STD prevention and control efforts also contribute to infectious disease prevalence. A woman's relationship status with her male partner, including concurrency of the relationship, may be an important predictor of her sexual health. ${ }^{1-3}$ In addition to poverty and lack of access to quality STD services, homelessness or unstable housing may influence a woman's sexual risk. ${ }^{4}$ For some women, maintaining the relationship with a partner may take a higher priority than STD risk reduction, ${ }^{5}$ affecting her sexual and reproductive health, as well as the health of her unborn baby. ${ }^{6,7}$ A woman can also be placed at risk for STIs through her partner's sexual encounter with an infected partner. Consequently, even a woman who has only one partner may be obliged to practice safer sex, such as using condoms. ${ }^{8}$

Chlamydia and gonorrhea disproportionately affect women because early infection may be asymptomatic and, if untreated, infection may ascend to the upper reproductive tract resulting in pelvic inflammatory disease (PID). Data from studies suggest that as much as $10 \%$ of untreated chlamydial infections progress to clinically diagnosed PID and the risk with untreated gonococcal infection may be even higher. ${ }^{9-11}$ PID is a major concern because it can result in inflammation and damage to the fallopian tubes, elevating the risk of infertility and ectopic pregnancy. Tubal factor infertility ranks among the most common causes of infertility, accounting for $30 \%$ of female infertility in the United States, ${ }^{12}$ and much of this damage results from previous episodes of PID. ${ }^{13}$

An important public health measure for preventing PID, and ultimately tubal factor infertility, is through the prevention and control of Chlamydia trachomatis and Neisseria gonorrhoeae. Strategies to improve the early detection and treatment of chlamydia and gonorrhea, as demonstrated in randomized controlled trials, ${ }^{10,14}$ has been shown to reduce a woman's risk for PID and ultimately protect the fertility of women. 
Human papillomavirus (HPV) infections are highly prevalent in the United States. ${ }^{15-16}$ Although most HPV infections in women appear to be transient and may not result in clinically significant sequelae, ${ }^{17}$ high-risk HPV-type infections can cause abnormal changes in the uterine cervical epithelium, ${ }^{17-18}$ which are detected by cytological examination of Papanicolaou (Pap) smears. ${ }^{19}$ Persistent high-risk HPV-type infections may lead to cervical cancer precursors, which, if undetected can result in cancer. ${ }^{18}$ Other lowrisk HPV-type infections can cause genital warts, ${ }^{18,20}$ low-grade Pap smear abnormalities, ${ }^{18,21}$ laryngeal papillomas, ${ }^{22}$ and, rarely, recurrent respiratory papillomatosis in children born to infected mothers. ${ }^{23-24}$ For more information on adolescent and adult HPV infections, see Other STDs.

\section{Impact on Maternal and Fetal Outcomes}

Similar to non-pregnant women, a high proportion of pregnant women with chlamydial and gonococcal infections are asymptomatic. Documented sequelae of untreated infections in pregnancy include premature delivery, premature rupture of the membranes, low birth weight, and stillbirth. Maternal infection can also affect the infant, leading to conjunctivitis infections (termed ophthalmia neonatorum in the first four weeks of life), and, in the case of C. trachomatis, pneumonia. Although topical prophylaxis of infants at delivery is effective for prevention of gonococcal ophthalmia neonatorum, prevention of neonatal pneumonia requires prenatal detection and treatment. The clinical presentation of conjunctivitis can be variable and these infections are especially important to treat promptly, as they can lead to visual impairment. ${ }^{25}$

Syphilis has long been known to be an important risk factor for adverse pregnancy outcomes. The consequences of untreated maternal infection include fetal death, preterm birth, and also congenital infection in a proportion of surviving infants resulting in both physical and mental developmental disabilities. Most cases of congenital syphilis are preventable if women are screened for syphilis and treated early during prenatal care. $^{26}$

Genital infections with herpes simplex virus (HSV) are extremely common, can cause painful outbreaks, and can have serious consequences for pregnant women and their infants. ${ }^{27}$ Neonatal herpes can be a severe illness presenting with vesicular lesions on the skin, eye, or mouth, seizures, respiratory collapse, and/or liver failure, following contact with infected cervical or vaginal secretions during delivery. ${ }^{27-28}$ Risk of transmission to the infant is greatest when the mother has a first-episode primary genital infection during pregnancy, especially if she acquires infection towards the end of her pregnancy. ${ }^{28-29}$ For more information on genital HSV infections among pregnant women, see Other STDs.

\section{Pelvic Inflammatory Disease}

Accurate estimates of PID and tubal factor infertility resulting from chlamydial and gonococcal infections are difficult to obtain, in part because definitive diagnoses of these conditions can be complex. Published data suggest overall declining rates of women diagnosed with PID in both hospital and ambulatory settings in the United States. ${ }^{30-32}$ The National Disease and Therapeutic Index (NDTI; see Section A2.5 in the Appendix) provides estimates of initial visits to office-based, private physicians for PID. NDTI estimated that from 2007-2016 the number of initial visits to such physicians for PID among women aged 15-44 years decreased by $38.3 \%$ from 146,000 to 90,000 visits (Figure A). The 2017 NDTI data was not obtained in time to include in this report. Similar declines have been observed in nationally representative data of emergency department (ED) visits from the Healthcare Cost and Utilization Project (HCUP), the largest all-payer publicly available database of ED visits across the US. The percentage

\section{Figure A. Pelvic Inflammatory Disease - Initial Visits to Physicians' Offices Among Women Aged 15-44 Years, United States, 2007-2016}

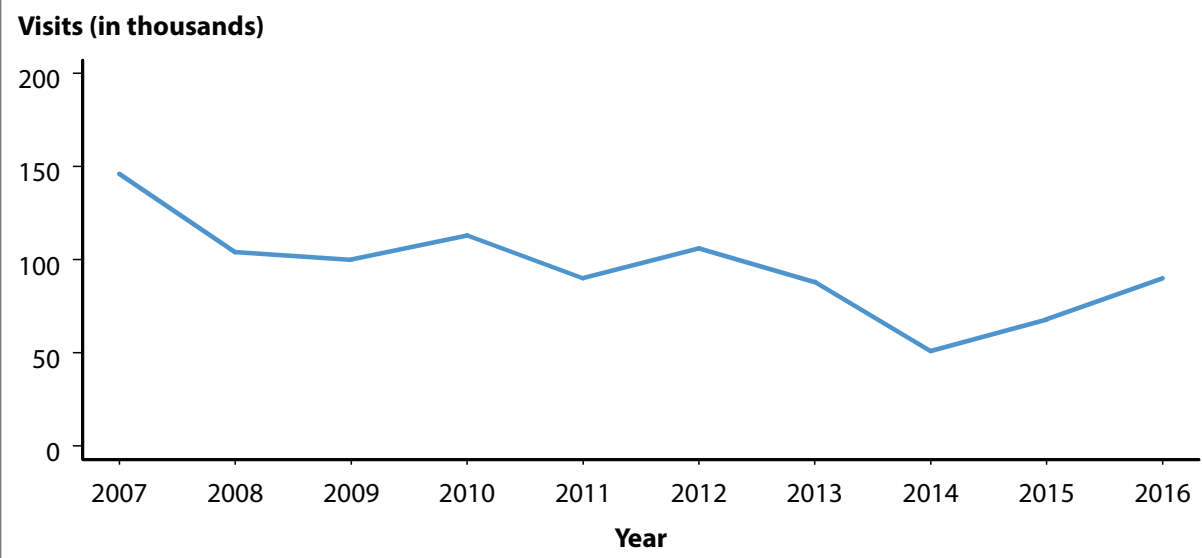

NOTE: The relative standard errors for these estimates are $23 \%-16 \%$. See section A2.5 in the Appendix and Table 44

SOURCE: National Disease and Therapeutic Index, IMS Health, Integrated Promotional Services ${ }^{\mathrm{TM}}$, IMS Health Report, 1966-2016. The 2017 data were not obtained in time to include them in this report. 
of ED visits with a PID diagnosis decreased during 2006-2013 among females aged 15-44 years, with the largest decreases among females aged 15-19 years (Figure B). ${ }^{33}$ It is not entirely clear what may be driving these declines, though several factors have been suggested including earlier identification and treatment of chlamydia and gonorrhea infection, and availability of singledose therapies that increase adherence to treatment. ${ }^{31-32,34}$ While PID is declining nationally, it is still a major cause of morbidity in women.

\section{Differences in self-reported}

lifetime diagnosis of PID by race/ Hispanic ethnicity in reproductive age women have been observed in earlier research..$^{35}$ Data from the 2013-2014 cycle of the National Health and Nutrition Examination Survey (NHANES) indicates that non-Hispanic Black and non-Hispanic White women reporting a previous STI diagnosis had nearly equal selfreported lifetime PID prevalence $(10.3 \%$ vs. $10.0 \%)$ (Figure C). ${ }^{36}$ However, the lifetime prevalence of PID among non-Hispanic Black women was 2.2 times that among non-Hispanic White women if no previous STI was diagnosed $(6.0 \%$ vs. $2.7 \%$ ). These findings suggest that PID is associated with previous STI diagnoses and it is therefore important for physicians to screen female patients for chlamydia and gonorrhea to reduce the incidence of PID. The racial disparities observed in PID diagnoses are consistent with the marked racial disparities observed for chlamydia and gonorrhea. However, because of the subjective methods by which PID is diagnosed, racial disparity data should be interpreted with caution.

\section{Ectopic Pregnancy}

Ectopic pregnancy is a potentially life-threatening condition that requires prompt evaluation and treatment. Up until the early 1990 's, a primary data source used to estimate the incidence of ectopic pregnancy was the National
Figure B. Trends in the Percentage of Acute Pelvic Inflammatory Disease (PID) Emergency Department (ED) Visits Among Women Aged 15-44 Years by Age Group, United States, 2006-2013

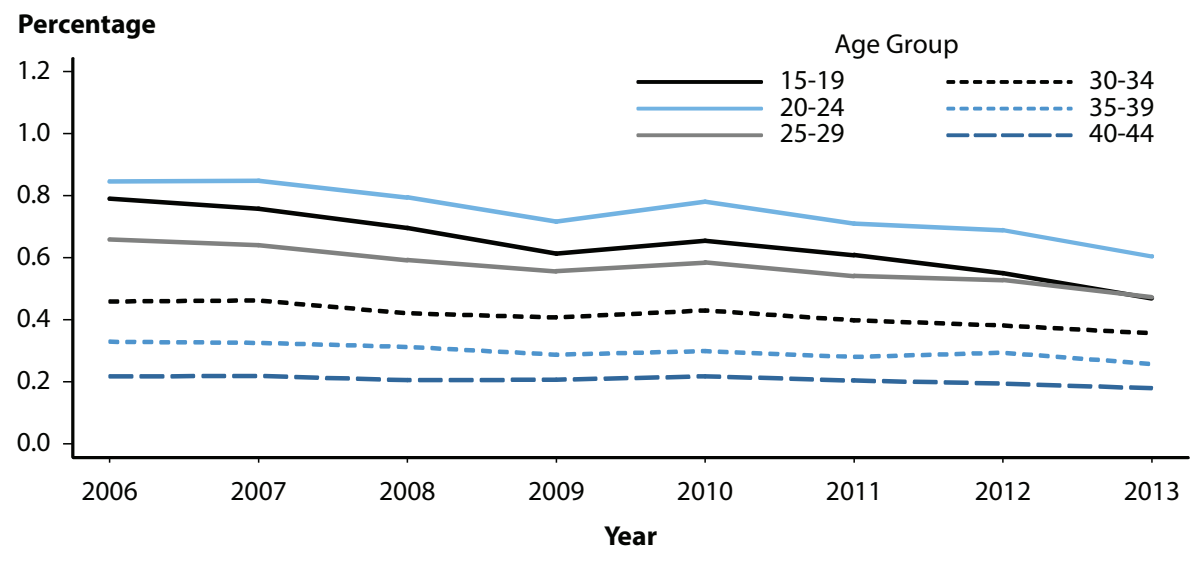

NOTE: Estimates were weighted using discharge weights representative of the reported total of emergency department visits in the US. Percent is calculated as the percent of visits where any PID was diagnosed where PID was the first diagnosis listed for the patient's ED visit.

SOURCE: Kreisel, K, Flagg, EW, Torrone E. Trends in pelvic inflammatory disease emergency department visits, United States, 2006-2013. Am J Obstet Gynecol 2018; 218(1): 117.e1-117.e10.

Figure C. Pelvic Inflammatory Disease - National Estimates of Lifetime Prevalence* Among Sexually Experienced Women ${ }^{\dagger}$ Aged 1844 Years by Race, Hispanic Ethnicity, and Previous STI Diagnosis, National Health and Nutrition Examination Survey (NHANES), 20132014

Prevalence, \%

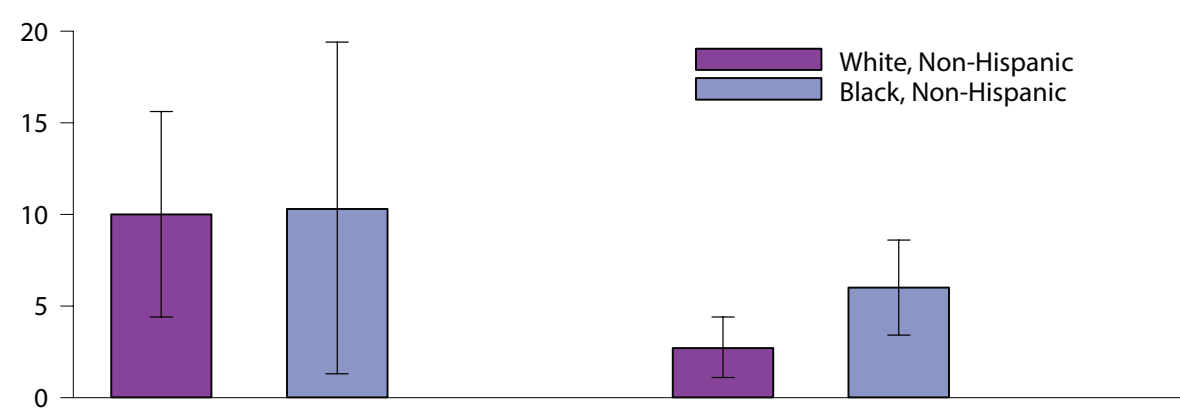

Yes

No

Previous STI Diagnosis

* Prevalence estimates based on response to the question, "Have you ever been treated for an infection in your fallopian tubes, uterus or ovaries, also called a pelvic infection, pelvic inflammatory disease, or PID?". Estimates were weighted to be nationally representative of the US population, accounting for unequal probabilities of selection and nonresponse.

" Based on a response of "Yes" to the question, "Have you ever had vaginal, anal, or oral sex?". ₹ $\mathrm{STI}=$ sexually transmitted infection. Participants who have been told by a doctor or other healthcare professional in the last 12 months that they had chlamydia or gonorrhea or have ever been told they have herpes, human papillomavirus, or genital warts.

NOTE: Error bars indicate $95 \%$ confidence intervals. Prevalence estimates among non-Hispanic Black women with a previous STI diagnosis have a relative standard error $>40 \%$ but $<50 \%$. SOURCE: Kreisel, K, Torrone, E, Bernstein, K, et al. Prevalence of pelvic inflammatory disease in sexually experienced women of reproductive age — United States, 2013-2014. MMWR Morb Mortal Wkly Rep 2017; 66(3):80-83. 
Hospital Discharge Survey, a sample of inpatient discharge records from select hospitals. However, the ability to ascertain the number of ectopic pregnancies occurring in the United States has been affected by a shift in clinical management from an inpatient to an outpatient event, making inpatient hospital surveillance data sources unreliable. As a result, alternative surveillance methods, including data from large administrative claims, ${ }^{36-37}$ have been used to evaluate trends and assess the continued public health burden of this condition. Data from MarketScan Commercial Claims and Encounters Database, a large administrative claims database of United States commercial health plans, indicate that the ratio of ectopic pregnancy diagnoses to all live births among women with live births aged 15-44 years during the period of 2005-2016 have marginally increased across all age groups (Figure D). As in previous years, in 2016, rates of ectopic pregnancy were highest among women in the 35-44 year age groups.

\section{Chlamydia}

Chlamydial infections in women are usually asymptomatic and screening is necessary to identify most infections. Routine chlamydia screening of sexually-active young women has been recommended by the CDC since $1993 .{ }^{38}$ Rates of reported cases of chlamydia among women increased steadily from the early 1990s, likely reflecting expanded screening coverage and use of more sensitive diagnostic tests (Table 1). During 2011-2013, chlamydia case rates decreased from 643.4 to 619.0 cases per 100,000 females and then increased $11.1 \%$ over the next 4 years, resulting in a rate of 687.4 cases per 100,000 females in 2017 (Table 4).

Chlamydia rates are highest among young women, the population targeted for screening (Figure 5, Table 10).

\section{Figure D. Ectopic Pregnancy — Ratio* Among Commercially Insured}

Women with Live Births Aged 15-44 Years by Age Group, 2005-2016

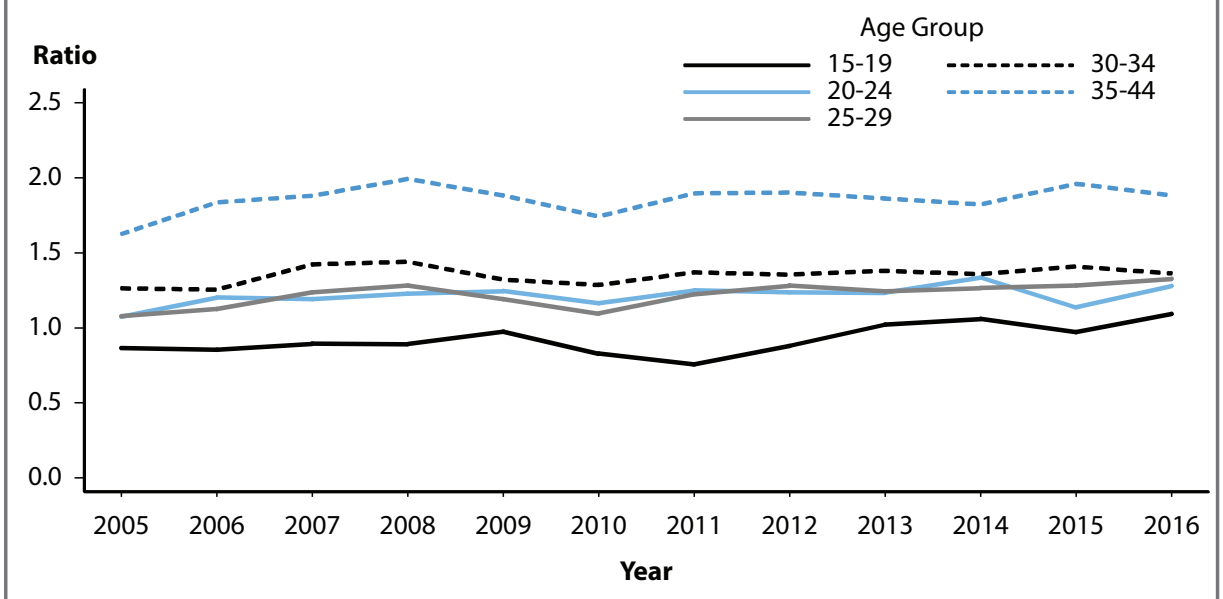

* Ratios represent the number of ectopic pregnancy diagnoses per 100,000 live births. SOURCE: MarketScan Commercial Claims and Encounters Database, Truven Health Analytics, Ann Arbor, Ml, 2005-2016.

\section{Figure E. Chlamydia - Rates of Reported Cases Among Women by} State, United States and Outlying Areas, 2017

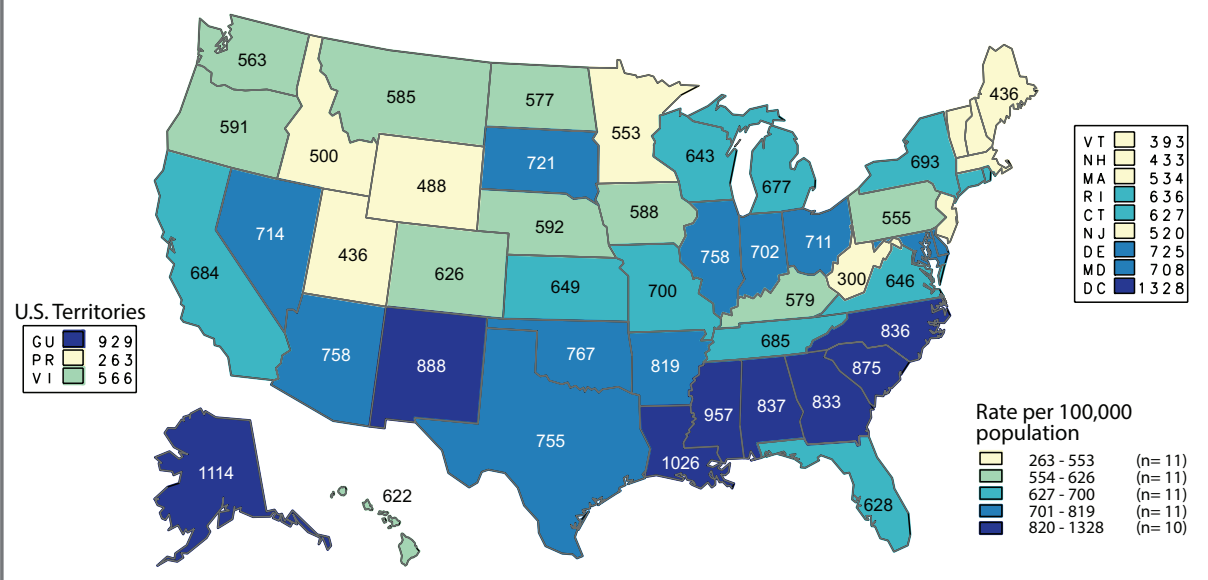

NOTE: The total rate of reported cases of chlamydia among women in the United States and outlying areas (including Guam, Puerto Rico, and the Virgin Islands) was 682.9 per 100,000 females. See Section A1.11 in the Appendix for more information on interpreting reported rates in the outlying areas.

ACRONYMS: $\mathrm{GU}=$ Guam; PR = Puerto Rico; $\mathrm{VI}=$ Virgin Islands.

During 2016-2017, rates of reported chlamydia cases increased $6.5 \%$ and $3.7 \%$ among females aged $15-19$ and 20-24 years, respectively (Figure 6). Regionally, chlamydia case rates were highest among women in the South, with a rate of 748.8 cases per 100,000 females in 2017 (Table 4). Rates of reported chlamydia cases exceeded gonorrhea case rates among women in all regions (Figures E and F, Tables 4 and 15). 
Figure F. Gonorrhea - Rates of Reported Cases Among Women by State, United States and Outlying Areas, 2017

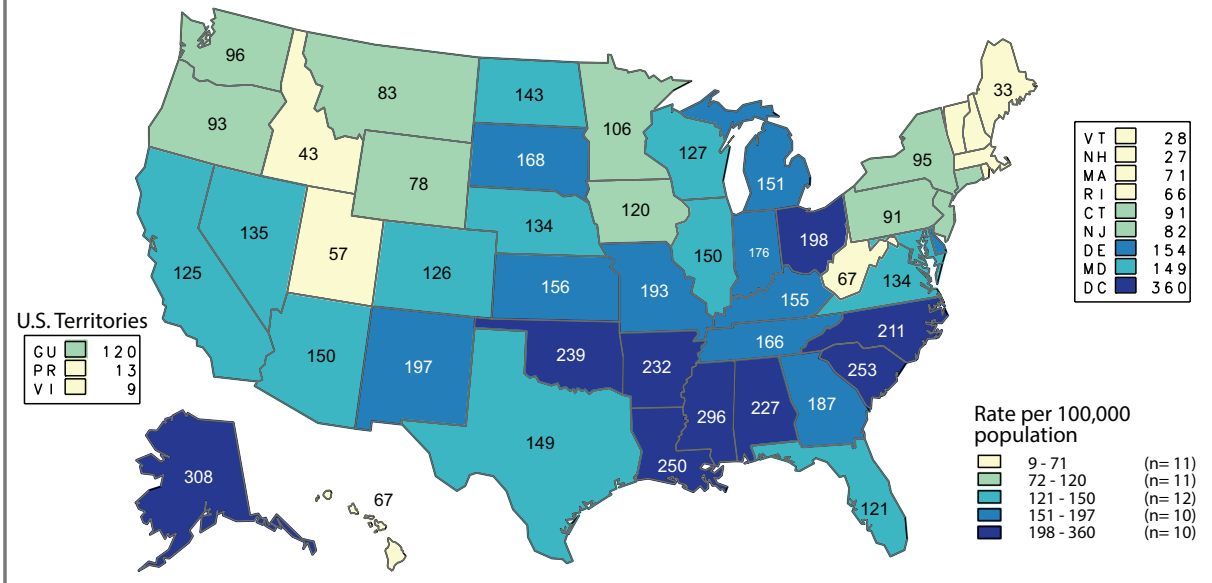

NOTE: The total rate of reported cases of gonorrhea among women in the United States and outlying areas (including Guam, Puerto Rico, and the Virgin Islands) was 140.3 per 100,000 females. See Section A1.2 in the Appendix for more information on estimating rates for outlying areas. ACRONYMS: GU = Guam; PR = Puerto Rico; VI = Virgin Islands. men declined steadily throughout the 1980s and early 1990s, and then declined more gradually in the late 1990 s and the 2000s. However, more recently, there have been increases in overall cases (Figure 14, Table 1).

After reaching a 40 -year low in 2009 (104.5 cases per 100,000 females), the rate of reported cases of gonorrhea for women increased slightly each year during 2009-2011, and then decreased during 2012-2014 (Figure 18). During 2015-2017, the gonorrhea rate among women increased $33.4 \%$ to 141.8 cases per 100,000 females (Figure 18, Table $15)$.

The gonorrhea case rate among women was slightly higher than the rate among men during 2008-2012; however, the rate among men was higher than the rate among women during 2013-2017 (Figure 18, Tables 15 and 16). During 2013-2017, gonorrhea rates among women were highest among those aged 15-24 years (Figure 20, Table 21). For women in this age group, rates were highest among 19-year olds in 2017

\section{Chlamydia Positivity in Selected Populations}

The STD Surveillance Network $(\mathrm{SSuN})$ is an ongoing collaboration of state, county, and city health departments from 10 participating jurisdictions where demographic, clinical, and laboratory data are collected from women aged 15-44 years attending facilities that provide family planning and reproductive health services (See Section A2.2 of the Appendix). Figure G shows chlamydia testing and positivity reported only among facilities that tested more than 100 women and more than $60 \%$ of young women aged 14-24 years. In 2017, the overall positivity of chlamydia among women aged 14-24 years was $9.6 \%$, but for women 14-19 years of age, chlamydia positivity was $10.8 \%$. For women between the ages of 14-24 years, chlamydia positivity among non-Hispanic Blacks was about 1.5 times those of non-Hispanic Whites or Hispanics.

\section{Gonorrhea}

Like chlamydia, gonorrhea is often asymptomatic in women. Therefore, gonorrhea screening is an important strategy for the identification of gonorrhea among women. Large-scale screening programs for gonorrhea in women began in the 1970s. After an initial increase in cases detected through screening, rates of reported gonorrhea cases for both women and
Figure G. Chlamydia - Positivity* Among Women Aged 14-39 Years by Race, Hispanic Ethnicity, and Age Group in Clinics ${ }^{\dagger}$ Providing Family Planning and Reproductive Health Services, STD Surveillance Network (SSuN), 2017

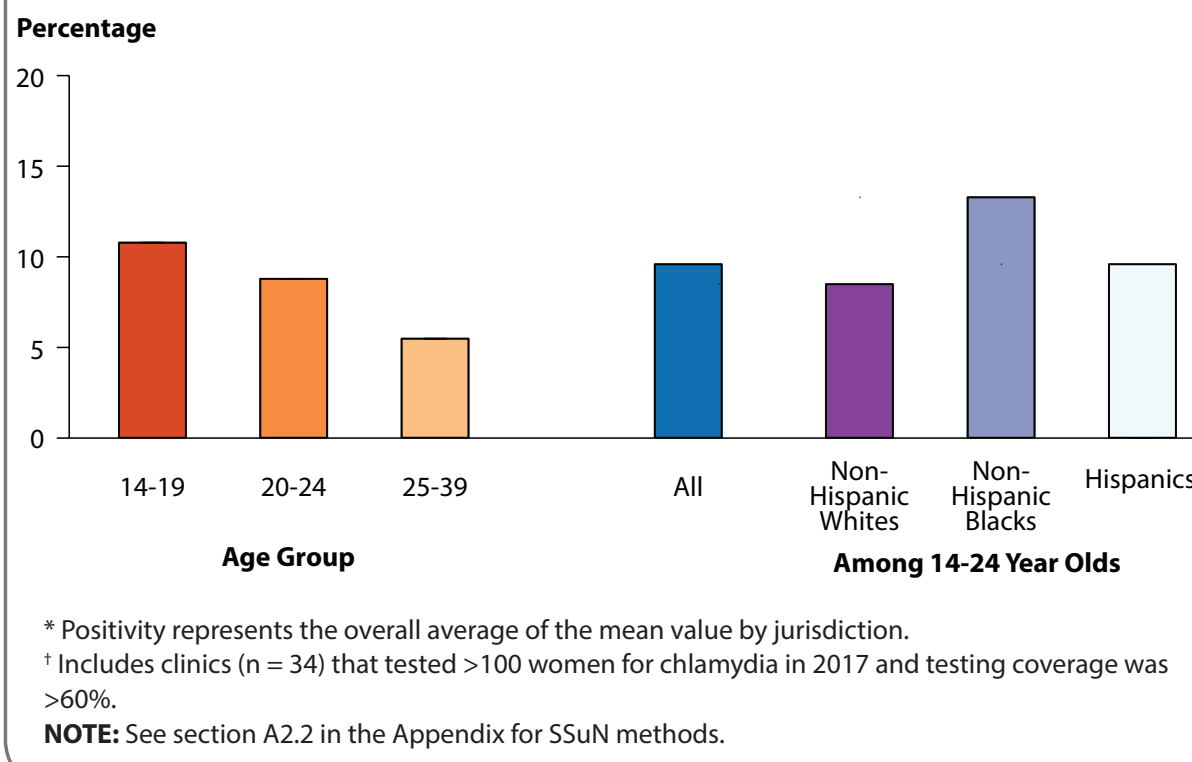


(872.2 cases per 100,000 females) (Table 23).

\section{Neonatal Conjunctivitis}

During 2013-2017, 461 chlamydia or gonorrhea cases among infants aged $<1$ year with a specimen source of either 'eye' or 'conjunctiva' (conjunctivitis infections) were reported to CDC. The overall reported rate of chlamydial conjunctivitis in infants was relatively stable during 2013-2017, ranging from 1.6 to 2.3 cases per 100,000 live births (Figure H). Similarly, the rate of gonococcal conjunctivitis in infants remained relatively constant and low during 2013-2017, at 0.4 cases or less per 100,000 live births each year. The rate of reported cases is heavily influenced by the completeness of reported data on specimen source. Of all cases reported to CDC of chlamydia or gonorrhea in infants aged $<1$ year during 2013-2017 $(n=2,567)$, nearly $82 \%$ did not have a specimen source of either 'eye' or 'conjunctiva'; of those, $56.2 \%$ had a specimen source of 'unknown' (42.1\%), 'other-not specified' (9.7\%), or was missing $(4.4 \%)$. When

evaluating rates including these cases, the rate of chlamydia and gonorrhea infections follows similar trends but is higher in all years, indicating potential missed cases for surveillance (Figure H).

\section{Congenital Syphilis}

Trends in congenital syphilis usually follow trends in primary and secondary $(\mathrm{P} \& \mathrm{~S})$ syphilis among reproductive-aged women (Figure 49). After plateauing at a relatively low rate $(0.9$ cases per 100,000 females) during 2011-2013, the rate of reported $\mathrm{P} \& \mathrm{~S}$ syphilis cases among all women increased each year since then. During 2013-2017, the rate among women increased $155.6 \%$, from 0.9 to 2.3 cases per 100,000 females (Table 28). During this same time, the rate among reproductive-aged women (women aged $15-44$ years) increased $142.8 \%$, from 2.1 to 5.1 cases per 100,000 females aged 15-44 years (Figure 49).

Similarly, the rate of reported congenital syphilis cases has increased each year since 2012 (Figure 49, Table 1). In 2017, there were 918 reported cases of congenital syphilis and the national congenital

\section{Figure H. Chlamydia and Gonorrhea - Rates of Reported Cases Among Infants < 1 Year of Age by Year and Specimen Source, United States, 2013-2017}

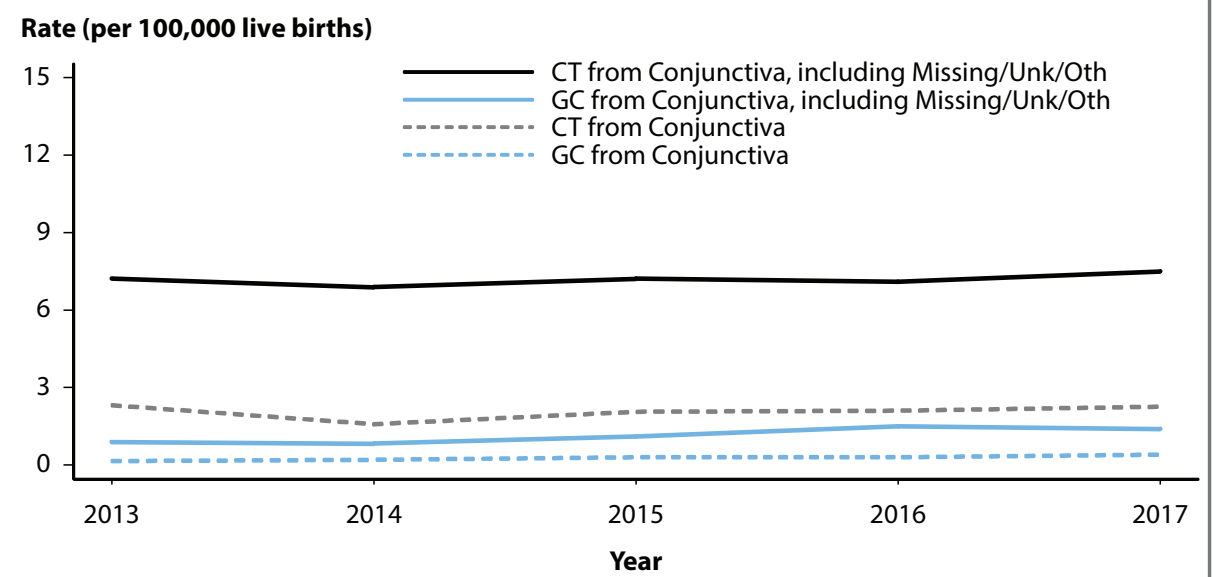

ADAPTED FROM: Kreisel K, Weston E, Braxton J, et al. Keeping an eye on chlamydia and gonorrhea conjunctivitis in infants in the United States, 2010-2015. Sex Transm Dis 2017; 44(6): 356-358. ACRONYMS: $\mathrm{CT}=$ Chlamydia; $\mathrm{GC}=$ Gonorrhea. syphilis rate was 23.3 cases per 100,000 live births, the highest rate reported since 1997. This increase in 2017 represents a $43.8 \%$ increase relative to 2016 and a $153.3 \%$ increase relative to 2013 (Table 41).

In 2017, the highest rates of P\&S syphilis among women and the highest rates of congenital syphilis were observed in the West and in the South (Figures I and J, Tables 28 and 41). The P\&S syphilis rates among women increased in every region during 2016-2017. During 2016-2017, the largest increases in the $\mathrm{P} \& \mathrm{~S}$ syphilis rates among women were seen in the West (29.6\%), followed by the South (22.7\%), Northeast (11.1\%), and Midwest $(8.3 \%)$ (Table 28). The congenital syphilis rate increased $60.3 \%$ in the South, $40.7 \%$ in the West, $5.7 \%$ in the Northeast, and $5.7 \%$ in the Midwest during 2016-2017 (Table 41).

Although most cases of congenital syphilis occur among infants whose mothers have had some prenatal care, late or limited prenatal care has been associated with congenital syphilis. Failure of health care providers to adhere to prenatal syphilis screening recommendations, as well as acquisition of infection during pregnancy after the initial screening test, also contribute to the occurrence of congenital syphilis. ${ }^{26}$

\section{Neonatal Herpes Simplex Virus}

Neonatal HSV infections, although relatively rare, cause significant morbidity and mortality. ${ }^{27}$ Most neonatal HSV infections result from perinatal transmission from mother to neonate, ${ }^{28}$ but postnatal infection can occur. ${ }^{39}$ Although reporting of neonatal HSV infection is required in a few jurisdictions, ${ }^{40-41}$ it is not a nationally reportable disease.

An examination of inpatient records of infants aged 60 days or younger at admission using the HCUP Kid's 
Figure I. Primary and Secondary Syphilis - Rates of Reported Cases Among Women by State, United States and Outlying Areas, 2017

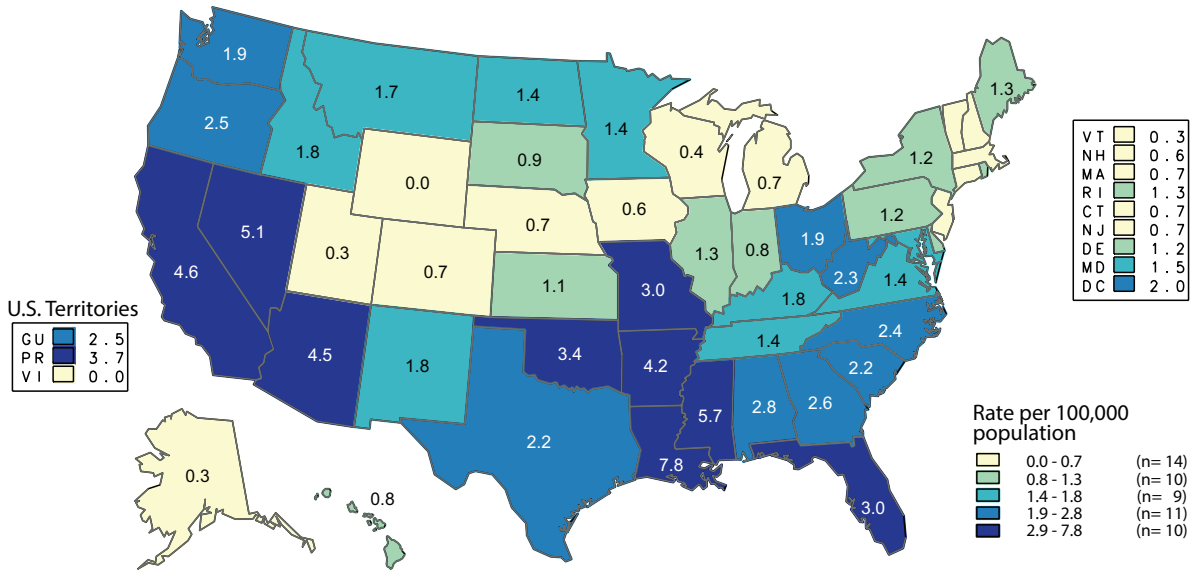

NOTE: The total rate of reported cases of primary and secondary syphilis among women in the United States and outlying areas (including Guam, Puerto Rico, and the Virgin Islands) was 2.3 per 100,000 females. See Section A1.11 in the Appendix for more information on interpreting reported rates in the outlying areas.

ACRONYMS: GU = Guam; PR = Puerto Rico; VI = Virgin Islands.

Figure J. Congenital Syphilis - Rates of Reported Cases Among Infants by Year of Birth and State, United States and Outlying Areas, 2017

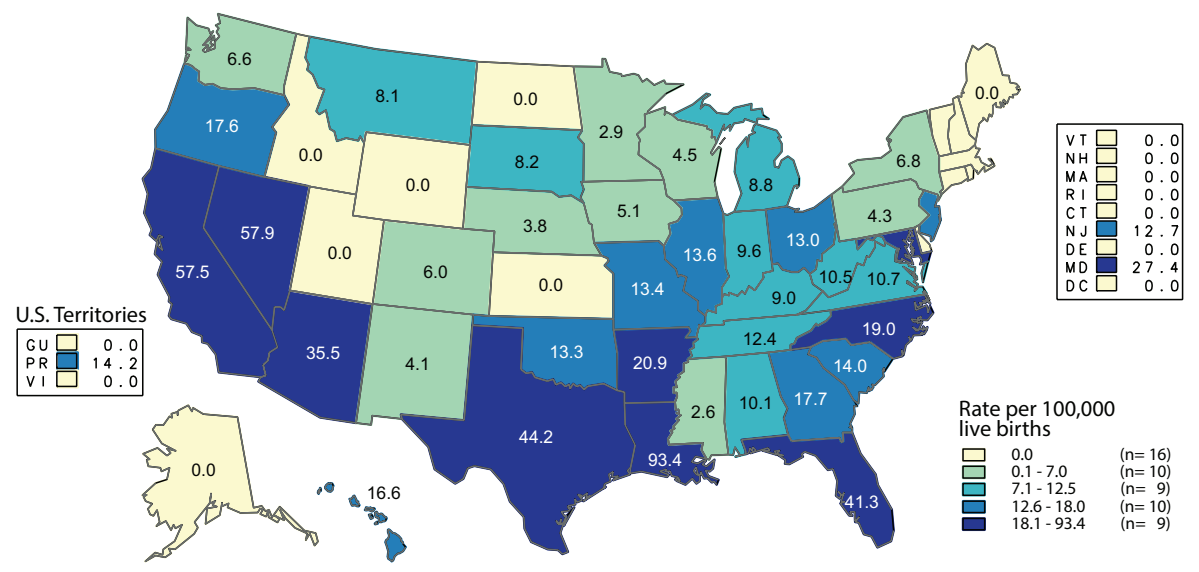

NOTE: The total rate of reported cases of congenital syphilis for infants by year of birth for the United States and outlying areas (including Guam, Puerto Rico, and the Virgin Islands) was 23.2 per 100,000 live births. See Section A1.2 in the Appendix for more information on estimating rates for outlying areas.

ACRONYMS: $\mathrm{GU}=$ Guam; PR = Puerto Rico; VI = Virgin Islands.

Inpatient Database showed an overall incidence of 9.6 cases per 100,000 live births in $2006 .{ }^{42}$ Rates did not vary significantly by region or by race and Hispanic ethnicity; however prevalence was significantly higher among cases for which the expected primary payer was Medicaid (15.1 cases per 100,000 live births) compared with private insurance or managed health care (5.4 cases per 100,000 live births).
In New York City, 76 cases of neonatal HSV infection were identified through population-based surveillance during a 4.5 year period (April 2006-September 2010), for an average annual incidence of 13.3 cases per 100,000 live births. ${ }^{43}$ Forty-one percent of the confirmed cases were infected with HSV type 1. A review of certificates of death or stillbirth issued in New York City during 1981-2013 identified 34 deaths due to neonatal HSV infection, or 0.82 deaths per 100,000 live births. ${ }^{42}$

For information on adolescent and adult HSV infections, see Other STDs.

\section{Summary}

STDs are an important health priority and what may often be overlooked is the substantial morbidity and mortality related to sequelae of STDs. This is particularly true for women and their infants. The overall rate of reported female chlamydia cases has increased $11.1 \%$ over the last four years, much of that attributed to increased screening and more complete national reporting. Gonorrhea infections have also increased $33.4 \%$ to 141.8 cases per 100,000 females in recent years. Surveillance data continues to show that numbers and rates of chlamydia and gonorrhea cases are highest in females between the ages of 15 and 24 and certain races are disproportionately impacted. Despite increases in chlamydia and gonorrhea, available data suggest an overall decline in the incidence of PID, largely attributed to an increase in effective screening and treatment of chlamydial and gonococcal infections in adolescents and young women. In contrast to declining PID rates, surveillance data suggests rates of ectopic pregnancy have marginally increased over time.

Mother to child transmission of STDs can result in serious adverse 
consequences. Potential adverse neonatal outcomes include neonatal ophthalmia, neonatal pneumonia, and prematurity. The rate of congenital syphilis in the United States has increased every year since 2013. In 2017, there were 918 reported cases of congenital syphilis and the national congenital syphilis rate was 23.3 cases per 100,000 live births, the highest rate in two decades. Despite current recommended STD testing during pregnancy, women remain underscreened for STDs during pregnancy, either because of a lack of or limited prenatal care, or infection outside of the testing window.

\section{References}

1. Jolly DH, Mueller MP, Chen M, et al. Concurrency and other sexual risk behaviors among black young adults in a southeastern city. AIDS Educ Prev 2016; 28(1):59-76.

2. Dolwick Grieb SM, Davey-Rothwell M, Latkin CA. Concurrent sexual partnerships among urban African American high risk women with main sex partners. AIDS Behav 2012; 16(2):323-333. DOI: 10.1007/s10461-0119954-6.

3. Hogben M, Leichliter JS. Social determinants and sexually transmitted disease disparities. Sex Transm Dis 2008; 35(12):S13-S18.

4. Kelly J, Cohen J, Grimes B, et al. High rates of herpes simplex virus type 2 infection in homeless women: Informing public health strategies. J Womens Health (Larchmt) 2016; 25(8):840-845. DOI: 10.1089/jwh.2015.5579.

5. Tschann JM, Flores E, de Groat CL, et al. Condom negotiation strategies and actual condom use among Latino youth. J Adolesc Health 2010; 47(3):254-262. DOI: 10.1016/j. jadohealth.2010.01.018.

6. Pulerwitz J, Amaro H, De Jong W, et al. Relationship power, condom use and HIV risk among women in the USA. AIDS Care 2002; 14(6):789-800.

7. McCree DH, Rompalo A. Biological and behavioral risk factors associated with STDs/ HIV in women: Implications for behavioral interventions. In: Aral SO, Douglas JM, Lipshutz JA, eds. Behavioral Interventions for Prevention and Control of Sexually Transmitted Diseases. New York, NY: Springer; 2007:310324.

8. Swartzendruber A, Zenilman JM, Niccolai LM, et al. It takes 2: Partner attributes associated with sexually transmitted infections among adolescents. STD 2013; 40(5):372-378.
9. Haggerty CL, Gottlieb SL, Taylor BD, et al. Risk of sequelae after Chlamydia trachomatis genital infection in women. J Infect Dis 2010; 201:S134-S155.

10. Oakeschott, P, Kerry S, Aghaizu A, et al. Randomised controlled trial of screening for Chlamydia trachomatis to prevent pelvic inflammatory disease: The POPI (prevention of pelvic infection) trial. BMJ 2010; 340:c1642.

11. Price MJ, Ades AE, De Angelis D, et al. Risk of pelvic inflammatory following Chlamydia trachomatis infection: Analysis of prospective studies with a multistate model. Am J Epidemiol 2013; 178(3):484-492.

12. Tsevat DG, Wiesenfeld HC, Parks C, et al. Sexually transmitted diseases and infertility. Am J Obstet Gynecol 2017; 216(1):1-9.

13. Ness RB, Trautmann G, Richter HE, et al. Effectiveness of treatment strategies of some women with pelvic inflammatory disease: A randomized trial. Obste Gyneco 2005; 106(3):573-580.

14. Gottlieb SL, Xu F, Brunham RC. Screening and treating chlamydia genital infections to prevent PID: Interpretation of findings from randomized controlled trials. Sex Transm Dis 2013; 40(2):97-102.

15. Satterwhite CL, Torrone E, Meites E, et al. Sexually transmitted infections among US women and men: Prevalence and incidence estimates, 2008. Sex Transm Dis 2013; 40(3):187-193.

16. McQuillan G, Kruszon-Moran D, Markowitz LE, et al. Prevalence of HPV in adults aged 18-69: United States, 2011-2014. NCHS Data Brief, No. 280. Hyattsville, MD: National Center for Health Statistics; 2017

17. Insinga RP, Perez G, Wheeler CM, et al. Incident cervical HPV infections in young women: Transition probabilities for CIN and infection clearance. Cancer Epidemiol Biomarkers Prev 2011; 20(2):287-296.

18. Doorbar J, Quint W, Banks L, et al. The biology and life-cycle of human papillomaviruses. Vaccine 2012; 30(Suppl 5):F55-F70.

19. Saslow D, Solomon D, Lawson HW, et al. American Cancer Society, American Society for Colposcopy and Cervical Pathology, and American Society for Clinical Pathology screening guidelines for the prevention and early detection of cervical cancer. J Low Genit Tract Dis 2012; 16(3):175-204.

20. Garland SM, Steben M, Sings HL, et al Natural history of genital warts: Analysis of the placebo arm of 2 randomized phase III trials of a quadrivalent human papillomavirus (Types $6,11,16$, and 18 ) vaccine. J Infect Dis 2009; 199(6):805-814.

21. Clifford GM, Rana RK, Franceschi S, et al. Human papillomavirus genotype distribution in low-grade cervical lesions: Comparison by geographic region and with cervical cancer. Cancer Epidemiol Biomarkers Prev 2005; 14(5):1157-1164.
22. Gissmann L, Wolnik L, Ikenberg H, et al. Human papillomavirus types 6 and 11 DNA sequences in genital and laryngeal papillomas and in some cervical cancers. Proc Natl Acad Sci USA 1983; 80(2): 560-563.

23. Donne AJ, Clarke R. Recurrent respiratory papillomatosis: An uncommon but potentially devastating effect of human papillomavirus in children. Int J STD AIDS 2010; 21(6):381-385.

24. Armstrong LR, Preston EJ, Reichert M, et al. Incidence and prevalence of recurrent respiratory papillomatosis among children in Atlanta and Seattle. Clin Infect Dis 2000; 31:107-109

25. Kohlhoff, SA, Hammerschlag, MR. Gonococcal and chlamydial infections in infants and children. In: Sexually Transmitted Diseases. 4th ed. New York, NY: McGraw Hill; 2007:16131627.

26. Kidd SE, Bowen VB, Torrone EA, et al. Use of national syphilis surveillance data to develop a congenital syphilis prevention cascade and estimate the number of potential congenital syphilis cases averted. Sex Transm Dis 2018; DOI: 10.1097/OLQ.0000000000000838. [Epub ahead of print].

27. Kimberlin DW. Herpes simplex virus infections of the newborn. Semin Perinatol 2007; 31(1):19-25.

28. Corey L, Wald A. Maternal and neonatal herpes simplex virus infections. N Engl J Med 2009; 361(14):1376-1385.

29. Kimberlin DW. The scarlet H. J Infect Dis 2014; 209(3):315-317.

30. Sutton MY, Sternberg M, Zaidi A, et al. Trends in pelvic inflammatory disease hospital discharges and ambulatory visits, United States, 1985-2001. Sex Transm Dis 2005; 32(12):778784 .

31. Bohm MK, Newman L, Satterwhite CL, et al. Pelvic inflammatory disease among privately insured women, United States, 2001-2005. Sex Transm Dis 2010; 37(3):131-136.

32. Whiteman MK, Kuklina E, Jamieson DJ, et al. Inpatient hospitalization for gynecologic disorders in the United States. Am J Obstet Gynecol 2010; 202(6):541.e1-6.

33. Kreisel K, Flagg EW, Torrone E. Trends in pelvic inflammatory disease emergency department visits, United States, 2006-2013. Am J Obstet Gynecol 2018; 218: 117.e1-10.

34. Owusu Edusei K Jr, Bohm MK, Chesson HW, et al. Chlamydia screening and pelvic inflammatory disease: Insights from exploratory time-series analyses. Am J Prev Med 2010; 38(6):652-657.

35. Leichliter JS., Chandra A., Aral SO. Correlates of self-reported pelvic inflammatory disease treatment in sexually experienced reproductiveaged women in the United States, 1995 and 2006-2010. Sex Transm Dis 2013; 40(5):413418 
36. Trabert B, Holt VL, Yu O, et al. Populationbased ectopic pregnancy trends, 1993-2007. Am J Prev Med 2011; 40(5):556-560.

37. Hoover KW, Tao G, Kent CK. Trends in the diagnosis and treatment of ectopic pregnancy in the United States. Obstet Gynecol 2010; 115(3):495-502.

38. Centers for Disease Control and Prevention. Recommendations for the prevention and management of Chlamydia trachomatis infections, 1993. MMWR Morb Mortal Wkly Rep 1993; 42(RR-12):1-39.

39. Centers for Disease Control and Prevention. Neonatal herpes simplex virus infection following Jewish ritual circumcisions that included direct orogenital suction - New York City, 2000-2011. MMWR Morb Mortal Wkly Rep 2012; 61(22):405-409.

40. Dinh T-H, Dunne EF, Markowitz LE, et al. Assessing neonatal herpes reporting in the United States, 2000-2005. Sex Transm Dis 2008; 35(1):19-21.

41. Handel S, Klingler EJ, Washburn K, et al. Population-based surveillance for neonatal herpes in New York City, April 2006-September 2010. Sex Transm Dis 2011; 38(8):705-711.

42. Flagg EW, Weinstock H. Incidence of neonatal herpes simplex virus infections in the United States, 2006. Pediatrics 2011; 127(1):e1-8.

43. Sampath A, Maduro G, Schillinger JA. Infant deaths due to herpes simplex virus, congenital syphilis, and HIV in New York City. Pediatrics 2016; 137(4):e20152387. 
This page intentionally left blank. 


\section{STDs in Adolescents and Young Adults}

\section{Background}

Incidence and prevalence estimates suggest that young people aged 15-24 years acquire half of all new STDs ${ }^{1}$ and that one in four sexually active adolescent females has an STD, such as chlamydia or human papillomavirus (HPV). ${ }^{2}$ Compared with older adults, sexually active adolescents aged $15-19$ years and young adults aged 20-24 years are at higher risk of acquiring STDs for a combination of behavioral, biological, and cultural reasons. For some STDs, such as chlamydia, adolescent females may have increased susceptibility to infection because of increased cervical ectopy. Cervical ectopy refers to columnar cells, which are typically found within the cervical canal, located on the outer surface of the cervix. Although this is a normal finding in adolescent and young women, these cells are more susceptible to infection. The higher prevalence of STDs among adolescents may also reflect multiple barriers to accessing quality STD prevention and management services, including inability to pay, lack of transportation, long waiting times, conflict between clinic hours and work and school schedules, embarrassment attached to seeking STD services, method of specimen collection, and concerns about confidentiality (e.g., Explanation of Benefits for services received mailed to parents or guardians). ${ }^{3}$

Traditionally, intervention efforts have targeted individual level factors associated with STD risk which do not address higher-level factors (e.g., peer norms and media influences) that may also influence behaviors. ${ }^{4}$ Interventions for atrisk adolescents and young adults that address underlying aspects of the social and cultural conditions affecting sexual risk-taking behaviors are needed, as are strategies designed to improve the underlying social conditions themselves. ${ }^{5,6}$ In addition, in designing STD programs, consideration should be given to the needs of adolescent and young adult populations including extended hours, optimizing privacy in waiting rooms, and urine based specimen collection. ${ }^{3}$

\section{Chlamydia}

In 2017 , there were $1,069,111$ reported cases of chlamydial infection among persons aged 15-24 years, representing $62.6 \%$ of all reported chlamydia cases. Among those aged 15-19 years, the rate of reported cases of chlamydia increased $7.5 \%$ during 2016-2017 (1,927.3 to 2,072.4 cases per 100,000 population) (Table 10). Among those aged 20-24 years, the rate increased 5.0\% during 2016$2017(2,686.1$ to $2,820.3$ cases per 100,000 population) (Table 10).

Among women aged 15-24 years, the population targeted for chlamydia screening, the overall rate of reported cases of chlamydia was 3,635.3 cases per 100,000 females (Table $12)$. This was a $4.9 \%$ increase from $2016(3,464.1$ cases per 100,000 females) and an $8.8 \%$ increase from 2013 (3,341.1 cases per 100,000 females). Among men aged 15-24 years, the overall rate of reported cases of chlamydia was $1,327.0$ cases per 100,000 males. This was an $8.9 \%$ increase from 2016 (1,219.0 cases per 100,000 males) and a $29.1 \%$ increase from $2013(1,027.8$ cases per 100,000 males). Rates varied by state for both males and females. The majority of states having the highest reported case rates were in the South (Figures K and L).

15-19 Year Old Females - In 2017, the rate of reported chlamydia cases among women aged 15-19 years was $3,265.7$ cases per 100,000 females, a $6.5 \%$ increase from the 2016 rate of 3065.8 cases per 100,000 females (Figures 5 and 6, Table 10). Increases in rates of reported cases of chlamydia during 2016-2017 were largest among 19-year old and $20-$ year old women $(8.4 \%$ and $6.8 \%$

\section{Figure K. Chlamydia - Rates of Reported Cases Among Women Aged 15-24 Years by State, United States and Outlying Areas, 2017}

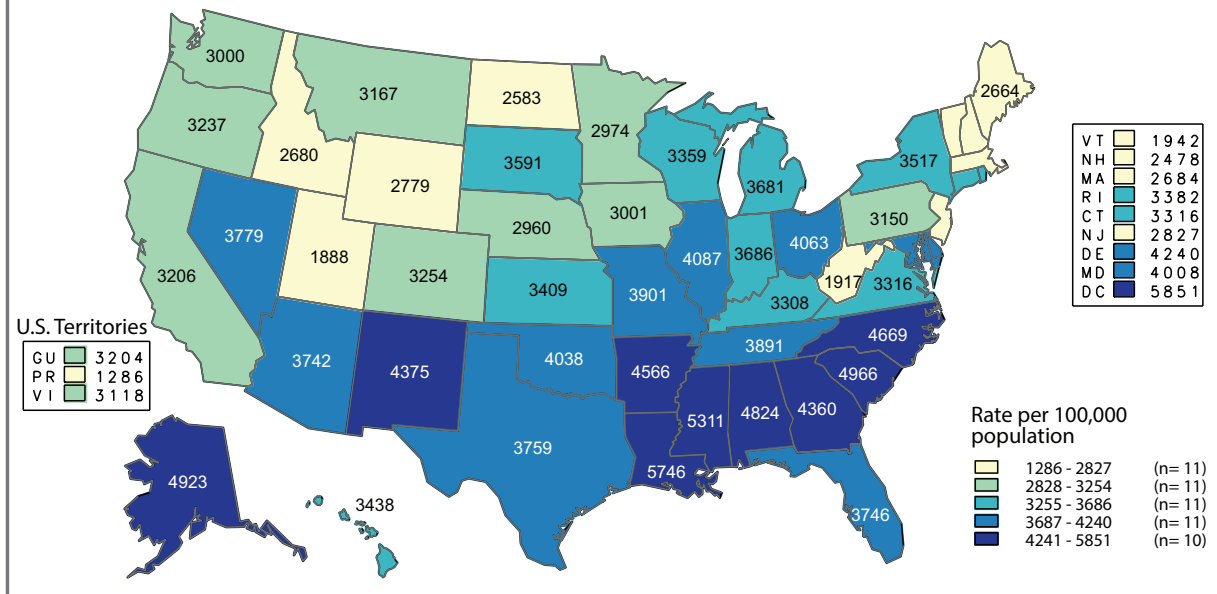

NOTE: Rates for Puerto Rico and the Virgin Islands were calculated by using the 2010 population estimates (see Section A1.2 in the Appendix).

ACRONYMS: $\mathrm{GU}=$ Guam; PR = Puerto Rico; VI = Virgin Islands 


\section{Figure L. Chlamydia - Rates of Reported Cases Among Men Aged 15-24 Years by State, United States and Outlying Areas, 2017}

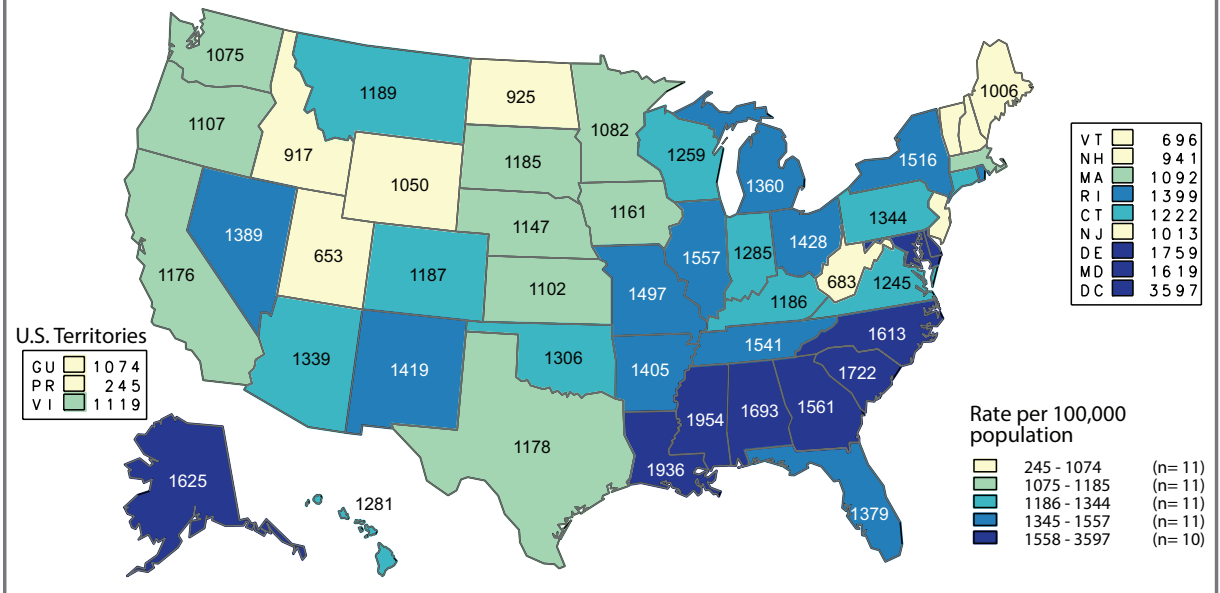

NOTE: Rates for Puerto Rico and the Virgin Islands were calculated by using the 2010 population estimates (see Section A1.2 in the Appendix).

ACRONYMS: GU = Guam; PR = Puerto Rico; VI = Virgin Islands. increases, respectively) (Table 12). During 2013-2017, the overall rate of reported cases for women aged 15-19 years increased $6.4 \%$ (Table 10).

20-24 Year Old Females - In 2017, women aged 20-24 years had the highest rate of reported chlamydia cases $(3,985.8$ cases per 100,000 females) compared with any other age group for either sex (Figures 5 and 6 , Table 10). The overall increase in the rate of reported chlamydia cases among females aged 20-24 years during 2016-2017 was 3.7\%, with the largest increase $(4.1 \%)$ observed among females aged 20 years. During 2013-2017, the rate of reported chlamydia cases in this population increased 10.9\% (Table 10).

\section{5-19 Year Old Males - In 2017,} the rate of reported chlamydia cases among men aged 15-19 years was 924.5 cases per 100,000 males, an $11.1 \%$ increase from 2016. During 2013-2017, the rate of reported chlamydia cases for men aged 15-19 years increased $27.9 \%$ (Figures 5 and 7, Table 10).

20-24 Year Old Males - In 2017, as in previous years, men aged 20-24 years had the highest rate of reported chlamydia cases among all men (1,705.4 cases per 100,000 males). The rate for men in this age group increased 7.8\% during 2016-2017 (Figures 5 and 7, Table 10). Similarly, during 2013-2017, the rate for men aged 20-24 years increased $30.1 \%$ (Table 10).

\section{Gonorrhea}

During 2016-2017, the rate of reported gonorrhea cases increased $15.5 \%$ for persons aged $15-19$ years and $12.8 \%$ for persons aged $20-24$ years (Table 21). In 2017, among women aged 15-24 years, the rate was 622.8 cases per 100,000 females (Table 23). This was a $14.3 \%$ increase from 2016 (545.0 cases per 100,000 females) and a $24.1 \%$ increase from 2013 (501.7 cases per 100,000 females). Among men aged 15-24 years, the rate was 520.1 cases per 100,000 males. This was a $13.4 \%$ increase from 2016 (458.8 cases per 100,000 males) and a $51.6 \%$ increase from 2013 (343.0 cases per 100,000 males). For both women and men, rates varied by state. The majority of states with the highest reported case rates were in the South (Figures M and $\mathrm{N}$ ).

15-19 Year Old Females - In 2017, women aged 15-19 years had the second highest rate of reported gonorrhea cases (557.4 cases per 100,000 females) compared with other age groups among women (Figures 19 and 20, Table 21). During 2016-2017, the rate of reported

\section{Figure M. Gonorrhea - Rates of Reported Cases Among Women Aged 15-24 Years by State, United States and Outlying Areas, 2017}

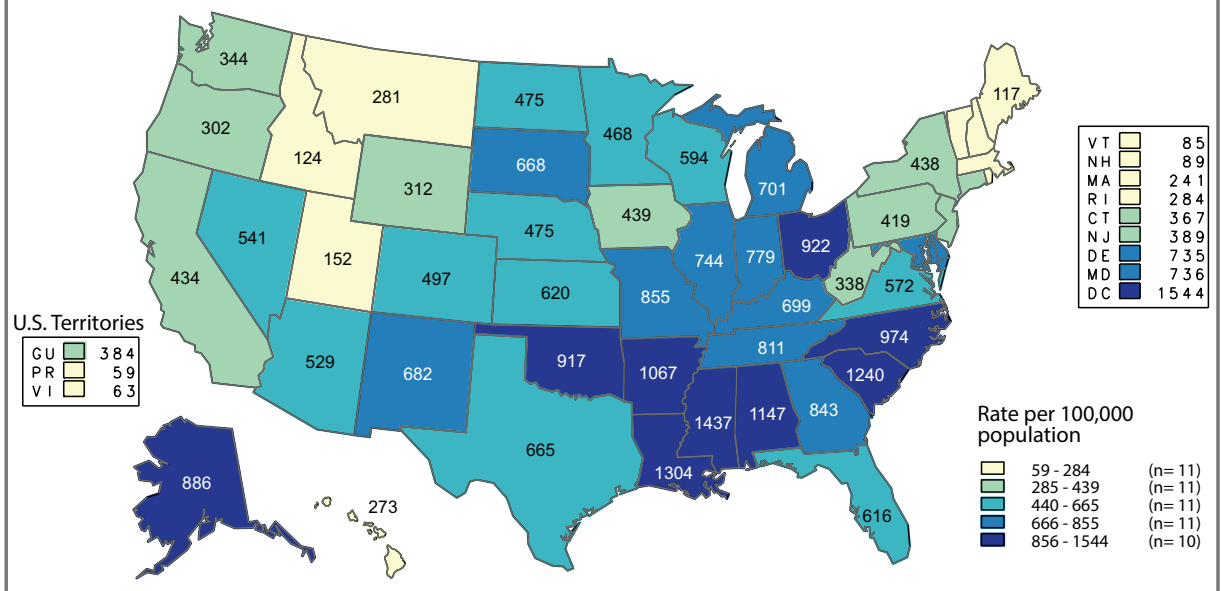

NOTE: Rates for Puerto Rico and the Virgin Islands were calculated by using the 2010 population estimates (see Section A1.2 in the Appendix).

ACRONYMS: $\mathrm{GU}=$ Guam; $\mathrm{PR}=$ Puerto Rico; $\mathrm{VI}=$ Virgin Islands. 


\section{Figure N. Gonorrhea - Rates of Reported Cases Among Men Aged 15-24 Years by State, United States and Outlying Areas, 2017}

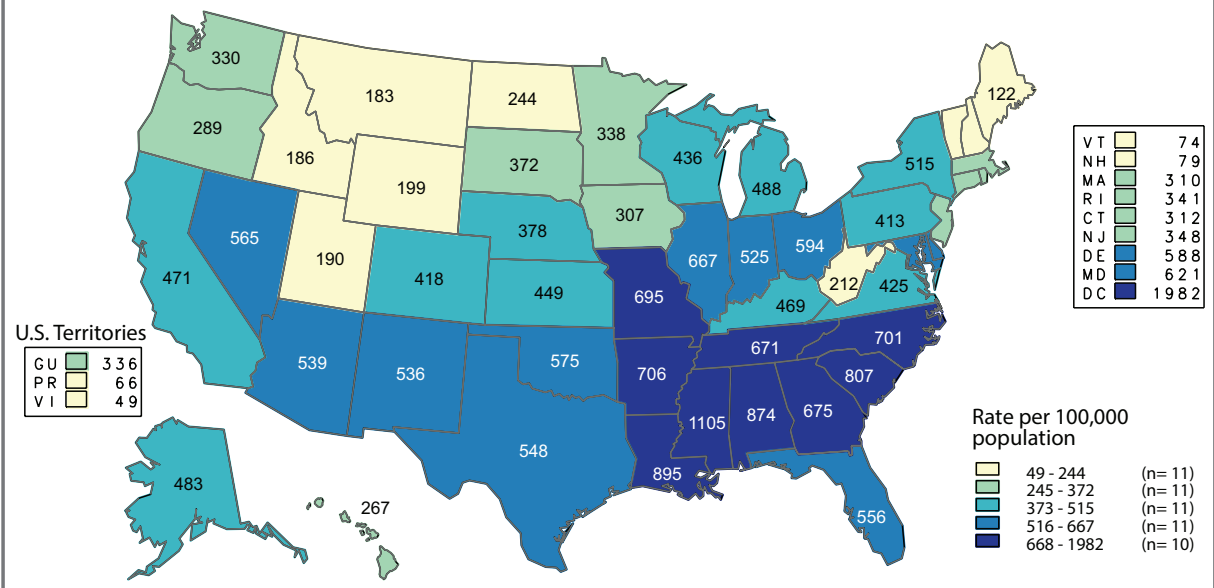

NOTE: Rates for Puerto Rico and the Virgin Islands were calculated by using the 2010 population estimates (see Section A1.2 in the Appendix).

ACRONYMS: $G U$ = Guam; PR = Puerto Rico; VI = Virgin Islands.

gonorrhea cases for women in this age group increased $15.8 \%$ and $20.4 \%$ during 2013-2017 (Table 21).

20-24 Year Old Females - In 2017, women aged 20-24 years had the highest rate of reported gonorrhea cases $(684.8$ cases per 100,000 females) compared with other age groups among women (Figures 19 and 20, Table 21). During 2016-2017, the rate of reported gonorrhea for women in this age group increased $13.1 \%$ and $27.4 \%$ during $2013-2017$ (Table 21).

15-19 Year Old Males — In 2017, the rate of reported gonorrhea cases among men aged 15-19 years was 323.3 cases per 100,000 males (Figures 19 and 21, Table 21). During 2016-2017, the rate of reported gonorrhea cases for men in this age group increased $15.2 \%$ and $44.8 \%$ during 2013-2017 (Table 21).

20-24 Year Old Males - In 2017, as in previous years, men aged 20-24 years had the highest rate of reported gonorrhea cases ( 705.2 cases per 100,000 males) compared with any other age group for either sex (Figures 19 and 21, Table 21). During
2016-2017, the rate of reported gonorrhea for men in this age group increased $12.6 \%$ and $55.2 \%$ during 2013-2017 (Table 21).

\section{Primary and Secondary Syphilis}

In 2017, among women aged 15-24 years, the rate of reported primary and secondary $(\mathrm{P} \& \mathrm{~S})$ syphilis was 5.5 cases per 100,000 females. This was a $7.8 \%$ increase from 2016 (5.1 cases per 100,000 females) and an $83.3 \%$ increase from 2013 (3.0 cases per 100,000 females). Among men aged 15-24 years, the rate was 26.1 cases per 100,000 males. This was an $8.3 \%$ increase from 2016 (24.1 cases per 100,000 males) and a 50.9\% increase from 2013 (17.3 cases per 100,000 males). During 2016-2017, the rate of reported $\mathrm{P} \& \mathrm{~S}$ syphilis cases increased 9.8\% among persons aged 15-19 years and $7.8 \%$ among persons aged 20-24 years (Table 34).

15-19 Year Old Females — The rate of reported P\&S syphilis cases among women aged 15-19 years increased each year during 2013-2016 (from 1.9 to 3.3 cases per 100,000 females) (Figure 43, Table 34). However, the rate slightly decreased in 2017. During 2016-2017, the rate decreased $3.0 \%$, from 3.3 to 3.2 cases per 100,000 females (Figure 43, Table 34). Despite this decline during 2016-2017, there was an overall increase in the rate of P\&S syphilis of $68.4 \%$ during 2013-2017 in women aged $15-19$ years.

20-24 Year Old Females - In 2017, women aged 20-24 years had the highest rate of $\mathrm{P} \& \mathrm{~S}$ syphilis (7.8 cases per 100,000 females) compared with other age groups among women (Figure 42, Table 34). The P\&S syphilis rate among women in this age group has increased each year since 2011 and has doubled since 2013 (Figure 43, Table 34). During 2016-2017, the rate increased $14.7 \%$.

15-19 Year Old Males — In 2017, the rate of reported $\mathrm{P} \& \mathrm{~S}$ syphilis cases among men aged 15-19 years was 10.1 cases per 100,000 males (Figure 42, Table 34). The P\&S syphilis rate among men in this age group has increased each year since 2011 , with an increase of $55.4 \%$ during 2013-2017 (Figure 44, Table 34). During 2016-2017, the rate increased $13.5 \%$.

20-24 Year Old Males - In 2017, men aged 20-24 years had the second highest rate of reported P\&S syphilis (41.1 cases per 100,000 males) compared with any other age group for either sex (Figure 42, Table 34). The P\&S syphilis rate among men in this age group has increased each year since 2006 , with a $50.0 \%$ increase during 2013-2017 (Figure 44, Table 34). During 2016-2017, the rate increased $7.0 \%$.

\section{Other STDs}

\section{Human papillomavirus}

HPV is the most common sexually transmitted infection in the United States. ${ }^{1}$ Starting in 2006, HPV vaccines have been recommended for routine use in United States females aged $11-12$ years, with 
catch-up vaccination through age $26 .^{7,8}$ Since late 2011, routine use of HPV vaccine has been recommended for males aged 11-12 years, with catch-up vaccination through age $21 .^{8-10}$ Vaccination through age 26 is recommended for gay, bisexual, and other men who have sex with men (collectively referred to as MSM) and persons who are immunocompromised (including those infected with HIV). ${ }^{8}$

Cervicovaginal prevalence of HPV vaccine types was examined using data from the National Health and Nutrition Examination Survey (NHANES; see Section A2.4 in the Appendix). ${ }^{11}$ Prevalence decreased significantly from 2003-2006 (the pre-vaccine era) to 2011-2014 in specimens from females aged 14-19 years and 20-24 years, the age groups most likely to benefit from HPV vaccination.

Health-care claims data from adolescents and adults with employerprovided private health insurance in the United States were used to examine the population effectiveness of HPV vaccination on two clinical sequelae of HPV infection: highgrade histologically-detected cervical intraepithelial neoplasia grades 2 and 3 (CIN2+), ${ }^{12}$ and anogenital warts. ${ }^{13}$ Prevalence of CIN2+ and of anogenital warts decreased significantly during 2007-2014 among females aged 15-19 and 20-24 years (Figures 51 and 52A); prevalence of anogenital warts also decreased significantly during 20092014 among women aged 25-29 years (Figure 52A). These declines provide ecologic evidence of population effectiveness of HPV vaccination in females. Anogenital wart prevalence also decreased significantly during 2009-2014 among men aged 20-24 years (Figure 52B); these declines among young men are consistent with herd protection from vaccination among females.
For more information on HPV infections, see Other STDs.

\section{Herpes simplex virus}

Herpes simplex virus (HSV) is among the most prevalent of sexually transmitted infections. ${ }^{1,14}$ Most genital HSV infections in the United States are caused by HSV type 2 (HSV-2), while HSV type 1 (HSV-1) infections are typically orolabial and acquired during childhood. ${ }^{15,16}$ NHANES data show that among adolescents aged 14-19 years, HSV-1 seroprevalence has significantly decreased by almost $23 \%$, from $39.0 \%$ during 1999-2004 to $30.1 \%$ during $2005-$ 2010, indicating declining orolabial infection in this age group. ${ }^{16} \mathrm{HSV}-2$ seroprevalence in this age group was much lower in both time periods. ${ }^{16}$

Other studies have found that genital HSV-1 infections are increasing among young adults. ${ }^{17,18}$ This has been attributed, in part, to the decline in orolabial HSV-1 infections, because those who lack HSV-1 antibodies at sexual debut are more susceptible to genital HSV-1 infection. ${ }^{16,19}$ Increasingly common oral sex behavior among adolescents and young adults also has been suggested as a contributing factor. ${ }^{16,20}$

For more information on genital HSV infections, see Other STDs.

\section{National Job Training Program}

The National Job Training Program (NJTP) is an educational program for socioeconomically disadvantaged youth aged 16-24 years and is administered at more than 100 sites throughout the country. The NJTP screens participants for chlamydia and gonorrhea within two days of entry to the program. All of NJTP's chlamydia screening tests and the majority of gonorrhea screening tests are conducted by a single national contract laboratory*.

To increase the stability of the 2017 estimates, chlamydia or gonorrhea prevalence data are presented when valid test results for 100 or more students per year are available for the population subgroup and state. Additional information about NJTP can be found in Section A2.1 in the Appendix.

Among women entering the program in 2017 in 44 states and Puerto Rico, the median state-specific chlamydia prevalence was $11.8 \%$ (range: $4.8 \%$ to $19.6 \%$ ) (Figure O). Among men entering the program in all 50 states, the District of Columbia, and Puerto Rico, the median state-specific chlamydia prevalence was $6.6 \%$ (range: $2.1 \%$ to $14.5 \%$ ) (Figure P).

Among women entering the program in 44 states and Puerto Rico, the median state-specific gonorrhea prevalence in 2017 was $2.6 \%$ (range: $0.0 \%$ to $6.3 \%$ ) (Figure Q). Among men entering the program in 44 states, the District of Columbia, and Puerto Rico, the median state-specific gonorrhea prevalence was $0.8 \%$ (range: $0.0 \%$ to $2.2 \%$ ) (Figure R).

* Laboratory tests are conducted by the Center for Disease Detection, LLC San Antonio, Texas.

\section{Summary}

The rate of reported cases of chlamydia, gonorrhea, and P\&S syphilis increased for both sexes in 15-24 year olds during 2016-2017. For chlamydia, rates of reported cases are consistently highest among women aged 15-24 years, likely reflecting targeted screening of young women; however, the rate of reported chlamydia in males aged $15-24$ years increased 29.1\% during 2013-2017, while the rate in females increased $8.8 \%$. Similarly, in 2017, the rate 


\section{Figure O. Chlamydia - Prevalence Among Women Aged 16-24 Years Entering the National Job Training Program (NJTP) by State of Residence, United States and Outlying Areas, 2017}

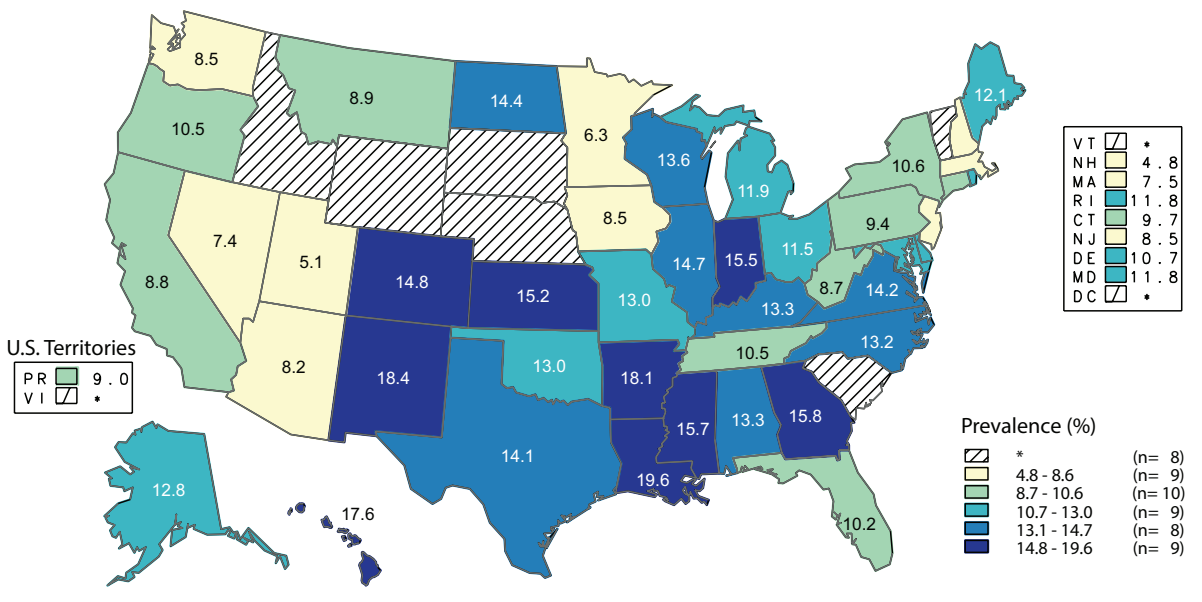

* Fewer than 100 women who resided in these states/areas and entered the NJTP were screened for chlamydia in 2017.

NOTE: See Section A2.1 in the Appendix for more information regarding NJTP methods. ACRONYMS: $\mathrm{PR}=$ Puerto Rico; $\mathrm{VI}=$ Virgin Islands

\section{Figure P. Chlamydia - Prevalence Among Men Aged 16-24 Years Entering the National Job Training Program (NJTP) by State of Residence, United States and Outlying Areas, 2017}

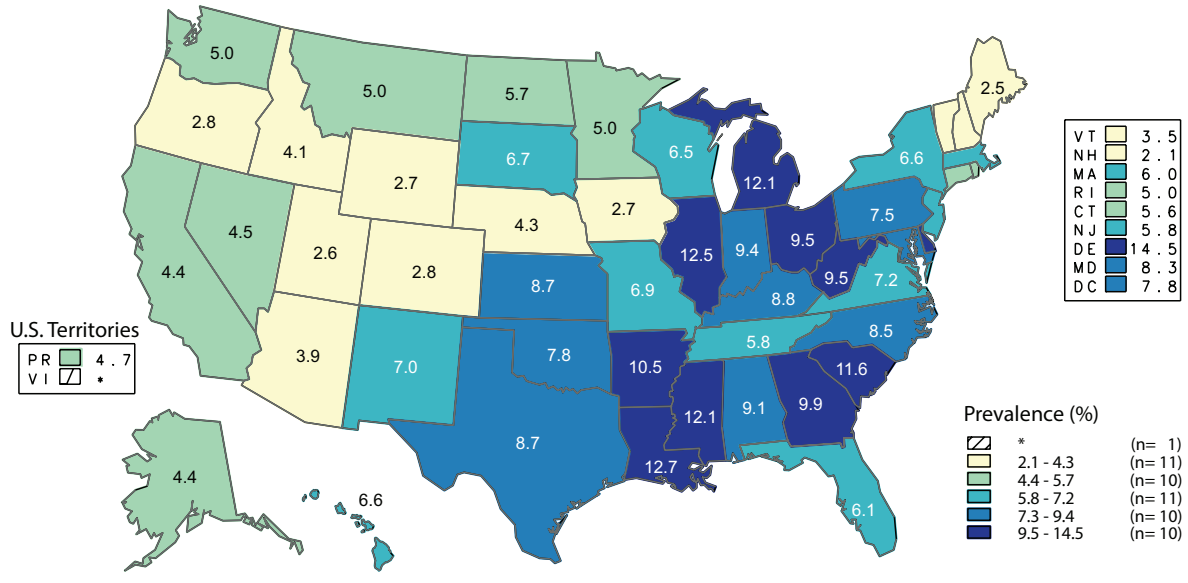

* Fewer than 100 men who resided in these states/areas and entered the NJTP were screened for chlamydia in 2017.

NOTE: See Section A2.1 in the Appendix for more information regarding NJTP methods. ACRONYMS: $\mathrm{PR}=$ Puerto Rico; $\mathrm{VI}=$ Virgin Islands

of reported cases of gonorrhea in females aged 15-24 years was higher than in men of the same age group; however, during 2013-2017, the rate of reported gonorrhea in males aged
15-24 years increased $51.6 \%$, while the rate in females increased $24.1 \%$. Increases in chlamydia and gonorrhea diagnoses among men likely reflect a combination of increased screening among young men, including extragenital screening, and increased incidence. Conversely, rates of reported cases of P\&S syphilis have been consistently higher among adolescent and young adult men compared to women; however, the largest increase in $\mathrm{P} \& \mathrm{~S}$ syphilis rates during 2013-2017 was among women of this age group. During 2013-2017, rates of reported $\mathrm{P} \& \mathrm{~S}$ syphilis cases increased $83.3 \%$ and $50.9 \%$ in 15-24 year old females and males, respectively.

\section{References}

1. Satterwhite CL, Torrone E, Meites E, et al Sexually transmitted infections among US women and men: Prevalence and incidence estimates, 2008. Sex Trans Dis 2013; 40(3): 187-193.

2. Forhan SE, Gottlieb SL, Sternberg MR, et al. Prevalence of sexually transmitted infections among female adolescents aged 14 to 19 in the United States. Pediatrics 2009; 124(6):15051512

3. Tilson EC, Sanchez V, Ford CL, et al. Barriers to asymptomatic screening and other STD services for adolescents and young adults: Focus group discussions. BMC Public Health 2004; 4(1):21.

4. DiClemente RJ, Salazar LF, Crosby RA. A review of STD/HIV preventive interventions for adolescents: Sustaining effects using an ecological approach. J Pediatr Psychol 2007; 32(8):888-906

5. Sieving RE, Bernat DH, Resnick MD, et al. A clinic-based youth development program to reduce sexual risk behaviors among adolescent girls: Prime time pilot study. Health Promot Pract 2012; 13(4):462-471.

6. Upchurch DM, Mason WM, Kusunoki Y, et al. Social and behavioral determinants of selfreported STD among adolescents. Perspect Sex Reprod Health 2004; 36(6):276-287.

7. Markowitz LE, Dunne EF, Saraiya M, et al. Quadrivalent human papillomavirus vaccine. Recommendations of the Advisory Committee on Immunization Practices (ACIP). MMWR Morb Mortal Wkly Rep Recomm Rep 2007; 56(RR-2):1-24.

8. Petrosky E, Bocchini Jr. JA, Hariri S, et al. Use of 9-valent human papillomavirus (HPV) vaccine: Updated HPV vaccination recommendations of the Advisory Committee on Immunization Practices. MMWR Morb Mortal Wkly Rep 2015; 64(11):300-304. 


\section{Figure Q. Gonorrhea - Prevalence Among Women Aged 16-24 Years Entering the National Job Training Program (NJTP) by State of Residence, United States and Outlying Areas, 2017}

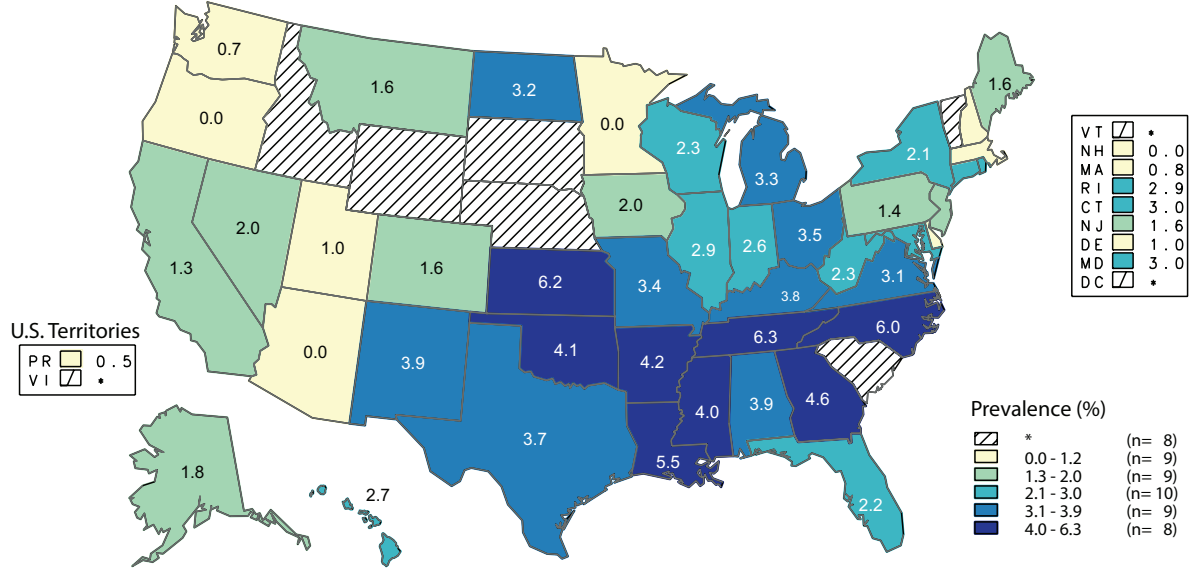

* Fewer than 100 women who resided in these states/areas and entered the NJTP were screened for gonorrhea in 2017.

NOTE: See Section A2.1 in the Appendix for more information regarding NJTP methods. ACRONYMS: PR = Puerto Rico; VI = Virgin Islands.

\section{Figure R. Gonorrhea - Prevalence Among Men Aged 16-24 Years Entering the National Job Training Program (NJTP) by State of Residence, United States and Outlying Areas, 2017}

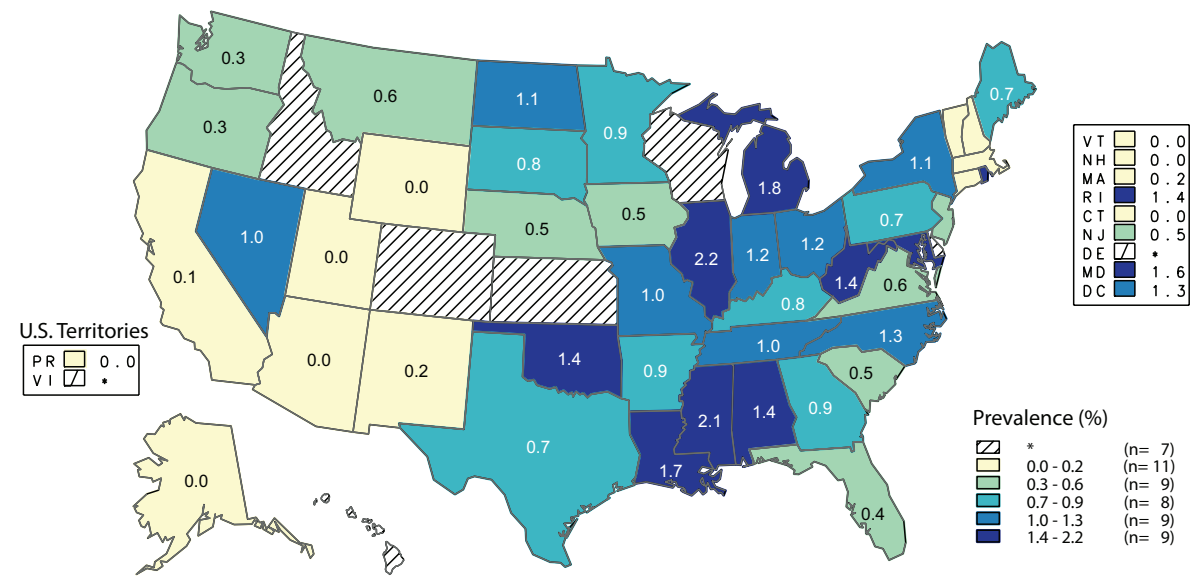

* Fewer than 100 men who resided in these states/areas and entered the NJTP were screened for gonorrhea in 2017.

NOTE: See Section A2.1 in the Appendix for more information regarding NJTP methods. ACRONYMS: $\mathrm{PR}=$ Puerto Rico; $\mathrm{VI}=$ Virgin Islands.
11. Oliver SE, Unger ER, Lewis R, et al. Prevalence of human papillomavirus among females after vaccine introduction - National Health and Nutrition Examination Survey, United States, 2003-2014. J Infect Dis 2017; 216(5):594-603.

12. Flagg EW, Torrone EA, Weinstock H. Ecological association of human papillomavirus vaccination with cervical dysplasia prevalence in the United States, 2007-2014. Am J Public Health 2016; 106(12):2211-2218.

13. Flagg EW, Torrone EA. Declines in anogenital warts among age groups most likely to be impacted by human papillomavirus vaccination, United States, 2006-2014. Am J Public Health 2018; 108(1):112-119.

14. Smith JS, Robinson NJ. Age-specific prevalence of infection with herpes simplex virus types 2 and 1: A global review. J Infect Dis 2002; 186(Suppl 1):S3-S28.

15. Corey L, Wald A. Genital Herpes. In: Holmes KK, Sparling FP, Stamm WE, et al., eds. Sexually Transmitted Diseases. 4th ed. New York, NY: McGraw-Hill; 2008:399-437.

16. Bradley H, Markowitz LE, Gibson T, et al. Seroprevalence of herpes simplex virus types 1 and 2 - United States, 1999-2010. J Infect Dis 2014; 209(3):325-333.

17. Bernstein DI, Bellamy AR, Hook EW III, et. al. Epidemiology, clinical presentation, and antibody response to primary infection with herpes simplex virus type 1 and type 2 in young women. Clin Infect Dis 2013; 56:344-351.

18. Roberts CM, Pfister JR, Spear SJ. Increasing proportion of herpes simplex virus type 1 as a cause of genital herpes infection in college students. Sex Transm Dis 2003; 30(10):797800 .

19. Kimberlin DW. The scarlet H. J Infect Dis 2014; 209(3):315-317.

20. Copen CE, Chandra A, Martinez G. Prevalence and timing of oral sex with opposite-sex partners among females and males aged 15-24 years: United States, 2007-2010. National Health Statistics Reports, No. 56. Hyattsville, MD: National Center for Health Statistics; 2012.
9. Centers for Disease Control and Prevention. Recommendations on the use of quadrivalent human papillomavirus vaccine in males Advisory Committee on Immunization Practices (ACIP), 2011. MMWR Morb Mortal Wkly Rep 2011; 60(50):1705-1708.
10. Meites E, Kempe A, Markowitz LE. Use of a 2-dose schedule for human papillomavirus vaccination - updated recommendations of the Advisory Committee on Immunization Practices. MMWR Morb Mortal Wkly Rep 2016; 65(49):1405-1408. 


\section{STDs in Racial and Ethnic Minorities}

\section{Background}

Disparities continue to persist in rates of STDs among some racial minority or Hispanic groups when compared with rates among Whites. ${ }^{1,2}$ This is also true across a wide variety of other health status indicators, providing evidence that race and Hispanic ethnicity in the United States are population characteristics strongly correlated with other factors affecting overall health status, such as income, employment, insurance coverage, and educational attainment..$^{3-5}$ In 2016, the most recent year for which national data on poverty and insurance status are available, the overall proportion of the United States population living in poverty was $12.7 \%$ (or 40.6 million), a slight decrease from 2015. Although the overall poverty rate has declined slightly over the last few years, many Americans continue to face challenges overcoming inequalities in economic opportunity; the poverty rate in 2016 for Whites was $8.8 \%$ (17.3 million), for Blacks it was $22.0 \%$ (or 9.2 million), and for Hispanics it was $19.4 \%$ (or 11.1 million). Significant differences by race and Hispanic ethnicity in the proportion of the population living in poverty persisted in 2016 and were even more acute for family households headed by women $(28.8 \%$ versus $10.7 \%$ for all households), regardless of other factors ${ }^{3}$. Those who cannot afford basic necessities often have trouble accessing and affording quality health care, including sexual health services. ${ }^{6}$

Access to, and routine use of, quality health care including STD prevention and treatment is key to reducing STD disparities in the United States. Of the estimated 19 million new cases of sexually transmitted infections (STIs) that occur each year, approximately half of all cases occur among people aged $15-24$ years. ${ }^{7}$ Although the overall proportion of adults without health insurance decreased from $13.3 \%$ in 2013 to $8.8 \%$ (or 28.2 million) in 2016, many people in the United States continue to struggle to afford full, routine access to health care. ${ }^{8}$ Among all races or ethnic groups in the United States, Hispanics had the lowest rate of health insurance coverage in 2016 at $84.0 \%$ (a slight increase from $83.8 \%$ in 2015). ${ }^{8}$

Even when health care is readily available to racial and ethnic minority populations, fear and distrust of health care institutions can negatively affect the health care-seeking experience. Social and cultural discrimination, language barriers, provider bias, or the perception that these may exist, likely discourage some people from seeking care. ${ }^{9,10}$ Moreover, the quality of care can differ substantially for minority patients. ${ }^{11}$ Broader inequities in social and economic conditions for minority communities are reflected in the profound disparities observed in the incidence of STDs by race and Hispanic ethnicity.

In communities where STD prevalence is higher because of these and other factors, people may experience difficulties reducing their risk for STIs. With each sexual encounter, they face a greater chance of encountering an infected partner than those in lower prevalence settings do, regardless of similar sexual behavior patterns. ${ }^{2}$ Acknowledging inequities in STD rates by race and Hispanic ethnicity is a critical first step toward empowering affected groups and the public health community to collaborate in addressing systemic inequities in the burden of disease with the ultimate goal of minimizing the health impacts of STDs on individuals and populations.

\section{STD Reporting Practices}

Surveillance data are based on cases of STDs reported to state and local health departments (see Section A.1 in the Appendix). In many state and local health departments, electronic laboratory reporting is increasingly becoming the primary source of initial case notifications. Laboratory reports are often missing race and Hispanic ethnicity of the patient; ascertainment of information on race and Hispanic ethnicity is therefore a function of active follow-up or dependent on previous information available about the patient in existing health department surveillance databases. Prevalence data from populationbased surveys, such as the National Health and Nutrition Examination Survey (NHANES) and the National Longitudinal Study of Adolescent Health, confirm the existence of marked disparities in some minority populations (see Other STDs below) for both reportable and non-nationally reportable STDs. ${ }^{12,13}$

\section{Method of Classifying Race and Hispanic Ethnicity}

Interpretation of racial and ethnic disparities among persons with STDs is influenced by data collection methods and by the categories by which these data are displayed. Race/Hispanic ethnicity data in this report are presented in Office of Management and Budget (OMB) race and Hispanic ethnicity categories according to the 1997 revised OMB standards. ${ }^{14}$ As of 2017 , most reporting jurisdictions are locally compliant with OMB standards and report in the standard categories, including the ability to collect more than one race per person. However, a small number of jurisdictions reported race in pre-1997 categories; while other jurisdictions were 
unable to report more than one race per person in 2017. All race and Hispanic ethnicity data reported by jurisdictions are summarized in tables, charts and interpretative text in this report regardless of local compliance with the 1997 OMB standards. No redistribution of cases is done; cases missing race and/or Hispanic ethnicity are not included in the calculation of rates by race and Hispanic ethnicity. See Section A1.5 of the Appendix for additional information on reporting of race and Hispanic ethnicity.

\section{Completeness of Race and Hispanic Ethnicity Data in 2017}

Chlamydia $-27.8 \%$ of chlamydia case reports were missing race or Hispanic ethnicity data, ranging by jurisdiction from $0.2 \%$ to $90.9 \%$ (Table A1).

Gonorrhea - $19.0 \%$ of gonorrhea case reports were missing information on race or Hispanic ethnicity, ranging by jurisdiction from $0.1 \%$ to $88.0 \%$ (Table A1).

Syphilis - $4.2 \%$ of all primary and secondary $(\mathrm{P} \& \mathrm{~S})$ syphilis case reports were missing information on race or Hispanic ethnicity, ranging by jurisdiction from no missing information to $30.3 \%$ (Table A1).

\section{Chlamydia}

During 2013-2017, rates of reported chlamydia cases increased among all racial and Hispanic ethnicity groups. Specifically, rates increased $3.7 \%$ among American Indians/ Alaska Natives (AI/AN), 29.6\% among Asians, 6.1\% among Blacks, 19.4\% among Native Hawaiians/ Other Pacific Islanders (NHOPI), 20.2\% among Whites, 59.9\% among Multirace, and 10.5\% among Hispanics (Figure 8).

Blacks - In 2017, the overall rate of reported chlamydia cases among Blacks in the United States was
$1,175.8$ cases per 100,000 population (Table 11B). The rate of reported chlamydia cases among Black women was 5.0 times the rate among White women $(1,419.9$ and 283.3 cases per 100,000 females, respectively) (Figure S and Table 11B). The rate of reported chlamydia cases among Black men was 6.6 times the rate among White men (907.3 and 137.1 cases per 100,000 males, respectively). Rates of reported cases of chlamydia were highest for Blacks aged 15-19 and 20-24 years in 2017 (Table 11B). The rate of reported chlamydia cases among Black women aged $15-19$ years $(6,771.6$ cases per 100,000 females) was 4.5 times the rate among White women in the same age group (1,518.5 cases per 100,000 females). The rate of reported chlamydia cases among Black women aged 20-24 years was 3.6 times the rate among White women in the same age group $(6,971.7$ and $1,936.0$ cases per 100,000 females, respectively) (Table 11B). Among females aged 15-24 years, the population targeted for screening, rates were highest among Blacks in all US regions (Figure T).
Similar racial disparities in reported chlamydia rates exist among men. Among men aged 15-19 years, the rate of reported chlamydia cases among Blacks was 8.9 times the rate among Whites $(2,589.3$ and 291.5 cases per 100,000 males, respectively) (Table 11B). The rate of reported chlamydia cases among Black men aged 20-24 years was 5.0 times the rate among White men of the same age group $(3,627.4$ and 726.8 cases per 100,000 males, respectively).

American Indians/Alaska Natives - In 2017, the rate of reported chlamydia cases among AI/AN was 781.2 cases per 100,000 population (Table 11B). Overall, the rate of reported chlamydia cases among $\mathrm{AI} / \mathrm{AN}$ in the United States was 3.7 times the rate among Whites.

\section{Native Hawaiians/Other Pacific} Islanders - In 2017, the rate of reported chlamydia cases among NHOPI was 715.4 cases per 100,000 population (Table 11B). The overall rate of reported chlamydia cases among NHOPI was 3.4 times the rate among Whites and 5.5 times the rate among Asians.

\section{Figure S. Chlamydia - Rates of Reported Cases by Race, Hispanic Ethnicity, and Sex, United States, 2017}

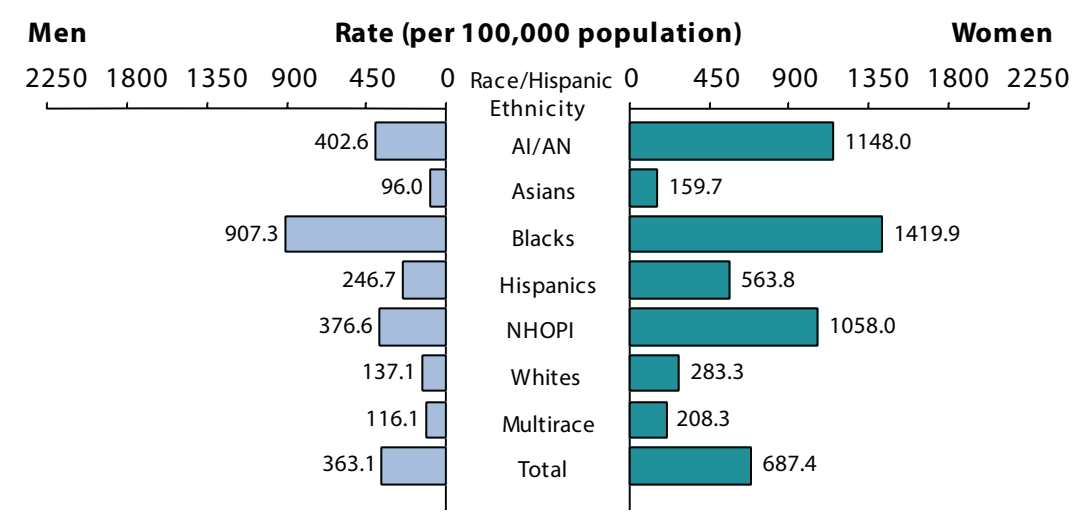

NOTE: Not all US jurisdictions reported cases in OMB-compliant race categories in 2017. This may minimally under- or overestimate rates for Asians, $\mathrm{NHOPI}$, or Multirace individuals. For completeness, data in this figure include cases reported from all jurisdictions. See Section A1.5 in the Appendix for information on race and Hispanic ethnicity in STD case reporting. ACRONYMS: AI/AN = American Indians/Alaska Natives; $\mathrm{NHOPI}=$ Native Hawaiians/Other Pacific Islanders; $\mathrm{OMB}=$ Office of Management and Budget. 


\section{Figure T. Chlamydia - Rate Ratios* Among Women Aged 15-24 Years by Race, Hispanic Ethnicity, and Region, United States, 2017}

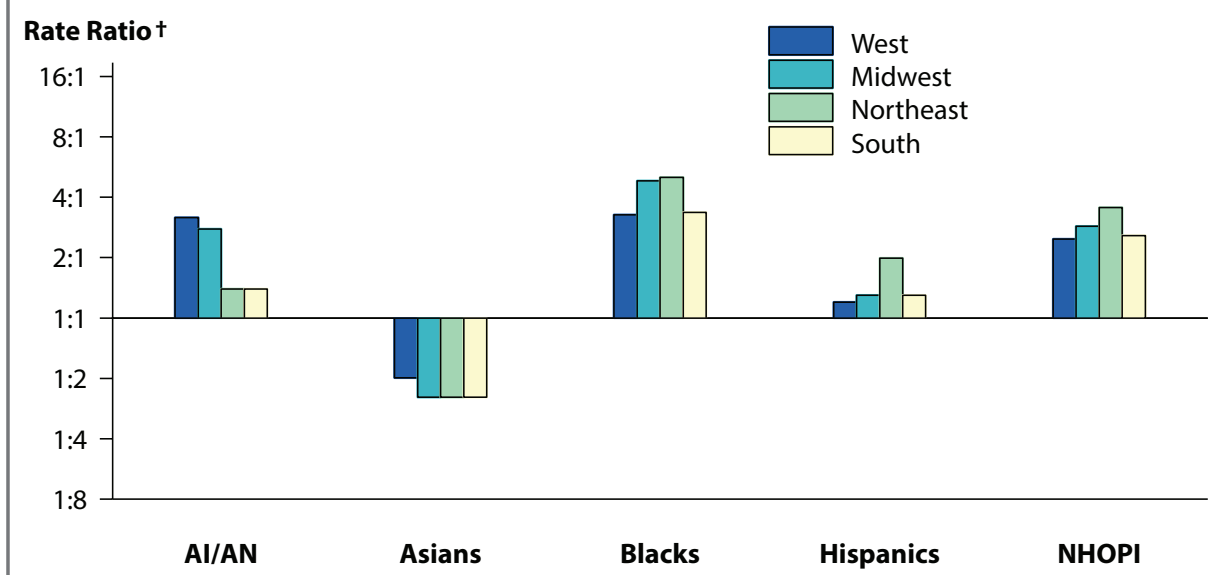

* Rate ratios are calculated as the rate of reported chlamydia cases per 100,000 population for a given racial or ethnic minority population divided by the rate of reported chlamydia cases per 100,000 population for Whites. Any population with a lower rate of reported cases of chlamydia than the White population will have a rate ratio of less than 1:1.

${ }^{+}$Y-axis is log scale.

NOTE: Not all US jurisdictions reported cases in OMB-compliant Race categories in 2017. This may minimally under- or overestimate rates for Asians, NHOPI, or Multirace individuals. For completeness, data in this figure include cases reported from all jurisdictions. See Section A1.5 in the Appendix for information on reporting STD case data for race and Hispanic ethnicity. ACRONYMS: AI/AN = American Indians/Alaska Natives; NHOPI = Native Hawaiians/Other Pacific Islanders; $\mathrm{OMB}=$ Office of Management and Budget.

Hispanics - In 2017, the rate of reported chlamydia cases among Hispanics was 404.1 cases per 100,000 population, which was 1.9 times the rate among Whites (Table 11B).

Asians - In 2017, the rate of reported chlamydia cases among Asians was 129.6 cases per 100,000 population (Table 11B). The overall rate of reported chlamydia cases among Whites was 1.6 times the rate among Asians.

\section{Gonorrhea}

During 2013-2017, rates of reported gonorrhea cases increased $176.6 \%$ among Multirace persons (27.8 to 76.9 cases per 100,000 population), $122.4 \%$ among Asians (15.6 to 34.7 cases per 100,000 population), $109.1 \%$ among NHOPI (89.8 to 187.8 cases per 100,000 population), $100.6 \%$ among Whites (33.1 to 66.4 cases per 100,00 population), $95.3 \%$
Considering all race, Hispanic ethnicity, and age categories, rates of reported gonorrhea cases were highest for Blacks aged 20-24, 15-19, and 25-29 years in 2017 (Table 22B). The rate of reported gonorrhea cases among Black women aged 20-24 years $(2,066.8$ cases per 100,000 females) was 7.4 times the rate among White women in the same age group ( 280.0 cases per 100,000 females). The rate of reported gonorrhea cases among Black women aged 15-19 years $(1,843.8$ cases per 100,000 females) was 9.3 times the rate among White women in the same age group (197.5 cases per 100,000 females). Among Black men aged 20-24 years, the rate of reported gonorrhea cases $(2,154.8$ cases per 100,000 males $)$ was 9.3 times the rate among White men in the same age group (231.3 cases per 100,000 males). The rate of reported gonorrhea cases among Black men aged 25-29 years $(1,863.1$ cases per 100,000 males) was 7.3 times the rate among White men in the same age group ( 253.5 cases per 100,000 males).

\section{American Indians/Alaska Natives}

- In 2017, the rate of reported gonorrhea cases among AI/AN (301.9 cases per 100,000 population) was 4.5 times the rate among Whites (Table 22B). The disparity between gonorrhea rates for $\mathrm{AI} / \mathrm{AN}$ and Whites was larger for $\mathrm{AI} / \mathrm{AN}$ women (6.2 times the rate among White women) than for AI/AN men (3.2 times the rate among White men) (Figure U, Table 22B). The disparity in gonorrhea rates for $\mathrm{AI} / \mathrm{AN}$ in 2017 was larger in the Midwest than in the West, Northeast, and South (Figure V).

\section{Native Hawaiians/Other Pacific} Islanders - In 2017, the rate of reported gonorrhea cases among NHOPI (187.8 cases per 100,000 population) was 2.8 times the rate among Whites (Table 22B). This disparity was similar for NHOPI women (3.0 times the rate among White women) and NHOPI men (2.7 
Figure U. Gonorrhea - Rates of Reported Cases by Race, Hispanic Ethnicity and Sex, United States, 2017

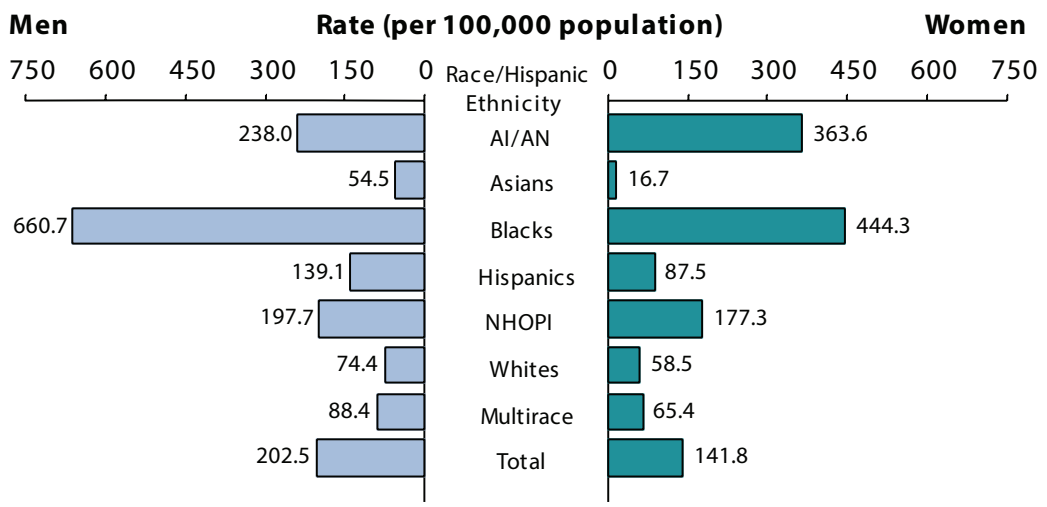

NOTE: Not all US jurisdictions reported cases in OMB-compliant race categories in 2017. This may minimally under- or overestimate rates for Asians, $\mathrm{NHOPI}$, or Multirace individuals. For completeness, data in this figure include cases reported from all jurisdictions. See Section A1.5 in the Appendix for information on race and Hispanic ethnicity in STD case reporting. ACRONYMS: $\mathrm{Al} / \mathrm{AN}=$ American Indians/Alaska Natives; $\mathrm{NHOPI}=$ Native Hawaiians/Other Pacific Islanders; $\mathrm{OMB}=$ Office of Management and Budget.

Figure V. Gonorrhea - Rate Ratios* by Race, Hispanic Ethnicity, and Region, United States, 2017

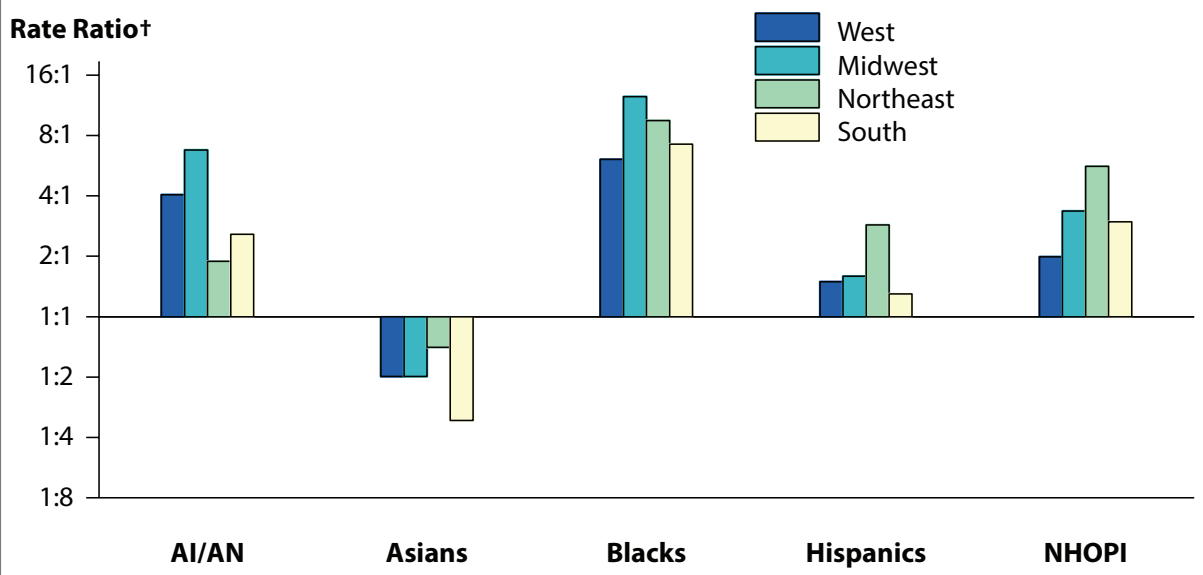

* Rate ratios are calculated as the rate of reported gonorrhea cases per 100,000 population for a given racial or ethnic minority population divided by the rate of reported gonorrhea cases per 100,000 population for Whites. Any population with a lower rate of reported cases of gonorrhea than the White population will have a rate ratio of less than 1:1.

${ }^{+}$Y-axis is log scale.

NOTE: Not all US jurisdictions reported cases in OMB-compliant race categories in 2017. This may minimally under- or overestimate rates for Asians, NHOPI, or Multirace individuals. For completeness, data in this figure include cases reported from all jurisdictions. See Section A1.5 in the Appendix for information on race and Hispanic ethnicity in STD case reporting.

ACRONYMS: Al/AN = American Indians/Alaska Natives; NHOPI = Native Hawaiians/Other Pacific Islanders; $\mathrm{OMB}=$ Office of Management and Budget. times the rate among White men) (Figure U, Table 22B). The disparity in gonorrhea rates for NHOPI in 2017 was lower in the West than in the Midwest, Northeast, or South (Figure V).

Hispanics - In 2017, the rate of reported gonorrhea cases among Hispanics was 113.7 cases per 100,000 population, which was 1.7 times the rate among Whites (Table 22B). This disparity was similar for Hispanic women (1.5 times the rate among White women) and Hispanic men (1.9 times the rate among White men) (Figure U, Table 22B). The disparity in gonorrhea rates for Hispanics in 2017 was higher in the Northeast than in the Midwest, South, or West (Figure V).

Asians - In 2017, the rate of reported gonorrhea cases among Asians (34.7 cases per 100,000 population) was 0.5 times the rate among Whites (Table 22B). This difference was larger for Asian women than for Asian men (Figure U, Table 22B). In 2017, gonorrhea rates among Asians were lower than rates among Whites in all four regions of the United States (Figure V).

\section{Primary and Secondary Syphilis}

During 2013-2017, rates of reported P\&S syphilis cases increased $192.6 \%$ among those who identified as Multirace (2.7 to 7.9 cases per 100,000 population), $141.3 \%$ among $\mathrm{AI} / \mathrm{AN}$ (4.6 to 11.1 cases per 100,000 population), $91.3 \%$ among Asians (2.3 to 4.4 cases per 100,000 population), $84.4 \%$ among Hispanics (6.4 to 11.8 cases per 100,000 population), $80.0 \%$ among Whites (3.0 to 5.4 cases per 100,000 population), $58.0 \%$ among NHOPI (8.8 to 13.9 cases per 100,000 population), and $44.0 \%$ among Blacks (16.8 to 24.2 cases per 100,000 population) (Figure 45). Across race and Hispanic ethnicity groups, MSM accounted for the highest proportion of P\&S syphilis cases (Figure W). 


\section{Figure W. Primary and Secondary Syphilis - Reported Cases* by Sex, Sexual Behavior, Race, and Hispanic Ethnicity, United States, 2017}

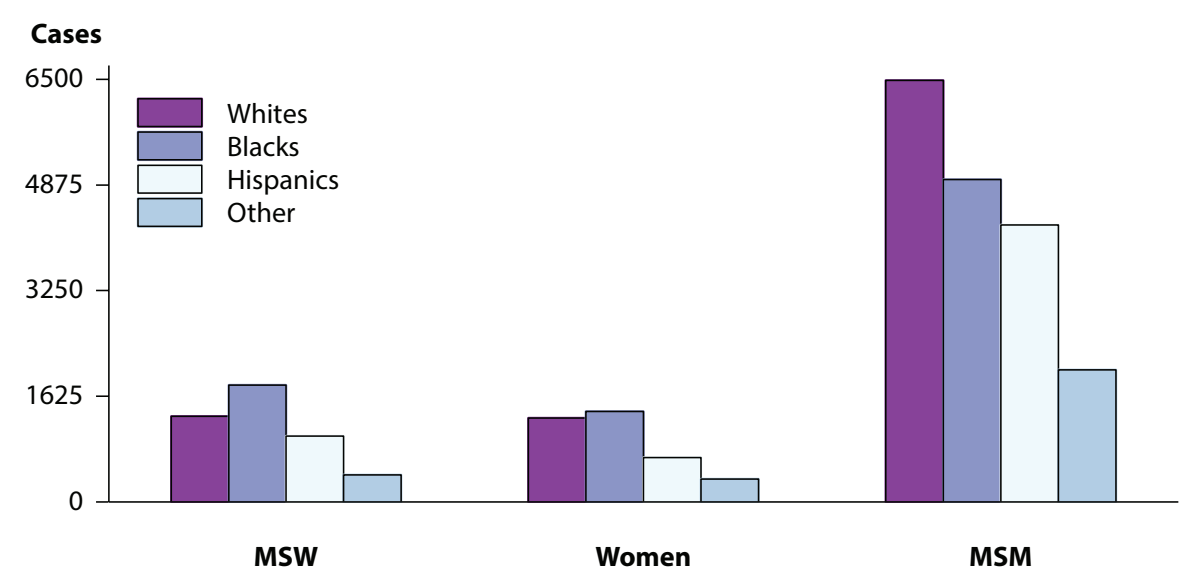

* Of all reported cases of primary and secondary syphilis, $15.0 \%$ were among men without data on sex of sex partner, and $0.1 \%$ were cases with unknown sex; $5.7 \%$ of all cases had missing or unknown race/Hispanic ethnicity. Cases with missing or unknown race/Hispanic ethnicity are included in the "Other" category.

NOTE: Not all US jurisdictions reported cases in OMB-compliant Race categories in 2017. This may minimally under- or overestimate rates for Asians, NHOPI, or Multirace individuals. For completeness, data in this figure include cases reported from all jurisdictions. See Section A1.5 in the Appendix for information on reporting STD case data for race and Hispanic ethnicity. ACRONYMS: AI/AN = American Indians/Alaska Natives; NHOPI = Native Hawaiians/Other Pacific Islanders; $\mathrm{OMB}=$ Office of Management and Budget.

Blacks - In 2017, 33.7\% of reported P\&S syphilis cases with known race and Hispanic ethnicity (excluding cases with missing information on race and Hispanic ethnicity, and cases whose reported race was 'Other' and Hispanic ethnicity was 'No' or 'Unknown') occurred among Blacks (Table 35A). The rate of reported $\mathrm{P} \& \mathrm{~S}$ syphilis cases among Blacks in 2017 (24.2 cases per 100,000 population) was 4.5 times the rate among Whites (5.4 cases per 100,000 population) (Table 35B). The disparity was greater for Black women (5.2 times the rate among White women) than for Black men (4.5 times the rate among White men) (Figure X, Table 35B). Similar disparities were seen in all regions of the United States (Figure Y).

Considering all race, Hispanic ethnicity, sex, and age categories, rates of reported $\mathrm{P} \& \mathrm{~S}$ syphilis cases were highest among Black men aged 25-29 years in 2017 (Table 35B). The rate of reported $\mathrm{P} \& \mathrm{~S}$ syphilis cases among Black men aged 25-29 years (142.4 cases per 100,000 males) was 5.8 times the rate among White men in the same age group (24.7 cases per 100,000 males).

\section{Native Hawaiians/Other Pacific} Islanders - In 2017, the rate of reported $\mathrm{P} \& \mathrm{~S}$ syphilis cases among NHOPI was 13.9 cases per 100,000 population, which was 2.6 times the rate among Whites (Table 35B). This disparity was similar for NHOPI women (2.5 times the rate among White women) and NHOPI men (2.6 times the rate among White men).

Hispanics - In 2017, the rate of reported P\&S syphilis cases among Hispanics (11.8 cases per 100,000 population) was 2.2 times the rate among Whites (Table 35B). This disparity was similar for Hispanic men (2.2 times the rate among White men) and Hispanic women (1.8 times the rate among White women).

\section{American Indians/Alaska Natives}

- In 2017, the rate of reported P\&S syphilis cases among AI/AN (11.1 cases per 100,000 population) was 2.1 times the rate among Whites (Table 35B). This disparity was greater for $\mathrm{AI} / \mathrm{AN}$ women (4.6 times the rate among White women) than for AI/AN men (1.7 times the rate among White men).
Figure X. Primary and Secondary Syphilis - Rates of Reported Cases by Race, Hispanic Ethnicity, and Sex, United States, 2017

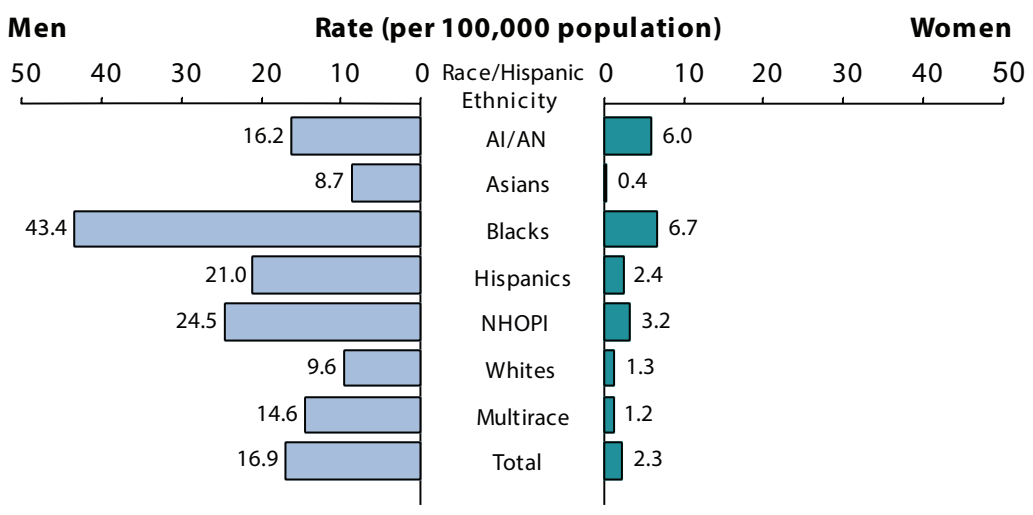

NOTE: Not all US jurisdictions reported cases in OMB-compliant Race categories in 2017. This may minimally under- or overestimate rates for Asians, NHOPI, or Multirace individuals. For completeness, data in this figure include cases reported from all jurisdictions. See Section A1.5 in the Appendix for information on reporting STD case data for race and Hispanic ethnicity. ACRONYMS: AI/AN = American Indians/Alaska Natives; NHOPI = Native Hawaiians/Other Pacific Islanders; $\mathrm{OMB}=$ Office of Management and Budget. 
Asians - In 2017, the rate of reported $\mathrm{P} \& \mathrm{~S}$ syphilis cases among Asians was 4.4 cases per 100,000 population, which was 0.8 times the rate among Whites (Table 35B). This difference was larger for Asian women (0.3 times the rate among White women) than for Asian men (0.9 times the rate among White men).

\section{Congenital Syphilis}

Race and Hispanic ethnicity for cases of congenital syphilis are based on the mother's information. During 2013-2017, rates of reported congenital syphilis cases increased in all population groups. Rates increased $234.5 \%$ among Whites, $225.2 \%$ among Hispanics, $177.3 \%$ among AI/AN, 87.6\% among Blacks, and $22.9 \%$ among Asians/Pacific Islanders (Figure Z, Table 42).

In $2017,39.1 \%$ of congenital syphilis cases with known race and Hispanic ethnicity (excluding cases with missing information on race and Hispanic ethnicity, and cases whose reported race was 'Other' and Hispanic ethnicity was 'No' or 'Unknown') occurred among Blacks (Table 42). The rate of reported cases of congenital syphilis among Blacks in 2017 (58.9 cases per 100,000 live births) was 6.1 times the rate among Whites (9.7 cases per 100,000 live births). The rate of reported cases of congenital syphilis was 35.5 cases per 100,000 live births among AI/AN (3.7 times the rate among Whites), 33.5 cases per 100,000 live births among Hispanics (3.5 times the rate among Whites), and 4.3 cases per 100,000 live births among Asians/Pacific Islanders (0.4 times the rate among Whites).

\section{Other STDs}

Data from the National Health and Nutrition Examination Survey (NHANES; see Section A2.4 in the Appendix) indicate the seroprevalence of herpes simplex virus type 2 (HSV2) in the United States has decreased from 1999-2000 to 2015-2016

\section{Figure Y. Primary and Secondary Syphilis - Rate Ratios* by Sex, Race, Hispanic Ethnicity, and Region, United States, 2017}

A. Male

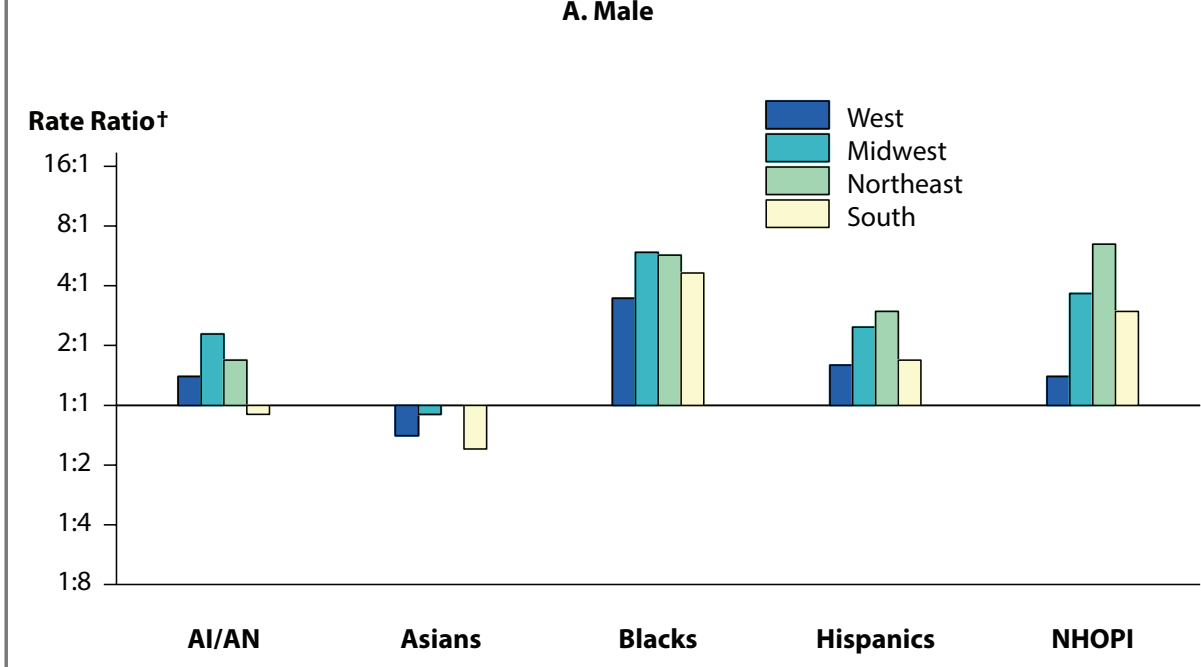

B. Female

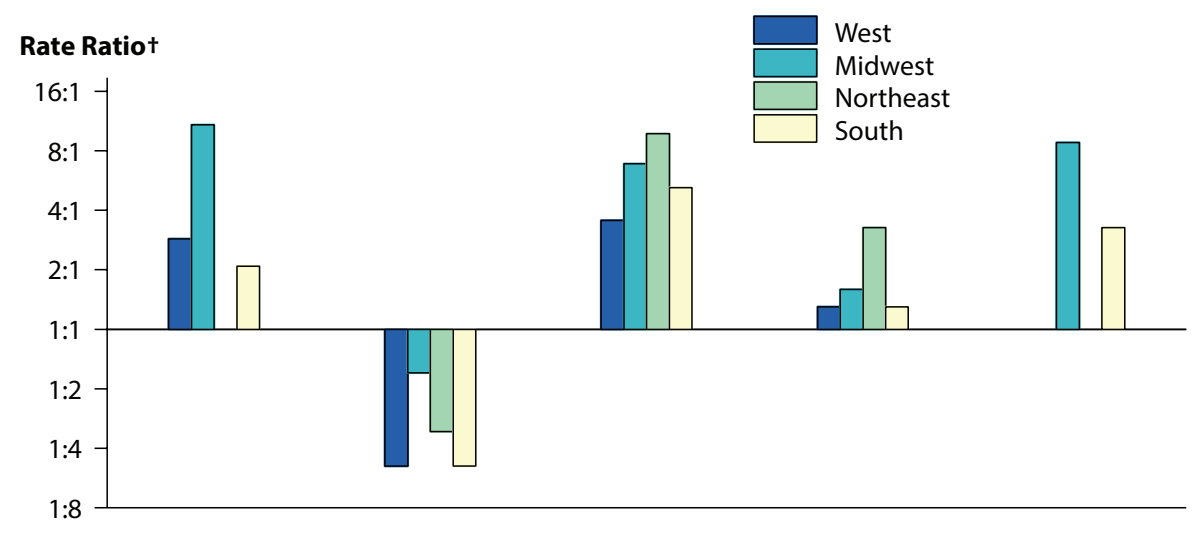

* Rate ratios are calculated as the rate of reported primary and secondary syphilis cases per 100,000 population for a given racial or ethnic minority population divided by the rate of reported primary and secondary syphilis cases per 100,000 population for Whites. Any population with a lower rate of reported cases of primary and secondary syphilis than the White population will have a rate ratio of less than 1:1.

${ }^{+} \mathrm{Y}$-axis is log scale.

NOTE: Not all US jurisdictions reported cases in OMB-compliant Race categories in 2017. This may minimally under- or overestimate rates for Asians, NHOPI, or Multirace individuals. For completeness, data in this figure include cases reported from all jurisdictions. See Section A1.5 in the Appendix for information on reporting STD case data for race and Hispanic ethnicity. ACRONYMS: AI/AN = American Indians/Alaska Natives; NHOPI = Native Hawaiians/Other Pacific Islanders; $\mathrm{OMB}=$ Office of Management and Budget.

for all race and Hispanic ethnicity STDs for more information on HSV groups (Figure 53); ${ }^{15}$ however, HSV-2 infections. seroprevalence was highest among non-Hispanic Blacks throughout the entire time period. See Other
Trichomonas vaginalis prevalence in urine specimens obtained from adult 


\section{Figure Z. Congenital Syphilis - Rates of Reported Cases by Year of Birth, Race, and Hispanic Ethnicity of Mother, United States, 2008- 2017}

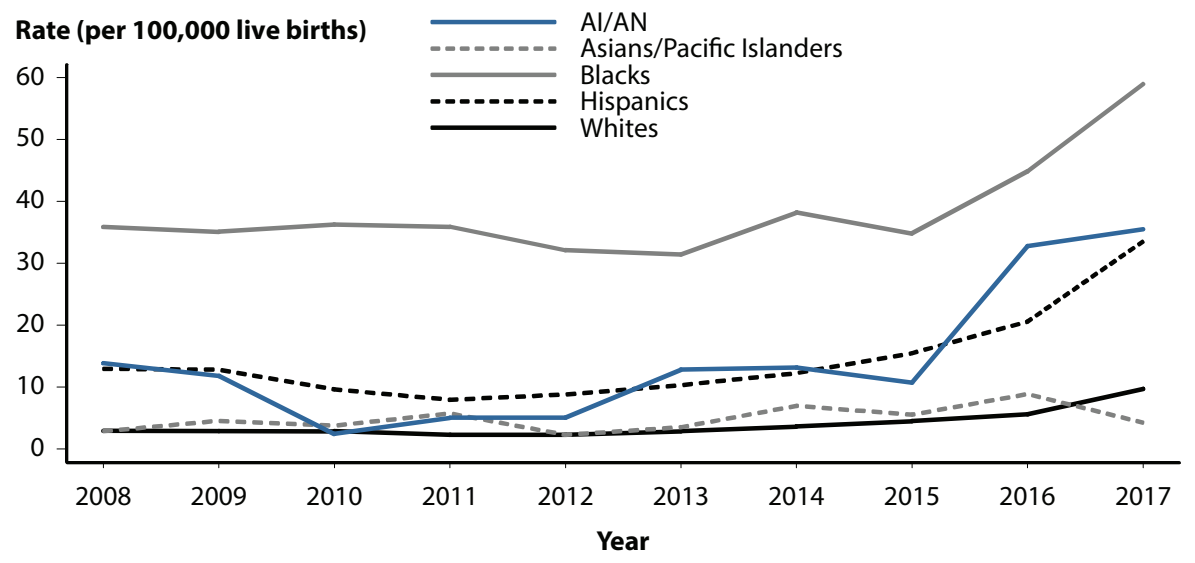

NOTE: National Center for Health Statistics bridged race categories are presented to allow the display of data across several years. Not all US jurisdictions reported cases in OMB-compliant Race categories in 2017. This may minimally under- or overestimate rates for Asians, NHOPI, or Multirace individuals. For completeness, data in this figure include cases reported from all jurisdictions. See Section A1.5 in the Appendix for information on reporting STD case data for race and Hispanic ethnicity.

ACRONYMS: $\mathrm{Al} / \mathrm{AN}=$ American Indians/Alaska Natives; $\mathrm{OMB}=$ Office of Management and Budget.
NHANES participants aged 18-59 years during 2013-2014 indicated a prevalence of $0.5 \%$ among males and $1.8 \%$ among females; highest rates were observed among nonHispanic Black males (4.2\%) and females $(8.9 \%) .{ }^{16}$ A separate analysis of NHANES data during 2013-2016 among men aged $18-59$ years also found higher prevalence among nonHispanic Blacks. ${ }^{17}$ An analysis of NHANES data from 2001-2004 from cervicovaginal swab specimens also found higher $T$. vaginalis prevalence among non-Hispanic Black females. ${ }^{18}$ See Other STDs for more information on $T$. vaginalis infections.

\section{Summary}

Inequities in the burden of disease for chlamydia, gonorrhea, syphilis and other STDs by race and Hispanic ethnicity continue to persist at unacceptable levels in the United States. These disparities are not explained by individual or populationlevel behavioral differences; rather they result in large measure from stubbornly entrenched systemic, societal, and cultural barriers to STD diagnoses, treatment and preventive services accessible on a routine basis. Some progress has been achieved in recent years in reducing the magnitude of disparities in some STDs, especially for Blacks, but much more needs to be done to address these issues through individual, group, and structural-level health care interventions. Continued monitoring of differences across groups in reported case incidence is also critical to the success of these efforts, including a sharpened focus on ascertainment of race and Hispanic ethnicity for persons diagnosed and reported with STDs.

\section{References}

1. Newman LM, Berman SM. Epidemiology of STD Disparities in African American Communities. Sex Transm Dis 2008; 35(12):S4-S12.

2. Hogben M, Leichliter JS. Social determinants and sexually transmitted disease disparities. Sex Transm Dis 2008; 35(12 Suppl):S13-18.

3. Semega JL, Fontenot KR, Kollar MA. U.S. Census Bureau, Current Population Reports, P60-259, Income and Poverty in the United States: 2016, U.S. Government Printing Office, Washington, DC, 2017. Available at: https:// www.census.gov/content/dam/Census/library/ publications/2017/demo/P60-259.pdf. Accessed July19, 2018.

4. Harling G, Subramanian SV, Barnighausen T, et al. Socioeconomic disparities in sexually transmitted infections among young adults in the United States: Examining the interaction between Income and race/ethnicity. Sex Transm Dis 2013; 40(7):575-581.

5. Centers for Disease Control and Prevention. CDC Health Disparities and Inequalities Report — United States 2013. MMWR Morb Mortal Wkly Rep 2013; 62(Suppl 3).

6. Institute of Medicine. The Hidden Epidemic: Confronting Sexually Transmitted Diseases. Washington, DC: National Academy Press; 1997

7. Satterwhite CL, Torrone E, Meites E, et al. Sexually transmitted infections among US women and men: Prevalence and incidence estimates, 2008. Sex Transm Dis 2013; 40(3):187-193.

8. Barnett JC, Berchick ER. Current Population Reports, P60-260, Health Insurance Coverage in the United States: 2016, U.S. Government Printing Office, Washington, DC,2017. Available at: https://www.census.gov/content/ dam/Census/library/publications/2017/demo/ p60-260.pdf. Accessed July 19, 2018.

9. Pérez-Escamilla R. Health care access among Latinos: Implications for social and health care reform. J Hispanic High Educ 2010: 9(1):43-60

10. Berk ML, Schur CL. The effect of fear on access to care among undocumented Latino immigrants. J Immigr Health 2001; 3(3):151156.

11. Institute of Medicine. Unequal Treatment: Confronting Racial and Ethnic Disparities in Health Care. Washington, DC: National Academies Press; 2002.

12. Datta SD, Sternberg M, Johnson RE, et al. Gonorrhea and chlamydia in the United States among persons 14 to 39 years of age, 1999 to 2002. Ann Intern Med 2007; 147(2):89-96.

13. Miller WC, Ford CA, Morris M, et al. Prevalence of chlamydial and gonococcal infections among young adults in the United States. JAMA 2004; 291(18):2229-2236. 
14. Office of Management and Budget. Provisional guidance on the implementation of the 1997 standards for federal data on race and ethnicity. Available at: https://www.whitehouse.gov/wpcontent/uploads/2017/11/Provisional-Guidancejan16-2001-1.pdf. Accessed July 20, 2018.

15. McQuillan G, Kruszon-Moran D, Flagg EW, et al. Prevalence of herpes simplex virus type 1 and type 2 in persons aged 14-49: United States, 2015-2016. NCHS Data Brief, No. 304. Hyattsville, MD: National Center for Health Statistics. 2018.

16. Patel EU, Gaydos CA, Packman ZR, et al. Prevalence and correlates of Trichomonas vaginalis infection among men and women in the United States. Clin Infect Dis 2018; 67(2):211-217.

17. Daugherty M, Glynn K, Byler T. The prevalence of Trichomonas vaginalis infection among US males, 2013-2016. Clin Infect Dis 2018; https://doi.org/10.1093/cid/ciy499.

18. Sutton M, Sternberg M, Koumans EH, et al. The prevalence of Trichomonas vaginalis infection among reproductive-age women in the United States, 2001-2004. Clin Infect Dis 2007; 45(10):1319-1326. 


\section{STDs in Men Who Have Sex with Men}

\section{Background}

The incidence of many STDs in gay, bisexual, and other men who have sex with men (collectively referred to as MSM) - including primary and secondary $(\mathrm{P} \& \mathrm{~S})$ syphilis and antimicrobial-resistant gonorrhea - is greater than that reported in women and men who have sex with women only (MSW). ${ }^{1-6}$ In addition to the negative effects of untreated STDs, elevated STD burden is of concern because it may indicate high risk for subsequent HIV infection. Annual increases in reported STD cases could reflect increased frequency of behaviors that transmit both STDs and HIV (e.g., condomless anal sex), and having an STD increases the risk of acquisition or transmission of HIV. ${ }^{7-14}$

The relatively high incidence of STD infection among MSM may be related to multiple factors, including individual behaviors and sexual network characteristics. ${ }^{15-17}$ The number of lifetime or recent sex partners, rate of partner exchange, and frequency of condomless sex each influence an individual's probability of exposure to STDs. ${ }^{15}$ However, MSM network characteristics such as high prevalence of STDs, interconnectedness and concurrency of sex partners, and possibly limited access to healthcare also affect the risk of acquiring an STD. ${ }^{15,18}$ Furthermore, experiences of stigma verbal harassment, discrimination, or physical assault based on attraction to men - are associated with increased sexual risk behavior among MSM. ${ }^{19}$

Disparities among MSM reflect those observed in the general population, with disproportionate incidence of STDs reported among racial minority and Hispanic MSM, MSM of lower socioeconomic status, and young MSM. ${ }^{20-24}$ The higher burden of STDs among MSM with these characteristics, relative to the general population of MSM, may suggest distinct mixing patterns in their sexual networks, reduced access to screening and treatment, and differential experiences of stigma and discrimination, rather than greater numbers of sexual partners or frequency of condomless sex. ${ }^{15,21-22 \text {, }}$ ${ }^{24-26}$ Furthermore, disparities may be more pronounced for racial minority and Hispanic MSM who are also unemployed, young, and/or of lower socioeconomic status. ${ }^{26-27}$

With the exception of reported syphilis cases, nationally notifiable STD surveillance data do not routinely include information on sexual behaviors, and these data are missing for the majority of gonorrhea and chlamydia cases reported to CDC. Therefore, trends in STDs among MSM in the United States are based on findings from sentinel and enhanced surveillance systems. Testing strategies are also evolving to include more extragenital STD screening, which may increase detection of asymptomatic infections. Until recently, testing for gonorrhea and chlamydia in MSM largely focused on detecting urethral infections, which are more likely to be symptomatic than pharyngeal or rectal infections. ${ }^{28}$

For data reported in this chapter, MSM were defined as men who either reported having one or more male sex partners or who self-reported as gay/ homosexual or bisexual. MSW were defined as men who reported having sex with women only or who did not report the sex of their sex partner, but reported that they considered themselves straight/heterosexual. Data presented in this chapter are derived from the National Notifiable Diseases Surveillance System (NNDSS), the Gonococcal Isolate Surveillance Project (GISP), and the
STD Surveillance Network (SSuN), a sentinel and enhanced surveillance project established in 2005 to provide supplemental information on STDs.

\section{Nationally Notifiable Diseases Surveillance System}

MSM accounted for $68.2 \%$ of reported $\mathrm{P} \& \mathrm{~S}$ syphilis cases among women or men with information about sex of sex partners in 2017 (Figure 39). Among men exclusively, MSM accounted for $79.6 \%$ of reported cases with information on sex of sex partners. Of MSM P\&S syphilis cases, $36.5 \%$ were White, $28.0 \%$ were Black, and $24.0 \%$ were Hispanic (Figure W). Relative to the percentage of the US population that is White $(61.2 \%)$, Black $(12.5 \%)$, and Hispanic (17.9\%), ${ }^{30}$ this represents a significant inequality in the burden of disease for non-White MSM, which was also evident among MSW and women. In addition, among MSM P\&S syphilis cases with known HIV status in 2017, 45.5\% were also reported to be HIV-positive (Figure 46).

In 2017, 43 states provided data to classify at least $70 \%$ of cases as MSM, MSW, or women. Among these areas, estimated rates of P\&S syphilis cases in MSM ranged from 55.7 cases per 100,000 MSM in Wyoming to 798.3 cases per 100,000 MSM in Nevada, with 27 states $(63 \%)$ estimated to have rates between 200 and 500 cases per 100,000 MSM (Figure AA).

When examining reported $\mathrm{P} \& \mathrm{~S}$ syphilis cases over time, 37 states were able to classify at least $70 \%$ of reported P\&S syphilis cases as MSM, MSW, or women each year during 2013-2017. In these states, cases among MSM increased 8.6\% during 2016-2017 and 64.2\% during 
Figure AA. Primary and Secondary Syphilis - Estimated Rates of Reported Cases Among MSM by State, United States, 2017

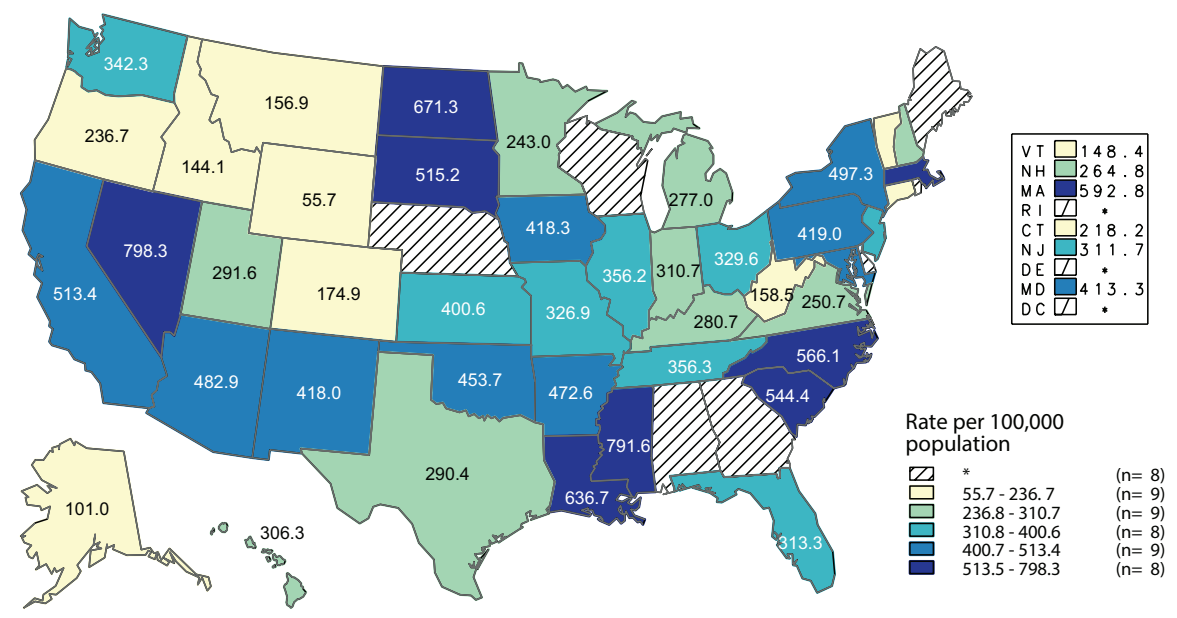

* States reporting less than 70\% of cases identified as MSM, MSW, or women in 2017 are suppressed.

NOTE: Estimates based on reported P\&S syphilis cases among MSM in 2017 (numerator) and a published method of estimating the population size of MSM (denominator) by state. See Section A1.2 in the Appendix for information on estimating MSM population sizes for rate denominators. ACRONYMS: MSM = Gay, bisexual, and other men who have sex with men (collectively referred to as MSM); MSW = Men who have sex with women only; P\&S = Primary and secondary.

2013-2017 (Figure 41). However, the percentage of $\mathrm{P} \& \mathrm{~S}$ syphilis cases that were attributed to MSM in those states fell slightly from $74.0 \%$ in 2013 to $66.5 \%$ in 2017 .

A description of the methods for estimating MSM population sizes for syphilis rate denominators can be found in Section A1.2 of the Appendix. More information about syphilis can be found in the Syphilis section of the National Profile.

\section{Gonococcal Isolate Surveillance Project}

GISP is a national sentinel surveillance system designed to monitor trends in antimicrobial susceptibilities of Neisseria gonorrhoeae strains in the United States. ${ }^{3}$ Overall, the proportion of isolates collected in selected STD clinics participating in GISP that were from MSM increased steadily, from $3.9 \%$ in 1989 to a high of $38.5 \%$ in 2017 (Figure BB). The reason for this increase over time is isolates from MSM are more likely to exhibit antimicrobial resistance than isolates from MSW. ${ }^{3,4}$ During 2011-2016, the proportion of isolates with elevated azithromycin minimum inhibitory concentrations (MICs) $(\geq 2.0 \mu \mathrm{g} / \mathrm{ml})$ and elevated ceftriaxone $\operatorname{MICS}(\geq 0.125 \mu \mathrm{g} / \mathrm{ml})$ was higher in isolates from MSM than from MSW (Figure CC). The proportion of isolates with elevated azithromycin MICs remained higher among MSM relative to MSW in 2017; however, no cases of elevated ceftriaxone MICs were identified among MSM in 2017.

Information on the antimicrobial susceptibility criteria used in GISP can be found in Section A2.3 of the Appendix. More information about GISP and additional data can be found at https://www.cdc.gov/std/ GISP.

\section{STD Surveillance Network}

$\mathrm{SSuN}$ is an ongoing collaboration of state, county, and city health departments collecting enhanced provider- and patient-based information among a random sample of reported gonorrhea cases, as well as clinical and behavioral information unclear, but might reflect changes in the epidemiology of gonorrhea or in healthcare-seeking behavior of men infected with gonorrhea. GISP has demonstrated that gonococcal

\section{Figure BB. Neisseria gonorrhoeae - Percentage of Urethral Isolates Obtained from MSM Attending STD Clinics, Gonococcal Isolate Surveillance Project (GISP), 1989-2017}

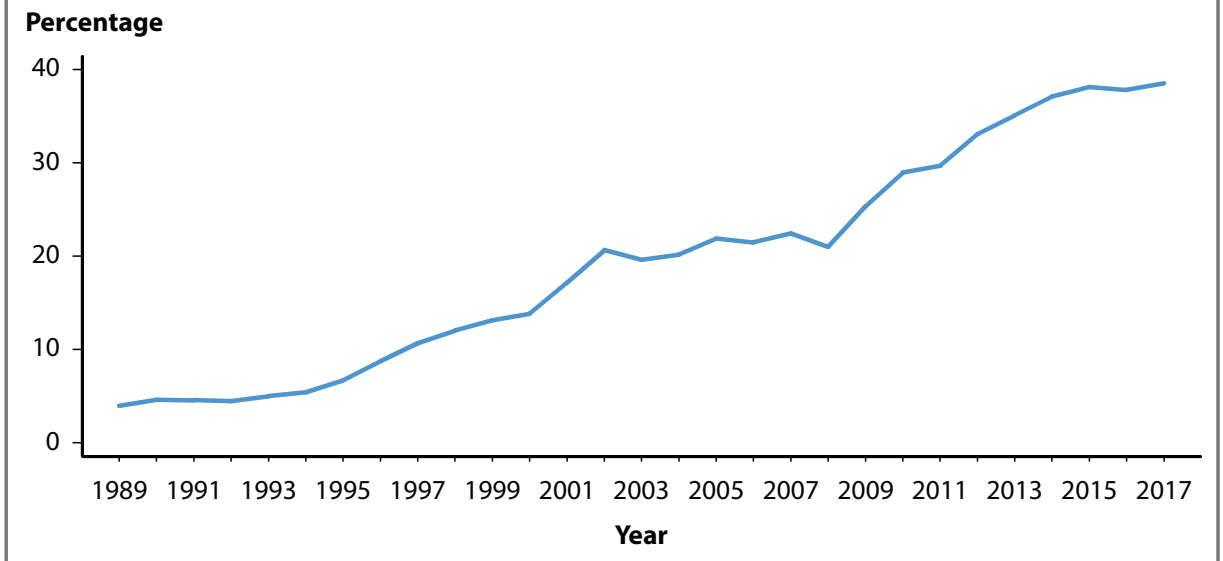

ACRONYMS: MSM = Gay, bisexual, and other men who have sex with men (collectively referred to as MSM). 
Figure CC. Neisseria gonorrhoeae - Percentage of Urethral Isolates with Elevated Azithromycin Minimum Inhibitory Concentrations (MICs) $(\geq 2.0 \mu \mathrm{g} / \mathrm{ml})$ and Elevated Ceftriaxone MICs $(\geq 0.125 \mu \mathrm{g} / \mathrm{ml})$ by Reported Sex of Sex Partners, Gonococcal Isolate Surveillance Project (GISP), 2008-2017

\section{A. Azithromycin}

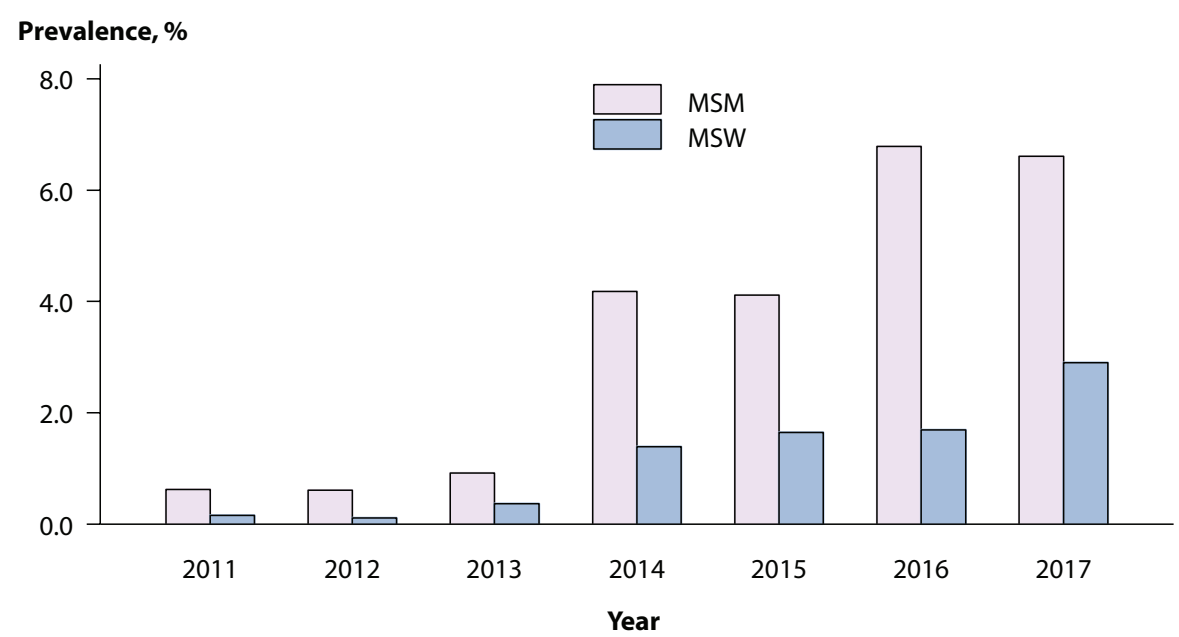

B. Ceftriaxone

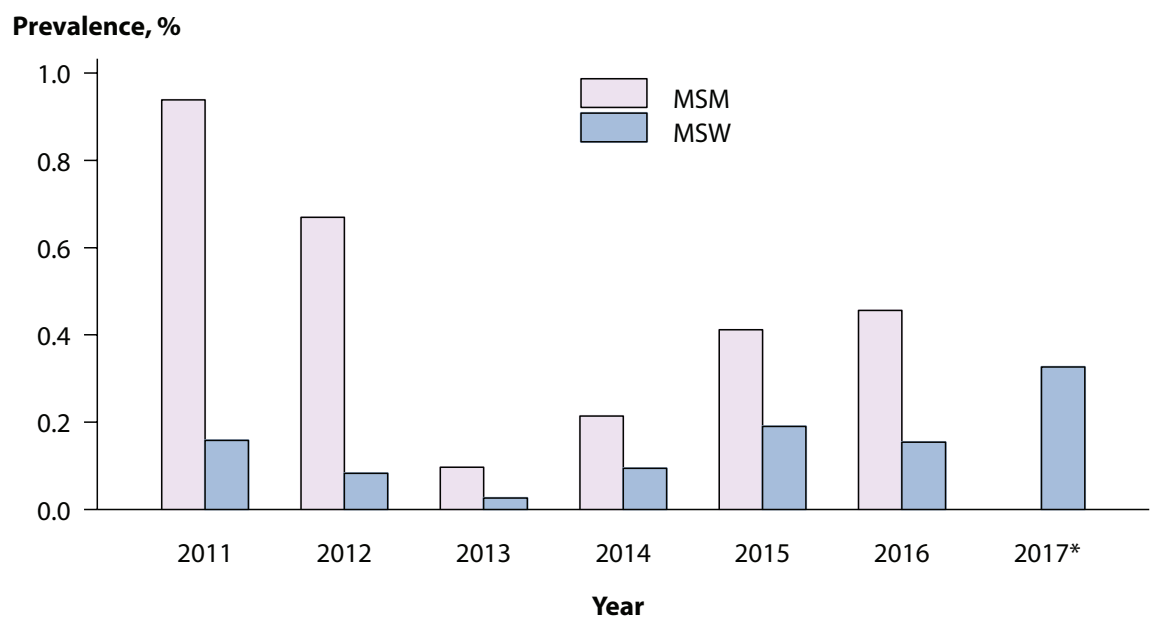

* No cases of elevated ceftriaxone MICs were reported among MSM in 2017.

ACRONYMS: MSM = Gay, bisexual, and other men who have sex with men (collectively referred to as MSM); MSW = Men who have sex with women only.

among all patients attending STD clinics in collaborating jurisdictions. ${ }^{29}$ Data for 2017 were obtained from 30 STD clinics in $10 \mathrm{SSuN}$ jurisdictions.

Estimated rates of reported gonorrhea among MSM based on SSuN data are provided in the Gonorrhea section of the National Profile (Figure 25).
Additional information about SSuN can be found in Section A2.2 of the Appendix.

\section{Gonorrhea and Chlamydia in STD Clinics, 2017}

In 2017, 31,052 unique MSM presented for care in the $30 \mathrm{STD}$ clinics in $10 \mathrm{SSuN}$ jurisdictions. In total, 27,430 unique MSM were tested for urogenital gonorrhea and/ or chlamydia $(27,407$ for gonorrhea, 27,337 for chlamydia). The proportion of men tested for urogenital infections was similar across SSuN jurisdictions, although the proportion who tested positive (positivity) varied by SSuN jurisdiction (Figure DD). Urogenital gonorrhea positivity was higher than urogenital chlamydia positivity in 6 of the 10 jurisdictions: Baltimore, Los Angeles, New York City, Philadelphia, San Francisco, and Seattle. Urogenital chlamydia positivity was higher than urogenital gonorrhea positivity in Florida; Massachusetts; Multnomah County, OR; and Minnesota. The median urogenital positivity for gonorrhea among MSM was $8.5 \%$ (range: $4.6 \%-13.5 \%$ ) and for chlamydia was $4.8 \%$ (range: $6.6 \%-10.7 \%$ ) across the 10 jurisdictions.

A total of 20,883 unique MSM were tested for rectal gonorrhea and/ or chlamydia in 2017 (20,861 for gonorrhea, 20,817 for chlamydia) (Figure EE). In most jurisdictions, similar proportions of MSM were tested for rectal gonorrhea and chlamydia, likely reflecting use of dual diagnostic tests. Compared to urogenital testing, a lower proportion of MSM were tested for rectal infection. The median positivity for rectal gonorrhea among MSM was $14.7 \%$ (range: $10.0 \%-24.4 \%$ ) and for rectal chlamydia was $16.8 \%$ (range: $12.8 \%-21.1 \%$ ) among the SSuN jurisdictions.

During 2017, 23,301 MSM were tested at the oropharyngeal site for gonorrhea (Figure FF). The median positivity for oropharyngeal gonorrhea among MSM was $13.4 \%$ (range: $6.9 \%-17.2 \%$ ) across the 10 jurisdictions. Oropharyngeal chlamydia data are not shown as some of the SSuN jurisdictions do not offer routine testing for oropharyngeal chlamydia infections. 
Figure DD. Gonorrhea and Chlamydia - Proportion* of MSM Attending STD Clinics Testing Positive for Urogenital ${ }^{\dagger}$ Gonorrhea and Chlamydia by Jurisdiction, STD Surveillance Network (SSuN), 2017

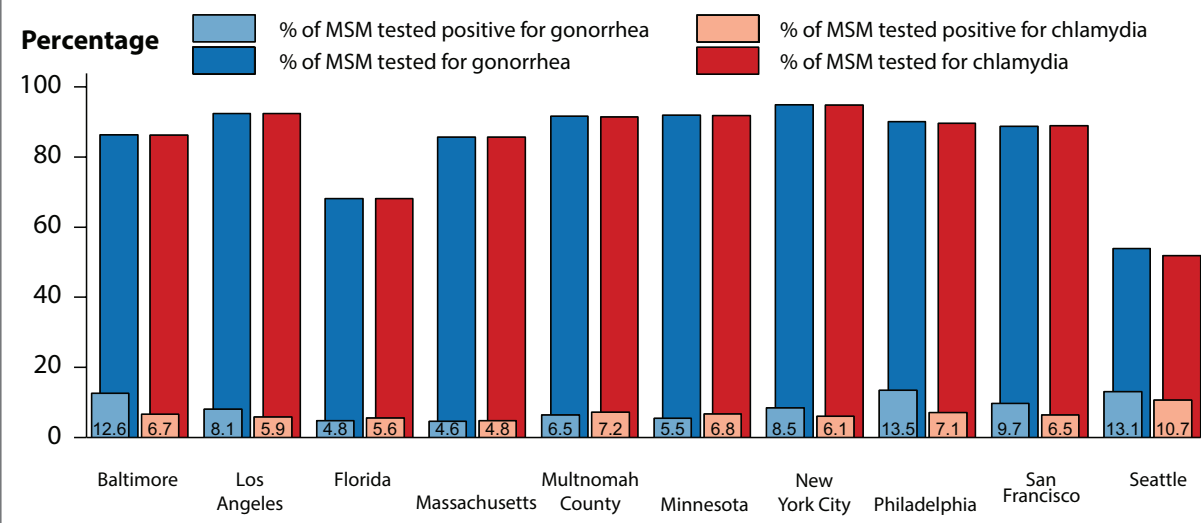

* Results based on data obtained from unique patients with known sexual behavior tested for urogenital gonorrhea $(n=27,407)$ and for urogenital chlamydia $(n=27,337) \geq 1$ times in 2017. ${ }^{\dagger}$ Includes results from both urethral and urine specimens.

NOTE: See section A2.2 in the Appendix for SSUN methods.

ACRONYMS: MSM = Gay, bisexual, and other men who have sex with men (collectively referred to as MSM).

Figure EE. Gonorrhea and Chlamydia - Proportion* of MSM Attending STD Clinics Testing Positive for Rectal Gonorrhea and Chlamydia by Jurisdiction, STD Surveillance Network (SSUN), 2017

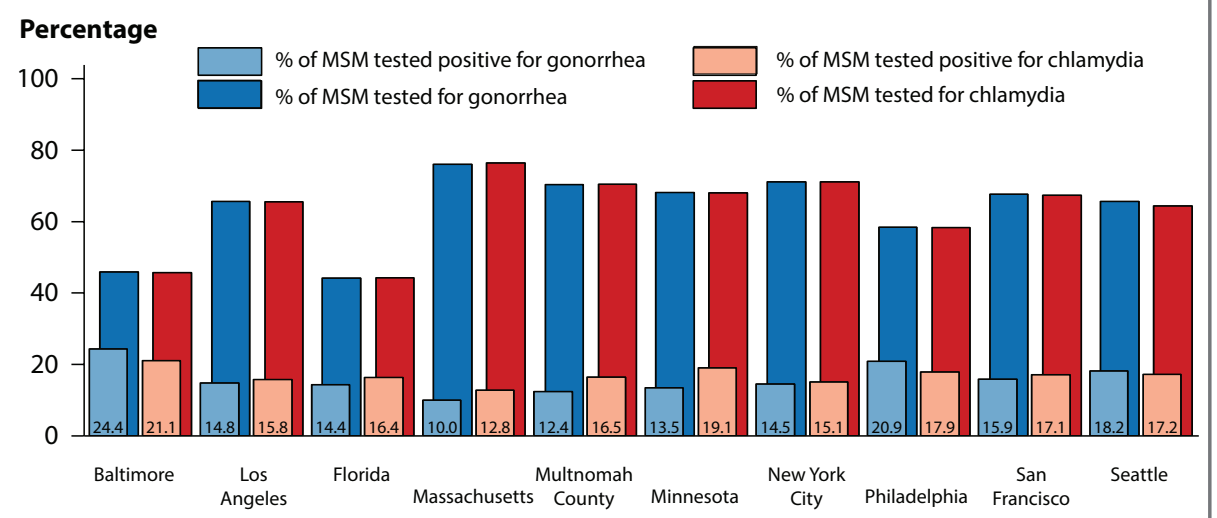

* Results based on data obtained from unique patients with known sexual behavior tested for rectal gonorrhea $(n=20,861)$ and for rectal chlamydia $(n=20,817) \geq 1$ times in 2017.

NOTE: See section A2.2 in the Appendix for SSuN methods.

ACRONYMS: MSM = Gay, bisexual, and other men who have sex with men (collectively referred to as MSM).
HIV Status and STDs in STD Clinics, 2017

Among HIV-positive MSM visiting SSuN STD clinics in 2017, urogenital chlamydia positivity was $7.1 \%$ and urogenital gonorrhea positivity was $12.0 \%$ (compared to $6.8 \%$ and $8.2 \%$, respectively, among HIV-negative MSM) (Figure GG). Among HIVpositive MSM, $8.5 \%$ were diagnosed with $\mathrm{P} \& \mathrm{~S}$ syphilis compared to $3.8 \%$ of HIV-negative MSM. Percentages represent the overall average of the mean value by jurisdiction.

\section{Summary}

The number of reported P\&S syphilis cases among MSM continued to rise in 2017 , and the majority of P\&S syphilis cases remained among MSM. Furthermore, the proportion of GISP isolates with elevated MICs to antimicrobials currently used to treat gonorrhea was higher among MSM than among MSW. Beyond STD burden in the general MSM population, the data indicated heterogeneity of STD prevalence among MSM according to geography, race, Hispanic ethnicity, and HIV status. Statespecific P\&S syphilis rate estimates among MSM varied from 55.7 to 798.3 cases per $100,000 \mathrm{MSM}$, and the prevalence of diagnosed STDs among MSM differed by SSuN jurisdiction. Reported P\&S syphilis was disproportionately prevalent among Black and Hispanic MSM, and data from MSM who attended SSuN clinics suggested that $\mathrm{P} \& \mathrm{~S}$ syphilis, urogenital gonorrhea, and urogenital chlamydia may be more prevalent among MSM living with diagnosed HIV infection than among HIVnegative MSM. 


\section{Figure FF. Gonorrhea - Proportion* of MSM Attending STD Clinics Testing Positive for Oropharyngeal Gonorrhea by Jurisdiction, STD Surveillance Network (SSuN), 2017}

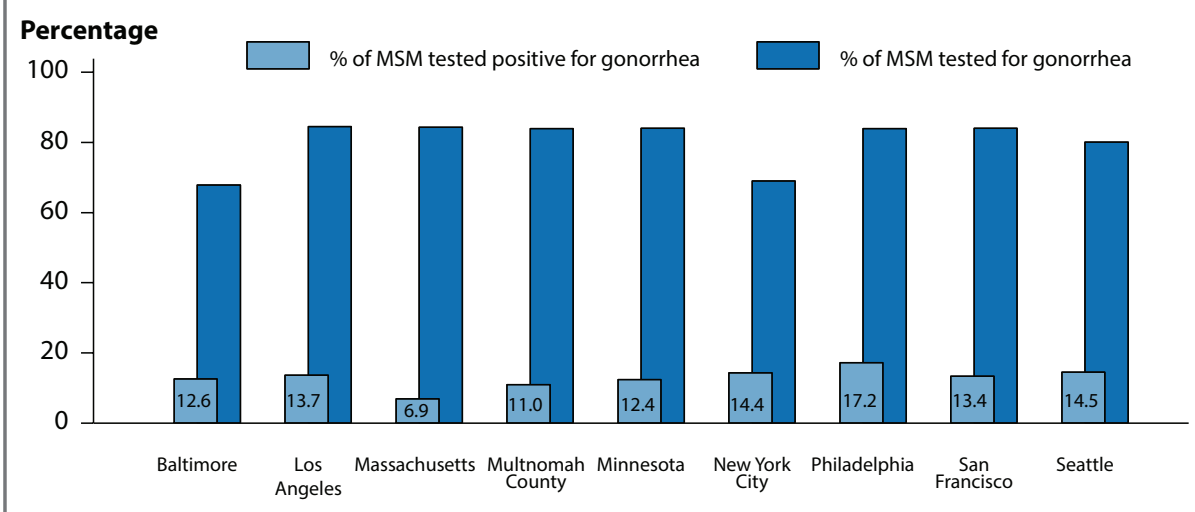

* Results based on data obtained from unique patients with known sexual behavior tested for oropharyngeal gonorrhea $(n=23,301) \geq 1$ times in 2017; data from Florida were not available. NOTE: See section A2.2 in the Appendix for SSuN methods.

ACRONYMS: MSM = Gay, bisexual, and other men who have sex with men (collectively referred to as MSM).

\section{Figure GG. Proportion* of MSM Attending STD Clinics with Primary and Secondary Syphilis ${ }^{\dagger}$, Urogenital ${ }^{\ddagger}$ Gonorrhea, or Urogenital ${ }^{\ddagger}$ Chlamydia by HIV Status ${ }^{\S}$, STD Surveillance Network (SSuN), 2017}

\section{Percentage}

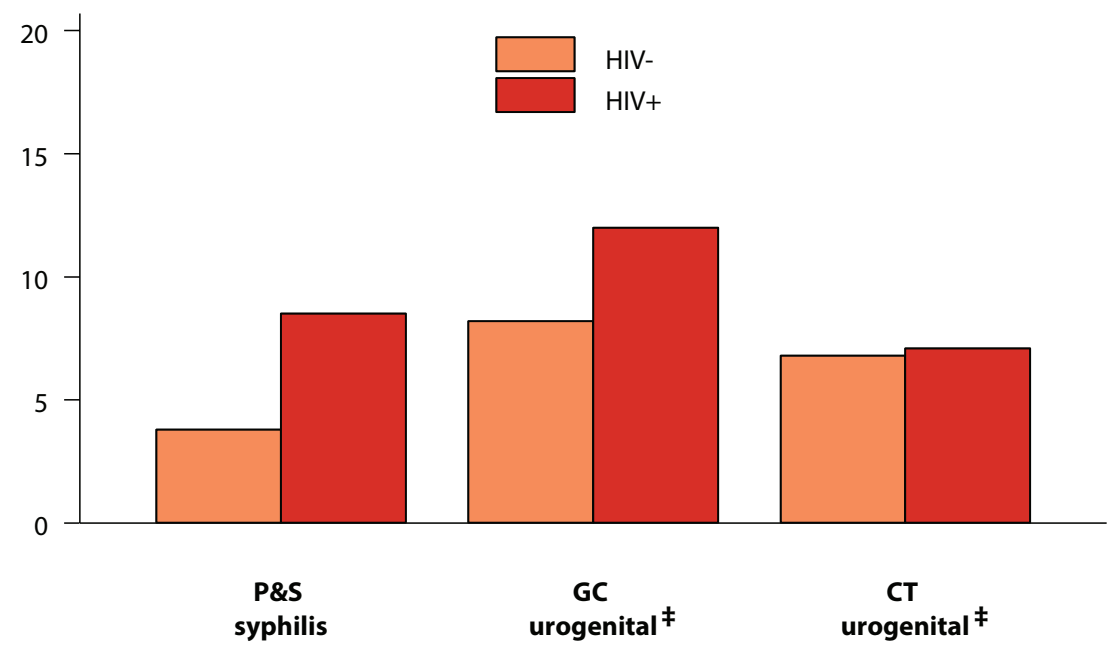

* Proportions represent the overall average of the mean proportions by jurisdiction.

+ Includes SSUN jurisdictions that reported data on at least 20 patients with a diagnosis of primary and secondary syphilis in 2017.

${ }^{\ddagger}$ Includes results from both urethral and urine specimens.

${ }^{\S}$ Excludes all persons for whom there was no laboratory documentation or self-report of HIV status. NOTE: See section A2.2 in the Appendix for SSuN methods.

ACRONYMS: MSM = Gay, bisexual, and other men who have sex with men (collectively referred to as MSM); P\&S = Primary and secondary; GC = Gonorrhea; $C T$ = Chlamydia.

\section{References}

1. An Q, Wejnert C, Bernstein K, et al. Syphilis screening and diagnosis among men who have sex with men, 2008-2014, 20 US cities. JAIDS 2017; 75(Suppl 3):S363-S369.

2. de Voux A, Kidd S, Grey JA, et al. State-specific rates of primary and secondary syphilis among men who have sex with men - United States, 2015. MMWR Morb Mortal Wkly Rep 2017; 66(13):349-354.

3. Kirkcaldy RD, Harvey A, Papp JR, et al. Neisseria gonorrhoeae antimicrobial susceptibility surveillance - the Gonococcal Isolate Surveillance Project, 27 sites, United States, 2014. MMWR Surveill Summ 2016; 65(SS-7):1-19.

4. Kirkcaldy RD, Zaidi A, Hook EW 3rd, et al. Neisseria gonorrhoeae antimicrobial resistance among men who have sex with men and men who have sex exclusively with women: The Gonococcal Isolate Surveillance Project, 2005-2010. Ann Intern Med 2013; 158(5 Pt 1):321-328.

5. Patton ME, Su JR, Nelson R, et al. Primary and secondary syphilis - United States, 20052013. MMWR Morb Mortal Wkly Rep 2014; 63(18):402-406.

6. Peterman TA, Su J, Bernstein KT, et al. Syphilis in the United States: on the rise? Expert Rev Anti Infect Ther 2015; 13(2):161-168.

7. Solomon MM, Mayer KH. Evolution of the syphilis epidemic among men who have sex with men. Sex Health 2014; 12(2):96-102.

8. Buchacz K, Patel P, Taylor M, et al. Syphilis increases HIV viral load and decreases CD4 cell counts in HIV-infected patients with new syphilis infections. AIDS 2004; 18(15):20752079.

9. Fleming DT, Wasserheit JN. From epidemiologic synergy to public health policy and practice: the contribution of other sexually transmitted diseases to sexual transmission of HIV infection. Sex Transm Infect 1999; 75(1):3-17.

10. Jarzebowski W, Caumes E, Dupin N, et al. Effect of early syphilis infection on plasma viral load and CD4 cell count in human immunodeficiency virus- infected men: Results from the FHDH-ANRS CO4 cohort. Arch Intern Med 2012; 172(16):1237-1243.

11. Katz DA, Dombrowski JC, Bell TR, et al. HIV incidence among men who have sex with men after diagnosis with sexually transmitted infections. Sex Transm Dis 2016; 43(4):249254.

12. Kelley CF, Vaughan AS, Luisi N, et al. The effect of high rates of bacterial sexually transmitted infections on HIV incidence in a cohort of black and white men who have sex with men in Atlanta, Georgia. AIDS Res Hum Retroviruses 2015; 31(6):587-592. 
13. Pathela P, Braunstein SL, Blank S, et al. HIV incidence among men with and those without sexually transmitted rectal infections: Estimates from matching against and HIV case registry. Clin Infect Dis 2013; 57(8):1203-1209.

14. Solomon MM, Mayer KH, Glidden DV, et al. Syphilis predicts HIV incidence among men and transgender women who have sex with men in a preexposure prophylaxis trial. Clin Infect Dis 2014; 59(7):1020-1026.

15. Glick SN, Morris M, Foxman B, et al. A comparison of sexual behavior patterns among men who have sex with men and heterosexual men and women. J Acquir Immune Defic Syndr 2012; 60(1):83-90.

16. Paz-Bailey G, Mendoza MCB, Finlayson T, et al. Trends in condom use among MSM in the United States: The role of antiretroviral therapy and seroadaptive strategies. AIDS 2016; 30(12):1985-1990.

17. Spicknall IH, Gift TL, Bernstein KT, et al, Sexual networks and infection transmission networks among men who have sex with men as causes of disparity and targets of prevention. Sex Transm Infect 2017; 93(5):307-308.

18. Alvy LM, McKirnan DJ, Du Bois SN, et al. Health care disparities and behavioral health among men who have sex with men. J Gay Lesbian Soc Serv 2011; 23(4):507-522.

19. Balaji AB, Bowles KE, Hess KL, et al. Association between enacted stigma and HIVrelated risk behavior among MSM, National HIV Behavioral Surveillance System, 2011. AIDS Behav 2017; 21(1):227-237.
20. Brewer TH, Schillinger J, Lewis FM, et al. Infectious syphilis among adolescent and young adult men: Implications for human immunodeficiency virus transmission and public health interventions. Sex Transm Dis 2011; 38(5):367-371.

21. Jeffries WL, Marks G, Lauby J. Homophobia is associated with sexual behavior that increases risk of acquiring and transmitting HIV infection among black men who have sex with men. AIDS Behav 2013; 17(4):1442-1453.

22. McKirnan DJ, Du Bois SN, Alvy LM, et al. Health care access and health behaviors among men who have sex with men: The cost of health disparities. Health Educ Behav 2013; 40(1):3241.

23. Su JR, Beltrami JF, Zaidi AA, et al. Primary and secondary syphilis among black and Hispanic men who have sex with men: Case report data from 27 States. Ann Intern Med 2011; 155(3):145-151

24. Díaz RM, Ayala G, Bein E. Sexual risk as an outcome of social oppression: Data from a probability sample of Latino gay men in three US cities. Cultur Divers Ethnic Minor Psychol 2004; 10(3):255-267.

25. Millett GA, Flores SA, Peterson JL, et al. Explaining disparities in HIV infection among black and white men who have sex with men: A meta-analysis of HIV risk behaviors. AIDS 2007; 21(15):2083-2091.
26. Sullivan PS, Peterson J, Rosenberg ES, et al. Understanding racial HIV/STI disparities in black and white men who have sex with men: A multilevel approach. PLoS One 2014; 9(3):e90514

27. Mayer KH, Wang L, Koblin B, et al. Concomitant socioeconomic, behavioral, and biological factors associated with the disproportionate HIV infection burden among black men who have sex with men in 6 US cities. PLoS One 2014; 9(1):e87298.

28. Patton ME, Kidd S, Llata E, et al. Extragenital gonorrhea and chlamydia testing and infection among men who have sex with men - STD Surveillance Network, United States, 2010 2012. Clin Infect Dis 2014; 58(11):1564-1570

29. Rietmeijer K, Donnelly J, Bernstein K, et al. Here comes the SSuN - early experiences with the STD Surveillance Network. Pub Health Rep 2009; 124(Suppl 2):72-77.

30. U.S. Census Bureau; Annual Estimates of the Resident Population by Sex, Race, and Hispanic Origin for the United States, States, and Counties: April 1, 2010 to July 1, 2017, Table PEPSR6H; generated by Jeremy Grey; using American FactFinder. Available at: https:// factfinder.census.gov/faces/nav/jsf/pages/index. xhtml. Accessed July 24, 2018.

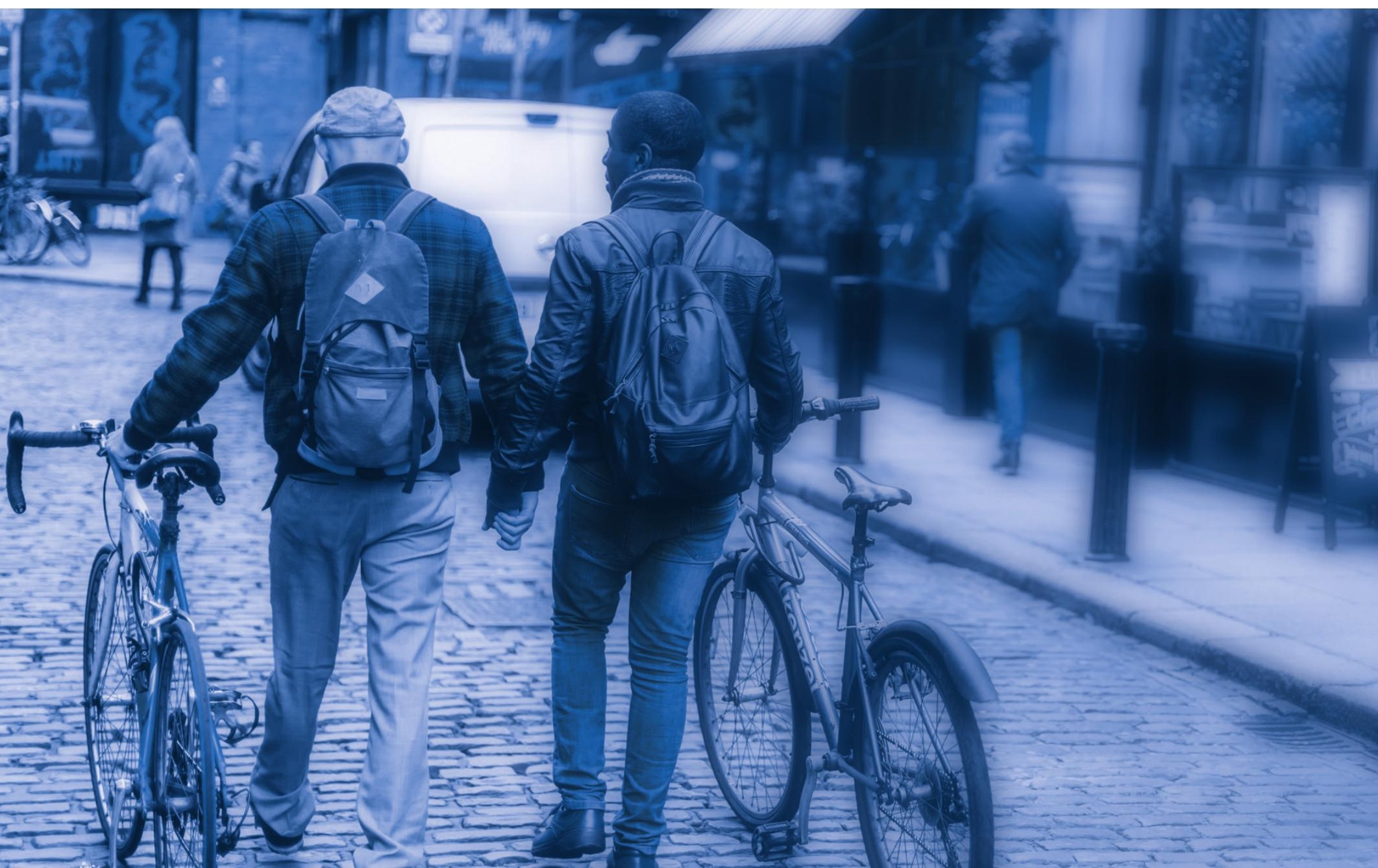




\section{Tables}

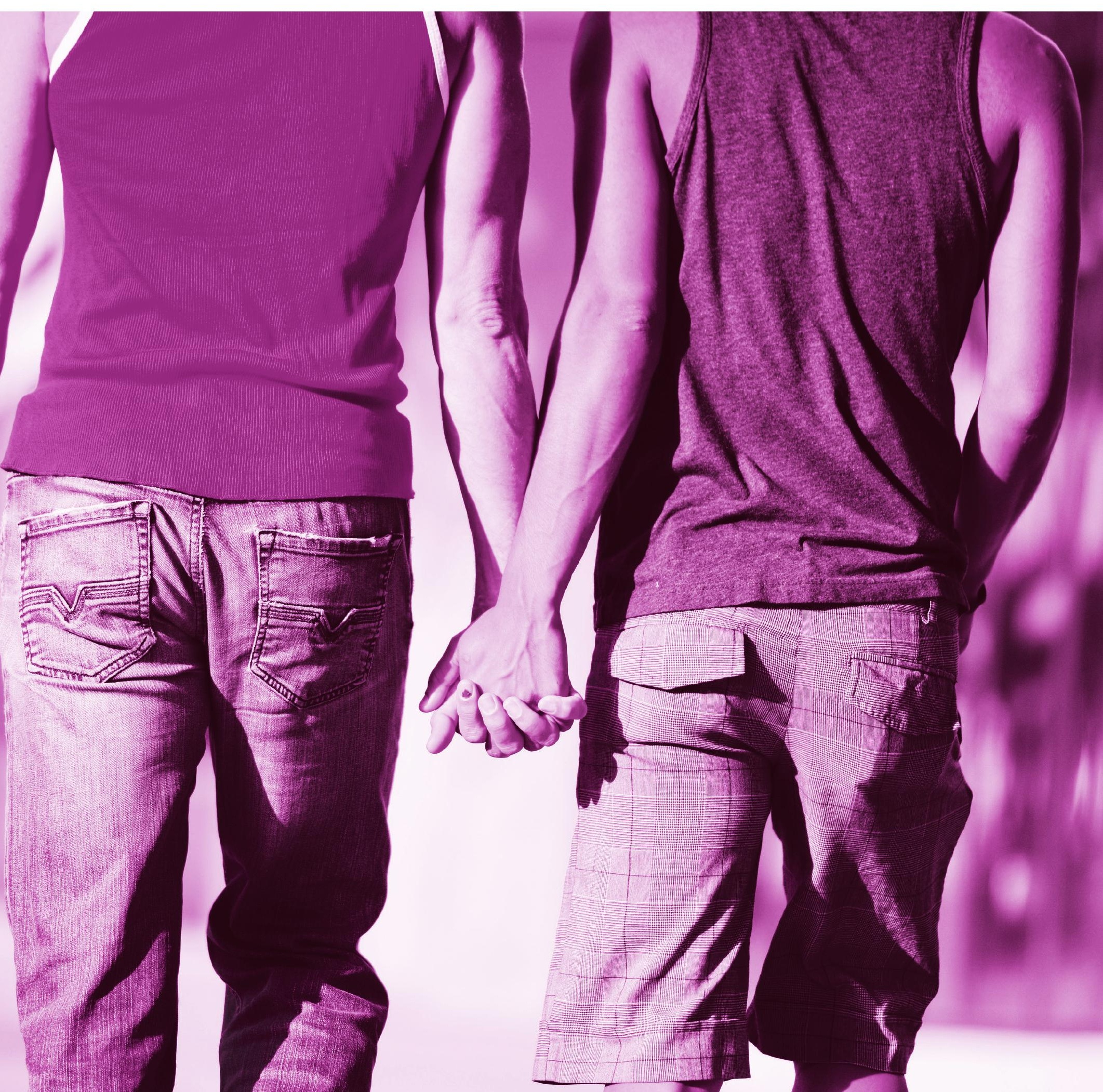


Table 1. Sexually Transmitted Diseases - Reported Cases and Rates of Reported Cases per 100,000 Population, United States, 1941-2017

\begin{tabular}{|c|c|c|c|c|c|c|c|c|c|c|c|c|c|c|c|c|}
\hline \multirow[b]{3}{*}{ Year* } & \multicolumn{10}{|c|}{ Syphilis } & & & & & & \\
\hline & \multicolumn{2}{|c|}{ All Stages $^{\dagger}$} & \multicolumn{2}{|c|}{$\begin{array}{c}\text { Primary and } \\
\text { Secondary }\end{array}$} & \multicolumn{2}{|c|}{$\begin{array}{l}\text { Early } \\
\text { Latent }\end{array}$} & \multicolumn{2}{|c|}{$\begin{array}{c}\text { Late and } \\
\text { Late Latent }^{\ddagger}\end{array}$} & \multicolumn{2}{|c|}{ Congenital } & \multicolumn{2}{|c|}{ Chlamydia } & \multicolumn{2}{|c|}{ Gonorrhea } & \multicolumn{2}{|c|}{ Chancroid } \\
\hline & Cases & Rate & Cases & Rate & Cases & Rate & Cases & Rate & Cases & Rate $^{\S}$ & Cases & Rate & Cases & Rate & Cases & Rate \\
\hline 1941 & 485,560 & 368.2 & 68,231 & 51.7 & 109,018 & 82.6 & 202,984 & 153.9 & 17,600 & 651.1 & NR & - & 193,468 & 146.7 & 3,384 & 2.5 \\
\hline 1942 & 479,601 & 363.4 & 75,312 & 57.0 & 116,245 & 88.0 & 202,064 & 153.1 & 16,918 & 566.0 & NR & - & 212,403 & 160.9 & 5,477 & 4.1 \\
\hline 1943 & 575,593 & 447.0 & 82,204 & 63.8 & 149,390 & 116.0 & 251,958 & 195.7 & 16,164 & 520.7 & NR & - & 275,070 & 213.6 & 8,354 & 6.4 \\
\hline 1944 & 467,755 & 367.9 & 78,443 & 61.6 & 123,038 & 96.7 & 202,848 & 159.6 & 13,578 & 462.0 & NR & - & 300,676 & 236.5 & 7,878 & 6.1 \\
\hline 1945 & 359,114 & 282.3 & 77,007 & 60.5 & 101,719 & 79.9 & 142,187 & 111.8 & 12,339 & 431.7 & NR & - & 287,181 & 225.8 & 5,515 & 4.3 \\
\hline 1946 & 363,647 & 271.7 & 94,957 & 70.9 & 107,924 & 80.6 & 125,248 & 93.6 & 12,106 & 354.9 & NR & - & 368,020 & 275.0 & 7,091 & 5.2 \\
\hline 1947 & 355,592 & 252.3 & 93,545 & 66.4 & 104,124 & 73.9 & 122,089 & 86.6 & 12,200 & 319.6 & NR & - & 380,666 & 270.0 & 9,515 & 6.7 \\
\hline 1948 & 314,313 & 218.2 & 68,174 & 47.3 & 90,598 & 62.9 & 123,312 & 85.6 & 13,931 & 383.0 & NR & - & 345,501 & 239.8 & 7,661 & 5.3 \\
\hline 1949 & 256,463 & 175.3 & 41,942 & 28.7 & 75,045 & 51.3 & 116,397 & 79.5 & 13,952 & 382.4 & NR & - & 317,950 & 217.3 & 6,707 & 4.6 \\
\hline 1950 & 217,558 & 146.0 & 23,939 & 16.7 & 59,256 & 39.7 & 113,569 & 70.2 & 13,377 & 368.3 & NR & - & 286,746 & 192.5 & 4,977 & 3.3 \\
\hline 1951 & 174,924 & 116.1 & 14,485 & 9.6 & 43,316 & 28.7 & 98,311 & 65.2 & 11,094 & 290.4 & NR & - & 254,470 & 168.9 & 4,233 & 2.8 \\
\hline 1952 & 167,762 & 110.2 & 10,449 & 6.9 & 36,454 & 24.0 & 105,238 & 69.1 & 8,553 & 218.8 & NR & - & 244,957 & 160.8 & 3,738 & 2.5 \\
\hline 1953 & 148,573 & 95.9 & 8,637 & 5.6 & 28,295 & 18.3 & 98,870 & 63.8 & 7,675 & 193.9 & NR & - & 238,340 & 153.9 & 3,338 & 2.2 \\
\hline 1954 & 130,697 & 82.9 & 7,147 & 4.5 & 23,861 & 15.1 & 89,123 & 56.5 & 6,676 & 164.0 & NR & - & 242,050 & 153.5 & 3,003 & 1.9 \\
\hline 1955 & 122,392 & 76.2 & 6,454 & 4.0 & 20,054 & 12.5 & 86,526 & 53.8 & 5,354 & 130.7 & NR & - & 236,197 & 147.0 & 2,649 & 1.7 \\
\hline 1956 & 130,201 & 78.7 & 6,392 & 3.9 & 19,783 & 12.0 & 95,097 & 57.5 & 5,491 & 130.4 & NR & - & 224,346 & 135.7 & 2,135 & 1.3 \\
\hline 1957 & 123,758 & 73.5 & 6,576 & 3.9 & 17,796 & 10.6 & 91,309 & 54.2 & 5,288 & 123.0 & NR & - & 214,496 & 127.4 & 1,637 & 1.0 \\
\hline 1958 & 113,884 & 66.4 & 7,176 & 4.2 & 16,556 & 9.7 & 83,027 & 48.4 & 4,866 & 114.6 & NR & - & 232,386 & 135.6 & 1,595 & 0.9 \\
\hline 1959 & 120,824 & 69.2 & 9,799 & 5.6 & 17,025 & 9.8 & 86,740 & 49.7 & 5,130 & 119.7 & NR & - & 240,254 & 137.6 & 1,537 & 0.9 \\
\hline 1960 & 122,538 & 68.8 & 16,145 & 9.1 & 18,017 & 10.1 & 81,798 & 45.9 & 4,416 & 103.7 & NR & - & 258,933 & 145.4 & 1,680 & 0.9 \\
\hline 1961 & 124,658 & 68.8 & 19,851 & 11.0 & 19,486 & 10.8 & 79,304 & 43.8 & 4,163 & 97.5 & NR & - & 264,158 & 145.8 & 1,438 & 0.8 \\
\hline 1962 & 126,245 & 68.7 & 21,067 & 11.5 & 19,585 & 10.7 & 79,533 & 43.3 & 4,070 & 97.7 & NR & - & 263,714 & 143.6 & 1,344 & 0.7 \\
\hline 1963 & 124,137 & 66.5 & 22,251 & 11.9 & 18,235 & 9.8 & 78,076 & 41.8 & 4,031 & 98.4 & NR & - & 278,289 & 149.0 & 1,220 & 0.7 \\
\hline 1964 & 114,325 & 60.4 & 22,969 & 12.1 & 17,781 & 9.4 & 68,629 & 36.3 & 3,516 & 87.3 & NR & - & 300,666 & 158.9 & 1,247 & 0.7 \\
\hline 1965 & 112,842 & 58.9 & 23,338 & 12.2 & 17,458 & 9.1 & 67,317 & 35.1 & 3,564 & 94.8 & NR & - & 324,925 & 169.5 & 982 & 0.5 \\
\hline 1966 & 105,159 & 54.2 & 21,414 & 11.0 & 15,950 & 8.2 & 63,541 & 32.7 & 3,170 & 87.9 & NR & - & 351,738 & 181.2 & 838 & 0.4 \\
\hline 1967 & 102,581 & 52.2 & 21,053 & 10.7 & 15,554 & 7.9 & 61,975 & 31.5 & 2,894 & 82.2 & NR & - & 404,836 & 205.9 & 784 & 0.4 \\
\hline 1968 & 96,271 & 48.4 & 19,019 & 9.6 & 15,150 & 7.6 & 58,564 & 29.4 & 2,381 & 68.0 & NR & - & 464,543 & 233.4 & 845 & 0.4 \\
\hline 1969 & 92,162 & 45.7 & 19,130 & 9.5 & 15,402 & 7.6 & 54,587 & 27.1 & 2,074 & 57.6 & NR & - & 534,872 & 265.4 & 1,104 & 0.5 \\
\hline 1970 & 91,382 & 44.8 & 21,982 & 10.8 & 16,311 & 8.0 & 50,348 & 24.7 & 1,953 & 52.3 & NR & - & 600,072 & 294.2 & 1,416 & 0.7 \\
\hline 1971 & 95,997 & 46.4 & 23,783 & 11.5 & 19,417 & 9.4 & 49,993 & 24.2 & 2,052 & 57.7 & NR & - & 670,268 & 324.1 & 1,320 & 0.6 \\
\hline 1972 & 91,149 & 43.6 & 24,429 & 11.7 & 20,784 & 9.9 & 43,456 & 20.8 & 1,758 & 54.0 & NR & - & 767,215 & 366.6 & 1,414 & 0.7 \\
\hline 1973 & 87,469 & 41.4 & 24,825 & 11.7 & 23,584 & 11.2 & 37,054 & 17.5 & 1,527 & 48.7 & NR & - & 842,621 & 398.7 & 1,165 & 0.6 \\
\hline 1974 & 83,771 & 39.3 & 25,385 & 11.9 & 25,124 & 11.8 & 31,854 & 14.9 & 1,138 & 36.0 & NR & - & 906,121 & 424.7 & 945 & 0.4 \\
\hline 1975 & 80,356 & 37.3 & 25,561 & 11.9 & 26,569 & 12.3 & 27,096 & 12.6 & 916 & 29.1 & NR & - & 999,937 & 464.1 & 700 & 0.3 \\
\hline 1976 & 71,761 & 33.0 & 23,731 & 10.9 & 25,363 & 11.7 & 21,905 & 10.1 & 626 & 19.8 & NR & - & $1,001,994$ & 460.6 & 628 & 0.3 \\
\hline 1977 & 64,621 & 29.4 & 20,399 & 9.3 & 21,329 & 9.7 & 22,313 & 10.2 & 463 & 13.9 & NR & - & $1,002,219$ & 456.0 & 455 & 0.2 \\
\hline 1978 & 64,875 & 29.2 & 21,656 & 9.8 & 19,628 & 8.8 & 23,038 & 10.4 & 434 & 13.0 & NR & - & $1,013,436$ & 456.3 & 521 & 0.2 \\
\hline 1979 & 67,049 & 29.9 & 24,874 & 11.1 & 20,459 & 9.1 & 21,301 & 9.5 & 332 & 9.5 & NR & - & $1,004,058$ & 447.1 & 840 & 0.4 \\
\hline 1980 & 68,832 & 30.3 & 27,204 & 12.0 & 20,297 & 8.9 & 20,979 & 9.2 & 277 & 7.7 & NR & - & $1,004,029$ & 442.1 & 788 & 0.3 \\
\hline 1981 & 72,799 & 31.7 & 31,266 & 13.6 & 21,033 & 9.2 & 20,168 & 8.8 & 287 & 7.9 & NR & - & 990,864 & 431.8 & 850 & 0.4 \\
\hline 1982 & 75,579 & 32.6 & 33,613 & 14.5 & 21,894 & 9.5 & 19,779 & 8.5 & 259 & 7.0 & NR & - & 960,633 & 414.7 & 1,392 & 0.6 \\
\hline
\end{tabular}

Continued on next page. 
Table 1. Sexually Transmitted Diseases - Reported Cases and Rates of Reported Cases per 100,000 Population, United States, 1941-2017 (continued)

\begin{tabular}{|c|c|c|c|c|c|c|c|c|c|c|c|c|c|c|c|c|}
\hline \multirow[b]{3}{*}{ Year* } & \multicolumn{10}{|c|}{ Syphilis } & & & & & & \\
\hline & \multicolumn{2}{|c|}{ All Stages ${ }^{\dagger}$} & \multicolumn{2}{|c|}{$\begin{array}{l}\text { Primary and } \\
\text { Secondary }\end{array}$} & \multicolumn{2}{|c|}{$\begin{array}{l}\text { Early } \\
\text { Latent }\end{array}$} & \multicolumn{2}{|c|}{$\begin{array}{c}\text { Late and } \\
\text { Late Latent }{ }^{\ddagger}\end{array}$} & \multicolumn{2}{|c|}{ Congenital } & \multicolumn{2}{|c|}{ Chlamydia } & \multicolumn{2}{|c|}{ Gonorrhea } & \multicolumn{2}{|c|}{ Chancroid } \\
\hline & Cases & Rate & Cases & Rate & Cases & Rate & Cases & Rate & Cases & Rate $^{\S}$ & Cases & Rate & Cases & Rate & Cases & Rate \\
\hline 1983 & 74,637 & 31.9 & 32,698 & 14.0 & 23,738 & 10.2 & 17,896 & 7.7 & 239 & 6.6 & NR & - & 900,435 & 385.1 & 847 & 0.4 \\
\hline 1984 & 69,872 & 29.6 & 28,607 & 12.1 & 23,131 & 9.8 & 17,829 & 7.6 & 305 & 8.3 & 7,594 & 6.5 & 878,556 & 372.5 & 665 & 0.3 \\
\hline 1985 & 67,563 & 28.4 & 27,131 & 11.4 & 21,689 & 9.1 & 18,414 & 7.7 & 329 & 8.7 & 25,848 & 17.4 & 911,419 & 383.0 & 2,067 & 0.9 \\
\hline 1986 & 67,779 & 28.2 & 27,667 & 11.5 & 21,656 & 9.0 & 18,046 & 7.5 & 410 & 10.9 & 58,001 & 35.2 & 892,229 & 371.5 & 3,045 & 1.3 \\
\hline 1987 & 87,286 & 36.0 & 35,585 & 14.7 & 28,233 & 11.7 & 22,988 & 9.5 & 480 & 12.6 & 91,913 & 50.8 & 787,532 & 325.0 & 4,986 & 2.1 \\
\hline 1988 & 104,546 & 42.8 & 40,474 & 16.6 & 35,968 & 14.7 & 27,363 & 11.2 & 741 & 19.0 & 157,854 & 87.1 & 738,160 & 301.9 & 4,891 & 2.0 \\
\hline 1989 & 115,089 & 46.6 & 45,826 & 18.6 & 45,394 & 18.4 & 22,032 & 8.9 & 1,837 & 45.5 & 200,904 & 102.5 & 733,294 & 297.1 & 4,697 & 1.9 \\
\hline 1990 & 135,590 & 54.3 & 50,578 & 20.3 & 55,397 & 22.2 & 25,750 & 10.3 & 3,865 & 92.9 & 323,663 & 160.2 & 690,042 & 276.4 & 4,212 & 1.7 \\
\hline 1991 & 128,719 & 50.9 & 42,950 & 17.0 & 53,855 & 21.3 & 27,490 & 10.9 & 4,424 & 107.6 & 381,228 & 179.7 & 621,918 & 245.8 & 3,476 & 1.4 \\
\hline 1992 & 114,730 & 44.7 & 34,009 & 13.3 & 49,929 & 19.5 & 26,725 & 10.4 & 4,067 & 100.0 & 409,694 & 182.3 & 502,858 & 196.0 & 1,906 & 0.7 \\
\hline 1993 & 102,612 & 39.5 & 26,527 & 10.2 & 41,919 & 16.1 & 30,746 & 11.8 & 3,420 & 85.5 & 405,332 & 178.0 & 444,649 & 171.1 & 1,292 & 0.5 \\
\hline 1994 & 82,713 & 31.4 & 20,641 & 7.8 & 32,017 & 12.2 & 27,603 & 10.5 & 2,452 & 62.0 & 451,785 & 192.5 & 419,602 & 163.9 & 782 & 0.3 \\
\hline 1995 & 69,359 & 26.0 & 16,543 & 6.2 & 26,657 & 10.0 & 24,296 & 9.1 & 1,863 & 47.8 & 478,577 & 187.8 & 392,651 & 147.5 & 607 & 0.2 \\
\hline 1996 & 53,240 & 19.8 & 11,405 & 4.2 & 20,187 & 7.5 & 20,366 & 7.6 & 1,282 & 32.9 & 492,631 & 190.6 & 328,169 & 121.8 & 386 & 0.1 \\
\hline 1997 & 46,716 & 17.1 & 8,556 & 3.1 & 16,631 & 6.1 & 20,447 & 7.5 & 1,082 & 27.9 & 537,904 & 205.5 & 327,665 & 120.2 & 246 & 0.1 \\
\hline 1998 & 38,289 & 13.9 & 7,007 & 2.5 & 12,696 & 4.6 & 17,743 & 6.4 & 843 & 21.4 & 614,250 & 231.8 & 356,492 & 129.2 & 189 & 0.1 \\
\hline 1999 & 35,386 & 12.7 & 6,617 & 2.4 & 11,534 & 4.1 & 16,655 & 6.0 & 580 & 14.6 & 662,647 & 247.2 & 360,813 & 129.3 & 110 & 0.0 \\
\hline 2000 & 31,618 & 11.2 & 5,979 & 2.1 & 9,465 & 3.4 & 15,594 & 5.5 & 580 & 14.3 & 709,452 & 251.4 & 363,136 & 128.7 & 78 & 0.0 \\
\hline 2001 & 32,286 & 11.3 & 6,103 & 2.1 & 8,701 & 3.0 & 16,976 & 5.9 & 506 & 12.6 & 783,242 & 274.5 & 361,705 & 126.8 & 38 & 0.0 \\
\hline 2002 & 32,919 & 11.4 & 6,862 & 2.4 & 8,429 & 2.9 & 17,168 & 6.0 & 460 & 11.4 & 834,555 & 289.4 & 351,852 & 122.0 & 48 & 0.0 \\
\hline 2003 & 34,289 & 11.8 & 7,177 & 2.5 & 8,361 & 2.9 & 18,319 & 6.3 & 432 & 10.6 & 877,478 & 301.7 & 335,104 & 115.2 & 54 & 0.0 \\
\hline 2004 & 33,423 & 11.4 & 7,980 & 2.7 & 7,768 & 2.6 & 17,300 & 5.9 & 375 & 9.1 & 929,462 & 316.5 & 330,132 & 112.4 & 30 & 0.0 \\
\hline 2005 & 33,288 & 11.2 & 8,724 & 2.9 & 8,176 & 2.8 & 16,049 & 5.4 & 339 & 8.2 & 976,445 & 329.4 & 339,593 & 114.6 & 17 & 0.0 \\
\hline 2006 & 36,958 & 12.3 & 9,756 & 3.3 & 9,186 & 3.1 & 17,644 & 5.9 & 372 & 8.7 & $1,030,911$ & 344.3 & 358,366 & 119.7 & 19 & 0.0 \\
\hline 2007 & 40,925 & 13.6 & 11,466 & 3.8 & 10,768 & 3.6 & 18,256 & 6.1 & 435 & 10.1 & $1,108,374$ & 367.5 & 355,991 & 118.0 & 23 & 0.0 \\
\hline 2008 & 46,292 & 15.2 & 13,500 & 4.4 & 12,401 & 4.1 & 19,945 & 6.6 & 446 & 10.5 & $1,210,523$ & 398.1 & 336,742 & 110.7 & 25 & 0.0 \\
\hline 2009 & 44,832 & 14.6 & 13,997 & 4.6 & 13,066 & 4.3 & 17,338 & 5.6 & 431 & 10.4 & $1,244,180$ & 405.3 & 301,174 & 98.1 & 28 & 0.0 \\
\hline 2010 & 45,844 & 14.8 & 13,774 & 4.5 & 13,604 & 4.4 & 18,079 & 5.9 & 387 & 9.7 & $1,307,893$ & 423.6 & 309,341 & 100.2 & 24 & 0.0 \\
\hline 2011 & 46,040 & 14.8 & 13,970 & 4.5 & 13,136 & 4.2 & 18,576 & 6.0 & 358 & 9.1 & $1,412,791$ & 453.4 & 321,849 & 103.3 & 8 & 0.0 \\
\hline 2012 & 49,915 & 15.9 & 15,667 & 5.0 & 14,503 & 4.6 & 19,411 & 6.2 & 334 & 8.4 & $1,422,976$ & 453.3 & 334,826 & 106.7 & 15 & 0.0 \\
\hline 2013 & 56,485 & 17.9 & 17,375 & 5.5 & 16,929 & 5.4 & 21,819 & 6.9 & 362 & 9.2 & $1,401,906$ & 443.5 & 333,004 & 105.3 & 10 & 0.0 \\
\hline 2014 & 63,454 & 19.9 & 19,999 & 6.3 & 19,452 & 6.1 & 23,541 & 7.4 & 462 & 11.6 & $1,441,789$ & 452.2 & 350,062 & 109.8 & 6 & 0.0 \\
\hline 2015 & 74,707 & 23.2 & 23,872 & 7.4 & 24,173 & 7.5 & 26,170 & 8.1 & 492 & 12.4 & $1,526,658$ & 475.0 & 395,216 & 123.0 & 11 & 0.0 \\
\hline 2016 & 88,053 & 27.3 & 27,814 & 8.6 & 28,924 & 9.0 & 30,676 & 9.5 & 639 & 16.2 & $1,598,354$ & 494.7 & 468,514 & 145.0 & 7 & 0.0 \\
\hline 2017 & 101,567 & 31.4 & 30,644 & 9.5 & 34,013 & 10.5 & 35,992 & 11.1 & 918 & 23.3 & $1,708,569$ & 528.8 & 555,608 & 171.9 & 7 & 0.0 \\
\hline
\end{tabular}

* For 1941-1946, data were reported for the federal fiscal year ending June 30 of the year indicated. From 1947 to the present, data were reported for the calendar year ending December 31. For 1941-1958, data for Alaska and Hawaii were not included.

${ }^{+}$Includes stage of syphilis not stated.

‡ Late and late latent syphilis includes late latent syphilis, latent syphilis of unknown duration, neurosyphilis, late syphilis with clinical manifestations other than neurosyphilis, and late syphilis with clinical manifestations (including late benign syphilis and cardiovascular syphilis).

${ }^{\S}$ Rates include all cases of congenitally acquired syphilis per 100,000 live births. As of 1995, cases of congenital syphilis are obtained in hardcopy and electronic format on the basis of case reporting form CDC 73.126.

$\mathrm{NR}=$ No report.

NOTE: Adjustments to the number of cases reported from state health departments were made for hardcopy forms and for electronic data submissions through June 19, 2018. The number of cases and the rates shown here supersede those published in previous reports. See Section A1.1 in the Appendix for more information. Cases and rates shown in this table exclude the outlying areas of Guam, Puerto Rico, and Virgin Islands. Case definitions have changed over time. See Section C.1 in the Appendix for more information. 
Table 2. Chlamydia - Reported Cases and Rates of Reported Cases by State, Ranked by Rates, United States, 2017

\begin{tabular}{|c|c|c|c|}
\hline Rank* & State & Cases & Rate per 100,000 Population \\
\hline 1 & Alaska & 5,934 & 799.8 \\
\hline 2 & Louisiana & 34,756 & 742.4 \\
\hline 3 & Mississippi & 21,149 & 707.6 \\
\hline 4 & New Mexico & 13,560 & 651.6 \\
\hline 5 & South Carolina & 32,235 & 649.8 \\
\hline 6 & Georgia & 65,104 & 631.4 \\
\hline 7 & North Carolina & 62,876 & 619.7 \\
\hline 8 & Alabama & 29,935 & 615.5 \\
\hline 9 & New York & 116,814 & 591.6 \\
\hline 10 & Illinois & 75,518 & 589.9 \\
\hline 11 & Arkansas & 17,320 & 579.6 \\
\hline 12 & Arizona & 39,598 & 571.3 \\
\hline 13 & Delaware & 5,392 & 566.3 \\
\hline 14 & California & 218,785 & 557.4 \\
\hline 15 & Maryland & 33,416 & 555.4 \\
\hline 16 & Oklahoma & 21,752 & 554.4 \\
\hline 17 & Nevada & 16,260 & 553.1 \\
\hline 18 & Texas & 151,533 & 543.9 \\
\hline \multirow[t]{2}{*}{19} & Missouri & 32,683 & 536.4 \\
\hline & U.S. TOTAL ${ }^{+}$ & $1,708,569$ & 528.8 \\
\hline 20 & Ohio & 61,389 & 528.6 \\
\hline 21 & Tennessee & 35,087 & 527.5 \\
\hline 22 & Indiana & 34,278 & 516.8 \\
\hline 23 & South Dakota & 4,437 & 512.7 \\
\hline 24 & Michigan & 50,595 & 509.6 \\
\hline 25 & Virginia & 42,374 & 503.7 \\
\hline 26 & Rhode Island & 5,282 & 500.0 \\
\hline 27 & Connecticut & 17,750 & 496.3 \\
\hline 28 & Colorado & 26,995 & 487.2 \\
\hline 29 & Florida & 100,018 & 485.2 \\
\hline 30 & Wisconsin & 27,740 & 480.0 \\
\hline 31 & Hawaii & 6,850 & 479.5 \\
\hline 32 & Kansas & 13,554 & 466.2 \\
\hline 33 & Oregon & 18,634 & 455.2 \\
\hline 34 & Nebraska & 8,595 & 450.7 \\
\hline 35 & lowa & 13,893 & 443.2 \\
\hline 36 & Washington & 32,231 & 442.2 \\
\hline 37 & Pennsylvania & 56,447 & 441.5 \\
\hline 38 & Montana & 4,560 & 437.4 \\
\hline 39 & Kentucky & 19,320 & 435.4 \\
\hline 40 & North Dakota & 3,278 & 432.5 \\
\hline 41 & Massachusetts & 29,315 & 430.4 \\
\hline 42 & Minnesota & 23,539 & 426.4 \\
\hline 43 & New Jersey & 35,239 & 394.0 \\
\hline 44 & Idaho & 6,200 & 368.4 \\
\hline 45 & Wyoming & 2,142 & 365.8 \\
\hline 46 & Maine & 4,555 & 342.1 \\
\hline 47 & Utah & 10,135 & 332.2 \\
\hline 48 & New Hampshire & 4,412 & 330.5 \\
\hline 49 & Vermont & 1,858 & 297.5 \\
\hline 50 & West Virginia & 4,140 & 226.1 \\
\hline
\end{tabular}

* States were ranked by rate, then by case count, then in alphabetical order, with rates shown rounded to the nearest tenth.

${ }^{\dagger}$ Total includes cases reported by the District of Columbia with 9,107 cases and a rate of 1337.0, but excludes outlying areas (Guam with 1,107 cases and rate of 663.3 , Puerto Rico with 5,961 cases and rate of 174.7, and Virgin Islands with 458 cases and rate of 425.2). 
Table 3. Chlamydia - Reported Cases and Rates of Reported Cases by State/Area and Region in Alphabetical Order, United States and Outlying Areas, 2013-2017

\begin{tabular}{|c|c|c|c|c|c|c|c|c|c|c|}
\hline \multirow[b]{2}{*}{ State/Area } & \multicolumn{5}{|c|}{ Cases } & \multicolumn{5}{|c|}{ Rates per 100,000 Population } \\
\hline & 2013 & 2014 & 2015 & 2016 & 2017 & 2013 & 2014 & 2015 & 2016 & 2017 \\
\hline Alabama & 29,464 & 29,010 & 26,359 & 26,901 & 29,935 & 609.6 & 598.2 & 542.5 & 553.1 & 615.5 \\
\hline Alaska & 5,774 & 5,789 & 5,660 & 5,698 & 5,934 & 785.4 & 785.8 & 766.5 & 768.0 & 799.8 \\
\hline Arizona & 30,564 & 32,397 & 32,387 & 34,923 & 39,598 & 461.2 & 481.3 & 474.3 & 503.9 & 571.3 \\
\hline Arkansas & 15,447 & 15,605 & 16,166 & 16,737 & 17,320 & 522.0 & 526.1 & 542.8 & 560.1 & 579.6 \\
\hline California & 167,346 & 176,308 & 189,170 & 198,155 & 218,785 & 436.6 & 454.4 & 483.3 & 504.9 & 557.4 \\
\hline Colorado & 20,386 & 21,863 & 23,857 & 25,569 & 26,995 & 387.0 & 408.2 & 437.2 & 461.5 & 487.2 \\
\hline Connecticut & 12,775 & 13,382 & 13,126 & 13,911 & 17,750 & 355.2 & 372.1 & 365.5 & 389.0 & 496.3 \\
\hline Delaware & 5,213 & 4,473 & 4,605 & 5,365 & 5,392 & 563.1 & 478.1 & 486.8 & 563.5 & 566.3 \\
\hline District of Columbia & 6,414 & 5,293 & 7,894 & 7,283 & 9,107 & 992.2 & 803.3 & $1,174.3$ & $1,069.2$ & $1,337.0$ \\
\hline Florida & 80,182 & 84,194 & 90,468 & 94,742 & 100,018 & 410.1 & 423.2 & 446.3 & 459.6 & 485.2 \\
\hline Georgia & 51,070 & 51,945 & 57,639 & 62,776 & 65,104 & 511.1 & 514.4 & 564.3 & 608.9 & 631.4 \\
\hline Hawaii & 6,640 & 6,419 & 7,074 & 6,902 & 6,850 & 472.9 & 452.2 & 494.1 & 483.1 & 479.5 \\
\hline Idaho & 5,428 & 5,442 & 5,631 & 5,897 & 6,200 & 336.7 & 333.0 & 340.3 & 350.4 & 368.4 \\
\hline Illinois & 63,797 & 66,536 & 69,610 & 72,201 & 75,518 & 495.2 & 516.6 & 541.3 & 564.0 & 589.9 \\
\hline Indiana & 28,023 & 28,519 & 28,886 & 30,847 & 34,278 & 426.5 & 432.3 & 436.4 & 465.0 & 516.8 \\
\hline lowa & 10,953 & 11,804 & 12,085 & 12,983 & 13,893 & 354.4 & 379.9 & 386.9 & 414.2 & 443.2 \\
\hline Kansas & 11,012 & 11,116 & 11,464 & 12,160 & 13,554 & 380.5 & 382.8 & 393.7 & 418.3 & 466.2 \\
\hline Kentucky & 17,134 & 17,664 & 17,444 & 18,286 & 19,320 & 389.8 & 400.2 & 394.2 & 412.1 & 435.4 \\
\hline Louisiana & 28,739 & 28,955 & 32,325 & 31,727 & 34,756 & 621.3 & 622.7 & 692.1 & 677.7 & 742.4 \\
\hline Maine & 3,438 & 3,530 & 3,965 & 4,156 & 4,555 & 258.8 & 265.4 & 298.3 & 312.1 & 342.1 \\
\hline Maryland & 26,723 & 27,424 & 27,450 & 30,658 & 33,416 & 450.7 & 458.9 & 457.0 & 509.6 & 555.4 \\
\hline Massachusetts & 23,210 & 21,271 & 24,100 & 26,807 & 29,315 & 346.8 & 315.3 & 354.7 & 393.5 & 430.4 \\
\hline Michigan & 44,835 & 44,256 & 46,486 & 45,936 & 50,595 & 453.1 & 446.6 & 468.5 & 462.7 & 509.6 \\
\hline Minnesota & 18,742 & 19,907 & 21,243 & 22,685 & 23,539 & 345.8 & 364.8 & 387.0 & 411.0 & 426.4 \\
\hline Mississippi & 17,464 & 19,605 & 17,371 & 20,112 & 21,149 & 583.8 & 654.8 & 580.5 & 672.9 & 707.6 \\
\hline Missouri & 27,328 & 27,981 & 28,948 & 30,843 & 32,683 & 452.1 & 461.5 & 475.8 & 506.2 & 536.4 \\
\hline Montana & 3,818 & 4,193 & 4,184 & 4,416 & 4,560 & 376.1 & 409.6 & 405.1 & 423.6 & 437.4 \\
\hline Nebraska & 7,301 & 7,499 & 7,956 & 8,197 & 8,595 & 390.7 & 398.6 & 419.6 & 429.8 & 450.7 \\
\hline Nevada & 11,781 & 11,841 & 12,925 & 14,649 & 16,260 & 422.2 & 417.1 & 447.1 & 498.3 & 553.1 \\
\hline New Hampshire & 3,119 & 3,586 & 3,095 & 3,467 & 4,412 & 235.7 & 270.3 & 232.6 & 259.7 & 330.5 \\
\hline New Jersey & 28,327 & 29,904 & 31,337 & 34,519 & 35,239 & 318.3 & 334.6 & 349.8 & 385.9 & 394.0 \\
\hline New Mexico & 12,249 & 11,558 & 12,632 & 13,108 & 13,560 & 587.4 & 554.2 & 605.8 & 629.9 & 651.6 \\
\hline New York & 95,803 & 98,814 & 103,615 & 109,433 & 116,814 & 487.5 & 500.4 & 523.4 & 554.2 & 591.6 \\
\hline North Carolina & 48,416 & 47,147 & 64,376 & 58,006 & 62,876 & 491.6 & 474.1 & 641.0 & 571.7 & 619.7 \\
\hline North Dakota & 2,932 & 3,451 & 3,159 & 3,455 & 3,278 & 405.3 & 466.7 & 417.3 & 455.8 & 432.5 \\
\hline Ohio & 53,121 & 54,858 & 56,726 & 60,496 & 61,389 & 459.1 & 473.2 & 488.5 & 520.9 & 528.6 \\
\hline Oklahoma & 18,278 & 20,662 & 21,025 & 21,449 & 21,752 & 474.7 & 532.8 & 537.5 & 546.7 & 554.4 \\
\hline Oregon & 14,181 & 15,508 & 16,305 & 17,425 & 18,634 & 360.8 & 390.6 & 404.7 & 425.7 & 455.2 \\
\hline Pennsylvania & 52,056 & 50,536 & 53,460 & 56,930 & 56,447 & 407.5 & 395.2 & 417.6 & 445.3 & 441.5 \\
\hline Rhode Island & 4,312 & 4,349 & 4,575 & 4,936 & 5,282 & 410.1 & 412.2 & 433.1 & 467.2 & 500.0 \\
\hline South Carolina & 25,594 & 28,087 & 27,538 & 28,179 & 32,235 & 536.0 & 581.2 & 562.4 & 568.0 & 649.8 \\
\hline South Dakota & 3,927 & 4,166 & 3,949 & 4,331 & 4,437 & 464.8 & 488.3 & 460.0 & 500.4 & 512.7 \\
\hline Tennessee & 30,370 & 30,793 & 31,272 & 32,304 & 35,087 & 467.5 & 470.2 & 473.8 & 485.7 & 527.5 \\
\hline Texas & 129,861 & 131,219 & 141,158 & 142,952 & 151,533 & 491.0 & 486.8 & 513.9 & 513.1 & 543.9 \\
\hline Utah & 7,535 & 8,223 & 8,633 & 9,457 & 10,135 & 259.7 & 279.4 & 288.2 & 309.9 & 332.2 \\
\hline Vermont & 1,842 & 2,237 & 1,901 & 1,690 & 1,858 & 294.0 & 357.0 & 303.7 & 270.6 & 297.5 \\
\hline Virginia & 33,316 & 36,048 & 35,349 & 39,666 & 42,374 & 403.3 & 432.9 & 421.7 & 471.6 & 503.7 \\
\hline Washington & 24,950 & 26,577 & 28,699 & 31,254 & 32,231 & 357.9 & 376.4 & 400.2 & 428.8 & 442.2 \\
\hline West Virginia & 5,139 & 4,719 & 4,958 & 4,821 & 4,140 & 277.1 & 255.0 & 268.9 & 263.3 & 226.1 \\
\hline Wisconsin & 23,572 & 23,154 & 24,381 & 26,894 & 27,740 & 410.5 & 402.1 & 422.4 & 465.4 & 480.0 \\
\hline Wyoming & 2,005 & 1,972 & 2,037 & 2,060 & 2,142 & 344.1 & 337.6 & 347.5 & 351.8 & 365.8 \\
\hline U.S. TOTAL & $1,401,906$ & $1,441,789$ & $1,526,658$ & $1,598,354$ & $1,708,569$ & 443.5 & 452.2 & 475.0 & 494.7 & 528.8 \\
\hline Northeast & 224,882 & 227,609 & 239,174 & 255,849 & 271,672 & 402.0 & 405.3 & 424.9 & 455.2 & 483.3 \\
\hline Midwest & 295,543 & 303,247 & 314,893 & 331,028 & 349,499 & 437.5 & 447.6 & 463.7 & 487.2 & 514.4 \\
\hline South & 568,824 & 582,843 & 623,397 & 641,964 & 685,514 & 480.5 & 486.6 & 514.4 & 524.8 & 560.4 \\
\hline West & 312,657 & 328,090 & 349,194 & 369,513 & 401,884 & 421.1 & 436.4 & 459.2 & 482.0 & 524.3 \\
\hline Guam & 937 & 839 & 881 & 934 & 1,107 & 584.2 & 521.1 & 544.5 & 559.6 & 663.3 \\
\hline Puerto Rico & 5,969 & 4,899 & 5,295 & 7,198 & 5,961 & 165.1 & 138.1 & 152.4 & 211.0 & 174.7 \\
\hline Virgin Islands & 775 & 791 & 743 & 571 & 458 & 739.9 & 759.3 & 721.7 & 530.1 & 425.2 \\
\hline OUTLYING AREAS & 7,681 & 6,529 & 6,919 & 8,703 & 7,526 & 198.0 & 171.2 & 185.1 & 236.1 & 204.2 \\
\hline TOTAL & $1,409,587$ & $1,448,318$ & $1,533,577$ & $1,607,057$ & $1,716,095$ & 440.5 & 448.9 & 471.6 & 491.7 & 525.1 \\
\hline
\end{tabular}

NOTE: See Section A1.11 in the Appendix for more information on interpreting case counts and rates in outlying areas. 
Table 4. Chlamydia Among Women - Reported Cases and Rates of Reported Cases by State/Area and Region in Alphabetical Order, United States and Outlying Areas, 2013-2017

\begin{tabular}{|c|c|c|c|c|c|c|c|c|c|c|}
\hline \multirow[b]{2}{*}{ State/Area } & \multicolumn{5}{|c|}{ Cases } & \multicolumn{5}{|c|}{ Rates per 100,000 Population } \\
\hline & 2013 & 2014 & 2015 & 2016 & 2017 & 2013 & 2014 & 2015 & 2016 & 2017 \\
\hline Alabama & 21,096 & 20,619 & 18,674 & 19,131 & 20,993 & 847.8 & 825.0 & 745.2 & 762.9 & 837.1 \\
\hline Alaska & 3,899 & 3,940 & 3,786 & 3,807 & 3,941 & $1,115.4$ & $1,127.5$ & $1,083.6$ & $1,076.1$ & $1,114.0$ \\
\hline Arizona & 21,950 & 22,747 & 22,299 & 23,693 & 26,453 & 658.9 & 671.5 & 648.9 & 679.2 & 758.4 \\
\hline Arkansas & 11,334 & 11,625 & 12,088 & 12,216 & 12,453 & 752.7 & 770.4 & 797.7 & 803.5 & 819.1 \\
\hline California & 112,460 & 115,339 & 121,387 & 123,906 & 135,040 & 583.7 & 590.5 & 616.1 & 627.2 & 683.5 \\
\hline Colorado & 14,336 & 14,906 & 16,151 & 16,945 & 17,251 & 546.8 & 559.4 & 595.4 & 615.1 & 626.2 \\
\hline Connecticut & 9,210 & 9,512 & 9,089 & 9,495 & 11,487 & 500.0 & 516.4 & 494.2 & 518.6 & 627.4 \\
\hline Delaware & 3,714 & 3,084 & 3,118 & 3,678 & 3,562 & 777.3 & 638.6 & 638.8 & 748.5 & 724.9 \\
\hline District of Columbia & 3,992 & 3,709 & 4,632 & 4,018 & 4,754 & $1,173.4$ & $1,071.1$ & $1,309.9$ & $1,122.5$ & $1,328.2$ \\
\hline Florida & 56,688 & 58,800 & 62,048 & 63,415 & 66,173 & 567.4 & 578.2 & 598.4 & 601.5 & 627.7 \\
\hline Georgia & 36,559 & 36,871 & 40,302 & 43,377 & 44,080 & 715.3 & 713.1 & 769.9 & 820.0 & 833.3 \\
\hline Hawaii & 4,646 & 4,469 & 4,720 & 4,480 & 4,424 & 669.0 & 637.1 & 668.3 & 630.1 & 622.3 \\
\hline Idaho & 3,885 & 3,895 & 3,963 & 4,022 & 4,198 & 482.6 & 477.3 & 479.7 & 479.0 & 500.0 \\
\hline Illinois & 45,764 & 46,516 & 47,268 & 48,128 & 49,336 & 698.1 & 709.1 & 722.1 & 739.3 & 757.9 \\
\hline Indiana & 20,307 & 20,586 & 20,385 & 21,664 & 23,595 & 609.0 & 615.0 & 607.1 & 644.1 & 701.5 \\
\hline lowa & 7,895 & 8,385 & 8,372 & 8,872 & 9,261 & 507.1 & 536.1 & 532.6 & 563.1 & 587.8 \\
\hline Kansas & 8,323 & 8,276 & 8,325 & 8,688 & 9,472 & 573.2 & 568.1 & 571.0 & 595.3 & 649.0 \\
\hline Kentucky & 12,086 & 12,404 & 12,140 & 12,345 & 13,035 & 541.5 & 553.7 & 540.5 & 548.6 & 579.2 \\
\hline Louisiana & 21,258 & 21,297 & 23,351 & 22,942 & 24,544 & 900.2 & 896.4 & 978.3 & 959.0 & $1,026.0$ \\
\hline Maine & 2,404 & 2,478 & 2,735 & 2,795 & 2,959 & 354.5 & 365.0 & 403.4 & 411.7 & 435.9 \\
\hline Maryland & 19,049 & 19,162 & 18,612 & 20,145 & 21,957 & 623.7 & 622.1 & 601.3 & 649.4 & 707.8 \\
\hline Massachusetts & 15,851 & 14,000 & 15,588 & 17,299 & 18,716 & 459.7 & 402.9 & 445.6 & 493.4 & 533.8 \\
\hline Michigan & 32,056 & 31,470 & 32,425 & 31,497 & 34,120 & 636.5 & 624.2 & 642.8 & 624.5 & 676.5 \\
\hline Minnesota & 12,950 & 13,484 & 14,112 & 14,967 & 15,338 & 474.9 & 491.2 & 511.4 & 539.9 & 553.3 \\
\hline Mississippi & 12,676 & 14,008 & 12,335 & 14,123 & 14,740 & 824.9 & 909.9 & 800.7 & 917.1 & 957.2 \\
\hline Missouri & 19,303 & 19,549 & 19,926 & 20,757 & 21,701 & 626.7 & 632.8 & 643.1 & 669.4 & 699.8 \\
\hline Montana & 2,701 & 2,878 & 2,846 & 2,962 & 3,030 & 534.5 & 564.9 & 554.1 & 572.1 & 585.2 \\
\hline Nebraska & 4,945 & 5,110 & 5,409 & 5,527 & 5,660 & 526.8 & 540.9 & 568.8 & 577.9 & 591.8 \\
\hline Nevada & 8,183 & 8,039 & 8,743 & 9,849 & 10,473 & 591.0 & 569.2 & 607.0 & 671.8 & 714.4 \\
\hline New Hampshire & 2,187 & 2,452 & 2,089 & 2,316 & 2,917 & 326.5 & 365.2 & 310.4 & 343.7 & 432.8 \\
\hline New Jersey & 20,771 & 21,556 & 22,274 & 24,021 & 23,811 & 456.0 & 471.0 & 485.7 & 524.9 & 520.3 \\
\hline New Mexico & 9,033 & 8,395 & 9,227 & 9,306 & 9,328 & 858.9 & 797.5 & 877.4 & 886.0 & 888.1 \\
\hline New York & 64,454 & 65,114 & 66,164 & 67,602 & 70,379 & 637.2 & 640.6 & 649.7 & 665.5 & 692.8 \\
\hline North Carolina & 37,146 & 35,494 & 47,178 & 41,085 & 43,580 & 735.9 & 696.0 & 915.9 & 788.0 & 835.9 \\
\hline North Dakota & 1,923 & 2,202 & 2,028 & 2,187 & 2,127 & 544.2 & 610.9 & 551.0 & 592.7 & 576.5 \\
\hline Ohio & 38,293 & 39,033 & 39,825 & 41,797 & 42,132 & 647.8 & 659.4 & 671.9 & 705.7 & 711.4 \\
\hline Oklahoma & 13,065 & 14,855 & 14,904 & 14,933 & 15,183 & 672.3 & 758.7 & 754.9 & 754.3 & 767.0 \\
\hline Oregon & 9,932 & 10,545 & 11,075 & 11,542 & 12,203 & 500.2 & 525.6 & 544.0 & 558.5 & 590.5 \\
\hline Pennsylvania & 35,657 & 34,170 & 35,201 & 37,030 & 36,201 & 545.9 & 523.0 & 538.4 & 567.7 & 555.0 \\
\hline Rhode Island & 3,044 & 3,037 & 3,064 & 3,278 & 3,454 & 561.5 & 558.5 & 562.9 & 603.3 & 635.7 \\
\hline South Carolina & 19,103 & 20,581 & 19,743 & 19,783 & 22,343 & 779.2 & 828.8 & 784.3 & 774.8 & 875.1 \\
\hline South Dakota & 2,793 & 2,942 & 2,831 & 3,072 & 3,094 & 664.2 & 694.1 & 663.8 & 715.8 & 720.9 \\
\hline Tennessee & 21,057 & 21,203 & 21,112 & 21,714 & 23,348 & 632.6 & 631.6 & 624.1 & 637.0 & 685.0 \\
\hline Texas & 96,923 & 96,959 & 102,141 & 101,618 & 105,995 & 728.7 & 714.3 & 738.5 & 724.2 & 755.4 \\
\hline Utah & 5,050 & 5,414 & 5,704 & 6,031 & 6,606 & 350.2 & 370.0 & 383.1 & 398.0 & 435.9 \\
\hline Vermont & 1,319 & 1,613 & 1,352 & 1,171 & 1,242 & 415.5 & 507.7 & 425.8 & 370.4 & 392.9 \\
\hline Virginia & 23,167 & 24,754 & 23,859 & 26,146 & 27,606 & 551.8 & 585.1 & 560.3 & 611.6 & 645.8 \\
\hline Washington & 17,452 & 18,193 & 19,047 & 20,276 & 20,515 & 500.2 & 515.1 & 531.2 & 556.0 & 562.6 \\
\hline West Virginia & 3,624 & 3,356 & 3,449 & 3,330 & 2,775 & 386.1 & 358.4 & 369.6 & 359.9 & 299.9 \\
\hline Wisconsin & 16,448 & 16,063 & 16,660 & 18,382 & 18,667 & 569.0 & 554.3 & 573.7 & 632.7 & 642.5 \\
\hline Wyoming & 1,387 & 1,352 & 1,387 & 1,356 & 1,399 & 486.0 & 472.5 & 482.9 & 473.2 & 488.2 \\
\hline U.S.TOTAL & 993,348 & $1,006,441$ & $1,045,143$ & $1,072,719$ & $1,127,651$ & 619.0 & 621.6 & 640.4 & 653.9 & 687.4 \\
\hline Northeast & 154,897 & 153,932 & 157,556 & 165,007 & 171,166 & 539.7 & 534.4 & 546.0 & 572.8 & 594.2 \\
\hline Midwest & 211,000 & 213,616 & 217,566 & 225,538 & 234,503 & 615.9 & 621.9 & 632.2 & 655.5 & 681.5 \\
\hline South & 412,537 & 418,781 & 439,686 & 443,999 & 467,121 & 684.1 & 685.9 & 711.7 & 711.8 & 748.8 \\
\hline West & 214,914 & 220,112 & 230,335 & 238,175 & 254,861 & 577.5 & 583.6 & 604.0 & 619.4 & 662.8 \\
\hline Guam & 700 & 595 & 618 & 654 & 753 & 885.8 & 749.6 & 774.4 & 806.8 & 929.0 \\
\hline Puerto Rico & 4,766 & 3,770 & 3,950 & 5,551 & 4,702 & 252.7 & 204.4 & 217.4 & 310.5 & 263.0 \\
\hline Virgin Islands & 579 & 590 & 563 & 405 & 318 & $1,037.1$ & $1,060.7$ & $1,020.6$ & 720.9 & 566.1 \\
\hline OUTLYING AREAS & 6,045 & 4,955 & 5,131 & 6,610 & 5,773 & 299.1 & 250.4 & 262.9 & 343.4 & 299.9 \\
\hline TOTAL & 999,393 & $1,011,396$ & $1,050,274$ & $1,079,329$ & $1,133,424$ & 615.0 & 617.1 & 636.0 & 650.3 & 682.9 \\
\hline
\end{tabular}

NOTE: Cases reported with unknown sex are not included in this table. See Section A1.11 in the Appendix for more information on interpreting case counts and rates in outlying areas. 
Table 5. Chlamydia Among Men - Reported Cases and Rates of Reported Cases by State/Area and Region in Alphabetical Order, United States and Outlying Areas, 2013-2017

\begin{tabular}{|c|c|c|c|c|c|c|c|c|c|c|}
\hline \multirow[b]{2}{*}{ State/Area } & \multicolumn{5}{|c|}{ Cases } & \multicolumn{5}{|c|}{ Rates per 100,000 Population } \\
\hline & 2013 & 2014 & 2015 & 2016 & 2017 & 2013 & 2014 & 2015 & 2016 & 2017 \\
\hline Alabama & 8,201 & 8,318 & 7,549 & 7,585 & 8,837 & 349.7 & 354.0 & 320.8 & 322.0 & 375.2 \\
\hline Alaska & 1,875 & 1,849 & 1,871 & 1,891 & 1,993 & 486.3 & 477.4 & 480.9 & 487.2 & 513.5 \\
\hline Arizona & 8,610 & 9,650 & 10,028 & 11,217 & 13,055 & 261.3 & 288.6 & 295.7 & 325.8 & 379.2 \\
\hline Arkansas & 4,104 & 3,964 & 4,078 & 4,521 & 4,866 & 282.3 & 272.0 & 278.8 & 308.0 & 331.5 \\
\hline California & 54,679 & 60,687 & 67,475 & 73,625 & 83,273 & 286.8 & 314.9 & 347.0 & 377.7 & 427.2 \\
\hline Colorado & 6,050 & 6,957 & 7,706 & 8,624 & 9,744 & 228.6 & 258.5 & 280.9 & 309.6 & 349.8 \\
\hline Connecticut & 3,481 & 3,757 & 3,926 & 4,268 & 5,618 & 198.4 & 214.1 & 224.1 & 244.5 & 321.8 \\
\hline Delaware & 1,499 & 1,389 & 1,487 & 1,687 & 1,830 & 334.6 & 306.8 & 324.8 & 366.2 & 397.2 \\
\hline District of Columbia & 2,400 & 1,555 & 3,108 & 3,112 & 4,279 & 783.7 & 497.4 & 975.5 & 962.8 & $1,323.8$ \\
\hline Florida & 23,300 & 25,239 & 28,332 & 31,275 & 33,811 & 243.7 & 259.6 & 286.1 & 310.6 & 335.8 \\
\hline Georgia & 14,063 & 14,736 & 17,212 & 19,338 & 20,837 & 288.1 & 299.1 & 345.6 & 385.2 & 415.0 \\
\hline Hawaii & 1,994 & 1,950 & 2,352 & 2,421 & 2,426 & 281.0 & 271.6 & 324.2 & 337.4 & 338.1 \\
\hline Idaho & 1,528 & 1,547 & 1,663 & 1,869 & 1,990 & 189.3 & 189.0 & 200.7 & 221.6 & 235.9 \\
\hline Illinois & 17,943 & 19,908 & 21,966 & 24,008 & 26,089 & 283.6 & 315.0 & 347.9 & 381.6 & 414.7 \\
\hline Indiana & 7,708 & 7,921 & 8,492 & 9,174 & 10,673 & 238.2 & 243.8 & 260.3 & 280.6 & 326.4 \\
\hline lowa & 3,058 & 3,419 & 3,712 & 4,111 & 4,631 & 199.4 & 221.6 & 239.2 & 263.7 & 297.0 \\
\hline Kansas & 2,689 & 2,840 & 3,139 & 3,472 & 4,082 & 186.5 & 196.2 & 215.9 & 239.8 & 282.0 \\
\hline Kentucky & 4,989 & 5,194 & 5,273 & 5,590 & 6,194 & 230.6 & 239.0 & 242.0 & 255.7 & 283.3 \\
\hline Louisiana & 7,481 & 7,655 & 8,974 & 8,784 & 10,212 & 330.4 & 336.6 & 392.9 & 383.7 & 446.1 \\
\hline Maine & 1,031 & 1,050 & 1,230 & 1,356 & 1,596 & 158.6 & 161.2 & 188.9 & 207.8 & 244.6 \\
\hline Maryland & 7,654 & 8,237 & 8,780 & 10,479 & 11,449 & 266.2 & 284.4 & 301.6 & 359.6 & 392.8 \\
\hline Massachusetts & 7,341 & 7,197 & 8,406 & 9,433 & 10,517 & 226.2 & 220.0 & 255.0 & 285.4 & 318.2 \\
\hline Michigan & 12,683 & 12,723 & 14,015 & 14,417 & 16,420 & 261.0 & 261.3 & 287.3 & 295.1 & 336.1 \\
\hline Minnesota & 5,791 & 6,414 & 7,122 & 7,703 & 8,183 & 215.0 & 236.5 & 260.9 & 280.4 & 297.8 \\
\hline Mississippi & 4,788 & 5,588 & 5,018 & 5,955 & 6,363 & 329.2 & 384.2 & 345.6 & 411.0 & 439.2 \\
\hline Missouri & 8,025 & 8,432 & 9,022 & 10,086 & 10,982 & 270.8 & 283.5 & 302.2 & 337.1 & 367.0 \\
\hline Montana & 1,116 & 1,314 & 1,338 & 1,454 & 1,528 & 218.9 & 255.6 & 257.6 & 277.1 & 291.2 \\
\hline Nebraska & 2,196 & 2,357 & 2,531 & 2,649 & 2,927 & 236.2 & 251.6 & 267.8 & 278.6 & 307.9 \\
\hline Nevada & 3,590 & 3,786 & 4,152 & 4,777 & 5,741 & 255.4 & 265.4 & 286.2 & 324.1 & 389.5 \\
\hline New Hampshire & 932 & 1,130 & 1,006 & 1,150 & 1,494 & 142.6 & 172.4 & 153.0 & 174.0 & 226.1 \\
\hline New Jersey & 7,476 & 8,272 & 9,025 & 10,435 & 11,362 & 172.1 & 189.6 & 206.4 & 238.9 & 260.1 \\
\hline New Mexico & 3,209 & 3,148 & 3,400 & 3,794 & 4,229 & 310.5 & 304.8 & 329.0 & 368.1 & 410.3 \\
\hline New York & 31,273 & 33,634 & 37,346 & 41,722 & 46,349 & 327.9 & 351.0 & 388.6 & 435.2 & 483.4 \\
\hline North Carolina & 11,254 & 11,638 & 17,195 & 16,918 & 19,295 & 234.4 & 240.2 & 351.5 & 343.0 & 391.1 \\
\hline North Dakota & 1,009 & 1,249 & 1,131 & 1,268 & 1,151 & 272.7 & 329.5 & 290.9 & 326.0 & 295.9 \\
\hline Ohio & 14,828 & 15,825 & 16,901 & 18,699 & 19,257 & 262.0 & 278.9 & 297.2 & 328.5 & 338.3 \\
\hline Oklahoma & 5,213 & 5,802 & 6,121 & 6,516 & 6,569 & 273.3 & 302.2 & 316.0 & 335.2 & 337.9 \\
\hline Oregon & 4,243 & 4,953 & 5,223 & 5,876 & 6,412 & 218.2 & 252.2 & 262.0 & 289.9 & 316.3 \\
\hline Pennsylvania & 16,360 & 16,315 & 18,201 & 19,840 & 20,184 & 262.1 & 260.9 & 290.5 & 316.9 & 322.4 \\
\hline Rhode Island & 1,268 & 1,312 & 1,511 & 1,656 & 1,825 & 248.9 & 256.6 & 295.1 & 322.8 & 355.7 \\
\hline South Carolina & 6,432 & 7,376 & 7,705 & 8,286 & 9,807 & 276.9 & 314.0 & 323.9 & 344.1 & 407.3 \\
\hline South Dakota & 1,134 & 1,224 & 1,118 & 1,259 & 1,343 & 267.2 & 285.1 & 258.8 & 288.6 & 307.8 \\
\hline Tennessee & 9,311 & 9,587 & 10,158 & 10,584 & 11,736 & 294.0 & 300.3 & 315.7 & 326.4 & 361.9 \\
\hline Texas & 31,980 & 34,110 & 38,539 & 40,992 & 45,170 & 243.2 & 254.9 & 282.6 & 296.4 & 326.6 \\
\hline Utah & 2,485 & 2,808 & 2,929 & 3,424 & 3,516 & 170.3 & 189.8 & 194.3 & 222.9 & 228.9 \\
\hline Vermont & 523 & 622 & 549 & 518 & 611 & 169.2 & 201.4 & 177.9 & 167.9 & 198.1 \\
\hline Virginia & 10,112 & 11,244 & 11,460 & 13,395 & 14,311 & 248.9 & 274.6 & 277.8 & 323.8 & 345.9 \\
\hline Washington & 7,498 & 8,384 & 9,651 & 10,975 & 11,713 & 215.3 & 237.5 & 269.2 & 301.4 & 321.7 \\
\hline West Virginia & 1,514 & 1,363 & 1,509 & 1,491 & 1,365 & 165.4 & 149.1 & 165.6 & 164.6 & 150.7 \\
\hline Wisconsin & 7,114 & 7,077 & 7,703 & 8,487 & 9,058 & 249.4 & 247.5 & 268.6 & 295.4 & 315.2 \\
\hline Wyoming & 617 & 619 & 643 & 704 & 741 & 207.5 & 207.7 & 215.1 & 235.5 & 247.9 \\
\hline U.S. TOTAL & 405,652 & 433,325 & 478,981 & 522,870 & 577,644 & 260.6 & 276.1 & 302.7 & 328.7 & 363.1 \\
\hline Northeast & 69,685 & 73,289 & 81,200 & 90,378 & 99,556 & 255.8 & 268.0 & 296.1 & 329.8 & 363.3 \\
\hline Midwest & 84,178 & 89,389 & 96,852 & 105,333 & 114,796 & 252.9 & 267.7 & 289.2 & 314.1 & 342.3 \\
\hline South & 154,295 & 162,995 & 182,498 & 196,508 & 216,931 & 265.7 & 277.6 & 307.2 & 327.8 & 361.9 \\
\hline West & 97,494 & 107,652 & 118,431 & 130,651 & 146,361 & 263.2 & 287.3 & 312.4 & 342.0 & 383.1 \\
\hline Guam & 234 & 244 & 263 & 280 & 354 & 287.6 & 298.9 & 320.8 & 326.2 & 412.4 \\
\hline Puerto Rico & 1,203 & 1,126 & 1,319 & 1,647 & 1,255 & 69.6 & 66.1 & 79.6 & 101.4 & 77.3 \\
\hline Virgin Islands & 196 & 201 & 180 & 166 & 140 & 400.7 & 414.0 & 376.7 & 322.1 & 271.7 \\
\hline OUTLYING AREAS & 1,633 & 1,571 & 1,762 & 2,093 & 1,749 & 87.8 & 85.6 & 98.6 & 118.8 & 99.3 \\
\hline TOTAL & 407,285 & 434,896 & 480,743 & 524,963 & 579,393 & 258.6 & 273.9 & 300.4 & 326.4 & 360.2 \\
\hline
\end{tabular}

NOTE: Cases reported with unknown sex are not included in this table. See Section A1.11 in the Appendix for more information on interpreting case counts and rates in outlying areas. 
Table 6. Chlamydia - Reported Cases and Rates of Reported Cases in Selected Metropolitan Statistical Areas (MSAs)* in Alphabetical Order, United States, 2013-2017

\begin{tabular}{|c|c|c|c|c|c|c|c|c|c|c|}
\hline \multirow[b]{2}{*}{ MSAs } & \multicolumn{5}{|c|}{ Cases } & \multicolumn{5}{|c|}{ Rates per 100,000 Population } \\
\hline & 2013 & 2014 & 2015 & 2016 & 2017 & 2013 & 2014 & 2015 & 2016 & 2017 \\
\hline Atlanta-Sandy Springs-Roswell, GA & $16,429^{\dagger}$ & 25,744 & $19,106^{+}$ & 33,273 & 34,189 & $297.5^{+}$ & 458.5 & $334.6^{+}$ & 574.7 & 590.5 \\
\hline Austin-Round Rock, TX & 10,138 & 10,920 & 11,679 & 12,299 & 13,015 & 538.4 & 561.9 & 583.7 & 598.1 & 632.9 \\
\hline Baltimore-Columbia-Towson, MD & 13,749 & 14,095 & 14,016 & 15,829 & 16,766 & 496.2 & 505.9 & 501.0 & 565.5 & 599.0 \\
\hline Birmingham-Hoover, AL & 6,552 & 6,309 & 5,839 & $4,982^{\ddagger}$ & 7,388 & 574.6 & 551.6 & 509.7 & $434.2^{\ddagger}$ & 643.9 \\
\hline Boston-Cambridge-Newton, MA-NH & 16,127 & $14,264^{+}$ & $14,378^{+}$ & $15,880^{\dagger}$ & $18,232^{+}$ & 344.3 & $301.4^{+}$ & $301.2^{+}$ & $331.2^{+}$ & $380.3^{\dagger}$ \\
\hline Buffalo-Cheektowaga-Niagara Falls, NY & 5,724 & 5,841 & 5,900 & 6,252 & 6,584 & 504.7 & 514.0 & 519.7 & 551.9 & 581.2 \\
\hline Charlotte-Concord-Gastonia, NC-SC & 11,418 & 11,766 & 16,284 & 14,314 & 15,901 & 488.9 & 494.3 & 671.1 & 578.5 & 642.6 \\
\hline Chicago-Naperville-Elgin, IL-IN-WI & 47,837 & 51,457 & 54,248 & 56,478 & 59,342 & 501.6 & 538.6 & 568.0 & 593.7 & 623.8 \\
\hline Cincinnati, OH-KY-IN & 10,207 & 10,516 & 11,219 & 11,392 & 11,205 & 477.5 & 489.2 & 519.9 & 526.2 & 517.5 \\
\hline Cleveland-Elyria, $\mathrm{OH}$ & 12,126 & 11,363 & 11,312 & 12,475 & 13,773 & 587.3 & 550.6 & 548.9 & 606.9 & 670.0 \\
\hline Columbus, $\mathrm{OH}$ & 9,734 & 10,258 & 11,327 & 12,113 & 11,921 & 494.8 & 514.3 & 560.3 & 593.3 & 583.9 \\
\hline Dallas-Fort Worth-Arlington, TX & 30,684 & 30,549 & 35,900 & 32,771 & 37,263 & 450.5 & 439.3 & 505.4 & 453.1 & 515.2 \\
\hline Denver-Aurora-Lakewood, CO & 12,131 & 13,346 & 13,942 & 14,282 & 15,520 & 449.7 & 484.6 & 495.4 & 500.6 & 544.0 \\
\hline Detroit-Warren-Dearborn, MI & 22,567 & 21,012 & 22,238 & 21,966 & 24,278 & 525.4 & 489.0 & 516.9 & 511.1 & 564.9 \\
\hline Hartford-West Hartford-East Hartford, CT & 4,311 & 4,713 & 4,689 & 4,898 & $6,153^{+}$ & 354.8 & 388.1 & 387.1 & 405.9 & $509.8^{\dagger}$ \\
\hline Houston-The Woodlands-Sugar Land, TX & 29,120 & 30,554 & 32,823 & 35,594 & 35,807 & 461.3 & 470.8 & 493.1 & 525.6 & 528.7 \\
\hline Indianapolis-Carmel-Anderson, IN & 11,835 & 11,952 & 11,544 & 12,794 & 13,701 & 605.7 & 606.3 & 580.4 & 638.3 & 683.6 \\
\hline Jacksonville, FL & 7,138 & 7,391 & 8,012 & 8,434 & 8,763 & 511.8 & 520.8 & 552.7 & 570.6 & 592.8 \\
\hline Kansas City, MO-KS & 9,513 & 9,866 & 10,240 & 11,043 & 11,868 & 463.0 & 476.4 & 490.5 & 524.7 & 563.9 \\
\hline Las Vegas-Henderson-Paradise, NV & 9,286 & 9,485 & 10,049 & 11,362 & 11,898 & 457.9 & 458.3 & 475.2 & 527.1 & 551.9 \\
\hline Los Angeles-Long Beach-Anaheim, CA & 59,386 & 64,263 & 68,285 & 71,943 & 78,312 & 452.2 & 484.6 & 511.9 & 540.5 & 588.3 \\
\hline Louisville-Jefferson County, KY-IN & 6,384 & 6,751 & 6,735 & 6,881 & 7,086 & 505.8 & 531.7 & 526.8 & 536.1 & 552.1 \\
\hline Memphis, TN-MS-AR & 10,763 & 10,554 & 10,342 & 10,365 & 11,718 & 802.2 & 785.7 & 769.4 & 771.9 & 872.6 \\
\hline Miami-Fort Lauderdale-West Palm Beach, FL & 22,821 & 24,599 & 26,746 & 28,070 & 29,430 & 391.6 & 414.8 & 444.9 & 462.7 & 485.1 \\
\hline Milwaukee-Waukesha-West Allis, WI & 10,754 & 10,303 & 10,645 & 11,891 & 12,187 & 685.1 & 655.3 & 675.6 & 756.2 & 775.0 \\
\hline Minneapolis-St. Paul-Bloomington, MN-WI & $12,227^{\dagger}$ & $13,589^{+}$ & $14,709^{\dagger}$ & 15,584 & 16,901 & $353.5^{\dagger}$ & $388.8^{+}$ & $417.3^{+}$ & 438.9 & 475.9 \\
\hline Nashville-Davidson-Murfreesboro-Franklin, TN & 7,356 & 7,878 & 8,066 & 8,196 & 8,928 & 418.5 & 439.5 & 440.7 & 439.4 & 478.6 \\
\hline New Orleans-Metairie, LA & 8,134 & 8,595 & 9,291 & 9,626 & 9,850 & 655.5 & 686.6 & 735.7 & 758.6 & 776.3 \\
\hline New York-Newark-Jersey City, NY-NJ-PA & 89,211 & 93,515 & 97,835 & 105,463 & 112,808 & 447.2 & 465.4 & 484.8 & 523.3 & 559.7 \\
\hline Oklahoma City, OK & 6,190 & 7,293 & 7,633 & 7,693 & 8,198 & 469.1 & 545.6 & 561.9 & 560.2 & 597.0 \\
\hline Orlando-Kissimmee-Sanford, FL & 10,230 & 11,001 & 12,026 & 12,492 & 13,870 & 451.1 & 473.9 & 503.8 & 511.7 & 568.1 \\
\hline Philadelphia-Camden-Wilmington, PA-NJ-DE-MD & 34,741 & 33,376 & 34,910 & 38,182 & 38,661 & 575.7 & 551.6 & 575.1 & 629.0 & 636.9 \\
\hline Phoenix-Mesa-Scottsdale, AZ & 20,164 & 21,576 & 21,795 & 23,567 & 26,939 & 458.4 & 480.6 & 476.4 & 505.6 & 577.9 \\
\hline Pittsburgh, PA & 8,605 & 8,059 & 8,604 & 8,623 & 7,801 & 364.5 & 342.1 & 365.7 & 368.1 & 333.0 \\
\hline Portland-Vancouver-Hillsboro, OR-WA & 8,536 & 9,283 & 9,982 & 11,052 & 11,461 & 368.8 & 395.3 & 417.8 & 455.8 & 472.6 \\
\hline Providence-Warwick, RI-MA & 5,828 & $5,695^{+}$ & $5,907^{+}$ & $6,668^{+}$ & $6,977^{\dagger}$ & 363.3 & $353.9^{+}$ & $366.2^{\dagger}$ & $412.9^{\dagger}$ & $432.1^{\dagger}$ \\
\hline Raleigh, NC & 4,966 & 5,126 & 6,911 & 6,493 & 7,243 & 408.9 & 412.4 & 542.6 & 498.3 & 555.9 \\
\hline Richmond, VA & 6,817 & 7,817 & 7,878 & 8,592 & 8,562 & 547.2 & 620.4 & 619.7 & 670.4 & 668.0 \\
\hline Riverside-San Bernardino-Ontario, CA & 19,819 & 19,560 & 20,778 & 20,081 & 24,294 & 452.4 & 440.4 & 462.8 & 443.5 & 536.5 \\
\hline Sacramento-Roseville-Arden-Arcade, CA & 9,771 & 9,674 & 10,621 & 10,892 & 12,408 & 441.0 & 431.0 & 467.0 & 474.3 & 540.3 \\
\hline Salt Lake City, UT & 3,947 & 4,423 & 4,751 & 5,264 & 5,516 & 346.1 & 383.5 & 406.0 & 443.8 & 465.0 \\
\hline San Antonio-New Braunfels, TX & 13,335 & 11,573 & 14,465 & 15,149 & 14,580 & 585.5 & 497.0 & 606.7 & 623.5 & 600.1 \\
\hline San Diego-Carlsbad, CA & 14,706 & 15,754 & 17,378 & 18,937 & 20,832 & 458.0 & 482.7 & 526.7 & 570.8 & 627.9 \\
\hline San Francisco-Oakland-Hayward, CA & 18,254 & 20,377 & 23,519 & 24,894 & 27,850 & 404.2 & 443.6 & 505.1 & 532.0 & 595.2 \\
\hline San Jose-Sunnyvale-Santa Clara, CA & 6,717 & 6,278 & 6,898 & 7,166 & 7,877 & 349.9 & 321.5 & 348.9 & 362.1 & 398.1 \\
\hline Seattle-Tacoma-Bellevue, WA & 12,971 & 13,861 & 15,257 & 16,886 & 17,832 & 359.3 & 377.5 & 408.6 & 444.5 & 469.4 \\
\hline St. Louis, MO-IL & 14,783 & 14,711 & 14,961 & 15,512 & 16,469 & 527.8 & 524.2 & 532.1 & 552.6 & 586.7 \\
\hline Tampa-St. Petersburg-Clearwater, FL & 12,752 & 12,952 & 13,472 & 13,996 & 14,512 & 444.2 & 444.2 & 452.8 & 461.6 & 478.6 \\
\hline Virginia Beach-Norfolk-Newport News, VA-NC & 11,852 & 12,192 & 11,281 & 13,223 & 13,571 & 694.2 & 710.2 & 654.0 & 765.7 & 785.9 \\
\hline Washington-Arlington-Alexandria, DC-VA-MD-WV & 23,531 & 18,342 & 18,890 & 21,269 & 33,134 & 395.5 & 304.0 & 309.8 & 346.9 & 540.3 \\
\hline SELECTED MSAs TOTAL & 792,177 & 820,371 & 865,316 & 923,081 & 999,374 & 458.3 & 469.1 & 489.6 & 518.8 & 561.7 \\
\hline
\end{tabular}

* MSAs were selected on the basis of the largest population in the 2010 U.S. Census.

${ }^{\dagger}$ The variable used to identify county, which is used to classify cases into MSAs, was complete for $\leq 95 \%$ of cases in a state contributing data to this MSA. See

Section A1.4 in the Appendix for more information.

${ }^{\ddagger} 2016$ county data for Alabama have been corrected and may not match previous reports. 
Table 7. Chlamydia Among Women - Reported Cases and Rates of Reported Cases in Selected Metropolitan Statistical Areas (MSAs)* in Alphabetical Order, United States, 2013-2017

\begin{tabular}{|c|c|c|c|c|c|c|c|c|c|c|}
\hline \multirow[b]{2}{*}{ MSAs } & \multicolumn{5}{|c|}{ Cases } & \multicolumn{5}{|c|}{ Rates per 100,000 Population } \\
\hline & 2013 & 2014 & 2015 & 2016 & 2017 & 2013 & 2014 & 2015 & 2016 & 2017 \\
\hline Atlanta-Sandy Springs-Roswell, GA & $11,221^{\dagger}$ & 17,564 & $12,640^{+}$ & 22,235 & 22,204 & $395.0^{+}$ & 607.2 & $429.0^{+}$ & 743.6 & 742.5 \\
\hline Austin-Round Rock, TX & 6,691 & 7,513 & 7,779 & 7,827 & 8,216 & 712.3 & 773.7 & 777.8 & 761.8 & 799.6 \\
\hline Baltimore-Columbia-Towson, MD & 9,848 & 9,780 & 9,453 & 10,359 & 11,007 & 686.9 & 678.0 & 652.7 & 714.5 & 759.2 \\
\hline Birmingham-Hoover, AL & 4,486 & 4,300 & 3,940 & $3,386^{\ddagger}$ & 4,985 & 759.2 & 724.5 & 662.3 & $568.9^{\ddagger}$ & 837.5 \\
\hline Boston-Cambridge-Newton, MA-NH & 10,791 & $9,243^{+}$ & $9,137^{+}$ & $9,987^{+}$ & $11,314^{+}$ & 447.5 & $379.6^{+}$ & $372.0^{+}$ & $405.1^{\dagger}$ & $458.9^{\dagger}$ \\
\hline Buffalo-Cheektowaga-Niagara Falls, NY & 4,024 & 4,077 & 4,035 & 4,142 & 4,383 & 688.0 & 696.2 & 690.1 & 710.1 & 751.4 \\
\hline Charlotte-Concord-Gastonia, NC-SC & 8,605 & 8,633 & 11,672 & 10,033 & 10,810 & 717.0 & 705.1 & 935.2 & 786.8 & 847.8 \\
\hline Chicago-Naperville-Elgin, IL-IN-WI & 34,216 & 35,696 & 36,547 & 37,102 & 38,191 & 702.5 & 730.9 & 749.1 & 764.1 & 786.5 \\
\hline Cincinnati, OH-KY-IN & 7,527 & 7,724 & 8,050 & 8,052 & 7,848 & 690.0 & 703.9 & 731.0 & 729.4 & 710.9 \\
\hline Cleveland-Elyria, $\mathrm{OH}$ & 8,550 & 7,914 & 7,815 & 8,601 & 9,167 & 799.4 & 740.8 & 732.5 & 809.0 & 862.3 \\
\hline Columbus, $\mathrm{OH}$ & 6,749 & 6,895 & 7,704 & 8,027 & 7,864 & 674.7 & 680.3 & 750.2 & 774.2 & 758.5 \\
\hline Dallas-Fort Worth-Arlington, TX & 22,744 & 22,213 & 25,902 & 22,719 & 25,893 & 658.7 & 628.6 & 717.3 & 617.7 & 704.0 \\
\hline Denver-Aurora-Lakewood, CO & 8,447 & 9,020 & 9,265 & 9,360 & 9,652 & 624.1 & 652.7 & 656.7 & 654.8 & 675.2 \\
\hline Detroit-Warren-Dearborn, MI & 16,152 & 14,822 & 15,410 & 14,957 & 16,198 & 730.5 & 670.3 & 696.5 & 677.4 & 733.6 \\
\hline Hartford-West Hartford-East Hartford, CT & 3,109 & 3,349 & 3,226 & 3,307 & $3,945^{\dagger}$ & 499.0 & 538.2 & 520.0 & 535.5 & $638.8^{\dagger}$ \\
\hline Houston-The Woodlands-Sugar Land, TX & 22,027 & 22,832 & 23,828 & 25,470 & 25,253 & 695.0 & 699.6 & 711.6 & 747.1 & 740.8 \\
\hline Indianapolis-Carmel-Anderson, IN & 8,149 & 8,398 & 7,816 & 8,604 & 9,090 & 816.1 & 832.9 & 768.7 & 839.5 & 887.0 \\
\hline Jacksonville, FL & 5,131 & 5,238 & 5,637 & 5,757 & 5,905 & 717.4 & 719.3 & 758.6 & 759.4 & 778.9 \\
\hline Kansas City, MO-KS & 6,795 & 6,991 & 7,108 & 7,544 & 7,947 & 649.2 & 662.4 & 668.8 & 703.9 & 741.5 \\
\hline Las Vegas-Henderson-Paradise, NV & 6,571 & 6,486 & 6,885 & 7,697 & 7,642 & 650.5 & 627.4 & 650.6 & 712.5 & 707.4 \\
\hline Los Angeles-Long Beach-Anaheim, CA & 38,456 & 40,401 & 42,385 & 43,278 & 46,649 & 578.5 & 600.7 & 626.6 & 641.1 & 691.0 \\
\hline Louisville-Jefferson County, KY-IN & 4,574 & 4,827 & 4,686 & 4,735 & 4,741 & 708.1 & 742.6 & 716.8 & 721.7 & 722.6 \\
\hline Memphis, TN-MS-AR & 7,717 & 7,758 & 7,238 & 7,324 & 8,164 & $1,105.5$ & $1,109.2$ & $1,033.5$ & $1,046.4$ & $1,166.4$ \\
\hline Miami-Fort Lauderdale-West Palm Beach, FL & 15,645 & 16,473 & 17,461 & 17,881 & 18,277 & 521.9 & 539.6 & 563.6 & 572.6 & 585.2 \\
\hline Milwaukee-Waukesha-West Allis, WI & 7,463 & 7,183 & 7,242 & 8,013 & 8,155 & 926.6 & 890.0 & 895.8 & 994.2 & $1,011.8$ \\
\hline Minneapolis-St. Paul-Bloomington, MN-WI & $8,293^{+}$ & $8,957^{\dagger}$ & $9,497^{+}$ & 9,958 & 10,694 & $474.2^{+}$ & $507.0^{+}$ & $533.5^{+}$ & 555.8 & 596.9 \\
\hline Nashville-Davidson-Murfreesboro-Franklin, TN & 5,084 & 5,278 & 5,322 & 5,374 & 5,846 & 565.0 & 574.5 & 567.4 & 562.0 & 611.4 \\
\hline New Orleans-Metairie, LA & 6,062 & 6,301 & 6,710 & 6,843 & 6,665 & 948.7 & 975.3 & $1,028.6$ & $1,044.5$ & $1,017.3$ \\
\hline New York-Newark-Jersey City, NY-NJ-PA & 60,539 & 62,097 & 62,905 & 65,594 & 68,082 & 587.7 & 598.4 & 603.9 & 630.7 & 654.6 \\
\hline Oklahoma City, OK & 4,430 & 5,255 & 5,420 & 5,308 & 5,669 & 662.5 & 775.0 & 786.4 & 762.5 & 814.3 \\
\hline Orlando-Kissimmee-Sanford, FL & 7,503 & 8,021 & 8,505 & 8,593 & 9,252 & 648.3 & 675.8 & 696.6 & 688.5 & 741.3 \\
\hline Philadelphia-Camden-Wilmington, PA-NJ-DE-MD & 23,532 & 22,317 & 22,967 & 24,813 & 24,713 & 754.4 & 713.7 & 732.2 & 791.6 & 788.4 \\
\hline Phoenix-Mesa-Scottsdale, AZ & 14,206 & 14,841 & 14,607 & 15,621 & 17,504 & 642.4 & 656.9 & 634.3 & 665.8 & 746.1 \\
\hline Pittsburgh, PA & 6,046 & 5,509 & 5,681 & 5,551 & 4,923 & 497.4 & 454.6 & 469.9 & 461.8 & 409.5 \\
\hline Portland-Vancouver-Hillsboro, OR-WA & 5,809 & 6,158 & 6,559 & 7,076 & 7,178 & 496.1 & 518.4 & 542.7 & 577.2 & 585.5 \\
\hline Providence-Warwick, RI-MA & 4,124 & $3,945^{+}$ & $3,989^{+}$ & $4,501^{\dagger}$ & $4,622^{+}$ & 498.7 & $475.6^{+}$ & $479.9^{+}$ & $541.6^{+}$ & $556.2^{+}$ \\
\hline Raleigh, NC & 3,490 & 3,502 & 4,685 & 4,244 & 4,721 & 561.5 & 550.1 & 718.3 & 634.9 & 706.3 \\
\hline Richmond, VA & 4,792 & 5,311 & 5,330 & 5,725 & 5,653 & 744.7 & 815.9 & 811.1 & 863.5 & 852.6 \\
\hline Riverside-San Bernardino-Ontario, CA & 14,536 & 13,988 & 14,693 & 13,893 & 16,830 & 661.1 & 626.6 & 651.4 & 610.5 & 739.5 \\
\hline Sacramento-Roseville-Arden-Arcade, CA & 6,915 & 6,686 & 7,284 & 7,282 & 8,123 & 611.8 & 583.6 & 627.3 & 620.5 & 692.1 \\
\hline Salt Lake City, UT & 2,541 & 2,873 & 3,067 & 3,275 & 3,520 & 448.0 & 500.3 & 526.4 & 554.6 & 596.1 \\
\hline San Antonio-New Braunfels, TX & 9,576 & 8,158 & 10,005 & 10,466 & 9,924 & 829.5 & 690.9 & 828.4 & 850.9 & 806.8 \\
\hline San Diego-Carlsbad, CA & 9,684 & 10,211 & 11,154 & 11,690 & 12,791 & 606.3 & 628.9 & 680.1 & 708.7 & 775.5 \\
\hline San Francisco-Oakland-Hayward, CA & 10,845 & 11,509 & 12,508 & 12,900 & 13,965 & 474.3 & 494.3 & 530.5 & 544.7 & 589.7 \\
\hline San Jose-Sunnyvale-Santa Clara, CA & 4,530 & 4,100 & 4,328 & 4,445 & 4,754 & 474.6 & 422.3 & 440.8 & 453.4 & 484.9 \\
\hline Seattle-Tacoma-Bellevue, WA & 8,411 & 8,751 & 9,306 & 10,085 & 10,454 & 465.4 & 476.5 & 498.4 & 530.8 & 550.2 \\
\hline St. Louis, MO-IL & 10,364 & 10,271 & 10,158 & 10,417 & 11,016 & 717.6 & 709.9 & 701.1 & 720.8 & 762.2 \\
\hline Tampa-St. Petersburg-Clearwater, FL & 8,948 & 9,066 & 9,269 & 9,360 & 9,743 & 604.2 & 602.5 & 603.7 & 598.4 & 622.9 \\
\hline Virginia Beach-Norfolk-Newport News, VA-NC & 8,259 & 8,425 & 7,677 & 8,791 & 8,873 & 952.5 & 966.9 & 877.4 & $1,002.4$ & $1,011.7$ \\
\hline Washington-Arlington-Alexandria, DC-VA-MD-WV & 15,768 & 12,501 & 12,517 & 13,635 & 20,072 & 518.1 & 405.0 & 401.3 & 434.6 & 639.7 \\
\hline SELECTED MSAs TOTAL & 549,975 & 559,361 & 577,004 & 601,837 & 639,087 & 623.8 & 626.7 & 639.8 & 663.0 & 704.1 \\
\hline
\end{tabular}

* MSAs were selected on the basis of the largest population in the 2010 U.S. Census.

'The variable used to identify county, which is used to classify cases into MSAs, was complete for $\leq 95 \%$ of cases in a state contributing data to this MSA. See Section A1.4 in the Appendix for more information.

₹ 2016 county data for Alabama have been corrected and may not match previous reports.

NOTE: Cases reported with unknown sex are not included in this table. 
Table 8. Chlamydia Among Men - Reported Cases and Rates of Reported Cases in Selected Metropolitan Statistical Areas (MSAs)* in Alphabetical Order, United States, 2013-2017

\begin{tabular}{|c|c|c|c|c|c|c|c|c|c|c|}
\hline \multirow[b]{2}{*}{ MSAs } & \multicolumn{5}{|c|}{ Cases } & \multicolumn{5}{|c|}{ Rates per 100,000 Population } \\
\hline & 2013 & 2014 & 2015 & 2016 & 2017 & 2013 & 2014 & 2015 & 2016 & 2017 \\
\hline Atlanta-Sandy Springs-Roswell, GA & $5,061^{\dagger}$ & 7,979 & $6,429^{+}$ & 11,000 & 11,888 & $188.7^{\dagger}$ & 293.2 & $232.6^{+}$ & 392.9 & 424.7 \\
\hline Austin-Round Rock, TX & 2,592 & 3,372 & 3,871 & 4,444 & 4,775 & 274.7 & 346.8 & 386.8 & 431.9 & 464.1 \\
\hline Baltimore-Columbia-Towson, MD & 3,889 & 4,294 & 4,514 & 5,446 & 5,755 & 290.9 & 319.6 & 334.6 & 403.7 & 426.6 \\
\hline Birmingham-Hoover, AL & 2,031 & 1,990 & 1,886 & $1,573^{\ddagger}$ & 2,377 & 369.7 & 361.6 & 342.4 & $284.9^{\ddagger}$ & 430.5 \\
\hline Boston-Cambridge-Newton, MA-NH & 5,328 & $4,988^{+}$ & $5,211^{+}$ & $5,861^{+}$ & $6,887^{+}$ & 234.4 & $217.2^{+}$ & $224.8^{+}$ & $251.7^{+}$ & $295.7^{\dagger}$ \\
\hline Buffalo-Cheektowaga-Niagara Falls, NY & 1,700 & 1,764 & 1,865 & 2,110 & 2,201 & 309.5 & 320.3 & 338.8 & 384.0 & 400.5 \\
\hline Charlotte-Concord-Gastonia, NC-SC & 2,804 & 3,125 & 4,607 & 4,278 & 5,085 & 247.0 & 270.4 & 391.0 & 356.7 & 424.0 \\
\hline Chicago-Naperville-Elgin, IL-IN-WI & 13,553 & 15,679 & 17,517 & 19,320 & 21,071 & 290.4 & 335.7 & 374.9 & 414.8 & 452.4 \\
\hline Cincinnati, OH-KY-IN & 2,676 & 2,787 & 3,168 & 3,333 & 3,352 & 255.7 & 264.9 & 299.9 & 314.1 & 315.9 \\
\hline Cleveland-Elyria, $\mathrm{OH}$ & 3,576 & 3,449 & 3,497 & 3,874 & 4,606 & 359.4 & 346.5 & 351.8 & 390.3 & 464.1 \\
\hline Columbus, $\mathrm{OH}$ & 2,985 & 3,363 & 3,623 & 4,086 & 4,057 & 308.7 & 342.8 & 364.2 & 406.7 & 403.8 \\
\hline Dallas-Fort Worth-Arlington, TX & 7,916 & 8,313 & 9,941 & 10,006 & 11,342 & 235.7 & 243.0 & 284.7 & 281.4 & 319.0 \\
\hline Denver-Aurora-Lakewood, CO & 3,684 & 4,326 & 4,677 & 4,922 & 5,868 & 274.1 & 315.2 & 333.3 & 345.8 & 412.2 \\
\hline Detroit-Warren-Dearborn, MI & 6,350 & 6,153 & 6,795 & 6,992 & 8,048 & 304.7 & 295.0 & 325.2 & 334.6 & 385.2 \\
\hline Hartford-West Hartford-East Hartford, CT & 1,181 & 1,333 & 1,443 & 1,541 & $1,982^{+}$ & 199.4 & 225.1 & 244.2 & 261.5 & $336.4^{+}$ \\
\hline Houston-The Woodlands-Sugar Land, TX & 7,078 & 7,700 & 8,939 & 10,004 & 10,434 & 225.1 & 238.6 & 270.2 & 297.4 & 310.2 \\
\hline Indianapolis-Carmel-Anderson, IN & 3,681 & 3,544 & 3,723 & 4,185 & 4,604 & 385.3 & 368.0 & 383.0 & 427.3 & 470.1 \\
\hline Jacksonville, FL & 1,989 & 2,138 & 2,367 & 2,672 & 2,854 & 292.7 & 309.4 & 335.1 & 371.1 & 396.3 \\
\hline Kansas City, MO-KS & 2,718 & 2,875 & 3,132 & 3,499 & 3,921 & 269.7 & 283.1 & 305.6 & 338.8 & 379.7 \\
\hline Las Vegas-Henderson-Paradise, NV & 2,708 & 2,986 & 3,139 & 3,646 & 4,228 & 266.1 & 288.3 & 297.1 & 339.1 & 393.2 \\
\hline Los Angeles-Long Beach-Anaheim, CA & 20,831 & 23,766 & 25,764 & 28,299 & 31,501 & 321.3 & 363.6 & 391.8 & 431.4 & 480.2 \\
\hline Louisville-Jefferson County, KY-IN & 1,781 & 1,896 & 2,031 & 2,131 & 2,323 & 289.0 & 305.9 & 325.1 & 339.7 & 370.3 \\
\hline Memphis, TN-MS-AR & 3,046 & 2,795 & 3,104 & 3,035 & 3,546 & 473.2 & 434.1 & 482.2 & 472.1 & 551.5 \\
\hline Miami-Fort Lauderdale-West Palm Beach, FL & 7,134 & 8,086 & 9,270 & 10,172 & 11,141 & 252.0 & 281.1 & 318.1 & 345.6 & 378.5 \\
\hline Milwaukee-Waukesha-West Allis, WI & 3,284 & 3,111 & 3,394 & 3,860 & 4,021 & 429.7 & 406.6 & 442.3 & 503.6 & 524.6 \\
\hline Minneapolis-St. Paul-Bloomington, MN-WI & $3,933^{+}$ & $4,623^{+}$ & $5,206^{+}$ & 5,614 & 6,192 & $229.9^{\dagger}$ & $267.5^{+}$ & $298.4^{+}$ & 319.1 & 351.9 \\
\hline Nashville-Davidson-Murfreesboro-Franklin, TN & 2,271 & 2,598 & 2,744 & 2,822 & 3,081 & 264.6 & 297.3 & 307.5 & 310.4 & 338.9 \\
\hline New Orleans-Metairie, LA & 2,072 & 2,294 & 2,581 & 2,783 & 3,185 & 344.2 & 378.7 & 422.7 & 453.4 & 518.9 \\
\hline New York-Newark-Jersey City, NY-NJ-PA & 28,546 & 31,310 & 34,799 & 39,725 & 44,597 & 295.8 & 322.3 & 356.3 & 407.3 & 457.3 \\
\hline Oklahoma City, OK & 1,760 & 2,036 & 2,213 & 2,385 & 2,529 & 270.4 & 309.1 & 330.7 & 352.3 & 373.5 \\
\hline Orlando-Kissimmee-Sanford, FL & 2,708 & 2,964 & 3,511 & 3,896 & 4,616 & 243.9 & 261.2 & 301.0 & 326.5 & 386.9 \\
\hline Philadelphia-Camden-Wilmington, PA-NJ-DE-MD & 11,178 & 11,010 & 11,912 & 13,315 & 13,889 & 383.4 & 376.5 & 406.1 & 453.5 & 473.1 \\
\hline Phoenix-Mesa-Scottsdale, AZ & 5,957 & 6,735 & 7,173 & 7,944 & 9,354 & 272.3 & 302.0 & 315.7 & 343.1 & 404.0 \\
\hline Pittsburgh, PA & 2,550 & 2,541 & 2,909 & 3,062 & 2,876 & 222.6 & 222.1 & 254.3 & 268.5 & 252.2 \\
\hline Portland-Vancouver-Hillsboro, OR-WA & 2,723 & 3,124 & 3,416 & 3,970 & 4,271 & 238.1 & 269.2 & 289.3 & 331.1 & 356.2 \\
\hline Providence-Warwick, RI-MA & 1,702 & $1,744^{+}$ & $1,913^{+}$ & $2,163^{+}$ & $2,350^{+}$ & 218.9 & $223.6^{+}$ & $244.7^{+}$ & $276.0^{+}$ & $299.9^{\dagger}$ \\
\hline Raleigh, NC & 1,474 & 1,622 & 2,226 & 2,248 & 2,522 & 248.6 & 267.5 & 358.3 & 354.3 & 397.5 \\
\hline Richmond, VA & 2,011 & 2,503 & 2,540 & 2,827 & 2,797 & 333.9 & 410.9 & 413.5 & 456.9 & 452.1 \\
\hline Riverside-San Bernardino-Ontario, CA & 5,271 & 5,542 & 6,070 & 6,141 & 7,415 & 241.5 & 250.8 & 271.8 & 272.7 & 329.3 \\
\hline Sacramento-Roseville-Arden-Arcade, CA & 2,846 & 2,976 & 3,322 & 3,580 & 4,255 & 262.2 & 270.9 & 298.5 & 318.9 & 379.0 \\
\hline Salt Lake City, UT & 1,406 & 1,550 & 1,684 & 1,989 & 1,991 & 245.2 & 267.7 & 286.6 & 333.9 & 334.2 \\
\hline San Antonio-New Braunfels, TX & 3,757 & 3,412 & 4,459 & 4,683 & 4,652 & 334.5 & 297.2 & 379.1 & 390.4 & 387.8 \\
\hline San Diego-Carlsbad, CA & 5,013 & 5,508 & 6,190 & 7,195 & 8,017 & 310.6 & 335.9 & 373.0 & 431.3 & 480.6 \\
\hline San Francisco-Oakland-Hayward, CA & 7,370 & 8,823 & 10,961 & 11,936 & 13,788 & 330.5 & 389.4 & 476.9 & 516.5 & 596.6 \\
\hline San Jose-Sunnyvale-Santa Clara, CA & 2,186 & 2,163 & 2,565 & 2,717 & 3,109 & 226.5 & 220.3 & 257.8 & 272.1 & 311.4 \\
\hline Seattle-Tacoma-Bellevue, WA & 4,560 & 5,110 & 5,951 & 6,799 & 7,375 & 253.0 & 278.5 & 318.8 & 358.0 & 388.4 \\
\hline St. Louis, MO-IL & 4,411 & 4,429 & 4,762 & 5,091 & 5,450 & 325.1 & 325.8 & 349.4 & 373.9 & 400.2 \\
\hline Tampa-St. Petersburg-Clearwater, FL & 3,752 & 3,835 & 4,195 & 4,632 & 4,768 & 270.0 & 271.8 & 291.3 & 315.5 & 324.8 \\
\hline Virginia Beach-Norfolk-Newport News, VA-NC & 3,584 & 3,748 & 3,595 & 4,406 & 4,593 & 426.5 & 443.4 & 423.0 & 518.4 & 540.4 \\
\hline Washington-Arlington-Alexandria, DC-VA-MD-WV & 7,725 & 5,813 & 6,359 & 7,595 & 12,856 & 265.8 & 197.2 & 213.5 & 253.6 & 429.3 \\
\hline SELECTED MSAs TOTAL & 240,342 & 259,825 & 287,163 & 319,807 & 358,395 & 283.8 & 303.4 & 331.8 & 367.0 & 411.3 \\
\hline
\end{tabular}

* MSAs were selected on the basis of the largest population in the 2010 U.S. Census,

'The variable used to identify county, which is used to classify cases into MSAs, was complete for $\leq 95 \%$ of cases in a state contributing data to this MSA. See Section A1.4 in the Appendix for more information.

${ }^{\ddagger} 2016$ county data for Alabama have been corrected and may not match previous reports.

NOTE: Cases reported with unknown sex are not included in this table. 
Table 9. Chlamydia - Reported Cases and Rates of Reported Cases in Counties and Independent Cities* Ranked by Number of Reported Cases, United States, 2017

\begin{tabular}{|c|c|c|c|c|}
\hline Rank* & County/Independent City & Cases & Rate per 100,000 Population & Cumulative Percentage \\
\hline 1 & Los Angeles County, CA & 64,302 & 634.3 & 3 \\
\hline 2 & Cook County, IL & 42,422 & 815.3 & 6 \\
\hline 3 & Harris County, TX & 27,556 & 600.4 & 7 \\
\hline 4 & Maricopa County, AZ & 25,336 & 597.1 & 9 \\
\hline 5 & Kings County, NY & 21,137 & 803.9 & 10 \\
\hline 6 & Philadelphia County, PA & 21,119 & $1,347.0$ & 11 \\
\hline 7 & San Diego County, CA & 20,832 & 627.9 & 13 \\
\hline 8 & Dallas County, TX & 18,417 & 715.2 & 14 \\
\hline 9 & Bronx County, NY & 17,711 & $1,216.6$ & 15 \\
\hline 10 & New York County, NY & 16,670 & $1,014.2$ & 16 \\
\hline 11 & Wayne County, MI & 15,636 & 893.8 & 17 \\
\hline 12 & Queens County, NY & 14,421 & 618.1 & 17 \\
\hline 13 & Orange County, CA & 14,010 & 441.6 & 18 \\
\hline 14 & San Bernardino County, CA & 13,144 & 614.2 & 19 \\
\hline 15 & Bexar County, TX & 12,986 & 673.3 & 20 \\
\hline 16 & Miami-Dade County, FL & 12,264 & 452.1 & 20 \\
\hline 17 & Clark County, NV & 11,898 & 551.9 & 21 \\
\hline 18 & Broward County, FL & 11,283 & 590.8 & 22 \\
\hline 19 & Riverside County, CA & 11,150 & 467.0 & 22 \\
\hline 20 & Cuyahoga County, $\mathrm{OH}$ & 11,044 & 884.0 & 23 \\
\hline 21 & Milwaukee County, WI & 10,928 & $1,148.6$ & 24 \\
\hline 22 & Marion County, IN & 10,536 & $1,119.4$ & 24 \\
\hline 23 & Sacramento County, CA & 9,852 & 650.5 & 25 \\
\hline 24 & King County, WA & 9,777 & 454.8 & 26 \\
\hline 25 & Orange County, FL & 9,523 & 724.5 & 26 \\
\hline 26 & Franklin County, $\mathrm{OH}$ & 9,409 & 744.1 & 27 \\
\hline 27 & Alameda County, CA & 9,200 & 558.4 & 27 \\
\hline 28 & San Francisco County, CA & 9,137 & $1,049.2$ & 28 \\
\hline 29 & Washington, D.C. & 9,107 & $1,337.0$ & 28 \\
\hline 30 & Shelby County, TN & 9,003 & 963.3 & 29 \\
\hline 31 & Travis County, TX & 8,946 & 745.9 & 29 \\
\hline 32 & Mecklenburg County, NC & 8,828 & 836.9 & 30 \\
\hline 33 & Tarrant County, TX & 8,625 & 427.6 & 30 \\
\hline 34 & Fulton County, GA & 8,510 & 831.6 & 31 \\
\hline 35 & Hillsborough County, FL & 8,323 & 604.8 & 31 \\
\hline 36 & Hennepin County, MN & 8,156 & 661.8 & 32 \\
\hline 37 & Santa Clara County, CA & 7,655 & 398.8 & 32 \\
\hline 38 & Baltimore (City), MD & 7,636 & $1,242.3$ & 33 \\
\hline 39 & Prince George's County, MD & 7,364 & 811.0 & 33 \\
\hline 40 & Fresno County, CA & 7,198 & 734.6 & 34 \\
\hline 41 & Denver County, CO & 6,928 & 999.6 & 34 \\
\hline 42 & Duval County, FL & 6,925 & 747.6 & 34 \\
\hline 43 & Kern County, CA & 6,866 & 776.0 & 35 \\
\hline 44 & Hamilton County, $\mathrm{OH}$ & 6,861 & 848.0 & 35 \\
\hline 45 & Pima County, AZ & 6,625 & 651.9 & 36 \\
\hline 46 & St. Louis County, MO & 6,161 & 617.0 & 36 \\
\hline 47 & Wake County, NC & 6,078 & 580.6 & 36 \\
\hline 48 & Essex County, NJ & 6,064 & 760.9 & 37 \\
\hline 49 & Jefferson County, $\mathrm{AL}$ & 6,031 & 914.5 & 37 \\
\hline 50 & Palm Beach County, FL & 5,883 & 407.5 & 37 \\
\hline 51 & Jackson County, MO & 5,856 & 846.5 & 38 \\
\hline 52 & DeKalb County, GA & 5,854 & 790.7 & 38 \\
\hline 53 & Contra Costa County, CA & 5,731 & 504.9 & 38 \\
\hline 54 & Oklahoma County, OK & 5,693 & 727.1 & 39 \\
\hline 55 & Erie County, NY & 5,571 & 604.9 & 39 \\
\hline 56 & Jefferson County, KY & 5,445 & 711.4 & 39 \\
\hline 57 & Pierce County, WA & 5,421 & 629.4 & 40 \\
\hline 58 & Honolulu County, $\mathrm{HI}$ & 5,389 & 542.9 & 40 \\
\hline 59 & Salt Lake County, UT & 5,322 & 474.6 & 40 \\
\hline 60 & Middlesex County, MA & 5,250 & 330.2 & 41 \\
\hline 61 & Hartford County, CT & 5,248 & 588.1 & 41 \\
\hline 62 & Allegheny County, PA & 5,223 & 426.2 & 41 \\
\hline 63 & Multnomah County, OR & 5,198 & 649.9 & 41 \\
\hline 64 & New Haven County, CT & 4,993 & 582.7 & 42 \\
\hline 65 & Monroe County, NY & 4,988 & 667.1 & 42 \\
\hline 66 & Guilford County, NC & 4,985 & 956.2 & 42 \\
\hline 67 & Bernalillo County, NM & 4,932 & 728.6 & 43 \\
\hline 68 & Suffolk County, NY & 4,917 & 329.4 & 43 \\
\hline 69 & El Paso County, TX & 4,880 & 582.4 & 43 \\
\hline 70 & Collin County, TX & 4,867 & 518.0 & 43 \\
\hline
\end{tabular}

* The top 70 counties and independent cities ranked in descending order by number of cases reported in 2017 then by rate are displayed.

NOTE: Relative rankings of counties may be impacted by completeness of the variable used to identify county. In 2017 , the variable used to identify county was complete for $\leq 95 \%$ of cases in Connecticut and Massachusetts. See Section A1.4 in the Appendix for more information. 
Table 10. Chlamydia - Reported Cases and Rates of Reported Cases by Age Group and Sex, United States, 2013-2017

\begin{tabular}{|c|c|c|c|c|c|c|c|c|}
\hline \multirow{2}{*}{$\begin{array}{l}\text { Age } \\
\text { Group }\end{array}$} & \multicolumn{4}{|c|}{ Cases } & \multicolumn{3}{|c|}{ Rates per 100,000 Population* } & \\
\hline & Total & Male & Female & Unknown Sex & Total & Male & Female & \\
\hline $0-4$ & 681 & 266 & 402 & 13 & 3.4 & 2.6 & 4.1 & \multirow{14}{*}{$\underset{\mathbf{\omega}}{\mathbf{0}}$} \\
\hline $5-9$ & 145 & 20 & 123 & 2 & 0.7 & 0.2 & 1.2 & \\
\hline $10-14$ & 12,585 & 1,554 & 11,001 & 30 & 60.9 & 14.7 & 108.9 & \\
\hline $15-19$ & 395,612 & 78,404 & 316,438 & 770 & $1,869.7$ & 722.9 & $3,068.4$ & \\
\hline $20-24$ & 553,658 & 153,102 & 399,545 & 1,011 & $2,428.8$ & $1,310.9$ & $3,594.2$ & \\
\hline $25-29$ & 233,429 & 82,190 & 150,733 & 506 & $1,081.7$ & 749.9 & $1,419.3$ & \\
\hline $30-34$ & 103,675 & 41,017 & 62,414 & 244 & 487.6 & 384.0 & 589.8 & \\
\hline $35-39$ & 46,991 & 20,157 & 26,720 & 114 & 239.7 & 206.0 & 272.1 & \\
\hline $40-44$ & 24,774 & 12,200 & 12,501 & 73 & 118.8 & 117.8 & 119.2 & \\
\hline $45-54$ & 21,511 & 12,180 & 9,299 & 32 & 49.1 & 56.5 & 41.9 & \\
\hline $55-64$ & 5,424 & 3,154 & 2,259 & 11 & 13.8 & 16.6 & 11.1 & \\
\hline $65+$ & 1,377 & 750 & 616 & 11 & 3.1 & 3.8 & 2.5 & \\
\hline Unknown Age & 2,044 & 658 & 1,297 & 89 & & & & \\
\hline TOTAL & $1,401,906$ & 405,652 & 993,348 & 2,906 & 443.5 & 260.6 & 619.0 & \\
\hline $0-4$ & 603 & 200 & 388 & 15 & 3.0 & 2.0 & 4.0 & \multirow{14}{*}{ 궁 } \\
\hline $5-9$ & 181 & 26 & 152 & 3 & 0.9 & 0.2 & 1.5 & \\
\hline $10-14$ & 11,406 & 1,342 & 10,041 & 23 & 55.2 & 12.7 & 99.2 & \\
\hline $15-19$ & 381,717 & 77,908 & 303,294 & 515 & $1,811.9$ & 722.4 & $2,949.3$ & \\
\hline $20-24$ & 566,385 & 159,804 & 405,876 & 705 & $2,472.0$ & $1,361.3$ & $3,632.7$ & \\
\hline $25-29$ & 253,825 & 91,729 & 161,793 & 303 & $1,154.4$ & 821.8 & $1,494.4$ & \\
\hline $30-34$ & 113,208 & 45,990 & 67,060 & 158 & 525.9 & 425.5 & 625.6 & \\
\hline $35-39$ & 52,536 & 22,894 & 29,545 & 97 & 263.7 & 230.3 & 296.0 & \\
\hline $40-44$ & 27,426 & 13,711 & 13,662 & 53 & 133.2 & 134.2 & 131.7 & \\
\hline $45-54$ & 24,773 & 14,318 & 10,424 & 31 & 57.0 & 66.8 & 47.3 & \\
\hline $55-64$ & 6,527 & 3,911 & 2,603 & 13 & 16.3 & 20.2 & 12.5 & \\
\hline $65+$ & 1,449 & 871 & 570 & 8 & 3.1 & 4.3 & 2.2 & \\
\hline Unknown Aqe & 1,753 & 621 & 1,033 & 99 & & & & \\
\hline TOTAL & $1,441,789$ & 433,325 & $1,006,441$ & 2,023 & 452.2 & 276.1 & 621.6 & \\
\hline $0-4$ & 518 & 196 & 322 & 0 & 2.6 & 1.9 & 3.3 & \multirow{14}{*}{$\underset{\mathrm{v}}{\mathrm{O}}$} \\
\hline $5-9$ & 148 & 18 & 130 & 0 & 0.7 & 0.2 & 1.3 & \\
\hline $10-14$ & 10,642 & 1,216 & 9,394 & 32 & 51.6 & 11.6 & 93.0 & \\
\hline $15-19$ & 391,396 & 82,775 & 307,937 & 684 & $1,854.2$ & 766.6 & $2,986.5$ & \\
\hline $20-24$ & 589,963 & 172,313 & 416,772 & 878 & $2,594.5$ & $1,476.8$ & $3,764.4$ & \\
\hline $25-29$ & 280,429 & 104,679 & 175,291 & 459 & $1,248.5$ & 917.5 & $1,586.0$ & \\
\hline $30-34$ & 123,866 & 52,019 & 71,653 & 194 & 571.5 & 477.7 & 664.3 & \\
\hline $35-39$ & 59,905 & 27,180 & 32,621 & 104 & 294.0 & 267.2 & 319.8 & \\
\hline $40-44$ & 30,379 & 15,210 & 15,118 & 51 & 150.3 & 151.6 & 148.4 & \\
\hline $45-54$ & 28,833 & 17,011 & 11,764 & 58 & 66.8 & 79.9 & 53.7 & \\
\hline $55-64$ & 7,756 & 4,901 & 2,840 & 15 & 19.0 & 24.9 & 13.4 & \\
\hline $65+$ & 1,596 & 1,043 & 546 & 7 & 3.3 & 4.9 & 2.0 & \\
\hline Unknown Age & 1,227 & 420 & 755 & 52 & & & & \\
\hline TOTAL & $1,526,658$ & 478,981 & $1,045,143$ & 2,534 & 475.0 & 302.7 & 640.4 & \\
\hline $0-4$ & 597 & 225 & 368 & 4 & 3.0 & 2.2 & 3.8 & \multirow{14}{*}{ 웅 } \\
\hline $5-9$ & 188 & 25 & 161 & 2 & 0.9 & 0.2 & 1.6 & \\
\hline $10-14$ & 10,571 & 1,341 & 9,206 & 24 & 51.3 & 12.7 & 91.2 & \\
\hline $15-19$ & 407,230 & 89,899 & 316,639 & 692 & $1,927.3$ & 832.3 & $3,065.8$ & \\
\hline $20-24$ & 601,173 & 181,857 & 418,388 & 928 & $2,686.1$ & $1,582.6$ & $3,842.0$ & \\
\hline $25-29$ & 298,176 & 114,484 & 183,222 & 470 & $1,302.6$ & 984.3 & $1,627.3$ & \\
\hline $30-34$ & 133,062 & 58,583 & 74,226 & 253 & 610.8 & 534.1 & 686.1 & \\
\hline $35-39$ & 66,669 & 31,671 & 34,872 & 126 & 320.9 & 305.2 & 335.4 & \\
\hline $40-44$ & 32,548 & 16,784 & 15,705 & 59 & 165.2 & 171.7 & 158.3 & \\
\hline $45-54$ & 32,316 & 19,569 & 12,683 & 64 & 75.5 & 92.7 & 58.5 & \\
\hline $55-64$ & 9,321 & 5,942 & 3,354 & 25 & 22.5 & 29.7 & 15.6 & \\
\hline $65+$ & 1,772 & 1,161 & 597 & 14 & 3.6 & 5.3 & 2.2 & \\
\hline Unknown Age & 4,731 & 1,329 & 3,298 & 104 & & & & \\
\hline TOTAL & $1,598,354$ & 522,870 & $1,072,719$ & 2,765 & 494.7 & 328.7 & 653.9 & \\
\hline $0-4$ & 514 & 188 & 323 & 3 & 2.6 & 1.8 & 3.3 & \multirow{14}{*}{$\begin{array}{l}\mathbf{O} \\
\overrightarrow{\mathbf{V}}\end{array}$} \\
\hline $5-9$ & 167 & 9 & 158 & 0 & 0.8 & 0.1 & 1.6 & \\
\hline $10-14$ & 10,726 & 1,252 & 9,454 & 20 & 52.0 & 11.9 & 93.6 & \\
\hline 15-19 & 437,904 & 99,864 & 337,290 & 750 & $2,072.4$ & 924.5 & $3,265.7$ & \\
\hline $20-24$ & 631,207 & 195,971 & 434,050 & 1,186 & $2,820.3$ & $1,705.4$ & $3,985.8$ & \\
\hline $25-29$ & 321,857 & 127,007 & 194,267 & 583 & $1,406.0$ & $1,091.9$ & $1,725.4$ & \\
\hline $30-34$ & 144,451 & 65,690 & 78,502 & 259 & 663.0 & 598.9 & 725.7 & \\
\hline $35-39$ & 74,202 & 36,427 & 37,621 & 154 & 357.2 & 351.1 & 361.8 & \\
\hline $40-44$ & 36,332 & 19,310 & 16,940 & 82 & 184.5 & 197.5 & 170.8 & \\
\hline $45-54$ & 36,229 & 22,431 & 13,713 & 85 & 84.7 & 106.3 & 63.3 & \\
\hline $55-64$ & 11,356 & 7,490 & 3,840 & 26 & 27.4 & 37.5 & 17.9 & \\
\hline $65+$ & 2,178 & 1,461 & 698 & 19 & 4.4 & 6.7 & 2.5 & \\
\hline Unknown Age & 1,446 & 544 & 795 & 107 & & & & \\
\hline TOTAL & $1,708,569$ & 577,644 & $1,127,651$ & 3,274 & 528.8 & 363.1 & 687.4 & \\
\hline
\end{tabular}

* No population data are available for unknown sex and age; therefore, rates are not calculated.

NOTE: This table should be used only for age comparisons. Cases in the 0-4 age group may include cases due to perinatal transmission. 
Table 11A. Chlamydia — Reported Cases by Race/Hispanic Ethnicity, Age Group, and Sex, United States, 2017

\begin{tabular}{|c|c|c|c|c|c|c|c|c|c|c|c|c|}
\hline \multirow{2}{*}{$\begin{array}{l}\text { Age } \\
\text { Group }\end{array}$} & \multicolumn{3}{|c|}{$\begin{array}{c}\text { American Indians/ } \\
\text { Alaska Natives }\end{array}$} & \multicolumn{3}{|c|}{ Asians } & \multicolumn{3}{|c|}{ Blacks } & \multicolumn{3}{|c|}{$\begin{array}{c}\text { Native Hawaiians/ } \\
\text { Other Pacific Islanders }\end{array}$} \\
\hline & Total* & Male & Female & Total* & Male & Female & Total* & Male & Female & Total* & Male & Female \\
\hline $0-4$ & 4 & 1 & 3 & 1 & 0 & 1 & 134 & 48 & 86 & 3 & 1 & 2 \\
\hline $5-9$ & 2 & 0 & 2 & 3 & 0 & 3 & 43 & 4 & 39 & 1 & 0 & 1 \\
\hline $10-14$ & 194 & 20 & 174 & 48 & 2 & 46 & 4,081 & 617 & 3,459 & 20 & 4 & 16 \\
\hline $15-19$ & 4,387 & 872 & 3,515 & 3,375 & 503 & 2,870 & 138,790 & 39,191 & 99,492 & 809 & 121 & 688 \\
\hline $20-24$ & 5,944 & 1,372 & 4,565 & 7,832 & 2,170 & 5,653 & 174,035 & 60,833 & 113,046 & 1,518 & 345 & 1,170 \\
\hline $25-29$ & 3,945 & 1,117 & 2,826 & 5,044 & 2,098 & 2,936 & 88,305 & 36,656 & 51,573 & 836 & 245 & 591 \\
\hline $30-34$ & 2,108 & 594 & 1,513 & 2,849 & 1,276 & 1,568 & 34,856 & 17,316 & 17,514 & 443 & 167 & 276 \\
\hline $35-39$ & 1,087 & 378 & 708 & 1,526 & 761 & 762 & 16,171 & 8,929 & 7,234 & 234 & 96 & 138 \\
\hline $40-44$ & 479 & 161 & 318 & 945 & 521 & 423 & 7,175 & 4,484 & 2,687 & 83 & 37 & 45 \\
\hline $45-54$ & 376 & 153 & 223 & 978 & 564 & 408 & 6,716 & 4,583 & 2,129 & 77 & 43 & 34 \\
\hline $55-64$ & 104 & 57 & 47 & 311 & 157 & 154 & 2,154 & 1,477 & 672 & 28 & 15 & 13 \\
\hline $65+$ & 14 & 9 & 5 & 61 & 43 & 18 & 326 & 227 & 98 & 2 & 1 & 1 \\
\hline Unknown Age & 7 & 1 & 6 & 24 & 10 & 13 & 246 & 119 & 123 & 4 & 1 & 3 \\
\hline TOTAL & 18,651 & 4,735 & 13,905 & 22,997 & 8,105 & 14,855 & 473,032 & 174,484 & 298,152 & 4,058 & 1,076 & 2,978 \\
\hline
\end{tabular}

\begin{tabular}{|c|c|c|c|c|c|c|c|c|c|c|c|c|}
\hline \multirow{2}{*}{$\begin{array}{l}\text { Age } \\
\text { Group }\end{array}$} & \multicolumn{3}{|c|}{ Whites } & \multicolumn{3}{|c|}{ Multirace } & \multicolumn{3}{|c|}{ Hispanics } & \multicolumn{3}{|c|}{$\begin{array}{l}\text { Other/ } \\
\text { Unknown }\end{array}$} \\
\hline & Total* & Male & Female & Total* & Male & Female & Total* & Male & Female & Total* & Male & Female \\
\hline $0-4$ & 86 & 37 & 49 & 2 & 2 & 0 & 61 & 17 & 44 & 223 & 82 & 138 \\
\hline $5-9$ & 22 & 2 & 20 & 2 & 0 & 2 & 36 & 1 & 35 & 58 & 2 & 56 \\
\hline $10-14$ & 1,895 & 95 & 1,797 & 100 & 7 & 93 & 1,370 & 135 & 1,234 & 3,018 & 372 & 2,635 \\
\hline $15-19$ & 101,211 & 17,027 & 84,129 & 3,075 & 564 & 2,511 & 56,164 & 11,094 & 45,033 & 130,093 & 30,492 & 99,052 \\
\hline $20-24$ & 159,863 & 45,452 & 114,290 & 3,989 & 1,114 & 2,873 & 83,245 & 22,760 & 60,423 & 194,781 & 61,925 & 132,030 \\
\hline $25-29$ & 77,826 & 30,193 & 47,575 & 1,902 & 848 & 1,052 & 45,461 & 16,751 & 28,655 & 98,538 & 39,099 & 59,059 \\
\hline $30-34$ & 35,739 & 16,283 & 19,437 & 907 & 562 & 345 & 21,961 & 9,360 & 12,577 & 45,588 & 20,132 & 25,272 \\
\hline $35-39$ & 18,463 & 9,331 & 9,119 & 475 & 324 & 150 & 11,759 & 5,207 & 6,530 & 24,487 & 11,401 & 12,980 \\
\hline $40-44$ & 8,768 & 4,907 & 3,849 & 244 & 174 & 69 & 5,996 & 2,817 & 3,171 & 12,642 & 6,209 & 6,378 \\
\hline $45-54$ & 10,095 & 7,063 & 3,026 & 248 & 205 & 43 & 4,810 & 2,745 & 2,053 & 12,929 & 7,075 & 5,797 \\
\hline $55-64$ & 3,484 & 2,750 & 734 & 64 & 60 & 4 & 1,011 & 560 & 450 & 4,200 & 2,414 & 1,766 \\
\hline $65+$ & 711 & 551 & 160 & 10 & 9 & 1 & 148 & 79 & 69 & 906 & 542 & 346 \\
\hline Unknown Age & 232 & 99 & 126 & 1 & 0 & 1 & 209 & 68 & 140 & 723 & 246 & 383 \\
\hline TOTAL & 418,395 & 133,790 & 284,311 & 11,019 & 3,869 & 7,144 & 232,231 & 71,594 & 160,414 & 528,186 & 179,991 & 345,892 \\
\hline
\end{tabular}

* Total includes cases reported with unknown sex.

NOTE: These tables should be used only for race/Hispanic ethnicity comparisons. See Table 10 for age-specific cases and rates and Tables 3-5 for total and sexspecific cases and rates. Cases in the 0-4 age group may include cases due to perinatal transmission. 
Table 11B. Chlamydia - Rates of Reported Cases per 100,000 Population by Race/Hispanic Ethnicity, Age Group, and Sex, United States, 2017

\begin{tabular}{|c|c|c|c|c|c|c|c|c|c|c|c|c|}
\hline \multirow{2}{*}{$\begin{array}{l}\text { Age } \\
\text { Group }\end{array}$} & \multicolumn{3}{|c|}{$\begin{array}{l}\text { American Indians/ } \\
\text { Alaska Natives }\end{array}$} & \multicolumn{3}{|c|}{ Asians } & \multicolumn{3}{|c|}{ Blacks } & \multicolumn{3}{|c|}{$\begin{array}{c}\text { Native Hawaiians/ } \\
\text { Other Pacific Islanders }\end{array}$} \\
\hline & Total* & Male & Female & Total* & Male & Female & Total* & Male & Female & Total* & Male & Female \\
\hline $0-4$ & 2.4 & 1.2 & 3.7 & 0.1 & 0.0 & 0.2 & 4.9 & 3.4 & 6.4 & 7.3 & 4.8 & 10.1 \\
\hline $5-9$ & 1.1 & 0.0 & 2.3 & 0.3 & 0.0 & 0.6 & 1.5 & 0.3 & 2.8 & 2.4 & 0.0 & 5.0 \\
\hline $10-14$ & 110.2 & 22.4 & 200.7 & 4.7 & 0.4 & 9.0 & 146.1 & 43.6 & 250.8 & 49.0 & 19.5 & 78.8 \\
\hline 15-19 & $2,411.9$ & 943.3 & $3,929.4$ & 321.8 & 95.1 & 552.1 & $4,653.0$ & $2,589.3$ & $6,771.6$ & $1,998.1$ & 581.5 & $3,495.9$ \\
\hline $20-24$ & $3,064.9$ & $1,380.4$ & $4,828.4$ & 620.1 & 338.5 & 908.9 & $5,276.1$ & $3,627.4$ & $6,971.7$ & $3,394.8$ & $1,498.7$ & $5,392.7$ \\
\hline $25-29$ & $2,071.6$ & $1,148.1$ & $3,034.1$ & 332.1 & 282.2 & 378.6 & $2,711.6$ & $2,264.3$ & $3,149.2$ & $1,604.8$ & 902.7 & $2,368.5$ \\
\hline $30-34$ & $1,278.1$ & 719.8 & 1,836.1 & 186.0 & 174.7 & 195.7 & $1,245.2$ & $1,280.4$ & $1,210.6$ & 888.6 & 654.8 & $1,133.5$ \\
\hline $35-39$ & 718.1 & 504.2 & 926.7 & 106.4 & 114.1 & 99.3 & 606.3 & 705.5 & 516.2 & 535.5 & 428.0 & 648.8 \\
\hline $40-44$ & 340.8 & 232.5 & 446.0 & 66.9 & 78.9 & 56.3 & 288.3 & 383.0 & 203.8 & 221.3 & 194.8 & 243.1 \\
\hline $45-54$ & 123.3 & 103.2 & 142.3 & 40.1 & 49.7 & 31.3 & 127.3 & 185.2 & 76.0 & 109.2 & 123.3 & 95.4 \\
\hline $55-64$ & 36.6 & 42.8 & 31.2 & 15.9 & 17.7 & 14.3 & 45.7 & 68.5 & 26.2 & 49.5 & 54.2 & 45.0 \\
\hline $65+$ & 5.4 & 7.7 & 3.5 & 2.9 & 4.7 & 1.5 & 7.4 & 12.8 & 3.7 & 4.1 & 4.4 & 3.8 \\
\hline \multicolumn{13}{|c|}{ Unknown Age } \\
\hline TOTAL & 781.2 & 402.6 & $1,148.0$ & 129.6 & 96.0 & 159.7 & $1,175.8$ & 907.3 & $1,419.9$ & 715.4 & 376.6 & $1,058.0$ \\
\hline
\end{tabular}

\begin{tabular}{|c|c|c|c|c|c|c|c|c|c|}
\hline \multirow{2}{*}{$\begin{array}{l}\text { Age } \\
\text { Group }\end{array}$} & \multicolumn{3}{|c|}{ Whites } & \multicolumn{3}{|c|}{ Multirace } & \multicolumn{3}{|c|}{ Hispanics } \\
\hline & Total* & Male & Female & Total* & Male & Female & Total* & Male & Female \\
\hline $0-4$ & 0.9 & 0.7 & 1.0 & 0.2 & 0.4 & 0.0 & 1.2 & 0.6 & 1.7 \\
\hline $5-9$ & 0.2 & 0.0 & 0.4 & 0.2 & 0.0 & 0.4 & 0.7 & 0.0 & 1.4 \\
\hline $10-14$ & 17.6 & 1.7 & 34.3 & 12.6 & 1.7 & 23.7 & 27.3 & 5.3 & 50.0 \\
\hline 15-19 & 889.3 & 291.5 & $1,518.5$ & 437.0 & 158.2 & 723.7 & $1,172.2$ & 453.0 & $1,922.4$ \\
\hline $20-24$ & $1,314.9$ & 726.8 & $1,936.0$ & 642.8 & 356.2 & 933.3 & $1,733.4$ & 916.4 & $2,605.7$ \\
\hline $25-29$ & 609.5 & 465.1 & 758.0 & 385.9 & 355.8 & 413.3 & 985.8 & 693.8 & $1,304.3$ \\
\hline $30-34$ & 288.4 & 260.1 & 317.0 & 230.8 & 302.3 & 166.6 & 493.0 & 401.6 & 592.2 \\
\hline $35-39$ & 155.9 & 156.3 & 155.2 & 140.1 & 203.5 & 83.4 & 273.9 & 235.0 & 314.4 \\
\hline $40-44$ & 77.0 & 85.8 & 68.0 & 87.0 & 131.8 & 46.4 & 151.6 & 140.4 & 162.6 \\
\hline $45-54$ & 36.7 & 51.5 & 21.9 & 49.8 & 87.2 & 16.4 & 71.9 & 81.4 & 62.0 \\
\hline $55-64$ & 11.8 & 19.0 & 4.9 & 15.8 & 31.2 & 1.9 & 22.6 & 25.9 & 19.5 \\
\hline $65+$ & 1.9 & 3.2 & 0.8 & 2.8 & 5.6 & 0.5 & 3.7 & 4.6 & 3.1 \\
\hline \multicolumn{10}{|c|}{ Unknown Age } \\
\hline TOTAL & 211.3 & 137.1 & 283.3 & 162.9 & 116.1 & 208.3 & 404.1 & 246.7 & 563.8 \\
\hline
\end{tabular}

* Total includes cases reported with unknown sex.

NOTE: These tables should be used only for race/Hispanic ethnicity comparisons. See Table 10 for age-specific cases and rates and Tables 3-5 for total and sexspecific cases and rates. Cases in the 0-4 age group may include cases due to perinatal transmission. No population data exist for unknown sex, unknown age, or unknown race; therefore rates are not calculated. 
Table 12. Chlamydia Among Women Aged 15-24 Years - Reported Cases and Rates of Reported Cases by Age, United States, 2013-2017

\begin{tabular}{|c|c|c|c|}
\hline & Age & Cases & Rate per 100,000 Population \\
\hline \multirow{11}{*}{$\underset{\text { m }}{\stackrel{m}{N}}$} & 15 & 21,680 & $1,070.3$ \\
\hline & 16 & 40,528 & $1,994.3$ \\
\hline & 17 & 61,666 & $3,018.5$ \\
\hline & 18 & 90,330 & $4,332.6$ \\
\hline & 19 & 102,234 & $4,806.0$ \\
\hline & 20 & 99,556 & $4,617.4$ \\
\hline & 21 & 93,713 & $4,219.8$ \\
\hline & 22 & 81,884 & $3,600.6$ \\
\hline & 23 & 68,600 & $3,013.5$ \\
\hline & 24 & 55,792 & $2,548.7$ \\
\hline & Total & 715,983 & $3,341.1$ \\
\hline \multirow{11}{*}{$\frac{+}{\text { 유 }}$} & 15 & 20,096 & 987.4 \\
\hline & 16 & 38,507 & $1,891.0$ \\
\hline & 17 & 58,940 & $2,880.9$ \\
\hline & 18 & 87,040 & $4,224.2$ \\
\hline & 19 & 98,711 & $4,688.0$ \\
\hline & 20 & 98,480 & $4,581.9$ \\
\hline & 21 & 94,204 & $4,323.2$ \\
\hline & 22 & 82,581 & $3,679.3$ \\
\hline & 23 & 71,535 & $3,112.0$ \\
\hline & 24 & 59,076 & $2,567.1$ \\
\hline & Total & 709,170 & $3,305.2$ \\
\hline \multirow{11}{*}{ 뭉 } & 15 & 19,643 & 945.9 \\
\hline & 16 & 37,786 & $1,847.7$ \\
\hline & 17 & 60,149 & $2,935.4$ \\
\hline & 18 & 89,481 & 4,339.6 \\
\hline & 19 & 100,878 & $4,854.1$ \\
\hline & 20 & 99,861 & $4,703.6$ \\
\hline & 21 & 95,927 & $4,427.2$ \\
\hline & 22 & 84,740 & $3,855.7$ \\
\hline & 23 & 73,686 & $3,254.1$ \\
\hline & 24 & 62,558 & $2,697.2$ \\
\hline & Total & 724,709 & $3,389.3$ \\
\hline \multirow{11}{*}{$\frac{0}{\stackrel{0}{0}}$} & 15 & 19,704 & 958.9 \\
\hline & 16 & 39,066 & $1,874.5$ \\
\hline & 17 & 61,406 & $2,988.7$ \\
\hline & 18 & 93,174 & $4,521.3$ \\
\hline & 19 & 103,289 & $4,980.6$ \\
\hline & 20 & 100,524 & $4,812.4$ \\
\hline & 21 & 96,723 & $4,532.8$ \\
\hline & 22 & 84,813 & $3,892.5$ \\
\hline & 23 & 73,054 & $3,304.7$ \\
\hline & 24 & 63,274 & $2,777.9$ \\
\hline & Total & 735,027 & $3,464.1$ \\
\hline \multirow{11}{*}{$\frac{n}{\stackrel{N}{N}}$} & 15 & 20,247 & 985.3 \\
\hline & 16 & 40,681 & $1,952.0$ \\
\hline & 17 & 65,496 & $3,187.8$ \\
\hline & 18 & 98,907 & $4,799.4$ \\
\hline & 19 & 111,959 & $5,398.6$ \\
\hline & 20 & 107,397 & $5,141.4$ \\
\hline & 21 & 100,160 & $4,693.9$ \\
\hline & 22 & 86,678 & $3,978.1$ \\
\hline & 23 & 75,046 & $3,394.8$ \\
\hline & 24 & 64,769 & $2,843.5$ \\
\hline & Total & 771,340 & $3,635.3$ \\
\hline
\end{tabular}

NOTE: This table should be used only for age comparisons. Cases reported with unknown sex are not included in this table. 
Table 13. Gonorrhea - Reported Cases and Rates of Reported Cases by State, Ranked by Rates, United States, 2017

\begin{tabular}{|c|c|c|c|}
\hline Rank* & State & Cases & Rate per 100,000 Population \\
\hline 1 & Mississippi & 9,258 & 309.8 \\
\hline 2 & Alaska & 2,189 & 295.1 \\
\hline 3 & Louisiana & 12,017 & 256.7 \\
\hline 4 & South Carolina & 12,623 & 254.4 \\
\hline 5 & Alabama & 11,948 & 245.7 \\
\hline 6 & Oklahoma & 9,081 & 231.4 \\
\hline 7 & North Carolina & 22,871 & 225.4 \\
\hline 8 & Arkansas & 6,710 & 224.5 \\
\hline 9 & Georgia & 22,667 & 219.8 \\
\hline 10 & New Mexico & 4,489 & 215.7 \\
\hline 11 & Missouri & 13,086 & 214.8 \\
\hline 12 & Ohio & 23,967 & 206.4 \\
\hline 13 & California & 75,348 & 192.0 \\
\hline 14 & Nevada & 5,520 & 187.8 \\
\hline 15 & Delaware & 1,784 & 187.4 \\
\hline 16 & Tennessee & 12,426 & 186.8 \\
\hline 17 & Illinois & 23,859 & 186.4 \\
\hline 18 & Maryland & 10,978 & 182.5 \\
\hline 19 & Arizona & 12,502 & 180.4 \\
\hline 20 & Indiana & 11,835 & 178.4 \\
\hline \multirow[t]{2}{*}{21} & New York & 34,099 & 172.7 \\
\hline & U.S. TOTAL ${ }^{\dagger}$ & 555,608 & 171.9 \\
\hline 22 & Texas & 47,409 & 170.2 \\
\hline 23 & Kentucky & 7,417 & 167.2 \\
\hline 24 & Michigan & 15,742 & 158.6 \\
\hline 25 & Kansas & 4,545 & 156.3 \\
\hline 26 & Florida & 31,683 & 153.7 \\
\hline 27 & Colorado & 8,478 & 153.0 \\
\hline 28 & Virginia & 12,596 & 149.7 \\
\hline 29 & South Dakota & 1,290 & 149.1 \\
\hline 30 & Nebraska & 2,653 & 139.1 \\
\hline 31 & Washington & 9,915 & 136.0 \\
\hline 32 & Wisconsin & 7,661 & 132.6 \\
\hline 33 & North Dakota & 966 & 127.4 \\
\hline 34 & Oregon & 5,022 & 122.7 \\
\hline 35 & lowa & 3,758 & 119.9 \\
\hline 36 & Pennsylvania & 15,244 & 119.2 \\
\hline 37 & Minnesota & 6,519 & 118.1 \\
\hline 38 & Massachusetts & 7,737 & 113.6 \\
\hline 39 & Connecticut & 3,913 & 109.4 \\
\hline 40 & New Jersey & 9,439 & 105.5 \\
\hline 41 & Rhode Island & 1,087 & 102.9 \\
\hline 42 & Hawaii & 1,358 & 95.1 \\
\hline 43 & Utah & 2,543 & 83.3 \\
\hline 44 & Montana & 782 & 75.0 \\
\hline 45 & West Virginia & 1,296 & 70.8 \\
\hline 46 & Wyoming & 412 & 70.4 \\
\hline 47 & Idaho & 987 & 58.6 \\
\hline 48 & Maine & 620 & 46.6 \\
\hline 49 & New Hampshire & 513 & 38.4 \\
\hline 50 & Vermont & 203 & 32.5 \\
\hline
\end{tabular}

* States were ranked by rate, then by case count, then in alphabetical order, with rates shown rounded to the nearest tenth.

${ }^{\dagger}$ Total includes cases reported by the District of Columbia with 4,563 cases and a rate of 669.9, but excludes outlying areas (Guam with 202 cases and rate of 121.0 , Puerto Rico with 588 cases and rate of 17.2, and Virgin Islands with 15 cases and rate of 13.9). 
Table 14. Gonorrhea - Reported Cases and Rates of Reported Cases by State/Area and Region in Alphabetical Order, United States and Outlying Areas, 2013-2017

\begin{tabular}{|c|c|c|c|c|c|c|c|c|c|c|}
\hline \multirow[b]{2}{*}{ State/Area } & \multicolumn{5}{|c|}{ Cases } & \multicolumn{5}{|c|}{ Rates per 100,000 Population } \\
\hline & 2013 & 2014 & 2015 & 2016 & 2017 & 2013 & 2014 & 2015 & 2016 & 2017 \\
\hline Alabama & 8,377 & 7,677 & 7,196 & 8,408 & 11,948 & 173.3 & 158.3 & 148.1 & 172.9 & 245.7 \\
\hline Alaska & 1,128 & 1,341 & 1,113 & 1,454 & 2,189 & 153.4 & 182.0 & 150.7 & 196.0 & 295.1 \\
\hline Arizona & 6,412 & 7,750 & 8,245 & 10,330 & 12,502 & 96.8 & 115.1 & 120.8 & 149.0 & 180.4 \\
\hline Arkansas & 4,007 & 4,539 & 4,780 & 5,732 & 6,710 & 135.4 & 153.0 & 160.5 & 191.8 & 224.5 \\
\hline California & 38,166 & 45,408 & 54,135 & 64,551 & 75,348 & 99.6 & 117.0 & 138.3 & 164.5 & 192.0 \\
\hline Colorado & 2,820 & 3,170 & 4,387 & 5,975 & 8,478 & 53.5 & 59.2 & 80.4 & 107.8 & 153.0 \\
\hline Connecticut & 2,860 & 2,333 & 2,088 & 2,731 & 3,913 & 79.5 & 64.9 & 58.1 & 76.4 & 109.4 \\
\hline Delaware & 1,390 & 1,279 & 1,310 & 1,702 & 1,784 & 150.1 & 136.7 & 138.5 & 178.8 & 187.4 \\
\hline District of Columbia & 2,478 & 1,883 & 2,742 & 3,226 & 4,563 & 383.3 & 285.8 & 407.9 & 473.6 & 669.9 \\
\hline Florida & 20,818 & 20,944 & 24,125 & 28,162 & 31,683 & 106.5 & 105.3 & 119.0 & 136.6 & 153.7 \\
\hline Georgia & 14,252 & 13,770 & 15,982 & 20,553 & 22,667 & 142.6 & 136.4 & 156.5 & 199.3 & 219.8 \\
\hline Hawaii & 718 & 1,020 & 1,239 & 1,467 & 1,358 & 51.1 & 71.9 & 86.5 & 102.7 & 95.1 \\
\hline Idaho & 211 & 443 & 472 & 635 & 987 & 13.1 & 27.1 & 28.5 & 37.7 & 58.6 \\
\hline Illinois & 16,464 & 15,970 & 17,130 & 21,199 & 23,859 & 127.8 & 124.0 & 133.2 & 165.6 & 186.4 \\
\hline Indiana & 7,144 & 7,289 & 7,843 & 9,451 & 11,835 & 108.7 & 110.5 & 118.5 & 142.5 & 178.4 \\
\hline lowa & 1,472 & 1,641 & 2,247 & 2,600 & 3,758 & 47.6 & 52.8 & 71.9 & 82.9 & 119.9 \\
\hline Kansas & 2,161 & 2,568 & 2,536 & 3,353 & 4,545 & 74.7 & 88.4 & 87.1 & 115.3 & 156.3 \\
\hline Kentucky & 4,315 & 4,353 & 4,678 & 5,812 & 7,417 & 98.2 & 98.6 & 105.7 & 131.0 & 167.2 \\
\hline Louisiana & 8,669 & 9,002 & 10,282 & 10,782 & 12,017 & 187.4 & 193.6 & 220.1 & 230.3 & 256.7 \\
\hline Maine & 245 & 237 & 417 & 451 & 620 & 18.4 & 17.8 & 31.4 & 33.9 & 46.6 \\
\hline Maryland & 5,989 & 6,108 & 6,858 & 9,523 & 10,978 & 101.0 & 102.2 & 114.2 & 158.3 & 182.5 \\
\hline Massachusetts & 3,106 & 3,817 & 3,817 & 4,980 & 7,737 & 46.4 & 56.6 & 56.2 & 73.1 & 113.6 \\
\hline Michigan & 10,569 & 9,688 & 10,330 & 12,450 & 15,742 & 106.8 & 97.8 & 104.1 & 125.4 & 158.6 \\
\hline Minnesota & 3,873 & 4,073 & 4,097 & 5,104 & 6,519 & 71.5 & 74.6 & 74.6 & 92.5 & 118.1 \\
\hline Mississippi & 5,096 & 5,625 & 5,775 & 7,157 & 9,258 & 170.4 & 187.9 & 193.0 & 239.5 & 309.8 \\
\hline Missouri & 7,546 & 7,387 & 8,942 & 11,479 & 13,086 & 124.8 & 121.8 & 147.0 & 188.4 & 214.8 \\
\hline Montana & 224 & 434 & 844 & 867 & 782 & 22.1 & 42.4 & 81.7 & 83.2 & 75.0 \\
\hline Nebraska & 1,385 & 1,459 & 1,703 & 2,156 & 2,653 & 74.1 & 77.5 & 89.8 & 113.1 & 139.1 \\
\hline Nevada & 2,714 & 3,188 & 3,630 & 4,380 & 5,520 & 97.3 & 112.3 & 125.6 & 149.0 & 187.8 \\
\hline New Hampshire & 121 & 226 & 245 & 456 & 513 & 9.1 & 17.0 & 18.4 & 34.2 & 38.4 \\
\hline New Jersey & 7,014 & 6,636 & 7,228 & 8,162 & 9,439 & 78.8 & 74.2 & 80.7 & 91.3 & 105.5 \\
\hline New Mexico & 1,918 & 2,246 & 2,489 & 3,516 & 4,489 & 92.0 & 107.7 & 119.4 & 169.0 & 215.7 \\
\hline New York & 19,919 & 20,758 & 25,561 & 29,000 & 34,099 & 101.4 & 105.1 & 129.1 & 146.9 & 172.7 \\
\hline North Carolina & 13,666 & 14,415 & 19,809 & 19,687 & 22,871 & 138.8 & 145.0 & 197.2 & 194.0 & 225.4 \\
\hline North Dakota & 492 & 694 & 684 & 1,000 & 966 & 68.0 & 93.8 & 90.4 & 131.9 & 127.4 \\
\hline Ohio & 16,619 & 16,237 & 16,564 & 20,487 & 23,967 & 143.6 & 140.0 & 142.6 & 176.4 & 206.4 \\
\hline Oklahoma & 5,303 & 6,137 & 6,542 & 7,574 & 9,081 & 137.7 & 158.2 & 167.3 & 193.0 & 231.4 \\
\hline Oregon & 1,729 & 2,320 & 3,232 & 4,353 & 5,022 & 44.0 & 58.4 & 80.2 & 106.3 & 122.7 \\
\hline Pennsylvania & 13,874 & 12,710 & 12,791 & 14,603 & 15,244 & 108.6 & 99.4 & 99.9 & 114.2 & 119.2 \\
\hline Rhode Island & 454 & 590 & 580 & 716 & 1,087 & 43.2 & 55.9 & 54.9 & 67.8 & 102.9 \\
\hline South Carolina & 7,194 & 8,253 & 8,206 & 9,194 & 12,623 & 150.7 & 170.8 & 167.6 & 185.3 & 254.4 \\
\hline South Dakota & 784 & 892 & 1,048 & 1,269 & 1,290 & 92.8 & 104.6 & 122.1 & 146.6 & 149.1 \\
\hline Tennessee & 7,376 & 7,199 & 8,386 & 10,179 & 12,426 & 113.5 & 109.9 & 127.1 & 153.0 & 186.8 \\
\hline Texas & 33,835 & 35,322 & 39,717 & 42,472 & 47,409 & 127.9 & 131.0 & 144.6 & 152.4 & 170.2 \\
\hline Utah & 951 & 1,441 & 1,562 & 2,100 & 2,543 & 32.8 & 49.0 & 52.1 & 68.8 & 83.3 \\
\hline Vermont & 97 & 84 & 155 & 126 & 203 & 15.5 & 13.4 & 24.8 & 20.2 & 32.5 \\
\hline Virginia & 6,952 & 8,250 & 8,099 & 11,084 & 12,596 & 84.2 & 99.1 & 96.6 & 131.8 & 149.7 \\
\hline Washington & 4,369 & 6,221 & 7,171 & 8,174 & 9,915 & 62.7 & 88.1 & 100.0 & 112.2 & 136.0 \\
\hline West Virginia & 1,063 & 841 & 769 & 919 & 1,296 & 57.3 & 45.5 & 41.7 & 50.2 & 70.8 \\
\hline Wisconsin & 4,599 & 4,078 & 5,260 & 6,498 & 7,661 & 80.1 & 70.8 & 91.1 & 112.4 & 132.6 \\
\hline Wyoming & 66 & 116 & 175 & 275 & 412 & 11.3 & 19.9 & 29.9 & 47.0 & 70.4 \\
\hline U.S. TOTAL & 333,004 & 350,062 & 395,216 & 468,514 & 555,608 & 105.3 & 109.8 & 123.0 & 145.0 & 171.9 \\
\hline Northeast & 47,690 & 47,391 & 52,882 & 61,225 & 72,855 & 85.2 & 84.4 & 94.0 & 108.9 & 129.6 \\
\hline Midwest & 73,108 & 71,976 & 78,384 & 97,046 & 115,881 & 108.2 & 106.2 & 115.4 & 142.8 & 170.6 \\
\hline South & 150,780 & 155,597 & 175,256 & 202,166 & 237,327 & 127.4 & 129.9 & 144.6 & 165.3 & 194.0 \\
\hline West & 61,426 & 75,098 & 88,694 & 108,077 & 129,545 & 82.7 & 99.9 & 116.6 & 141.0 & 169.0 \\
\hline Guam & 92 & 99 & 147 & 133 & 202 & 57.4 & 61.5 & 90.9 & 79.7 & 121.0 \\
\hline Puerto Rico & 356 & 454 & 620 & 744 & 588 & 9.8 & 12.8 & 17.8 & 21.8 & 17.2 \\
\hline Virgin Islands & 58 & 84 & 52 & 35 & 15 & 55.4 & 80.6 & 50.5 & 32.5 & 13.9 \\
\hline OUTLYING AREAS & 506 & 637 & 819 & 912 & 805 & 13.0 & 16.7 & 21.9 & 24.7 & 21.8 \\
\hline TOTAL & 333,510 & 350,699 & 396,035 & 469,426 & 556,413 & 104.2 & 108.7 & 121.8 & 143.6 & 170.3 \\
\hline
\end{tabular}

NOTE: See Section A1.11 in the Appendix for more information on interpreting case counts and rates in outlying areas. 
Table 15. Gonorrhea Among Women - Reported Cases and Rates of Reported Cases by State/Area and Region in Alphabetical Order, United States and Outlying Areas, 2013-2017

\begin{tabular}{|c|c|c|c|c|c|c|c|c|c|c|}
\hline \multirow[b]{2}{*}{ State/Area } & \multicolumn{5}{|c|}{ Cases } & \multicolumn{5}{|c|}{ Rates per 100,000 Population } \\
\hline & 2013 & 2014 & 2015 & 2016 & 2017 & 2013 & 2014 & 2015 & 2016 & 2017 \\
\hline Alabama & 4,668 & 4,090 & 3,629 & 4,088 & 5,693 & 187.6 & 163.6 & 144.8 & 163.0 & 227.0 \\
\hline Alaska & 589 & 665 & 567 & 738 & 1,090 & 168.5 & 190.3 & 162.3 & 208.6 & 308.1 \\
\hline Arizona & 3,102 & 3,564 & 3,505 & 4,315 & 5,219 & 93.1 & 105.2 & 102.0 & 123.7 & 149.6 \\
\hline Arkansas & 2,160 & 2,527 & 2,510 & 2,964 & 3,525 & 143.4 & 167.5 & 165.6 & 195.0 & 231.9 \\
\hline California & 14,258 & 16,009 & 18,404 & 20,914 & 24,599 & 74.0 & 82.0 & 93.4 & 105.9 & 124.5 \\
\hline Colorado & 1,243 & 1,318 & 1,832 & 2,323 & 3,465 & 47.4 & 49.5 & 67.5 & 84.3 & 125.8 \\
\hline Connecticut & 1,419 & 1,108 & 851 & 1,165 & 1,660 & 77.0 & 60.1 & 46.3 & 63.6 & 90.7 \\
\hline Delaware & 763 & 693 & 641 & 799 & 757 & 159.7 & 143.5 & 131.3 & 162.6 & 154.1 \\
\hline District of Columbia & 953 & 858 & 874 & 857 & 1,287 & 280.1 & 247.8 & 247.2 & 239.4 & 359.6 \\
\hline Florida & 9,718 & 9,228 & 10,078 & 11,488 & 12,769 & 97.3 & 90.7 & 97.2 & 109.0 & 121.1 \\
\hline Georgia & 7,060 & 6,552 & 7,322 & 9,156 & 9,873 & 138.1 & 126.7 & 139.9 & 173.1 & 186.6 \\
\hline Hawaii & 264 & 350 & 446 & 551 & 476 & 38.0 & 49.9 & 63.2 & 77.5 & 67.0 \\
\hline Idaho & 87 & 196 & 197 & 237 & 357 & 10.8 & 24.0 & 23.8 & 28.2 & 42.5 \\
\hline Illinois & 8,574 & 7,559 & 7,698 & 8,920 & 9,750 & 130.8 & 115.2 & 117.6 & 137.0 & 149.8 \\
\hline Indiana & 3,796 & 3,819 & 3,984 & 4,811 & 5,931 & 113.8 & 114.1 & 118.6 & 143.0 & 176.3 \\
\hline lowa & 812 & 862 & 1,122 & 1,267 & 1,887 & 52.2 & 55.1 & 71.4 & 80.4 & 119.8 \\
\hline Kansas & 1,222 & 1,464 & 1,262 & 1,695 & 2,269 & 84.2 & 100.5 & 86.6 & 116.1 & 155.5 \\
\hline Kentucky & 2,331 & 2,270 & 2,242 & 2,716 & 3,478 & 104.4 & 101.3 & 99.8 & 120.7 & 154.5 \\
\hline Louisiana & 4,927 & 5,049 & 5,535 & 5,493 & 5,978 & 208.6 & 212.5 & 231.9 & 229.6 & 249.9 \\
\hline Maine & 119 & 98 & 143 & 134 & 225 & 17.5 & 14.4 & 21.1 & 19.7 & 33.1 \\
\hline Maryland & 2,841 & 2,793 & 3,090 & 3,944 & 4,629 & 93.0 & 90.7 & 99.8 & 127.1 & 149.2 \\
\hline Massachusetts & 1,168 & 1,215 & 1,027 & 1,390 & 2,495 & 33.9 & 35.0 & 29.4 & 39.6 & 71.2 \\
\hline Michigan & 5,865 & 5,129 & 5,191 & 6,201 & 7,604 & 116.5 & 101.7 & 102.9 & 122.9 & 150.8 \\
\hline Minnesota & 2,037 & 1,802 & 1,675 & 2,214 & 2,939 & 74.7 & 65.6 & 60.7 & 79.9 & 106.0 \\
\hline Mississippi & 2,726 & 2,987 & 3,131 & 3,665 & 4,563 & 177.4 & 194.0 & 203.2 & 238.0 & 296.3 \\
\hline Missouri & 3,944 & 3,620 & 4,187 & 5,228 & 5,997 & 128.0 & 117.2 & 135.1 & 168.6 & 193.4 \\
\hline Montana & 127 & 221 & 462 & 493 & 427 & 25.1 & 43.4 & 89.9 & 95.2 & 82.5 \\
\hline Nebraska & 694 & 770 & 870 & 1,055 & 1,285 & 73.9 & 81.5 & 91.5 & 110.3 & 134.4 \\
\hline Nevada & 1,203 & 1,294 & 1,402 & 1,611 & 1,972 & 86.9 & 91.6 & 97.3 & 109.9 & 134.5 \\
\hline New Hampshire & 52 & 91 & 65 & 132 & 179 & 7.8 & 13.6 & 9.7 & 19.6 & 26.6 \\
\hline New Jersey & 3,484 & 3,082 & 3,110 & 3,338 & 3,731 & 76.5 & 67.3 & 67.8 & 72.9 & 81.5 \\
\hline New Mexico & 823 & 961 & 1,087 & 1,542 & 2,073 & 78.3 & 91.3 & 103.4 & 146.8 & 197.4 \\
\hline New York & 8,020 & 7,077 & 8,593 & 8,709 & 9,649 & 79.3 & 69.6 & 84.4 & 85.7 & 95.0 \\
\hline North Carolina & 7,547 & 7,759 & 10,064 & 9,527 & 11,013 & 149.5 & 152.2 & 195.4 & 182.7 & 211.2 \\
\hline North Dakota & 301 & 385 & 375 & 516 & 528 & 85.2 & 106.8 & 101.9 & 139.8 & 143.1 \\
\hline Ohio & 9,176 & 8,735 & 8,466 & 10,130 & 11,736 & 155.2 & 147.6 & 142.8 & 171.0 & 198.2 \\
\hline Oklahoma & 3,000 & 3,451 & 3,580 & 4,052 & 4,721 & 154.4 & 176.3 & 181.3 & 204.7 & 238.5 \\
\hline Oregon & 566 & 786 & 1,158 & 1,519 & 1,920 & 28.5 & 39.2 & 56.9 & 73.5 & 92.9 \\
\hline Pennsylvania & 7,206 & 6,164 & 5,889 & 6,135 & 5,947 & 110.3 & 94.3 & 90.1 & 94.1 & 91.2 \\
\hline Rhode Island & 192 & 218 & 172 & 221 & 357 & 35.4 & 40.1 & 31.6 & 40.7 & 65.7 \\
\hline South Carolina & 4,050 & 4,527 & 4,401 & 4,709 & 6,470 & 165.2 & 182.3 & 174.8 & 184.4 & 253.4 \\
\hline South Dakota & 464 & 557 & 621 & 757 & 722 & 110.3 & 131.4 & 145.6 & 176.4 & 168.2 \\
\hline Tennessee & 3,617 & 3,419 & 3,809 & 4,681 & 5,667 & 108.7 & 101.8 & 112.6 & 137.3 & 166.3 \\
\hline Texas & 17,206 & 17,253 & 17,843 & 18,620 & 20,963 & 129.4 & 127.1 & 129.0 & 132.7 & 149.4 \\
\hline Utah & 373 & 565 & 507 & 717 & 865 & 25.9 & 38.6 & 34.1 & 47.3 & 57.1 \\
\hline Vermont & 46 & 35 & 85 & 32 & 87 & 14.5 & 11.0 & 26.8 & 10.1 & 27.5 \\
\hline Virginia & 3,678 & 4,361 & 4,007 & 5,056 & 5,734 & 87.6 & 103.1 & 94.1 & 118.3 & 134.1 \\
\hline Washington & 1,704 & 2,504 & 2,797 & 2,943 & 3,488 & 48.8 & 70.9 & 78.0 & 80.7 & 95.7 \\
\hline West Virginia & 539 & 461 & 365 & 422 & 618 & 57.4 & 49.2 & 39.1 & 45.6 & 66.8 \\
\hline Wisconsin & 2,455 & 2,046 & 2,557 & 3,189 & 3,696 & 84.9 & 70.6 & 88.1 & 109.8 & 127.2 \\
\hline Wyoming & 39 & 61 & 86 & 120 & 224 & 13.7 & 21.3 & 29.9 & 41.9 & 78.2 \\
\hline U.S. TOTAL & 163,208 & 162,608 & 173,514 & 197,499 & 232,587 & 101.7 & 100.4 & 106.3 & 120.4 & 141.8 \\
\hline Northeast & 21,706 & 19,088 & 19,935 & 21,256 & 24,330 & 75.6 & 66.3 & 69.1 & 73.8 & 84.5 \\
\hline Midwest & 39,340 & 36,748 & 38,008 & 45,983 & 54,344 & 114.8 & 107.0 & 110.4 & 133.6 & 157.9 \\
\hline South & 77,784 & 78,278 & 83,121 & 92,237 & 107,738 & 129.0 & 128.2 & 134.5 & 147.9 & 172.7 \\
\hline West & 24,378 & 28,494 & 32,450 & 38,023 & 46,175 & 65.5 & 75.6 & 85.1 & 98.9 & 120.1 \\
\hline Guam & 43 & 47 & 67 & 59 & 97 & 54.4 & 59.2 & 84.0 & 72.8 & 119.7 \\
\hline Puerto Rico & 120 & 161 & 259 & 313 & 230 & 6.4 & 8.7 & 14.3 & 17.5 & 12.9 \\
\hline Virgin Islands & 41 & 54 & 28 & 14 & 5 & 73.4 & 97.1 & 50.8 & 24.9 & 8.9 \\
\hline OUTLYING AREAS & 204 & 262 & 354 & 386 & 332 & 10.1 & 13.2 & 18.1 & 20.1 & 17.2 \\
\hline TOTAL & 163,412 & 162,870 & 173,868 & 197,885 & 232,919 & 100.6 & 99.4 & 105.3 & 119.2 & 140.3 \\
\hline
\end{tabular}

NOTE: Cases reported with unknown sex are not included in this table. See Section A1.11 in the Appendix for more information on interpreting case counts and rates in outlying areas. 
Table 16. Gonorrhea Among Men - Reported Cases and Rates of Reported Cases by State/Area and Region in Alphabetical Order, United States and Outlying Areas, 2013-2017

\begin{tabular}{|c|c|c|c|c|c|c|c|c|c|c|}
\hline \multirow[b]{2}{*}{ State/Area } & \multicolumn{5}{|c|}{ Cases } & \multicolumn{5}{|c|}{ Rates per 100,000 Population } \\
\hline & 2013 & 2014 & 2015 & 2016 & 2017 & 2013 & 2014 & 2015 & 2016 & 2017 \\
\hline Alabama & 3,680 & 3,563 & 3,519 & 4,265 & 6,214 & 156.9 & 151.6 & 149.5 & 181.1 & 263.8 \\
\hline Alaska & 539 & 676 & 546 & 716 & 1,099 & 139.8 & 174.5 & 140.4 & 184.5 & 283.2 \\
\hline Arizona & 3,310 & 4,186 & 4,724 & 6,011 & 7,256 & 100.5 & 125.2 & 139.3 & 174.6 & 210.8 \\
\hline Arkansas & 1,843 & 2,007 & 2,270 & 2,768 & 3,184 & 126.8 & 137.7 & 155.2 & 188.6 & 216.9 \\
\hline California & 23,849 & 29,310 & 35,644 & 43,259 & 50,577 & 125.1 & 152.1 & 183.3 & 221.9 & 259.5 \\
\hline Colorado & 1,577 & 1,852 & 2,555 & 3,652 & 5,013 & 59.6 & 68.8 & 93.1 & 131.1 & 179.9 \\
\hline Connecticut & 1,440 & 1,219 & 1,237 & 1,564 & 2,246 & 82.1 & 69.5 & 70.6 & 89.6 & 128.7 \\
\hline Delaware & 627 & 586 & 669 & 903 & 1,027 & 140.0 & 129.4 & 146.1 & 196.0 & 222.9 \\
\hline District of Columbia & 1,519 & 1,011 & 1,817 & 2,298 & 3,254 & 496.0 & 323.4 & 570.3 & 710.9 & 1006.7 \\
\hline Florida & 11,049 & 11,686 & 14,039 & 16,661 & 18,904 & 115.5 & 120.2 & 141.8 & 165.4 & 187.7 \\
\hline Georgia & 7,075 & 7,137 & 8,631 & 11,378 & 12,741 & 144.9 & 144.9 & 173.3 & 226.6 & 253.8 \\
\hline Hawaii & 454 & 669 & 793 & 914 & 882 & 64.0 & 93.2 & 109.3 & 127.4 & 122.9 \\
\hline Idaho & 124 & 247 & 275 & 396 & 629 & 15.4 & 30.2 & 33.2 & 46.9 & 74.6 \\
\hline Illinois & 7,872 & 8,386 & 9,335 & 12,255 & 14,057 & 124.4 & 132.7 & 147.8 & 194.8 & 223.4 \\
\hline Indiana & 3,347 & 3,465 & 3,854 & 4,636 & 5,899 & 103.4 & 106.6 & 118.2 & 141.8 & 180.4 \\
\hline lowa & 660 & 779 & 1,122 & 1,332 & 1,870 & 43.0 & 50.5 & 72.3 & 85.4 & 119.9 \\
\hline Kansas & 939 & 1,104 & 1,274 & 1,658 & 2,276 & 65.1 & 76.3 & 87.6 & 114.5 & 157.2 \\
\hline Kentucky & 1,966 & 2,068 & 2,430 & 3,006 & 3,906 & 90.9 & 95.2 & 111.5 & 137.5 & 178.6 \\
\hline Louisiana & 3,742 & 3,953 & 4,747 & 5,289 & 6,039 & 165.3 & 173.8 & 207.9 & 231.0 & 263.8 \\
\hline Maine & 126 & 137 & 274 & 316 & 392 & 19.4 & 21.0 & 42.1 & 48.4 & 60.1 \\
\hline Maryland & 3,145 & 3,304 & 3,755 & 5,573 & 6,346 & 109.4 & 114.1 & 129.0 & 191.2 & 217.7 \\
\hline Massachusetts & 1,932 & 2,590 & 2,768 & 3,575 & 5,206 & 59.5 & 79.2 & 84.0 & 108.1 & 157.5 \\
\hline Michigan & 4,694 & 4,551 & 5,129 & 6,245 & 8,121 & 96.6 & 93.5 & 105.1 & 127.8 & 166.3 \\
\hline Minnesota & 1,835 & 2,260 & 2,420 & 2,881 & 3,568 & 68.1 & 83.3 & 88.6 & 104.9 & 129.9 \\
\hline Mississippi & 2,370 & 2,637 & 2,638 & 3,486 & 4,681 & 162.9 & 181.3 & 181.7 & 240.6 & 323.1 \\
\hline Missouri & 3,602 & 3,767 & 4,755 & 6,251 & 7,088 & 121.5 & 126.7 & 159.3 & 208.9 & 236.9 \\
\hline Montana & 97 & 213 & 381 & 374 & 355 & 19.0 & 41.4 & 73.4 & 71.3 & 67.6 \\
\hline Nebraska & 674 & 686 & 833 & 1,097 & 1,366 & 72.5 & 73.2 & 88.1 & 115.4 & 143.7 \\
\hline Nevada & 1,509 & 1,892 & 2,218 & 2,763 & 3,539 & 107.4 & 132.6 & 152.9 & 187.4 & 240.1 \\
\hline New Hampshire & 69 & 135 & 180 & 324 & 334 & 10.6 & 20.6 & 27.4 & 49.0 & 50.5 \\
\hline New Jersey & 3,514 & 3,544 & 4,108 & 4,810 & 5,699 & 80.9 & 81.2 & 94.0 & 110.1 & 130.5 \\
\hline New Mexico & 1,095 & 1,284 & 1,401 & 1,971 & 2,412 & 105.9 & 124.3 & 135.6 & 191.2 & 234.0 \\
\hline New York & 11,844 & 13,624 & 16,893 & 20,224 & 24,383 & 124.2 & 142.2 & 175.8 & 210.9 & 254.3 \\
\hline North Carolina & 6,113 & 6,652 & 9,744 & 10,160 & 11,857 & 127.3 & 137.3 & 199.2 & 206.0 & 240.4 \\
\hline North Dakota & 191 & 309 & 309 & 484 & 438 & 51.6 & 81.5 & 79.5 & 124.4 & 112.6 \\
\hline Ohio & 7,443 & 7,502 & 8,098 & 10,357 & 12,231 & 131.5 & 132.2 & 142.4 & 182.0 & 214.9 \\
\hline Oklahoma & 2,303 & 2,685 & 2,962 & 3,521 & 4,360 & 120.7 & 139.8 & 152.9 & 181.1 & 224.3 \\
\hline Oregon & 1,163 & 1,532 & 2,073 & 2,834 & 3,095 & 59.8 & 78.0 & 104.0 & 139.8 & 152.7 \\
\hline Pennsylvania & 6,659 & 6,543 & 6,892 & 8,449 & 9,285 & 106.7 & 104.6 & 110.0 & 134.9 & 148.3 \\
\hline Rhode Island & 262 & 372 & 408 & 495 & 729 & 51.4 & 72.7 & 79.7 & 96.5 & 142.1 \\
\hline South Carolina & 3,133 & 3,689 & 3,781 & 4,436 & 6,122 & 134.9 & 157.0 & 158.9 & 184.2 & 254.2 \\
\hline South Dakota & 320 & 335 & 427 & 512 & 568 & 75.4 & 78.0 & 98.8 & 117.4 & 130.2 \\
\hline Tennessee & 3,758 & 3,778 & 4,577 & 5,497 & 6,758 & 118.7 & 118.4 & 142.3 & 169.5 & 208.4 \\
\hline Texas & 16,410 & 18,035 & 21,792 & 23,779 & 26,344 & 124.8 & 134.8 & 159.8 & 171.9 & 190.5 \\
\hline Utah & 578 & 876 & 1,055 & 1,383 & 1,672 & 39.6 & 59.2 & 70.0 & 90.0 & 108.9 \\
\hline Vermont & 51 & 49 & 70 & 94 & 116 & 16.5 & 15.9 & 22.7 & 30.5 & 37.6 \\
\hline Virginia & 3,272 & 3,879 & 4,085 & 5,996 & 6,779 & 80.5 & 94.7 & 99.0 & 144.9 & 163.9 \\
\hline Washington & 2,665 & 3,717 & 4,374 & 5,231 & 6,425 & 76.5 & 105.3 & 122.0 & 143.7 & 176.4 \\
\hline West Virginia & 524 & 380 & 404 & 497 & 678 & 57.2 & 41.6 & 44.3 & 54.9 & 74.8 \\
\hline Wisconsin & 2,140 & 2,027 & 2,697 & 3,302 & 3,961 & 75.0 & 70.9 & 94.1 & 114.9 & 137.8 \\
\hline Wyoming & 27 & 55 & 88 & 155 & 188 & 9.1 & 18.5 & 29.4 & 51.8 & 62.9 \\
\hline U.S. TOTAL & 169,130 & 186,943 & 221,070 & 270,033 & 322,169 & 108.7 & 119.1 & 139.7 & 169.7 & 202.5 \\
\hline Northeast & 25,897 & 28,213 & 32,830 & 39,851 & 48,390 & 95.1 & 103.2 & 119.7 & 145.4 & 176.6 \\
\hline Midwest & 33,717 & 35,171 & 40,253 & 51,010 & 61,443 & 101.3 & 105.3 & 120.2 & 152.1 & 183.2 \\
\hline South & 72,529 & 77,050 & 91,860 & 109,513 & 129,194 & 124.9 & 131.2 & 154.6 & 182.7 & 215.5 \\
\hline West & 36,987 & 46,509 & 56,127 & 69,659 & 83,142 & 99.9 & 124.1 & 148.1 & 182.3 & 217.6 \\
\hline Guam & 49 & 52 & 80 & 74 & 105 & 60.2 & 63.7 & 97.6 & 86.2 & 122.3 \\
\hline Puerto Rico & 236 & 293 & 359 & 431 & 356 & 13.6 & 17.2 & 21.7 & 26.5 & 21.9 \\
\hline Virgin Islands & 17 & 30 & 24 & 21 & 10 & 34.8 & 61.8 & 50.2 & 40.8 & 19.4 \\
\hline OUTLYING AREAS & 302 & 375 & 463 & 526 & 471 & 16.2 & 20.4 & 25.9 & 29.9 & 26.7 \\
\hline TOTAL & 169,432 & 187,318 & 221,533 & 270,559 & 322,640 & 107.6 & 118.0 & 138.4 & 168.2 & 200.6 \\
\hline
\end{tabular}

NOTE: Cases reported with unknown sex are not included in this table. See Section A1.11 in the Appendix for more information on interpreting case counts and rates in outlying areas. 
Table 17. Gonorrhea - Reported Cases and Rates of Reported Cases in Selected Metropolitan Statistical Areas (MSAs)* in Alphabetical Order, United States, 2013-2017

\begin{tabular}{|c|c|c|c|c|c|c|c|c|c|c|}
\hline \multirow[b]{2}{*}{ MSAs } & \multicolumn{5}{|c|}{ Cases } & \multicolumn{5}{|c|}{ Rates per 100,000 Population } \\
\hline & 2013 & 2014 & 2015 & 2016 & 2017 & 2013 & 2014 & 2015 & 2016 & 2017 \\
\hline Atlanta-Sandy Springs-Roswell, GA & $5,452^{+}$ & 7,256 & $6,471^{\dagger}$ & 11,670 & 12,373 & $98.7^{+}$ & 129.2 & $113.3^{+}$ & 201.6 & 213.7 \\
\hline Austin-Round Rock, TX & 2,570 & 2,860 & 3,199 & 3,670 & 4,414 & 136.5 & 147.2 & 159.9 & 178.5 & 214.6 \\
\hline Baltimore-Columbia-Towson, MD & 3,233 & 3,459 & 4,179 & 5,854 & 6,892 & 116.7 & 124.2 & 149.4 & 209.2 & 246.2 \\
\hline Birmingham-Hoover, AL & 2,130 & 1,957 & 2,088 & $2,025^{\ddagger}$ & 3,486 & 186.8 & 171.1 & 182.3 & $176.5^{\ddagger}$ & 303.8 \\
\hline Boston-Cambridge-Newton, MA-NH & 2,372 & $2,716^{+}$ & $2,487^{\dagger}$ & $3,002^{+}$ & $4,385^{+}$ & 50.6 & $57.4^{+}$ & $52.1^{\dagger}$ & $62.6^{+}$ & $91.5^{+}$ \\
\hline Buffalo-Cheektowaga-Niagara Falls, NY & 1,232 & 1,342 & 1,982 & 2,180 & 2,448 & 108.6 & 118.1 & 174.6 & 192.4 & 216.1 \\
\hline Charlotte-Concord-Gastonia, NC-SC & 3,058 & 3,645 & 4,673 & 4,749 & 5,411 & 130.9 & 153.1 & 192.6 & 191.9 & 218.7 \\
\hline Chicago-Naperville-Elgin, IL-IN-WI & 12,793 & 12,630 & 13,529 & 16,634 & 18,558 & 134.1 & 132.2 & 141.6 & 174.9 & 195.1 \\
\hline Cincinnati, OH-KY-IN & 3,229 & 3,346 & 3,713 & 4,096 & 4,716 & 151.1 & 155.7 & 172.1 & 189.2 & 217.8 \\
\hline Cleveland-Elyria, $\mathrm{OH}$ & 4,155 & 3,802 & 3,428 & 4,205 & 5,843 & 201.2 & 184.2 & 166.3 & 204.6 & 284.2 \\
\hline Columbus, $\mathrm{OH}$ & 3,220 & 3,260 & 3,676 & 4,821 & 5,197 & 163.7 & 163.4 & 181.8 & 236.1 & 254.6 \\
\hline Dallas-Fort Worth-Arlington, TX & 8,354 & 9,195 & 11,334 & 11,092 & 12,846 & 122.7 & 132.2 & 159.6 & 153.3 & 177.6 \\
\hline Denver-Aurora-Lakewood, CO & 1,828 & 2,016 & 2,838 & 3,848 & 5,408 & 67.8 & 73.2 & 100.8 & 134.9 & 189.5 \\
\hline Detroit-Warren-Dearborn, MI & 6,564 & 5,311 & 5,494 & 6,816 & 8,668 & 152.8 & 123.6 & 127.7 & 158.6 & 201.7 \\
\hline Hartford-West Hartford-East Hartford, CT & 1,065 & 894 & $726^{+}$ & $963^{+}$ & 1,831 & 87.6 & 73.6 & $59.9^{+}$ & $79.8^{+}$ & 151.7 \\
\hline Houston-The Woodlands-Sugar Land, TX & 7,783 & 8,299 & 9,290 & 10,378 & 10,789 & 123.3 & 127.9 & 139.6 & 153.2 & 159.3 \\
\hline Indianapolis-Carmel-Anderson, IN & 3,616 & 3,759 & 3,716 & 4,808 & 5,430 & 185.1 & 190.7 & 186.8 & 239.9 & 270.9 \\
\hline Jacksonville, FL & 2,321 & 2,608 & 2,740 & 3,168 & 3,721 & 166.4 & 183.8 & 189.0 & 214.3 & 251.7 \\
\hline Kansas City, MO-KS & 2,696 & 2,642 & 2,943 & 4,009 & 5,274 & 131.2 & 127.6 & 141.0 & 190.5 & 250.6 \\
\hline Las Vegas-Henderson-Paradise, NV & 2,256 & 2,653 & 2,975 & 3,653 & 4,430 & 111.2 & 128.2 & 140.7 & 169.5 & 205.5 \\
\hline Los Angeles-Long Beach-Anaheim, CA & 14,449 & 17,130 & 19,867 & 25,438 & 29,669 & 110.0 & 129.2 & 148.9 & 191.1 & 222.9 \\
\hline Louisville-Jefferson County, KY-IN & 2,063 & 1,962 & 2,187 & 2,957 & 3,413 & 163.4 & 154.5 & 171.1 & 230.4 & 265.9 \\
\hline Memphis, TN-MS-AR & 3,086 & 2,625 & 3,143 & 3,746 & 4,653 & 230.0 & 195.4 & 233.8 & 279.0 & 346.5 \\
\hline Miami-Fort Lauderdale-West Palm Beach, FL & 5,801 & 6,128 & 6,905 & 7,984 & 8,848 & 99.5 & 103.3 & 114.8 & 131.6 & 145.9 \\
\hline Milwaukee-Waukesha-West Allis, WI & 3,179 & 2,584 & 3,719 & 4,454 & 4,910 & 202.5 & 164.4 & 236.0 & 283.2 & 312.2 \\
\hline Minneapolis-St. Paul-Bloomington, MN-WI & $3,188^{+}$ & 3,341 & 3,289 & 4,123 & 5,260 & $92.2^{+}$ & 95.6 & 93.3 & 116.1 & 148.1 \\
\hline Nashville-Davidson-Murfreesboro-Franklin, TN & 1,806 & 1,922 & 2,200 & 2,695 & 2,706 & 102.7 & 107.2 & 120.2 & 144.5 & 145.1 \\
\hline New Orleans-Metairie, LA & 2,448 & 2,667 & 2,929 & 3,414 & 3,638 & 197.3 & 213.0 & 231.9 & 269.1 & 286.7 \\
\hline New York-Newark-Jersey City, NY-NJ-PA & 19,319 & 20,054 & 23,721 & 26,186 & 32,018 & 96.8 & 99.8 & 117.5 & 129.9 & 158.9 \\
\hline Oklahoma City, OK & 2,352 & 2,366 & 2,403 & 2,953 & 3,418 & 178.2 & 177.0 & 176.9 & 215.0 & 248.9 \\
\hline Orlando-Kissimmee-Sanford, FL & 2,514 & 2,571 & 3,073 & 3,393 & 4,290 & 110.9 & 110.8 & 128.7 & 139.0 & 175.7 \\
\hline Philadelphia-Camden-Wilmington, PA-NJ-DE-MD & 10,557 & 9,618 & 9,724 & 11,394 & 12,096 & 174.9 & 158.9 & 160.2 & 187.7 & 199.3 \\
\hline Phoenix-Mesa-Scottsdale, AZ & 4,918 & 5,944 & 6,495 & 8,086 & 9,433 & 111.8 & 132.4 & 142.0 & 173.5 & 202.4 \\
\hline Pittsburgh, PA & 2,827 & 2,602 & 2,422 & 2,601 & 2,370 & 119.7 & 110.4 & 102.9 & 111.0 & 101.2 \\
\hline Portland-Vancouver-Hillsboro, OR-WA & 1,199 & 1,499 & 2,386 & 3,177 & 3,444 & 51.8 & 63.8 & 99.9 & 131.0 & 142.0 \\
\hline Providence-Warwick, RI-MA & 593 & $913^{+}$ & $834^{+}$ & $1,108^{+}$ & $1,561^{+}$ & 37.0 & $56.7^{+}$ & $51.7^{+}$ & $68.6^{+}$ & $96.7^{+}$ \\
\hline Raleigh, NC & 1,384 & 1,408 & 2,018 & 1,915 & 2,491 & 114.0 & 113.3 & 158.5 & 147.0 & 191.2 \\
\hline Richmond, VA & 1,658 & 2,173 & 2,200 & 3,198 & 3,200 & 133.1 & 172.5 & 173.0 & 249.5 & 249.7 \\
\hline Riverside-San Bernardino-Ontario, CA & 3,273 & 4,292 & 4,904 & 5,958 & 7,329 & 74.7 & 96.6 & 109.2 & 131.6 & 161.9 \\
\hline Sacramento-Roseville-Arden-Arcade, CA & 2,597 & 2,616 & 3,317 & 3,402 & 4,057 & 117.2 & 116.6 & 145.9 & 148.1 & 176.7 \\
\hline Salt Lake City, UT & 690 & 1,026 & 1,078 & 1,462 & 1,693 & 60.5 & 89.0 & 92.1 & 123.3 & 142.7 \\
\hline San Antonio-New Braunfels, TX & 3,352 & 3,155 & 4,160 & 4,779 & 4,891 & 147.2 & 135.5 & 174.5 & 196.7 & 201.3 \\
\hline San Diego-Carlsbad, CA & 2,825 & 3,420 & 3,691 & 4,989 & 5,973 & 88.0 & 104.8 & 111.9 & 150.4 & 180.0 \\
\hline San Francisco-Oakland-Hayward, CA & 5,681 & 7,110 & 9,330 & 10,669 & 12,169 & 125.8 & 154.8 & 200.4 & 228.0 & 260.1 \\
\hline San Jose-Sunnyvale-Santa Clara, CA & 1,145 & 1,552 & 1,857 & 1,976 & 2,528 & 59.6 & 79.5 & 93.9 & 99.9 & 127.8 \\
\hline Seattle-Tacoma-Bellevue, WA & 2,990 & 3,931 & 4,766 & 5,149 & 6,667 & 82.8 & 107.1 & 127.7 & 135.5 & 175.5 \\
\hline St. Louis, MO-IL & 4,492 & 4,346 & 5,257 & 6,558 & 6,699 & 160.4 & 154.9 & 187.0 & 233.6 & 238.7 \\
\hline Tampa-St. Petersburg-Clearwater, FL & 3,660 & 3,455 & 3,916 & 4,408 & 4,661 & 127.5 & 118.5 & 131.6 & 145.4 & 153.7 \\
\hline Virginia Beach-Norfolk-Newport News, VA-NC & 2,581 & 3,206 & 3,300 & 4,320 & 4,785 & 151.2 & 186.8 & 191.3 & 250.2 & 277.1 \\
\hline Washington-Arlington-Alexandria, DC-VA-MD-WV & 5,616 & 2,974 & 3,008 & 4,335 & 9,682 & 94.4 & 49.3 & 49.3 & 70.7 & 157.9 \\
\hline SELECTED MSAs TOTAL & 202,170 & 212,240 & 239,580 & 288,468 & 340,672 & 117.0 & 121.4 & 135.6 & 162.1 & 191.5 \\
\hline
\end{tabular}

* MSAs were selected on the basis of the largest population in the 2010 U.S. Census.

${ }^{\dagger}$ The variable used to identify county, which is used to classify cases into MSAs, was complete for $\leq 95 \%$ of cases in a state contributing data to this MSA. See Section A1.4 in the Appendix for more information.

₹ 2016 county data for Alabama have been corrected and may not match previous reports. 


\begin{tabular}{|c|c|c|c|c|c|c|c|c|c|c|}
\hline \multirow[b]{2}{*}{ MSAs } & \multicolumn{5}{|c|}{ Cases } & \multicolumn{5}{|c|}{ Rates per 100,000 Population } \\
\hline & 2013 & 2014 & 2015 & 2016 & 2017 & 2013 & 2014 & 2015 & 2016 & 2017 \\
\hline Atlanta-Sandy Springs-Roswell, GA & $2,458^{+}$ & 3,030 & $2,578^{+}$ & 4,696 & 4,723 & $86.5^{+}$ & 104.7 & $87.5^{+}$ & 157.0 & 157.9 \\
\hline Austin-Round Rock, TX & 1,078 & 1,213 & 1,039 & 1,162 & 1,522 & 114.8 & 124.9 & 103.9 & 113.1 & 148.1 \\
\hline Baltimore-Columbia-Towson, MD & 1,542 & 1,608 & 1,964 & 2,492 & 3,059 & 107.5 & 111.5 & 135.6 & 171.9 & 211.0 \\
\hline Birmingham-Hoover, AL & 1,099 & 970 & 999 & $943^{\ddagger}$ & 1,644 & 186.0 & 163.4 & 167.9 & $158.4^{\ddagger}$ & 276.2 \\
\hline Boston-Cambridge-Newton, MA-NH & 828 & $844^{+}$ & $601^{+}$ & $720^{+}$ & $1,242^{+}$ & 34.3 & $34.7^{+}$ & $24.5^{+}$ & $29.2^{+}$ & $50.4^{+}$ \\
\hline Buffalo-Cheektowaga-Niagara Falls, NY & 594 & 664 & 959 & 985 & 1,157 & 101.6 & 113.4 & 164.0 & 168.9 & 198.4 \\
\hline Charlotte-Concord-Gastonia, NC-SC & 1,700 & 1,962 & 2,419 & 2,296 & 2,554 & 141.7 & 160.2 & 193.8 & 180.1 & 200.3 \\
\hline Chicago-Naperville-Elgin, IL-IN-WI & 6,374 & 5,662 & 5,696 & 6,481 & 6,997 & 130.9 & 115.9 & 116.8 & 133.5 & 144.1 \\
\hline Cincinnati, OH-KY-IN & 1,932 & 1,913 & 2,020 & 2,183 & 2,481 & 177.1 & 174.3 & 183.4 & 197.7 & 224.7 \\
\hline Cleveland-Elyria, $\mathrm{OH}$ & 2,328 & 2,021 & 1,745 & 2,134 & 3,043 & 217.6 & 189.2 & 163.6 & 200.7 & 286.2 \\
\hline Columbus, $\mathrm{OH}$ & 1,500 & 1,473 & 1,638 & 2,105 & 2,165 & 150.0 & 145.3 & 159.5 & 203.0 & 208.8 \\
\hline Dallas-Fort Worth-Arlington, TX & 3,921 & 4,153 & 4,963 & 4,577 & 5,504 & 113.6 & 117.5 & 137.4 & 124.5 & 149.7 \\
\hline Denver-Aurora-Lakewood, CO & 724 & 780 & 1,096 & 1,403 & 2,083 & 53.5 & 56.4 & 77.7 & 98.1 & 145.7 \\
\hline Detroit-Warren-Dearborn, MI & 3,614 & 2,698 & 2,592 & 3,190 & 4,008 & 163.5 & 122.0 & 117.2 & 144.5 & 181.5 \\
\hline Hartford-West Hartford-East Hartford, CT & 543 & 425 & $310^{+}$ & $412^{+}$ & 796 & 87.2 & 68.3 & $50.0^{+}$ & $66.7^{+}$ & 128.9 \\
\hline Houston-The Woodlands-Sugar Land, TX & 4,033 & 4,151 & 4,113 & 4,422 & 4,422 & 127.2 & 127.2 & 122.8 & 129.7 & 129.7 \\
\hline Indianapolis-Carmel-Anderson, IN & 1,761 & 1,828 & 1,791 & 2,264 & 2,533 & 176.4 & 181.3 & 176.1 & 220.9 & 247.2 \\
\hline Jacksonville, FL & 1,121 & 1,288 & 1,237 & 1,402 & 1,643 & 156.7 & 176.9 & 166.5 & 184.9 & 216.7 \\
\hline Kansas City, MO-KS & 1,424 & 1,361 & 1,409 & 1,875 & 2,448 & 136.0 & 128.9 & 132.6 & 174.9 & 228.4 \\
\hline Las Vegas-Henderson-Paradise, NV & 1,015 & 1,039 & 1,129 & 1,341 & 1,518 & 100.5 & 100.5 & 106.7 & 124.1 & 140.5 \\
\hline Los Angeles-Long Beach-Anaheim, CA & 4,578 & 5,029 & 5,778 & 7,083 & 8,808 & 68.9 & 74.8 & 85.4 & 104.9 & 130.5 \\
\hline Louisville-Jefferson County, KY-IN & 1,079 & 992 & 1,012 & 1,278 & 1,493 & 167.0 & 152.6 & 154.8 & 194.8 & 227.5 \\
\hline Memphis, TN-MS-AR & 1,550 & 1,371 & 1,469 & 1,818 & 2,304 & 222.0 & 196.0 & 209.8 & 259.7 & 329.2 \\
\hline Miami-Fort Lauderdale-West Palm Beach, FL & 2,225 & 2,123 & 2,252 & 2,606 & 2,699 & 74.2 & 69.5 & 72.7 & 83.4 & 86.4 \\
\hline Milwaukee-Waukesha-West Allis, WI & 1,655 & 1,298 & 1,850 & 2,208 & 2,346 & 205.5 & 160.8 & 228.8 & 274.0 & 291.1 \\
\hline Minneapolis-St. Paul-Bloomington, MN-WI & $1,641^{+}$ & 1,388 & 1,229 & 1,676 & 2,256 & $93.8^{+}$ & 78.6 & 69.0 & 93.5 & 125.9 \\
\hline Nashville-Davidson-Murfreesboro-Franklin, TN & 838 & 790 & 888 & 1,129 & 1,036 & 93.1 & 86.0 & 94.7 & 118.1 & 108.3 \\
\hline New Orleans-Metairie, LA & 1,317 & 1,339 & 1,423 & 1,527 & 1,512 & 206.1 & 207.2 & 218.1 & 233.1 & 230.8 \\
\hline New York-Newark-Jersey City, NY-NJ-PA & 7,615 & 6,544 & 7,349 & 7,134 & 8,210 & 73.9 & 63.1 & 70.6 & 68.6 & 78.9 \\
\hline Oklahoma City, OK & 1,305 & 1,310 & 1,267 & 1,558 & 1,771 & 195.1 & 193.2 & 183.8 & 223.8 & 254.4 \\
\hline Orlando-Kissimmee-Sanford, FL & 1,114 & 1,109 & 1,254 & 1,326 & 1,569 & 96.3 & 93.4 & 102.7 & 106.2 & 125.7 \\
\hline Philadelphia-Camden-Wilmington, PA-NJ-DE-MD & 5,052 & 4,461 & 4,277 & 4,640 & 4,544 & 162.0 & 142.7 & 136.4 & 148.0 & 145.0 \\
\hline Phoenix-Mesa-Scottsdale, AZ & 2,318 & 2,658 & 2,708 & 3,259 & 3,807 & 104.8 & 117.6 & 117.6 & 138.9 & 162.3 \\
\hline Pittsburgh, PA & 1,715 & 1,415 & 1,239 & 1,153 & 948 & 141.1 & 116.8 & 102.5 & 95.9 & 78.9 \\
\hline Portland-Vancouver-Hillsboro, OR-WA & 325 & 382 & 764 & 1,005 & 1,208 & 27.8 & 32.2 & 63.2 & 82.0 & 98.5 \\
\hline Providence-Warwick, RI-MA & 261 & $325^{+}$ & $260^{+}$ & $368^{+}$ & $559^{+}$ & 31.6 & $39.2^{+}$ & $31.3^{+}$ & $44.3^{+}$ & $67.3^{+}$ \\
\hline Raleigh, NC & 677 & 638 & 857 & 785 & 1,101 & 108.9 & 100.2 & 131.4 & 117.4 & 164.7 \\
\hline Richmond, VA & 957 & 1,194 & 1,141 & 1,473 & 1,432 & 148.7 & 183.4 & 173.6 & 222.2 & 216.0 \\
\hline Riverside-San Bernardino-Ontario, CA & 1,576 & 1,966 & 2,208 & 2,515 & 3,101 & 71.7 & 88.1 & 97.9 & 110.5 & 136.3 \\
\hline Sacramento-Roseville-Arden-Arcade, CA & 1,323 & 1,246 & 1,538 & 1,463 & 1,613 & 117.0 & 108.8 & 132.5 & 124.7 & 137.4 \\
\hline Salt Lake City, UT & 263 & 376 & 325 & 480 & 549 & 46.4 & 65.5 & 55.8 & 81.3 & 93.0 \\
\hline San Antonio-New Braunfels, TX & 1,624 & 1,445 & 1,785 & 1,981 & 2,228 & 140.7 & 122.4 & 147.8 & 161.1 & 181.1 \\
\hline San Diego-Carlsbad, CA & 827 & 1,038 & 1,018 & 1,479 & 1,593 & 51.8 & 63.9 & 62.1 & 89.7 & 96.6 \\
\hline San Francisco-Oakland-Hayward, CA & 1,491 & 1,836 & 2,101 & 2,183 & 2,646 & 65.2 & 78.9 & 89.1 & 92.2 & 111.7 \\
\hline San Jose-Sunnyvale-Santa Clara, CA & 446 & 557 & 642 & 614 & 772 & 46.7 & 57.4 & 65.4 & 62.6 & 78.7 \\
\hline Seattle-Tacoma-Bellevue, WA & 988 & 1,412 & 1,662 & 1,556 & 2,011 & 54.7 & 76.9 & 89.0 & 81.9 & 105.8 \\
\hline St. Louis, MO-IL & 2,313 & 2,087 & 2,436 & 2,911 & 2,969 & 160.2 & 144.3 & 168.1 & 201.4 & 205.4 \\
\hline Tampa-St. Petersburg-Clearwater, FL & 1,774 & 1,619 & 1,676 & 1,815 & 1,955 & 119.8 & 107.6 & 109.2 & 116.0 & 125.0 \\
\hline Virginia Beach-Norfolk-Newport News, VA-NC & 1,341 & 1,711 & 1,701 & 2,118 & 2,340 & 154.7 & 196.4 & 194.4 & 241.5 & 266.8 \\
\hline Washington-Arlington-Alexandria, DC-VA-MD-WV & 2,278 & 1,163 & 1,064 & 1,489 & 3,031 & 74.8 & 37.7 & 34.1 & 47.5 & 96.6 \\
\hline SELECTED MSAs TOTAL & 92,055 & 89,905 & 95,471 & 109,713 & 127,953 & 104.4 & 100.7 & 105.9 & 120.9 & 141.0 \\
\hline
\end{tabular}

* MSAs were selected on the basis of the largest population in the 2010 U.S. Census.

${ }^{\dagger}$ The variable used to identify county, which is used to classify cases into MSAs, was complete for $\leq 95 \%$ of cases in a state contributing data to this MSA. See Section A1.4 in the Appendix for more information.

₹ 2016 county data for Alabama have been corrected and may not match previous reports.

NOTE: Cases reported with unknown sex are not included in this table. 
Table 19. Gonorrhea Among Men - Reported Cases and Rates of Reported Cases in Selected Metropolitan Statistical Areas (MSAs)* in Alphabetical Order, United States, 2013-2017

\begin{tabular}{|c|c|c|c|c|c|c|c|c|c|c|}
\hline \multirow[b]{2}{*}{ MSAs } & \multicolumn{5}{|c|}{ Cases } & \multicolumn{5}{|c|}{ Rates per 100,000 Population } \\
\hline & 2013 & 2014 & 2015 & 2016 & 2017 & 2013 & 2014 & 2015 & 2016 & 2017 \\
\hline Atlanta-Sandy Springs-Roswell, GA & $2,952^{+}$ & 4,177 & $3,885^{+}$ & 6,960 & 7,622 & $110.1^{+}$ & 153.5 & $140.5^{+}$ & 248.6 & 272.3 \\
\hline Austin-Round Rock, TX & 1,295 & 1,635 & 2,144 & 2,499 & 2,882 & 137.2 & 168.2 & 214.2 & 242.9 & 280.1 \\
\hline Baltimore-Columbia-Towson, MD & 1,690 & 1,840 & 2,202 & 3,359 & 3,833 & 126.4 & 137.0 & 163.2 & 249.0 & 284.1 \\
\hline Birmingham-Hoover, AL & 1,029 & 979 & 1,075 & $1,068^{\ddagger}$ & 1,827 & 187.3 & 177.9 & 195.2 & $193.4^{\ddagger}$ & 330.9 \\
\hline Boston-Cambridge-Newton, MA-NH & 1,541 & $1,870^{+}$ & $1,881^{+}$ & $2,274^{+}$ & $3,120^{+}$ & 67.8 & $81.4^{\dagger}$ & $81.1^{\dagger}$ & $97.6^{+}$ & $134.0^{+}$ \\
\hline Buffalo-Cheektowaga-Niagara Falls, NY & 638 & 678 & 1,023 & 1,195 & 1,291 & 116.2 & 123.1 & 185.8 & 217.5 & 234.9 \\
\hline Charlotte-Concord-Gastonia, NC-SC & 1,356 & 1,683 & 2,251 & 2,451 & 2,856 & 119.4 & 145.6 & 191.0 & 204.4 & 238.2 \\
\hline Chicago-Naperville-Elgin, IL-IN-WI & 6,407 & 6,947 & 7,780 & 10,133 & 11,514 & 137.3 & 148.7 & 166.5 & 217.6 & 247.2 \\
\hline Cincinnati, OH-KY-IN & 1,297 & 1,431 & 1,693 & 1,913 & 2,233 & 123.9 & 136.0 & 160.2 & 180.3 & 210.4 \\
\hline Cleveland-Elyria, $\mathrm{OH}$ & 1,827 & 1,781 & 1,683 & 2,071 & 2,800 & 183.6 & 178.9 & 169.3 & 208.7 & 282.1 \\
\hline Columbus, $\mathrm{OH}$ & 1,720 & 1,787 & 2,038 & 2,716 & 3,032 & 177.9 & 182.2 & 204.9 & 270.3 & 301.8 \\
\hline Dallas-Fort Worth-Arlington, TX & 4,426 & 5,033 & 6,357 & 6,498 & 7,331 & 131.8 & 147.1 & 182.0 & 182.8 & 206.2 \\
\hline Denver-Aurora-Lakewood, CO & 1,104 & 1,236 & 1,742 & 2,445 & 3,325 & 82.1 & 90.1 & 124.1 & 171.8 & 233.6 \\
\hline Detroit-Warren-Dearborn, MI & 2,942 & 2,606 & 2,894 & 3,622 & 4,648 & 141.2 & 125.0 & 138.5 & 173.3 & 222.4 \\
\hline Hartford-West Hartford-East Hartford, CT & 522 & 466 & $416^{+}$ & $551^{+}$ & 1,033 & 88.1 & 78.7 & $70.4^{+}$ & $93.5^{+}$ & 175.3 \\
\hline Houston-The Woodlands-Sugar Land, TX & 3,749 & 4,146 & 5,167 & 5,930 & 6,343 & 119.3 & 128.5 & 156.2 & 176.3 & 188.6 \\
\hline Indianapolis-Carmel-Anderson, IN & 1,854 & 1,927 & 1,920 & 2,542 & 2,892 & 194.0 & 200.1 & 197.5 & 259.5 & 295.3 \\
\hline Jacksonville, FL & 1,198 & 1,316 & 1,501 & 1,764 & 2,076 & 176.3 & 190.5 & 212.5 & 245.0 & 288.3 \\
\hline Kansas City, MO-KS & 1,272 & 1,281 & 1,534 & 2,134 & 2,826 & 126.2 & 126.1 & 149.7 & 206.6 & 273.6 \\
\hline Las Vegas-Henderson-Paradise, NV & 1,239 & 1,612 & 1,837 & 2,306 & 2,905 & 121.8 & 155.6 & 173.9 & 214.4 & 270.2 \\
\hline Los Angeles-Long Beach-Anaheim, CA & 9,849 & 12,071 & 14,066 & 18,060 & 20,803 & 151.9 & 184.7 & 213.9 & 275.3 & 317.1 \\
\hline Louisville-Jefferson County, KY-IN & 969 & 961 & 1,170 & 1,670 & 1,910 & 157.2 & 155.1 & 187.3 & 266.2 & 304.5 \\
\hline Memphis, TN-MS-AR & 1,536 & 1,254 & 1,674 & 1,926 & 2,347 & 238.6 & 194.8 & 260.0 & 299.6 & 365.0 \\
\hline Miami-Fort Lauderdale-West Palm Beach, FL & 3,564 & 3,999 & 4,651 & 5,374 & 6,146 & 125.9 & 139.0 & 159.6 & 182.6 & 208.8 \\
\hline Milwaukee-Waukesha-West Allis, WI & 1,521 & 1,281 & 1,864 & 2,241 & 2,560 & 199.0 & 167.4 & 242.9 & 292.4 & 334.0 \\
\hline Minneapolis-St. Paul-Bloomington, MN-WI & $1,546^{+}$ & 1,942 & 2,058 & 2,439 & 2,993 & $90.4^{+}$ & 112.4 & 118.0 & 138.6 & 170.1 \\
\hline Nashville-Davidson-Murfreesboro-Franklin, TN & 968 & 1,130 & 1,312 & 1,566 & 1,670 & 112.8 & 129.3 & 147.0 & 172.3 & 183.7 \\
\hline New Orleans-Metairie, LA & 1,131 & 1,328 & 1,506 & 1,887 & 2,126 & 187.9 & 219.2 & 246.7 & 307.5 & 346.4 \\
\hline New York-Newark-Jersey City, NY-NJ-PA & 11,639 & 13,448 & 16,290 & 18,976 & 23,737 & 120.6 & 138.4 & 166.8 & 194.6 & 243.4 \\
\hline Oklahoma City, OK & 1,047 & 1,056 & 1,136 & 1,394 & 1,647 & 160.8 & 160.3 & 169.7 & 205.9 & 243.3 \\
\hline Orlando-Kissimmee-Sanford, FL & 1,399 & 1,461 & 1,818 & 2,067 & 2,720 & 126.0 & 128.8 & 155.9 & 173.2 & 228.0 \\
\hline Philadelphia-Camden-Wilmington, PA-NJ-DE-MD & 5,501 & 5,152 & 5,439 & 6,736 & 7,540 & 188.7 & 176.2 & 185.4 & 229.4 & 256.8 \\
\hline Phoenix-Mesa-Scottsdale, AZ & 2,600 & 3,286 & 3,780 & 4,824 & 5,602 & 118.9 & 147.4 & 166.4 & 208.3 & 241.9 \\
\hline Pittsburgh, PA & 1,110 & 1,187 & 1,182 & 1,448 & 1,422 & 96.9 & 103.7 & 103.3 & 127.0 & 124.7 \\
\hline Portland-Vancouver-Hillsboro, OR-WA & 874 & 1,116 & 1,621 & 2,172 & 2,236 & 76.4 & 96.2 & 137.3 & 181.1 & 186.5 \\
\hline Providence-Warwick, RI-MA & 331 & $587^{\dagger}$ & $572^{+}$ & $740^{+}$ & $1,001^{\dagger}$ & 42.6 & $75.3^{+}$ & $73.2^{\dagger}$ & $94.4^{+}$ & $127.7^{\dagger}$ \\
\hline Raleigh, NC & 707 & 770 & 1,161 & 1,130 & 1,390 & 119.2 & 127.0 & 186.9 & 178.1 & 219.1 \\
\hline Richmond, VA & 701 & 978 & 1,058 & 1,712 & 1,743 & 116.4 & 160.6 & 172.3 & 276.7 & 281.7 \\
\hline Riverside-San Bernardino-Ontario, CA & 1,695 & 2,321 & 2,692 & 3,437 & 4,207 & 77.7 & 105.0 & 120.5 & 152.6 & 186.8 \\
\hline Sacramento-Roseville-Arden-Arcade, CA & 1,271 & 1,362 & 1,771 & 1,934 & 2,433 & 117.1 & 124.0 & 159.1 & 172.3 & 216.7 \\
\hline Salt Lake City, UT & 427 & 650 & 753 & 982 & 1,144 & 74.5 & 112.3 & 128.1 & 164.8 & 192.0 \\
\hline San Antonio-New Braunfels, TX & 1,728 & 1,710 & 2,375 & 2,798 & 2,663 & 153.9 & 149.0 & 201.9 & 233.2 & 222.0 \\
\hline San Diego-Carlsbad, CA & 1,995 & 2,354 & 2,668 & 3,498 & 4,365 & 123.6 & 143.5 & 160.8 & 209.7 & 261.6 \\
\hline San Francisco-Oakland-Hayward, CA & 4,167 & 5,261 & 7,201 & 8,441 & 9,482 & 186.9 & 232.2 & 313.3 & 365.3 & 410.3 \\
\hline San Jose-Sunnyvale-Santa Clara, CA & 699 & 995 & 1,212 & 1,361 & 1,756 & 72.4 & 101.3 & 121.8 & 136.3 & 175.9 \\
\hline Seattle-Tacoma-Bellevue, WA & 2,002 & 2,519 & 3,104 & 3,593 & 4,654 & 111.1 & 137.3 & 166.3 & 189.2 & 245.1 \\
\hline St. Louis, MO-IL & 2,178 & 2,256 & 2,802 & 3,646 & 3,726 & 160.5 & 166.0 & 205.6 & 267.8 & 273.6 \\
\hline Tampa-St. Petersburg-Clearwater, FL & 1,871 & 1,823 & 2,240 & 2,592 & 2,704 & 134.6 & 129.2 & 155.6 & 176.6 & 184.2 \\
\hline Virginia Beach-Norfolk-Newport News, VA-NC & 1,239 & 1,489 & 1,595 & 2,195 & 2,423 & 147.5 & 176.2 & 187.7 & 258.3 & 285.1 \\
\hline Washington-Arlington-Alexandria, DC-VA-MD-WV & 3,330 & 1,811 & 1,943 & 2,838 & 6,613 & 114.6 & 61.4 & 65.2 & 94.8 & 220.9 \\
\hline SELECTED MSAs TOTAL & 109,653 & 121,989 & 143,737 & 178,108 & 212,152 & 129.5 & 142.5 & 166.1 & 204.4 & 243.4 \\
\hline
\end{tabular}

* MSAs were selected on the basis of the largest population in the 2010 U.S. Census.

${ }^{\dagger}$ The variable used to identify county, which is used to classify cases into MSAs, was complete for $\leq 95 \%$ of cases in a state contributing data to this MSA. See Section A1.4 in the Appendix for more information.

${ }^{\ddagger} 2016$ county data for Alabama have been corrected and may not match previous reports.

NOTE: Cases reported with unknown sex are not included in this table. 
Table 20. Gonorrhea - Reported Cases and Rates of Reported Cases in Counties and Independent Cities* Ranked by Number of Reported Cases, United States, 2017

\begin{tabular}{|c|c|c|c|c|}
\hline Rank* & County/Independent City & Cases & Rate per 100,000 Population & Cumulative Percentage \\
\hline 1 & Los Angeles County, CA & 26,103 & 257.5 & 4 \\
\hline 2 & Cook County, IL & 14,920 & 286.7 & 7 \\
\hline 3 & Maricopa County, AZ & 8,907 & 209.9 & 8 \\
\hline 4 & Harris County, TX & 8,826 & 192.3 & 10 \\
\hline 5 & New York County, NY & 7,845 & 477.3 & 11 \\
\hline 6 & Philadelphia County, PA & 7,288 & 464.8 & 13 \\
\hline 7 & Kings County, NY & 6,824 & 259.6 & 14 \\
\hline 8 & Dallas County, TX & 6,800 & 264.1 & 15 \\
\hline 9 & Wayne County, MI & 6,349 & 362.9 & 16 \\
\hline 10 & San Diego County, CA & 5,973 & 180.0 & 17 \\
\hline 11 & San Francisco County, CA & 5,775 & 663.1 & 19 \\
\hline 12 & Cuyahoga County, OH & 5,097 & 408.0 & 19 \\
\hline 13 & Bronx County, NY & 4,941 & 339.4 & 20 \\
\hline 14 & Milwaukee County, WI & 4,706 & 494.6 & 21 \\
\hline 15 & Marion County, IN & 4,691 & 498.4 & 22 \\
\hline 16 & Washington, D.C. & 4,563 & 669.9 & 23 \\
\hline 17 & Bexar County, TX & 4,496 & 233.1 & 24 \\
\hline 18 & Franklin County, OH & 4,444 & 351.4 & 24 \\
\hline 19 & Clark County, NV & 4,430 & 205.5 & 25 \\
\hline 20 & Baltimore (City), MD & 4,231 & 688.3 & 26 \\
\hline 21 & King County, WA & 4,154 & 193.2 & 27 \\
\hline 22 & Fulton County, GA & 4,017 & 392.5 & 27 \\
\hline 23 & San Bernardino County, CA & 3,978 & 185.9 & 28 \\
\hline 24 & Broward County, FL & 3,933 & 206.0 & 29 \\
\hline 25 & Shelby County, TN & 3,694 & 395.2 & 30 \\
\hline 26 & Alameda County, CA & 3,597 & 218.3 & 30 \\
\hline 27 & Orange County, CA & 3,566 & 112.4 & 31 \\
\hline 28 & Miami-Dade County, FL & 3,538 & 130.4 & 31 \\
\hline 29 & Queens County, NY & 3,513 & 150.6 & 32 \\
\hline 30 & Hamilton County, OH & 3,381 & 417.9 & 33 \\
\hline 31 & Riverside County, CA & 3,351 & 140.3 & 33 \\
\hline 32 & Sacramento County, CA & 3,342 & 220.7 & 34 \\
\hline 33 & Travis County, TX & 3,306 & 275.7 & 35 \\
\hline 34 & Duval County, FL & 3,239 & 349.7 & 35 \\
\hline 35 & Mecklenburg County, NC & 3,181 & 301.6 & 36 \\
\hline 36 & Jackson County, MO & 3,146 & 454.8 & 36 \\
\hline 37 & Orange County, FL & 3,100 & 235.9 & 37 \\
\hline 38 & Hennepin County, MN & 3,028 & 245.7 & 37 \\
\hline 39 & Jefferson County, AL & 2,920 & 442.7 & 38 \\
\hline 40 & Tarrant County, TX & 2,851 & 141.4 & 38 \\
\hline 41 & Denver County, CO & 2,821 & 407.0 & 39 \\
\hline 42 & Jefferson County, KY & 2,790 & 364.5 & 39 \\
\hline 43 & Oklahoma County, OK & 2,606 & 332.8 & 40 \\
\hline 44 & St. Louis County, MO & 2,599 & 260.3 & 40 \\
\hline 45 & DeKalb County, GA & 2,550 & 344.4 & 41 \\
\hline 46 & Santa Clara County, CA & 2,466 & 128.5 & 41 \\
\hline 47 & Hillsborough County, FL & 2,450 & 178.0 & 42 \\
\hline 48 & Kern County, CA & 2,264 & 255.9 & 42 \\
\hline 49 & Fresno County, CA & 2,261 & 230.7 & 42 \\
\hline 50 & St. Louis (City), MO & 2,191 & 703.6 & 43 \\
\hline 51 & Orleans Parish, LA & 2,123 & 542.3 & 43 \\
\hline 52 & Tulsa County, OK & 2,111 & 328.3 & 44 \\
\hline 53 & Multnomah County, OR & 2,086 & 260.8 & 44 \\
\hline 54 & Wake County, NC & 2,082 & 198.9 & 44 \\
\hline 55 & Bernalillo County, NM & 2,079 & 307.1 & 45 \\
\hline 56 & Erie County, NY & 2,039 & 221.4 & 45 \\
\hline 57 & Essex County, NJ & 2,015 & 252.9 & 45 \\
\hline 58 & Prince George's County, MD & 2,001 & 220.4 & 46 \\
\hline 59 & Guilford County, NC & 1,907 & 365.8 & 46 \\
\hline 60 & Monroe County, NY & 1,891 & 252.9 & 47 \\
\hline 61 & Allegheny County, PA & 1,794 & 146.4 & 47 \\
\hline 62 & Pierce County, WA & 1,774 & 206.0 & 47 \\
\hline 63 & Contra Costa County, CA & 1,760 & 155.0 & 48 \\
\hline 64 & Lucas County, $\mathrm{OH}$ & 1,693 & 391.5 & 48 \\
\hline 65 & Richland County, SC & 1,669 & 407.5 & 48 \\
\hline 66 & Davidson County, TN & 1,656 & 242.0 & 48 \\
\hline 67 & Hartford County, CT & 1,656 & 185.6 & 49 \\
\hline 68 & Salt Lake County, UT & 1,651 & 147.2 & 49 \\
\hline 69 & Collin County, TX & 1,641 & 174.7 & 49 \\
\hline 70 & Pulaski County, AR & 1,632 & 415.0 & 50 \\
\hline
\end{tabular}

* The top 70 counties and independent cities ranked in descending order by number of cases reported in 2017 then by rate are displayed.

NOTE: Relative rankings of counties may be impacted by completeness of the variable used to identify county. In 2017, the variable used to identify county was complete for $\leq 95 \%$ of cases in Massachusetts. See Section A1.4 in the Appendix for more information. 
Table 21. Gonorrhea - Reported Cases and Rates of Reported Cases by Age Group and Sex, United States, 2013-2017

\begin{tabular}{|c|c|c|c|c|c|c|c|c|}
\hline \multirow{2}{*}{$\begin{array}{l}\text { Age } \\
\text { Group }\end{array}$} & \multicolumn{4}{|c|}{ Cases } & \multicolumn{3}{|c|}{ Rates per 100,000 Population* } & \\
\hline & Total & Male & Female & Unknown Sex & Total & Male & Female & \\
\hline $0-4$ & 172 & 60 & 111 & 1 & 0.9 & 0.6 & 1.1 & \multirow{14}{*}{$\stackrel{\text { O }}{\mathbf{\omega}}$} \\
\hline $5-9$ & 75 & 11 & 64 & 0 & 0.4 & 0.1 & 0.6 & \\
\hline $10-14$ & 2,637 & 508 & 2,122 & 7 & 12.8 & 4.8 & 21.0 & \\
\hline $15-19$ & 72,092 & 24,212 & 47,749 & 131 & 340.7 & 223.2 & 463.0 & \\
\hline $20-24$ & 113,035 & 53,055 & 59,760 & 220 & 495.9 & 454.3 & 537.6 & \\
\hline $25-29$ & 62,102 & 34,718 & 27,266 & 118 & 287.8 & 316.8 & 256.7 & \\
\hline $30-34$ & 34,065 & 20,855 & 13,143 & 67 & 160.2 & 195.2 & 124.2 & \\
\hline $35-39$ & 18,034 & 11,850 & 6,145 & 39 & 92.0 & 121.1 & 62.6 & \\
\hline $40-44$ & 11,817 & 8,590 & 3,192 & 35 & 56.7 & 82.9 & 30.4 & \\
\hline $45-54$ & 13,823 & 11,087 & 2,714 & 22 & 31.6 & 51.4 & 12.2 & \\
\hline $55-64$ & 3,802 & 3,176 & 621 & 5 & 9.7 & 16.8 & 3.1 & \\
\hline $65+$ & 825 & 696 & 128 & 1 & 1.8 & 3.6 & 0.5 & \\
\hline Unknown Age & 525 & 312 & 193 & 20 & & & & \\
\hline TOTAL & 333,004 & 169,130 & 163,208 & 666 & 105.3 & 108.7 & 101.7 & \\
\hline $0-4$ & 154 & 47 & 105 & 2 & 0.8 & 0.5 & 1.1 & \multirow{14}{*}{$\underset{\mathbf{O}}{\mathbf{O}}$} \\
\hline $5-9$ & 53 & 7 & 46 & 0 & 0.3 & 0.1 & 0.5 & \\
\hline $10-14$ & 2,450 & 440 & 2,005 & 5 & 11.9 & 4.2 & 19.8 & \\
\hline $15-19$ & 68,468 & 23,981 & 44,399 & 88 & 325.0 & 222.4 & 431.7 & \\
\hline $20-24$ & 116,200 & 56,714 & 59,329 & 157 & 507.2 & 483.1 & 531.0 & \\
\hline $25-29$ & 69,587 & 40,602 & 28,899 & 86 & 316.5 & 363.8 & 266.9 & \\
\hline $30-34$ & 38,393 & 24,349 & 13,988 & 56 & 178.3 & 225.3 & 130.5 & \\
\hline $35-39$ & 20,803 & 14,129 & 6,654 & 20 & 104.4 & 142.1 & 66.7 & \\
\hline $40-44$ & 12,687 & 9,349 & 3,320 & 18 & 61.6 & 91.5 & 32.0 & \\
\hline $45-54$ & 15,322 & 12,388 & 2,917 & 17 & 35.3 & 57.8 & 13.2 & \\
\hline $55-64$ & 4,549 & 3,859 & 680 & 10 & 11.4 & 20.0 & 3.3 & \\
\hline $65+$ & 911 & 790 & 121 & 0 & 2.0 & 3.9 & 0.5 & \\
\hline Unknown Age & 485 & 288 & 145 & 52 & & & & \\
\hline TOTAL & 350,062 & 186,943 & 162,608 & 511 & 109.8 & 119.1 & 100.4 & \\
\hline $0-4$ & 148 & 47 & 98 & 3 & 0.7 & 0.5 & 1.0 & \multirow{14}{*}{$\underset{\mathbf{U}}{\mathbf{v}}$} \\
\hline $5-9$ & 78 & 11 & 66 & 1 & 0.4 & 0.1 & 0.7 & \\
\hline $10-14$ & 2,312 & 385 & 1,923 & 4 & 11.2 & 3.7 & 19.0 & \\
\hline $15-19$ & 72,001 & 26,401 & 45,477 & 123 & 341.1 & 244.5 & 441.1 & \\
\hline $20-24$ & 124,592 & 63,289 & 61,105 & 198 & 547.9 & 542.4 & 551.9 & \\
\hline $25-29$ & 82,867 & 50,089 & 32,662 & 116 & 368.9 & 439.0 & 295.5 & \\
\hline $30-34$ & 45,681 & 29,751 & 15,867 & 63 & 210.7 & 273.2 & 147.1 & \\
\hline $35-39$ & 26,137 & 18,198 & 7,897 & 42 & 128.3 & 178.9 & 77.4 & \\
\hline $40-44$ & 15,042 & 11,116 & 3,898 & 28 & 74.4 & 110.8 & 38.3 & \\
\hline $45-54$ & 18,779 & 15,379 & 3,375 & 25 & 43.5 & 72.2 & 15.4 & \\
\hline $55-64$ & 6,035 & 5,175 & 849 & 11 & 14.8 & 26.2 & 4.0 & \\
\hline $65+$ & 1,191 & 1,032 & 158 & 1 & 2.5 & 4.9 & 0.6 & \\
\hline Unknown Age & 353 & 197 & 139 & 17 & & & & \\
\hline TOTAL & 395,216 & 221,070 & 173,514 & 632 & 123.0 & 139.7 & 106.3 & \\
\hline $0-4$ & 187 & 72 & 113 & 2 & 0.9 & 0.7 & 1.2 & \multirow{14}{*}{ 全 } \\
\hline $5-9$ & 98 & 16 & 81 & 1 & 0.5 & 0.2 & 0.8 & \\
\hline $10-14$ & 2,436 & 498 & 1,929 & 9 & 11.8 & 4.7 & 19.1 & \\
\hline $15-19$ & 80,172 & 30,316 & 49,710 & 146 & 379.4 & 280.7 & 481.3 & \\
\hline $20-24$ & 138,130 & 71,967 & 65,930 & 233 & 617.2 & 626.3 & 605.4 & \\
\hline $25-29$ & 101,283 & 62,189 & 38,881 & 213 & 442.5 & 534.7 & 345.3 & \\
\hline $30-34$ & 57,646 & 38,193 & 19,306 & 147 & 264.6 & 348.2 & 178.5 & \\
\hline $35-39$ & 34,058 & 23,744 & 10,230 & 84 & 163.9 & 228.8 & 98.4 & \\
\hline $40-44$ & 19,104 & 14,116 & 4,935 & 53 & 97.0 & 144.4 & 49.7 & \\
\hline $45-54$ & 24,142 & 19,762 & 4,330 & 50 & 56.4 & 93.6 & 20.0 & \\
\hline $55-64$ & 8,138 & 6,947 & 1,178 & 13 & 19.6 & 34.7 & 5.5 & \\
\hline $65+$ & 1,599 & 1,403 & 191 & 5 & 3.2 & 6.4 & 0.7 & \\
\hline Unknown Age & 1,521 & 810 & 685 & 26 & & & & \\
\hline TOTAL & 468,514 & 270,033 & 197,499 & 982 & 145.0 & 169.7 & 120.4 & \\
\hline $0-4$ & 203 & 56 & 144 & 3 & 1.0 & 0.5 & 1.5 & \multirow{14}{*}{$\underset{\mathbf{V}}{\mathbf{V}}$} \\
\hline $5-9$ & 110 & 19 & 90 & 1 & 0.5 & 0.2 & 0.9 & \\
\hline $10-14$ & 2,725 & 507 & 2,212 & 6 & 13.2 & 4.8 & 21.9 & \\
\hline $15-19$ & 92,608 & 34,918 & 57,573 & 117 & 438.3 & 323.3 & 557.4 & \\
\hline $20-24$ & 155,862 & 81,036 & 74,578 & 248 & 696.4 & 705.2 & 684.8 & \\
\hline $25-29$ & 121,880 & 75,123 & 46,577 & 180 & 532.4 & 645.9 & 413.7 & \\
\hline $30-34$ & 71,603 & 47,342 & 24,157 & 104 & 328.7 & 431.6 & 223.3 & \\
\hline $35-39$ & 43,792 & 30,277 & 13,448 & 67 & 210.8 & 291.8 & 129.3 & \\
\hline $40-44$ & 24,108 & 17,753 & 6,331 & 24 & 122.4 & 181.6 & 63.8 & \\
\hline $45-54$ & 29,428 & 23,803 & 5,580 & 45 & 68.8 & 112.8 & 25.7 & \\
\hline $55-64$ & 10,867 & 9,311 & 1,538 & 18 & 26.2 & 46.6 & 7.2 & \\
\hline $65+$ & 2,063 & 1,818 & 233 & 12 & 4.2 & 8.3 & 0.8 & \\
\hline Unknown Age & 359 & 206 & 126 & 27 & & & & \\
\hline TOTAL & 555,608 & 322,169 & 232,587 & 852 & 171.9 & 202.5 & 141.8 & \\
\hline
\end{tabular}

* No population data are available for unknown sex and age; therefore, rates are not calculated.

NOTE: This table should be used only for age comparisons. Cases in the $0-4$ age group may include cases due to perinatal transmission. 
Table 22A. Gonorrhea — Reported Cases by Race/Hispanic Ethnicity, Age Group, and Sex, United States, 2017

\begin{tabular}{|c|c|c|c|c|c|c|c|c|c|c|c|c|}
\hline \multirow{2}{*}{$\begin{array}{l}\text { Age } \\
\text { Group }\end{array}$} & \multicolumn{3}{|c|}{$\begin{array}{l}\text { American Indians/ } \\
\text { Alaska Natives }\end{array}$} & \multicolumn{3}{|c|}{ Asians } & \multicolumn{3}{|c|}{ Blacks } & \multicolumn{3}{|c|}{$\begin{array}{l}\text { Native Hawaiians/ } \\
\text { Other Pacific Islanders }\end{array}$} \\
\hline & Total* & Male & Female & Total* & Male & Female & Total* & Male & Female & Total* & Male & Female \\
\hline $0-4$ & 3 & 0 & 3 & 1 & 0 & 1 & 71 & 17 & 54 & 4 & 3 & 1 \\
\hline $5-9$ & 3 & 1 & 2 & 1 & 0 & 1 & 36 & 8 & 28 & 0 & 0 & 0 \\
\hline $10-14$ & 46 & 6 & 40 & 12 & 0 & 12 & 1,398 & 302 & 1,095 & 5 & 2 & 3 \\
\hline $15-19$ & 1,022 & 300 & 721 & 460 & 187 & 272 & 45,197 & 18,077 & 27,090 & 151 & 53 & 98 \\
\hline $20-24$ & 1,797 & 632 & 1,165 & 1,433 & 986 & 447 & 69,695 & 36,136 & 33,513 & 284 & 117 & 167 \\
\hline $25-29$ & 1,756 & 671 & 1,085 & 1,469 & 1,175 & 293 & 48,288 & 30,162 & 18,087 & 239 & 140 & 98 \\
\hline $30-34$ & 1,157 & 482 & 675 & 1,067 & 849 & 214 & 23,600 & 16,494 & 7,087 & 153 & 97 & 56 \\
\hline $35-39$ & 680 & 336 & 341 & 653 & 513 & 138 & 13,171 & 9,726 & 3,436 & 120 & 72 & 48 \\
\hline $40-44$ & 373 & 160 & 213 & 446 & 368 & 78 & 6,924 & 5,523 & 1,398 & 47 & 31 & 16 \\
\hline $45-54$ & 266 & 132 & 133 & 462 & 395 & 66 & 8,227 & 7,093 & 1,131 & 46 & 38 & 8 \\
\hline $55-64$ & 83 & 60 & 23 & 131 & 105 & 26 & 3,297 & 2,977 & 318 & 11 & 9 & 2 \\
\hline $65+$ & 17 & 17 & 0 & 24 & 20 & 4 & 531 & 488 & 42 & 4 & 3 & 1 \\
\hline Unknown Age & 5 & 2 & 3 & 4 & 4 & 0 & 71 & 47 & 24 & 1 & 0 & 1 \\
\hline TOTAL & 7,208 & 2,799 & 4,404 & 6,163 & 4,602 & 1,552 & 220,506 & 127,050 & 93,303 & 1,065 & 565 & 499 \\
\hline
\end{tabular}

\begin{tabular}{|c|c|c|c|c|c|c|c|c|c|c|c|c|}
\hline \multirow{2}{*}{$\begin{array}{l}\text { Age } \\
\text { Group }\end{array}$} & \multicolumn{3}{|c|}{ Whites } & \multicolumn{3}{|c|}{ Multirace } & \multicolumn{3}{|c|}{ Hispanics } & \multicolumn{3}{|c|}{$\begin{array}{c}\text { Other/ } \\
\text { Unknown }\end{array}$} \\
\hline & Total* & Male & Female & Total* & Male & Female & Total* & Male & Female & Total* & Male & Female \\
\hline $0-4$ & 46 & 10 & 36 & 0 & 0 & 0 & 13 & 4 & 9 & 65 & 22 & 40 \\
\hline $5-9$ & 30 & 5 & 25 & 0 & 0 & 0 & 9 & 1 & 8 & 31 & 4 & 26 \\
\hline $10-14$ & 364 & 29 & 335 & 28 & 2 & 26 & 234 & 32 & 202 & 638 & 134 & 499 \\
\hline $15-19$ & 15,154 & 4,203 & 10,941 & 821 & 225 & 595 & 9,366 & 3,559 & 5,801 & 20,437 & 8,314 & 12,055 \\
\hline $20-24$ & 31,018 & 14,464 & 16,528 & 1,455 & 626 & 826 & 18,095 & 10,199 & 7,874 & 32,085 & 17,876 & 14,058 \\
\hline $25-29$ & 29,396 & 16,460 & 12,918 & 1,107 & 693 & 411 & 15,237 & 10,178 & 5,031 & 24,388 & 15,644 & 8,654 \\
\hline $30-34$ & 20,428 & 12,159 & 8,255 & 788 & 571 & 217 & 9,497 & 6,691 & 2,794 & 14,913 & 9,999 & 4,859 \\
\hline $35-39$ & 13,244 & 8,456 & 4,783 & 412 & 326 & 85 & 5,863 & 4,163 & 1,694 & 9,649 & 6,685 & 2,923 \\
\hline $40-44$ & 7,316 & 5,016 & 2,296 & 238 & 193 & 45 & 3,121 & 2,384 & 734 & 5,643 & 4,078 & 1,551 \\
\hline $45-54$ & 9,886 & 7,895 & 1,984 & 263 & 231 & 31 & 3,057 & 2,440 & 607 & 7,221 & 5,579 & 1,620 \\
\hline $55-64$ & 3,749 & 3,228 & 516 & 72 & 65 & 7 & 731 & 610 & 121 & 2,793 & 2,257 & 525 \\
\hline $65+$ & 733 & 667 & 66 & 16 & 14 & 2 & 98 & 79 & 19 & 640 & 530 & 99 \\
\hline Unknown Age & 56 & 35 & 20 & 0 & 0 & 0 & 46 & 31 & 14 & 176 & 87 & 64 \\
\hline TOTAL & 131,420 & 72,627 & 58,703 & 5,200 & 2,946 & 2,245 & 65,367 & 40,371 & 24,908 & 118,679 & 71,209 & 46,973 \\
\hline
\end{tabular}

* Total includes cases reported with unknown sex.

NOTE: These tables should be used only for race/Hispanic ethnicity comparisons. See Table 21 for age-specific cases and rates and Tables $14-16$ for total and sexspecific cases and rates. Cases in the $0-4$ age group may include cases due to perinatal transmission. 
Table 22B. Gonorrhea - Rates of Reported Cases per 100,000 Population by Race/Hispanic Ethnicity, Age Group, and Sex, United States, 2017

\begin{tabular}{|c|c|c|c|c|c|c|c|c|c|c|c|c|}
\hline \multirow{2}{*}{$\begin{array}{l}\text { Age } \\
\text { Group }\end{array}$} & \multicolumn{3}{|c|}{$\begin{array}{l}\text { American Indians/ } \\
\text { Alaska Natives }\end{array}$} & \multicolumn{3}{|c|}{ Asians } & \multicolumn{3}{|c|}{ Blacks } & \multicolumn{3}{|c|}{$\begin{array}{l}\text { Native Hawaiians/ } \\
\text { Other Pacific Islanders }\end{array}$} \\
\hline & Total* & Male & Female & Total* & Male & Female & Total* & Male & Female & Total* & Male & Female \\
\hline $0-4$ & 1.8 & 0.0 & 3.7 & 0.1 & 0.0 & 0.2 & 2.6 & 1.2 & 4.0 & 9.8 & 14.3 & 5.0 \\
\hline $5-9$ & 1.7 & 1.1 & 2.3 & 0.1 & 0.0 & 0.2 & 1.3 & 0.6 & 2.0 & 0.0 & 0.0 & 0.0 \\
\hline 10-14 & 26.1 & 6.7 & 46.1 & 1.2 & 0.0 & 2.3 & 50.0 & 21.3 & 79.4 & 12.3 & 9.8 & 14.8 \\
\hline $15-19$ & 561.9 & 324.5 & 806.0 & 43.9 & 35.4 & 52.3 & $1,515.2$ & $1,194.3$ & $1,843.8$ & 373.0 & 254.7 & 498.0 \\
\hline $20-24$ & 926.6 & 635.9 & $1,232.2$ & 113.5 & 153.8 & 71.9 & $2,112.9$ & $2,154.8$ & $2,066.8$ & 635.1 & 508.3 & 769.7 \\
\hline $25-29$ & 922.1 & 689.7 & $1,164.9$ & 96.7 & 158.1 & 37.8 & $1,482.8$ & $1,863.1$ & $1,104.4$ & 458.8 & 515.8 & 392.7 \\
\hline $30-34$ & 701.5 & 584.1 & 819.1 & 69.7 & 116.2 & 26.7 & 843.1 & $1,219.6$ & 489.9 & 306.9 & 380.3 & 230.0 \\
\hline 35-39 & 449.2 & 448.1 & 446.3 & 45.5 & 76.9 & 18.0 & 493.8 & 768.4 & 245.2 & 274.6 & 321.0 & 225.7 \\
\hline $40-44$ & 265.4 & 231.1 & 298.7 & 31.6 & 55.7 & 10.4 & 278.2 & 471.8 & 106.0 & 125.3 & 163.2 & 86.4 \\
\hline $45-54$ & 87.3 & 89.1 & 84.9 & 18.9 & 34.8 & 5.1 & 156.0 & 286.6 & 40.4 & 65.3 & 109.0 & 22.5 \\
\hline $55-64$ & 29.2 & 45.0 & 15.3 & 6.7 & 11.8 & 2.4 & 69.9 & 138.1 & 12.4 & 19.4 & 32.5 & 6.9 \\
\hline $65+$ & 6.6 & 14.6 & 0.0 & 1.1 & 2.2 & 0.3 & 12.1 & 27.6 & 1.6 & 8.1 & 13.1 & 3.8 \\
\hline \multicolumn{13}{|c|}{ Unknown Age } \\
\hline TOTAL & 301.9 & 238.0 & 363.6 & 34.7 & 54.5 & 16.7 & 548.1 & 660.7 & 444.3 & 187.8 & 197.7 & 177.3 \\
\hline
\end{tabular}

\begin{tabular}{|c|c|c|c|c|c|c|c|c|c|}
\hline \multirow{2}{*}{$\begin{array}{l}\text { Age } \\
\text { Group }\end{array}$} & \multicolumn{3}{|c|}{ Whites } & \multicolumn{3}{|c|}{ Multirace } & \multicolumn{3}{|c|}{ Hispanics } \\
\hline & Total* & Male & Female & Total* & Male & Female & Total* & Male & Female \\
\hline $0-4$ & 0.5 & 0.2 & 0.7 & 0.0 & 0.0 & 0.0 & 0.3 & 0.2 & 0.4 \\
\hline $5-9$ & 0.3 & 0.1 & 0.5 & 0.0 & 0.0 & 0.0 & 0.2 & 0.0 & 0.3 \\
\hline $10-14$ & 3.4 & 0.5 & 6.4 & 3.5 & 0.5 & 6.6 & 4.7 & 1.3 & 8.2 \\
\hline $15-19$ & 133.2 & 72.0 & 197.5 & 116.7 & 63.1 & 171.5 & 195.5 & 145.3 & 247.6 \\
\hline $20-24$ & 255.1 & 231.3 & 280.0 & 234.5 & 200.2 & 268.3 & 376.8 & 410.7 & 339.6 \\
\hline $25-29$ & 230.2 & 253.5 & 205.8 & 224.6 & 290.7 & 161.5 & 330.4 & 421.6 & 229.0 \\
\hline $30-34$ & 164.8 & 194.2 & 134.6 & 200.5 & 307.1 & 104.8 & 213.2 & 287.1 & 131.5 \\
\hline $35-39$ & 111.8 & 141.6 & 81.4 & 121.5 & 204.8 & 47.3 & 136.6 & 187.9 & 81.5 \\
\hline $40-44$ & 64.3 & 87.7 & 40.6 & 84.8 & 146.2 & 30.3 & 78.9 & 118.8 & 37.6 \\
\hline $45-54$ & 35.9 & 57.6 & 14.4 & 52.8 & 98.3 & 11.8 & 45.7 & 72.4 & 18.3 \\
\hline $55-64$ & 12.7 & 22.4 & 3.4 & 17.8 & 33.8 & 3.3 & 16.4 & 28.2 & 5.3 \\
\hline $65+$ & 1.9 & 3.9 & 0.3 & 4.4 & 8.7 & 1.0 & 2.5 & 4.6 & 0.8 \\
\hline \multicolumn{10}{|c|}{ Unknown Age } \\
\hline TOTAL & 66.4 & 74.4 & 58.5 & 76.9 & 88.4 & 65.4 & 113.7 & 139.1 & 87.5 \\
\hline
\end{tabular}

* Total includes cases reported with unknown sex.

NOTE: These tables should be used only for race/Hispanic ethnicity comparisons. See Table 21 for age-specific cases and rates and Tables 14-16 for total and sexspecific cases and rates. Cases in the 0-4 age group may include cases due to perinatal transmission. No population data exist for unknown sex, unknown age, or unknown race; therefore rates are not calculated. 
Table 23. Gonorrhea Among Women Aged 15-24 Years - Reported Cases and Rates of Reported Cases by Age, United States, 2013-2017

\begin{tabular}{|c|c|c|c|}
\hline & Age & Cases & Rate per 100,000 Population \\
\hline \multirow{11}{*}{$\frac{m}{\text { m }}$} & 15 & 3,776 & 186.4 \\
\hline & 16 & 6,503 & 320.0 \\
\hline & 17 & 9,374 & 458.8 \\
\hline & 18 & 13,393 & 642.4 \\
\hline & 19 & 14,703 & 691.2 \\
\hline & 20 & 14,420 & 668.8 \\
\hline & 21 & 13,394 & 603.1 \\
\hline & 22 & 12,272 & 539.6 \\
\hline & 23 & 10,819 & 475.3 \\
\hline & 24 & 8,855 & 404.5 \\
\hline & Total & 107,509 & 501.7 \\
\hline \multirow{11}{*}{$\frac{+}{\text { 유 }}$} & 15 & 3,487 & 171.3 \\
\hline & 16 & 6,188 & 303.9 \\
\hline & 17 & 8,830 & 431.6 \\
\hline & 18 & 12,196 & 591.9 \\
\hline & 19 & 13,698 & 650.5 \\
\hline & 20 & 13,801 & 642.1 \\
\hline & 21 & 13,324 & 611.5 \\
\hline & 22 & 12,031 & 536.0 \\
\hline & 23 & 10,746 & 467.5 \\
\hline & 24 & 9,427 & 409.6 \\
\hline & Total & 103,728 & 483.4 \\
\hline \multirow{11}{*}{ ำ } & 15 & 3,477 & 167.4 \\
\hline & 16 & 6,090 & 297.8 \\
\hline & 17 & 9,117 & 444.9 \\
\hline & 18 & 12,769 & 619.3 \\
\hline & 19 & 14,024 & 674.8 \\
\hline & 20 & 13,835 & 651.7 \\
\hline & 21 & 13,331 & 615.2 \\
\hline & 22 & 12,597 & 573.2 \\
\hline & 23 & 11,271 & 497.7 \\
\hline & 24 & 10,071 & 434.2 \\
\hline & Total & 106,582 & 498.5 \\
\hline \multirow{11}{*}{$\frac{0}{\circ}$} & 15 & 3,678 & 179.0 \\
\hline & 16 & 6,573 & 315.4 \\
\hline & 17 & 9,855 & 479.7 \\
\hline & 18 & 14,304 & 694.1 \\
\hline & 19 & 15,300 & 737.8 \\
\hline & 20 & 14,657 & 701.7 \\
\hline & 21 & 14,149 & 663.1 \\
\hline & 22 & 13,540 & 621.4 \\
\hline & 23 & 12,245 & 553.9 \\
\hline & 24 & 11,339 & 497.8 \\
\hline & Total & 115,640 & 545.0 \\
\hline \multirow{11}{*}{$\underset{\mathbf{N}}{\mathbf{N}}$} & 15 & 3,976 & 193.5 \\
\hline & 16 & 7,600 & 364.7 \\
\hline & 17 & 11,479 & 558.7 \\
\hline & 18 & 16,429 & 797.2 \\
\hline & 19 & 18,089 & 872.2 \\
\hline & 20 & 16,950 & 811.4 \\
\hline & 21 & 15,968 & 748.3 \\
\hline & 22 & 14,933 & 685.4 \\
\hline & 23 & 14,046 & 635.4 \\
\hline & 24 & 12,681 & 556.7 \\
\hline & Total & 132,151 & 622.8 \\
\hline
\end{tabular}

NOTE: This table should be used only for age comparisons. Cases reported with unknown sex are not included in this table. 
Table 24. All Stages of Syphilis* - Reported Cases and Rates of Reported Cases by State/Area and Region in Alphabetical Order, United States and Outlying Areas, 2013-2017

\begin{tabular}{|c|c|c|c|c|c|c|c|c|c|c|}
\hline \multirow[b]{2}{*}{ State/Area } & \multicolumn{5}{|c|}{ Cases } & \multicolumn{5}{|c|}{ Rates per 100,000 Population } \\
\hline & 2013 & 2014 & 2015 & 2016 & 2017 & 2013 & 2014 & 2015 & 2016 & 2017 \\
\hline Alabama & 679 & 475 & 657 & 905 & 1,202 & 14.0 & 9.8 & 13.5 & 18.6 & 24.7 \\
\hline Alaska & 35 & 45 & 24 & 24 & 28 & 4.8 & 6.1 & 3.3 & 3.2 & 3.8 \\
\hline Arizona & 963 & 1,459 & 1,496 & 1,905 & 2,422 & 14.5 & 21.7 & 21.9 & 27.5 & 34.9 \\
\hline Arkansas & 527 & 390 & 500 & 567 & 726 & 17.8 & 13.1 & 16.8 & 19.0 & 24.3 \\
\hline California & 9,973 & 11,443 & 14,449 & 17,603 & 21,804 & 26.0 & 29.5 & 36.9 & 44.8 & 55.6 \\
\hline Colorado & 475 & 355 & 553 & 739 & 817 & 9.0 & 6.6 & 10.1 & 13.3 & 14.7 \\
\hline Connecticut & 133 & 169 & 220 & 217 & 283 & 3.7 & 4.7 & 6.1 & 6.1 & 7.9 \\
\hline Delaware & 146 & 110 & 110 & 149 & 194 & 15.8 & 11.8 & 11.6 & 15.7 & 20.4 \\
\hline District of Columbia & 609 & 281 & 322 & 568 & 845 & 94.2 & 42.6 & 47.9 & 83.4 & 124.1 \\
\hline Florida & 5,022 & 6,103 & 7,132 & 8,333 & 8,951 & 25.7 & 30.7 & 35.2 & 40.4 & 43.4 \\
\hline Georgia & 2,990 & 3,384 & 4,156 & 4,112 & 4,310 & 29.9 & 33.5 & 40.7 & 39.9 & 41.8 \\
\hline Hawaii & 87 & 106 & 163 & 215 & 165 & 6.2 & 7.5 & 11.4 & 15.1 & 11.6 \\
\hline Idaho & 42 & 46 & 102 & 127 & 151 & 2.6 & 2.8 & 6.2 & 7.5 & 9.0 \\
\hline Illinois & 2,661 & 2,796 & 3,290 & 4,039 & 3,837 & 20.7 & 21.7 & 25.6 & 31.6 & 30.0 \\
\hline Indiana & 543 & 475 & 699 & 778 & 788 & 8.3 & 7.2 & 10.6 & 11.7 & 11.9 \\
\hline lowa & 226 & 239 & 232 & 276 & 290 & 7.3 & 7.7 & 7.4 & 8.8 & 9.3 \\
\hline Kansas & 196 & 200 & 240 & 303 & 338 & 6.8 & 6.9 & 8.2 & 10.4 & 11.6 \\
\hline Kentucky & 395 & 447 & 433 & 572 & 721 & 9.0 & 10.1 & 9.8 & 12.9 & 16.2 \\
\hline Louisiana & 2,006 & 2,173 & 2,466 & 2,599 & 2,856 & 43.4 & 46.7 & 52.8 & 55.5 & 61.0 \\
\hline Maine & 21 & 23 & 38 & 64 & 132 & 1.6 & 1.7 & 2.9 & 4.8 & 9.9 \\
\hline Maryland & 1,361 & 1,475 & 1,870 & 1,842 & 2,059 & 23.0 & 24.7 & 31.1 & 30.6 & 34.2 \\
\hline Massachusetts & 990 & 813 & 1,263 & 1,446 & 1,474 & 14.8 & 12.1 & 18.6 & 21.2 & 21.6 \\
\hline Michigan & 1,068 & 1,095 & 1,089 & 1,092 & 1,267 & 10.8 & 11.0 & 11.0 & 11.0 & 12.8 \\
\hline Minnesota & 541 & 631 & 653 & 853 & 934 & 10.0 & 11.6 & 11.9 & 15.5 & 16.9 \\
\hline Mississippi & 293 & 642 & 760 & 925 & 937 & 9.8 & 21.4 & 25.4 & 30.9 & 31.4 \\
\hline Missouri & 609 & 771 & 778 & 955 & 1,337 & 10.1 & 12.7 & 12.8 & 15.7 & 21.9 \\
\hline Montana & 8 & 9 & 20 & 24 & 85 & 0.8 & 0.9 & 1.9 & 2.3 & 8.2 \\
\hline Nebraska & 95 & 96 & 81 & 121 & 118 & 5.1 & 5.1 & 4.3 & 6.3 & 6.2 \\
\hline Nevada & 523 & 893 & 915 & 1,313 & 1,681 & 18.7 & 31.5 & 31.7 & 44.7 & 57.2 \\
\hline New Hampshire & 79 & 79 & 84 & 100 & 109 & 6.0 & 6.0 & 6.3 & 7.5 & 8.2 \\
\hline New Jersey & 968 & 1,172 & 1,306 & 1,620 & 1,866 & 10.9 & 13.1 & 14.6 & 18.1 & 20.9 \\
\hline New Mexico & 247 & 283 & 332 & 470 & 510 & 11.8 & 13.6 & 15.9 & 22.6 & 24.5 \\
\hline New York & 6,173 & 7,129 & 7,795 & 9,456 & 9,877 & 31.4 & 36.1 & 39.4 & 47.9 & 50.0 \\
\hline North Carolina & 1,153 & 1,998 & 2,741 & 2,655 & 2,947 & 11.7 & 20.1 & 27.3 & 26.2 & 29.0 \\
\hline North Dakota & 25 & 51 & 42 & 61 & 78 & 3.5 & 6.9 & 5.5 & 8.0 & 10.3 \\
\hline Ohio & 1,096 & 1,229 & 1,348 & 1,600 & 1,900 & 9.5 & 10.6 & 11.6 & 13.8 & 16.4 \\
\hline Oklahoma & 383 & 414 & 521 & 696 & 953 & 9.9 & 10.7 & 13.3 & 17.7 & 24.3 \\
\hline Oregon & 527 & 582 & 783 & 810 & 848 & 13.4 & 14.7 & 19.4 & 19.8 & 20.7 \\
\hline Pennsylvania & 1,486 & 1,523 & 1,788 & 2,037 & 2,234 & 11.6 & 11.9 & 14.0 & 15.9 & 17.5 \\
\hline Rhode Island & 94 & 160 & 163 & 234 & 221 & 8.9 & 15.2 & 15.4 & 22.2 & 20.9 \\
\hline South Carolina & 753 & 750 & 834 & 974 & 1,096 & 15.8 & 15.5 & 17.0 & 19.6 & 22.1 \\
\hline South Dakota & 61 & 95 & 71 & 57 & 73 & 7.2 & 11.1 & 8.3 & 6.6 & 8.4 \\
\hline Tennessee & 980 & 977 & 1,241 & 1,448 & 1,453 & 15.1 & 14.9 & 18.8 & 21.8 & 21.8 \\
\hline Texas & 7,044 & 7,805 & 8,250 & 9,564 & 12,124 & 26.6 & 29.0 & 30.0 & 34.3 & 43.5 \\
\hline Utah & 172 & 149 & 169 & 259 & 299 & 5.9 & 5.1 & 5.6 & 8.5 & 9.8 \\
\hline Vermont & 10 & 12 & 15 & 37 & 26 & 1.6 & 1.9 & 2.4 & 5.9 & 4.2 \\
\hline Virginia & 1,001 & 702 & 1,023 & 1,304 & 1,757 & 12.1 & 8.4 & 12.2 & 15.5 & 20.9 \\
\hline Washington & 711 & 854 & 1,109 & 1,414 & 1,751 & 10.2 & 12.1 & 15.5 & 19.4 & 24.0 \\
\hline West Virginia & 39 & 55 & 109 & 151 & 123 & 2.1 & 3.0 & 5.9 & 8.2 & 6.7 \\
\hline Wisconsin & 257 & 285 & 262 & 423 & 551 & 4.5 & 5.0 & 4.5 & 7.3 & 9.5 \\
\hline Wyoming & 9 & 6 & 10 & 17 & 19 & 1.5 & 1.0 & 1.7 & 2.9 & 3.2 \\
\hline U.S. TOTAL & 56,485 & 63,454 & 74,707 & 88,053 & 101,567 & 17.9 & 19.9 & 23.2 & 27.3 & 31.4 \\
\hline Northeast & 9,954 & 11,080 & 12,672 & 15,211 & 16,222 & 17.8 & 19.7 & 22.5 & 27.1 & 28.9 \\
\hline Midwest & 7,378 & 7,963 & 8,785 & 10,558 & 11,511 & 10.9 & 11.8 & 12.9 & 15.5 & 16.9 \\
\hline South & 25,381 & 28,181 & 33,125 & 37,364 & 43,254 & 21.4 & 23.5 & 27.3 & 30.5 & 35.4 \\
\hline West & 13,772 & 16,230 & 20,125 & 24,920 & 30,580 & 18.5 & 21.6 & 26.5 & 32.5 & 39.9 \\
\hline Guam & 24 & 13 & 22 & 13 & 21 & 15.0 & 8.1 & 13.6 & 7.8 & 12.6 \\
\hline Puerto Rico & 811 & 960 & 1,267 & 1,185 & 1,052 & 22.4 & 27.1 & 36.5 & 34.7 & 30.8 \\
\hline Virgin Islands & 9 & 6 & 25 & 2 & 0 & 8.6 & 5.8 & 24.3 & 1.9 & 0.0 \\
\hline OUTLYING AREAS & 844 & 979 & 1,314 & 1,200 & 1,073 & 21.8 & 25.7 & 35.1 & 32.6 & 29.1 \\
\hline TOTAL & 57,329 & 64,433 & 76,021 & 89,253 & 102,640 & 17.9 & 20.0 & 23.4 & 27.3 & 31.4 \\
\hline
\end{tabular}

* See Section A1.9 in the Appendix for definition.

NOTE: See Section A1.11 in the Appendix for more information on interpreting case counts and rates in outlying areas. 
Table 25. All Stages of Syphilis* — Reported Cases and Rates of Reported Cases in Selected Metropolitan Statistical Areas (MSAs) ${ }^{\dagger}$ in Alphabetical Order, United States, 2013-2017

\begin{tabular}{|c|c|c|c|c|c|c|c|c|c|c|}
\hline \multirow[b]{2}{*}{ MSAs } & \multicolumn{5}{|c|}{ Cases } & \multicolumn{5}{|c|}{ Rates per 100,000 Population } \\
\hline & 2013 & 2014 & 2015 & 2016 & 2017 & 2013 & 2014 & 2015 & 2016 & 2017 \\
\hline Atlanta-Sandy Springs-Roswell, GA & 2,257 & 2,669 & 3,106 & 3,219 & 3,281 & 40.9 & 47.5 & 54.4 & 55.6 & 56.7 \\
\hline Austin-Round Rock, TX & 500 & 681 & 623 & 825 & 1,012 & 26.6 & 35.0 & 31.1 & 40.1 & 49.2 \\
\hline Baltimore-Columbia-Towson, MD & 732 & 816 & 1,015 & 1,031 & 1,084 & 26.4 & 29.3 & 36.3 & 36.8 & 38.7 \\
\hline Birmingham-Hoover, AL & 236 & 157 & 197 & $248^{\S}$ & 314 & 20.7 & 13.7 & 17.2 & $21.6^{5}$ & 27.4 \\
\hline Boston-Cambridge-Newton, MA-NH & 758 & 597 & $827^{\ddagger}$ & $1,000^{\ddagger}$ & 1,068 & 16.2 & 12.6 & $17.3^{+}$ & $20.9^{\ddagger}$ & 22.3 \\
\hline Buffalo-Cheektowaga-Niagara Falls, NY & 115 & 135 & 182 & 146 & 176 & 10.1 & 11.9 & 16.0 & 12.9 & 15.5 \\
\hline Charlotte-Concord-Gastonia, NC-SC & 360 & 533 & 732 & 851 & 876 & 15.4 & 22.4 & 30.2 & 34.4 & 35.4 \\
\hline Chicago-Naperville-Elgin, IL-IN-WI & 2,499 & 2,559 & 3,060 & 3,805 & 3,536 & 26.2 & 26.8 & 32.0 & 40.0 & 37.2 \\
\hline Cincinnati, OH-KY-IN & 437 & 381 & 319 & 269 & 326 & 20.4 & 17.7 & 14.8 & 12.4 & 15.1 \\
\hline Cleveland-Elyria, $\mathrm{OH}$ & 111 & 199 & 229 & 378 & 430 & 5.4 & 9.6 & 11.1 & 18.4 & 20.9 \\
\hline Columbus, $\mathrm{OH}$ & 342 & 441 & 518 & 578 & 700 & 17.4 & 22.1 & 25.6 & 28.3 & 34.3 \\
\hline Dallas-Fort Worth-Arlington, TX & 2,093 & 2,231 & 2,261 & 2,661 & 3,848 & 30.7 & 32.1 & 31.8 & 36.8 & 53.2 \\
\hline Denver-Aurora-Lakewood, CO & 382 & 298 & 426 & 552 & 618 & 14.2 & 10.8 & 15.1 & 19.3 & 21.7 \\
\hline Detroit-Warren-Dearborn, MI & 830 & 804 & 790 & 751 & 802 & 19.3 & 18.7 & 18.4 & 17.5 & 18.7 \\
\hline Hartford-West Hartford-East Hartford, CT & 43 & 52 & 84 & 55 & 66 & 3.5 & 4.3 & 6.9 & 4.6 & 5.5 \\
\hline Houston-The Woodlands-Sugar Land, TX & 1,891 & 2,316 & 2,568 & 2,817 & 3,242 & 30.0 & 35.7 & 38.6 & 41.6 & 47.9 \\
\hline Indianapolis-Carmel-Anderson, IN & 340 & 285 & 408 & 415 & 457 & 17.4 & 14.5 & 20.5 & 20.7 & 22.8 \\
\hline Jacksonville, FL & 189 & 270 & 435 & 445 & 644 & 13.6 & 19.0 & 30.0 & 30.1 & 43.6 \\
\hline Kansas City, MO-KS & 320 & 406 & 365 & 434 & 470 & 15.6 & 19.6 & 17.5 & 20.6 & 22.3 \\
\hline Las Vegas-Henderson-Paradise, NV & 438 & 830 & 826 & 1,194 & 1,501 & 21.6 & 40.1 & 39.1 & 55.4 & 69.6 \\
\hline Los Angeles-Long Beach-Anaheim, CA & 4,537 & 4,739 & 5,813 & 7,098 & 8,705 & 34.6 & 35.7 & 43.6 & 53.3 & 65.4 \\
\hline Louisville-Jefferson County, KY-IN & 210 & 239 & 270 & 381 & 421 & 16.6 & 18.8 & 21.1 & 29.7 & 32.8 \\
\hline Memphis, TN-MS-AR & 578 & 475 & 575 & 762 & 661 & 43.1 & 35.4 & 42.8 & 56.7 & 49.2 \\
\hline Miami-Fort Lauderdale-West Palm Beach, FL & 2,740 & 3,314 & 3,640 & 4,102 & 4,279 & 47.0 & 55.9 & 60.5 & 67.6 & 70.5 \\
\hline Milwaukee-Waukesha-West Allis, WI & 153 & 184 & 148 & 228 & 295 & 9.7 & 11.7 & 9.4 & 14.5 & 18.8 \\
\hline Minneapolis-St. Paul-Bloomington, MN-WI & 487 & 585 & 592 & 742 & 791 & 14.1 & 16.7 & 16.8 & 20.9 & 22.3 \\
\hline Nashville-Davidson-Murfreesboro-Franklin, TN & 239 & 305 & 359 & 383 & 363 & 13.6 & 17.0 & 19.6 & 20.5 & 19.5 \\
\hline New Orleans-Metairie, LA & 634 & 736 & 765 & 849 & 855 & 51.1 & 58.8 & 60.6 & 66.9 & 67.4 \\
\hline New York-Newark-Jersey City, NY-NJ-PA & 6,506 & 7,481 & 8,172 & 10,116 & 10,576 & 32.6 & 37.2 & 40.5 & 50.2 & 52.5 \\
\hline Oklahoma City, OK & 213 & 231 & 264 & 406 & 534 & 16.1 & 17.3 & 19.4 & 29.6 & 38.9 \\
\hline Orlando-Kissimmee-Sanford, FL & 631 & 784 & 916 & 1,183 & 1,196 & 27.8 & 33.8 & 38.4 & 48.5 & 49.0 \\
\hline Philadelphia-Camden-Wilmington, PA-NJ-DE-MD & 1,333 & 1,273 & 1,393 & 1,647 & 1,820 & 22.1 & 21.0 & 22.9 & 27.1 & 30.0 \\
\hline Phoenix-Mesa-Scottsdale, AZ & 714 & 1,065 & 1,126 & 1,501 & 1,881 & 16.2 & 23.7 & 24.6 & 32.2 & 40.4 \\
\hline Pittsburgh, PA & 95 & 154 & 273 & 236 & 192 & 4.0 & 6.5 & 11.6 & 10.1 & 8.2 \\
\hline Portland-Vancouver-Hillsboro, OR-WA & 475 & 473 & 604 & 665 & 654 & 20.5 & 20.1 & 25.3 & 27.4 & 27.0 \\
\hline Providence-Warwick, RI-MA & 138 & 205 & $233^{\ddagger}$ & $288^{\ddagger}$ & 295 & 8.6 & 12.7 & $14.4^{\ddagger}$ & $17.8^{\ddagger}$ & 18.3 \\
\hline Raleigh, NC & 179 & 317 & 410 & 384 & 438 & 14.7 & 25.5 & 32.2 & 29.5 & 33.6 \\
\hline Richmond, VA & 204 & 145 & 207 & 305 & 394 & 16.4 & 11.5 & 16.3 & 23.8 & 30.7 \\
\hline Riverside-San Bernardino-Ontario, CA & 803 & 950 & 1,165 & 1,554 & 1,960 & 18.3 & 21.4 & 26.0 & 34.3 & 43.3 \\
\hline Sacramento-Roseville-Arden-Arcade, CA & 289 & 371 & 609 & 611 & 807 & 13.0 & 16.5 & 26.8 & 26.6 & 35.1 \\
\hline Salt Lake City, UT & 136 & 109 & 122 & 188 & 203 & 11.9 & 9.5 & 10.4 & 15.8 & 17.1 \\
\hline San Antonio-New Braunfels, TX & 1,167 & 1,017 & 988 & 1,126 & 1,444 & 51.2 & 43.7 & 41.4 & 46.3 & 59.4 \\
\hline San Diego-Carlsbad, CA & 792 & 987 & 1,209 & 1,419 & 1,722 & 24.7 & 30.2 & 36.6 & 42.8 & 51.9 \\
\hline San Francisco-Oakland-Hayward, CA & 1,892 & 2,111 & 2,355 & 2,427 & 2,947 & 41.9 & 46.0 & 50.6 & 51.9 & 63.0 \\
\hline San Jose-Sunnyvale-Santa Clara, CA & 276 & 304 & 360 & 504 & 661 & 14.4 & 15.6 & 18.2 & 25.5 & 33.4 \\
\hline Seattle-Tacoma-Bellevue, WA & 539 & 590 & 759 & 968 & 1,185 & 14.9 & 16.1 & 20.3 & 25.5 & 31.2 \\
\hline St. Louis, MO-IL & 338 & 412 & 417 & 528 & 782 & 12.1 & 14.7 & 14.8 & 18.8 & 27.9 \\
\hline Tampa-St. Petersburg-Clearwater, FL & 632 & 807 & 958 & 1,129 & 1,061 & 22.0 & 27.7 & 32.2 & 37.2 & 35.0 \\
\hline Virginia Beach-Norfolk-Newport News, VA-NC & 302 & 220 & 375 & 516 & 565 & 17.7 & 12.8 & 21.7 & 29.9 & 32.7 \\
\hline Washington-Arlington-Alexandria, DC-VA-MD-WV & 1,543 & 811 & 1,058 & 1,107 & 2,295 & 25.9 & 13.4 & 17.4 & 18.1 & 37.4 \\
\hline SELECTED MSAs TOTAL & 43,648 & 48,054 & 55,106 & 65,027 & 74,433 & 25.3 & 27.5 & 31.2 & 36.5 & 41.8 \\
\hline
\end{tabular}

* See Section A1.9 in the Appendix for definition.

${ }^{+}$MSAs were selected on the basis of the largest population in the 2010 U.S. Census.

${ }^{\ddagger}$ The variable used to identify county, which is used to classify cases into MSAs, was complete for $\leq 95 \%$ of cases in a state contributing data to this MSA. See

Section A1.4 in the Appendix for more information.

$\$ 2016$ county data for Alabama have been corrected and may not match previous reports. 
Table 26. Primary and Secondary Syphilis - Reported Cases and Rates of Reported Cases by State, Ranked by Rates, United States, 2017

\begin{tabular}{|c|c|c|c|}
\hline Rank* & State & Cases & Rate per 100,000 Population \\
\hline 1 & Nevada & 587 & 20.0 \\
\hline 2 & California & 6,708 & 17.1 \\
\hline 3 & Louisiana & 679 & 14.5 \\
\hline 4 & Georgia & 1,489 & 14.4 \\
\hline 5 & Arizona & 943 & 13.6 \\
\hline 6 & New York & 2,355 & 11.9 \\
\hline 7 & Florida & 2,390 & 11.6 \\
\hline 8 & North Carolina & 1,138 & 11.2 \\
\hline 9 & Mississippi & 310 & 10.4 \\
\hline 10 & Illinois & 1,225 & 9.6 \\
\hline 11 & Maryland & 573 & 9.5 \\
\hline \multirow[t]{2}{*}{12} & Oklahoma & 373 & 9.5 \\
\hline & U.S. TOTAL ${ }^{\dagger}$ & 30,644 & 9.5 \\
\hline 13 & Washington & 677 & 9.3 \\
\hline 14 & New Mexico & 193 & 9.3 \\
\hline 15 & Alabama & 424 & 8.7 \\
\hline 16 & Oregon & 352 & 8.6 \\
\hline 17 & Missouri & 507 & 8.3 \\
\hline 18 & Texas & 2,233 & 8.0 \\
\hline 19 & Massachusetts & 538 & 7.9 \\
\hline 20 & Arkansas & 234 & 7.8 \\
\hline 21 & Tennessee & 488 & 7.3 \\
\hline 22 & South Carolina & 361 & 7.3 \\
\hline 23 & Ohio & 832 & 7.2 \\
\hline 24 & Rhode Island & 71 & 6.7 \\
\hline 25 & Hawaii & 94 & 6.6 \\
\hline 26 & Virginia & 536 & 6.4 \\
\hline 27 & Pennsylvania & 793 & 6.2 \\
\hline 28 & Delaware & 57 & 6.0 \\
\hline 29 & Kentucky & 262 & 5.9 \\
\hline 30 & North Dakota & 44 & 5.8 \\
\hline 31 & New Jersey & 499 & 5.6 \\
\hline 32 & Minnesota & 292 & 5.3 \\
\hline 33 & Colorado & 292 & 5.3 \\
\hline 34 & Maine & 65 & 4.9 \\
\hline 35 & Michigan & 480 & 4.8 \\
\hline 36 & Indiana & 319 & 4.8 \\
\hline 37 & Montana & 48 & 4.6 \\
\hline 38 & Kansas & 133 & 4.6 \\
\hline 39 & Utah & 117 & 3.8 \\
\hline 40 & South Dakota & 33 & 3.8 \\
\hline 41 & Idaho & 64 & 3.8 \\
\hline 42 & West Virginia & 62 & 3.4 \\
\hline 43 & lowa & 101 & 3.2 \\
\hline 44 & New Hampshire & 43 & 3.2 \\
\hline 45 & Connecticut & 110 & 3.1 \\
\hline 46 & Wisconsin & 173 & 3.0 \\
\hline 47 & Nebraska & 43 & 2.3 \\
\hline 48 & Vermont & 13 & 2.1 \\
\hline 49 & Alaska & 13 & 1.8 \\
\hline 50 & Wyoming & 4 & 0.7 \\
\hline
\end{tabular}

* States were ranked by rate, then by case count, then in alphabetical order, with rates shown rounded to the nearest tenth.

${ }^{+}$Total includes cases reported by the District of Columbia with 274 cases and a rate of 40.2 , but excludes outlying areas (Guam with 13 cases and rate of 7.8 , Puerto Rico with 411 cases and rate of 12.0, and Virgin Islands with 0 cases and rate of 0.0). 
Table 27. Primary and Secondary Syphilis - Reported Cases and Rates of Reported Cases by State/Area and Region in Alphabetical Order, United States and Outlying Areas, 2013-2017

\begin{tabular}{|c|c|c|c|c|c|c|c|c|c|c|}
\hline \multirow[b]{2}{*}{ State/Area } & \multicolumn{5}{|c|}{ Cases } & \multicolumn{5}{|c|}{ Rates per 100,000 Population } \\
\hline & 2013 & 2014 & 2015 & 2016 & 2017 & 2013 & 2014 & 2015 & 2016 & 2017 \\
\hline Alabama & 183 & 161 & 280 & 376 & 424 & 3.8 & 3.3 & 5.8 & 7.7 & 8.7 \\
\hline Alaska & 23 & 15 & 8 & 8 & 13 & 3.1 & 2.0 & 1.1 & 1.1 & 1.8 \\
\hline Arizona & 287 & 577 & 589 & 721 & 943 & 4.3 & 8.6 & 8.6 & 10.4 & 13.6 \\
\hline Arkansas & 177 & 121 & 134 & 150 & 234 & 6.0 & 4.1 & 4.5 & 5.0 & 7.8 \\
\hline California & 3,532 & 3,835 & 4,908 & 5,891 & 6,708 & 9.2 & 9.9 & 12.5 & 15.0 & 17.1 \\
\hline Colorado & 163 & 186 & 245 & 250 & 292 & 3.1 & 3.5 & 4.5 & 4.5 & 5.3 \\
\hline Connecticut & 56 & 86 & 92 & 110 & 110 & 1.6 & 2.4 & 2.6 & 3.1 & 3.1 \\
\hline Delaware & 52 & 47 & 41 & 58 & 57 & 5.6 & 5.0 & 4.3 & 6.1 & 6.0 \\
\hline District of Columbia & 168 & 116 & 95 & 161 & 274 & 26.0 & 17.6 & 14.1 & 23.6 & 40.2 \\
\hline Florida & 1,513 & 1,740 & 2,083 & 2,406 & 2,390 & 7.7 & 8.7 & 10.3 & 11.7 & 11.6 \\
\hline Georgia & 1,017 & 1,234 & 1,413 & 1,350 & 1,489 & 10.2 & 12.2 & 13.8 & 13.1 & 14.4 \\
\hline Hawaii & 46 & 68 & 91 & 112 & 94 & 3.3 & 4.8 & 6.4 & 7.8 & 6.6 \\
\hline Idaho & 15 & 12 & 57 & 50 & 64 & 0.9 & 0.7 & 3.4 & 3.0 & 3.8 \\
\hline Illinois & 798 & 863 & 1,085 & 1,260 & 1,225 & 6.2 & 6.7 & 8.4 & 9.8 & 9.6 \\
\hline Indiana & 215 & 168 & 285 & 326 & 319 & 3.3 & 2.5 & 4.3 & 4.9 & 4.8 \\
\hline lowa & 106 & 72 & 75 & 89 & 101 & 3.4 & 2.3 & 2.4 & 2.8 & 3.2 \\
\hline Kansas & 51 & 60 & 87 & 124 & 133 & 1.8 & 2.1 & 3.0 & 4.3 & 4.6 \\
\hline Kentucky & 122 & 158 & 145 & 219 & 262 & 2.8 & 3.6 & 3.3 & 4.9 & 5.9 \\
\hline Louisiana & 423 & 575 & 696 & 750 & 679 & 9.1 & 12.4 & 14.9 & 16.0 & 14.5 \\
\hline Maine & 10 & 16 & 28 & 42 & 65 & 0.8 & 1.2 & 2.1 & 3.2 & 4.9 \\
\hline Maryland & 456 & 449 & 509 & 509 & 573 & 7.7 & 7.5 & 8.5 & 8.5 & 9.5 \\
\hline Massachusetts & 360 & 301 & 418 & 489 & 538 & 5.4 & 4.5 & 6.2 & 7.2 & 7.9 \\
\hline Michigan & 487 & 421 & 403 & 365 & 480 & 4.9 & 4.2 & 4.1 & 3.7 & 4.8 \\
\hline Minnesota & 193 & 257 & 246 & 306 & 292 & 3.6 & 4.7 & 4.5 & 5.5 & 5.3 \\
\hline Mississippi & 78 & 189 & 219 & 326 & 310 & 2.6 & 6.3 & 7.3 & 10.9 & 10.4 \\
\hline Missouri & 251 & 352 & 307 & 400 & 507 & 4.2 & 5.8 & 5.0 & 6.6 & 8.3 \\
\hline Montana & 5 & 8 & 13 & 14 & 48 & 0.5 & 0.8 & 1.3 & 1.3 & 4.6 \\
\hline Nebraska & 41 & 50 & 45 & 67 & 43 & 2.2 & 2.7 & 2.4 & 3.5 & 2.3 \\
\hline Nevada & 205 & 357 & 335 & 444 & 587 & 7.3 & 12.6 & 11.6 & 15.1 & 20.0 \\
\hline New Hampshire & 28 & 36 & 40 & 40 & 43 & 2.1 & 2.7 & 3.0 & 3.0 & 3.2 \\
\hline New Jersey & 233 & 297 & 372 & 472 & 499 & 2.6 & 3.3 & 4.2 & 5.3 & 5.6 \\
\hline New Mexico & 78 & 126 & 118 & 189 & 193 & 3.7 & 6.0 & 5.7 & 9.1 & 9.3 \\
\hline New York & 1,459 & 1,727 & 2,006 & 2,455 & 2,355 & 7.4 & 8.7 & 10.1 & 12.4 & 11.9 \\
\hline North Carolina & 404 & 733 & 1,196 & 1,082 & 1,138 & 4.1 & 7.4 & 11.9 & 10.7 & 11.2 \\
\hline North Dakota & 12 & 13 & 11 & 33 & 44 & 1.7 & 1.8 & 1.5 & 4.4 & 5.8 \\
\hline Ohio & 436 & 568 & 560 & 716 & 832 & 3.8 & 4.9 & 4.8 & 6.2 & 7.2 \\
\hline Oklahoma & 118 & 151 & 209 & 264 & 373 & 3.1 & 3.9 & 5.3 & 6.7 & 9.5 \\
\hline Oregon & 267 & 272 & 345 & 327 & 352 & 6.8 & 6.9 & 8.6 & 8.0 & 8.6 \\
\hline Pennsylvania & 471 & 532 & 655 & 755 & 793 & 3.7 & 4.2 & 5.1 & 5.9 & 6.2 \\
\hline Rhode Island & 45 & 71 & 77 & 90 & 71 & 4.3 & 6.7 & 7.3 & 8.5 & 6.7 \\
\hline South Carolina & 271 & 250 & 294 & 316 & 361 & 5.7 & 5.2 & 6.0 & 6.4 & 7.3 \\
\hline South Dakota & 44 & 53 & 39 & 26 & 33 & 5.2 & 6.2 & 4.5 & 3.0 & 3.8 \\
\hline Tennessee & 214 & 237 & 349 & 390 & 488 & 3.3 & 3.6 & 5.3 & 5.9 & 7.3 \\
\hline Texas & 1,475 & 1,636 & 1,680 & 1,955 & 2,233 & 5.6 & 6.1 & 6.1 & 7.0 & 8.0 \\
\hline Utah & 74 & 47 & 65 & 92 & 117 & 2.6 & 1.6 & 2.2 & 3.0 & 3.8 \\
\hline Vermont & 3 & 5 & 9 & 23 & 13 & 0.5 & 0.8 & 1.4 & 3.7 & 2.1 \\
\hline Virginia & 315 & 289 & 334 & 459 & 536 & 3.8 & 3.5 & 4.0 & 5.5 & 6.4 \\
\hline Washington & 284 & 344 & 445 & 565 & 677 & 4.1 & 4.9 & 6.2 & 7.8 & 9.3 \\
\hline West Virginia & 15 & 28 & 52 & 53 & 62 & 0.8 & 1.5 & 2.8 & 2.9 & 3.4 \\
\hline Wisconsin & 95 & 86 & 79 & 132 & 173 & 1.7 & 1.5 & 1.4 & 2.3 & 3.0 \\
\hline Wyoming & 1 & 4 & 5 & 7 & 4 & 0.2 & 0.7 & 0.9 & 1.2 & 0.7 \\
\hline U.S. TOTAL & 17,375 & 19,999 & 23,872 & 27,814 & 30,644 & 5.5 & 6.3 & 7.4 & 8.6 & 9.5 \\
\hline Northeast & 2,665 & 3,071 & 3,697 & 4,476 & 4,487 & 4.8 & 5.5 & 6.6 & 8.0 & 8.0 \\
\hline Midwest & 2,729 & 2,963 & 3,222 & 3,844 & 4,182 & 4.0 & 4.4 & 4.7 & 5.7 & 6.2 \\
\hline South & 7,001 & 8,114 & 9,729 & 10,824 & 11,883 & 5.9 & 6.8 & 8.0 & 8.8 & 9.7 \\
\hline West & 4,980 & 5,851 & 7,224 & 8,670 & 10,092 & 6.7 & 7.8 & 9.5 & 11.3 & 13.2 \\
\hline Guam & 6 & 7 & 2 & 2 & 13 & 3.7 & 4.3 & 1.2 & 1.2 & 7.8 \\
\hline Puerto Rico & 385 & 484 & 531 & 493 & 411 & 10.6 & 13.6 & 15.3 & 14.5 & 12.0 \\
\hline Virgin Islands & 2 & 2 & 8 & 0 & 0 & 1.9 & 1.9 & 7.8 & 0.0 & 0.0 \\
\hline OUTLYING AREAS & 393 & 493 & 541 & 495 & 424 & 10.1 & 12.9 & 14.5 & 13.4 & 11.5 \\
\hline TOTAL & 17,768 & 20,492 & 24,413 & 28,309 & 31,068 & 5.6 & 6.4 & 7.5 & 8.7 & 9.5 \\
\hline
\end{tabular}

NOTE: See Section A1.11 in the Appendix for more information on interpreting case counts and rates in outlying areas. 
Table 28. Primary and Secondary Syphilis Among Women - Reported Cases and Rates of Reported Cases by State/Area and Region in Alphabetical Order, United States and Outlying Areas, 2013-2017

\begin{tabular}{|c|c|c|c|c|c|c|c|c|c|c|}
\hline \multirow[b]{2}{*}{ State/Area } & \multicolumn{5}{|c|}{ Cases } & \multicolumn{5}{|c|}{ Rates per 100,000 Population } \\
\hline & 2013 & 2014 & 2015 & 2016 & 2017 & 2013 & 2014 & 2015 & 2016 & 2017 \\
\hline Alabama & 22 & 17 & 37 & 80 & 70 & 0.9 & 0.7 & 1.5 & 3.2 & 2.8 \\
\hline Alaska & 2 & 1 & 1 & 0 & 1 & 0.6 & 0.3 & 0.3 & 0.0 & 0.3 \\
\hline Arizona & 27 & 50 & 47 & 80 & 158 & 0.8 & 1.5 & 1.4 & 2.3 & 4.5 \\
\hline Arkansas & 44 & 23 & 26 & 37 & 64 & 2.9 & 1.5 & 1.7 & 2.4 & 4.2 \\
\hline California & 210 & 318 & 476 & 744 & 902 & 1.1 & 1.6 & 2.4 & 3.8 & 4.6 \\
\hline Colorado & 4 & 6 & 6 & 16 & 18 & 0.2 & 0.2 & 0.2 & 0.6 & 0.7 \\
\hline Connecticut & 8 & 7 & 15 & 12 & 13 & 0.4 & 0.4 & 0.8 & 0.7 & 0.7 \\
\hline Delaware & 3 & 2 & 2 & 3 & 6 & 0.6 & 0.4 & 0.4 & 0.6 & 1.2 \\
\hline District of Columbia & 19 & 5 & 6 & 5 & 7 & 5.6 & 1.4 & 1.7 & 1.4 & 2.0 \\
\hline Florida & 137 & 137 & 210 & 266 & 321 & 1.4 & 1.3 & 2.0 & 2.5 & 3.0 \\
\hline Georgia & 87 & 96 & 94 & 113 & 139 & 1.7 & 1.9 & 1.8 & 2.1 & 2.6 \\
\hline Hawaii & 0 & 2 & 1 & 10 & 6 & 0.0 & 0.3 & 0.1 & 1.4 & 0.8 \\
\hline Idaho & 0 & 0 & 9 & 6 & 15 & 0.0 & 0.0 & 1.1 & 0.7 & 1.8 \\
\hline Illinois & 66 & 81 & 84 & 95 & 85 & 1.0 & 1.2 & 1.3 & 1.5 & 1.3 \\
\hline Indiana & 18 & 11 & 28 & 36 & 27 & 0.5 & 0.3 & 0.8 & 1.1 & 0.8 \\
\hline lowa & 10 & 6 & 5 & 6 & 9 & 0.6 & 0.4 & 0.3 & 0.4 & 0.6 \\
\hline Kansas & 4 & 14 & 17 & 12 & 16 & 0.3 & 1.0 & 1.2 & 0.8 & 1.1 \\
\hline Kentucky & 17 & 22 & 23 & 21 & 41 & 0.8 & 1.0 & 1.0 & 0.9 & 1.8 \\
\hline Louisiana & 115 & 132 & 189 & 192 & 186 & 4.9 & 5.6 & 7.9 & 8.0 & 7.8 \\
\hline Maine & 1 & 3 & 6 & 3 & 9 & 0.1 & 0.4 & 0.9 & 0.4 & 1.3 \\
\hline Maryland & 61 & 49 & 58 & 54 & 47 & 2.0 & 1.6 & 1.9 & 1.7 & 1.5 \\
\hline Massachusetts & 17 & 23 & 25 & 24 & 26 & 0.5 & 0.7 & 0.7 & 0.7 & 0.7 \\
\hline Michigan & 29 & 31 & 34 & 31 & 34 & 0.6 & 0.6 & 0.7 & 0.6 & 0.7 \\
\hline Minnesota & 12 & 21 & 39 & 37 & 38 & 0.4 & 0.8 & 1.4 & 1.3 & 1.4 \\
\hline Mississippi & 19 & 17 & 32 & 58 & 88 & 1.2 & 1.1 & 2.1 & 3.8 & 5.7 \\
\hline Missouri & 19 & 34 & 54 & 66 & 93 & 0.6 & 1.1 & 1.7 & 2.1 & 3.0 \\
\hline Montana & 1 & 2 & 1 & 1 & 9 & 0.2 & 0.4 & 0.2 & 0.2 & 1.7 \\
\hline Nebraska & 4 & 4 & 3 & 5 & 7 & 0.4 & 0.4 & 0.3 & 0.5 & 0.7 \\
\hline Nevada & 14 & 23 & 23 & 50 & 75 & 1.0 & 1.6 & 1.6 & 3.4 & 5.1 \\
\hline New Hampshire & 1 & 4 & 3 & 4 & 4 & 0.1 & 0.6 & 0.4 & 0.6 & 0.6 \\
\hline New Jersey & 13 & 16 & 26 & 24 & 34 & 0.3 & 0.3 & 0.6 & 0.5 & 0.7 \\
\hline New Mexico & 20 & 14 & 11 & 27 & 19 & 1.9 & 1.3 & 1.0 & 2.6 & 1.8 \\
\hline New York & 44 & 49 & 59 & 115 & 121 & 0.4 & 0.5 & 0.6 & 1.1 & 1.2 \\
\hline North Carolina & 36 & 68 & 112 & 109 & 127 & 0.7 & 1.3 & 2.2 & 2.1 & 2.4 \\
\hline North Dakota & 1 & 5 & 0 & 3 & 5 & 0.3 & 1.4 & 0.0 & 0.8 & 1.4 \\
\hline Ohio & 63 & 76 & 68 & 94 & 112 & 1.1 & 1.3 & 1.1 & 1.6 & 1.9 \\
\hline Oklahoma & 13 & 15 & 21 & 41 & 67 & 0.7 & 0.8 & 1.1 & 2.1 & 3.4 \\
\hline Oregon & 12 & 22 & 35 & 35 & 52 & 0.6 & 1.1 & 1.7 & 1.7 & 2.5 \\
\hline Pennsylvania & 26 & 47 & 52 & 62 & 76 & 0.4 & 0.7 & 0.8 & 1.0 & 1.2 \\
\hline Rhode Island & 1 & 5 & 4 & 3 & 7 & 0.2 & 0.9 & 0.7 & 0.6 & 1.3 \\
\hline South Carolina & 39 & 23 & 37 & 52 & 56 & 1.6 & 0.9 & 1.5 & 2.0 & 2.2 \\
\hline South Dakota & 15 & 34 & 7 & 6 & 4 & 3.6 & 8.0 & 1.6 & 1.4 & 0.9 \\
\hline Tennessee & 22 & 34 & 23 & 56 & 47 & 0.7 & 1.0 & 0.7 & 1.6 & 1.4 \\
\hline Texas & 179 & 242 & 230 & 230 & 313 & 1.3 & 1.8 & 1.7 & 1.6 & 2.2 \\
\hline Utah & 2 & 1 & 2 & 6 & 4 & 0.1 & 0.1 & 0.1 & 0.4 & 0.3 \\
\hline Vermont & 0 & 0 & 0 & 3 & 1 & 0.0 & 0.0 & 0.0 & 0.9 & 0.3 \\
\hline Virginia & 17 & 17 & 17 & 47 & 60 & 0.4 & 0.4 & 0.4 & 1.1 & 1.4 \\
\hline Washington & 13 & 18 & 30 & 51 & 70 & 0.4 & 0.5 & 0.8 & 1.4 & 1.9 \\
\hline West Virginia & 4 & 6 & 9 & 10 & 21 & 0.4 & 0.6 & 1.0 & 1.1 & 2.3 \\
\hline Wisconsin & 9 & 7 & 0 & 7 & 12 & 0.3 & 0.2 & 0.0 & 0.2 & 0.4 \\
\hline Wyoming & 0 & 0 & 1 & 1 & 0 & 0.0 & 0.0 & 0.3 & 0.3 & 0.0 \\
\hline U.S. TOTAL & 1,500 & 1,840 & 2,298 & 3,049 & 3,722 & 0.9 & 1.1 & 1.4 & 1.9 & 2.3 \\
\hline Northeast & 111 & 154 & 190 & 250 & 291 & 0.4 & 0.5 & 0.7 & 0.9 & 1.0 \\
\hline Midwest & 250 & 324 & 339 & 398 & 442 & 0.7 & 0.9 & 1.0 & 1.2 & 1.3 \\
\hline South & 834 & 905 & 1,126 & 1,374 & 1,660 & 1.4 & 1.5 & 1.8 & 2.2 & 2.7 \\
\hline West & 305 & 457 & 643 & 1,027 & 1,329 & 0.8 & 1.2 & 1.7 & 2.7 & 3.5 \\
\hline Guam & 5 & 2 & 0 & 0 & 2 & 6.3 & 2.5 & 0.0 & 0.0 & 2.5 \\
\hline Puerto Rico & 35 & 30 & 70 & 86 & 66 & 1.9 & 1.6 & 3.9 & 4.8 & 3.7 \\
\hline Virgin Islands & 1 & 1 & 4 & 0 & 0 & 1.8 & 1.8 & 7.3 & 0.0 & 0.0 \\
\hline OUTLYING AREAS & 41 & 33 & 74 & 86 & 68 & 2.0 & 1.7 & 3.8 & 4.5 & 3.5 \\
\hline TOTAL & 1,541 & 1,873 & 2,372 & 3,135 & 3,790 & 0.9 & 1.1 & 1.4 & 1.9 & 2.3 \\
\hline
\end{tabular}

NOTE: Cases reported with unknown sex are not included in this table. See Section A1.11 in the Appendix for more information on interpreting case counts and rates in outlying areas. 
Table 29. Primary and Secondary Syphilis Among Men - Reported Cases and Rates of Reported Cases by State/Area and Region in Alphabetical Order, United States and Outlying Areas, 2013-2017

\begin{tabular}{|c|c|c|c|c|c|c|c|c|c|c|}
\hline \multirow[b]{2}{*}{ State/Area } & \multicolumn{5}{|c|}{ Cases } & \multicolumn{5}{|c|}{ Rates per 100,000 Population } \\
\hline & 2013 & 2014 & 2015 & 2016 & 2017 & 2013 & 2014 & 2015 & 2016 & 2017 \\
\hline Alabama & 161 & 144 & 243 & 296 & 354 & 6.9 & 6.1 & 10.3 & 12.6 & 15.0 \\
\hline Alaska & 21 & 14 & 7 & 8 & 12 & 5.4 & 3.6 & 1.8 & 2.1 & 3.1 \\
\hline Arizona & 260 & 527 & 542 & 641 & 785 & 7.9 & 15.8 & 16.0 & 18.6 & 22.8 \\
\hline Arkansas & 133 & 98 & 108 & 113 & 170 & 9.1 & 6.7 & 7.4 & 7.7 & 11.6 \\
\hline California & 3,319 & 3,515 & 4,430 & 5,143 & 5,804 & 17.4 & 18.2 & 22.8 & 26.4 & 29.8 \\
\hline Colorado & 159 & 180 & 239 & 234 & 274 & 6.0 & 6.7 & 8.7 & 8.4 & 9.8 \\
\hline Connecticut & 48 & 79 & 77 & 98 & 97 & 2.7 & 4.5 & 4.4 & 5.6 & 5.6 \\
\hline Delaware & 49 & 45 & 39 & 55 & 51 & 10.9 & 9.9 & 8.5 & 11.9 & 11.1 \\
\hline District of Columbia & 149 & 106 & 83 & 152 & 264 & 48.7 & 33.9 & 26.1 & 47.0 & 81.7 \\
\hline Florida & 1,376 & 1,602 & 1,873 & 2,140 & 2,069 & 14.4 & 16.5 & 18.9 & 21.3 & 20.5 \\
\hline Georgia & 930 & 1,138 & 1,319 & 1,237 & 1,350 & 19.1 & 23.1 & 26.5 & 24.6 & 26.9 \\
\hline Hawaii & 46 & 66 & 90 & 102 & 88 & 6.5 & 9.2 & 12.4 & 14.2 & 12.3 \\
\hline Idaho & 15 & 12 & 48 & 44 & 49 & 1.9 & 1.5 & 5.8 & 5.2 & 5.8 \\
\hline Illinois & 731 & 782 & 1,001 & 1,165 & 1,140 & 11.6 & 12.4 & 15.9 & 18.5 & 18.1 \\
\hline Indiana & 197 & 157 & 257 & 290 & 292 & 6.1 & 4.8 & 7.9 & 8.9 & 8.9 \\
\hline lowa & 96 & 66 & 70 & 83 & 92 & 6.3 & 4.3 & 4.5 & 5.3 & 5.9 \\
\hline Kansas & 47 & 46 & 70 & 112 & 117 & 3.3 & 3.2 & 4.8 & 7.7 & 8.1 \\
\hline Kentucky & 105 & 136 & 122 & 198 & 221 & 4.9 & 6.3 & 5.6 & 9.1 & 10.1 \\
\hline Louisiana & 308 & 443 & 507 & 558 & 493 & 13.6 & 19.5 & 22.2 & 24.4 & 21.5 \\
\hline Maine & 9 & 13 & 22 & 39 & 54 & 1.4 & 2.0 & 3.4 & 6.0 & 8.3 \\
\hline Maryland & 395 & 400 & 451 & 455 & 526 & 13.7 & 13.8 & 15.5 & 15.6 & 18.0 \\
\hline Massachusetts & 343 & 277 & 391 & 464 & 512 & 10.6 & 8.5 & 11.9 & 14.0 & 15.5 \\
\hline Michigan & 458 & 390 & 369 & 334 & 446 & 9.4 & 8.0 & 7.6 & 6.8 & 9.1 \\
\hline Minnesota & 178 & 235 & 207 & 267 & 252 & 6.6 & 8.7 & 7.6 & 9.7 & 9.2 \\
\hline Mississippi & 59 & 172 & 187 & 268 & 222 & 4.1 & 11.8 & 12.9 & 18.5 & 15.3 \\
\hline Missouri & 232 & 318 & 253 & 334 & 414 & 7.8 & 10.7 & 8.5 & 11.2 & 13.8 \\
\hline Montana & 4 & 6 & 12 & 13 & 39 & 0.8 & 1.2 & 2.3 & 2.5 & 7.4 \\
\hline Nebraska & 37 & 46 & 42 & 62 & 36 & 4.0 & 4.9 & 4.4 & 6.5 & 3.8 \\
\hline Nevada & 191 & 334 & 312 & 394 & 512 & 13.6 & 23.4 & 21.5 & 26.7 & 34.7 \\
\hline New Hampshire & 27 & 32 & 37 & 36 & 39 & 4.1 & 4.9 & 5.6 & 5.4 & 5.9 \\
\hline New Jersey & 220 & 281 & 346 & 448 & 465 & 5.1 & 6.4 & 7.9 & 10.3 & 10.6 \\
\hline New Mexico & 58 & 112 & 107 & 162 & 174 & 5.6 & 10.8 & 10.4 & 15.7 & 16.9 \\
\hline New York & 1,408 & 1,675 & 1,933 & 2,319 & 2,214 & 14.8 & 17.5 & 20.1 & 24.2 & 23.1 \\
\hline North Carolina & 368 & 665 & 1,084 & 973 & 1,011 & 7.7 & 13.7 & 22.2 & 19.7 & 20.5 \\
\hline North Dakota & 11 & 8 & 11 & 30 & 39 & 3.0 & 2.1 & 2.8 & 7.7 & 10.0 \\
\hline Ohio & 373 & 492 & 492 & 622 & 720 & 6.6 & 8.7 & 8.7 & 10.9 & 12.7 \\
\hline Oklahoma & 105 & 136 & 188 & 223 & 306 & 5.5 & 7.1 & 9.7 & 11.5 & 15.7 \\
\hline Oregon & 255 & 250 & 310 & 292 & 298 & 13.1 & 12.7 & 15.6 & 14.4 & 14.7 \\
\hline Pennsylvania & 445 & 485 & 602 & 693 & 717 & 7.1 & 7.8 & 9.6 & 11.1 & 11.5 \\
\hline Rhode Island & 44 & 66 & 73 & 87 & 64 & 8.6 & 12.9 & 14.3 & 17.0 & 12.5 \\
\hline South Carolina & 232 & 227 & 257 & 264 & 305 & 10.0 & 9.7 & 10.8 & 11.0 & 12.7 \\
\hline South Dakota & 29 & 19 & 32 & 20 & 29 & 6.8 & 4.4 & 7.4 & 4.6 & 6.6 \\
\hline Tennessee & 192 & 203 & 326 & 334 & 441 & 6.1 & 6.4 & 10.1 & 10.3 & 13.6 \\
\hline Texas & 1,296 & 1,394 & 1,450 & 1,725 & 1,920 & 9.9 & 10.4 & 10.6 & 12.5 & 13.9 \\
\hline Utah & 72 & 46 & 63 & 86 & 113 & 4.9 & 3.1 & 4.2 & 5.6 & 7.4 \\
\hline Vermont & 3 & 5 & 9 & 20 & 12 & 1.0 & 1.6 & 2.9 & 6.5 & 3.9 \\
\hline Virginia & 298 & 272 & 316 & 403 & 471 & 7.3 & 6.6 & 7.7 & 9.7 & 11.4 \\
\hline Washington & 271 & 326 & 414 & 514 & 606 & 7.8 & 9.2 & 11.5 & 14.1 & 16.6 \\
\hline West Virginia & 11 & 22 & 43 & 43 & 41 & 1.2 & 2.4 & 4.7 & 4.7 & 4.5 \\
\hline Wisconsin & 86 & 79 & 79 & 125 & 161 & 3.0 & 2.8 & 2.8 & 4.4 & 5.6 \\
\hline Wyoming & 1 & 4 & 4 & 6 & 4 & 0.3 & 1.3 & 1.3 & 2.0 & 1.3 \\
\hline U.S.TOTAL & 15,861 & 18,146 & 21,547 & 24,724 & 26,885 & 10.2 & 11.6 & 13.6 & 15.5 & 16.9 \\
\hline Northeast & 2,547 & 2,913 & 3,490 & 4,204 & 4,174 & 9.3 & 10.7 & 12.7 & 15.3 & 15.2 \\
\hline Midwest & 2,475 & 2,638 & 2,883 & 3,444 & 3,738 & 7.4 & 7.9 & 8.6 & 10.3 & 11.1 \\
\hline South & 6,167 & 7,203 & 8,596 & 9,437 & 10,215 & 10.6 & 12.3 & 14.5 & 15.7 & 17.0 \\
\hline West & 4,672 & 5,392 & 6,578 & 7,639 & 8,758 & 12.6 & 14.4 & 17.4 & 20.0 & 22.9 \\
\hline Guam & 1 & 5 & 2 & 2 & 11 & 1.2 & 6.1 & 2.4 & 2.3 & 12.8 \\
\hline Puerto Rico & 350 & 454 & 461 & 407 & 345 & 20.2 & 26.6 & 27.8 & 25.1 & 21.2 \\
\hline Virgin Islands & 1 & 1 & 4 & 0 & 0 & 2.0 & 2.1 & 8.4 & 0.0 & 0.0 \\
\hline OUTLYING AREAS & 352 & 460 & 467 & 409 & 356 & 18.9 & 25.1 & 26.1 & 23.2 & 20.2 \\
\hline TOTAL & 16,213 & 18,606 & 22,014 & 25,133 & 27,241 & 10.3 & 11.7 & 13.8 & 15.6 & 16.9 \\
\hline
\end{tabular}

NOTE: Cases reported with unknown sex are not included in this table. See Section A1.11 in the Appendix for more information on interpreting case counts and rates in outlying areas. 
Table 30. Primary and Secondary Syphilis - Reported Cases and Rates of Reported Cases in Selected Metropolitan Statistical Areas (MSAs)* in Alphabetical Order, United States, 2013-2017

\begin{tabular}{|c|c|c|c|c|c|c|c|c|c|c|}
\hline \multirow[b]{2}{*}{ MSAs } & \multicolumn{5}{|c|}{ Cases } & \multicolumn{5}{|c|}{ Rates per 100,000 Population } \\
\hline & 2013 & 2014 & 2015 & 2016 & 2017 & 2013 & 2014 & 2015 & 2016 & 2017 \\
\hline Atlanta-Sandy Springs-Roswell, GA & 789 & 996 & 1,097 & 1,018 & 1,095 & 14.3 & 17.7 & 19.2 & 17.6 & 18.9 \\
\hline Austin-Round Rock, TX & 145 & 227 & 203 & 317 & 359 & 7.7 & 11.7 & 10.1 & 15.4 & 17.5 \\
\hline Baltimore-Columbia-Towson, MD & 288 & 282 & 343 & 332 & 342 & 10.4 & 10.1 & 12.3 & 11.9 & 12.2 \\
\hline Birmingham-Hoover, AL & 69 & 58 & 74 & $109^{\ddagger}$ & 122 & 6.1 & 5.1 & 6.5 & $9.5^{\ddagger}$ & 10.6 \\
\hline Boston-Cambridge-Newton, MA-NH & 268 & 227 & $271^{+}$ & $320^{+}$ & 373 & 5.7 & 4.8 & $5.7^{+}$ & $6.7^{+}$ & 7.8 \\
\hline Buffalo-Cheektowaga-Niagara Falls, NY & 38 & 49 & 92 & 63 & 60 & 3.4 & 4.3 & 8.1 & 5.6 & 5.3 \\
\hline Charlotte-Concord-Gastonia, NC-SC & 134 & 220 & 333 & 378 & 355 & 5.7 & 9.2 & 13.7 & 15.3 & 14.3 \\
\hline Chicago-Naperville-Elgin, IL-IN-WI & 763 & 811 & 1,047 & 1,202 & 1,140 & 8.0 & 8.5 & 11.0 & 12.6 & 12.0 \\
\hline Cincinnati, OH-KY-IN & 166 & 153 & 93 & 81 & 103 & 7.8 & 7.1 & 4.3 & 3.7 & 4.8 \\
\hline Cleveland-Elyria, $\mathrm{OH}$ & 32 & 80 & 72 & 156 & 180 & 1.5 & 3.9 & 3.5 & 7.6 & 8.8 \\
\hline Columbus, $\mathrm{OH}$ & 167 & 250 & 274 & 316 & 353 & 8.5 & 12.5 & 13.6 & 15.5 & 17.3 \\
\hline Dallas-Fort Worth-Arlington, TX & 445 & 508 & 476 & 542 & 699 & 6.5 & 7.3 & 6.7 & 7.5 & 9.7 \\
\hline Denver-Aurora-Lakewood, CO & 135 & 153 & 192 & 187 & 216 & 5.0 & 5.6 & 6.8 & 6.6 & 7.6 \\
\hline Detroit-Warren-Dearborn, MI & 394 & 317 & 284 & 253 & 291 & 9.2 & 7.4 & 6.6 & 5.9 & 6.8 \\
\hline Hartford-West Hartford-East Hartford, CT & 14 & 26 & 44 & 32 & 32 & 1.2 & 2.1 & 3.6 & 2.7 & 2.7 \\
\hline Houston-The Woodlands-Sugar Land, TX & 363 & 414 & 439 & 411 & 386 & 5.7 & 6.4 & 6.6 & 6.1 & 5.7 \\
\hline Indianapolis-Carmel-Anderson, IN & 146 & 109 & 172 & 158 & 186 & 7.5 & 5.5 & 8.6 & 7.9 & 9.3 \\
\hline Jacksonville, FL & 40 & 69 & 91 & 118 & 194 & 2.9 & 4.9 & 6.3 & 8.0 & 13.1 \\
\hline Kansas City, MO-KS & 155 & 220 & 191 & 201 & 214 & 7.5 & 10.6 & 9.1 & 9.6 & 10.2 \\
\hline Las Vegas-Henderson-Paradise, NV & 164 & 318 & 305 & 398 & 519 & 8.1 & 15.4 & 14.4 & 18.5 & 24.1 \\
\hline Los Angeles-Long Beach-Anaheim, CA & 1,299 & 1,407 & 1,832 & 2,123 & 2,365 & 9.9 & 10.6 & 13.7 & 15.9 & 17.8 \\
\hline Louisville-Jefferson County, KY-IN & 71 & 83 & 87 & 149 & 150 & 5.6 & 6.5 & 6.8 & 11.6 & 11.7 \\
\hline Memphis, TN-MS-AR & 105 & 94 & 121 & 173 & 153 & 7.8 & 7.0 & 9.0 & 12.9 & 11.4 \\
\hline Miami-Fort Lauderdale-West Palm Beach, FL & 762 & 821 & 884 & 925 & 919 & 13.1 & 13.8 & 14.7 & 15.2 & 15.1 \\
\hline Milwaukee-Waukesha-West Allis, WI & 54 & 52 & 39 & 50 & 79 & 3.4 & 3.3 & 2.5 & 3.2 & 5.0 \\
\hline Minneapolis-St. Paul-Bloomington, MN-WI & 181 & 243 & 228 & 261 & 248 & 5.2 & 7.0 & 6.5 & 7.3 & 7.0 \\
\hline Nashville-Davidson-Murfreesboro-Franklin, TN & 57 & 74 & 116 & 115 & 160 & 3.2 & 4.1 & 6.3 & 6.2 & 8.6 \\
\hline New Orleans-Metairie, LA & 103 & 221 & 218 & 235 & 210 & 8.3 & 17.7 & 17.3 & 18.5 & 16.5 \\
\hline New York-Newark-Jersey City, NY-NJ-PA & 1,491 & 1,721 & 2,037 & 2,551 & 2,461 & 7.5 & 8.6 & 10.1 & 12.7 & 12.2 \\
\hline Oklahoma City, OK & 78 & 91 & 113 & 162 & 219 & 5.9 & 6.8 & 8.3 & 11.8 & 15.9 \\
\hline Orlando-Kissimmee-Sanford, FL & 201 & 239 & 299 & 384 & 318 & 8.9 & 10.3 & 12.5 & 15.7 & 13.0 \\
\hline Philadelphia-Camden-Wilmington, PA-NJ-DE-MD & 396 & 446 & 458 & 639 & 636 & 6.6 & 7.4 & 7.5 & 10.5 & 10.5 \\
\hline Phoenix-Mesa-Scottsdale, AZ & 219 & 416 & 452 & 574 & 775 & 5.0 & 9.3 & 9.9 & 12.3 & 16.6 \\
\hline Pittsburgh, PA & 39 & 78 & 150 & 100 & 71 & 1.7 & 3.3 & 6.4 & 4.3 & 3.0 \\
\hline Portland-Vancouver-Hillsboro, OR-WA & 240 & 206 & 261 & 263 & 255 & 10.4 & 8.8 & 10.9 & 10.8 & 10.5 \\
\hline Providence-Warwick, RI-MA & 65 & 93 & $111^{+}$ & $115^{+}$ & 98 & 4.1 & 5.8 & $6.9^{+}$ & $7.1^{+}$ & 6.1 \\
\hline Raleigh, NC & 70 & 129 & 168 & 137 & 136 & 5.8 & 10.4 & 13.2 & 10.5 & 10.4 \\
\hline Richmond, VA & 70 & 68 & 73 & 104 & 124 & 5.6 & 5.4 & 5.7 & 8.1 & 9.7 \\
\hline Riverside-San Bernardino-Ontario, CA & 203 & 288 & 341 & 445 & 495 & 4.6 & 6.5 & 7.6 & 9.8 & 10.9 \\
\hline Sacramento-Roseville-Arden-Arcade, CA & 147 & 162 & 265 & 272 & 343 & 6.6 & 7.2 & 11.7 & 11.8 & 14.9 \\
\hline Salt Lake City, UT & 65 & 39 & 49 & 69 & 87 & 5.7 & 3.4 & 4.2 & 5.8 & 7.3 \\
\hline San Antonio-New Braunfels, TX & 310 & 247 & 237 & 243 & 329 & 13.6 & 10.6 & 9.9 & 10.0 & 13.5 \\
\hline San Diego-Carlsbad, CA & 333 & 371 & 493 & 524 & 585 & 10.4 & 11.4 & 14.9 & 15.8 & 17.6 \\
\hline San Francisco-Oakland-Hayward, CA & 814 & 767 & 830 & 872 & 1,030 & 18.0 & 16.7 & 17.8 & 18.6 & 22.0 \\
\hline San Jose-Sunnyvale-Santa Clara, CA & 146 & 120 & 134 & 222 & 217 & 7.6 & 6.1 & 6.8 & 11.2 & 11.0 \\
\hline Seattle-Tacoma-Bellevue, WA & 211 & 235 & 311 & 397 & 440 & 5.8 & 6.4 & 8.3 & 10.5 & 11.6 \\
\hline St. Louis, MO-IL & 108 & 153 & 112 & 215 & 278 & 3.9 & 5.5 & 4.0 & 7.7 & 9.9 \\
\hline Tampa-St. Petersburg-Clearwater, FL & 226 & 320 & 393 & 436 & 364 & 7.9 & 11.0 & 13.2 & 14.4 & 12.0 \\
\hline Virginia Beach-Norfolk-Newport News, VA-NC & 102 & 85 & 117 & 206 & 170 & 6.0 & 5.0 & 6.8 & 11.9 & 9.8 \\
\hline Washington-Arlington-Alexandria, DC-VA-MD-WV & 418 & 226 & 230 & 273 & 640 & 7.0 & 3.7 & 3.8 & 4.5 & 10.4 \\
\hline SELECTED MSAs TOTAL & 13,299 & 14,982 & 17,252 & 19,826 & 21,574 & 7.7 & 8.6 & 9.8 & 11.1 & 12.1 \\
\hline
\end{tabular}

* MSAs were selected on the basis of the largest population in the 2010 U.S. Census.

${ }^{\dagger}$ The variable used to identify county, which is used to classify cases into MSAs, was complete for $\leq 95 \%$ of cases in a state contributing data to this MSA. See Section A1.4 in the Appendix for more information.

₹ 2016 county data for Alabama have been corrected and may not match previous reports. 
Table 31. Primary and Secondary Syphilis Among Women - Reported Cases and Rates of Reported Cases in Selected Metropolitan Statistical Areas (MSAs)* in Alphabetical Order, United States, 2013-2017

\begin{tabular}{|c|c|c|c|c|c|c|c|c|c|c|}
\hline \multirow[b]{2}{*}{ MSAs } & \multicolumn{5}{|c|}{ Cases } & \multicolumn{5}{|c|}{ Rates per 100,000 Population } \\
\hline & 2013 & 2014 & 2015 & 2016 & 2017 & 2013 & 2014 & 2015 & 2016 & 2017 \\
\hline Atlanta-Sandy Springs-Roswell, GA & 52 & 58 & 61 & 61 & 78 & 1.8 & 2.0 & 2.1 & 2.0 & 2.6 \\
\hline Austin-Round Rock, TX & 6 & 14 & 16 & 17 & 29 & 0.6 & 1.4 & 1.6 & 1.7 & 2.8 \\
\hline Baltimore-Columbia-Towson, MD & 46 & 42 & 49 & 46 & 39 & 3.2 & 2.9 & 3.4 & 3.2 & 2.7 \\
\hline Birmingham-Hoover, AL & 5 & 5 & 8 & $25^{\ddagger}$ & 21 & 0.8 & 0.8 & 1.3 & $4.2^{\ddagger}$ & 3.5 \\
\hline Boston-Cambridge-Newton, MA-NH & 10 & 14 & $13^{+}$ & $9^{+}$ & 13 & 0.4 & 0.6 & $0.5^{+}$ & $0.4^{+}$ & 0.5 \\
\hline Buffalo-Cheektowaga-Niagara Falls, NY & 2 & 1 & 0 & 1 & 6 & 0.3 & 0.2 & 0.0 & 0.2 & 1.0 \\
\hline Charlotte-Concord-Gastonia, NC-SC & 6 & 6 & 16 & 25 & 31 & 0.5 & 0.5 & 1.3 & 2.0 & 2.4 \\
\hline Chicago-Naperville-Elgin, IL-IN-WI & 65 & 76 & 86 & 88 & 79 & 1.3 & 1.6 & 1.8 & 1.8 & 1.6 \\
\hline Cincinnati, OH-KY-IN & 31 & 33 & 21 & 11 & 11 & 2.8 & 3.0 & 1.9 & 1.0 & 1.0 \\
\hline Cleveland-Elyria, $\mathrm{OH}$ & 2 & 5 & 1 & 12 & 23 & 0.2 & 0.5 & 0.1 & 1.1 & 2.2 \\
\hline Columbus, $\mathrm{OH}$ & 18 & 34 & 27 & 48 & 45 & 1.8 & 3.4 & 2.6 & 4.6 & 4.3 \\
\hline Dallas-Fort Worth-Arlington, TX & 41 & 63 & 56 & 62 & 75 & 1.2 & 1.8 & 1.6 & 1.7 & 2.0 \\
\hline Denver-Aurora-Lakewood, CO & 3 & 4 & 6 & 11 & 15 & 0.2 & 0.3 & 0.4 & 0.8 & 1.0 \\
\hline Detroit-Warren-Dearborn, MI & 25 & 23 & 26 & 26 & 20 & 1.1 & 1.0 & 1.2 & 1.2 & 0.9 \\
\hline Hartford-West Hartford-East Hartford, CT & 0 & 2 & 9 & 2 & 8 & 0.0 & 0.3 & 1.5 & 0.3 & 1.3 \\
\hline Houston-The Woodlands-Sugar Land, TX & 59 & 64 & 58 & 49 & 63 & 1.9 & 2.0 & 1.7 & 1.4 & 1.8 \\
\hline Indianapolis-Carmel-Anderson, IN & 9 & 5 & 12 & 15 & 12 & 0.9 & 0.5 & 1.2 & 1.5 & 1.2 \\
\hline Jacksonville, FL & 5 & 9 & 8 & 26 & 50 & 0.7 & 1.2 & 1.1 & 3.4 & 6.6 \\
\hline Kansas City, MO-KS & 9 & 23 & 41 & 39 & 43 & 0.9 & 2.2 & 3.9 & 3.6 & 4.0 \\
\hline Las Vegas-Henderson-Paradise, NV & 6 & 17 & 17 & 40 & 55 & 0.6 & 1.6 & 1.6 & 3.7 & 5.1 \\
\hline Los Angeles-Long Beach-Anaheim, CA & 50 & 67 & 108 & 144 & 177 & 0.8 & 1.0 & 1.6 & 2.1 & 2.6 \\
\hline Louisville-Jefferson County, KY-IN & 11 & 14 & 10 & 12 & 16 & 1.7 & 2.2 & 1.5 & 1.8 & 2.4 \\
\hline Memphis, TN-MS-AR & 17 & 22 & 13 & 25 & 21 & 2.4 & 3.1 & 1.9 & 3.6 & 3.0 \\
\hline Miami-Fort Lauderdale-West Palm Beach, FL & 65 & 44 & 75 & 66 & 71 & 2.2 & 1.4 & 2.4 & 2.1 & 2.3 \\
\hline Milwaukee-Waukesha-West Allis, WI & 5 & 6 & 0 & 1 & 5 & 0.6 & 0.7 & 0.0 & 0.1 & 0.6 \\
\hline Minneapolis-St. Paul-Bloomington, MN-WI & 9 & 19 & 37 & 31 & 24 & 0.5 & 1.1 & 2.1 & 1.7 & 1.3 \\
\hline Nashville-Davidson-Murfreesboro-Franklin, TN & 4 & 5 & 4 & 12 & 10 & 0.4 & 0.5 & 0.4 & 1.3 & 1.0 \\
\hline New Orleans-Metairie, LA & 10 & 18 & 29 & 25 & 36 & 1.6 & 2.8 & 4.4 & 3.8 & 5.5 \\
\hline New York-Newark-Jersey City, NY-NJ-PA & 46 & 52 & 69 & 123 & 119 & 0.4 & 0.5 & 0.7 & 1.2 & 1.1 \\
\hline Oklahoma City, OK & 5 & 7 & 14 & 27 & 39 & 0.7 & 1.0 & 2.0 & 3.9 & 5.6 \\
\hline Orlando-Kissimmee-Sanford, FL & 9 & 8 & 14 & 21 & 24 & 0.8 & 0.7 & 1.1 & 1.7 & 1.9 \\
\hline Philadelphia-Camden-Wilmington, PA-NJ-DE-MD & 24 & 39 & 43 & 49 & 63 & 0.8 & 1.2 & 1.4 & 1.6 & 2.0 \\
\hline Phoenix-Mesa-Scottsdale, AZ & 22 & 42 & 39 & 56 & 128 & 1.0 & 1.9 & 1.7 & 2.4 & 5.5 \\
\hline Pittsburgh, PA & 2 & 6 & 11 & 11 & 6 & 0.2 & 0.5 & 0.9 & 0.9 & 0.5 \\
\hline Portland-Vancouver-Hillsboro, OR-WA & 9 & 9 & 19 & 20 & 31 & 0.8 & 0.8 & 1.6 & 1.6 & 2.5 \\
\hline Providence-Warwick, RI-MA & 3 & 8 & $8^{+}$ & $6^{+}$ & 8 & 0.4 & 1.0 & $1.0^{+}$ & $0.7^{+}$ & 1.0 \\
\hline Raleigh, NC & 9 & 6 & 13 & 10 & 17 & 1.4 & 0.9 & 2.0 & 1.5 & 2.5 \\
\hline Richmond, VA & 4 & 2 & 4 & 15 & 18 & 0.6 & 0.3 & 0.6 & 2.3 & 2.7 \\
\hline Riverside-San Bernardino-Ontario, CA & 7 & 15 & 17 & 47 & 67 & 0.3 & 0.7 & 0.8 & 2.1 & 2.9 \\
\hline Sacramento-Roseville-Arden-Arcade, CA & 10 & 11 & 26 & 34 & 43 & 0.9 & 1.0 & 2.2 & 2.9 & 3.7 \\
\hline Salt Lake City, UT & 1 & 1 & 1 & 5 & 0 & 0.2 & 0.2 & 0.2 & 0.8 & 0.0 \\
\hline San Antonio-New Braunfels, TX & 44 & 47 & 43 & 43 & 54 & 3.8 & 4.0 & 3.6 & 3.5 & 4.4 \\
\hline San Diego-Carlsbad, CA & 10 & 20 & 17 & 20 & 31 & 0.6 & 1.2 & 1.0 & 1.2 & 1.9 \\
\hline San Francisco-Oakland-Hayward, CA & 40 & 34 & 46 & 50 & 58 & 1.7 & 1.5 & 2.0 & 2.1 & 2.4 \\
\hline San Jose-Sunnyvale-Santa Clara, CA & 9 & 12 & 10 & 32 & 29 & 0.9 & 1.2 & 1.0 & 3.3 & 3.0 \\
\hline Seattle-Tacoma-Bellevue, WA & 10 & 10 & 6 & 15 & 5 & 0.6 & 0.5 & 0.3 & 0.8 & 0.3 \\
\hline St. Louis, MO-IL & 6 & 17 & 14 & 24 & 34 & 0.4 & 1.2 & 1.0 & 1.7 & 2.4 \\
\hline Tampa-St. Petersburg-Clearwater, FL & 31 & 41 & 44 & 48 & 42 & 2.1 & 2.7 & 2.9 & 3.1 & 2.7 \\
\hline Virginia Beach-Norfolk-Newport News, VA-NC & 5 & 8 & 5 & 22 & 24 & 0.6 & 0.9 & 0.6 & 2.5 & 2.7 \\
\hline Washington-Arlington-Alexandria, DC-VA-MD-WV & 35 & 7 & 10 & 8 & 19 & 1.2 & 0.2 & 0.3 & 0.3 & 0.6 \\
\hline SELECTED MSAs TOTAL & 912 & 1,095 & 1,280 & 1,615 & 1,935 & 1.0 & 1.2 & 1.4 & 1.8 & 2.1 \\
\hline
\end{tabular}

* MSAs were selected on the basis of the largest population in the 2010 U.S. Census.

${ }^{\dagger}$ The variable used to identify county, which is used to classify cases into MSAs, was complete for $\leq 95 \%$ of cases in a state contributing data to this MSA. See Section A1.4 in the Appendix for more information.

₹2016 county data for Alabama have been corrected and may not match previous reports.

NOTE: Cases reported with unknown sex are not included in this table. 
Table 32. Primary and Secondary Syphilis Among Men - Reported Cases and Rates of Reported Cases in Selected Metropolitan Statistical Areas (MSAs)* in Alphabetical Order, United States, 2013-2017

\begin{tabular}{|c|c|c|c|c|c|c|c|c|c|c|}
\hline \multirow[b]{2}{*}{ MSAs } & \multicolumn{5}{|c|}{ Cases } & \multicolumn{5}{|c|}{ Rates per 100,000 Population } \\
\hline & 2013 & 2014 & 2015 & 2016 & 2017 & 2013 & 2014 & 2015 & 2016 & 2017 \\
\hline Atlanta-Sandy Springs-Roswell, GA & 737 & 938 & 1,036 & 957 & 1,017 & 27.5 & 34.5 & 37.5 & 34.2 & 36.3 \\
\hline Austin-Round Rock, TX & 139 & 213 & 187 & 300 & 330 & 14.7 & 21.9 & 18.7 & 29.2 & 32.1 \\
\hline Baltimore-Columbia-Towson, MD & 242 & 240 & 294 & 286 & 303 & 18.1 & 17.9 & 21.8 & 21.2 & 22.5 \\
\hline Birmingham-Hoover, AL & 64 & 53 & 66 & $84^{\ddagger}$ & 101 & 11.6 & 9.6 & 12.0 & $15.2^{\ddagger}$ & 18.3 \\
\hline Boston-Cambridge-Newton, MA-NH & 258 & 213 & $257^{+}$ & $311^{+}$ & 360 & 11.4 & 9.3 & $11.1^{+}$ & $13.4^{+}$ & 15.5 \\
\hline Buffalo-Cheektowaga-Niagara Falls, NY & 36 & 48 & 92 & 62 & 54 & 6.6 & 8.7 & 16.7 & 11.3 & 9.8 \\
\hline Charlotte-Concord-Gastonia, NC-SC & 128 & 214 & 317 & 353 & 324 & 11.3 & 18.5 & 26.9 & 29.4 & 27.0 \\
\hline Chicago-Naperville-Elgin, IL-IN-WI & 697 & 735 & 961 & 1,114 & 1,061 & 14.9 & 15.7 & 20.6 & 23.9 & 22.8 \\
\hline Cincinnati, OH-KY-IN & 135 & 120 & 72 & 70 & 92 & 12.9 & 11.4 & 6.8 & 6.6 & 8.7 \\
\hline Cleveland-Elyria, $\mathrm{OH}$ & 30 & 75 & 71 & 144 & 157 & 3.0 & 7.5 & 7.1 & 14.5 & 15.8 \\
\hline Columbus, $\mathrm{OH}$ & 149 & 216 & 247 & 268 & 308 & 15.4 & 22.0 & 24.8 & 26.7 & 30.7 \\
\hline Dallas-Fort Worth-Arlington, TX & 404 & 445 & 420 & 480 & 624 & 12.0 & 13.0 & 12.0 & 13.5 & 17.6 \\
\hline Denver-Aurora-Lakewood, CO & 132 & 149 & 186 & 176 & 201 & 9.8 & 10.9 & 13.3 & 12.4 & 14.1 \\
\hline Detroit-Warren-Dearborn, MI & 369 & 294 & 258 & 227 & 271 & 17.7 & 14.1 & 12.3 & 10.9 & 13.0 \\
\hline Hartford-West Hartford-East Hartford, CT & 14 & 24 & 35 & 30 & 24 & 2.4 & 4.1 & 5.9 & 5.1 & 4.1 \\
\hline Houston-The Woodlands-Sugar Land, TX & 304 & 350 & 381 & 362 & 323 & 9.7 & 10.8 & 11.5 & 10.8 & 9.6 \\
\hline Indianapolis-Carmel-Anderson, IN & 137 & 104 & 160 & 143 & 174 & 14.3 & 10.8 & 16.5 & 14.6 & 17.8 \\
\hline Jacksonville, FL & 35 & 60 & 83 & 92 & 144 & 5.2 & 8.7 & 11.7 & 12.8 & 20.0 \\
\hline Kansas City, MO-KS & 146 & 197 & 150 & 162 & 171 & 14.5 & 19.4 & 14.6 & 15.7 & 16.6 \\
\hline Las Vegas-Henderson-Paradise, NV & 158 & 301 & 288 & 358 & 464 & 15.5 & 29.1 & 27.3 & 33.3 & 43.2 \\
\hline Los Angeles-Long Beach-Anaheim, CA & 1,248 & 1,340 & 1,724 & 1,978 & 2,188 & 19.2 & 20.5 & 26.2 & 30.2 & 33.4 \\
\hline Louisville-Jefferson County, KY-IN & 60 & 69 & 77 & 137 & 134 & 9.7 & 11.1 & 12.3 & 21.8 & 21.4 \\
\hline Memphis, TN-MS-AR & 88 & 72 & 108 & 148 & 132 & 13.7 & 11.2 & 16.8 & 23.0 & 20.5 \\
\hline Miami-Fort Lauderdale-West Palm Beach, FL & 697 & 777 & 809 & 859 & 848 & 24.6 & 27.0 & 27.8 & 29.2 & 28.8 \\
\hline Milwaukee-Waukesha-West Allis, WI & 49 & 46 & 39 & 49 & 74 & 6.4 & 6.0 & 5.1 & 6.4 & 9.7 \\
\hline Minneapolis-St. Paul-Bloomington, MN-WI & 169 & 223 & 191 & 228 & 222 & 9.9 & 12.9 & 10.9 & 13.0 & 12.6 \\
\hline Nashville-Davidson-Murfreesboro-Franklin, TN & 53 & 69 & 112 & 103 & 150 & 6.2 & 7.9 & 12.5 & 11.3 & 16.5 \\
\hline New Orleans-Metairie, LA & 93 & 203 & 189 & 210 & 174 & 15.4 & 33.5 & 31.0 & 34.2 & 28.4 \\
\hline New York-Newark-Jersey City, NY-NJ-PA & 1,438 & 1,666 & 1,954 & 2,407 & 2,322 & 14.9 & 17.1 & 20.0 & 24.7 & 23.8 \\
\hline Oklahoma City, OK & 73 & 84 & 99 & 135 & 180 & 11.2 & 12.8 & 14.8 & 19.9 & 26.6 \\
\hline Orlando-Kissimmee-Sanford, FL & 192 & 231 & 285 & 363 & 294 & 17.3 & 20.4 & 24.4 & 30.4 & 24.6 \\
\hline Philadelphia-Camden-Wilmington, PA-NJ-DE-MD & 372 & 407 & 415 & 590 & 573 & 12.8 & 13.9 & 14.1 & 20.1 & 19.5 \\
\hline Phoenix-Mesa-Scottsdale, AZ & 197 & 374 & 413 & 518 & 647 & 9.0 & 16.8 & 18.2 & 22.4 & 27.9 \\
\hline Pittsburgh, PA & 37 & 72 & 139 & 89 & 65 & 3.2 & 6.3 & 12.1 & 7.8 & 5.7 \\
\hline Portland-Vancouver-Hillsboro, OR-WA & 231 & 197 & 242 & 243 & 224 & 20.2 & 17.0 & 20.5 & 20.3 & 18.7 \\
\hline Providence-Warwick, RI-MA & 62 & 84 & $103^{+}$ & $109^{+}$ & 90 & 8.0 & 10.8 & $13.2^{+}$ & $13.9^{+}$ & 11.5 \\
\hline Raleigh, NC & 61 & 123 & 155 & 127 & 119 & 10.3 & 20.3 & 24.9 & 20.0 & 18.8 \\
\hline Richmond, VA & 66 & 66 & 69 & 87 & 103 & 11.0 & 10.8 & 11.2 & 14.1 & 16.6 \\
\hline Riverside-San Bernardino-Ontario, CA & 196 & 273 & 324 & 398 & 428 & 9.0 & 12.4 & 14.5 & 17.7 & 19.0 \\
\hline Sacramento-Roseville-Arden-Arcade, CA & 137 & 151 & 239 & 238 & 300 & 12.6 & 13.7 & 21.5 & 21.2 & 26.7 \\
\hline Salt Lake City, UT & 64 & 38 & 48 & 64 & 87 & 11.2 & 6.6 & 8.2 & 10.7 & 14.6 \\
\hline San Antonio-New Braunfels, TX & 266 & 200 & 194 & 200 & 275 & 23.7 & 17.4 & 16.5 & 16.7 & 22.9 \\
\hline San Diego-Carlsbad, CA & 323 & 351 & 476 & 504 & 554 & 20.0 & 21.4 & 28.7 & 30.2 & 33.2 \\
\hline San Francisco-Oakland-Hayward, CA & 773 & 731 & 783 & 820 & 970 & 34.7 & 32.3 & 34.1 & 35.5 & 42.0 \\
\hline San Jose-Sunnyvale-Santa Clara, CA & 137 & 108 & 124 & 190 & 188 & 14.2 & 11.0 & 12.5 & 19.0 & 18.8 \\
\hline Seattle-Tacoma-Bellevue, WA & 201 & 225 & 305 & 382 & 434 & 11.2 & 12.3 & 16.3 & 20.1 & 22.9 \\
\hline St. Louis, MO-IL & 102 & 136 & 98 & 191 & 244 & 7.5 & 10.0 & 7.2 & 14.0 & 17.9 \\
\hline Tampa-St. Petersburg-Clearwater, FL & 195 & 278 & 349 & 388 & 322 & 14.0 & 19.7 & 24.2 & 26.4 & 21.9 \\
\hline Virginia Beach-Norfolk-Newport News, VA-NC & 97 & 77 & 112 & 178 & 144 & 11.5 & 9.1 & 13.2 & 20.9 & 16.9 \\
\hline Washington-Arlington-Alexandria, DC-VA-MD-WV & 383 & 219 & 219 & 264 & 618 & 13.2 & 7.4 & 7.4 & 8.8 & 20.6 \\
\hline SELECTED MSAs TOTAL & 12,374 & 13,879 & 15,955 & 18,176 & 19,606 & 14.6 & 16.2 & 18.4 & 20.9 & 22.5 \\
\hline
\end{tabular}

* MSAs were selected on the basis of the largest population in the 2010 U.S. Census.

'The variable used to identify county, which is used to classify cases into MSAs, was complete for $\leq 95 \%$ of cases in a state contributing data to this MSA. See Section A1.4 in the Appendix for more information.

₹ 2016 county data for Alabama have been corrected and may not match previous reports.

NOTE: Cases reported with unknown sex are not included in this table. 
Table 33. Primary and Secondary Syphilis - Reported Cases and Rates of Reported Cases in Counties and Independent Cities* Ranked by Number of Reported Cases, United States, 2017

\begin{tabular}{|c|c|c|c|c|}
\hline Rank* & County/Independent City & Cases & Rate per 100,000 Population & Cumulative Percentage \\
\hline 1 & Los Angeles County, CA & 1,996 & 19.7 & 6 \\
\hline 2 & Cook County, IL & 980 & 18.8 & 9 \\
\hline 3 & Maricopa County, AZ & 730 & 17.2 & 12 \\
\hline 4 & New York County, NY & 596 & 36.3 & 14 \\
\hline 5 & San Diego County, CA & 585 & 17.6 & 15 \\
\hline 6 & San Francisco County, CA & 584 & 67.1 & 17 \\
\hline 7 & Clark County, NV & 519 & 24.1 & 19 \\
\hline 8 & Kings County, NY & 519 & 19.7 & 21 \\
\hline 9 & Miami-Dade County, FL & 481 & 17.7 & 22 \\
\hline 10 & Philadelphia County, PA & 459 & 29.3 & 24 \\
\hline 11 & Fulton County, GA & 424 & 41.4 & 25 \\
\hline 12 & Dallas County, TX & 417 & 16.2 & 27 \\
\hline 13 & Bronx County, NY & 388 & 26.7 & 28 \\
\hline 14 & San Joaquin County, CA & 379 & 51.7 & 29 \\
\hline 15 & Orange County, CA & 369 & 11.6 & 30 \\
\hline 16 & Broward County, FL & 328 & 17.2 & 31 \\
\hline 17 & Harris County, TX & 328 & 7.1 & 32 \\
\hline 18 & Franklin County, $\mathrm{OH}$ & 321 & 25.4 & 33 \\
\hline 19 & King County, WA & 321 & 14.9 & 34 \\
\hline 20 & Bexar County, TX & 314 & 16.3 & 36 \\
\hline 21 & Travis County, TX & 310 & 25.8 & 37 \\
\hline 22 & Sacramento County, CA & 287 & 19.0 & 37 \\
\hline 23 & DeKalb County, GA & 281 & 38.0 & 38 \\
\hline 24 & Fresno County, CA & 279 & 28.5 & 39 \\
\hline 25 & Washington, D.C. & 274 & 40.2 & 40 \\
\hline 26 & Queens County, NY & 267 & 11.4 & 41 \\
\hline 27 & Mecklenburg County, NC & 265 & 25.1 & 42 \\
\hline 28 & Riverside County, CA & 253 & 10.6 & 43 \\
\hline 29 & Kern County, CA & 251 & 28.4 & 44 \\
\hline 30 & Orange County, FL & 243 & 18.5 & 44 \\
\hline 31 & San Bernardino County, CA & 242 & 11.3 & 45 \\
\hline 32 & Alameda County, CA & 230 & 14.0 & 46 \\
\hline 33 & Santa Clara County, CA & 215 & 11.2 & 47 \\
\hline 34 & Baltimore (City), MD & 210 & 34.2 & 47 \\
\hline 35 & Tarrant County, TX & 197 & 9.8 & 48 \\
\hline 36 & Hillsborough County, FL & 185 & 13.4 & 49 \\
\hline 37 & Wayne County, MI & 179 & 10.2 & 49 \\
\hline 38 & Oklahoma County, OK & 176 & 22.5 & 50 \\
\hline 39 & Duval County, FL & 170 & 18.4 & 50 \\
\hline 40 & Marion County, IN & 168 & 17.8 & 51 \\
\hline 41 & Pinellas County, FL & 160 & 16.7 & 51 \\
\hline 42 & Multnomah County, OR & 159 & 19.9 & 52 \\
\hline 43 & Cuyahoga County, OH & 158 & 12.6 & 52 \\
\hline 44 & Suffolk County, MA & 152 & 19.4 & 53 \\
\hline 45 & Hennepin County, MN & 152 & 12.3 & 53 \\
\hline 46 & Jackson County, MO & 146 & 21.1 & 54 \\
\hline 47 & Prince George's County, MD & 143 & 15.7 & 54 \\
\hline 48 & Shelby County, TN & 135 & 14.4 & 55 \\
\hline 49 & Orleans Parish, LA & 133 & 34.0 & 55 \\
\hline 50 & Denver County, CO & 133 & 19.2 & 56 \\
\hline 51 & Contra Costa County, CA & 133 & 11.7 & 56 \\
\hline 52 & Jefferson County, KY & 129 & 16.9 & 56 \\
\hline 53 & Wake County, NC & 126 & 12.0 & 57 \\
\hline 54 & Middlesex County, MA & 121 & 7.6 & 57 \\
\hline 55 & Stanislaus County, CA & 119 & 22.0 & 58 \\
\hline 56 & Gwinnett County, GA & 116 & 12.8 & 58 \\
\hline 57 & St. Louis County, MO & 114 & 11.4 & 58 \\
\hline 58 & Guilford County, NC & 113 & 21.7 & 59 \\
\hline 59 & Palm Beach County, FL & 110 & 7.6 & 59 \\
\hline 60 & Davidson County, TN & 109 & 15.9 & 59 \\
\hline 61 & Bernalillo County, NM & 107 & 15.8 & 60 \\
\hline 62 & El Paso County, TX & 106 & 12.7 & 60 \\
\hline 63 & St. Louis (City), MO & 101 & 32.4 & 61 \\
\hline 64 & Hudson County, NJ & 101 & 14.9 & 61 \\
\hline 65 & Pima County, AZ & 100 & 9.8 & 61 \\
\hline 66 & Essex County, NJ & 98 & 12.3 & 61 \\
\hline 67 & Manatee County, FL & 97 & 25.8 & 62 \\
\hline 68 & Jefferson County, AL & 97 & 14.7 & 62 \\
\hline 69 & Cobb County, GA & 97 & 13.0 & 62 \\
\hline 70 & Salt Lake County, UT & 86 & 7.7 & 63 \\
\hline
\end{tabular}

* The top 70 counties and independent cities ranked in descending order by number of cases reported in 2017 then by rate are displayed.

NOTE: Relative rankings of counties may be impacted by completeness of the variable used to identify county. See Section A1.4 in the Appendix for more information. 
Table 34. Primary and Secondary Syphilis - Reported Cases and Rates of Reported Cases by Age Group and Sex, United States, 2013-2017

\begin{tabular}{|c|c|c|c|c|c|c|c|c|}
\hline \multirow{2}{*}{$\begin{array}{l}\text { Age } \\
\text { Group }\end{array}$} & \multicolumn{4}{|c|}{ Cases } & \multicolumn{3}{|c|}{ Rates per 100,000 Population* } & \\
\hline & Total & Male & Female & Unknown Sex & Total & Male & Female & \\
\hline $0-4$ & 5 & 2 & 3 & 0 & 0.0 & 0.0 & 0.0 & \multirow{14}{*}{$\stackrel{\mathbf{0}}{\mathbf{\omega}}$} \\
\hline $5-9$ & 0 & 0 & 0 & 0 & 0.0 & 0.0 & 0.0 & \\
\hline $10-14$ & 23 & 14 & 9 & 0 & 0.1 & 0.1 & 0.1 & \\
\hline $15-19$ & 900 & 700 & 200 & 0 & 4.3 & 6.5 & 1.9 & \\
\hline $20-24$ & 3,642 & 3,204 & 435 & 3 & 16.0 & 27.4 & 3.9 & \\
\hline $25-29$ & 3,329 & 3,037 & 286 & 6 & 15.4 & 27.7 & 2.7 & \\
\hline $30-34$ & 2,447 & 2,272 & 172 & 3 & 11.5 & 21.3 & 1.6 & \\
\hline $35-39$ & 1,800 & 1,674 & 125 & 1 & 9.2 & 17.1 & 1.3 & \\
\hline $40-44$ & 1,693 & 1,587 & 105 & 1 & 8.1 & 15.3 & 1.0 & \\
\hline $45-54$ & 2,614 & 2,495 & 119 & 0 & 6.0 & 11.6 & 0.5 & \\
\hline $55-64$ & 750 & 716 & 34 & 0 & 1.9 & 3.8 & 0.2 & \\
\hline $65+$ & 162 & 152 & 10 & 0 & 0.4 & 0.8 & 0.0 & \\
\hline Unknown Age & 10 & 8 & 2 & 0 & & & & \\
\hline TOTAL & 17,375 & 15,861 & 1,500 & 14 & 5.5 & 10.2 & 0.9 & \\
\hline $0-4$ & 0 & 0 & 0 & 0 & 0.0 & 0.0 & 0.0 & \multirow{14}{*}{$\stackrel{\mathbf{O}}{\overrightarrow{0}}$} \\
\hline $5-9$ & 0 & 0 & 0 & 0 & 0.0 & 0.0 & 0.0 & \\
\hline $10-14$ & 12 & 4 & 8 & 0 & 0.1 & 0.0 & 0.1 & \\
\hline $15-19$ & 1,023 & 761 & 262 & 0 & 4.9 & 7.1 & 2.5 & \\
\hline $20-24$ & 4,137 & 3,632 & 503 & 2 & 18.1 & 30.9 & 4.5 & \\
\hline $25-29$ & 4,092 & 3,727 & 361 & 4 & 18.6 & 33.4 & 3.3 & \\
\hline $30-34$ & 2,887 & 2,635 & 248 & 4 & 13.4 & 24.4 & 2.3 & \\
\hline $35-39$ & 2,045 & 1,868 & 177 & 0 & 10.3 & 18.8 & 1.8 & \\
\hline $40-44$ & 1,758 & 1,654 & 103 & 1 & 8.5 & 16.2 & 1.0 & \\
\hline $45-54$ & 2,966 & 2,830 & 135 & 1 & 6.8 & 13.2 & 0.6 & \\
\hline $55-64$ & 897 & 860 & 36 & 1 & 2.2 & 4.5 & 0.2 & \\
\hline $65+$ & 176 & 169 & 7 & 0 & 0.4 & 0.8 & 0.0 & \\
\hline Unknown Age & 6 & 6 & 0 & 0 & & & & \\
\hline TOTAL & 19,999 & 18,146 & 1,840 & 13 & 6.3 & 11.6 & 1.1 & \\
\hline $0-4$ & 2 & 0 & 1 & 1 & 0.0 & 0.0 & 0.0 & \multirow{14}{*}{$\underset{\mathbf{U}}{\mathbf{O}}$} \\
\hline $5-9$ & 1 & 0 & 1 & 0 & 0.0 & 0.0 & 0.0 & \\
\hline $10-14$ & 9 & 1 & 8 & 0 & 0.0 & 0.0 & 0.1 & \\
\hline $15-19$ & 1,148 & 865 & 283 & 0 & 5.4 & 8.0 & 2.7 & \\
\hline $20-24$ & 4,766 & 4,186 & 573 & 7 & 21.0 & 35.9 & 5.2 & \\
\hline $25-29$ & 5,168 & 4,671 & 491 & 6 & 23.0 & 40.9 & 4.4 & \\
\hline $30-34$ & 3,549 & 3,234 & 311 & 4 & 16.4 & 29.7 & 2.9 & \\
\hline $35-39$ & 2,482 & 2,249 & 229 & 4 & 12.2 & 22.1 & 2.2 & \\
\hline $40-44$ & 1,897 & 1,744 & 152 & 1 & 9.4 & 17.4 & 1.5 & \\
\hline $45-54$ & 3,488 & 3,294 & 190 & 4 & 8.1 & 15.5 & 0.9 & \\
\hline $55-64$ & 1,153 & 1,099 & 54 & 0 & 2.8 & 5.6 & 0.3 & \\
\hline $65+$ & 207 & 202 & 5 & 0 & 0.4 & 1.0 & 0.0 & \\
\hline Unknown Age & 2 & 2 & 0 & 0 & & & & \\
\hline TOTAL & 23,872 & 21,547 & 2,298 & 27 & 7.4 & 13.6 & 1.4 & \\
\hline $0-4$ & 2 & 0 & 2 & 0 & 0.0 & 0.0 & 0.0 & \multirow{14}{*}{ O } \\
\hline $5-9$ & 2 & 1 & 1 & 0 & 0.0 & 0.0 & 0.0 & \\
\hline $10-14$ & 15 & 6 & 9 & 0 & 0.1 & 0.1 & 0.1 & \\
\hline $15-19$ & 1,298 & 957 & 340 & 1 & 6.1 & 8.9 & 3.3 & \\
\hline $20-24$ & 5,172 & 4,418 & 744 & 10 & 23.1 & 38.4 & 6.8 & \\
\hline $25-29$ & 6,177 & 5,538 & 624 & 15 & 27.0 & 47.6 & 5.5 & \\
\hline $30-34$ & 4,278 & 3,806 & 464 & 8 & 19.6 & 34.7 & 4.3 & \\
\hline $35-39$ & 3,043 & 2,729 & 311 & 3 & 14.6 & 26.3 & 3.0 & \\
\hline $40-44$ & 2,140 & 1,944 & 193 & 3 & 10.9 & 19.9 & 1.9 & \\
\hline $45-54$ & 3,953 & 3,691 & 261 & 1 & 9.2 & 17.5 & 1.2 & \\
\hline $55-64$ & 1,418 & 1,338 & 80 & 0 & 3.4 & 6.7 & 0.4 & \\
\hline $65+$ & 279 & 269 & 10 & 0 & 0.6 & 1.2 & 0.0 & \\
\hline Unknown Age & 37 & 27 & 10 & 0 & & & & \\
\hline TOTAL & 27,814 & 24,724 & 3,049 & 41 & 8.6 & 15.5 & 1.9 & \\
\hline $0-4$ & 5 & 0 & 5 & 0 & 0.0 & 0.0 & 0.1 & \multirow{14}{*}{$\underset{v}{v}$} \\
\hline $5-9$ & 1 & 0 & 1 & 0 & 0.0 & 0.0 & 0.0 & \\
\hline $10-14$ & 20 & 6 & 14 & 0 & 0.1 & 0.1 & 0.1 & \\
\hline $15-19$ & 1,421 & 1,092 & 327 & 2 & 6.7 & 10.1 & 3.2 & \\
\hline $20-24$ & 5,580 & 4,728 & 848 & 4 & 24.9 & 41.1 & 7.8 & \\
\hline $25-29$ & 6,838 & 6,033 & 795 & 10 & 29.9 & 51.9 & 7.1 & \\
\hline $30-34$ & 4,870 & 4,313 & 549 & 8 & 22.4 & 39.3 & 5.1 & \\
\hline $35-39$ & 3,580 & 3,145 & 431 & 4 & 17.2 & 30.3 & 4.1 & \\
\hline $40-44$ & 2,290 & 2,005 & 282 & 3 & 11.6 & 20.5 & 2.8 & \\
\hline $45-54$ & 4,091 & 3,753 & 334 & 4 & 9.6 & 17.8 & 1.5 & \\
\hline $55-64$ & 1,586 & 1,468 & 117 & 1 & 3.8 & 7.3 & 0.5 & \\
\hline $65+$ & 349 & 329 & 19 & 1 & 0.7 & 1.5 & 0.1 & \\
\hline Unknown Age & 13 & 13 & 0 & 0 & & & & \\
\hline TOTAL & 30,644 & 26,885 & 3,722 & 37 & 9.5 & 16.9 & 2.3 & \\
\hline
\end{tabular}

* No population data are available for unknown sex and age; therefore, rates are not calculated. NOTE: This table should be used only for age comparisons. 
Table 35A. Primary and Secondary Syphilis - Reported Cases by Race/Hispanic Ethnicity, Age Group, and Sex, United States, 2017

\begin{tabular}{|c|c|c|c|c|c|c|c|c|c|c|c|c|}
\hline \multirow{2}{*}{$\begin{array}{l}\text { Age } \\
\text { Group }\end{array}$} & \multicolumn{3}{|c|}{$\begin{array}{l}\text { American Indians/ } \\
\text { Alaska Natives }\end{array}$} & \multicolumn{3}{|c|}{ Asians } & \multicolumn{3}{|c|}{ Blacks } & \multicolumn{3}{|c|}{$\begin{array}{c}\text { Native Hawaiians/ } \\
\text { Other Pacific Islanders }\end{array}$} \\
\hline & Total* & Male & Female & Total* & Male & Female & Total* & Male & Female & Total* & Male & Female \\
\hline $0-4$ & 0 & 0 & 0 & 0 & 0 & 0 & 2 & 0 & 2 & 0 & 0 & 0 \\
\hline $5-9$ & 1 & 0 & 1 & 0 & 0 & 0 & 0 & 0 & 0 & 0 & 0 & 0 \\
\hline $10-14$ & 0 & 0 & 0 & 1 & 1 & 0 & 13 & 3 & 10 & 0 & 0 & 0 \\
\hline $15-19$ & 17 & 12 & 5 & 20 & 17 & 3 & 626 & 465 & 160 & 2 & 1 & 1 \\
\hline $20-24$ & 60 & 42 & 18 & 148 & 141 & 7 & 2,194 & 1,791 & 402 & 14 & 12 & 2 \\
\hline $25-29$ & 66 & 49 & 17 & 181 & 173 & 8 & 2,621 & 2,306 & 311 & 11 & 11 & 0 \\
\hline $30-34$ & 44 & 30 & 14 & 124 & 116 & 8 & 1,541 & 1,378 & 160 & 21 & 19 & 2 \\
\hline $35-39$ & 27 & 19 & 8 & 95 & 91 & 4 & 973 & 845 & 126 & 13 & 9 & 4 \\
\hline $40-44$ & 23 & 19 & 4 & 71 & 69 & 1 & 601 & 515 & 86 & 7 & 7 & 0 \\
\hline $45-54$ & 20 & 15 & 5 & 99 & 94 & 5 & 812 & 716 & 96 & 7 & 7 & 0 \\
\hline $55-64$ & 5 & 4 & 1 & 27 & 25 & 2 & 320 & 280 & 40 & 3 & 3 & 0 \\
\hline $65+$ & 1 & 1 & 0 & 8 & 8 & 0 & 46 & 40 & 6 & 1 & 1 & 0 \\
\hline Unknown Age & 0 & 0 & 0 & 1 & 1 & 0 & 5 & 5 & 0 & 0 & 0 & 0 \\
\hline TOTAL & 264 & 191 & 73 & 775 & 736 & 38 & 9,754 & 8,344 & 1,399 & 79 & 70 & 9 \\
\hline
\end{tabular}

\begin{tabular}{|c|c|c|c|c|c|c|c|c|c|c|c|c|}
\hline \multirow{2}{*}{$\begin{array}{l}\text { Age } \\
\text { Group }\end{array}$} & \multicolumn{3}{|c|}{ Whites } & \multicolumn{3}{|c|}{ Multirace } & \multicolumn{3}{|c|}{ Hispanics } & \multicolumn{3}{|c|}{$\begin{array}{l}\text { Other/ } \\
\text { Unknown }\end{array}$} \\
\hline & Total* & Male & Female & Total* & Male & Female & Total* & Male & Female & Total* & Male & Female \\
\hline $0-4$ & 2 & 0 & 2 & 1 & 0 & 1 & 0 & 0 & 0 & 0 & 0 & 0 \\
\hline $5-9$ & 0 & 0 & 0 & 0 & 0 & 0 & 0 & 0 & 0 & 0 & 0 & 0 \\
\hline $10-14$ & 2 & 0 & 2 & 0 & 0 & 0 & 3 & 2 & 1 & 1 & 0 & 1 \\
\hline $15-19$ & 333 & 247 & 86 & 18 & 16 & 2 & 333 & 278 & 55 & 72 & 56 & 15 \\
\hline $20-24$ & 1,408 & 1,167 & 239 & 81 & 68 & 12 & 1,359 & 1,226 & 133 & 316 & 281 & 35 \\
\hline $25-29$ & 1,884 & 1,601 & 281 & 138 & 123 & 12 & 1,593 & 1,455 & 137 & 344 & 315 & 29 \\
\hline $30-34$ & 1,590 & 1,359 & 230 & 121 & 115 & 5 & 1,152 & 1,046 & 103 & 277 & 250 & 27 \\
\hline $35-39$ & 1,353 & 1,194 & 158 & 65 & 60 & 5 & 851 & 747 & 103 & 203 & 180 & 23 \\
\hline $40-44$ & 869 & 766 & 103 & 33 & 31 & 2 & 555 & 481 & 73 & 131 & 117 & 13 \\
\hline $45-54$ & 2,125 & 1,975 & 149 & 59 & 57 & 2 & 704 & 644 & 58 & 265 & 245 & 19 \\
\hline $55-64$ & 922 & 871 & 50 & 13 & 12 & 1 & 204 & 184 & 20 & 92 & 89 & 3 \\
\hline $65+$ & 219 & 211 & 8 & 4 & 4 & 0 & 36 & 33 & 2 & 34 & 31 & 3 \\
\hline Unknown Age & 6 & 6 & 0 & 0 & 0 & 0 & 0 & 0 & 0 & 1 & 1 & 0 \\
\hline TOTAL & 10,713 & 9,397 & 1,308 & 533 & 486 & 42 & 6,790 & 6,096 & 685 & 1,736 & 1,565 & 168 \\
\hline
\end{tabular}

* Total includes cases reported with unknown sex.

NOTE: These tables should be used only for race/Hispanic ethnicity comparisons. See Table 34 for age-specific cases and rates and Tables $27-29$ for total and sexspecific cases and rates. 
Table 35B. Primary and Secondary Syphilis - Rates of Reported Cases per 100,000 Population by Race/ Hispanic Ethnicity, Age Group, and Sex, United States, 2017

\begin{tabular}{|c|c|c|c|c|c|c|c|c|c|c|c|c|}
\hline \multirow{2}{*}{$\begin{array}{l}\text { Age } \\
\text { Group }\end{array}$} & \multicolumn{3}{|c|}{$\begin{array}{c}\text { American Indians/ } \\
\text { Alaska Natives }\end{array}$} & \multicolumn{3}{|c|}{ Asians } & \multicolumn{3}{|c|}{ Blacks } & \multicolumn{3}{|c|}{$\begin{array}{l}\text { Native Hawaiians/ } \\
\text { Other Pacific Islanders }\end{array}$} \\
\hline & Total* & Male & Female & Total* & Male & Female & Total* & Male & Female & Total* & Male & Female \\
\hline $0-4$ & 0.0 & 0.0 & 0.0 & 0.0 & 0.0 & 0.0 & 0.1 & 0.0 & 0.1 & 0.0 & 0.0 & 0.0 \\
\hline $5-9$ & 0.6 & 0.0 & 1.2 & 0.0 & 0.0 & 0.0 & 0.0 & 0.0 & 0.0 & 0.0 & 0.0 & 0.0 \\
\hline $10-14$ & 0.0 & 0.0 & 0.0 & 0.1 & 0.2 & 0.0 & 0.5 & 0.2 & 0.7 & 0.0 & 0.0 & 0.0 \\
\hline $15-19$ & 9.3 & 13.0 & 5.6 & 1.9 & 3.2 & 0.6 & 21.0 & 30.7 & 10.9 & 4.9 & 4.8 & 5.1 \\
\hline $20-24$ & 30.9 & 42.3 & 19.0 & 11.7 & 22.0 & 1.1 & 66.5 & 106.8 & 24.8 & 31.3 & 52.1 & 9.2 \\
\hline $25-29$ & 34.7 & 50.4 & 18.3 & 11.9 & 23.3 & 1.0 & 80.5 & 142.4 & 19.0 & 21.1 & 40.5 & 0.0 \\
\hline $30-34$ & 26.7 & 36.4 & 17.0 & 8.1 & 15.9 & 1.0 & 55.1 & 101.9 & 11.1 & 42.1 & 74.5 & 8.2 \\
\hline 35-39 & 17.8 & 25.3 & 10.5 & 6.6 & 13.6 & 0.5 & 36.5 & 66.8 & 9.0 & 29.7 & 40.1 & 18.8 \\
\hline $40-44$ & 16.4 & 27.4 & 5.6 & 5.0 & 10.4 & 0.1 & 24.1 & 44.0 & 6.5 & 18.7 & 36.9 & 0.0 \\
\hline $45-54$ & 6.6 & 10.1 & 3.2 & 4.1 & 8.3 & 0.4 & 15.4 & 28.9 & 3.4 & 9.9 & 20.1 & 0.0 \\
\hline $55-64$ & 1.8 & 3.0 & 0.7 & 1.4 & 2.8 & 0.2 & 6.8 & 13.0 & 1.6 & 5.3 & 10.8 & 0.0 \\
\hline $65+$ & 0.4 & 0.9 & 0.0 & 0.4 & 0.9 & 0.0 & 1.0 & 2.3 & 0.2 & 2.0 & 4.4 & 0.0 \\
\hline \multicolumn{13}{|c|}{ Unknown Age } \\
\hline TOTAL & 11.1 & 16.2 & 6.0 & 4.4 & 8.7 & 0.4 & 24.2 & 43.4 & 6.7 & 13.9 & 24.5 & 3.2 \\
\hline
\end{tabular}

\begin{tabular}{|c|c|c|c|c|c|c|c|c|c|}
\hline \multirow{2}{*}{$\begin{array}{l}\text { Age } \\
\text { Group }\end{array}$} & \multicolumn{3}{|c|}{ Whites } & \multicolumn{3}{|c|}{ Multirace } & \multicolumn{3}{|c|}{ Hispanics } \\
\hline & Total* & Male & Female & Total* & Male & Female & Total* & Male & Female \\
\hline $0-4$ & 0.0 & 0.0 & 0.0 & 0.1 & 0.0 & 0.2 & 0.0 & 0.0 & 0.0 \\
\hline $5-9$ & 0.0 & 0.0 & 0.0 & 0.0 & 0.0 & 0.0 & 0.0 & 0.0 & 0.0 \\
\hline $10-14$ & 0.0 & 0.0 & 0.0 & 0.0 & 0.0 & 0.0 & 0.1 & 0.1 & 0.0 \\
\hline $15-19$ & 2.9 & 4.2 & 1.6 & 2.6 & 4.5 & 0.6 & 6.9 & 11.4 & 2.3 \\
\hline $20-24$ & 11.6 & 18.7 & 4.0 & 13.1 & 21.7 & 3.9 & 28.3 & 49.4 & 5.7 \\
\hline $25-29$ & 14.8 & 24.7 & 4.5 & 28.0 & 51.6 & 4.7 & 34.5 & 60.3 & 6.2 \\
\hline $30-34$ & 12.8 & 21.7 & 3.8 & 30.8 & 61.9 & 2.4 & 25.9 & 44.9 & 4.8 \\
\hline $35-39$ & 11.4 & 20.0 & 2.7 & 19.2 & 37.7 & 2.8 & 19.8 & 33.7 & 5.0 \\
\hline $40-44$ & 7.6 & 13.4 & 1.8 & 11.8 & 23.5 & 1.3 & 14.0 & 24.0 & 3.7 \\
\hline $45-54$ & 7.7 & 14.4 & 1.1 & 11.9 & 24.3 & 0.8 & 10.5 & 19.1 & 1.8 \\
\hline $55-64$ & 3.1 & 6.0 & 0.3 & 3.2 & 6.2 & 0.5 & 4.6 & 8.5 & 0.9 \\
\hline $65+$ & 0.6 & 1.2 & 0.0 & 1.1 & 2.5 & 0.0 & 0.9 & 1.9 & 0.1 \\
\hline \multicolumn{10}{|c|}{ Unknown Age } \\
\hline TOTAL & 5.4 & 9.6 & 1.3 & 7.9 & 14.6 & 1.2 & 11.8 & 21.0 & 2.4 \\
\hline
\end{tabular}

* Total includes cases reported with unknown sex.

NOTE: These tables should be used only for race/Hispanic ethnicity comparisons. See Table 34 for age-specific cases and rates and Tables $27-29$ for total and sexspecific cases and rates. No population data exist for unknown sex, unknown age, or unknown race; therefore rates are not calculated. 
Table 36. Early Latent Syphilis - Reported Cases and Rates of Reported Cases by State/Area and Region in Alphabetical Order, United States and Outlying Areas, 2013-2017

\begin{tabular}{|c|c|c|c|c|c|c|c|c|c|c|}
\hline \multirow[b]{2}{*}{ State/Area } & \multicolumn{5}{|c|}{ Cases } & \multicolumn{5}{|c|}{ Rates per 100,000 Population } \\
\hline & 2013 & 2014 & 2015 & 2016 & 2017 & 2013 & 2014 & 2015 & 2016 & 2017 \\
\hline Alabama & 202 & 144 & 177 & 293 & 425 & 4.2 & 3.0 & 3.6 & 6.0 & 8.7 \\
\hline Alaska & 8 & 25 & 13 & 13 & 9 & 1.1 & 3.4 & 1.8 & 1.8 & 1.2 \\
\hline Arizona & 207 & 311 & 361 & 488 & 620 & 3.1 & 4.6 & 5.3 & 7.0 & 8.9 \\
\hline Arkansas & 163 & 152 & 216 & 280 & 328 & 5.5 & 5.1 & 7.3 & 9.4 & 11.0 \\
\hline California & 2,844 & 3,396 & 4,435 & 5,289 & 7,028 & 7.4 & 8.8 & 11.3 & 13.5 & 17.9 \\
\hline Colorado & 195 & 164 & 212 & 274 & 281 & 3.7 & 3.1 & 3.9 & 4.9 & 5.1 \\
\hline Connecticut & 55 & 62 & 97 & 84 & 145 & 1.5 & 1.7 & 2.7 & 2.3 & 4.1 \\
\hline Delaware & 30 & 33 & 47 & 57 & 49 & 3.2 & 3.5 & 5.0 & 6.0 & 5.1 \\
\hline District of Columbia & 243 & 142 & 200 & 355 & 341 & 37.6 & 21.6 & 29.8 & 52.1 & 50.1 \\
\hline Florida & 1,540 & 1,886 & 2,288 & 2,634 & 3,033 & 7.9 & 9.5 & 11.3 & 12.8 & 14.7 \\
\hline Georgia & 863 & 1,078 & 1,477 & 1,263 & 1,218 & 8.6 & 10.7 & 14.5 & 12.2 & 11.8 \\
\hline Hawaii & 22 & 25 & 56 & 89 & 58 & 1.6 & 1.8 & 3.9 & 6.2 & 4.1 \\
\hline Idaho & 6 & 12 & 24 & 33 & 41 & 0.4 & 0.7 & 1.5 & 2.0 & 2.4 \\
\hline Illinois & 809 & 819 & 889 & 1,138 & 1,192 & 6.3 & 6.4 & 6.9 & 8.9 & 9.3 \\
\hline Indiana & 157 & 129 & 220 & 247 & 250 & 2.4 & 2.0 & 3.3 & 3.7 & 3.8 \\
\hline lowa & 63 & 82 & 69 & 59 & 91 & 2.0 & 2.6 & 2.2 & 1.9 & 2.9 \\
\hline Kansas & 84 & 92 & 153 & 178 & 202 & 2.9 & 3.2 & 5.3 & 6.1 & 6.9 \\
\hline Kentucky & 167 & 169 & 164 & 189 & 236 & 3.8 & 3.8 & 3.7 & 4.3 & 5.3 \\
\hline Louisiana & 276 & 372 & 439 & 568 & 623 & 6.0 & 8.0 & 9.4 & 12.1 & 13.3 \\
\hline Maine & 6 & 7 & 10 & 6 & 36 & 0.5 & 0.5 & 0.8 & 0.5 & 2.7 \\
\hline Maryland & 387 & 529 & 594 & 598 & 683 & 6.5 & 8.9 & 9.9 & 9.9 & 11.4 \\
\hline Massachusetts & 350 & 282 & 355 & 538 & 549 & 5.2 & 4.2 & 5.2 & 7.9 & 8.1 \\
\hline Michigan & 204 & 243 & 282 & 290 & 330 & 2.1 & 2.5 & 2.8 & 2.9 & 3.3 \\
\hline Minnesota & 139 & 159 & 185 & 251 & 313 & 2.6 & 2.9 & 3.4 & 4.5 & 5.7 \\
\hline Mississippi & 184 & 336 & 405 & 490 & 555 & 6.2 & 11.2 & 13.5 & 16.4 & 18.6 \\
\hline Missouri & 220 & 240 & 247 & 276 & 423 & 3.6 & 4.0 & 4.1 & 4.5 & 6.9 \\
\hline Montana & 2 & 1 & 5 & 6 & 23 & 0.2 & 0.1 & 0.5 & 0.6 & 2.2 \\
\hline Nebraska & 14 & 19 & 5 & 19 & 26 & 0.7 & 1.0 & 0.3 & 1.0 & 1.4 \\
\hline Nevada & 232 & 389 & 439 & 510 & 498 & 8.3 & 13.7 & 15.2 & 17.3 & 16.9 \\
\hline New Hampshire & 21 & 22 & 16 & 33 & 37 & 1.6 & 1.7 & 1.2 & 2.5 & 2.8 \\
\hline New Jersey & 539 & 612 & 714 & 755 & 865 & 6.1 & 6.8 & 8.0 & 8.4 & 9.7 \\
\hline New Mexico & 67 & 76 & 71 & 118 & 120 & 3.2 & 3.6 & 3.4 & 5.7 & 5.8 \\
\hline New York & 1,945 & 2,307 & 2,802 & 3,504 & 3,914 & 9.9 & 11.7 & 14.2 & 17.7 & 19.8 \\
\hline North Carolina & 236 & 468 & 753 & 799 & 771 & 2.4 & 4.7 & 7.5 & 7.9 & 7.6 \\
\hline North Dakota & 2 & 22 & 17 & 12 & 12 & 0.3 & 3.0 & 2.2 & 1.6 & 1.6 \\
\hline Ohio & 211 & 265 & 326 & 389 & 454 & 1.8 & 2.3 & 2.8 & 3.3 & 3.9 \\
\hline Oklahoma & 237 & 198 & 222 & 339 & 478 & 6.2 & 5.1 & 5.7 & 8.6 & 12.2 \\
\hline Oregon & 127 & 149 & 214 & 250 & 205 & 3.2 & 3.8 & 5.3 & 6.1 & 5.0 \\
\hline Pennsylvania & 581 & 641 & 770 & 982 & 1,100 & 4.5 & 5.0 & 6.0 & 7.7 & 8.6 \\
\hline Rhode Island & 22 & 49 & 38 & 63 & 71 & 2.1 & 4.6 & 3.6 & 6.0 & 6.7 \\
\hline South Carolina & 415 & 467 & 496 & 613 & 687 & 8.7 & 9.7 & 10.1 & 12.4 & 13.8 \\
\hline South Dakota & 5 & 23 & 11 & 14 & 19 & 0.6 & 2.7 & 1.3 & 1.6 & 2.2 \\
\hline Tennessee & 267 & 236 & 312 & 337 & 412 & 4.1 & 3.6 & 4.7 & 5.1 & 6.2 \\
\hline Texas & 1,902 & 1,984 & 2,471 & 2,872 & 3,680 & 7.2 & 7.4 & 9.0 & 10.3 & 13.2 \\
\hline Utah & 47 & 41 & 31 & 61 & 85 & 1.6 & 1.4 & 1.0 & 2.0 & 2.8 \\
\hline Vermont & 2 & 7 & 6 & 14 & 13 & 0.3 & 1.1 & 1.0 & 2.2 & 2.1 \\
\hline Virginia & 354 & 274 & 410 & 602 & 659 & 4.3 & 3.3 & 4.9 & 7.2 & 7.8 \\
\hline Washington & 204 & 198 & 293 & 446 & 588 & 2.9 & 2.8 & 4.1 & 6.1 & 8.1 \\
\hline West Virginia & 10 & 23 & 40 & 51 & 34 & 0.5 & 1.2 & 2.2 & 2.8 & 1.9 \\
\hline Wisconsin & 62 & 91 & 95 & 150 & 199 & 1.1 & 1.6 & 1.6 & 2.6 & 3.4 \\
\hline Wyoming & 1 & 1 & 1 & 5 & 4 & 0.2 & 0.2 & 0.2 & 0.9 & 0.7 \\
\hline U.S. TOTAL & 16,929 & 19,452 & 24,173 & 28,924 & 34,013 & 5.4 & 6.1 & 7.5 & 9.0 & 10.5 \\
\hline Northeast & 3,521 & 3,989 & 4,808 & 5,979 & 6,730 & 6.3 & 7.1 & 8.5 & 10.6 & 12.0 \\
\hline Midwest & 1,970 & 2,184 & 2,499 & 3,023 & 3,511 & 2.9 & 3.2 & 3.7 & 4.4 & 5.2 \\
\hline South & 7,476 & 8,491 & 10,711 & 12,340 & 14,212 & 6.3 & 7.1 & 8.8 & 10.1 & 11.6 \\
\hline West & 3,962 & 4,788 & 6,155 & 7,582 & 9,560 & 5.3 & 6.4 & 8.1 & 9.9 & 12.5 \\
\hline Guam & 3 & 1 & 2 & 1 & 3 & 1.9 & 0.6 & 1.2 & 0.6 & 1.8 \\
\hline Puerto Rico & 270 & 375 & 565 & 570 & 527 & 7.5 & 10.6 & 16.3 & 16.7 & 15.4 \\
\hline Virgin Islands & 2 & 0 & 7 & 2 & 0 & 1.9 & 0.0 & 6.8 & 1.9 & 0.0 \\
\hline OUTLYING AREAS & 275 & 376 & 574 & 573 & 530 & 7.1 & 9.9 & 15.4 & 15.5 & 14.4 \\
\hline TOTAL & 17,204 & 19,828 & 24,747 & 29,497 & 34,543 & 5.4 & 6.1 & 7.6 & 9.0 & 10.6 \\
\hline
\end{tabular}

NOTE: See Section A1.11 in the Appendix for more information on interpreting case counts and rates in outlying areas. 
Table 37. Early Latent Syphilis - Reported Cases and Rates of Reported Cases in Selected Metropolitan Statistical Areas (MSAs)* in Alphabetical Order, United States, 2013-2017

\begin{tabular}{|c|c|c|c|c|c|c|c|c|c|c|}
\hline \multirow[b]{2}{*}{ MSAs } & \multicolumn{5}{|c|}{ Cases } & \multicolumn{5}{|c|}{ Rates per 100,000 Population } \\
\hline & 2013 & 2014 & 2015 & 2016 & 2017 & 2013 & 2014 & 2015 & 2016 & 2017 \\
\hline Atlanta-Sandy Springs-Roswell, GA & 672 & 863 & 1,067 & 1,053 & 1,027 & 12.2 & 15.4 & 18.7 & 18.2 & 17.7 \\
\hline Austin-Round Rock, TX & 220 & 207 & 242 & 291 & 424 & 11.7 & 10.7 & 12.1 & 14.2 & 20.6 \\
\hline Baltimore-Columbia-Towson, MD & 216 & 296 & 344 & 358 & 337 & 7.8 & 10.6 & 12.3 & 12.8 & 12.0 \\
\hline Birmingham-Hoover, AL & 71 & 46 & 60 & $79^{\ddagger}$ & 124 & 6.2 & 4.0 & 5.2 & $6.9^{\ddagger}$ & 10.8 \\
\hline Boston-Cambridge-Newton, MA-NH & 278 & 208 & $235^{+}$ & $408^{+}$ & 429 & 5.9 & 4.4 & $4.9^{+}$ & $8.5^{+}$ & 8.9 \\
\hline Buffalo-Cheektowaga-Niagara Falls, NY & 15 & 19 & 37 & 29 & 32 & 1.3 & 1.7 & 3.3 & 2.6 & 2.8 \\
\hline Charlotte-Concord-Gastonia, NC-SC & 74 & 129 & 206 & 264 & 259 & 3.2 & 5.4 & 8.5 & 10.7 & 10.5 \\
\hline Chicago-Naperville-Elgin, IL-IN-WI & 751 & 734 & 814 & 1,058 & 1,091 & 7.9 & 7.7 & 8.5 & 11.1 & 11.5 \\
\hline Cincinnati, OH-KY-IN & 70 & 98 & 92 & 74 & 83 & 3.3 & 4.6 & 4.3 & 3.4 & 3.8 \\
\hline Cleveland-Elyria, $\mathrm{OH}$ & 14 & 31 & 37 & 63 & 73 & 0.7 & 1.5 & 1.8 & 3.1 & 3.6 \\
\hline Columbus, $\mathrm{OH}$ & 71 & 82 & 130 & 149 & 183 & 3.6 & 4.1 & 6.4 & 7.3 & 9.0 \\
\hline Dallas-Fort Worth-Arlington, TX & 550 & 644 & 932 & 1,038 & 1,256 & 8.1 & 9.3 & 13.1 & 14.4 & 17.4 \\
\hline Denver-Aurora-Lakewood, CO & 166 & 145 & 175 & 212 & 213 & 6.2 & 5.3 & 6.2 & 7.4 & 7.5 \\
\hline Detroit-Warren-Dearborn, MI & 152 & 163 & 206 & 194 & 196 & 3.5 & 3.8 & 4.8 & 4.5 & 4.6 \\
\hline Hartford-West Hartford-East Hartford, CT & 19 & 16 & 31 & 20 & 31 & 1.6 & 1.3 & 2.6 & 1.7 & 2.6 \\
\hline Houston-The Woodlands-Sugar Land, TX & 348 & 444 & 522 & 585 & 740 & 5.5 & 6.8 & 7.8 & 8.6 & 10.9 \\
\hline Indianapolis-Carmel-Anderson, IN & 104 & 91 & 143 & 165 & 161 & 5.3 & 4.6 & 7.2 & 8.2 & 8.0 \\
\hline Jacksonville, FL & 73 & 69 & 162 & 137 & 191 & 5.2 & 4.9 & 11.2 & 9.3 & 12.9 \\
\hline Kansas City, MO-KS & 111 & 132 & 133 & 140 & 158 & 5.4 & 6.4 & 6.4 & 6.7 & 7.5 \\
\hline Las Vegas-Henderson-Paradise, NV & 218 & 375 & 413 & 470 & 451 & 10.8 & 18.1 & 19.5 & 21.8 & 20.9 \\
\hline Los Angeles-Long Beach-Anaheim, CA & 1,520 & 1,619 & 2,052 & 2,403 & 3,119 & 11.6 & 12.2 & 15.4 & 18.1 & 23.4 \\
\hline Louisville-Jefferson County, KY-IN & 85 & 82 & 90 & 118 & 143 & 6.7 & 6.5 & 7.0 & 9.2 & 11.1 \\
\hline Memphis, TN-MS-AR & 188 & 143 & 195 & 246 & 244 & 14.0 & 10.6 & 14.5 & 18.3 & 18.2 \\
\hline Miami-Fort Lauderdale-West Palm Beach, FL & 885 & 1,094 & 1,220 & 1,282 & 1,569 & 15.2 & 18.4 & 20.3 & 21.1 & 25.9 \\
\hline Milwaukee-Waukesha-West Allis, WI & 43 & 69 & 66 & 108 & 130 & 2.7 & 4.4 & 4.2 & 6.9 & 8.3 \\
\hline Minneapolis-St. Paul-Bloomington, MN-WI & 131 & 155 & 170 & 226 & 280 & 3.8 & 4.4 & 4.8 & 6.4 & 7.9 \\
\hline Nashville-Davidson-Murfreesboro-Franklin, TN & 62 & 83 & 82 & 72 & 102 & 3.5 & 4.6 & 4.5 & 3.9 & 5.5 \\
\hline New Orleans-Metairie, LA & 81 & 122 & 171 & 242 & 244 & 6.5 & 9.7 & 13.5 & 19.1 & 19.2 \\
\hline New York-Newark-Jersey City, NY-NJ-PA & 2,299 & 2,681 & 3,210 & 4,008 & 4,405 & 11.5 & 13.3 & 15.9 & 19.9 & 21.9 \\
\hline Oklahoma City, OK & 124 & 107 & 114 & 195 & 270 & 9.4 & 8.0 & 8.4 & 14.2 & 19.7 \\
\hline Orlando-Kissimmee-Sanford, FL & 175 & 180 & 266 & 377 & 359 & 7.7 & 7.8 & 11.1 & 15.4 & 14.7 \\
\hline Philadelphia-Camden-Wilmington, PA-NJ-DE-MD & 497 & 512 & 616 & 736 & 835 & 8.2 & 8.5 & 10.1 & 12.1 & 13.8 \\
\hline Phoenix-Mesa-Scottsdale, AZ & 150 & 240 & 268 & 381 & 440 & 3.4 & 5.3 & 5.9 & 8.2 & 9.4 \\
\hline Pittsburgh, PA & 45 & 63 & 111 & 125 & 109 & 1.9 & 2.7 & 4.7 & 5.3 & 4.7 \\
\hline Portland-Vancouver-Hillsboro, OR-WA & 117 & 124 & 170 & 226 & 187 & 5.1 & 5.3 & 7.1 & 9.3 & 7.7 \\
\hline Providence-Warwick, RI-MA & 28 & 64 & $48^{+}$ & $71^{+}$ & 92 & 1.7 & 4.0 & $3.0^{+}$ & $4.4^{+}$ & 5.7 \\
\hline Raleigh, NC & 41 & 77 & 115 & 135 & 130 & 3.4 & 6.2 & 9.0 & 10.4 & 10.0 \\
\hline Richmond, VA & 75 & 68 & 98 & 162 & 157 & 6.0 & 5.4 & 7.7 & 12.6 & 12.2 \\
\hline Riverside-San Bernardino-Ontario, CA & 159 & 223 & 311 & 379 & 513 & 3.6 & 5.0 & 6.9 & 8.4 & 11.3 \\
\hline Sacramento-Roseville-Arden-Arcade, CA & 33 & 74 & 137 & 133 & 194 & 1.5 & 3.3 & 6.0 & 5.8 & 8.4 \\
\hline Salt Lake City, UT & 37 & 31 & 23 & 43 & 55 & 3.2 & 2.7 & 2.0 & 3.6 & 4.6 \\
\hline San Antonio-New Braunfels, TX & 381 & 308 & 258 & 339 & 485 & 16.7 & 13.2 & 10.8 & 14.0 & 20.0 \\
\hline San Diego-Carlsbad, CA & 211 & 299 & 343 & 461 & 550 & 6.6 & 9.2 & 10.4 & 13.9 & 16.6 \\
\hline San Francisco-Oakland-Hayward, CA & 656 & 839 & 964 & 919 & 1,218 & 14.5 & 18.3 & 20.7 & 19.6 & 26.0 \\
\hline San Jose-Sunnyvale-Santa Clara, CA & 60 & 58 & 96 & 135 & 200 & 3.1 & 3.0 & 4.9 & 6.8 & 10.1 \\
\hline Seattle-Tacoma-Bellevue, WA & 167 & 143 & 221 & 303 & 424 & 4.6 & 3.9 & 5.9 & 8.0 & 11.2 \\
\hline St. Louis, MO-IL & 125 & 139 & 138 & 151 & 245 & 4.5 & 5.0 & 4.9 & 5.4 & 8.7 \\
\hline Tampa-St. Petersburg-Clearwater, FL & 176 & 227 & 258 & 364 & 296 & 6.1 & 7.8 & 8.7 & 12.0 & 9.8 \\
\hline Virginia Beach-Norfolk-Newport News, VA-NC & 112 & 90 & 167 & 245 & 270 & 6.6 & 5.2 & 9.7 & 14.2 & 15.6 \\
\hline Washington-Arlington-Alexandria, DC-VA-MD-WV & 520 & 286 & 320 & 355 & 820 & 8.7 & 4.7 & 5.2 & 5.8 & 13.4 \\
\hline SELECTED MSAs TOTAL & 13,376 & 14,992 & 18,279 & 21,727 & 25,544 & 7.7 & 8.6 & 10.3 & 12.2 & 14.4 \\
\hline
\end{tabular}

* MSAs were selected on the basis of the largest population in the 2010 U.S. Census.

${ }^{\dagger}$ The variable used to identify county, which is used to classify cases into MSAs, was complete for $\leq 95 \%$ of cases in a state contributing data to this MSA. See Section A1.4 in the Appendix for more information.

‡ 2016 county data for Alabama have been corrected and may not match previous reports. 
Table 38. Late and Late Latent Syphilis* - Reported Cases and Rates of Reported Cases by State/Area and Region in Alphabetical Order, United States and Outlying Areas, 2013-2017

\begin{tabular}{|c|c|c|c|c|c|c|c|c|c|c|}
\hline \multirow[b]{2}{*}{ State/Area } & \multicolumn{5}{|c|}{ Cases } & \multicolumn{5}{|c|}{ Rates per 100,000 Population } \\
\hline & 2013 & 2014 & 2015 & 2016 & 2017 & 2013 & 2014 & 2015 & 2016 & 2017 \\
\hline Alabama & 292 & 167 & 197 & 232 & 347 & 6.0 & 3.4 & 4.1 & 4.8 & 7.1 \\
\hline Alaska & 3 & 5 & 3 & 3 & 6 & 0.4 & 0.7 & 0.4 & 0.4 & 0.8 \\
\hline Arizona & 455 & 558 & 532 & 680 & 829 & 6.9 & 8.3 & 7.8 & 9.8 & 12.0 \\
\hline Arkansas & 175 & 110 & 145 & 131 & 156 & 5.9 & 3.7 & 4.9 & 4.4 & 5.2 \\
\hline California & 3,539 & 4,110 & 4,966 & 6,216 & 7,787 & 9.2 & 10.6 & 12.7 & 15.8 & 19.8 \\
\hline Colorado & 117 & 5 & 96 & 211 & 240 & 2.2 & 0.1 & 1.8 & 3.8 & 4.3 \\
\hline Connecticut & 22 & 21 & 30 & 23 & 28 & 0.6 & 0.6 & 0.8 & 0.6 & 0.8 \\
\hline Delaware & 63 & 30 & 21 & 34 & 88 & 6.8 & 3.2 & 2.2 & 3.6 & 9.2 \\
\hline District of Columbia & 196 & 23 & 26 & 51 & 230 & 30.3 & 3.5 & 3.9 & 7.5 & 33.8 \\
\hline Florida & 1,934 & 2,429 & 2,723 & 3,233 & 3,435 & 9.9 & 12.2 & 13.4 & 15.7 & 16.7 \\
\hline Georgia & 1,090 & 1,055 & 1,245 & 1,478 & 1,580 & 10.9 & 10.4 & 12.2 & 14.3 & 15.3 \\
\hline Hawaii & 19 & 13 & 14 & 13 & 10 & 1.4 & 0.9 & 1.0 & 0.9 & 0.7 \\
\hline Idaho & 21 & 22 & 21 & 44 & 46 & 1.3 & 1.3 & 1.3 & 2.6 & 2.7 \\
\hline Illinois & 1,031 & 1,087 & 1,285 & 1,623 & 1,399 & 8.0 & 8.4 & 10.0 & 12.7 & 10.9 \\
\hline Indiana & 171 & 170 & 189 & 197 & 211 & 2.6 & 2.6 & 2.9 & 3.0 & 3.2 \\
\hline lowa & 57 & 84 & 88 & 127 & 96 & 1.8 & 2.7 & 2.8 & 4.1 & 3.1 \\
\hline Kansas & 61 & 48 & 0 & 0 & 3 & 2.1 & 1.7 & 0.0 & 0.0 & 0.1 \\
\hline Kentucky & 102 & 117 & 123 & 159 & 218 & 2.3 & 2.7 & 2.8 & 3.6 & 4.9 \\
\hline Louisiana & 1,267 & 1,180 & 1,277 & 1,233 & 1,495 & 27.4 & 25.4 & 27.3 & 26.3 & 31.9 \\
\hline Maine & 5 & 0 & 0 & 16 & 31 & 0.4 & 0.0 & 0.0 & 1.2 & 2.3 \\
\hline Maryland & 504 & 481 & 749 & 719 & 783 & 8.5 & 8.0 & 12.5 & 12.0 & 13.0 \\
\hline Massachusetts & 276 & 227 & 486 & 416 & 387 & 4.1 & 3.4 & 7.2 & 6.1 & 5.7 \\
\hline Michigan & 368 & 416 & 393 & 424 & 447 & 3.7 & 4.2 & 4.0 & 4.3 & 4.5 \\
\hline Minnesota & 209 & 215 & 220 & 289 & 327 & 3.9 & 3.9 & 4.0 & 5.2 & 5.9 \\
\hline Mississippi & 31 & 116 & 136 & 107 & 71 & 1.0 & 3.9 & 4.5 & 3.6 & 2.4 \\
\hline Missouri & 135 & 178 & 220 & 271 & 397 & 2.2 & 2.9 & 3.6 & 4.4 & 6.5 \\
\hline Montana & 1 & 0 & 2 & 4 & 13 & 0.1 & 0.0 & 0.2 & 0.4 & 1.2 \\
\hline Nebraska & 40 & 26 & 31 & 34 & 48 & 2.1 & 1.4 & 1.6 & 1.8 & 2.5 \\
\hline Nevada & 84 & 142 & 133 & 347 & 575 & 3.0 & 5.0 & 4.6 & 11.8 & 19.6 \\
\hline New Hampshire & 30 & 21 & 28 & 27 & 29 & 2.3 & 1.6 & 2.1 & 2.0 & 2.2 \\
\hline New Jersey & 196 & 263 & 220 & 381 & 489 & 2.2 & 2.9 & 2.5 & 4.3 & 5.5 \\
\hline New Mexico & 100 & 80 & 141 & 160 & 196 & 4.8 & 3.8 & 6.8 & 7.7 & 9.4 \\
\hline New York & 2,758 & 3,073 & 2,975 & 3,484 & 3,592 & 14.0 & 15.6 & 15.0 & 17.6 & 18.2 \\
\hline North Carolina & 509 & 791 & 783 & 756 & 1,015 & 5.2 & 8.0 & 7.8 & 7.5 & 10.0 \\
\hline North Dakota & 11 & 16 & 14 & 16 & 22 & 1.5 & 2.2 & 1.8 & 2.1 & 2.9 \\
\hline Ohio & 431 & 381 & 445 & 483 & 596 & 3.7 & 3.3 & 3.8 & 4.2 & 5.1 \\
\hline Oklahoma & 28 & 59 & 83 & 90 & 95 & 0.7 & 1.5 & 2.1 & 2.3 & 2.4 \\
\hline Oregon & 133 & 159 & 218 & 227 & 283 & 3.4 & 4.0 & 5.4 & 5.5 & 6.9 \\
\hline Pennsylvania & 431 & 346 & 356 & 295 & 335 & 3.4 & 2.7 & 2.8 & 2.3 & 2.6 \\
\hline Rhode Island & 27 & 40 & 48 & 81 & 79 & 2.6 & 3.8 & 4.5 & 7.7 & 7.5 \\
\hline South Carolina & 66 & 28 & 41 & 36 & 40 & 1.4 & 0.6 & 0.8 & 0.7 & 0.8 \\
\hline South Dakota & 12 & 16 & 21 & 15 & 20 & 1.4 & 1.9 & 2.4 & 1.7 & 2.3 \\
\hline Tennessee & 497 & 502 & 575 & 713 & 543 & 7.7 & 7.7 & 8.7 & 10.7 & 8.2 \\
\hline Texas & 3,593 & 4,110 & 4,047 & 4,666 & 6,035 & 13.6 & 15.2 & 14.7 & 16.7 & 21.7 \\
\hline Utah & 51 & 61 & 73 & 106 & 97 & 1.8 & 2.1 & 2.4 & 3.5 & 3.2 \\
\hline Vermont & 5 & 0 & 0 & 0 & 0 & 0.8 & 0.0 & 0.0 & 0.0 & 0.0 \\
\hline Virginia & 329 & 137 & 276 & 235 & 551 & 4.0 & 1.6 & 3.3 & 2.8 & 6.6 \\
\hline Washington & 223 & 310 & 366 & 400 & 480 & 3.2 & 4.4 & 5.1 & 5.5 & 6.6 \\
\hline West Virginia & 14 & 4 & 17 & 45 & 25 & 0.8 & 0.2 & 0.9 & 2.5 & 1.4 \\
\hline Wisconsin & 100 & 108 & 88 & 140 & 176 & 1.7 & 1.9 & 1.5 & 2.4 & 3.0 \\
\hline Wyoming & 7 & 1 & 4 & 5 & 11 & 1.2 & 0.2 & 0.7 & 0.9 & 1.9 \\
\hline U.S.TOTAL & 21,819 & 23,541 & 26,170 & 30,676 & 35,992 & 6.9 & 7.4 & 8.1 & 9.5 & 11.1 \\
\hline Northeast & 3,750 & 3,991 & 4,143 & 4,723 & 4,970 & 6.7 & 7.1 & 7.4 & 8.4 & 8.8 \\
\hline Midwest & 2,626 & 2,745 & 2,994 & 3,619 & 3,742 & 3.9 & 4.1 & 4.4 & 5.3 & 5.5 \\
\hline South & 10,690 & 11,339 & 12,464 & 13,918 & 16,707 & 9.0 & 9.5 & 10.3 & 11.4 & 13.7 \\
\hline West & 4,753 & 5,466 & 6,569 & 8,416 & 10,573 & 6.4 & 7.3 & 8.6 & 11.0 & 13.8 \\
\hline Guam & 14 & 5 & 16 & 10 & 5 & 8.7 & 3.1 & 9.9 & 6.0 & 3.0 \\
\hline Puerto Rico & 154 & 101 & 166 & 117 & 110 & 4.3 & 2.8 & 4.8 & 3.4 & 3.2 \\
\hline Virgin Islands & 5 & 4 & 10 & 0 & 0 & 4.8 & 3.8 & 9.7 & 0.0 & 0.0 \\
\hline OUTLYING AREAS & 173 & 110 & 192 & 127 & 115 & 4.5 & 2.9 & 5.1 & 3.4 & 3.1 \\
\hline TOTAL & 21,992 & 23,651 & 26,362 & 30,803 & 36,107 & 6.9 & 7.3 & 8.1 & 9.4 & 11.0 \\
\hline
\end{tabular}

* Late and late latent syphilis includes late latent syphilis, latent syphilis of unknown duration, and late syphilis with clinical manifestations (including late benign syphilis and cardiovascular syphilis).

NOTE: See Section A1.11 in the Appendix for more information on interpreting case counts and rates in outlying areas. 
Table 39. Late and Late Latent Syphilis* - Reported Cases and Rates of Reported Cases in Selected Metropolitan Statistical Areas (MSAs) ${ }^{\dagger}$ in Alphabetical Order, United States, 2013-2017

\begin{tabular}{|c|c|c|c|c|c|c|c|c|c|c|}
\hline \multirow[b]{2}{*}{ MSAs } & \multicolumn{5}{|c|}{ Cases } & \multicolumn{5}{|c|}{ Rates per 100,000 Population } \\
\hline & 2013 & 2014 & 2015 & 2016 & 2017 & 2013 & 2014 & 2015 & 2016 & 2017 \\
\hline Atlanta-Sandy Springs-Roswell, GA & 782 & 804 & $927^{\S}$ & 1,135 & 1,146 & 14.2 & 14.3 & $16.2^{\S}$ & 19.6 & 19.8 \\
\hline Austin-Round Rock, TX & 134 & 246 & 175 & 212 & 226 & 7.1 & 12.7 & 8.7 & 10.3 & 11.0 \\
\hline Baltimore-Columbia-Towson, MD & 218 & 226 & 316 & 327 & 389 & 7.9 & 8.1 & 11.3 & 11.7 & 13.9 \\
\hline Birmingham-Hoover, AL & 96 & 53 & 62 & $57^{\ddagger}$ & 68 & 8.4 & 4.6 & 5.4 & $5.0^{\ddagger}$ & 5.9 \\
\hline Boston-Cambridge-Newton, MA-NH & 211 & $159^{\S}$ & $319^{\S}$ & $271^{\S}$ & 266 & 4.5 & $3.4^{5}$ & $6.7^{5}$ & $5.7^{\S}$ & 5.5 \\
\hline Buffalo-Cheektowaga-Niagara Falls, NY & 62 & 67 & 53 & 54 & 83 & 5.5 & 5.9 & 4.7 & 4.8 & 7.3 \\
\hline Charlotte-Concord-Gastonia, NC-SC & 151 & 183 & 191 & 201 & 252 & 6.5 & 7.7 & 7.9 & 8.1 & 10.2 \\
\hline Chicago-Naperville-Elgin, IL-IN-WI & 963 & 988 & 1,169 & 1,529 & 1,288 & 10.1 & 10.3 & 12.2 & 16.1 & 13.5 \\
\hline Cincinnati, OH-KY-IN & 191 & 124 & 129 & 105 & 136 & 8.9 & 5.8 & 6.0 & 4.8 & 6.3 \\
\hline Cleveland-Elyria, $\mathrm{OH}$ & 64 & 88 & 120 & 159 & 176 & 3.1 & 4.3 & 5.8 & 7.7 & 8.6 \\
\hline Columbus, $\mathrm{OH}$ & 98 & 101 & 106 & 109 & 155 & 5.0 & 5.1 & 5.2 & 5.3 & 7.6 \\
\hline Dallas-Fort Worth-Arlington, TX & 1,081 & 1,065 & 837 & 1,069 & 1,854 & 15.9 & 15.3 & 11.8 & 14.8 & 25.6 \\
\hline Denver-Aurora-Lakewood, CO & 81 & 0 & 59 & 150 & 185 & 3.0 & 0.0 & 2.1 & 5.3 & 6.5 \\
\hline Detroit-Warren-Dearborn, MI & 277 & 312 & 291 & 295 & 312 & 6.4 & 7.3 & 6.8 & 6.9 & 7.3 \\
\hline Hartford-West Hartford-East Hartford, CT & 10 & 10 & 8 & 3 & 3 & 0.8 & 0.8 & 0.7 & 0.2 & 0.2 \\
\hline Houston-The Woodlands-Sugar Land, TX & 1,154 & 1,430 & 1,592 & 1,805 & 2,060 & 18.3 & 22.0 & 23.9 & 26.7 & 30.4 \\
\hline Indianapolis-Carmel-Anderson, IN & 90 & 83 & 93 & 87 & 106 & 4.6 & 4.2 & 4.7 & 4.3 & 5.3 \\
\hline Jacksonville, FL & 75 & 128 & 179 & 181 & 249 & 5.4 & 9.0 & 12.3 & 12.2 & 16.8 \\
\hline Kansas City, MO-KS & 54 & 54 & 40 & 87 & 92 & 2.6 & 2.6 & 1.9 & 4.1 & 4.4 \\
\hline Las Vegas-Henderson-Paradise, NV & 54 & 133 & 102 & 315 & 513 & 2.7 & 6.4 & 4.8 & 14.6 & 23.8 \\
\hline Los Angeles-Long Beach-Anaheim, CA & 1,705 & 1,679 & 1,902 & 2,532 & 3,164 & 13.0 & 12.7 & 14.3 & 19.0 & 23.8 \\
\hline Louisville-Jefferson County, KY-IN & 52 & 70 & 92 & 112 & 127 & 4.1 & 5.5 & 7.2 & 8.7 & 9.9 \\
\hline Memphis, TN-MS-AR & 283 & 236 & 256 & 336 & 257 & 21.1 & 17.6 & 19.0 & 25.0 & 19.1 \\
\hline Miami-Fort Lauderdale-West Palm Beach, FL & 1,075 & 1,371 & 1,524 & 1,868 & 1,745 & 18.4 & 23.1 & 25.3 & 30.8 & 28.8 \\
\hline Milwaukee-Waukesha-West Allis, WI & 56 & 63 & 43 & 70 & 84 & 3.6 & 4.0 & 2.7 & 4.5 & 5.3 \\
\hline Minneapolis-St. Paul-Bloomington, MN-WI & 175 & 187 & 192 & 251 & 261 & 5.1 & 5.4 & 5.4 & 7.1 & 7.3 \\
\hline Nashville-Davidson-Murfreesboro-Franklin, TN & 120 & 148 & 161 & 196 & 101 & 6.8 & 8.3 & 8.8 & 10.5 & 5.4 \\
\hline New Orleans-Metairie, LA & 442 & 383 & 370 & 363 & 391 & 35.6 & 30.6 & 29.3 & 28.6 & 30.8 \\
\hline New York-Newark-Jersey City, NY-NJ-PA & 2,707 & 3,057 & 2,915 & 3,534 & 3,687 & 13.6 & 15.2 & 14.4 & 17.5 & 18.3 \\
\hline Oklahoma City, OK & 11 & 31 & 32 & 47 & 42 & 0.8 & 2.3 & 2.4 & 3.4 & 3.1 \\
\hline Orlando-Kissimmee-Sanford, FL & 250 & 364 & 344 & 416 & 509 & 11.0 & 15.7 & 14.4 & 17.0 & 20.8 \\
\hline Philadelphia-Camden-Wilmington, PA-NJ-DE-MD & 439 & 311 & 314 & 266 & 342 & 7.3 & 5.1 & 5.2 & 4.4 & 5.6 \\
\hline Phoenix-Mesa-Scottsdale, AZ & 335 & 397 & 394 & 534 & 645 & 7.6 & 8.8 & 8.6 & 11.5 & 13.8 \\
\hline Pittsburgh, PA & 11 & 13 & 11 & 11 & 12 & 0.5 & 0.6 & 0.5 & 0.5 & 0.5 \\
\hline Portland-Vancouver-Hillsboro, OR-WA & 118 & 143 & 171 & 173 & 207 & 5.1 & 6.1 & 7.2 & 7.1 & 8.5 \\
\hline Providence-Warwick, RI-MA & 45 & $48^{5}$ & $74^{\S}$ & $102^{\S}$ & $105^{\varsigma}$ & 2.8 & $3.0^{5}$ & $4.6^{5}$ & $6.3^{\S}$ & $6.5^{\S}$ \\
\hline Raleigh, NC & 68 & 111 & 125 & 109 & 169 & 5.6 & 8.9 & 9.8 & 8.4 & 13.0 \\
\hline Richmond, VA & 58 & 9 & 36 & 35 & 111 & 4.7 & 0.7 & 2.8 & 2.7 & 8.7 \\
\hline Riverside-San Bernardino-Ontario, CA & 433 & 432 & 508 & 707 & 911 & 9.9 & 9.7 & 11.3 & 15.6 & 20.1 \\
\hline Sacramento-Roseville-Arden-Arcade, CA & 107 & 134 & 205 & 199 & 264 & 4.8 & 6.0 & 9.0 & 8.7 & 11.5 \\
\hline Salt Lake City, UT & 34 & 39 & 50 & 76 & 61 & 3.0 & 3.4 & 4.3 & 6.4 & 5.1 \\
\hline San Antonio-New Braunfels, TX & 457 & 448 & 483 & 531 & 612 & 20.1 & 19.2 & 20.3 & 21.9 & 25.2 \\
\hline San Diego-Carlsbad, CA & 245 & 310 & 367 & 425 & 576 & 7.6 & 9.5 & 11.1 & 12.8 & 17.4 \\
\hline San Francisco-Oakland-Hayward, CA & 421 & 502 & 553 & 629 & 691 & 9.3 & 10.9 & 11.9 & 13.4 & 14.8 \\
\hline San Jose-Sunnyvale-Santa Clara, CA & 69 & 125 & 129 & 145 & 237 & 3.6 & 6.4 & 6.5 & 7.3 & 12.0 \\
\hline Seattle-Tacoma-Bellevue, WA & 161 & 211 & 224 & 268 & 321 & 4.5 & 5.7 & 6.0 & 7.1 & 8.4 \\
\hline St. Louis, MO-IL & 104 & 119 & 165 & 160 & 254 & 3.7 & 4.2 & 5.9 & 5.7 & 9.0 \\
\hline Tampa-St. Petersburg-Clearwater, FL & 227 & 255 & 299 & 326 & 392 & 7.9 & 8.7 & 10.0 & 10.8 & 12.9 \\
\hline Virginia Beach-Norfolk-Newport News, VA-NC & 86 & 45 & 90 & 65 & 122 & 5.0 & 2.6 & 5.2 & 3.8 & 7.1 \\
\hline Washington-Arlington-Alexandria, DC-VA-MD-WV & 599 & 295 & 501 & 474 & 827 & 10.1 & 4.9 & 8.2 & 7.7 & 13.5 \\
\hline SELECTED MSAs TOTAL & 16,739 & 17,790 & 19,298 & 23,111 & 26,786 & 9.7 & 10.2 & 10.9 & 13.0 & 15.1 \\
\hline
\end{tabular}

* Late and late latent syphilis includes late latent syphilis, latent syphilis of unknown duration, and late syphilis with clinical manifestations (including late benign syphilis and cardiovascular syphilis).

${ }^{+}$MSAs were selected on the basis of the largest population in the 2010 U.S. Census.

₹ 2016 county data for Alabama have been corrected and may not match previous reports.

${ }^{\S}$ The variable used to identify county, which is used to classify cases into MSAs, was complete for $\leq 95 \%$ of cases in a state contributing data to this MSA. See Appendix A1.4 for more information. 
Table 40. Congenital Syphilis - Reported Cases and Rates of Reported Cases by State ${ }^{\dagger}$, Ranked by Rates, United States, 2017

\begin{tabular}{|c|c|c|c|}
\hline Rank* & State $^{\dagger}$ & Cases & Rate per 100,000 Live Births \\
\hline 1 & Louisiana & 59 & 93.4 \\
\hline 2 & Nevada & 21 & 57.9 \\
\hline 3 & California & 281 & 57.5 \\
\hline 4 & Texas & 176 & 44.2 \\
\hline 5 & Florida & 93 & 41.3 \\
\hline 6 & Arizona & 30 & 35.5 \\
\hline \multirow[t]{2}{*}{7} & Maryland & 20 & 27.3 \\
\hline & U.S. TOTAL ${ }^{\ddagger}$ & 918 & 23.3 \\
\hline 8 & Arkansas & 8 & 20.9 \\
\hline 9 & North Carolina & 23 & 19.0 \\
\hline 10 & Georgia & 23 & 17.7 \\
\hline 11 & Oregon & 8 & 17.6 \\
\hline 12 & Hawaii & 3 & 16.6 \\
\hline 13 & South Carolina & 8 & 14.0 \\
\hline 14 & Illinois & 21 & 13.6 \\
\hline 15 & Missouri & 10 & 13.4 \\
\hline 16 & Oklahoma & 7 & 13.3 \\
\hline 17 & Ohio & 18 & 13.0 \\
\hline 18 & New Jersey & 13 & 12.7 \\
\hline 19 & Tennessee & 10 & 12.4 \\
\hline 20 & Virginia & 11 & 10.7 \\
\hline 21 & West Virginia & 2 & 10.5 \\
\hline 22 & Alabama & 6 & 10.1 \\
\hline \multirow[t]{2}{*}{23} & Indiana & 8 & 9.6 \\
\hline & HP 2020 TARGET & & 9.6 \\
\hline 24 & Kentucky & 5 & 9.0 \\
\hline 25 & Michigan & 10 & 8.8 \\
\hline 26 & South Dakota & 1 & 8.1 \\
\hline 27 & Montana & 1 & 8.1 \\
\hline 28 & New York & 16 & 6.8 \\
\hline 29 & Washington & 6 & 6.6 \\
\hline 30 & Colorado & 4 & 6.0 \\
\hline 31 & lowa & 2 & 5.1 \\
\hline 32 & Wisconsin & 3 & 4.5 \\
\hline 33 & Pennsylvania & 6 & 4.3 \\
\hline 34 & New Mexico & 1 & 4.0 \\
\hline 35 & Nebraska & 1 & 3.8 \\
\hline 36 & Minnesota & 2 & 2.9 \\
\hline \multirow[t]{14}{*}{37} & Mississippi & 1 & 2.6 \\
\hline & Alaska & 0 & 0.0 \\
\hline & Connecticut & 0 & 0.0 \\
\hline & Delaware & 0 & 0.0 \\
\hline & Idaho & 0 & 0.0 \\
\hline & Kansas & 0 & 0.0 \\
\hline & Maine & 0 & 0.0 \\
\hline & Massachusetts & 0 & 0.0 \\
\hline & New Hampshire & 0 & 0.0 \\
\hline & North Dakota & 0 & 0.0 \\
\hline & Rhode Island & 0 & 0.0 \\
\hline & Utah & 0 & 0.0 \\
\hline & Vermont & 0 & 0.0 \\
\hline & Wyoming & 0 & 0.0 \\
\hline
\end{tabular}

* States were ranked by rate, then by case count, then in alphabetical order, with rates shown rounded to the nearest tenth.

${ }^{\dagger}$ Mother's state of residence was used to assign case.

₹ Total includes cases reported by the District of Columbia with 0 cases and a rate of 0.0 , but excludes outlying areas (Guam with 0 cases and rate of 0.0 , Puerto Rico with 4 cases and rate of 14.2, and Virgin Islands with 0 cases and rate of 0.0 ). 
Table 41. Congenital Syphilis - Reported Cases and Rates of Reported Cases by Year of Birth, State/Area* and Region in Alphabetical Order, United States and Outlying Areas, 2013-2017

\begin{tabular}{|c|c|c|c|c|c|c|c|c|c|c|}
\hline \multirow[b]{2}{*}{ State/Area* } & \multicolumn{5}{|c|}{ Cases } & \multicolumn{5}{|c|}{ Rates per 100,000 Live Births } \\
\hline & 2013 & 2014 & 2015 & 2016 & 2017 & 2013 & 2014 & 2015 & 2016 & 2017 \\
\hline Alabama & 2 & 3 & 3 & 4 & 6 & 3.4 & 5.0 & 5.0 & 6.8 & 10.1 \\
\hline Alaska & 1 & 0 & 0 & 0 & 0 & 8.7 & 0.0 & 0.0 & 0.0 & 0.0 \\
\hline Arizona & 14 & 13 & 14 & 16 & 30 & 16.4 & 15.0 & 16.4 & 18.9 & 35.5 \\
\hline Arkansas & 12 & 7 & 5 & 6 & 8 & 31.7 & 18.2 & 12.9 & 15.7 & 20.9 \\
\hline California & 58 & 102 & 140 & 207 & 281 & 11.7 & 20.3 & 28.5 & 42.3 & 57.5 \\
\hline Colorado & 0 & 0 & 0 & 4 & 4 & 0.0 & 0.0 & 0.0 & 6.0 & 6.0 \\
\hline Connecticut & 0 & 0 & 1 & 0 & 0 & 0.0 & 0.0 & 2.8 & 0.0 & 0.0 \\
\hline Delaware & 1 & 0 & 1 & 0 & 0 & 9.2 & 0.0 & 9.0 & 0.0 & 0.0 \\
\hline District of Columbia & 2 & 0 & 1 & 1 & 0 & 21.5 & 0.0 & 10.4 & 10.1 & 0.0 \\
\hline Florida & 35 & 48 & 38 & 60 & 93 & 16.2 & 21.8 & 16.9 & 26.7 & 41.3 \\
\hline Georgia & 20 & 17 & 21 & 21 & 23 & 15.5 & 13.0 & 16.0 & 16.1 & 17.7 \\
\hline Hawaii & 0 & 0 & 2 & 1 & 3 & 0.0 & 0.0 & 10.9 & 5.5 & 16.6 \\
\hline Idaho & 0 & 0 & 0 & 0 & 0 & 0.0 & 0.0 & 0.0 & 0.0 & 0.0 \\
\hline Illinois & 23 & 27 & 31 & 18 & 21 & 14.7 & 17.0 & 19.6 & 11.7 & 13.6 \\
\hline Indiana & 0 & 8 & 5 & 8 & 8 & 0.0 & 9.5 & 5.9 & 9.6 & 9.6 \\
\hline lowa & 0 & 1 & 0 & 1 & 2 & 0.0 & 2.5 & 0.0 & 2.5 & 5.1 \\
\hline Kansas & 0 & 0 & 0 & 1 & 0 & 0.0 & 0.0 & 0.0 & 2.6 & 0.0 \\
\hline Kentucky & 4 & 3 & 1 & 5 & 5 & 7.2 & 5.3 & 1.8 & 9.0 & 9.0 \\
\hline Louisiana & 40 & 46 & 54 & 48 & 59 & 63.3 & 71.3 & 83.5 & 76.0 & 93.4 \\
\hline Maine & 0 & 0 & 0 & 0 & 0 & 0.0 & 0.0 & 0.0 & 0.0 & 0.0 \\
\hline Maryland & 14 & 16 & 18 & 16 & 20 & 19.5 & 21.6 & 24.5 & 21.9 & 27.3 \\
\hline Massachusetts & 4 & 3 & 4 & 3 & 0 & 5.6 & 4.2 & 5.6 & 4.2 & 0.0 \\
\hline Michigan & 9 & 15 & 11 & 13 & 10 & 7.9 & 13.1 & 9.7 & 11.5 & 8.8 \\
\hline Minnesota & 0 & 0 & 2 & 7 & 2 & 0.0 & 0.0 & 2.9 & 10.0 & 2.9 \\
\hline Mississippi & 0 & 1 & 0 & 2 & 1 & 0.0 & 2.6 & 0.0 & 5.3 & 2.6 \\
\hline Missouri & 3 & 1 & 4 & 8 & 10 & 4.0 & 1.3 & 5.3 & 10.7 & 13.4 \\
\hline Montana & 0 & 0 & 0 & 0 & 1 & 0.0 & 0.0 & 0.0 & 0.0 & 8.1 \\
\hline Nebraska & 0 & 1 & 0 & 1 & 1 & 0.0 & 3.7 & 0.0 & 3.8 & 3.8 \\
\hline Nevada & 2 & 5 & 8 & 12 & 21 & 5.7 & 13.9 & 22.0 & 33.1 & 57.9 \\
\hline New Hampshire & 0 & 0 & 0 & 0 & 0 & 0.0 & 0.0 & 0.0 & 0.0 & 0.0 \\
\hline New Jersey & 0 & 0 & 0 & 12 & 13 & 0.0 & 0.0 & 0.0 & 11.7 & 12.7 \\
\hline New Mexico & 2 & 1 & 2 & 3 & 1 & 7.6 & 3.8 & 7.7 & 12.1 & 4.0 \\
\hline New York & 11 & 22 & 12 & 13 & 16 & 4.6 & 9.2 & 5.1 & 5.5 & 6.8 \\
\hline North Carolina & 4 & 6 & 9 & 18 & 23 & 3.4 & 5.0 & 7.4 & 14.9 & 19.0 \\
\hline North Dakota & 0 & 0 & 0 & 0 & 0 & 0.0 & 0.0 & 0.0 & 0.0 & 0.0 \\
\hline Ohio & 18 & 15 & 17 & 12 & 18 & 13.0 & 10.8 & 12.2 & 8.7 & 13.0 \\
\hline Oklahoma & 0 & 6 & 7 & 3 & 7 & 0.0 & 11.2 & 13.2 & 5.7 & 13.3 \\
\hline Oregon & 0 & 2 & 6 & 6 & 8 & 0.0 & 4.4 & 13.1 & 13.2 & 17.6 \\
\hline Pennsylvania & 3 & 4 & 7 & 5 & 6 & 2.1 & 2.8 & 5.0 & 3.6 & 4.3 \\
\hline Rhode Island & 0 & 0 & 0 & 0 & 0 & 0.0 & 0.0 & 0.0 & 0.0 & 0.0 \\
\hline South Carolina & 1 & 5 & 3 & 9 & 8 & 1.8 & 8.7 & 5.2 & 15.7 & 14.0 \\
\hline South Dakota & 0 & 3 & 0 & 2 & 1 & 0.0 & 24.4 & 0.0 & 16.3 & 8.1 \\
\hline Tennessee & 2 & 2 & 5 & 8 & 10 & 2.5 & 2.5 & 6.1 & 9.9 & 12.4 \\
\hline Texas & 74 & 75 & 52 & 71 & 176 & 19.1 & 18.8 & 12.9 & 17.8 & 44.2 \\
\hline Utah & 0 & 0 & 0 & 0 & 0 & 0.0 & 0.0 & 0.0 & 0.0 & 0.0 \\
\hline Vermont & 0 & 0 & 0 & 0 & 0 & 0.0 & 0.0 & 0.0 & 0.0 & 0.0 \\
\hline Virginia & 3 & 2 & 3 & 8 & 11 & 2.9 & 1.9 & 2.9 & 7.8 & 10.7 \\
\hline Washington & 0 & 2 & 5 & 3 & 6 & 0.0 & 2.3 & 5.6 & 3.3 & 6.6 \\
\hline West Virginia & 0 & 0 & 0 & 2 & 2 & 0.0 & 0.0 & 0.0 & 10.5 & 10.5 \\
\hline Wisconsin & 0 & 0 & 0 & 1 & 3 & 0.0 & 0.0 & 0.0 & 1.5 & 4.5 \\
\hline Wyoming & 0 & 0 & 0 & 0 & 0 & 0.0 & 0.0 & 0.0 & 0.0 & 0.0 \\
\hline U.S. TOTAL & 362 & 462 & 492 & 639 & 918 & 9.2 & 11.6 & 12.4 & 16.2 & 23.3 \\
\hline Northeast & 18 & 29 & 24 & 33 & 35 & 2.9 & 4.6 & 3.8 & 5.3 & 5.6 \\
\hline Midwest & 53 & 71 & 70 & 72 & 76 & 6.4 & 8.5 & 8.4 & 8.7 & 9.2 \\
\hline South & 214 & 237 & 221 & 282 & 452 & 14.2 & 15.4 & 14.3 & 18.4 & 29.5 \\
\hline West & 77 & 125 & 177 & 252 & 355 & 8.0 & 12.8 & 18.4 & 26.3 & 37.0 \\
\hline Guam & 1 & 0 & 2 & 0 & 0 & 30.4 & 0.0 & 59.4 & 0.0 & 0.0 \\
\hline Puerto Rico & 2 & 0 & 5 & 5 & 4 & 5.5 & 0.0 & 16.0 & 17.7 & 14.2 \\
\hline Virgin Islands & 0 & 0 & 0 & 0 & 0 & 0.0 & 0.0 & 0.0 & 0.0 & 0.0 \\
\hline OUTLYING AREAS & 3 & 0 & 7 & 5 & 4 & 7.3 & 0.0 & 19.5 & 15.2 & 12.2 \\
\hline TOTAL & 365 & 462 & 499 & 644 & 922 & 9.2 & 11.5 & 12.4 & 16.2 & 23.2 \\
\hline
\end{tabular}

* Mother's state of residence was used to assign case.

NOTE: See Section A1.11 in the Appendix for more information on interpreting case counts and rates in outlying areas. 
Table 42. Congenital Syphilis - Reported Cases and Rates of Reported Cases per 100,000 Live Births by Year of Birth and Race/Hispanic Ethnicity of Mother, United States, 2013-2017

\begin{tabular}{|c|c|c|c|c|c|c|c|c|c|c|c|c|c|c|c|c|}
\hline \multirow[b]{2}{*}{ Year of Birth } & \multicolumn{2}{|c|}{ Whites } & \multicolumn{2}{|c|}{ Blacks } & \multicolumn{2}{|c|}{ Hispanics } & \multicolumn{2}{|c|}{$\begin{array}{c}\text { Asians/ } \\
\text { Pacific } \\
\text { Islanders }\end{array}$} & \multicolumn{2}{|c|}{$\begin{array}{c}\text { American } \\
\text { Indians/ } \\
\text { Alaska Natives }\end{array}$} & \multicolumn{2}{|c|}{ Other } & \multicolumn{2}{|c|}{ Unknown } & \multicolumn{2}{|c|}{ Total } \\
\hline & Cases & Rates & Cases & Rates & Cases & Rates & Cases & Rates & Cases & Rates & Cases & Rates & Cases & Rates & Cases & Rates \\
\hline 2013 & 62 & 2.9 & 185 & 31.4 & 93 & 10.3 & 9 & 3.5 & 5 & 12.8 & 3 & NA & 5 & NA & 362 & 9.2 \\
\hline 2014 & 79 & 3.6 & 227 & 38.2 & 112 & 12.3 & 19 & 7.0 & 5 & 13.2 & 9 & NA & 11 & NA & 462 & 11.6 \\
\hline 2015 & 97 & 4.5 & 207 & 34.8 & 143 & 15.5 & 15 & 5.5 & 4 & 10.7 & 7 & NA & 19 & NA & 492 & 12.4 \\
\hline 2016 & 119 & 5.6 & 264 & 44.8 & 189 & 20.6 & 25 & 8.9 & 12 & 32.8 & 8 & NA & 22 & NA & 639 & 16.2 \\
\hline 2017 & 206 & 9.7 & 347 & 58.9 & 308 & 33.5 & 12 & 4.3 & 13 & 35.5 & 12 & NA & 20 & NA & 918 & 23.3 \\
\hline
\end{tabular}

$\mathrm{NA}=$ Not applicable. 
Table 43. Chancroid - Reported Cases and Rates of Reported Cases by State/Area in Alphabetical Order, United States and Outlying Areas, 2013-2017

\begin{tabular}{|c|c|c|c|c|c|c|c|c|c|c|}
\hline \multirow[b]{2}{*}{ State/Area } & \multicolumn{5}{|c|}{ Cases } & \multicolumn{5}{|c|}{ Rates per 100,000 Population } \\
\hline & 2013 & 2014 & 2015 & 2016 & 2017 & 2013 & 2014 & 2015 & 2016 & 2017 \\
\hline Alabama & 1 & 0 & 0 & 1 & 0 & 0.0 & 0.0 & 0.0 & 0.0 & 0.0 \\
\hline Alaska & 0 & 0 & 0 & 0 & 0 & 0.0 & 0.0 & 0.0 & 0.0 & 0.0 \\
\hline Arizona & 0 & 0 & 1 & 0 & 0 & 0.0 & 0.0 & 0.0 & 0.0 & 0.0 \\
\hline Arkansas & 0 & 0 & 0 & 0 & 0 & 0.0 & 0.0 & 0.0 & 0.0 & 0.0 \\
\hline California & 6 & 4 & 2 & 2 & 1 & 0.0 & 0.0 & 0.0 & 0.0 & 0.0 \\
\hline Colorado & 0 & 0 & 0 & 1 & 0 & 0.0 & 0.0 & 0.0 & 0.0 & 0.0 \\
\hline Connecticut & 0 & 0 & 0 & 0 & 0 & 0.0 & 0.0 & 0.0 & 0.0 & 0.0 \\
\hline Delaware & 0 & 0 & 0 & 0 & 0 & 0.0 & 0.0 & 0.0 & 0.0 & 0.0 \\
\hline District of Columbia & 0 & 0 & 0 & 0 & 0 & 0.0 & 0.0 & 0.0 & 0.0 & 0.0 \\
\hline Florida & 0 & 0 & 0 & 0 & 0 & 0.0 & 0.0 & 0.0 & 0.0 & 0.0 \\
\hline Georgia & 0 & 0 & 0 & 0 & 0 & 0.0 & 0.0 & 0.0 & 0.0 & 0.0 \\
\hline Hawaii & 0 & 0 & 0 & 0 & 0 & 0.0 & 0.0 & 0.0 & 0.0 & 0.0 \\
\hline Idaho & 0 & 0 & 0 & 0 & 0 & 0.0 & 0.0 & 0.0 & 0.0 & 0.0 \\
\hline Illinois & 0 & 0 & 0 & 0 & 0 & 0.0 & 0.0 & 0.0 & 0.0 & 0.0 \\
\hline Indiana & 0 & 0 & 1 & 0 & 0 & 0.0 & 0.0 & 0.0 & 0.0 & 0.0 \\
\hline lowa & 0 & 0 & 0 & 0 & 0 & 0.0 & 0.0 & 0.0 & 0.0 & 0.0 \\
\hline Kansas & 0 & 0 & 0 & 0 & 0 & 0.0 & 0.0 & 0.0 & 0.0 & 0.0 \\
\hline Kentucky & 0 & 0 & 0 & 0 & 0 & 0.0 & 0.0 & 0.0 & 0.0 & 0.0 \\
\hline Louisiana & 0 & 0 & 0 & 0 & 0 & 0.0 & 0.0 & 0.0 & 0.0 & 0.0 \\
\hline Maine & 0 & 0 & 0 & 0 & 0 & 0.0 & 0.0 & 0.0 & 0.0 & 0.0 \\
\hline Maryland & 0 & 0 & 0 & 0 & 0 & 0.0 & 0.0 & 0.0 & 0.0 & 0.0 \\
\hline Massachusetts & 2 & 1 & 3 & 1 & 2 & 0.0 & 0.0 & 0.0 & 0.0 & 0.0 \\
\hline Michigan & 0 & 0 & 0 & 0 & 1 & 0.0 & 0.0 & 0.0 & 0.0 & 0.0 \\
\hline Minnesota & 0 & 0 & 0 & 0 & 0 & 0.0 & 0.0 & 0.0 & 0.0 & 0.0 \\
\hline Mississippi & 0 & 0 & 0 & 0 & 0 & 0.0 & 0.0 & 0.0 & 0.0 & 0.0 \\
\hline Missouri & 0 & 0 & 0 & 0 & 0 & 0.0 & 0.0 & 0.0 & 0.0 & 0.0 \\
\hline Montana & 0 & 0 & 0 & 0 & 0 & 0.0 & 0.0 & 0.0 & 0.0 & 0.0 \\
\hline Nebraska & 0 & 0 & 0 & 0 & 0 & 0.0 & 0.0 & 0.0 & 0.0 & 0.0 \\
\hline Nevada & 0 & 0 & 0 & 0 & 0 & 0.0 & 0.0 & 0.0 & 0.0 & 0.0 \\
\hline New Hampshire & 0 & 0 & 0 & 0 & 0 & 0.0 & 0.0 & 0.0 & 0.0 & 0.0 \\
\hline New Jersey & 0 & 0 & 0 & 0 & 0 & 0.0 & 0.0 & 0.0 & 0.0 & 0.0 \\
\hline New Mexico & 0 & 0 & 0 & 0 & 0 & 0.0 & 0.0 & 0.0 & 0.0 & 0.0 \\
\hline New York & 0 & 0 & 0 & 0 & 0 & 0.0 & 0.0 & 0.0 & 0.0 & 0.0 \\
\hline North Carolina & 0 & 0 & 0 & 1 & 1 & 0.0 & 0.0 & 0.0 & 0.0 & 0.0 \\
\hline North Dakota & 0 & 0 & 0 & 0 & 0 & 0.0 & 0.0 & 0.0 & 0.0 & 0.0 \\
\hline Ohio & 0 & 0 & 0 & 0 & 0 & 0.0 & 0.0 & 0.0 & 0.0 & 0.0 \\
\hline Oklahoma & 0 & 0 & 0 & 0 & 0 & 0.0 & 0.0 & 0.0 & 0.0 & 0.0 \\
\hline Oregon & 0 & 0 & 0 & 0 & 0 & 0.0 & 0.0 & 0.0 & 0.0 & 0.0 \\
\hline Pennsylvania & 0 & 0 & 0 & 0 & 0 & 0.0 & 0.0 & 0.0 & 0.0 & 0.0 \\
\hline Rhode Island & 0 & 0 & 0 & 0 & 0 & 0.0 & 0.0 & 0.0 & 0.0 & 0.0 \\
\hline South Carolina & 0 & 0 & 0 & 1 & 0 & 0.0 & 0.0 & 0.0 & 0.0 & 0.0 \\
\hline South Dakota & 0 & 0 & 0 & 0 & 0 & 0.0 & 0.0 & 0.0 & 0.0 & 0.0 \\
\hline Tennessee & 0 & 0 & 0 & 0 & 0 & 0.0 & 0.0 & 0.0 & 0.0 & 0.0 \\
\hline Texas & 1 & 1 & 2 & 0 & 2 & 0.0 & 0.0 & 0.0 & 0.0 & 0.0 \\
\hline Utah & 0 & 0 & 0 & 0 & 0 & 0.0 & 0.0 & 0.0 & 0.0 & 0.0 \\
\hline Vermont & 0 & 0 & 0 & 0 & 0 & 0.0 & 0.0 & 0.0 & 0.0 & 0.0 \\
\hline Virginia & 0 & 0 & 0 & 0 & 0 & 0.0 & 0.0 & 0.0 & 0.0 & 0.0 \\
\hline Washington & 0 & 0 & 1 & 0 & 0 & 0.0 & 0.0 & 0.0 & 0.0 & 0.0 \\
\hline West Virginia & 0 & 0 & 0 & 0 & 0 & 0.0 & 0.0 & 0.0 & 0.0 & 0.0 \\
\hline Wisconsin & 0 & 0 & 0 & 0 & 0 & 0.0 & 0.0 & 0.0 & 0.0 & 0.0 \\
\hline Wyoming & 0 & 0 & 1 & 0 & 0 & 0.0 & 0.0 & 0.2 & 0.0 & 0.0 \\
\hline U.S.TOTAL & 10 & 6 & 11 & 7 & 7 & 0.0 & 0.0 & 0.0 & 0.0 & 0.0 \\
\hline Guam & 0 & 0 & 0 & 0 & 0 & 0.0 & 0.0 & 0.0 & 0.0 & 0.0 \\
\hline Puerto Rico & 0 & 0 & 0 & 0 & 0 & 0.0 & 0.0 & 0.0 & 0.0 & 0.0 \\
\hline Virgin Islands & 0 & 0 & 0 & 0 & 0 & 0.0 & 0.0 & 0.0 & 0.0 & 0.0 \\
\hline OUTLYING AREAS & 0 & 0 & 0 & 0 & 0 & 0.0 & 0.0 & 0.0 & 0.0 & 0.0 \\
\hline TOTAL & 10 & 6 & 11 & 7 & 7 & 0.0 & 0.0 & 0.0 & 0.0 & 0.0 \\
\hline
\end{tabular}

NOTE: See Section A1.11 in the Appendix for more information on interpreting case counts and rates in outlying areas. 
Table 44. Selected STDs and Complications - Initial Visits to Physicians' Offices, National Disease and Therapeutic Index (NDTI), United States, 1966-2016

\begin{tabular}{|c|c|c|c|}
\hline Year & $\begin{array}{c}\text { Trichomonas vaginalis } \\
\text { Infections* }\end{array}$ & $\begin{array}{c}\text { Other } \\
\text { Vaginal } \\
\text { Infections* }\end{array}$ & $\begin{array}{c}\text { Pelvic } \\
\text { Inflammatory } \\
\text { Disease }^{\dagger}\end{array}$ \\
\hline 1966 & 579,000 & $1,155,000$ & NA \\
\hline 1967 & 515,000 & $1,277,000$ & NA \\
\hline 1968 & 463,000 & $1,460,000$ & NA \\
\hline 1969 & 421,000 & $1,390,000$ & NA \\
\hline 1970 & 529,000 & $1,500,000$ & NA \\
\hline 1971 & 484,000 & $1,281,000$ & NA \\
\hline 1972 & 574,000 & $1,810,000$ & NA \\
\hline 1973 & 466,000 & $1,858,000$ & NA \\
\hline 1974 & 427,000 & $1,907,000$ & NA \\
\hline 1975 & 500,000 & $1,919,000$ & NA \\
\hline 1976 & 473,000 & $1,690,000$ & NA \\
\hline 1977 & 324,000 & $1,713,000$ & NA \\
\hline 1978 & 329,000 & $2,149,000$ & NA \\
\hline 1979 & 363,000 & $1,662,000$ & NA \\
\hline 1980 & 358,000 & $1,670,000$ & 423,000 \\
\hline 1981 & 369,000 & $1,742,000$ & 283,000 \\
\hline 1982 & 268,000 & $1,859,000$ & 374,000 \\
\hline 1983 & 424,000 & $1,932,000$ & 424,000 \\
\hline 1984 & 381,000 & $2,450,000$ & 381,000 \\
\hline 1985 & 291,000 & $2,728,000$ & 425,000 \\
\hline 1986 & 338,000 & $3,118,000$ & 457,000 \\
\hline 1987 & 293,000 & $3,087,000$ & 403,000 \\
\hline 1988 & 191,000 & $3,583,000$ & 431,000 \\
\hline 1989 & 165,000 & $3,374,000$ & 413,000 \\
\hline 1990 & 213,000 & $4,474,000$ & 358,000 \\
\hline 1991 & 198,000 & $3,822,000$ & 377,000 \\
\hline 1992 & 182,000 & $3,428,000$ & 335,000 \\
\hline 1993 & 207,000 & $3,755,000$ & 407,000 \\
\hline 1994 & 199,000 & $4,123,000$ & 332,000 \\
\hline 1995 & 141,000 & $3,927,000$ & 262,000 \\
\hline 1996 & 245,000 & $3,472,000$ & 286,000 \\
\hline 1997 & 176,000 & $3,100,000$ & 260,000 \\
\hline 1998 & 164,000 & $3,200,000$ & 233,000 \\
\hline 1999 & 171,000 & $3,077,000$ & 250,000 \\
\hline 2000 & 222,000 & $3,470,000$ & 254,000 \\
\hline 2001 & 210,000 & $3,365,000$ & 244,000 \\
\hline 2002 & 150,000 & $3,315,000$ & 197,000 \\
\hline 2003 & 179,000 & $3,516,000$ & 123,000 \\
\hline 2004 & 221,000 & $3,602,000$ & 132,000 \\
\hline 2005 & 165,000 & $4,071,000$ & 176,000 \\
\hline 2006 & 200,000 & $3,891,000$ & 106,000 \\
\hline 2007 & 205,000 & $3,723,000$ & 146,000 \\
\hline 2008 & 204,000 & $3,571,000$ & 104,000 \\
\hline 2009 & 216,000 & $3,063,000$ & 100,000 \\
\hline 2010 & 149,000 & $3,192,000$ & 113,000 \\
\hline 2011 & 168,000 & $3,102,000$ & 90,000 \\
\hline 2012 & 219,000 & $3,452,000$ & 106,000 \\
\hline 2013 & 225,000 & $3,278,000$ & 88,000 \\
\hline 2014 & 155,000 & $3,419,000$ & 51,000 \\
\hline 2015 & 139,000 & $3,215,000$ & 68,000 \\
\hline 2016 & 222,000 & $4,112,000$ & 90,000 \\
\hline
\end{tabular}

* Women only.

${ }^{+}$Women aged 15-44 years only.

NA $=$ Not available.

NOTE: Standard errors for estimates under 100,000 are not available. The relative standard errors for estimates 100,000-299,999 are from 23\% to $19 \% ; 300,000-599,999$ are from $19 \%$ to $16 \% ; 600,000-999,999$ are from $16 \%$ to $13 \%$; and $1,000,000-5,000,000$ are from $13 \%$ to $7 \%$.

SOURCE: National Disease and Therapeutic Index, IMS Health, Integrated Promotional Services. IMS Health report, 1966-2016. The 2017 data were not obtained in time to include them in this report. See Section A2.5 in the Appendix for more information. 


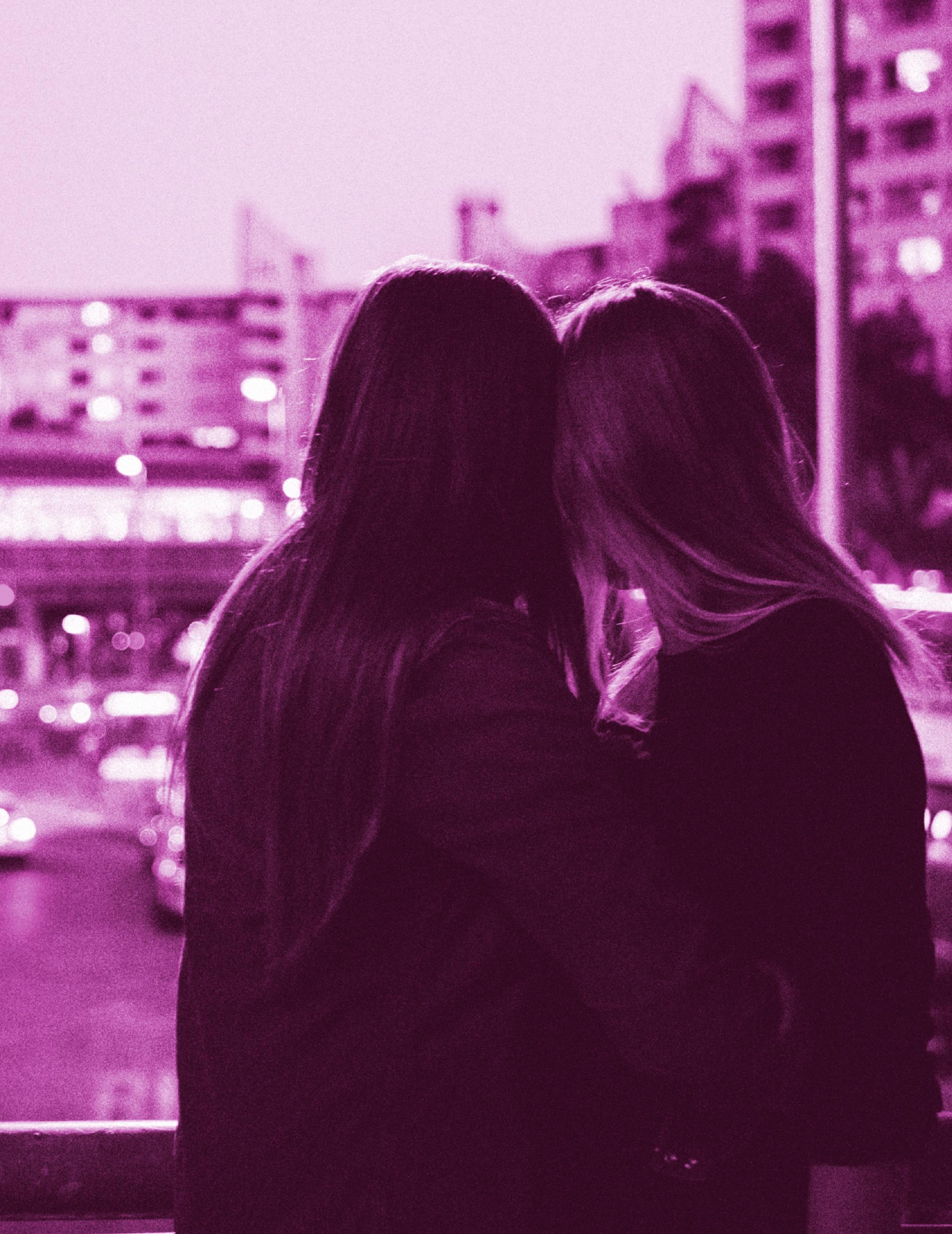




\section{Summary Report Forms}

The following hard copy forms were used to report national STD morbidity data:

1. FORM CDC 73.998: Monthly Surveillance Report of Early Syphilis. This monthly hard copy reporting form was used during 1984-2002 to report summary data for primary and secondary (P\&S) syphilis and early latent syphilis by county and state.

2. FORM CDC 73.688: Sexually Transmitted Disease Morbidity Report. This quarterly hard copy reporting form was used during 1963-2002 to report summary data for all stages of syphilis, congenital syphilis, gonorrhea, chancroid, chlamydia, and other STDs by sex and source of report (private versus public) for all 50 states, the District of Columbia, 64 selected cities (including San Juan, Puerto Rico), and outlying areas of the United States.

Note: Chlamydial infection became a nationally notifiable condition in 1995 and the form was modified to support reporting of chlamydia that year. Congenital syphilis was dropped from this aggregate form in 1995 and replaced by the case-specific CDC 73.126 form described later in this section.

3. FORM CDC 73.2638: Report of Civilian Cases of Primary \& Secondary Syphilis, Gonorrhea, and Chlamydia by Reporting Source, Sex, Race/Ethnicity, and Age Group. This annual hard copy form was used during 1981-2002 to report summary data for $\mathrm{P} \& \mathrm{~S}$ syphilis, gonorrhea, and chlamydia by age, race, sex, and source (public versus private) for all 50 states, seven large cities (Baltimore, Chicago, New York City, Los Angeles, Philadelphia, San Francisco, and the District of Columbia), and outlying areas of the United States.

Note: Chlamydial infection became a nationally notifiable condition in 1995, and the form was modified to support reporting of chlamydia that year.

4. FORM CDC 73.126: Congenital Syphilis (CS) Case Investigation and Reporting. This case-specific hard copy form was first used in 1983 and continues to be used to report detailed case-specific data for congenital syphilis in some areas.

\section{National Electronic Telecommunications System for Surveillance}

Notifiable STD data reported electronically through NETSS make up the nationally notifiable disease information published in CDC's Morbidity and Mortality Weekly Report.

As of December 31, 2003, all 50 states and the District of Columbia had converted from summary hard copy reporting to electronic submission of line-listed (i.e., case-specific) STD data through NETSS (41 reporting areas submitted congenital syphilis surveillance data through NETSS in 2017). Puerto Rico converted to electronic reporting in 2006 for all STDs excluding congenital syphilis. Guam and the Virgin Islands continue to report STD data through summary hard copy forms.

Surveillance data and updates sent to CDC through NETSS and on hard copy forms through June 19, 2018, are included in this report. The data presented in the figures and tables in this report supersede those in all earlier publications.

\section{A1.2 Population Denominators and Rate Calculations}

\section{0-2017 Rates and Population}

For those figures and tables presenting race using the 1997 Office of Management and Budget (OMB) standards, nonbridged-race data provided directly by the United States Census Bureau were used to calculate race. The latest available

year for population estimates at the time this report was written was 2016. Thus, 2016 population estimates were used to calculate 2017 rates.

Once published, the 2017 population estimates will be used to calculate 2017 rates in Sexually Transmitted Disease Surveillance 2018. 
Population estimates for Puerto Rico were obtained from the US Census Bureau Web site at:

https://factfinder.census.gov.

Population estimates for Guam and the Virgin Islands were obtained from the US Census Bureau International Programs Web site at: www.census.gov/programs-surveys/international-programs.html.

The 2017 rates by age and sex for Guam and the Virgin Islands were calculated using the latest population estimates available at: https://factfinder.census.gov/faces/nav/jsf/pages/index.xhtml.

Because of the use of the updated population data, rates for 2000-2016 may be different from those presented in previous STD surveillance reports.

Several figures throughout this report depict state- or county-specific rates of reported cases of STDs. Rates were grouped and displayed by quintiles in Figures 3, 4, 16, 17, 37, E, F, I, J, K, L, M, N, O, P, Q, R, and AA. Rates were grouped and displayed in 4 categories - zero cases and tertiles - in Figure 38.

\section{0-1999 Rates and Population}

The population counts for 1990 through 1999 incorporated the bridged single-race estimates of the April 1, 2000, US resident population. These files were prepared by the US Census Bureau with support from the National Cancer Institute.

\section{1-1989 Rates and Population}

Rates were calculated by using US Census Bureau population estimates for 1981 through 1989. ${ }^{1,2}$

\section{1-1980 Rates and Population}

Rates for 1941 through 1980 were based on population estimates from the US Census Bureau and are currently maintained by CDC's Division of STD Prevention.

\section{1-2017 Congenital Syphilis Rates and Live Births}

The congenital syphilis data in Table 1 of this report represent the number of congenital syphilis cases per 100,000 live births for all years during 1941-2017. Previous publications presented congenital syphilis rates per 100,000 population during 1941-1994 and rates for cases diagnosed at younger than 1 year of age per 100,000 live births during 19952005. To allow for trends in congenital syphilis rates to be compared for the period of 1941 through 2017, live births now are used as the denominator for congenital syphilis and case counts are no longer limited to those diagnosed within the first year of life. Congenital syphilis morbidity is assigned by year of birth. Rates of congenital syphilis for 1963 through 1988 were calculated by using published live birth data. ${ }^{3}$ Congenital syphilis rates for 1989 through 2016 were calculated by using live birth data based on information coded by the states and provided to the National Center for Health Statistics (NCHS) through the Vital Statistics Cooperative Program. Rates for 2017 were calculated by using live birth data for 2016 .

\section{0-2017 Gay, Bisexual, and Other Men Who Have Sex with Men Rates and Population}

Figures 26 and AA show rates of reported cases of gonorrhea and $\mathrm{P} \& \mathrm{~S}$ syphilis among gay, bisexual, and other men who have sex with men (collectively referred to as MSM). Population estimates of MSM are based on a method that combines published estimates of the prevalence of same-sex behavior among adult men with housing and population data from the American Community Survey 5-year summary file (2012-2016) $\cdot{ }^{4-7}$ County-specific estimates begin with MSM prevalence estimates that are determined by their urbanicity according to the NCHS urban-rural classification scheme for counties and their United States region. ${ }^{8}$ Estimates are then multiplied by a modified ratio of each county's percentage of male same-sex households to the total percentage of male same-sex households among all counties at the same level of urbanicity and within the same region. Thus, the final estimate for each county reflects what would be expected based on the county's geography, urban-rural classification, and observed concentration of households with a male head of household and a male partner. 


\section{A1.3 Reporting Practices}

Although most state and local STD programs generally adhere to the national notifiable STD case definitions collaboratively developed by the Council of State and Territorial Epidemiologists (CSTE) and CDC, differences in policies and systems for collecting surveillance data may exist. Thus, comparisons of case numbers and rates between jurisdictions should be interpreted with caution. However, because case definitions and surveillance activities within a given area remain relatively stable over time, trends should be minimally affected by these differences.

\section{A1.4 Reporting of Surveillance Data by Metropolitan Statistical Area}

Sexually Transmitted Disease Surveillance 2017 continues the presentation of STD incidence data and rates for the 50 metropolitan statistical areas (MSA) with the largest populations according to 2010 United States census data. MSAs are defined by the OMB to provide nationally consistent definitions for collecting, tabulating, and publishing federal statistics for a set of geographic areas. ${ }^{9}$ An MSA is associated with at least one urbanized area that has a population of at least 50,000. The MSA comprises the central county or counties containing the central county, plus adjacent, outlying counties that have a high degree of social and economic integration with the central county as measured through commuting. The title of an MSA includes the name of the principal city with the largest 2010 census population. If there are multiple principal cities, the names of the second largest and third largest principal cities appear in the title in order of descending population size.

Reported cases are assigned to MSAs based on the reported county; cases reported with a missing a value for the county variable cannot be assigned to an MSA. Consequently, if a jurisdiction reports cases missing values for the county variable, reported rates for MSAs in their jurisdiction may be incomplete. Additionally, relative rankings of case counts by counties may be impacted by completeness of the variable used to identify county. Table A1 reports the percentage of cases reported with missing county information in each state for P\&S syphilis, chlamydia, and gonorrhea.

The MSA concept has been used as a statistical representation of the social and economic links between urban cores and outlying, integrated areas. However, MSAs do not equate to an urban-rural classification; all counties included in MSAs and many other counties contain both urban and rural territory and populations. STD programs that treat all parts of an MSA as if they were as urban as the densely settled core ignore the rural conditions that may exist in some parts of the area. In short, MSAs are not intended to be a general purpose geographic framework for nonstatistical activities or for use in program funding formulas.

For more information on the MSA definitions used in this report, go to: https://www.census.gov/programs-surveys/metro-micro.html.

\section{A1.5 Reporting of Data for Race/Hispanic Ethnicity}

In April 2008, the NETSS record layout was updated to conform to the OMB's current government-wide standard for race/Hispanic ethnicity data. The OMB standards were first issued in $1997 .{ }^{10}$ Beginning with the publication of Sexually Transmitted Disease Surveillance 2012, the race/Hispanic ethnicity data are presented according to the current OMB standard categories: American Indian or Alaska Native, Asian, Black or African American, Hispanic or Latino, Native Hawaiian or Other Pacific Islander, White, and Multirace. As of 2017, most reporting jurisdictions are locally compliant with current OMB standards and report in the current OMB standard race categories, including Multirace. However, a small number of jurisdictions reported race in pre-1997 single race categories; while other jurisdictions were using current OMB standards categories but were unable to report more than one race per person in 2017.

For this report, all race and Hispanic ethnicity data reported by jurisdictions are summarized in tables, charts, and interpretative text regardless of local compliance with the 1997 OMB standards. However, a small number of cases reported in the legacy 'Asian/Pacific Islander' category from non-compliant jurisdictions are re-coded to 'Unknown' because these cases cannot be properly re-coded into an appropriate current OMB standards category of 'Asian' or 'Native Hawaiian/Other Pacific Islander.' No redistribution of cases is done; cases missing race and/or Hispanic ethnicity are not included in the calculation of rates by race and Hispanic ethnicity. As a consequence, rate data presented in this report underestimate actual case incidence in these population categories by a roughly similar proportion to the overall percentage of cases missing/unknown race and Hispanic ethnicity. 
All states and reporting jurisdictions are encouraged to continue efforts to upgrade local surveillance systems to be fully compliant with OMB standards for the collection of race and Hispanic ethnicity, to redouble efforts to ascertain complete information for all cases, and to implement CDC's HL7 case reporting guides at the earliest opportunity.

\section{A1.6 Management of Unknown, Missing, or Invalid Data for Age Group, Race/Hispanic Ethnicity, and Sex}

The percentage of unknown, missing, or invalid data for age group, race/Hispanic ethnicity, and sex varies from year to year, state to state, and by disease for reported STDs (Table A1).

Prior to the publication of Sexually Transmitted Disease Surveillance 2010, when the percentage of unknown, missing, or invalid values for age group, race/Hispanic ethnicity, and sex exceeded $50 \%$ for any state, the state's incidence and population data were excluded from the tables that presented data stratified by one or more of these variables. For the states for which $50 \%$ or more of their data were valid for age group, race/Hispanic ethnicity, and sex, the values for unknown, missing, or invalid data were redistributed on the basis of the state's distribution of known age group, race/ Hispanic ethnicity, and sex data. Beginning with the publication of Sexually Transmitted Disease Surveillance 2010, redistribution methodology is not applied to any of the data. The counts presented in this report are summations of all valid data reported in reporting year 2017.

As a result, rate data that are stratified by one or more of these variables reflect rates based on reported data only; caution should be used in interpreting specific rate data points as these may underestimate reported case incidence by race and Hispanic ethnicity due to the exclusion of cases missing these important demographic data.

\section{A1.7 Classification of STD Morbidity Reporting Sources}

Before 1996, states classified the source of case reports as either private source (including private physicians, hospitals, and institutions) or public source (primarily STD clinics). As states began reporting morbidity data electronically in 1996, the classification categories for source of case reports expanded to include the following data sources: STD clinics, HIV counseling and testing sites, drug treatment clinics, family planning clinics, prenatal/obstetrics clinics, tuberculosis clinics, private physicians/health maintenance organizations (HMOs), hospitals (inpatient), emergency rooms, correctional facilities, laboratories, blood banks, the National Job Training Program (NJTP), school-based clinics, mental health providers, the military, the Indian Health Service, and other unspecified sources. Figures 9, 10, 23, and 24 display trends in the proportion of cases reported in 2017 categorized by reporting source. Categories displayed vary across these figures and include the five most commonly reported sources for the population included in the figure, along with trends for all other reporting sources combined into the "All Other" category, and trends in the proportion of cases with unknown reporting source.

\section{A1.8 Interpreting Chlamydia Case Reporting}

Trends in rates of reported cases of chlamydia are influenced by changes in incidence of infection, as well as changes in diagnostic, screening, and reporting practices. As chlamydial infections are usually asymptomatic, the number of infections identified and reported can increase as more people are screened even when incidence is flat or decreasing. During 2000-2011, the expanded use of more sensitive diagnostic tests (e.g., nucleic acid amplification tests [NAATs]) likely increased the number of infections identified and reported independently of increases in incidence. Also, although chlamydia has been a nationally notifiable condition since 1994, it was not until 2000 that all 50 states and the District of Columbia required reporting of chlamydia cases. National case rates prior to 2000 reflect incomplete reporting. The increased use of electronic laboratory reporting over the last decade or so also likely increased the proportion of diagnosed cases reported. Consequently, an increasing chlamydia case rate over time may reflect increases in incidence of infection, screening coverage, and use of more sensitive tests, as well as more complete reporting. Likewise, decreases in chlamydia case rates may suggest decreases in incidence of infection or screening coverage.

\section{A1.9 Syphilis Morbidity Reporting}

The category of "total syphilis" or "all stages of syphilis" includes primary syphilis, secondary syphilis, early latent syphilis, late latent syphilis, late syphilis with clinical manifestations (including late benign syphilis and cardiovascular syphilis), and congenital syphilis. 
Although neurosyphilis can occur at almost any stage of syphilis, during 1996-2005 it was classified and reported as one of several mutually exclusive stages of syphilis. Beginning in 2005, neurosyphilis was no longer classified or reported as a distinct stage of syphilis.

\section{A1.10 Congenital Syphilis Morbidity Reporting}

In 1988, the surveillance case definition for congenital syphilis was changed. This case definition has greater sensitivity than the former definition. ${ }^{11}$ In addition, many state and local STD programs have greatly enhanced active case finding for congenital syphilis since 1988. For these reasons, as well as because of increasing morbidity, the number of reported cases increased dramatically during 1989-1991. All reporting areas had implemented the new case definition for reporting congenital syphilis by January 1, 1992. In addition to changing the case definition for congenital syphilis, CDC introduced a new data collection form (CDC 73.126) in 1990 (revised February 2013). Since 1995, the data collected on this form have been used for reporting congenital syphilis cases and associated rates. This form is used to collect individual case information, which allows more thorough analysis of case characteristics. For the purpose of analyzing race/Hispanic ethnicity, cases are classified by the race/Hispanic ethnicity of the mother. Similarly, since 1995 , congenital syphilis cases are reported by state and city of residence of the mother.

Congenital syphilis reporting may be delayed as a result of case investigation and validation. Cases for previous years are added to CDC's surveillance databases throughout the year. Congenital syphilis data reported after publication of the current annual STD surveillance report will appear in subsequent reports and are assigned by the case patient's year of birth.

\section{A1.11 Interpreting Surveillance Data from Outlying Areas}

There are a number of issues affecting the STD surveillance data reported to CDC from the US outlying areas, including test kit stock-outs, resulting in an inability to test or screen for undetermined periods of time, as well as a variety of data collection, entry, and transmission issues. As such, the data likely underestimate the total STD burden in these areas and should be interpreted cautiously.

\section{A2. Other Sources of Surveillance Data}

\section{A2.1 National Job Training Program}

Chlamydia and gonorrhea prevalence was calculated for men and women entering the NJTP. To increase the stability of the estimates, chlamydia or gonorrhea prevalence data are presented when valid test results for 100 or more students per year are available for the population subgroup and state. The majority of NJTP's chlamydia screening tests are conducted by a single national contract laboratory, which provides these data to CDC. Gonorrhea screening tests for male and female students in many training centers are conducted by local laboratories; these data are not available to CDC. Test results for students at centers that submit specimens to the national contract laboratory are included only if the number of gonorrhea tests submitted is greater than $90 \%$ of the number of chlamydia tests submitted from the same center for the same period. Prevalence data for state-specific figures were published with permission from the Department of Labor. Prevalence data are presented in figures O, P, Q, and R.

\section{A2.2 STD Surveillance Network}

In 2005, CDC established the STD Surveillance Network (SSuN) as a collaborative network of state, county and/or city health departments following protocols to conduct sentinel and enhanced STD surveillance activities. The purpose of SSuN is to improve the capacity of national, state and local STD programs to detect, monitor, and respond to trends in STDs through enhanced collection, reporting, analysis, visualization, and interpretation of disease information.

Cycle 3 (2013-2018) of SSuN provides funding to 10 jurisdictions to conduct two core STD surveillance activities including; (1) sentinel facility component, providing clinical and demographic information on a full census of patients attending categorical STD clinics and women aged 15-44 years presenting for care in reproductive health settings, and, (2) population component, conducting enhanced health department look-back, provider, and patient investigations on a probability sample of all persons diagnosed and reported with gonorrhea. Funded jurisdictions for both core activities in SSuN Cycle 3 include Baltimore City (Maryland), California (excluding San Francisco County), Florida, Massachusetts, Minnesota, Multnomah County (Oregon), Philadelphia City (Pennsylvania), New York City (New York), San Francisco County (California), and Washington State. 
In both the facility and population components of SSuN Cycle 3, unique patients can be anonymously followed using unique, coded IDs to provide longitudinal information. In the facility component, the primary unit of analysis is the patient visit, which is merged with multiple laboratory, diagnostic, and treatment observations. In the population component, the primary unit of analysis is a reported episode of gonorrhea for a unique person merged with multiple laboratory observations, health department disease registry history, provider-based clinical information, and patient demographic and behavioral interview data. For analysis in the population component, cases in the probability sample are weighted to reflect study design and to adjust for non-response by demographic category of the patient. Weighted analyses provide estimates of case and person characteristics representative of all reported cases in the collaborating jurisdictions.

MSM are defined in all SSuN data collection activities as men who either reported having sex with another man in the preceding 2-3 months, reported current history of male sex partners, and/or those who reported that they considered themselves gay/homosexual or bisexual. Men who have sex with women (MSW) are defined as men who reported having sex with women only or who did not report the sex of their sex partners but reported that they considered themselves to be straight/heterosexual.

Data presented in this report from the facility component of SSuN are from nine participating jurisdictions (Baltimore City [Maryland], California [excluding San Francisco County], Massachusetts, Minnesota, Multnomah County [Oregon], New York City [New York], Philadelphia City [Pennsylvania], San Francisco County [California] and Washington State). Figures 13, 27, DD, EE, FF, and GG are based on STD clinic data and Figure G is based on data from facilities that provide family planning and reproductive health services. Data presented in this report from the population component of SSuN include Figures 25 and 26. Figure 25 presents data collected January-December 2017 showing the proportion of cases attributable to MSM, MSW, and women for all ten SSuN jurisdictions. Figure 26 presents data collected January 2010-June 2013 and June 2015-December 2017 for six SSuN jurisdictions collaborating in both SSuN Cycle 2 and SSuN Cycle 3 (Baltimore City [Maryland], California [excluding San Francisco County], New York City [New York], Philadelphia City [Pennsylvania], San Francisco County [California], and Washington State).

\section{A2.3 Gonococcal Isolate Surveillance Project}

Data on antimicrobial susceptibility in Neisseria gonorrhoeae were collected through the Gonococcal Isolate Surveillance Project (GISP), a sentinel system of selected STD clinics located at 25-30 GISP sentinel sites and regional laboratories in the United States. For more details on findings from GISP, go to: https://www.cdc.gov/std/GISP/.

For 2017, the antimicrobial agents tested by GISP were ceftriaxone, cefixime, azithromycin, ciprofloxacin, penicillin, tetracycline, and gentamicin.

The antimicrobial susceptibility criteria used in GISP for 2017 are as follows:

- Ceftriaxone, minimum inhibitory concentration (MIC) $\geq 0.5 \mu \mathrm{g} / \mathrm{ml}$ (decreased susceptibility)*

- Ceftriaxone, MIC $\geq 0.125 \mu \mathrm{g} / \mathrm{ml}$ (elevated MICs)*

- Cefixime, MIC $\geq 0.5 \mu \mathrm{g} / \mathrm{ml}$ (decreased susceptibility)*

- Cefixime, $\mathrm{MIC} \geq 0.25 \mu \mathrm{g} / \mathrm{ml}$ (elevated MICs)*

- Azithromycin, MIC $\geq 2.0 \mu \mathrm{g} / \mathrm{ml}$ (elevated MICs)*

- Ciprofloxacin, $\mathrm{MIC} \geq 1.0 \mu \mathrm{g} / \mathrm{ml}$ (resistance)

- Ciprofloxacin, MIC 0.125-0.5 $\mu \mathrm{g} / \mathrm{ml}$ (intermediate resistance)

- Penicillin, MIC $\geq 2.0 \mu \mathrm{g} / \mathrm{ml}$ (resistance)

- Tetracycline, $\mathrm{MIC} \geq 2.0 \mu \mathrm{g} / \mathrm{ml}$ (resistance)

- Gentamicin (MIC values correlated with susceptibility and resistance have not been established)*

The majority of these criteria are also recommended by the Clinical and Laboratory Standards Institute (CLSI). ${ }^{12}$

\footnotetext{
* The CLSI criteria for decreased susceptibility and resistance to ceftriaxone, cefixime, gentamicin, and azithromycin and for susceptibility to gentamicin and azithromycin have not been established for $N$. gonorrhoeae.
} 


\section{A2.4 National Health and Nutrition Examination Survey}

The National Health and Nutrition Examination Survey (NHANES) is a series of cross-sectional surveys designed to provide national statistics on the health and nutritional status of the general household population in the United States. Data are collected through household interviews, standardized physical examinations, and the collection of biological samples in special mobile examination centers. In 1999, NHANES became a continuous survey with data released every two years. The sampling plan of the survey is a stratified, multistage, probability cluster design that selects a sample representative of the United States civilian, non-institutionalized population. For more information, see: https://www.cdc.gov/nchs/nhanes.htm.

\section{A2.5 National Disease and Therapeutic Index}

The information on the number of initial visits to private physicians' offices for STDs was based on analysis of data from the National Disease and Therapeutic Index (NDTI) (machine-readable files or summary statistics for 1966 through 2016; the 2017 NDTI data were not obtained in time to include them in this report). NDTI is a probability sample survey of private physicians' clinical management practices. For more information on this database, contact IMS Health, e-mail: ServiceCenter@us.imshealth.com; Telephone: (800) 523-5334.

\section{References}

1. US Census Bureau. United States population estimates by age, sex and race: 1980-1988. In: Current population reports [Series P-25, No. 1045]. Washington, DC: US Government Printing Office; 1990.

2. US Census Bureau. United States population estimates by age, sex and race: 1989. In: Current population reports [Series P-25, No. 1057]. Washington, DC: US Government Printing Office; 1990.

3. Centers for Disease Control and Prevention. Vital statistics of the United States 1988. vol.1 — natality. Hyattsville (MD): US Department of Health and Human Services; 1990.

4. American Community Survey. 5-year summary file, 2012-2016. US Census Bureau: 2016.

5. Grey JA, Bernstein KT, Sullivan PS, et al. Estimating the population sizes of men who have sex with men in US states and counties using data from the American Community Survey. JMIR Public Health Surveill 2016; 2(1):e14.

6. Oster AM, Sternberg M, Lansky A, et al. Population size estimates for men who have sex with men and persons who inject drugs. J Urban Health 2015; 92(4):733-743.

7. Purcell DW, Johnson CH, Lansky A, et al. Estimating the population size of men who have sex with men in the United States to obtain HIV and syphilis rates. Open AIDS J 2012; 6(Suppl 1):98-107.

8. Ingram DD, Franco SJ. 2013 NCHS urban-rural classification scheme for counties. National Center for Health Statistics. Vital Health Stat 2014; 2(166):1-81.

9. Office of Management and Budget. Standards for defining metropolitan and micropolitan statistical areas. Federal Register 2000; 65(249):82228-82238.

10. Office of Management and Budget. Revisions to the Standards for Classification of Federal Data on Race and Ethnicity. Federal Register Notice. October 30, 1997.

11. Kaufman RE, Jones OG, Blount JH, et al. Questionnaire survey of reported early congenital syphilis: Problems in diagnosis, prevention, and treatment. Sex Transm Dis 1977; 4(4):135-139.

12. Clinical and Laboratory Standards Institute. Performance standards for antimicrobial susceptibility testing; Twenty-fifth informational supplement. M100-S25, 35(3). Wayne (PA): Clinical and Laboratory Standards Institute; 2015. 
Table A1. Selected STDs - Percentage of Unknown, Missing, or Invalid Values for Selected Variables by State and by Nationally Notifiable STD, 2017

\begin{tabular}{|c|c|c|c|c|c|}
\hline \multirow[b]{2}{*}{ State } & \multicolumn{5}{|c|}{ Primary and Secondary Syphilis } \\
\hline & $\begin{array}{c}\text { Percentage } \\
\text { Unknown } \\
\text { Race/Hispanic } \\
\text { Ethnicity }\end{array}$ & $\begin{array}{c}\text { Percentage } \\
\text { Unknown } \\
\text { Age }\end{array}$ & $\begin{array}{l}\text { Percentage } \\
\text { Unknown } \\
\text { Sex }\end{array}$ & $\begin{array}{c}\text { Percentage } \\
\text { Unknown } \\
\text { Sex } \\
\text { Partner }\end{array}$ & $\begin{array}{c}\text { Percentage } \\
\text { Unknown } \\
\text { County }\end{array}$ \\
\hline Alabama & 0.5 & 0.9 & 0.0 & 39.6 & 0.0 \\
\hline Alaska & 0.0 & 0.0 & 0.0 & 0.0 & 0.0 \\
\hline Arizona & 2.5 & 0.0 & 0.0 & 6.4 & 0.0 \\
\hline Arkansas & 0.4 & 0.0 & 0.0 & 8.5 & 0.0 \\
\hline California & 5.8 & 0.1 & 0.0 & 12.7 & 0.0 \\
\hline Colorado & 6.8 & 0.0 & 0.0 & 8.9 & 0.0 \\
\hline Connecticut & 5.5 & 0.0 & 0.0 & 10.9 & 0.0 \\
\hline Delaware & 7.0 & 0.0 & 0.0 & 78.9 & 0.0 \\
\hline District of Columbia & 30.3 & 0.0 & 1.1 & 47.8 & 0.0 \\
\hline Florida & 4.7 & 0.0 & 0.0 & 11.6 & 0.0 \\
\hline Georgia & 4.0 & 0.0 & 0.0 & 30.3 & 0.1 \\
\hline Hawaii & 2.1 & 0.0 & 0.0 & 18.1 & 0.0 \\
\hline Idaho & 7.8 & 3.1 & 0.0 & 28.1 & 0.0 \\
\hline Illinois & 5.2 & 0.0 & 0.0 & 23.9 & 0.5 \\
\hline Indiana & 0.6 & 0.0 & 0.0 & 5.6 & 0.0 \\
\hline lowa & 0.0 & 0.0 & 0.0 & 9.9 & 0.0 \\
\hline Kansas & 0.0 & 0.0 & 0.0 & 4.5 & 0.0 \\
\hline Kentucky & 0.4 & 0.0 & 0.0 & 16.4 & 0.0 \\
\hline Louisiana & 0.0 & 0.0 & 0.0 & 2.7 & 0.0 \\
\hline Maine & 1.5 & 1.5 & 3.1 & 96.9 & 4.6 \\
\hline Maryland & 4.4 & 0.0 & 0.0 & 8.7 & 0.0 \\
\hline Massachusetts & 2.6 & 0.0 & 0.0 & 6.7 & 4.8 \\
\hline Michigan & 0.2 & 0.0 & 0.0 & 11.5 & 0.0 \\
\hline Minnesota & 1.7 & 0.0 & 0.7 & 4.1 & 0.0 \\
\hline Mississippi & 0.0 & 0.0 & 0.0 & 3.9 & 0.0 \\
\hline Missouri & 1.0 & 0.0 & 0.0 & 11.8 & 0.0 \\
\hline Montana & 0.0 & 0.0 & 0.0 & 27.1 & 0.0 \\
\hline Nebraska & 18.6 & 0.0 & 0.0 & 46.5 & 0.0 \\
\hline Nevada & 3.1 & 0.0 & 0.0 & 11.1 & 0.0 \\
\hline New Hampshire & 25.6 & 0.0 & 0.0 & 4.7 & 0.0 \\
\hline New Jersey & 4.0 & 0.0 & 0.0 & 30.9 & 0.0 \\
\hline New Mexico & 12.4 & 0.0 & 0.0 & 14.0 & 0.0 \\
\hline New York & 5.8 & 0.1 & 0.8 & 25.6 & 0.0 \\
\hline North Carolina & 0.0 & 0.0 & 0.0 & 8.3 & 0.0 \\
\hline North Dakota & 0.0 & 0.0 & 0.0 & 11.4 & 0.0 \\
\hline Ohio & 0.2 & 0.0 & 0.0 & 7.0 & 0.1 \\
\hline Oklahoma & 0.0 & 0.0 & 0.0 & 2.4 & 0.0 \\
\hline Oregon & 6.5 & 0.0 & 0.6 & 17.0 & 0.0 \\
\hline Pennsylvania & 6.7 & 0.0 & 0.0 & 9.2 & 0.0 \\
\hline Rhode Island & 4.2 & 0.0 & 0.0 & 33.8 & 0.0 \\
\hline South Carolina & 3.9 & 0.0 & 0.0 & 6.4 & 0.0 \\
\hline South Dakota & 0.0 & 0.0 & 0.0 & 6.1 & 0.0 \\
\hline Tennessee & 0.2 & 0.0 & 0.0 & 4.3 & 0.0 \\
\hline Texas & 1.6 & 0.0 & 0.0 & 6.1 & 0.0 \\
\hline Utah & 3.4 & 0.0 & 0.0 & 7.7 & 1.7 \\
\hline Vermont & 0.0 & 0.0 & 0.0 & 15.4 & 0.0 \\
\hline Virginia & 9.7 & 0.0 & 0.9 & 14.0 & 0.0 \\
\hline Washington & 5.5 & 0.0 & 0.1 & 4.4 & 0.0 \\
\hline West Virginia & 0.0 & 0.0 & 0.0 & 11.3 & 0.0 \\
\hline Wisconsin & 2.9 & 0.0 & 0.0 & 59.5 & 0.0 \\
\hline Wyoming* & 0.0 & 0.0 & 0.0 & 0.0 & 0.0 \\
\hline U.S. TOTAL & 4.2 & 0.0 & 0.1 & 14.3 & 0.1 \\
\hline
\end{tabular}

Continued on next page. 
Table A1. Selected STDs - Percentage of Unknown, Missing, or Invalid Values for Selected Variables by State and by Nationally Notifiable STD, 2017 (continued)

\begin{tabular}{|c|c|c|c|c|c|c|c|c|}
\hline \multirow[b]{2}{*}{ State } & \multicolumn{4}{|c|}{ Gonorrhea } & \multicolumn{4}{|c|}{ Chlamydia } \\
\hline & $\begin{array}{c}\text { Percentage } \\
\text { Unknown } \\
\text { Race/Hispanic } \\
\text { Ethnicity }\end{array}$ & $\begin{array}{c}\text { Percentage } \\
\text { Unknown } \\
\text { Age }\end{array}$ & $\begin{array}{l}\text { Percentage } \\
\text { Unknown } \\
\text { Sex }\end{array}$ & $\begin{array}{c}\text { Percentage } \\
\text { Unknown } \\
\text { County }\end{array}$ & $\begin{array}{c}\text { Percentage } \\
\text { Unknown } \\
\text { Race/Hispanic } \\
\text { Ethnicity }\end{array}$ & $\begin{array}{l}\text { Percentage } \\
\text { Unknown } \\
\text { Age }\end{array}$ & $\begin{array}{l}\text { Percentage } \\
\text { Unknown } \\
\text { Sex }\end{array}$ & $\begin{array}{l}\text { Percentage } \\
\text { Unknown } \\
\text { County }\end{array}$ \\
\hline Alabama & 32.8 & 0.2 & 0.3 & 0.0 & 37.3 & 0.2 & 0.4 & 0.0 \\
\hline Alaska & 0.5 & 0.0 & 0.0 & 0.0 & 3.5 & 0.0 & 0.0 & 0.3 \\
\hline Arizona & 21.1 & 0.0 & 0.2 & 0.0 & 29.9 & 0.0 & 0.2 & 0.0 \\
\hline Arkansas & 5.1 & 0.0 & 0.0 & 0.0 & 6.5 & 0.0 & 0.0 & 0.0 \\
\hline California & 21.4 & 0.2 & 0.2 & 0.0 & 34.2 & 0.2 & 0.2 & 0.0 \\
\hline Colorado & 33.4 & 0.0 & 0.0 & 0.0 & 48.0 & 0.0 & 0.0 & 0.0 \\
\hline Connecticut & 28.7 & 0.0 & 0.2 & 3.0 & 76.8 & 0.2 & 3.6 & 5.7 \\
\hline Delaware & 4.1 & 0.0 & 0.0 & 0.0 & 6.0 & 0.0 & 0.0 & 0.0 \\
\hline District of Columbia & 88.0 & 0.2 & 0.5 & 0.0 & 90.9 & 0.5 & 0.8 & 0.0 \\
\hline Florida & 11.6 & 0.0 & 0.0 & 0.0 & 17.8 & 0.0 & 0.0 & 0.0 \\
\hline Georgia & 27.5 & 0.1 & 0.2 & 3.1 & 39.8 & 0.1 & 0.3 & 4.1 \\
\hline Hawaii & 33.3 & 0.1 & 0.0 & 0.1 & 45.2 & 0.1 & 0.0 & 0.1 \\
\hline Idaho & 21.7 & 0.9 & 0.1 & 0.0 & 32.9 & 0.1 & 0.2 & 0.0 \\
\hline Illinois & 14.0 & 0.0 & 0.2 & 0.0 & 17.9 & 0.0 & 0.1 & 0.0 \\
\hline Indiana & 6.2 & 0.0 & 0.0 & 0.0 & 9.7 & 0.0 & 0.0 & 0.0 \\
\hline lowa & 2.8 & 0.0 & 0.0 & 0.0 & 4.4 & 0.0 & 0.0 & 0.0 \\
\hline Kansas & 10.4 & 0.0 & 0.0 & 0.0 & 28.6 & 0.0 & 0.0 & 0.0 \\
\hline Kentucky & 25.3 & 0.1 & 0.4 & 0.0 & 34.6 & 0.1 & 0.5 & 0.0 \\
\hline Louisiana & 0.1 & 0.0 & 0.0 & 0.0 & 0.2 & 0.0 & 0.0 & 0.1 \\
\hline Maine & 4.8 & 1.0 & 0.5 & 1.0 & 27.3 & 0.4 & 0.0 & 4.6 \\
\hline Maryland & 23.7 & 0.0 & 0.0 & 0.0 & 40.6 & 0.0 & 0.0 & 0.0 \\
\hline Massachusetts & 37.0 & 0.1 & 0.5 & 11.7 & 68.1 & 0.1 & 0.3 & 14.7 \\
\hline Michigan & 18.3 & 0.1 & 0.1 & 0.1 & 23.8 & 0.1 & 0.1 & 0.0 \\
\hline Minnesota & 15.5 & 0.0 & 0.2 & 1.3 & 19.7 & 0.0 & 0.1 & 2.0 \\
\hline Mississippi & 16.8 & 0.0 & 0.2 & 0.0 & 26.0 & 0.0 & 0.2 & 0.0 \\
\hline Missouri & 8.2 & 0.0 & 0.0 & 0.0 & 13.0 & 0.0 & 0.0 & 0.0 \\
\hline Montana & 4.1 & 0.4 & 0.0 & 0.0 & 3.1 & 0.3 & 0.0 & 0.0 \\
\hline Nebraska & 15.8 & 0.0 & 0.1 & 0.0 & 24.1 & 0.1 & 0.1 & 0.0 \\
\hline Nevada & 35.1 & 0.0 & 0.2 & 4.0 & 44.4 & 0.0 & 0.3 & 4.9 \\
\hline New Hampshire & 14.2 & 0.0 & 0.0 & 0.0 & 29.5 & 0.0 & 0.0 & 0.0 \\
\hline New Jersey & 35.8 & 0.5 & 0.1 & 0.9 & 51.1 & 0.4 & 0.2 & 0.8 \\
\hline New Mexico & 25.7 & 0.0 & 0.1 & 0.0 & 30.3 & 0.0 & 0.0 & 0.0 \\
\hline New York & 27.8 & 0.1 & 0.2 & 0.0 & 42.2 & 0.2 & 0.1 & 0.0 \\
\hline North Carolina & 14.4 & 0.0 & 0.0 & 0.0 & 19.5 & 0.0 & 0.0 & 0.0 \\
\hline North Dakota & 3.1 & 0.0 & 0.0 & 0.0 & 8.7 & 0.0 & 0.0 & 0.0 \\
\hline Ohio & 17.6 & 0.1 & 0.0 & 1.7 & 23.4 & 0.1 & 0.0 & 1.8 \\
\hline Oklahoma & 10.6 & 0.0 & 0.0 & 0.0 & 15.0 & 0.0 & 0.0 & 0.0 \\
\hline Oregon & 10.3 & 0.0 & 0.1 & 0.0 & 23.1 & 0.1 & 0.1 & 0.0 \\
\hline Pennsylvania & 21.9 & 0.0 & 0.1 & 0.0 & 32.0 & 0.0 & 0.1 & 0.0 \\
\hline Rhode Island & 15.5 & 0.0 & 0.1 & 3.9 & 17.0 & 0.0 & 0.1 & 2.5 \\
\hline South Carolina & 30.2 & 0.0 & 0.2 & 0.1 & 35.2 & 0.0 & 0.3 & 0.1 \\
\hline South Dakota & 0.9 & 0.0 & 0.0 & 0.0 & 7.3 & 0.0 & 0.0 & 0.0 \\
\hline Tennessee & 2.2 & 0.0 & 0.0 & 0.0 & 3.4 & 0.0 & 0.0 & 0.0 \\
\hline Texas & 11.9 & 0.0 & 0.2 & 0.0 & 15.2 & 0.0 & 0.2 & 0.0 \\
\hline Utah & 5.4 & 0.0 & 0.2 & 0.1 & 6.5 & 0.0 & 0.1 & 0.1 \\
\hline Vermont & 3.9 & 0.0 & 0.0 & 0.0 & 12.8 & 0.0 & 0.3 & 0.0 \\
\hline Virginia & 28.0 & 0.2 & 0.7 & 0.0 & 39.9 & 0.2 & 1.1 & 0.0 \\
\hline Washington & 10.2 & 0.0 & 0.0 & 0.0 & 16.0 & 0.0 & 0.0 & 0.0 \\
\hline West Virginia & 10.3 & 0.0 & 0.0 & 0.0 & 16.6 & 0.0 & 0.0 & 0.0 \\
\hline Wisconsin & 24.3 & 0.0 & 0.1 & 0.0 & 20.8 & 0.0 & 0.1 & 0.1 \\
\hline Wyoming* & 17.0 & 0.0 & 0.0 & 0.0 & 38.1 & 0.0 & 0.1 & 0.0 \\
\hline U.S. TOTAL & 19.0 & 0.1 & 0.2 & 0.5 & 27.8 & 0.1 & 0.2 & 0.7 \\
\hline
\end{tabular}

* Percentages for primary and secondary syphilis are based on fewer than 10 cases.

NOTE: For all categories, unknown included cases reported as unknown or missing. 
Table A2. Reported Cases of STDs by Reporting Source and Sex, United States, 2017

\begin{tabular}{|c|c|c|c|c|c|c|c|c|c|}
\hline \multirow[b]{2}{*}{ Disease } & \multicolumn{3}{|c|}{ Non-STD Clinic } & \multicolumn{3}{|c|}{ STD Clinic } & \multicolumn{3}{|c|}{ Total } \\
\hline & Male & Female & Total* & Male & Female & Total* & Male $^{+}$ & Female $^{\dagger}$ & Total $^{\ddagger}$ \\
\hline Chlamydia & 432,467 & 909,467 & $1,343,625$ & 56,172 & 43,168 & 99,469 & 577,644 & $1,127,651$ & $1,708,569$ \\
\hline Gonorrhea & 241,098 & 184,210 & 425,921 & 36,935 & 14,996 & 51,982 & 322,169 & 232,587 & 555,608 \\
\hline Primary Syphilis & 6,554 & 819 & 7,377 & 1,807 & 132 & 1,940 & 9,539 & 1,078 & 10,623 \\
\hline Secondary Syphilis & 12,542 & 2,026 & 14,589 & 2,906 & 345 & 3,255 & 17,346 & 2,644 & 20,021 \\
\hline Early Latent Syphilis & 21,142 & 3,487 & 24,691 & 4,022 & 612 & 4,638 & 29,251 & 4,684 & 34,013 \\
\hline Late and Late Latent Syphilis ${ }^{\S}$ & 17,956 & 7,019 & 25,030 & 2,265 & 677 & 2,948 & 26,178 & 9,740 & 35,992 \\
\hline Chancroid & 3 & 3 & 6 & 0 & 0 & 0 & 4 & 3 & 7 \\
\hline
\end{tabular}

* Total includes cases reported with unknown sex.

${ }^{\dagger}$ Total includes cases reported with unknown reporting source.

₹ Total includes cases reported with unknown sex and reporting source.

${ }^{\S}$ Late and late latent syphilis includes late latent syphilis and late syphilis with clinical manifestations (including late benign syphilis and cardiovascular syphilis). 
This page intentionally left blank. 


\section{B. National Objectives and Goals}

\section{B1. Healthy People 2020 Objectives}

For three decades, Healthy People has provided a comprehensive set of national 10-year health promotion and disease prevention objectives aimed at improving the health of all Americans. ${ }^{1}$ It is grounded in the principle that establishing objectives and providing benchmarks to track and monitor progress over time can motivate, guide, and focus action.

Healthy People 2020 (HP2020) continues in the tradition of its ambitious, yet achievable, 10-year agenda for improving the Nation's health. HP2020 is the result of a multiyear process that reflects input from a diverse group of individuals and organizations. HP2020 is organized into 42 topic areas, with more than 1,200 measures designed to drive action that will support its four overarching goals:

- Attain high-quality, longer lives free of preventable disease, disability, injury, and premature death.

- Achieve health equity, eliminate disparities, and improve the health of all groups.

- Create social and physical environments that promote good health for all.

- Promote quality of life, healthy development, and healthy behaviors across all life stages.

The topic area, Sexually Transmitted Diseases, contains objectives and measures related to STDs. Baselines, HP2020 targets, and annual progress toward the targets are reported in Table B1. The year 2020 targets for the diseases addressed in this report are as follows: primary and secondary (P\&S) syphilis (males), 6.7 cases per 100,000 males; P\&S syphilis (females), 1.3 cases per 100,000 females; congenital syphilis, 9.6 cases per 100,000 live births; gonorrhea (females aged 15-44 years), 251.9 cases per 100,000 females and gonorrhea (males aged 15-44 years), 194.8 cases per 100,000 males. The majority of the STD-related HP2020 targets were set using a standard percentage improvement with a standard default of a "10 percent improvement over the baseline."

\section{B2. Government Performance and Results Act of 1993}

The Government Performance and Results Act (GPRA) of 1993 was enacted by Congress to increase confidence in the capability of the federal government to increase the effectiveness and accountability of federal programs, to improve service delivery, to provide federal agencies a uniform tool for internal management, and to help Congress make decisions.

GPRA requires each agency to have a performance plan with long-term outcomes and annual, measurable performance goals and to report on these plans annually, comparing results with annual goals. There are two GPRA goals for STD: reducing pelvic inflammatory disease (PID) and eliminating congenital syphilis. Each of these goals has specific measures of progress, which are outlined in Table B2.

\section{References}

1. U.S. Department of Health and Human Services. Healthy People 2020 (Healthy People 2020 Web site). Available at https://www.healthypeople.gov/ Accessed on August 16, 2018. 
Table B1. Healthy People 2020 (HP 2020) Sexually Transmitted Diseases Objectives

\begin{tabular}{|c|c|c|c|c|c|c|c|}
\hline & HP2020 Objectives & $\begin{array}{l}\text { Baseline } \\
\text { Year }\end{array}$ & Baseline & 2015 & 2016 & 2017 & $\begin{array}{l}2020 \\
\text { Target }\end{array}$ \\
\hline \multirow[t]{4}{*}{1} & $\begin{array}{l}\text { Reduce the proportion of adolescents and young adults with } \\
\text { Chlamydia trachomatis infections }\end{array}$ & & & & & & \\
\hline & a. Among females aged 15 to 24 years attending family planning clinics & 2008 & $7.4 \%$ & $8.6 \%$ & $8.4 \%$ & $9.6 \%$ & $6.7 \%$ \\
\hline & $\begin{array}{l}\text { b. Among females aged } 24 \text { years and under enrolled in a National Job } \\
\text { Training Program }\end{array}$ & 2008 & $12.8 \%$ & $12.7 \%$ & $11.4 \%$ & $11.8 \%$ & $11.5 \%$ \\
\hline & $\begin{array}{l}\text { c. Among males aged } 24 \text { years and under enrolled in a National Job } \\
\text { Training Program }\end{array}$ & 2008 & $7.0 \%$ & $7.5 \%$ & $7.1 \%$ & $6.6 \%$ & $6.3 \%$ \\
\hline \multirow[t]{3}{*}{2} & $\begin{array}{l}\text { Increase the proportion of sexually active females aged } 24 \text { years } \\
\text { and under enrolled in Medicaid plans who are screened for genital } \\
\text { Chlamydia infections during the measurement year }\end{array}$ & & & & & & \\
\hline & a. Females aged 16 to 20 years & 2008 & $52.7 \%$ & $51.2 \%$ & $53.9 \%$ & $\mathrm{~N} / \mathrm{A}$ & $70.9 \%$ \\
\hline & b. Females aged 21 to 24 years & 2008 & $59.4 \%$ & $60.1 \%$ & $62.2 \%$ & $\mathrm{~N} / \mathrm{A}$ & $80.0 \%$ \\
\hline \multirow[t]{3}{*}{3} & $\begin{array}{l}\text { Increase the proportion of sexually active females aged } 24 \text { years } \\
\text { and under enrolled in commercial health insurance plans who are } \\
\text { screened for genital Chlamydia infections during the measurement } \\
\text { year }\end{array}$ & & & & & & \\
\hline & a. Females aged 16 to 20 years & 2008 & $40.1 \%$ & $42.4 \%$ & $44.3 \%$ & $N / A$ & $61.3 \%$ \\
\hline & b. Females aged 21 to 24 years & 2008 & $43.5 \%$ & $52.4 \%$ & $54.8 \%$ & $\mathrm{~N} / \mathrm{A}$ & $74.6 \%$ \\
\hline 4 & $\begin{array}{l}\text { Reduce the proportion of females aged } 15 \text { to } 44 \text { years who have ever } \\
\text { required treatment for pelvic inflammatory disease (PID) }\end{array}$ & $2006-2010$ & $4.2 \%$ & $3.0 \%$ & $\mathrm{~N} / \mathrm{A}$ & $\mathrm{N} / \mathrm{A}$ & $3.8 \%$ \\
\hline \multirow[t]{3}{*}{5} & Reduce gonorrhea rates & & & & & & \\
\hline & a. Females aged 15 to 44 years & 2008 & 279.9 & 263.4 & 297.1 & 350.0 & 251.9 \\
\hline & b. Males aged 15 to 44 years & 2008 & 216.5 & 307.6 & 370.2 & 440.4 & 194.8 \\
\hline \multirow[t]{3}{*}{6} & $\begin{array}{l}\text { Reduce sustained domestic transmission of primary and secondary } \\
\text { syphilis }\end{array}$ & & & & & & \\
\hline & a. Among females & 2008 & 1.4 & 1.4 & 1.9 & 2.3 & 1.3 \\
\hline & b. Among males & 2008 & 7.4 & 13.7 & 15.6 & 16.9 & 6.7 \\
\hline 7 & Reduce congenital syphilis & 2008 & 10.7 & 12.4 & 15.7 & 23.3 & 9.6 \\
\hline 8 & $\begin{array}{l}\text { Reduce the proportion of young adults with genital herpes infection } \\
\text { due to herpes simplex type } 2\end{array}$ & $2005-2008$ & $10.5 \%$ & N/A & $\mathrm{N} / \mathrm{A}$ & $\mathrm{N} / \mathrm{A}$ & $9.5 \%$ \\
\hline
\end{tabular}

\begin{tabular}{cl}
$\begin{array}{c}\text { HP2020 } \\
\text { Objective }\end{array}$ & \multicolumn{1}{c}{ Data Source } \\
\hline $1 a$ & STD Surveillance Network (SSuN), CDC \\
\hline $1 b, 1 c$ & National Job Training Program (NJTP) \\
\hline $2 a, 2 b$ & Healthcare Effectiveness Data and Information Set (HEDIS), National Committee for Quality Assurance (NCQA) \\
\hline $3 a, 3 b$ & Healthcare Effectiveness Data and Information Set (HEDIS), National Committee for Quality Assurance (NCQA) \\
\hline 4 & National Survey of Family Growth (NSFG), CDC \\
\hline $5 a, 5 b$ & National Notifiable Disease Surveillance System (NNDSS), CDC \\
\hline $6 a, 6 b$ & National Notifiable Disease Surveillance System (NNDSS), CDC \\
\hline 7 & National Notifiable Disease Surveillance System (NNDSS), CDC \\
\hline 8 & National Health and Nutrition Examination Survey (NHANES), CDC \\
\hline
\end{tabular}

NOTE: Data presented in this table reflect data reported to HP2020 in current and prior years. Data for years prior to 2016 may not match estimates presented in other sections of this report. More information about HP2020 is available at: https://www.healthypeople.gov/

$\mathrm{N} / \mathrm{A}=$ Not available. 
Table B2. Government Performance and Results Act (GPRA) Sexually Transmitted Diseases Goals, Measures, and Target

\begin{tabular}{|c|c|c|c|c|c|}
\hline \multirow{2}{*}{\multicolumn{2}{|c|}{ GPRA Goals }} & \multicolumn{3}{|c|}{ Actual } & \multirow{2}{*}{$\begin{array}{r}\text { Target } \\
2018\end{array}$} \\
\hline & & 2015 & 2016 & 2017 & \\
\hline \multicolumn{2}{|c|}{ Goal 1: Reduction in PID (as measured by initial visits to physicians in women 15-44 years of age) } & 68,000 & 90,000 & N/A & 68,000 \\
\hline \multicolumn{2}{|c|}{ a. Proportion of high-risk women aged 16-20 infected with chlamydia* } & $13.4 \%$ & $12.3 \%$ & $13.1 \%$ & $11.7 \%$ \\
\hline \multicolumn{2}{|c|}{ b. Proportion of high-risk women aged 21-24 infected with chlamydia*t } & $8.5 \%$ & $7.4 \%$ & $7.9 \%$ & $8.3 \%$ \\
\hline \multicolumn{2}{|c|}{ c. Rate of gonorrhea/100,000 population in women aged $16-20$} & 537.0 & 586.0 & 680.8 & 523.9 \\
\hline \multicolumn{2}{|c|}{ d. Rate of gonorrhea/100,000 population in women aged $21-24$} & 523.9 & 573.0 & 654.8 & 511.8 \\
\hline \multicolumn{2}{|c|}{ e. Black: White ratio of gonorrhea in women aged 16-24 } & 9.5 & 8.4 & 7.8 & $9.5: 1$ \\
\hline \multicolumn{2}{|c|}{$\begin{array}{l}\text { f. Proportion of sexually active females aged } 16-20 \text { years enrolled in Medicaid who are screened for } \\
\text { chlamydia infections }\end{array}$} & $51.2 \%$ & $53.9 \%$ & N/A & $62.5 \%$ \\
\hline \multicolumn{2}{|c|}{$\begin{array}{l}\text { g. Proportion of sexually active females aged } 21-24 \text { years enrolled in Medicaid who are screened for } \\
\text { chlamydia infections }\end{array}$} & $60.1 \%$ & $62.2 \%$ & N/A & $66.0 \%$ \\
\hline \multicolumn{2}{|c|}{$\begin{array}{l}\text { h. Proportion of sexually active females aged } 16-20 \text { years enrolled in commercial health insurance plans } \\
\text { who are screened for chlamydia infections }\end{array}$} & $42.4 \%$ & $42.5 \%$ & N/A & $43.5 \%$ \\
\hline \multicolumn{2}{|c|}{$\begin{array}{l}\text { i. Proportion of sexually active females aged } 21-24 \text { years enrolled in commercial health insurance plans } \\
\text { who are screened for chlamydia infections }\end{array}$} & $52.4 \%$ & $53.3 \%$ & N/A & $52.7 \%$ \\
\hline \multicolumn{6}{|c|}{ Goal 2: Elimination of Congenital Syphilis } \\
\hline \multicolumn{2}{|c|}{ a. Incidence of P\&S syphilis/100,000 population in women aged 15-44 } & 3.2 & 4.2 & 5.1 & 0.8 \\
\hline \multicolumn{2}{|c|}{ b. Incidence of congenital syphilis/100,000 live births } & 12.4 & 15.7 & 23.3 & 6.2 \\
\hline \multicolumn{2}{|c|}{ c. Proportion of pregnant women that are screened for syphilis at least one month before delivery } & $84.0 \%$ & N/A & N/A & $87.2 \%$ \\
\hline GPRA Goals & \multicolumn{5}{|l|}{ Data Source } \\
\hline 1 & \multicolumn{5}{|l|}{ National Disease and Therapeutic Index (IMS Health) } \\
\hline $1 \mathrm{a}, 1 \mathrm{~b}$ & \multicolumn{5}{|l|}{ National Job Training Program (NJTP) } \\
\hline $1 c, 1 d, 1 e$ & \multicolumn{5}{|l|}{ National Notifiable Disease Surveillance System (NNDSS), CDC } \\
\hline $1 \mathrm{f}, 1 \mathrm{~g}, 1 \mathrm{~h}, 1 \mathrm{i}$ & \multicolumn{5}{|c|}{ Healthcare Effectiveness Data and Information Set (HEDIS), National Committee for Quality Assurance (NCQA) } \\
\hline $2 a, 2 b$ & \multicolumn{5}{|l|}{ National Notifiable Disease Surveillance System (NNDSS),CDC } \\
\hline $2 c$ & \multicolumn{5}{|l|}{ MarketScan Commercial Claims and Encounters Database, Truven Health Analytics } \\
\hline
\end{tabular}

NOTE: Data presented in this table reflect data reported to GPRA in current and prior years. Data for years prior to 2016 may not match estimates presented in other sections of this report.

* Median state-specific chlamydia prevalence/positivity among states with $>100$ females in this age group entering the National Job Training Program.

${ }^{+}$In FY 2013, CDC improved the calculation of these data to increase the stability of estimates over time. Data for 2010 and later years reflect this improved calculation method.

$\mathrm{N} / \mathrm{A}=$ Not available; GPRA = Government Performance and Results Act; PID = pelvic inflammatory disease; P\&S = primary and secondary. 
This page intentionally left blank. 


\section{STD Surveillance Case Definitions}

\section{C1. Case Definitions For Nationally Notifiable Infectious Diseases}

The Council of State and Territorial Epidemiologists (CSTE) recommends that state health departments report cases of selected diseases to CDC's National Notifiable Diseases Surveillance System (NNDSS). Case definitions are periodically revised using CSTE's Position Statements and provide uniform criteria of nationally notifiable conditions for reporting purposes. The most current surveillance case definitions for nationally notifiable STDs are listed below. Please see the NNDSS website (https://wwwn.cdc.gov/nndss/case-definitions.html) for historical case definitions.

\section{C1.1 Chancroid (Revised 9/96)}

\section{Clinical description}

A sexually transmitted disease characterized by painful genital ulceration and inflammatory inguinal adenopathy. The disease is caused by infection with Haemophilus ducreyi.

\section{Laboratory criteria for diagnosis}

- Isolation of H. ducreyi from a clinical specimen.

\section{Case classification}

Probable: a clinically compatible case with both a) no evidence of Treponema pallidum infection by darkfield microscopic examination of ulcer exudate or by a serologic test for syphilis performed $\geq 7$ days after onset of ulcers and b) either a clinical presentation of the ulcer(s) not typical of disease caused by herpes simplex virus (HSV) or a culture negative for HSV.

Confirmed: a clinically compatible case that is laboratory confirmed.

\section{C1.2 Chlamydia trachomatis Infection (Effective 1/10)}

\section{Clinical description}

Infection with Chlamydia trachomatis may result in urethritis, epididymitis, cervicitis, acute salpingitis, or other syndromes when sexually transmitted; however, the infection is often asymptomatic in women. Perinatal infections may result in inclusion conjunctivitis and pneumonia in newborns. Other syndromes caused by C. trachomatis include lymphogranuloma venereum (see Lymphogranuloma Venereum) and trachoma.

\section{Laboratory criteria for diagnosis}

- Isolation of C. trachomatis by culture or

- Demonstration of C. trachomatis in a clinical specimen by detection of antigen or nucleic acid

\section{Case classification}

Confirmed: a case that is laboratory confirmed. 


\section{C1.3 Gonorrhea (Effective 1/14)}

\section{Clinical description}

A sexually transmitted infection commonly manifested by urethritis, cervicitis, proctitis, salpingitis, or pharyngitis. Infection may be asymptomatic.

\section{Laboratory criteria for diagnosis}

- Observation of gram-negative intracellular diplococci in a urethral smear obtained from a male or an endocervical smear obtained from a female, or

- Isolation of typical gram-negative, oxidase-positive diplococci by culture (presumptive Neisseria gonorrhoeae) from a clinical specimen, or

- Demonstration of N. gonorrhoeae in a clinical specimen by detection of antigen or nucleic acid

\section{Case classification}

Probable: demonstration of gram-negative intracellular diplococci in a urethral smear obtained from a male or an endocervical smear obtained from a female.

Confirmed: a person with laboratory isolation of typical gram-negative, oxidase-positive diplococci by culture (presumptive $N$. gonorrhoeae) from a clinical specimen, or demonstration of $N$. gonorrhoeae in a clinical specimen by detection of antigen or detection of nucleic acid via nucleic acid amplification (e.g., polymerase chain reaction [PCR]) or hybridization with a nucleic acid probe.

\section{C1.4 Syphilis (Effective 1/14)}

Syphilis is a complex sexually transmitted disease that has a highly variable clinical course. Adherence to the following surveillance case definitions will facilitate understanding the epidemiology of this disease across the US.

\section{Syphilis, primarv (Effective 1/14)}

\section{Clinical description}

A stage of infection with Treponema pallidum characterized by one or more ulcerative lesions (e.g. chancre), which might differ considerably in clinical appearance.

\section{Laboratory criteria for diagnosis}

Demonstration of T. pallidum in clinical specimens by darkfield microscopy, or by PCR or equivalent direct molecular methods.

\section{Case classification}

Probable: a case that meets the clinical description of primary syphilis with a reactive serologic test (nontreponemal: Venereal Disease Research Laboratory [VDRL], rapid plasma reagin [RPR], or equivalent serologic methods; treponemal: fluorescent treponemal antibody absorbed [FTA-ABS], T. pallidum particle agglutination [TP-PA], enzyme immunoassay [EIA], chemiluminescence immunoassay [CIA], or equivalent serologic methods). These treponemal tests supersede older testing technologies, including microhemagglutination assay for antibody to T. pallidum [MHA-TP].

Confirmed: a case that meets the clinical description of primary syphilis that is laboratory confirmed. 


\section{Syphilis, secondary (Effective 1/14)}

\section{Clinical description}

A stage of infection caused by T. pallidum characterized by localized or diffuse mucocutaneous lesions (e.g., rash such as non-pruritic macular, maculopapular, popular, or pustular lesions), often with generalized lymphadenopathy. Other symptoms can include mucous patches, condyloma lata, and alopecia. The primary ulcerative lesion may still be present. Because of the wide array of symptoms possibly indicating secondary syphilis, serologic tests for syphilis and a thorough sexual history and physical examination are crucial to determining if a case should be classified as secondary syphilis.

\section{Laboratory criteria for diagnosis}

Demonstration of T. pallidum in clinical specimens by darkfield microscopy, or by PCR or equivalent direct molecular methods.

\section{Case classification}

Probable: a case that meets the clinical description of secondary syphilis with a nontreponemal (VDRL, RPR, or equivalent serologic methods) titer $\geq 4$ and a reactive treponemal test (FTA-ABS, TP-PA, EIA, CIA, or equivalent serologic methods).

Confirmed: a case that meets the clinical description of secondary syphilis (with at least one sign or symptom) that is laboratory confirmed.

\section{Syphilis, early latent (Effective 1/14)}

\section{Clinical description}

A subcategory of latent syphilis (a stage of infection caused by T. pallidum in which organisms persist in the body of the infected person without causing symptoms or signs) when initial infection has occurred within the previous 12 months.

\section{Case classification}

Probable: A person with no clinical signs or symptoms of syphilis who has one of the following:

- No past diagnosis of syphilis, and a reactive nontreponemal test (e.g., VDRL, RPR, or equivalent serologic methods), and a reactive treponemal test (e.g., FTA-ABS, TP-PA, EIA, CIA, or equivalent serologic methods), or

- A current nontreponemal test titer demonstrating fourfold or greater increase from the last nontreponemal test titer

AND evidence of having acquired the infection within the previous 12 months based on one or more of the following criteria:

- Documented seroconversion or fourfold or greater increase in titer of a nontreponemal test during the previous 12 months

- Documented seroconversion of a treponemal test during the previous 12 months

- A history of symptoms consistent with primary or secondary syphilis during the previous 12 months

- A history of sexual exposure to a partner within the previous 12 months who had primary, secondary, or early latent syphilis (documented independently as duration $<12$ months)

- Only sexual contact was within the last 12 months (sexual debut).

There is no confirmed case classification for early latent syphilis. 


\section{Clinical description}

A subcategory of latent syphilis (a stage of infection caused by T. pallidum in which organisms persist in the body of the infected person without causing symptoms or signs) when initial infection has occurred $>12$ months previously.

\section{Case classification}

Probable: a person with no clinical signs or symptoms of syphilis who has one of the following:

- No past diagnosis of syphilis, and a reactive nontreponemal test (e.g., VDRL, RPR, or equivalent serologic methods), and a reactive treponemal test (e.g., FTA-ABS, TP-PA, EIA, CIA, or equivalent serologic methods), or

- A past history of syphilis therapy and a current nontreponemal test titer demonstrating fourfold or greater increase from the last nontreponemal test titer.

AND who has no evidence of having acquired the disease within the preceding 12 months (see Syphilis, early latent).

There is no confirmed case classification for late latent syphilis.

\section{Neurosyphilis (Effective 1/14)}

Neurosyphilis can occur at any stage of syphilis. If the patient has neurologic manifestations of syphilis, the case should be reported with the appropriate stage of infection (as if neurologic manifestations were not present) and neurologic manifestations should be noted in the case report data. If no other stage is appropriate, the case should be staged as "late, with clinical manifestations".

Neurosyphilis can apply to all stages of infection of syphilis listed, including: primary syphilis, secondary syphilis, early latent syphilis, late latent syphilis, and late syphilis with clinical manifestations.

\section{Clinical description}

Infection of the central nervous system with T. pallidum, as evidenced by manifestations including syphilitic meningitis, meningovascular syphilis, optical involvement including interstitial keratitis and uveitis, general paresis, including dementia, and tabes dorsalis.

\section{Laboratory criteria for diagnosis}

- A reactive VDRL in cerebrospinal fluid (CSF) and either (1) a reactive treponemal serologic test for syphilis (e.g., FTA-ABS, TP-PA, EIA, CIA, or equivalent serologic methods) or (2) a reactive nontreponemal serologic test for syphilis (VDRL, RPR, or equivalent serologic method).

\section{Case classification}

Probable: syphilis of any stage with a negative VDRL test in CSF specimen and either (1) a reactive treponemal serologic test for syphilis (e.g., FTA-ABS, TP-PA, EIA, CIA, or equivalent serologic methods) or (2) a reactive nontreponemal serologic test for syphilis (VDRL, RPR, or equivalent serologic method), and both of the following:

- Elevated CSF protein ( $>50 \mathrm{mg} / \mathrm{dL} 2)$ or leukocyte count ( $>5$ white blood cells/cubic millimeter CSF) in the absence of other known causes of these abnormalities, and

- Clinical symptoms or signs consistent with neurosyphilis without other known causes for these clinical abnormalities.

Confirmed: syphilis of any stage that meets the laboratory criteria for neurosyphilis. 


\section{Clinical description}

Clinical manifestations of late syphilis may include inflammatory lesions of the cardiovascular system (e.g., aortitis, coronary vessel disease), skin (e.g., gummatous lesions) bone (e.g., osteitis) or other tissue. Rarely, other structures (e.g., the upper and lower respiratory tracts, mouth, eye, abdominal organs, reproductive organs, lymph nodes, and skeletal muscle) may be involved. Late syphilis usually becomes clinically manifest only after a period of 15-30 years of untreated infection. If only neurologic manifestations of syphilis (e.g., tabes dorsalis, dementia) are present and infection occurred more than 12 months ago, the case should be reported as "late syphilis".

\section{Laboratory criteria for diagnosis}

Demonstration of T. pallidum in late lesions by special stains (although organisms are rarely visualized in late lesions), or equivalent methods, or by PCR or equivalent direct molecular methods.

\section{Case classification}

Probable: characteristic abnormalities or lesions of the cardiovascular system (e.g., aortitis, coronary vessel disease), skin (e.g., gummatous lesions), bone (e.g., osteitis), or other tissue and a reactive treponemal test (e.g., FTA-ABS, TP-PA, EIA, CIA, or equivalent serologic methods), in the absence of other known causes of these abnormalities. CSF abnormalities and clinical symptoms or signs consistent with neurologic manifestations of syphilis might be present.

Confirmed: a case that meets the clinical description of late syphilis that is laboratory confirmed.

\section{Syphilis, Conqenital (Effective 1/15)}

\section{Clinical description}

A condition caused by infection in utero with T. pallidum. A wide spectrum of severity exists, from inapparent infection to severe cases that are clinically apparent at birth. An infant or child (aged less than 2 years) may have signs such as hepatosplenomegaly, rash, condyloma lata, snuffles, jaundice (nonviral hepatitis), pseudoparalysis, anemia, or edema (nephrotic syndrome and/or malnutrition). An older child may have stigmata (e.g., interstitial keratitis, nerve deafness, anterior bowing of shins, frontal bossing, mulberry molars, Hutchinson teeth, saddle nose, rhagades, or Clutton joints).

\section{Laboratory criteria for diagnosis}

- Demonstration of T. pallidum by darkfield microscopy of lesions, body fluids, or neonatal nasal discharge, or

- PCR or other equivalent direct molecular methods of lesions, placenta, umbilical cord, or autopsy material, or

- Immunohistochemistry (IHC), or special stains (e.g., silver staining) of specimens from lesions, neonatal nasal discharge, placenta, umbilical cord, or autopsy material.

\section{Case classification}

Probable: a condition affecting an infant whose mother had untreated or inadequately treated* syphilis at delivery, regardless of signs in the infant, or an infant or child who has a reactive non-treponemal test for syphilis (VDRL, RPR, or equivalent serologic methods) AND any one of the following:

- Any evidence of congenital syphilis on physical examination (see Clinical description)

- Any evidence of congenital syphilis on radiographs of long bones

- A reactive CSF VDRL test

- In a nontraumatic lumbar puncture, an elevated CSF leukocyte (white blood cell [WBC]) count or protein (without other cause):

\footnotetext{
* Adequate treatment is defined as completion of a penicillin-based regimen, in accordance with CDC treatment guidelines, appropriate for stage of infection,
} initiated 30 or more days before delivery. 
Suggested parameters for abnormal CSF WBC and protein values:

- During the first 30 days of life, a CSF WBC count of $>15 \mathrm{WBC} / \mathrm{mm} 3$ or a CSF protein $>120 \mathrm{mg} / \mathrm{dL}$.

- After the first 30 days of life, a CSF WBC count of $>5 \mathrm{WBC} \mathrm{mm} 3$ or a CSF protein $>40 \mathrm{mg} / \mathrm{dL}$, regardless of CSF serology.

- The treating clinician should be consulted to interpret the CSF values for the specific patient.

Confirmed: a case that is laboratory confirmed.

\section{Syphilitic Stillbirth}

\section{Clinical case definition}

A fetal death that occurs after a 20 -week gestation or in which the fetus weighs greater than $500 \mathrm{~g}$ and the mother had untreated or inadequately treated* syphilis at delivery.

\section{Comment}

Congenital and acquired syphilis may be difficult to distinguish when a child is seropositive after infancy. Signs of congenital syphilis may not be obvious, and stigmata may not yet have developed. Abnormal values for CSF VDRL, WBC cell count, and protein may be found in either congenital or acquired syphilis. Findings on radiographs of long bones may help because radiographic changes in the metaphysis and epiphysis are considered classic signs of congenitally acquired syphilis. While maternal antibodies can complicate interpretation of serologic tests in an infant, reactive tests past 18 months of age are considered to reflect the status of the child. The decision may ultimately be based on maternal history and clinical judgment. In a young child, the possibility of sexual abuse should be considered as a cause of acquired rather than congenital syphilis, depending on the clinical picture. For reporting purposes, congenital syphilis includes cases of congenitally acquired syphilis among infants and children as well as syphilitic stillbirths.

\footnotetext{
* Adequate treatment is defined as completion of a penicillin-based regimen, in accordance with CDC treatment guidelines, appropriate for stage of infection, initiated 30 or more days before delivery.
}

\section{C2. Case Definitions For Non-Notifiable Infectious Diseases}

Although the conditions below are not currently nationally notifiable, they may be reportable in some jurisdictions. To provide uniform criteria for those jurisdictions, case definitions are provided by CSTE. Case definitions are periodically revised. The most current surveillance case definitions for non-notifiable STDs are listed below. Please see the NNDSS website (https://wwwn.cdc.gov/nndss/case-definitions.html) for historical case definitions.

\section{C2.1 Genital Herpes (Herpes Simplex Virus) (Revised 9/96)}

\section{Clinical description}

A condition characterized by visible, painful genital or anal lesions.

\section{Laboratory criteria for diagnosis}

- Isolation of herpes simplex virus from cervix, urethra, or anogenital lesion, or

- Demonstration of virus by antigen detection technique in clinical specimens from cervix, urethra, or anogenital lesion, or

- Demonstration of multinucleated giant cells on a Tzanck smear of scrapings from an anogenital lesion. 


\section{Case classification}

Probable: a clinically compatible case (in which primary and secondary syphilis have been excluded by appropriate serologic tests and darkfield microscopy, when available) with either a diagnosis of genital herpes based on clinical presentation (without laboratory confirmation) or a history of one or more previous episodes of similar genital lesions.

Confirmed: a clinically compatible case that is laboratory confirmed.

\section{Comment}

Genital herpes should be reported only once per patient. The first diagnosis for a patient with no previous diagnosis should be reported.

\section{C2.2 Genital Warts (Revised 9/96)}

\section{Clinical description}

An infection characterized by the presence of visible, exophytic (raised) growths on the internal or external genitalia, perineum, or perianal region.

\section{Laboratory criteria for diagnosis}

- Histopathologic changes characteristic of human papillomavirus infection in specimens obtained by biopsy or exfoliative cytology or

- Demonstration of virus by antigen or nucleic acid detection in a lesion biopsy.

\section{Case classification}

Probable: a clinically compatible case without histopathologic diagnosis and without microscopic or serologic evidence that the growth is the result of secondary syphilis.

Confirmed: a clinically compatible case that is laboratory confirmed.

\section{Comment}

Genital warts should be reported only once per patient. The first diagnosis for a patient with no previous diagnosis should be reported.

\section{C2.3 Granuloma Inguinale}

\section{Clinical description}

A slowly progressive ulcerative disease of the skin and lymphatics of the genital and perianal area caused by infection with Calymmatobacterium granulomatis. A clinically compatible case would have one or more painless or minimally painful granulomatous lesions in the anogenital area.

\section{Laboratory criteria for diagnosis}

Demonstration of intracytoplasmic Donovan bodies in Wright or Giemsa-stained smears or biopsies of granulation tissue.

\section{Case classification}

Confirmed: a clinically compatible case that is laboratory confirmed. 


\section{C2.4 Lymphogranuloma Venereum}

\section{Clinical description}

Infection with L1, L2, or, L3 serovars of C. trachomatis may result in a disease characterized by genital lesions, suppurative regional lymphadenopathy, or hemorrhagic proctitis. The infection is usually sexually transmitted.

\section{Laboratory criteria for diagnosis}

Isolation of C. trachomatis, serotype L1, L2, or L3 from clinical specimen, or

- Demonstration by immunofluorescence of inclusion bodies in leukocytes of an inguinal lymph node (bubo) aspirate, or

- Positive microimmunofluorescent serologic test for a lymphogranuloma venereum strain of C. trachomatis.

\section{Case classification}

Probable: a clinically compatible case with one or more tender fluctuant inguinal lymph nodes or characteristic proctogenital lesions with supportive laboratory findings of a single C. trachomatis complement fixation titer of $>64$.

Confirmed: a clinically compatible case that is laboratory confirmed.

\section{C2.5 Mucopurulent Cervicitis (Revised 9/96)}

\section{Clinical description}

Cervical inflammation that is not the result of infection with $N$. gonorrhoeae or Trichomonas vaginalis. Cervical inflammation is defined by the presence of one of the following criteria:

- Mucopurulent secretion (from the endocervix) that is yellow or green when viewed on a white, cotton-tipped swab (positive swab test)

- Induced endocervical bleeding (bleeding when the first swab is placed in the endocervix).

\section{Laboratory criteria for diagnosis}

No evidence of $N$. gonorrhoeae by culture, Gram stain, or antigen or nucleic acid detection, and no evidence of $T$. vaginalis on wet mount.

\section{Case classification}

Confirmed: a clinically compatible case in a female who does not have either gonorrhea or trichomoniasis.

\section{Comment}

Mucopurulent cervicitis (MPC) is a clinical diagnosis of exclusion. The syndrome may result from infection with any of several agents (see C. trachomatis). If gonorrhea, trichomoniasis, and chlamydia are excluded, a clinically compatible illness should be classified as MPC. An illness in a female that meets the case definition of MPC and C. trachomatis infection should be classified as chlamydia. 


\section{C2.6 Nongonococcal Urethritis (Revised 9/96)}

\section{Clinical description}

Urethral inflammation that is not the result of infection with $N$. gonorrhoeae. Urethral inflammation may be diagnosed by the presence of one of the following criteria:

- A visible abnormal urethral discharge, or

- A positive leukocyte esterase test from a male aged $<60$ years who does not have a history of kidney disease or bladder infection, prostate enlargement, urogenital anatomic anomaly, or recent urinary tract instrumentation, or

- Microscopic evidence of urethritis ( $\geq 5$ white blood cells per high-power field) on a Gram stain of a urethral smear.

\section{Laboratory criteria for diagnosis}

No evidence of $N$. gonorrhoeae infection by culture, Gram stain, or antigen or nucleic acid detection.

\section{Case classification}

Confirmed: a clinically compatible case in a male in whom gonorrhea is not found, either by culture, Gram stain, or antigen or nucleic acid detection.

\section{Comment}

Nongonococcal urethritis (NGU) is a clinical diagnosis of exclusion. The syndrome may result from infection with any of several agents (see C. trachomatis). If gonorrhea and chlamydia are excluded, a clinically compatible illness should be classified as NGU. An illness in a male that meets the case definition of NGU and C. trachomatis infection should be classified as chlamydia.

\section{C2.7 Pelvic Inflammatory Disease (Revised 9/96)}

\section{Clinical case definition}

A clinical syndrome resulting from the ascending spread of microorganisms from the vagina and endocervix to the endometrium, fallopian tubes, and/or contiguous structures. In a female who has lower abdominal pain and who has not been diagnosed as having an established cause other than pelvic inflammatory disease (PID) (e.g., ectopic pregnancy, acute appendicitis, and functional pain), all the following clinical criteria must be present:

- Lower abdominal tenderness, and

- Tenderness with motion of the cervix, and

- Adnexal tenderness.

In addition to the preceding criteria, at least one of the following findings must also be present:

- Meets the surveillance case definition of C. trachomatis infection or gonorrhea

- Temperature $>100.4 \mathrm{~F}(>38.0 \mathrm{C})$

- Leukocytosis $>10,000 \mathrm{WBC} / \mathrm{mm} 3$

- Purulent material in the peritoneal cavity obtained by culdocentesis or laparoscopy

- Pelvic abscess or inflammatory complex detected by bimanual examination or by sonography

- Patient is a sexual contact of a person known to have gonorrhea, chlamydia, or nongonococcal urethritis.

\section{Case classification}

Confirmed: a case that meets the clinical case definition.

\section{Comment}

For reporting purposes, a clinician's report of PID should be counted as a case. 
$1.42 x-1=11$

cose 1110

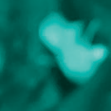

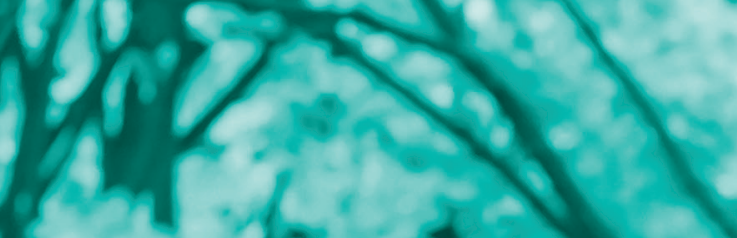

\section{$y_{3} x^{2}=3$}

24 - 19 bx ans. $3 x-35=42 \times 5$ $4 x^{2}+2$ in 


\section{Contributors}

We gratefully acknowledge the contributions of state STD project directors, STD program managers, state and territorial epidemiologists, and laboratory directors. The persons listed were in the positions shown as of July 9, 2018.

\begin{tabular}{|c|c|c|c|c|}
\hline State/City/Outlying Area & STD Project Directors & STD Program Managers & State Epidemiologists & Laboratory Directors \\
\hline Alabama & Harrison Wallace & Thomas Lee & Sherri L. Davidson & Sharon Massingale \\
\hline Alaska & Susan Jones & Tracy Smith & Joe McLaughlin & Bernard Jilly \\
\hline Arizona & Rebecca Scranton & Rebecca Scranton & Kenneth Komatsu & Victor Waddell \\
\hline Arkansas & Rene Montgomery & Brandi Roberts & Dirk Haselow & Glen Baker \\
\hline California & Heidi Bauer & Romni Neiman & Gil F. Chavez & Paul Kimsey \\
\hline Los Angeles & Mario Perez & Leo Moore & Gil F. Chavez & Nicole Green \\
\hline San Francisco & Susan Philip & Trang Nguyen & Gil F. Chavez & Godfred Masinde \\
\hline Colorado & Daniel Shodell & Yesenia Mendez & Rachel K. Herlihy & Hugh Maguire \\
\hline Connecticut & Lynn Sosa & Heidi Jenkins & Matthew Cartter & Jafar Razeq \\
\hline Delaware & Catherine Mosley & Catherine Mosley & Tabatha Offutt-Powell & Sergio Huerta \\
\hline District of Columbia & Michael Kharfen & Adam Visconti & John Davies-Cole & Anthony Tran \\
\hline Florida & Craig Wilson & Lisa Thompson & Carina Blackmore & Carina Blackmore \\
\hline Georgia & LaTasha Terry & Mildred Banks & Cherie Drenzek & Elizabeth Franko \\
\hline Hawaii & Peter Whiticar & Gerald “Luke”" Hasty, Jr. & Sarah Park & A. Christian Whelen \\
\hline Idaho & Aimee Shipman & Kimberly Matulonis & Christine Hahn & Christopher Ball \\
\hline Illinois & Danny Brikshavana & Danny Brikshavana & Jennifer Layden & Matt Charles \\
\hline Chicago & Irina, Tabidze & Irina, Tabidze & Jennifer Layden & Massimo Pacilli (Lab Liaison) \\
\hline Indiana & Caitlin Conrad & Caitlin Conrad & Pamela Pontones & Judith Lovchik \\
\hline lowa & Randy Mayer & George Walton & Patricia Quinlisk & Michael Pentella \\
\hline Kansas & Jennifer VandeVelde & Jennifer VandeVelde & Farah Ahmed & N. Myron Gunsalus \\
\hline Kentucky & Robert Brawley & Chang Lee & Douglas Thoroughman & Jeremy Hart \\
\hline Louisiana & DeAnn Gruber & Chaquetta Johnson & Raoult Ratard & Stephen Martin \\
\hline Maine & Jayson Hunt & Emer Smith & Siiri Bennett & Kenneth Pote \\
\hline Maryland & Kenneth Ruby & Marcia Pearlowitz & David Blythe & Robert Myers \\
\hline Baltimore & Adena Greenbaum & Arielle Juberg & David Blythe & $\mathrm{N} / \mathrm{A}$ \\
\hline Massachusetts & Kathleen Roosevelt & David Goudreau & Catherine Brown & Sandra Smole \\
\hline Michigan & Kathryn Macomber & Kristine Judd-Tuinier & Sarah Lyon-Callo & Sandip Shah \\
\hline Minnesota & Krissie Guerard & Krissie Guerard & Ruth Lynfield & Joanne Bartkus \\
\hline Mississippi & James Stewart & David Peyton & Paul Byers & Daphne Ware \\
\hline Missouri & Nicole Massey & Craig Highfill & George Turabelidze & Bill Whitmar \\
\hline Montana & Cara Murolo & Cara Murolo & Laura Williamson & Ron Paul \\
\hline Nebraska & Jeri Weberg-Bryce & Jeri Weberg-Bryce & Tom Safranek & Peter Iwen \\
\hline Nevada & Elizabeth Kessler & Brian Parrish & Sandra Larson/ Melissa Bullock & Stephanie Van Hooser \\
\hline New Hampshire & Lisa Morris & Lindsay Pierce & Benjamin Chan & Christine Bean \\
\hline New Jersey & Greta Anschuetz & Greta Anschuetz & Christina Tan & Christopher Rinn \\
\hline New Mexico & Andrew Gans & Janine Waters & Michael Landen & Lixia Liu \\
\hline New York & Travis O'Donnell & Margaret Carroll & Debra Blog & Jill Taylor \\
\hline New York City & Susan Blank & Kate Washburn & Debra Blog & Jennifer Rakeman \\
\hline North Carolina & Jacquelyn Clymore & Roger Follas & Zack Moore & Scott Zimmerman \\
\hline North Dakota & Lindsey VanderBusch & Lindsey VanderBusch & Tracy Miller & Jim Quarnstrom \\
\hline Ohio & Laurie Rickert & Laurie Rickert & Sietske de Fijter & Quanta Brown (Acting) \\
\hline Oklahoma & Jan Fox & Kristen Eberly & Kristy Bradley & Samuel T. Dunn \\
\hline Oregon & Annick Benson-Scott & Joshua Ferrer & Katrina Hedberg & John Fontana \\
\hline Pennsylvania & Beth Butler & Kristine King & Sharon Watkins & Dongxiang Xia \\
\hline Philadelphia & Caroline Carlson Johnson & Cherie Walker-Baban & Sharon Watkins & Kerry Buchs \\
\hline
\end{tabular}




\begin{tabular}{|c|c|c|c|c|}
\hline State/City/Outlying Area & STD Project Directors & STD Program Managers & State Epidemiologists & Laboratory Directors \\
\hline Rhode Island & Thomas Bertrand & Valentina Adamova & Utpala Bandy & Ewa King \\
\hline South Carolina & Terri Stephens & Bernard Gilliard & Linda Bell & Robert Brent Dixon \\
\hline South Dakota & Colleen Winter & Amanda Gill & Joshua Clayton & Timothy Southern \\
\hline Tennessee & Carolyn Wester & Leo Parker & Tim Jones & Richard Steece \\
\hline Texas & Shelley Lucas & Tammy Foskey & Linda Gaul & Grace Kubin \\
\hline Utah & Amelia Self & Erin Fratto & Angela Dunn & Robyn Atkinson-Dunn \\
\hline Vermont & Daniel Daltry & Daniel Daltry & Patsy Kelso/Lori Cragin & Mary Celotti \\
\hline Virginia & Diana Prat & Oana Vasiliu & Laurie Forlano & Denise Toney \\
\hline Washington & Elisabeth Crutsinger-Perry & Emalie Huriaux & Scott Lindquist/Cathy Wasserman & Romesh Gautom \\
\hline West Virginia & Loretta Haddy & Pamela Reynolds & Loretta Haddy & Sharon Lee Cibrik \\
\hline Wisconsin & Stephanie Smiley & Anthony Wade & Jon Meiman & James Schauer \\
\hline Wyoming & Debi Anderson & Brittany Wardle & Alexia Harrist & Sarah Buss \\
\hline American Samoa & Fara M. Utu & Fetaui V. Saelua & Scott Anesi & June Vaifanua-Leo \\
\hline Federated States of Micronesia & Mayleen Ekiek & Mayleen Ekiek & Magdalena Walter & $\begin{array}{l}\text { Maria Marfel, Kasian } \\
\text { Otoko, Maopa } \\
\text { Raikabula, William } \\
\text { Nena }\end{array}$ \\
\hline Government of the Marshall Islands & Mailynn K. Lang & Adela Sibok & N/A & Paul Lalita \\
\hline Northern Marianas (CNMI) & John Dax Moreno & John Dax Moreno & Paul White & Philip Dauterman \\
\hline Guam & Josephine O'Mallan & Bernadette P. Schumann & Josephine O'Mallan & Josephine O'Mallan \\
\hline Puerto Rico & Greduvel Duran Guzman & Javier Vazquez Melendez & Carmen Deseda & Gonzalo Gonzalez \\
\hline Republic of Palau & Sherilynn Madraisau & Columbo Sakuma & Tmong Cheryl Udui & Clarette Matlab \\
\hline Virgin Islands & Vanessa Farrell & Jasper Lettsome & Esther Ellis & Joseph Mark \\
\hline
\end{tabular}


This page intentionally left blank. 


MEDIA MAIL
POSTAGE \& FEES PAID
CDC
Permit No. G-284

U.S. Department of Health \& Human Services Centers for Disease Control and Prevention, MS US12-2 Atlanta, Georgia 30329-4027

OFFICIAL BUSINESS Penalty for Private Use $\$ 300$ 